Wolfgang Eckstein

Reflexion (Rückstreuung)

Reflection (Backscattering)

IPP 17/12

August, 2009 



\title{
Reflection (Backscattering)
}

\author{
W.Eckstein \\ Max-Planck-Institut für Plasmaphysik \\ Boltzmannstr.2, D-85748 Garching \\ wolfgang.eckstein@ipp.mpg.de
}

August 13, 2009

\section{Abstract}

This report gives an overview of available calculated and measured reflection coefficients. The energy and angular dependence of the particle and energy reflection coefficients are fitted by simple formulas for a large number of elemental targets and light (hydrogen and helium) and heavy (mainly noble gases and selfbombardment) bombarding atoms. The incident energy covers a range from 10 to $10^{5} \mathrm{eV}$, in some cases to $\mathrm{MeV}$ energies. Calculated values of the reflection coefficients are compared with experimental data from the literature. 


\section{Introduction}

Scattering always occurs if two (moving) atoms come close enough. In many cases one atom is at rest, and the moving atom is scattered in a forward direction if the moving atom has a larger mass than the atom at rest. If the moving atom has a lower mass, then the moving atom can be scattered forward and backward. This backscattering is the basic process in analytic tools as Ion Surface Scattering (ISS) and Rutherford Backscattering (RBS). But an energetic ion or atom can be reflected also by multiple collisions even if its mass is larger than the mass of atoms in the solid. The energetic atom looses energy by nuclear and electronic collisions so that they can escape the solid with different energies and angles.

Summing up all escaping atoms with different emission energies and angles and charge states provides the particle (number) reflection coefficient, $R_{N}\left(E_{0}, \alpha\right)$, which is dependent on the ion energy, $E_{0}$, and on the angle of incidence, $\alpha$. Integration over the emission energies and angles of all reflected atoms give the energy reflection coefficient, $R_{E}\left(E_{0}, \alpha\right)$.

$$
R_{E}=\frac{1}{E_{0}} \int_{0}^{E_{0}} \int_{0}^{\pi / 2} \int_{0}^{2 \pi} f(E, \beta, \phi) d \phi d \beta d E
$$

where $f(E, \beta, \phi)$ is the energy/angular distribution of reflected atoms, which is dependent on the emission energy, $\mathrm{E}$, the polar emission angle, $\beta$, and the azimuthal emission angle, $\phi$. The mean energy, $\left\langle E\left(E_{0}, \alpha\right)\right\rangle$, of reflected atoms can be determined by

$$
<E\left(E_{0}, \alpha\right)>=E_{0} \frac{R_{E}}{R_{N}}
$$

The reflection plays an important role in fusion plasma devices, because reflection of hydrogen from the vessel walls contributes to the recycling of hydrogen and the plasma density in the device. The interest from this field triggered a number of surveys about reflection coefficients $[1,2,3,4,5,6,7,8]$ mainly for the light ions, hydrogen and helium and some selfbombardment cases, but up to

now a systematic comparison of experimental and calculated values for heavier ions does not exist. A general survey about backscattering and reflection is given in [9].

\section{Experimental Methods}

\subsection{Particle Reflection Coefficient}

The first method to measure the particle reflection coefficient was a radioactive tracer method [10]. The reflected radioactive atoms are collected on a catcher, and then the collected amount in the catcher and in the target is measured by the radiation of this species. A possible error is the sticking of the species on the catcher. 
A similar method is to measure the amount of an implanted species by a nuclear reaction. Special cases are the implantation of $\mathrm{D}$ or ${ }^{3} \mathrm{He}$ in materials [11]. The particle reflection coefficient is unity minus the implanted amount normalized to the incident fluence.

Another possibility is the measurement of the energy distribution of reflected atoms under a specific emission angle, usually at $45^{\circ}$ [12]. It is assumed, that the angular distribution is a cosine distribution (for easy integration) for normal incidence. A further complication is that at low energies the reflected species are neutral (about $90 \%$ at $1 \mathrm{keV}$ ). A stripping cell is used to ionize the neutral hydrogen or helium for energy analysis. This method breaks down below about $200 \mathrm{eV}$, so that the integration of the energy distribution may leed to a too small value of $R_{N}$ because of the neglect of low backscattered energies energies, which becomes especially severe at low incident energies. Another problem may be oxidation of the surface leeding to smaller values of $R_{N}$ due to a smaller scattering of oxygen compared with heavier target atoms.

A field ion microscope (FIM) has also been applied to determine the particle reflection coefficient [13]. The implanted amount is measured by successive removal from the tip. This method is limited to some materials, but has the advantage of using a clean surface.

\subsection{Energy Reflection Coefficient}

The energy reflection coefficient, $R_{E}$, can be determined by the energy deposited into the target by comparing the incident energy (beam intensity times beam current) and the energy left in the target by a caloric method for example [14]. The main error of this method is the energy carried away by secondary electrons, which is dependent on the beam energy and its angle of incidence on the target, and also on the target surface conditions as oxide layers and surface roughness. A further complication is the energy transport of sputtered atoms, the sputtered energy coefficient, $Y_{E}$. Therefore, the measurement of the energy deposition into the target determines $R_{E}+Y_{E}$. For light incident ions as $\mathrm{H}$ the energy reflection coefficient is (much) larger than the sputtered energy coefficient, for heavy ions the opposite is true. The influence of $R_{E}$ and $Y_{E}$ on the mass ratio and their angular dependence was discussed theoretically in [15].

Another method is the integration of the energy distribution of backscattered atoms $[12,16]$, for example in the same way as described for the determination

of the particle reflection coefficient. Also the same possible errors as mentioned above occur.

It should be mentioned, that for the determination of the reflection coefficients from elements the incident ion dose should be small to avoid errors due the implanted species which will change reflection and sputtering. 


\section{Calculational Methods}

The calculated results reported in this review are determined with a Monte Carlo program based on the binary collision model [5]. The programs used are TRIM (Transport of Ions in Matter) [17], TRIM.SP [5, 18] and the newest version SDTrimSP [19]. The most important input for the program is the interaction potential. In the programs mentioned, mostly the WHB $(\mathrm{KrC})$ potential [20] is applied; but other potentials such as the ZBL [21] and Moliere [22] potentials are also used sometimes. All these potentials are purely repulsive and only dependent on the interatomic distance. For Si a special potential with an attractive part has been used in a few cases [23]. Reflection coefficients determined with different potentials agree usually better than a factor of two. Another important input is the electronic (inelastic) energy loss. Here, an equipartition of the local Oen-Robinson model [24] and the nonlocal velocity proportional loss $[25,26]$ is applied at low energies for hydrogen, helium, and for heavy ions in the whole energy range ( 1 to $10^{5} \mathrm{eV}$ ) investigated. At high energies the formulae of Ziegler [27] for helium and the formulae of Andersen and Ziegler [28] for hydrogen are used. Other input parameters for the calculations [5] are the surface binding energy of target atoms, $E_{s b}$, which is only important in reflection for selfbombardment, the binding energy of projectiles, $E_{s p}$, to the target surface (about $1 \mathrm{eV}$ ), which is only of importance for hydrogen and nitrogen (for most elements but not for $\mathrm{Cu}, \mathrm{Ag}, \mathrm{Au}$ ), and finally a cutoff energy of projectiles of $0.5 \mathrm{eV}$. Most reflection coefficient values calculated with TRIM, TRIM.SP and SDTrimSP are documented in $[8,29,30]$, which implies that all calculated values are only valid for zero fluence (static case) or in other words, the influence of implanted species are not taken into account. The absolute error depends on the applied interaction potential and on the inelastic energy loss model; it should be smaller than a factor ot two, in most cases better than $30 \%$. The calculational error is usually better than a few percent, in many cases better than $1 \%$. References $[29,30]$ contain more but not enough calculated values to make a good fit; these values do not appear in this report. For other calculational approaches see [5].

The implantation of bombarding species in the target and the resulting change of the reflection coefficients with fluence can be treated by a dynamical program like TRIDYN [31] or in the dynamic mode of SDTrimSP [19]. Examples are reported for example in $[32,33]$.

Theoretical descriptions for the energy dependence of the reflection coefficients can be found in [34] and of the particle reflection coefficient in [35]. The angular dependence of both reflection coefficients has been treated in [36].

\section{$5 \quad$ Fitting}

Fitting of experimental and calculated reflection coefficients has been done at the IAEA for the purpose of data needs for fusion $[6,7]$. The adopted fitting formulae asked for at least six fitting parameters. With the new amount of 
calculated values new fitting formulae with less fitting parameters are proposed.

The following empirical formula applies to selfbombardment:

$$
R_{N}\left(R_{E}\right)=\frac{\exp \left(a_{1} \varepsilon^{a_{2}}\right)}{1+\exp \left(a_{3} \varepsilon^{a_{4}}\right)}
$$

with

$$
\begin{gathered}
\varepsilon=E_{0} / \varepsilon_{L} \quad, \quad \varepsilon_{L}=\frac{M_{1}+M_{2}}{M_{2}} \frac{Z_{1} Z_{2} e^{2}}{a_{L}} \\
a_{L}=\left(\frac{9 \pi^{2}}{128}\right)^{1 / 3} a_{B}\left(Z_{1}^{2 / 3}+Z_{2}^{2 / 3}\right)^{-1 / 2} \quad, \quad a_{B}=0.0529177 \mathrm{~nm}
\end{gathered}
$$

where $E_{0}$ is the energy (eV) of the incident ion (neutral), $Z_{1}, Z_{2}$ and $M_{1}, M_{2}$ are the atomic numbers and masses of the ion and target atom, respectively. $a_{L}$ is the Lindhard screening length, $a_{B}$ the Bohr radius, and $e^{2}=14.399651 \mathrm{eV} \AA$.

Another empirical formula applies to light ion reflection (hydrogen and helium isotopes):

$$
R_{N}\left(R_{E}\right)=\frac{a_{1} \varepsilon^{a_{2}}}{1+a_{3} \varepsilon^{a_{4}}}
$$

For high energies $E_{0}>10^{5}$ and light ions formula (2) has to be modified:

$$
R_{N}\left(R_{E}\right)=\frac{b_{1} \varepsilon^{b_{2}}}{1+b_{3} \varepsilon^{b_{4}}}\left(1+b_{5} \varepsilon^{b_{6}}\right)
$$

The formulae above use less fitting parameters as in $[6,7]$. It should be mentioned, that the fit formulae are only valid in energy range given in the plots.

For the angular dependence of the reflection coefficients another fit formula can describe the results reasonably well:

$$
R_{N}\left(R_{E}\right)=c_{1}+c_{2} \tanh \left(c_{3} \alpha \pi / 180-c_{4}\right)
$$

where $\alpha$ is the angle of incidence (counted from the surface normal in degree). Instead of the function tanh also the function atan could be used, but the tanh gives better fits at normal incidence and smaller fit values (close to unity), The function atan gives better fits close to $90^{\circ}$, but at these large angles surface roughness will probably always show discrepancies between experimental data and calculated values.

Other empirical formulas for the reflection coefficients as well as for the energy distributions have been proposed in [37]. 


\section{Energy Dependence of the Reflection Coeffi- cients}

Generally, for each elemental target and several incident ions two plots are provided, one for the particle reflection coefficient and one for the energy reflection coefficient, showing calculated values and the corresponding fits (if available). In case, that other calculated values or experimental data exist, additional plots show the comparison for the individual ion-target combination. The energy range covered starts at $10 \mathrm{eV}$ and goes up to $10^{5} \mathrm{eV}$. In a few cases the energy range is extended into the $\mathrm{MeV}$ range (neglecting nuclear reactions). Calculated values are only included in these plots if the whole energy range is covered. More values in a smaller, limited energy range can be found in $[29,30]$.

Fitting parameters for the particle reflection coefficients, $R_{N}$, are provided in tables 1 to 7 , and for the energy reflection coefficient, $R_{E}$, in tables 7 to 14. A good fit could not be found in all cases, especially if the mass ratio is smaller than unity and for selfbombardment. Besides the fit parameters, the tables provide $\varepsilon_{L}, E_{s p}$, and the applied fitting formula.

The calculated energy dependence of both reflection coefficients show a more complex behaviour for heavy than for light ions, especially for targets of light elements, see for example Ne on Be (Fig.2).

Some discrepancies between experimental data and calculated values occur due to the effect, that for heavy ions the energy carried away from the target by sputtered atoms $\left(Y_{E}\right)$ is larger (sometimes by orders of magnitude) than the reflected energy $\left(R_{E}\right)$, as for example in the case for Neon on carbon. Some experiments measure the total energy removed from the target $\left(R_{E}+Y_{E}\right)$. This was demonstrated in [38]. For light ions (hydrogen and helium) $Y_{E}$ is usually smaller than $R_{E}$, see for example the case of helium on carbon; or more generally, for mass ratios $M_{2} / M_{1}>2, Y_{E}$ is larger than $R_{E}$, besides the in the threshold region for sputtering, where $R_{E}>Y_{E}$.

The influence of the application of a surface binding energy, $E_{s p}$, for projectiles on the reflection coefficients in calculations is discussed in [5, 39]. A value of $E_{s p}=1 \mathrm{eV}$ (for $\mathrm{H}$ or $\mathrm{N}$ ) shows a decrease of the reflection coefficients below an incident energy of $10 \mathrm{eV}$. This is supported by a different calculational approach using the Emmbedded Atom Method (EAM) [40]. A decrease of the reflection coefficients at low energies in the case of noble gas ions cannot be attributed to a binding of ions to a target $\left(E_{s p}=0\right)$. The decrease of the reflection coefficients at low energies for selfbombardment is caused by the surface binding represented by the surface binding energy, $E_{s b}$, in the same way as for the sputtering yield [41].

Light ion reflection at high energies $\left(>10^{5} \mathrm{eV}\right)$ has been treated analytically and by computer simulation in [42], Simulation results are given for hydrogen and helium reflection from $\mathrm{C}, \mathrm{Nb}, \mathrm{W}$ and $\mathrm{Au}$, see figures $6,33,51,57$.

Heavy (mass) impurities in a target of light atoms will increase the reflection coefficients, light impurities in a target of heavy atoms will decrease $R_{N}$ and $R_{E}$. Typical cases are oxides, see, for example, the plots for Ta and $\mathrm{W}$ [43], where 
the calculated reflection coefficients for pure $\mathrm{W}$ and a $1.5 \mathrm{~nm} \mathrm{WO}_{3}$ layer on $\mathrm{W}$ are compared. Other examples are metal hydrides [44, 45], borides, carbides, and nitrides [46].

The $\varepsilon$-scaling of the reflection coefficients, often regarded in earlier publications, is only valid for large mass ratios and at not too high incident energies, where the electronic energy loss is the main slowing-down process.

The use of different interaction potentials in the calculations leed to different values of the reflection coefficients; these differences are usually smaller than a factor of two, for the mostly used potentials (WHB, ZBL) these differences are in most cases smaller than 20\%, see the cases of Si on Si (Figs. 77 and 78) and of $\mathrm{Ar}$ on $\mathrm{Ag}$ (Fig. 41).

Calculations with the program MARLOWE have shown strong channeling effects of the particle reflection coefficient for the bombardment of $\mathrm{Cu}(001)$ with Ar [47].

Fig. 62 shows the fitting parameters of formula (6) for the energy dependence versus the atomic numbers of target atoms for the bombardment with $\mathrm{H}$, $\mathrm{He}$ and Ne. 

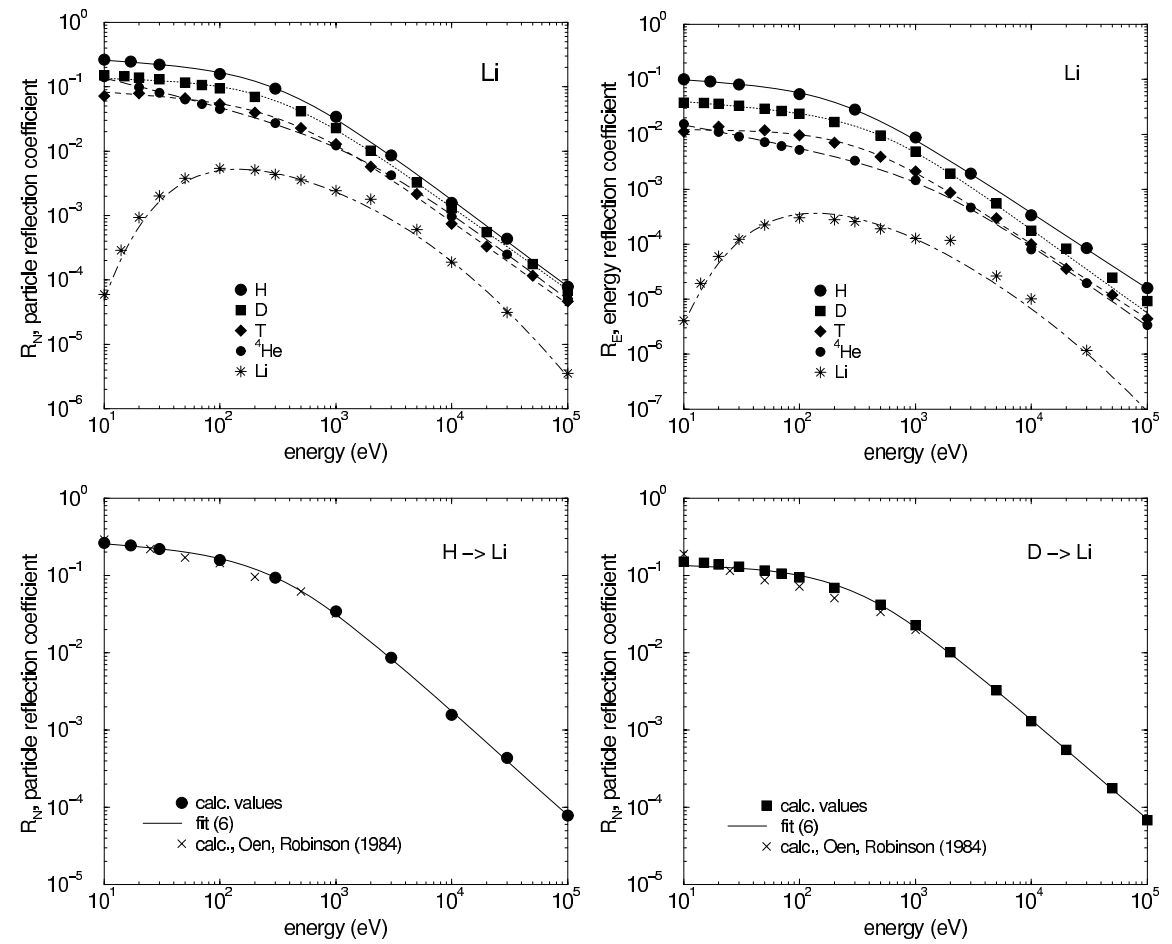

Figure 1: Calculated values for the energy dependence of the particle and energy reflection coefficients at normal incidence for the bombardment of $\mathrm{Li}$ with $\mathrm{H}$ [30], D, T, ${ }^{4} \mathrm{He}[29]$. Lines are fits to the calculated values (see Table 1). Other calculated values for $\mathrm{H}$ and $\mathrm{D}$ are given in [48] 

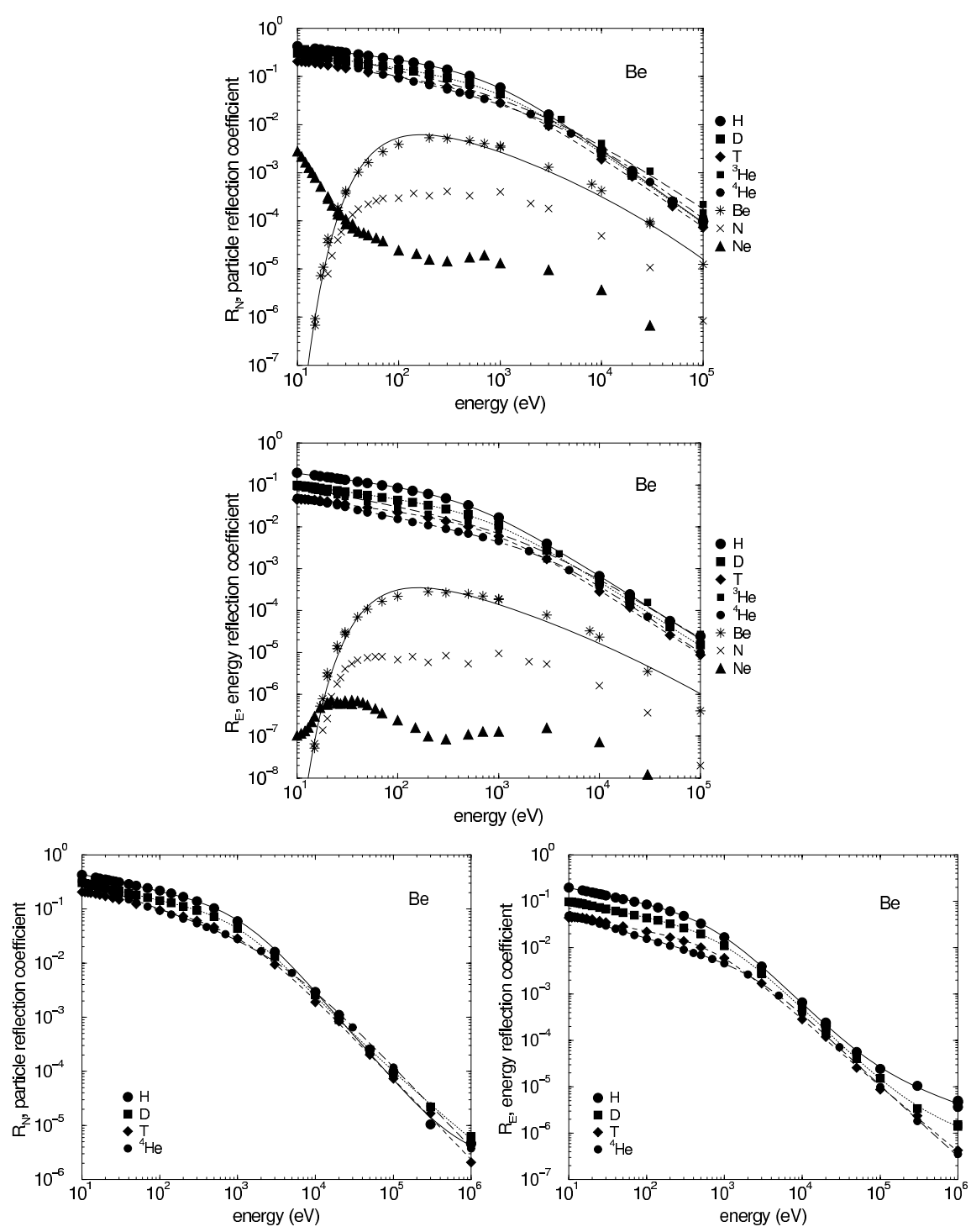

Figure 2: Calculated values for energy dependence of the particle and energy reflection coefficients at normal incidence for the bombardment of Be with $\mathrm{H}$, $\mathrm{D}, \mathrm{T},{ }^{3} \mathrm{He},{ }^{4} \mathrm{He}, \mathrm{Be}, \mathrm{Ne}[8], \mathrm{N}[30]$, and for the extension of the energy range to $1 \mathrm{MeV}$ for H, D, T, ${ }^{4} \mathrm{He}$. Lines are fits to the calculated values (see Tables 1 and 9,7 and 15$)$ 

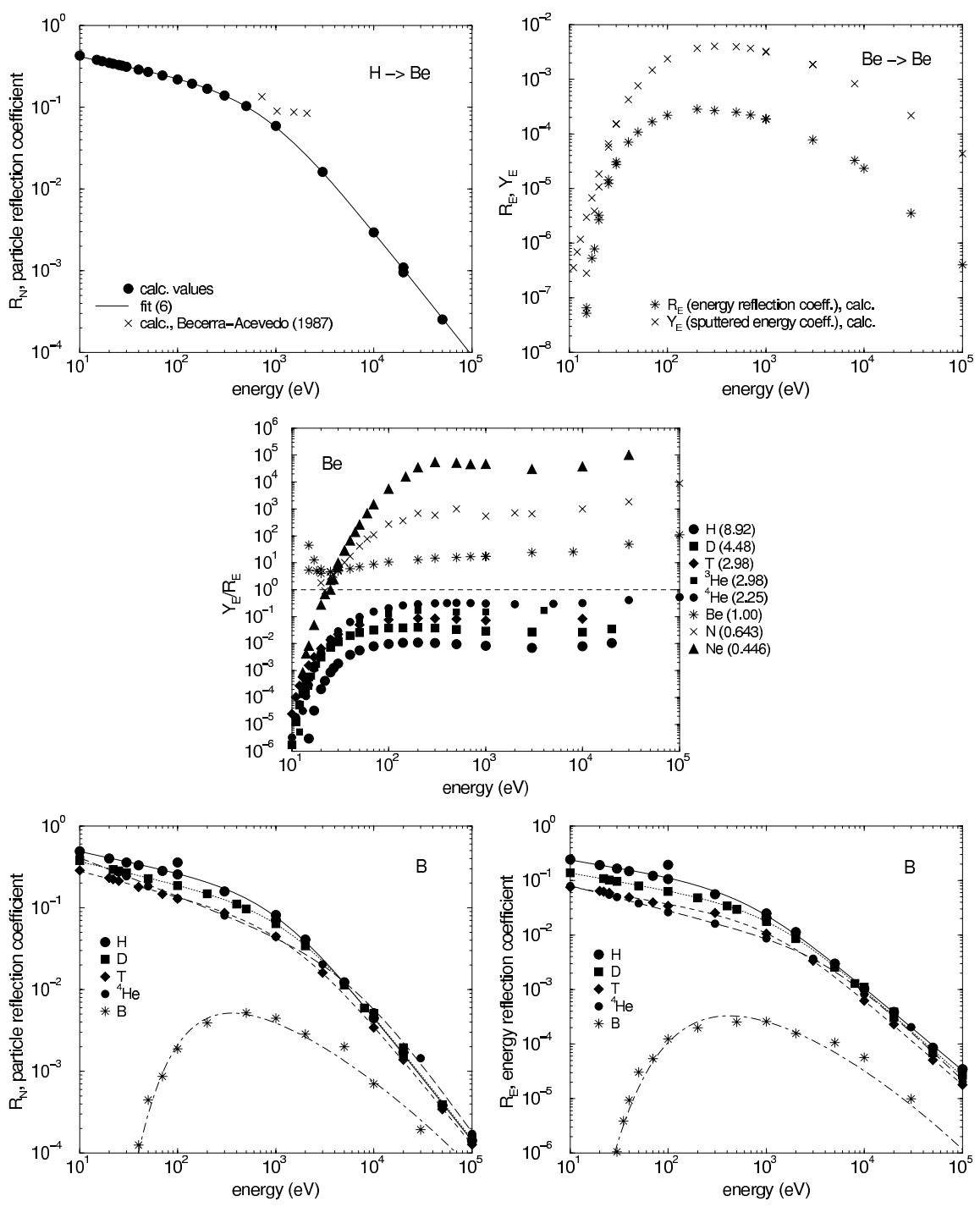

Figure 3: Calculated energy dependence of the particle reflection coefficient at normal incidence for the bombardment of $\mathrm{Be}$ with $\mathrm{H}[8,49]$ and a comparison of the energy dependence at normal incidence of the energy reflection and sputtered energy coefficients for $\mathrm{Be}$ on $\mathrm{Be}$ [8]. In addition, calculated values of the ratio of the sputtered energy coefficient, $Y_{E}$, divided by the energy reflection coefficient, $R_{E}$, for Be and different incident ions are provided. Furthermore, calculated values for energy dependence of the particle and energy reflection coefficients at normal incidence for the bombardment of B with $\mathrm{H}, \mathrm{D}, \mathrm{T},{ }^{4} \mathrm{He}$, and B [29] are shown; lines are fits to the calculated values (see Tables 1 and 9 ) 

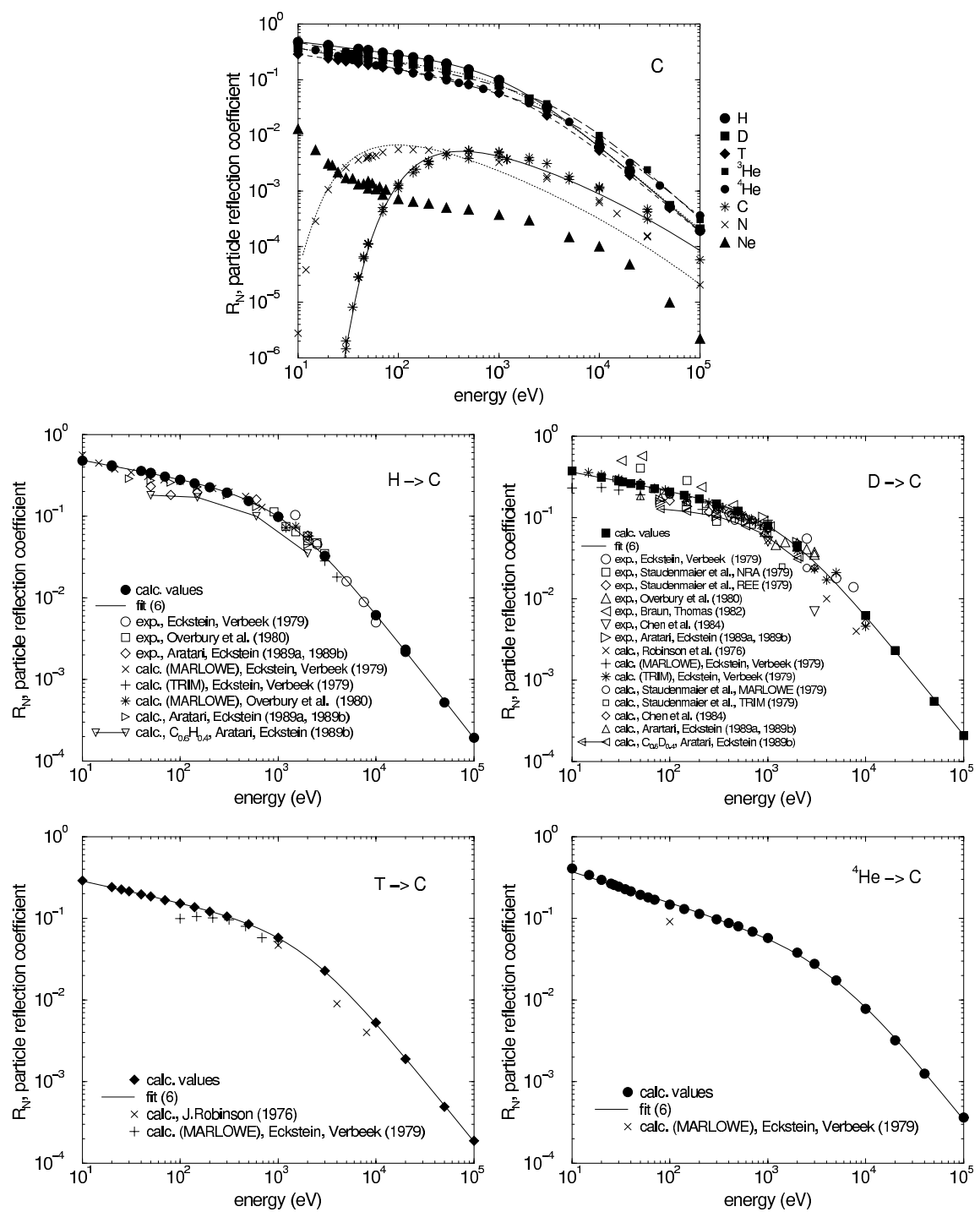

Figure 4: Calculated energy dependence of the particle reflection coefficient at normal incidence for the bombardment of $\mathrm{C}$ with different incident ions [8]. Lines are fits to the calculated values (see Table 1 ). Other calculated an experimental values are given for $\mathrm{H}[12,50,51,52], \mathrm{D}[11,12,50,53,54,55], \mathrm{T}[12,54],{ }^{4} \mathrm{He}$ $[12]$ 

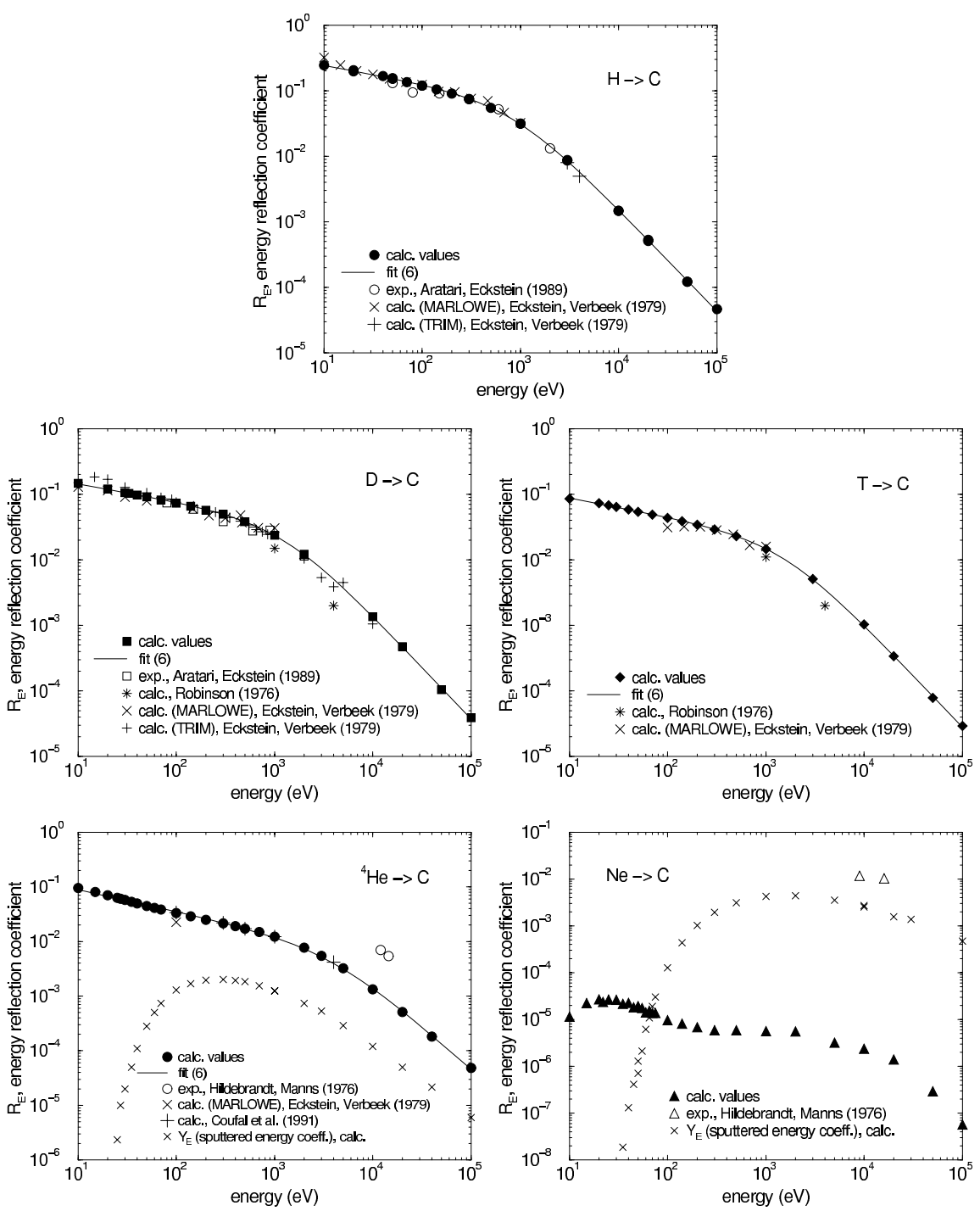

Figure 5: Calculated energy dependence of the energy reflection coefficient at normal incidence for the bombardment of $\mathrm{C}$ with different incident ions [8]. Lines are fits to the calculated values (see Table 9). Other calculated values are given for $\mathrm{H}[12], \mathrm{D}[12,54], \mathrm{T}[12,54]$ and ${ }^{4} \mathrm{He}[12,38]$ experimental values for $\mathrm{H}$ [52], D [52], ${ }^{4} \mathrm{He}[56]$ and $\mathrm{Ne}[56]$. For ${ }^{4} \mathrm{He}$ and Ne also the sputtered energy coefficient is given 

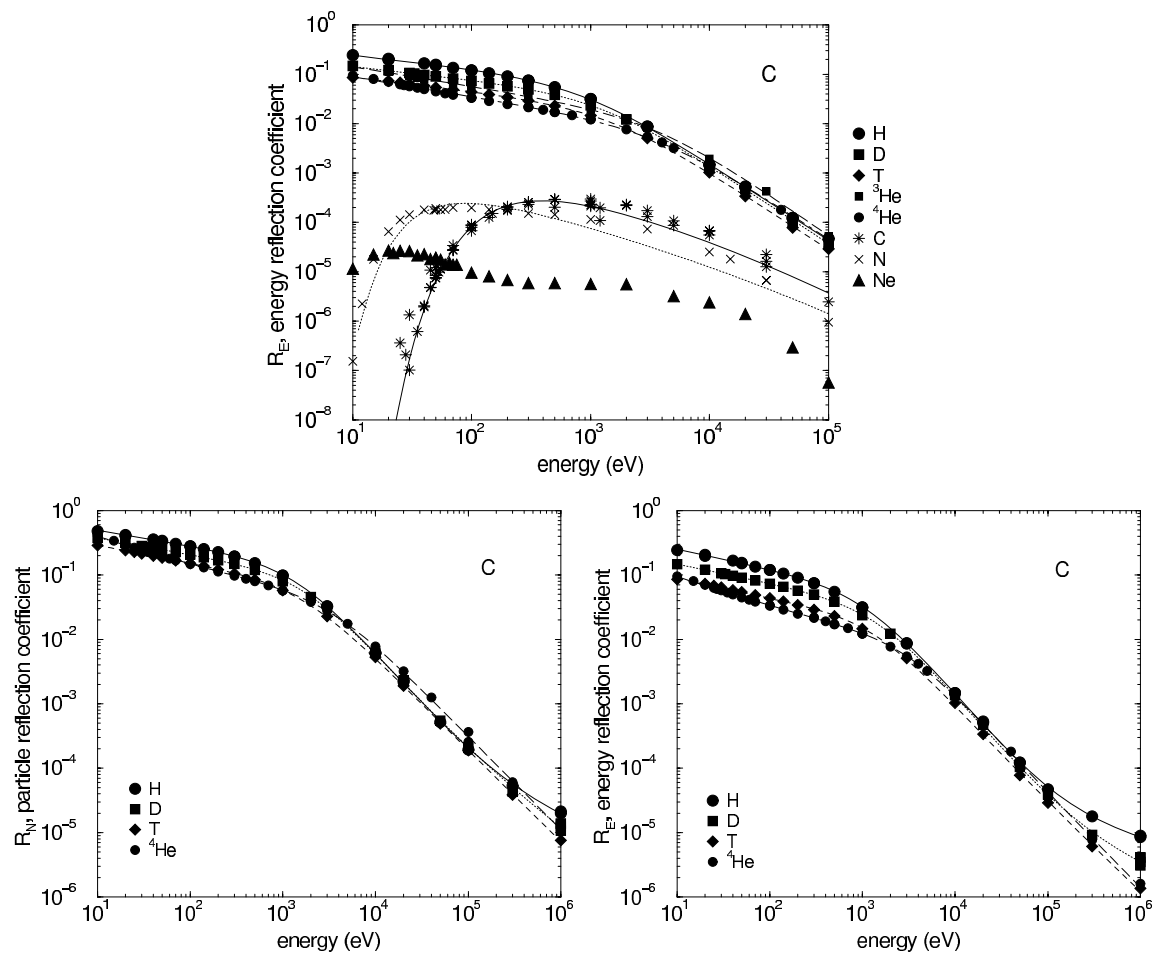

Figure 6: Calculated energy dependence of the energy reflection coefficient at normal incidence for the bombardment of $\mathrm{C}$ with $\mathrm{H}, \mathrm{D}, \mathrm{T},{ }^{3} \mathrm{He},{ }^{4} \mathrm{He}, \mathrm{C}, \mathrm{N}, \mathrm{Ne}$ [8] and the extension of $R_{N}$ and $R_{E}$ to high energies (up to $1 \mathrm{MeV}$ ) for $\mathrm{H}, \mathrm{D}$, $\mathrm{T},{ }^{4} \mathrm{He}$. Lines are fits to the calculated values (see Tables 9,7 and 15 ) 

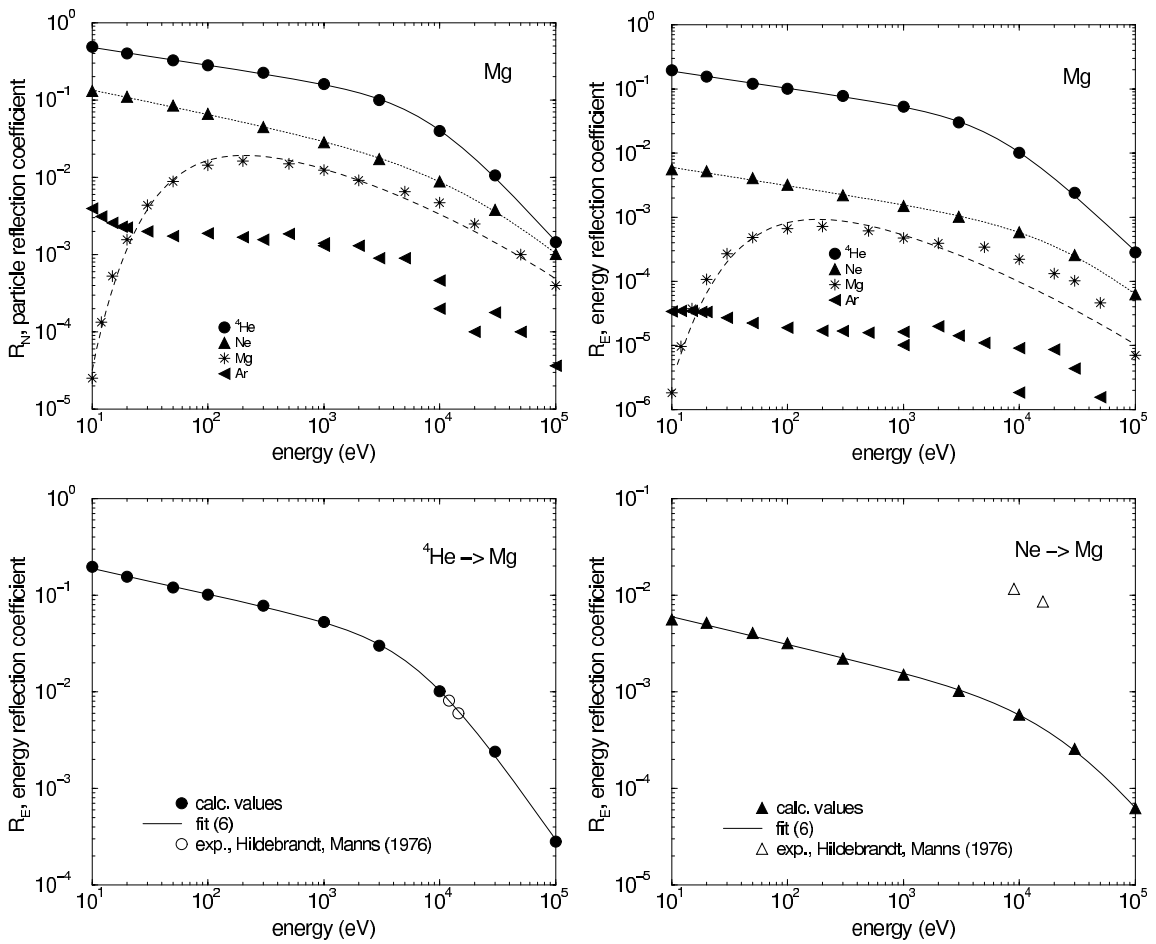

Figure 7: Calculated energy dependence of the particle and energy reflection coefficients at normal incidence for the bombardment of $\mathrm{Mg}$ with ${ }^{4} \mathrm{He}, \mathrm{Ne}, \mathrm{Mg}, \mathrm{Ar}$ [30]. Lines are fits to the calculated values (see Tables 1 and 9). Experimental values for $R_{E}$ are given for ${ }^{4} \mathrm{He}[56]$ and $\mathrm{Ne}$ [56] on $\mathrm{Mg}$ 

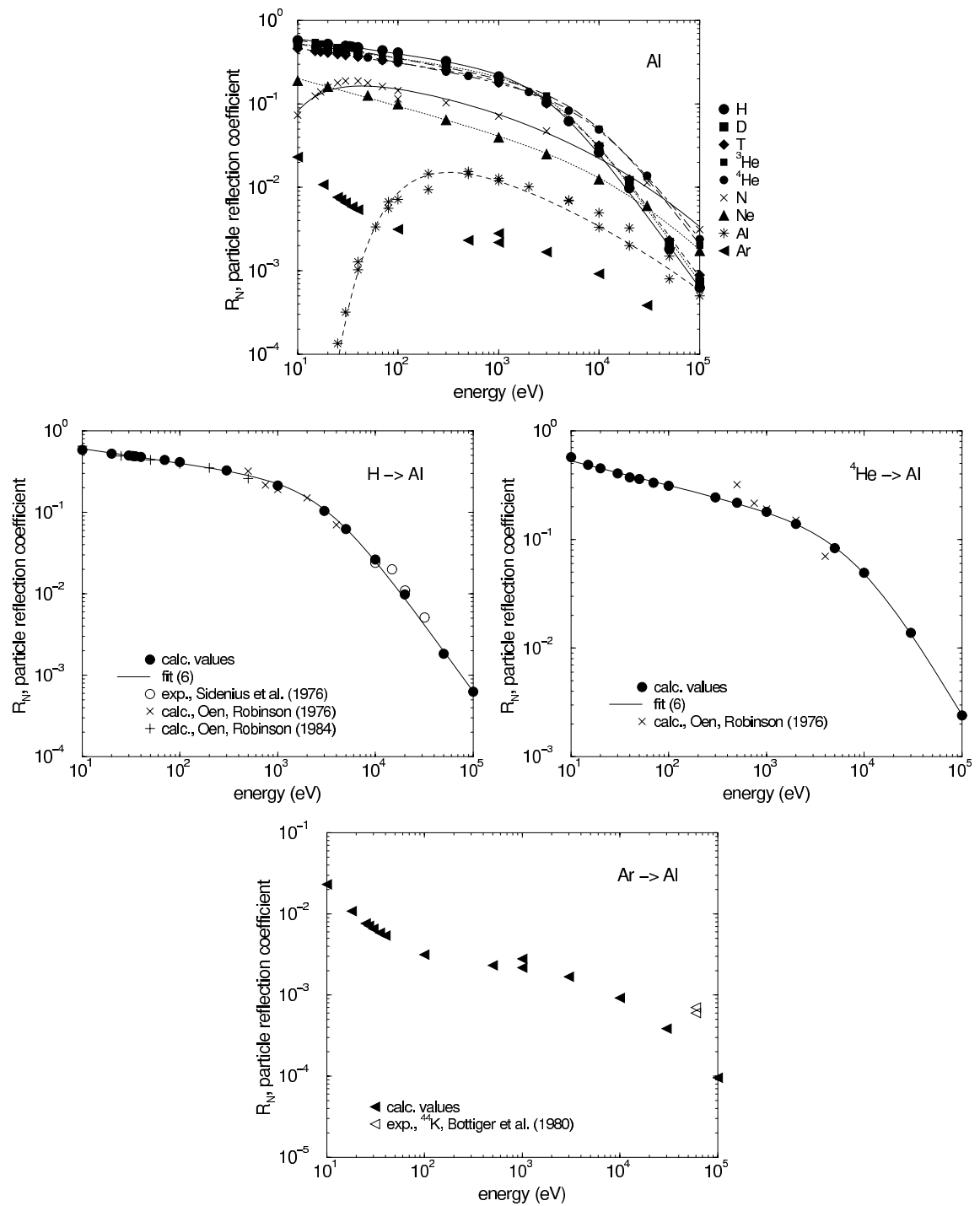

Figure 8: Calculated energy dependence of the particle reflection coefficient at normal incidence for the bombardment of $\mathrm{Al}$ with $\mathrm{H}, \mathrm{D}, \mathrm{T},{ }^{3} \mathrm{He},{ }^{4} \mathrm{He}, \mathrm{N}$, Ne, $\mathrm{Al}, \mathrm{Ar}[29,30]$. Lines are fits to the calculated values (see Table 1). Other calculated and experimental values are given for $\mathrm{H}[16,24,48],{ }^{4} \mathrm{He}[24]$ and $\mathrm{K}$ (Ar) $[57]$ 

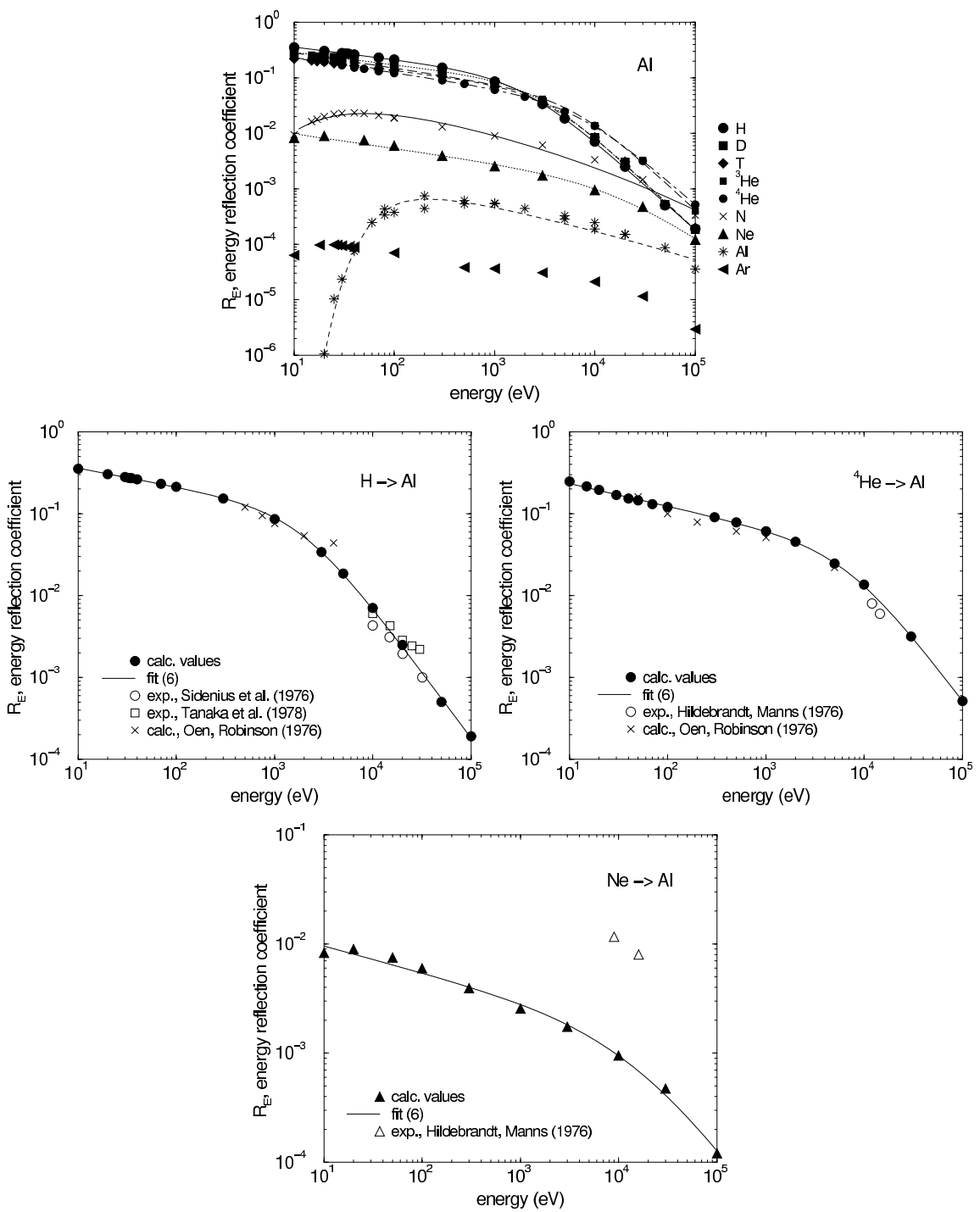

Figure 9: Calculated energy dependence of the energy reflection coefficient at normal incidence for the bombardment of $\mathrm{Al}$ with $\mathrm{H}, \mathrm{D}, \mathrm{T},{ }^{3} \mathrm{He},{ }^{4} \mathrm{He}, \mathrm{N}, \mathrm{Ne}$, $\mathrm{Al}, \operatorname{Ar}[29,30]$. Lines are fits to the calculated values (see Table 9). Other calculated and experimental values are given for $\mathrm{H}[16,24,58],{ }^{4} \mathrm{He}[24,56]$ and Ne [56] 

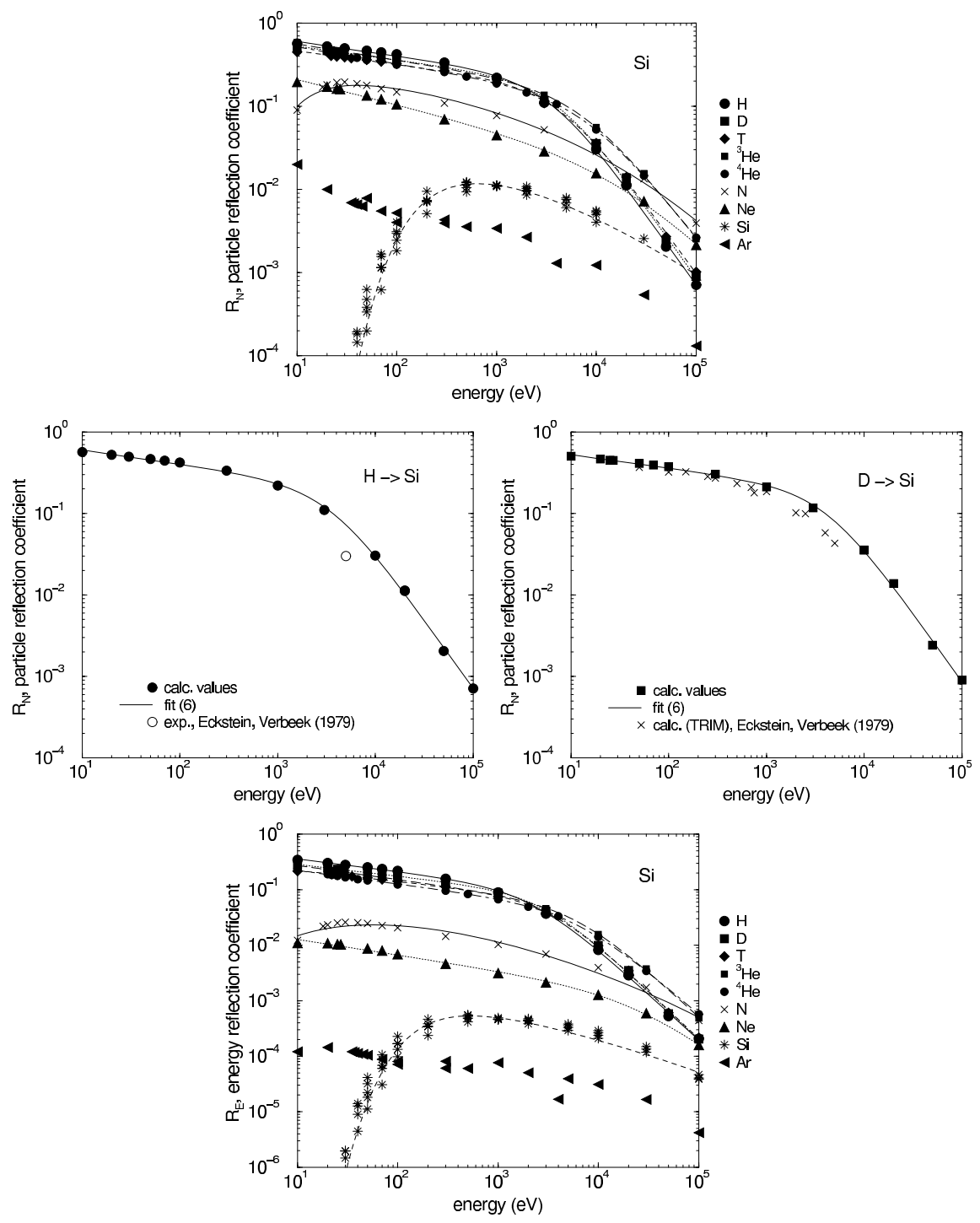

Figure 10: Calculated energy dependence of the particle and energy reflection coefficients at normal incidence for the bombardment of Si with $\mathrm{H}, \mathrm{D}, \mathrm{T},{ }^{3} \mathrm{He}$, ${ }^{4} \mathrm{He}, \mathrm{N}, \mathrm{Ne}, \mathrm{Si}, \mathrm{Ar}[29,30]$. Lines are fits to the calculated values (see Table 1 and 9). Other calculated and experimental values of $R_{N}$ are given for $\mathrm{H}$ and $\mathrm{D}[12]$ 

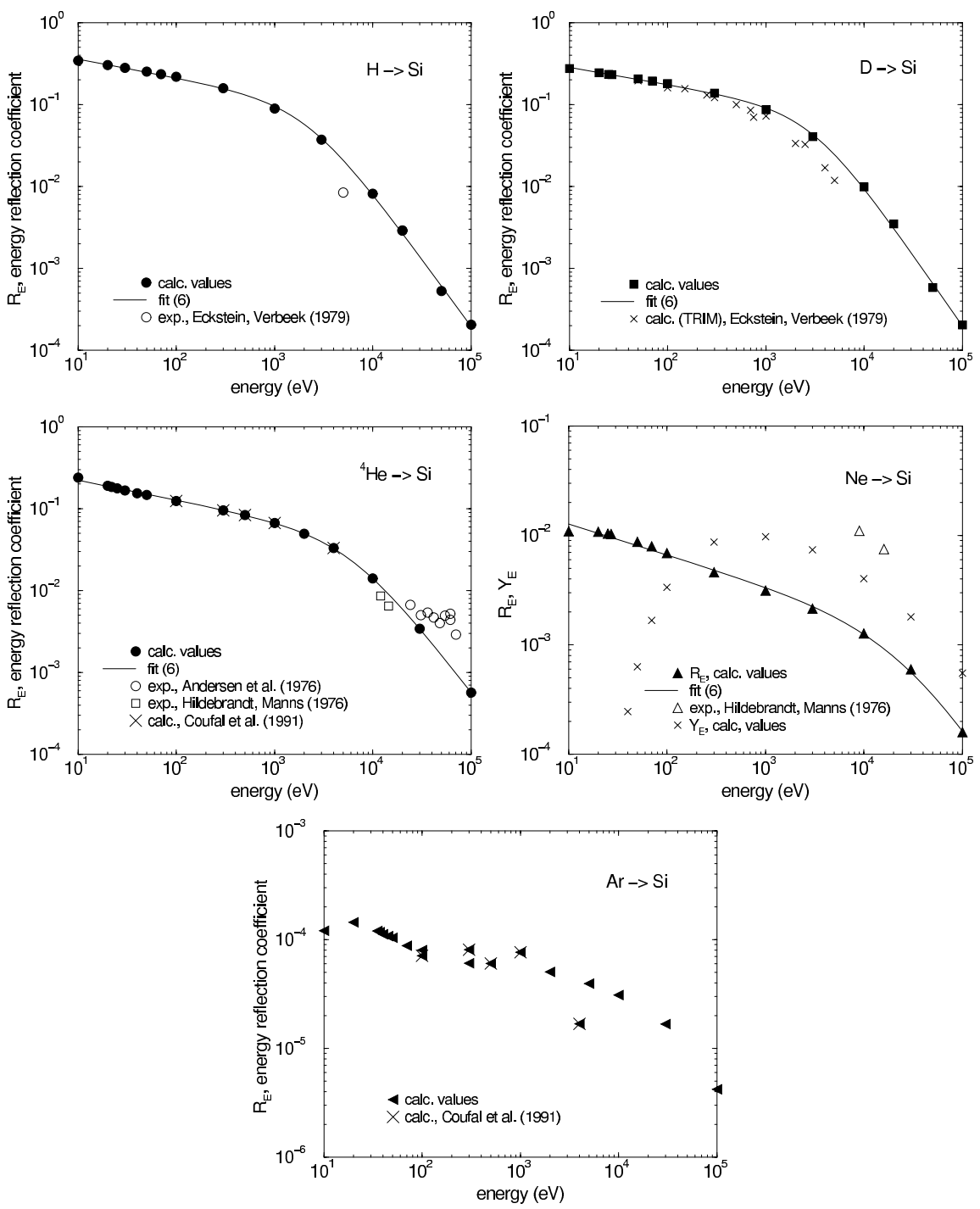

Figure 11: Calculated energy dependence of the energy reflection coefficient at normal incidence for the bombardment of $\mathrm{Si}$ with $\mathrm{H}, \mathrm{D}, \mathrm{T},{ }^{3} \mathrm{He},{ }^{4} \mathrm{He}, \mathrm{N}, \mathrm{Ne}, \mathrm{Si}$, $\operatorname{Ar}[29,30]$. Lines are fits to the calculated values (see Table 9). Additional calculated values are given for D [12], ${ }^{4} \mathrm{He}[38]$, Ar [38], experimental data for $\mathrm{H}[12],{ }^{4} \mathrm{He}[14,56]$, Ne [56] 

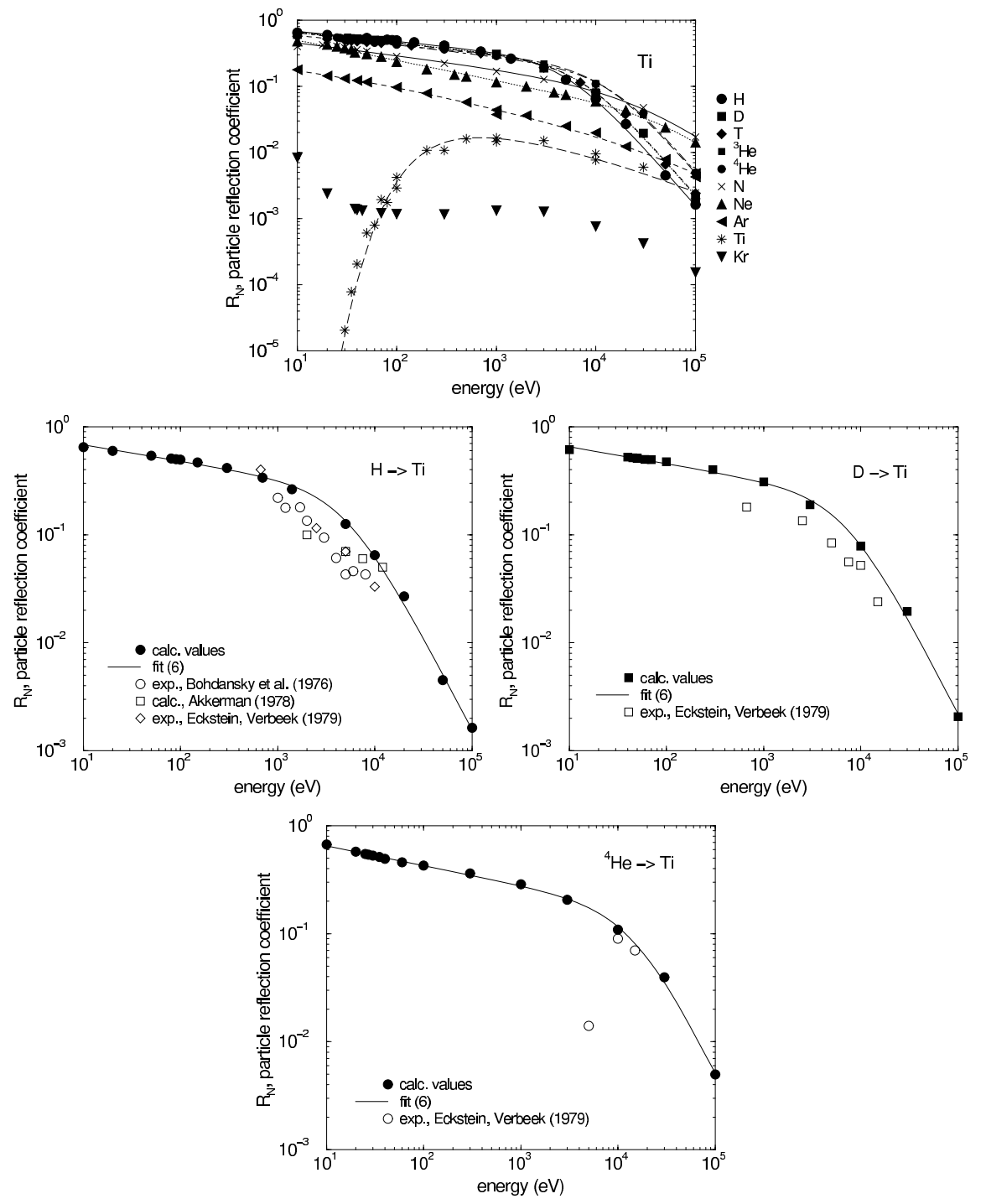

Figure 12: Calculated energy dependence of the particle reflection coefficient at normal incidence for the bombardment of $\mathrm{Ti}$ with $\mathrm{H}, \mathrm{D}, \mathrm{T},{ }^{3} \mathrm{He},{ }^{4} \mathrm{He}, \mathrm{N}, \mathrm{Ne}$, $\mathrm{Ar}, \mathrm{Ti}, \mathrm{Kr}[29,30]$. Lines are fits to the calculated values (see Table 2). Other calculated and experimental values are given for $\mathrm{H}[12,59,60], \mathrm{D}[12],{ }^{4} \mathrm{He}[12]$ 

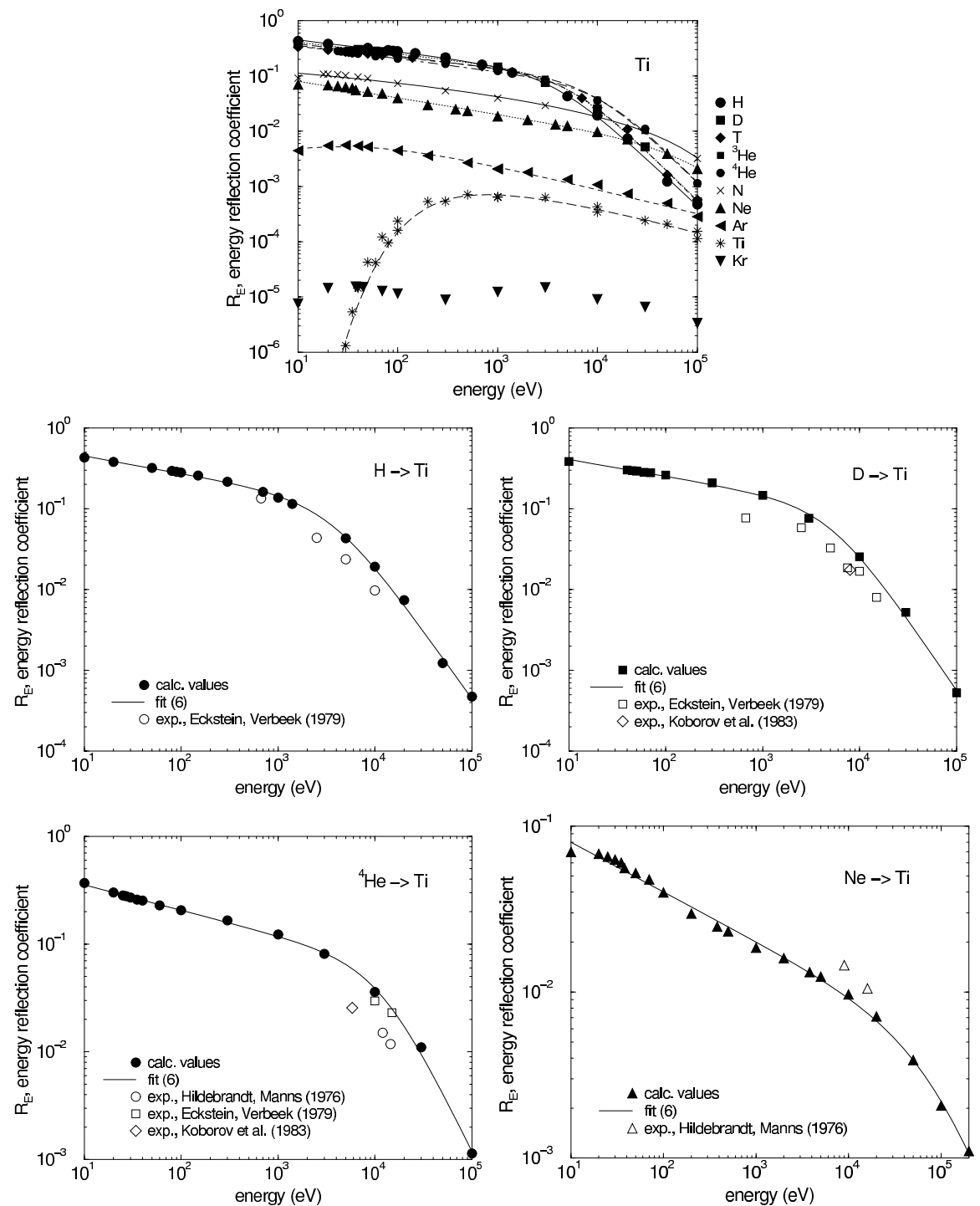

Figure 13: Calculated energy dependence of the energy reflection coefficient at normal incidence for the bombardment of $\mathrm{Ti}$ with $\mathrm{H}, \mathrm{D}, \mathrm{T},{ }^{3} \mathrm{He},{ }^{4} \mathrm{He}, \mathrm{N}, \mathrm{Ne}$, $\mathrm{Ar}, \mathrm{Ti}, \mathrm{Kr}[29,30]$. Lines are fits to the calculated values (see Table 10). Experimental data are given for $\mathrm{H}[12], \mathrm{D}[12,61],{ }^{4} \mathrm{He}[12,56,61]$, Ne [56] 

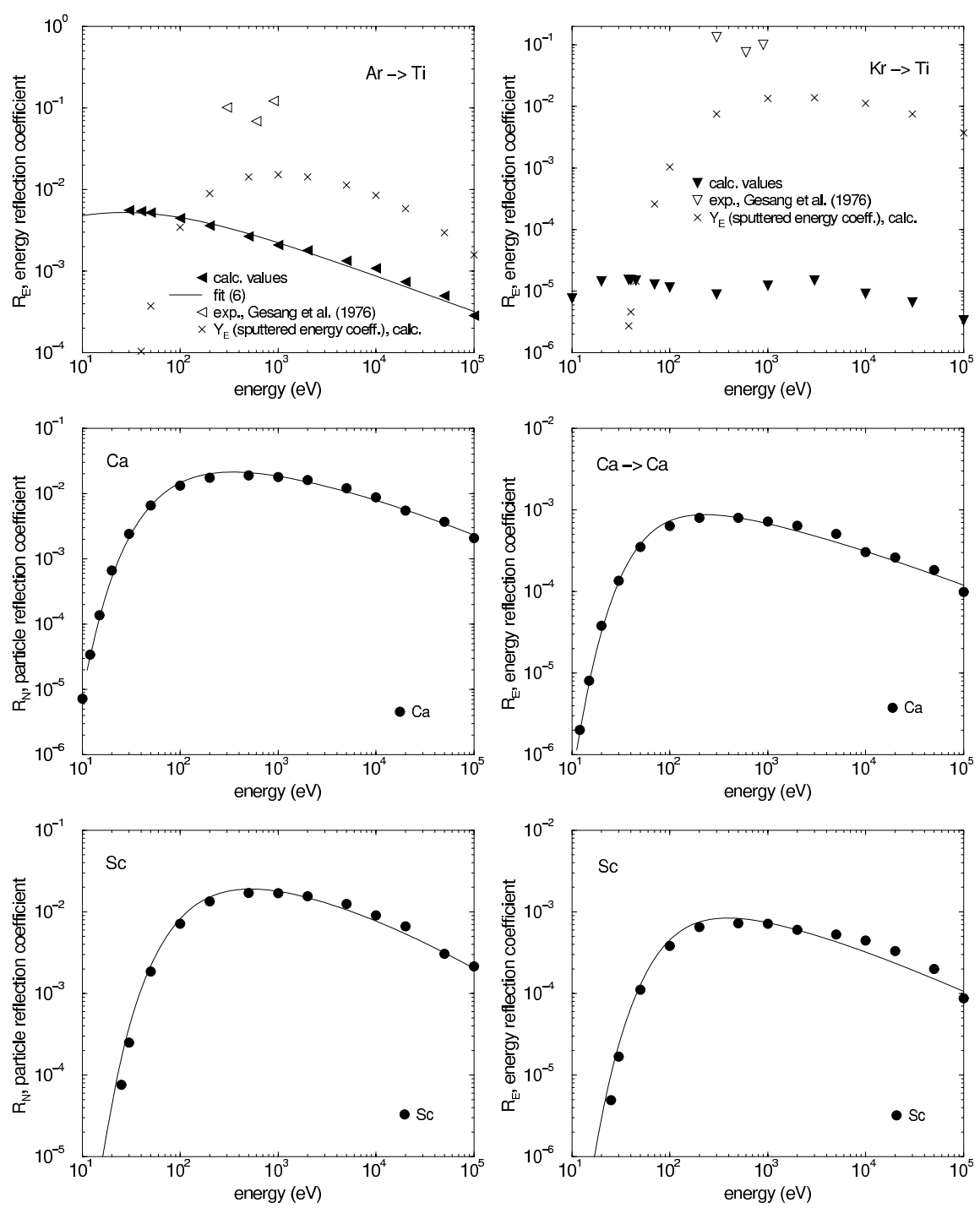

Figure 14: Calculated energy dependence of the energy reflection and sputtered energy coefficients at normal incidence for the bombardment of $\mathrm{Ti}$ with $\mathrm{Ar}$ and $\mathrm{Kr}[30]$. The line is a fit to the calculated values (see Table 10). Experimental data are given for $\operatorname{Ar}[62]$ and $\mathrm{Kr}[62]$ 

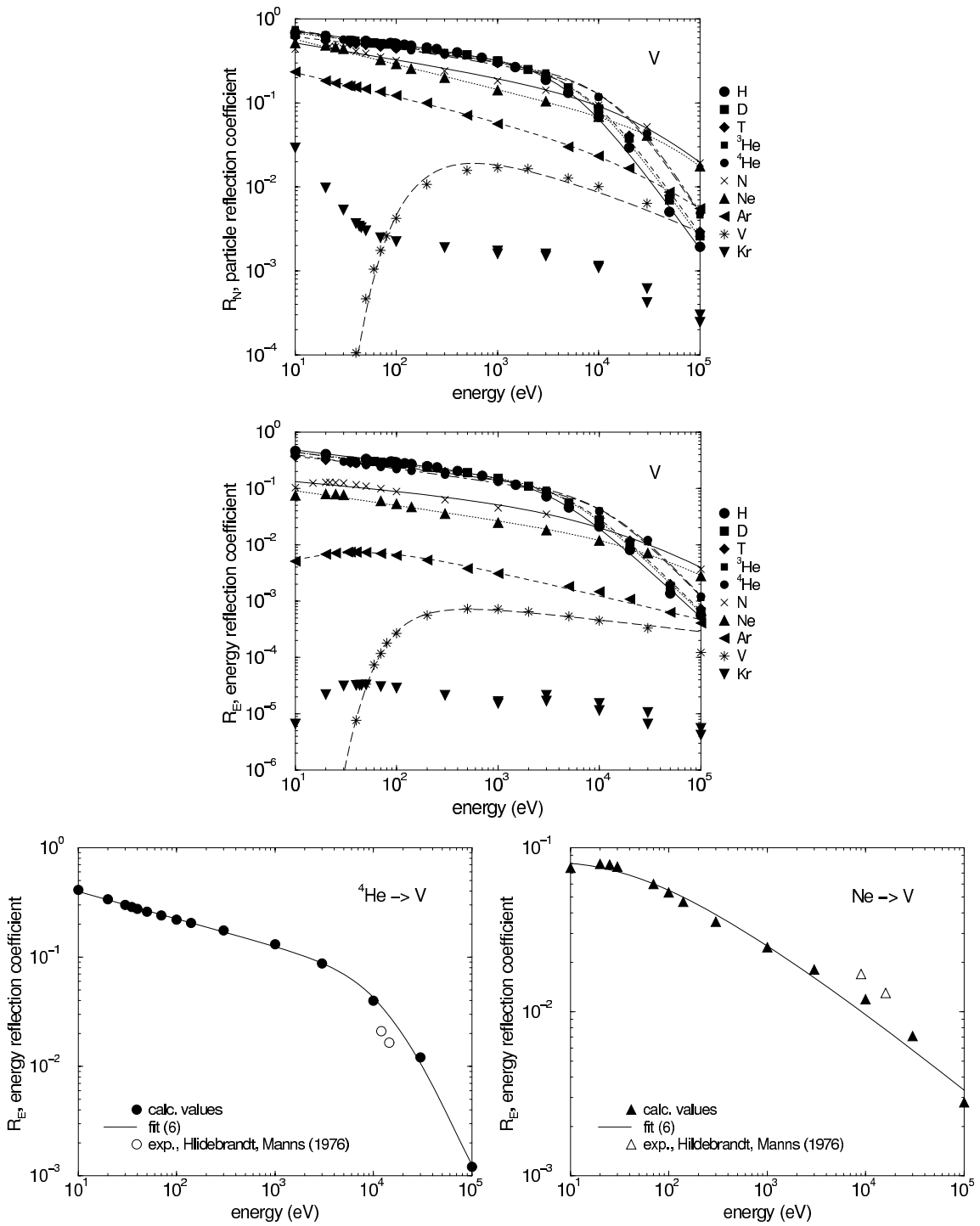

Figure 15: Calculated energy dependence of the particle and energy reflection coefficients at normal incidence for the bombardment of $\mathrm{V}$ with $\mathrm{H}, \mathrm{D}, \mathrm{T},{ }^{3} \mathrm{He}$, ${ }^{4} \mathrm{He}, \mathrm{N}, \mathrm{Ne}, \mathrm{Ar}, \mathrm{V}, \mathrm{Kr}[29,30]$. Lines are fits to the calculated values (see Table 2 and 10). Experimental data of $R_{E}$ are given for ${ }^{4} \mathrm{He}$ [56] and Ne [56] 

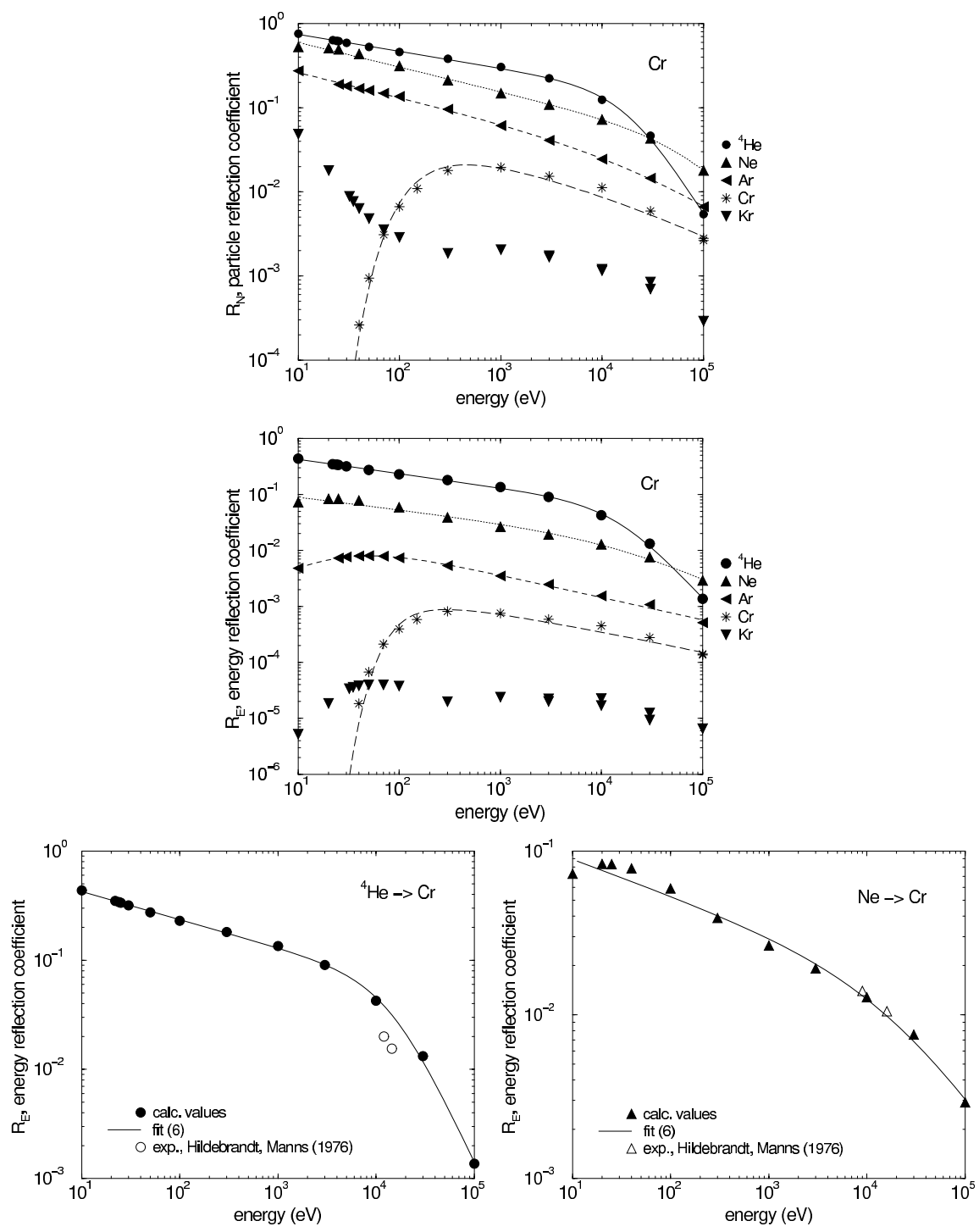

Figure 16: Calculated energy dependence of the particle and energy reflection coefficients at normal incidence for the bombardment of $\mathrm{Cr}$ with ${ }^{4} \mathrm{He}, \mathrm{Ne}, \mathrm{Ar}$, $\mathrm{Cr}, \mathrm{Kr}$ [30]. Lines are fits to the calculated values (see Table 2 and 10). Experimental data of $R_{E}$ are given for ${ }^{4} \mathrm{He}$ [56] and $\mathrm{Ne}$ [56] 

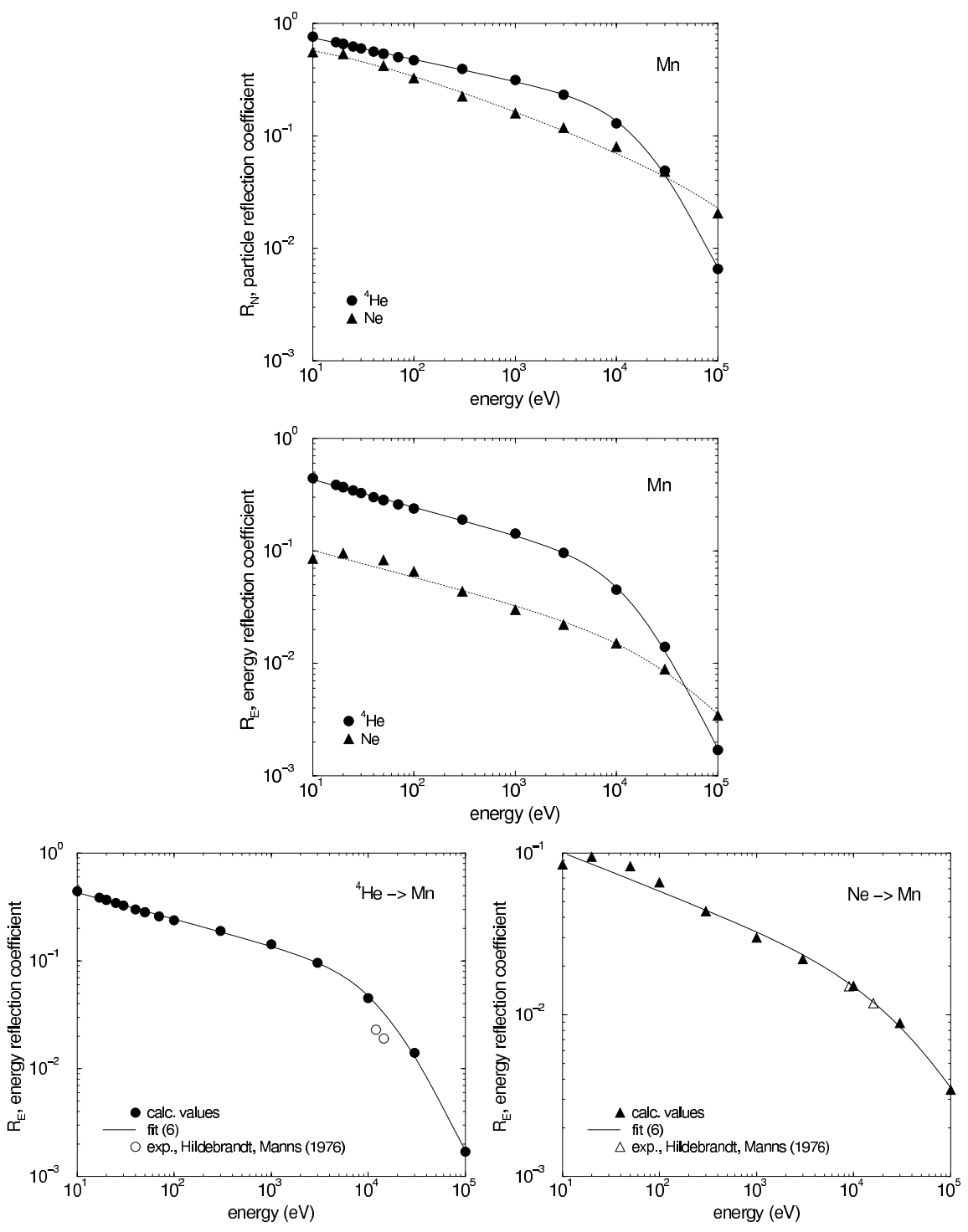

Figure 17: Calculated energy dependence of the particle and energy reflection coefficients at normal incidence for the bombardment of Mn with ${ }^{4} \mathrm{He}$, Ne [30]. Lines are fits to the calculated values (see Table 2 and 10). Experimental data of $R_{E}$ are given for ${ }^{4} \mathrm{He}$ [56] and $\mathrm{Ne}$ [56] 

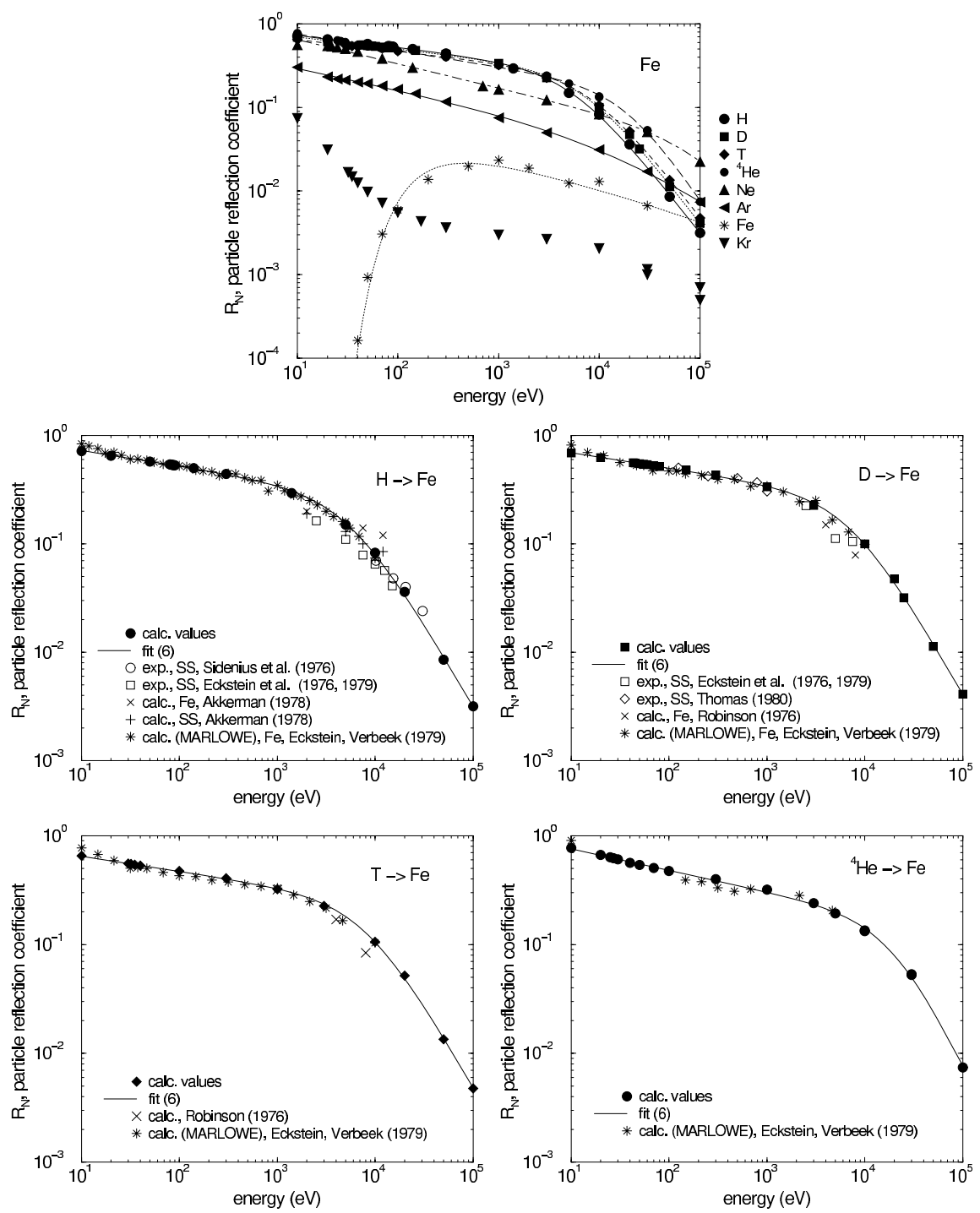

Figure 18: Calculated energy dependence of the particle reflection coefficient at normal incidence for the bombardment of Fe with $\mathrm{H}, \mathrm{D}, \mathrm{T},{ }^{4} \mathrm{He}, \mathrm{Ne}, \mathrm{Ar}, \mathrm{Fe}$, $\operatorname{Kr}[29,30]$. Lines are fits to the calculated values (see Table 2). Experimental data of $R_{N}$ are given for $\mathrm{H}[12,16,63], \mathrm{D}[12,63,64]$, calculated values for $\mathrm{H}$ $[12,59], \mathrm{D}[12,54], \mathrm{T}[12,54],{ }^{4} \mathrm{He}[12]$ 

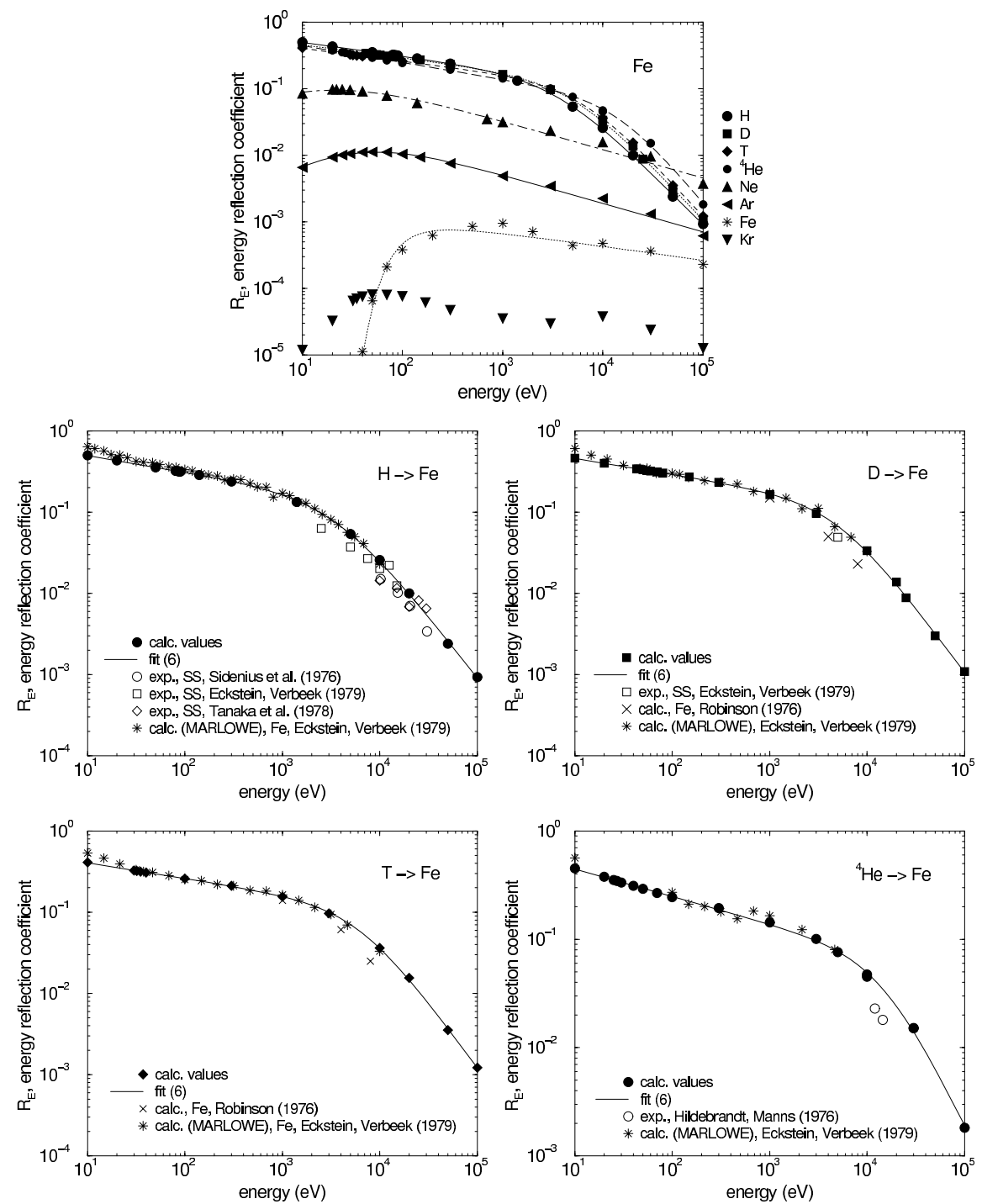

Figure 19: Calculated energy dependence of the energy reflection coefficient at normal incidence for the bombardment of Fe with $\mathrm{H}, \mathrm{D}, \mathrm{T},{ }^{4} \mathrm{He}, \mathrm{Ne}, \mathrm{Ar}, \mathrm{Fe}, \mathrm{Kr}$ $[29,30]$. Lines are fits to the calculated values (see Table 10). Experimental data of $R_{E}$ are given for $\mathrm{H}[12,16,58], \mathrm{D}$ [12], ${ }^{4} \mathrm{He}$ [56], calculated values for $\mathrm{H}$ $[12], \mathrm{D}[12,54], \mathrm{T}[12,54],{ }^{4} \mathrm{He}[12]$ 

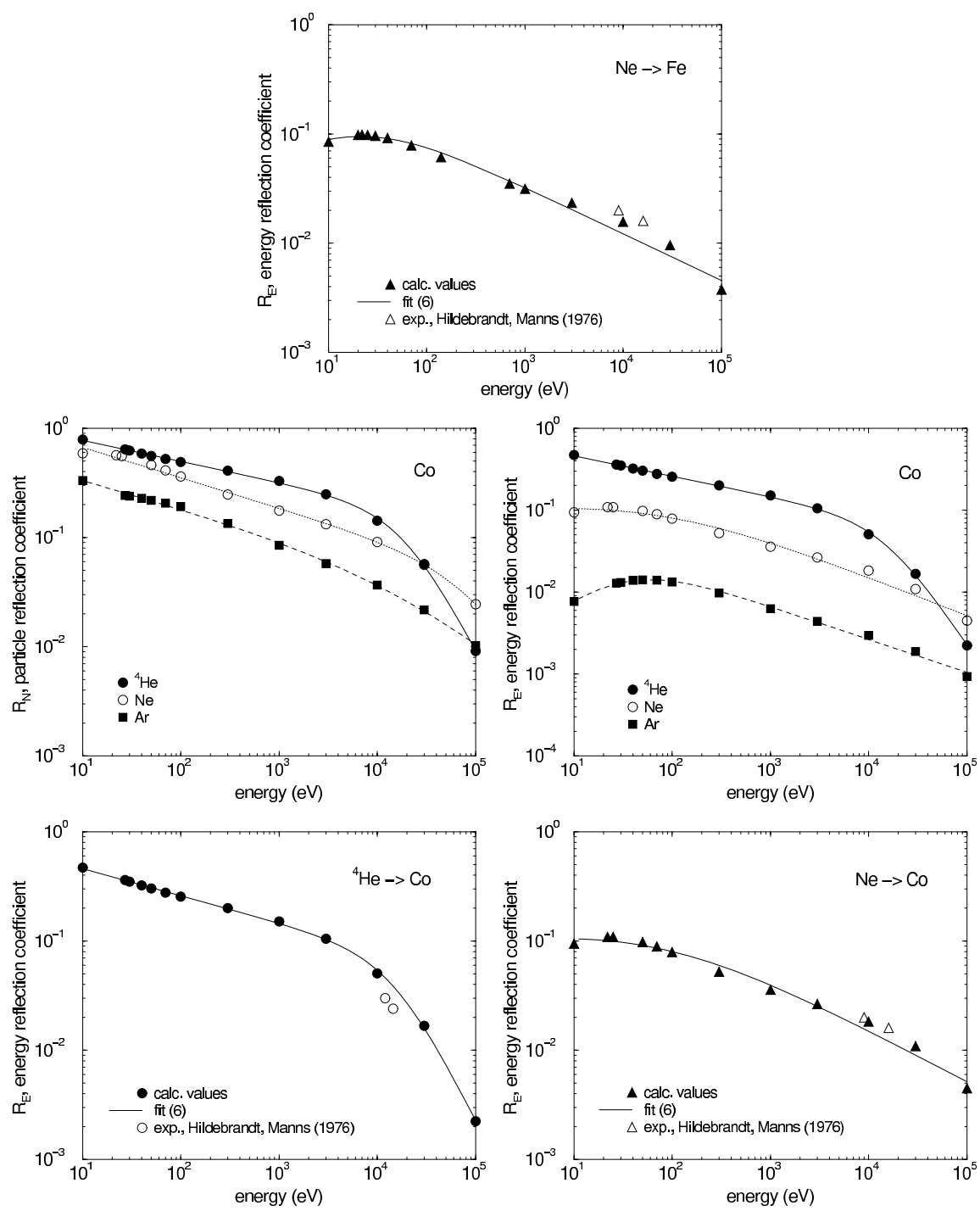

Figure 20: Calculated energy dependence of the particle and energy reflection coefficients at normal incidence for the bombardment of Co with ${ }^{4} \mathrm{He}, \mathrm{Ne}, \mathrm{Ar}$ [30]. Experimental data of $R_{E}$ are given for $\mathrm{Ne}$ on $\mathrm{Fe}$ [56] and for ${ }^{4} \mathrm{He}$ and $\mathrm{Ne}$ on Co [56]. Lines are fits to the calculated values (see Tables 2 and 10) 

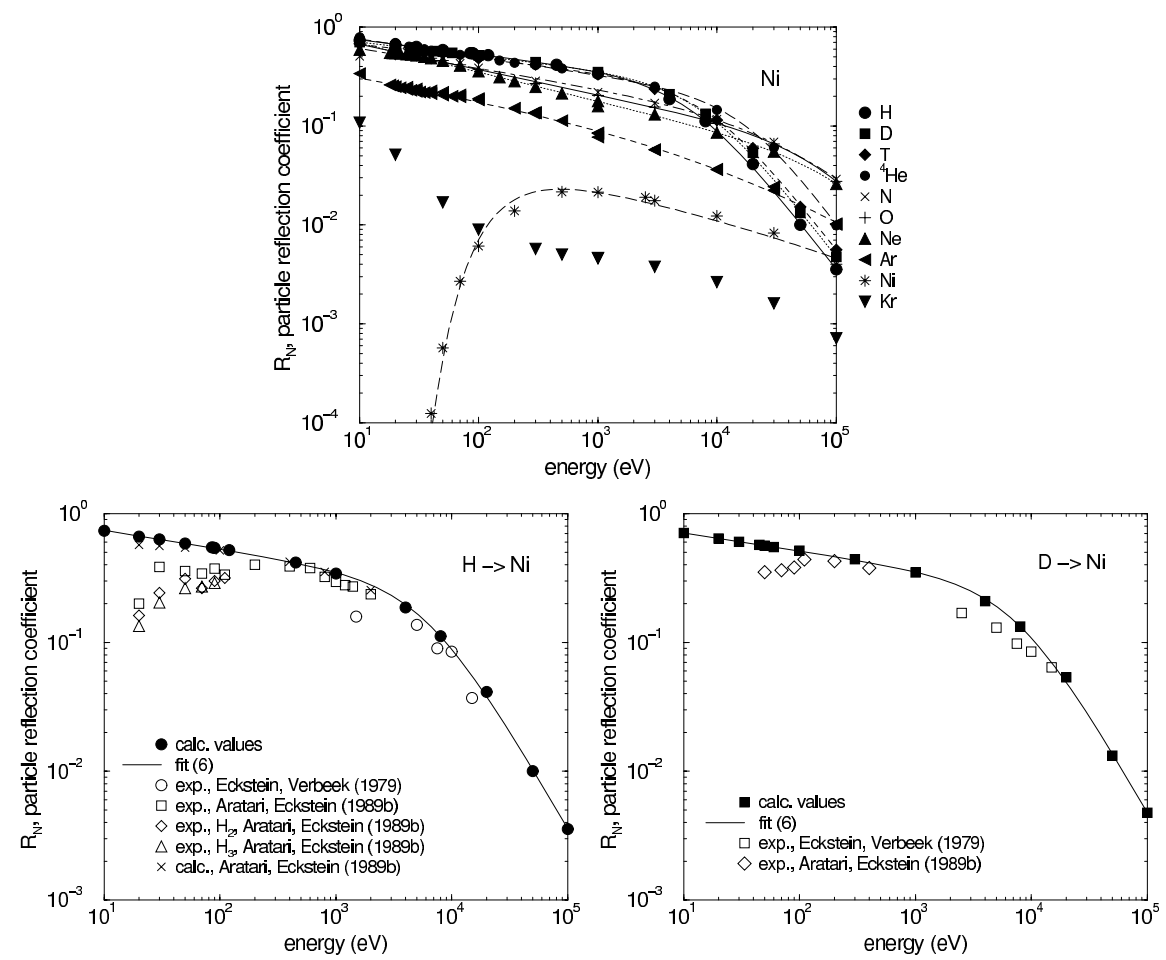

Figure 21: Calculated energy dependence of the particle reflection coefficient at normal incidence for the bombardment of $\mathrm{Ni}$ with $\mathrm{H}, \mathrm{D}, \mathrm{T},{ }^{4} \mathrm{He}, \mathrm{N}, \mathrm{O}, \mathrm{Ne}, \mathrm{Ar}$, $\mathrm{Ni}, \mathrm{Kr}[29,30]$. Experimental data of $R_{N}$ are given for $\mathrm{H}, \mathrm{D}$ on $\mathrm{Ni}[12,52]$. Lines are fits to the calculated values (see Tables 2 and 10) 

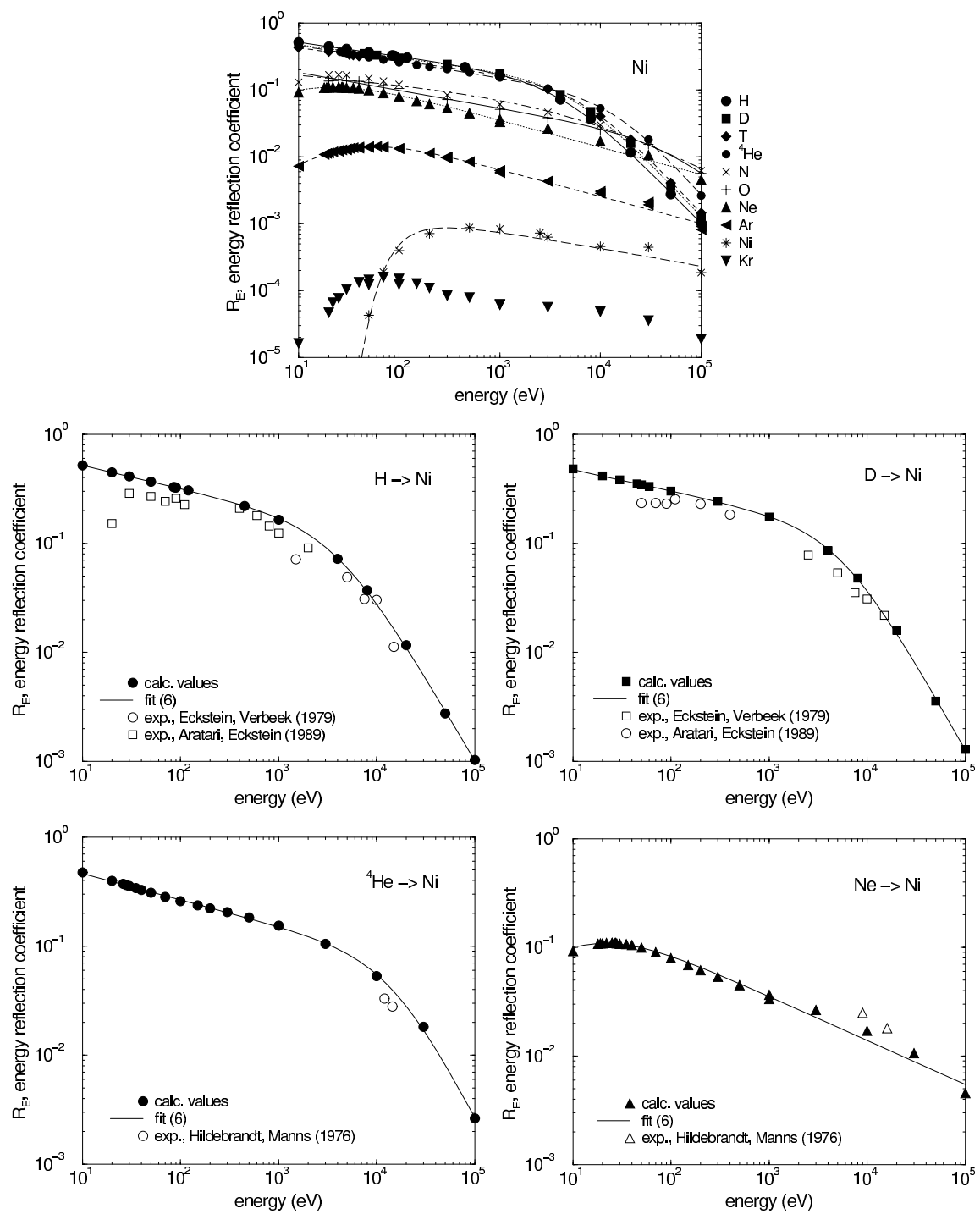

Figure 22: Calculated energy dependence of the energy reflection coefficient at normal incidence for the bombardment of $\mathrm{Ni}$ with $\mathrm{H}, \mathrm{D}, \mathrm{T},{ }^{4} \mathrm{He}, \mathrm{N}, \mathrm{O}, \mathrm{Ne}$, Ar, Ni, Kr [29, 30]. Lines are fits to the calculated values (see Table 10). Experimental data of $R_{E}$ are given for $\mathrm{H}[12,52]$, D [12, 52], ${ }^{4} \mathrm{He}$ [56], Ne [56] 

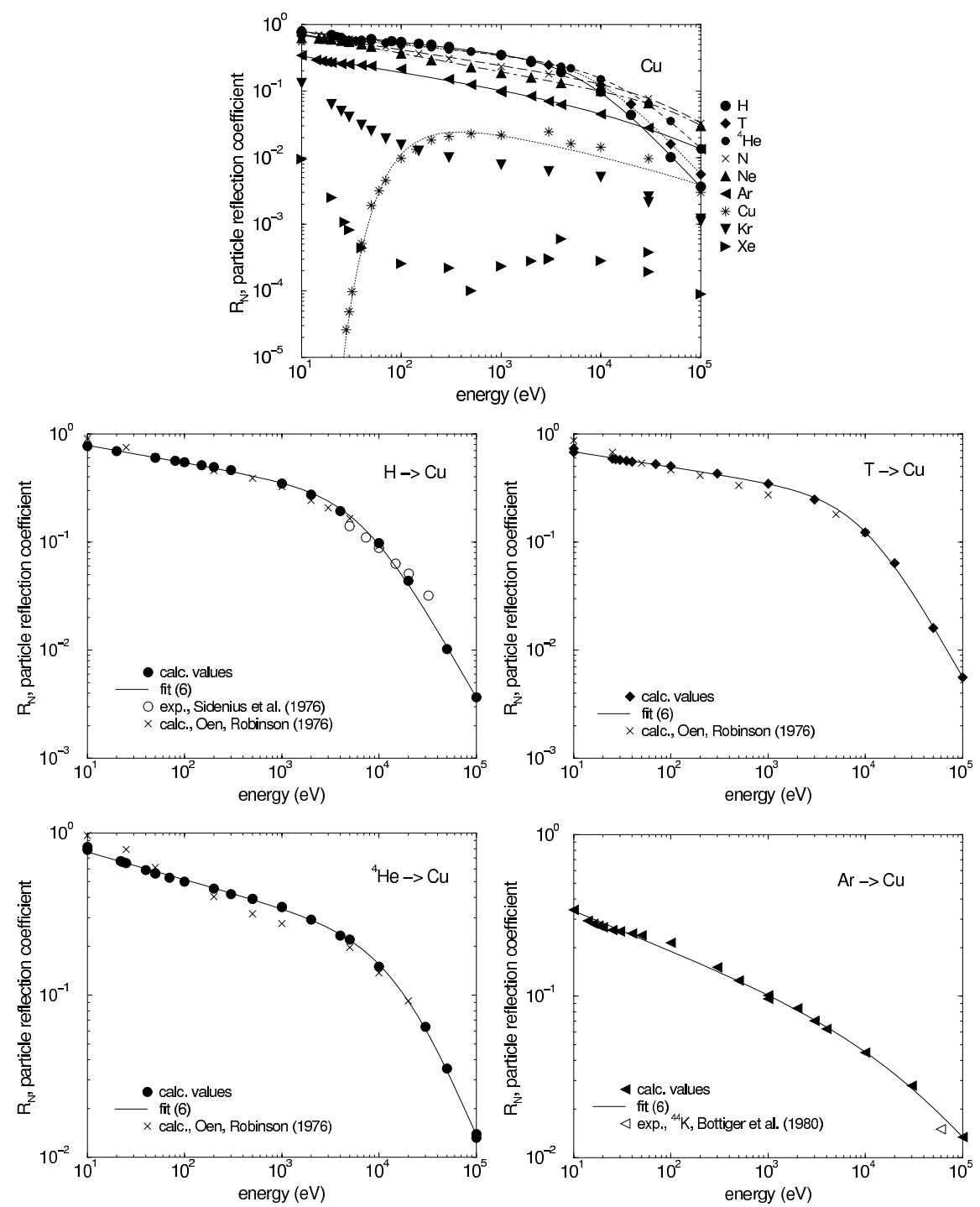

Figure 23: Calculated energy dependence of the particle reflection coefficient at normal incidence for the bombardment of $\mathrm{Cu}$ with $\mathrm{H}, \mathrm{T},{ }^{4} \mathrm{He}, \mathrm{N}, \mathrm{Ne}, \mathrm{Ar}, \mathrm{Cu}, \mathrm{Kr}$, $\mathrm{Xe}[29,30]$. Lines are fits to the calculated values (see Table 2). Experimental data of $R_{N}$ are given for $\mathrm{H}$ [16], calculated values for $\mathrm{H}$ [24], [65] (not shown), $\mathrm{T}[24],{ }^{4} \mathrm{He}[24]$ 

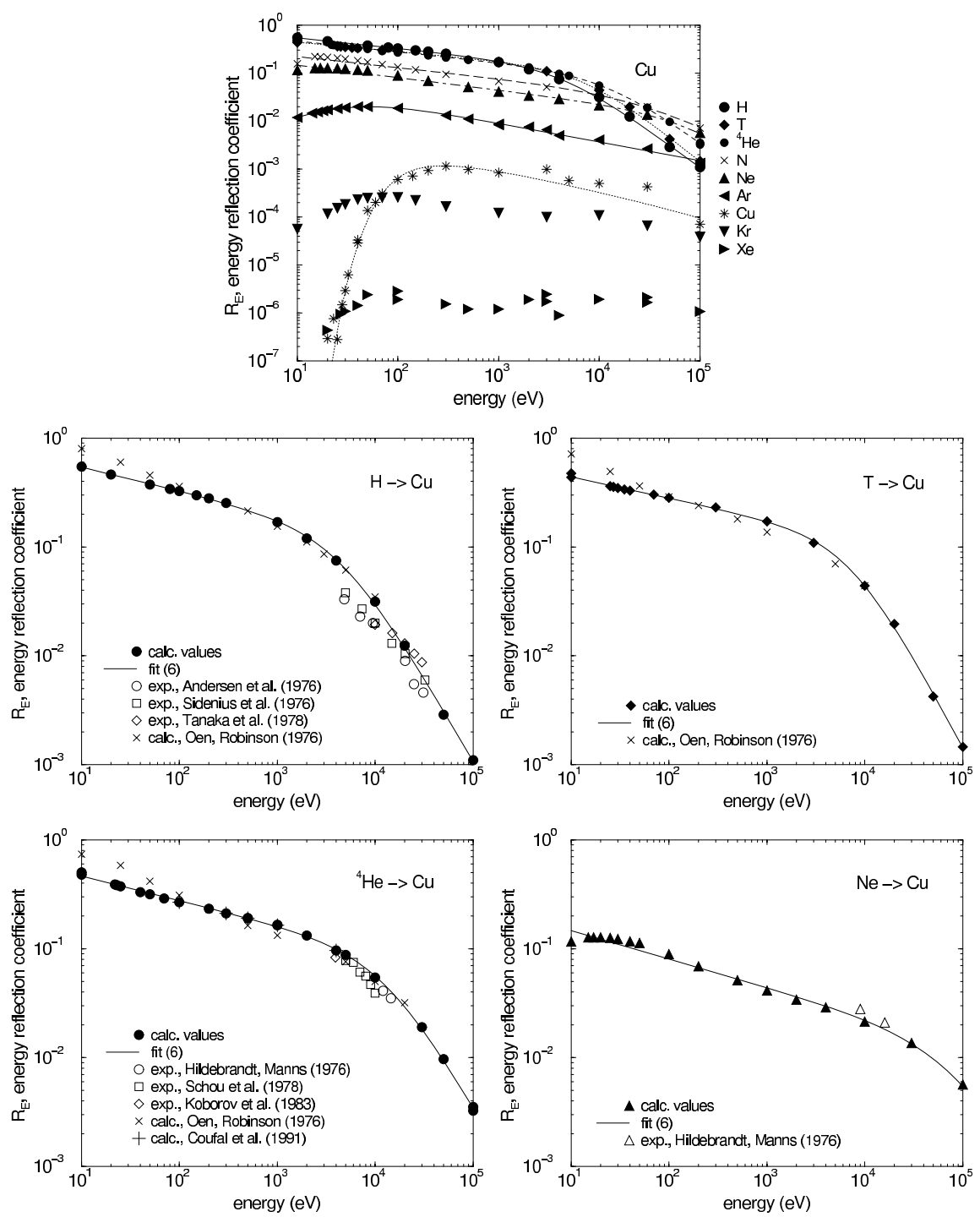

Figure 24: Calculated energy dependence of the energy reflection coefficient at normal incidence for the bombardment of $\mathrm{Cu}$ with $\mathrm{H}, \mathrm{T},{ }^{4} \mathrm{He}, \mathrm{N}, \mathrm{Ne}, \mathrm{Ar}, \mathrm{Cu}, \mathrm{Kr}$, Xe $[29,30]$. Lines are fits to the calculated values (see Table 10). Experimental data of $R_{E}$ are given for $\mathrm{H}[14,16,58],{ }^{4} \mathrm{He}[56,61,66]$, Ne [56], calculated values for $\mathrm{H}[24], \mathrm{T}[24],{ }^{4} \mathrm{He}[24,38]$ 

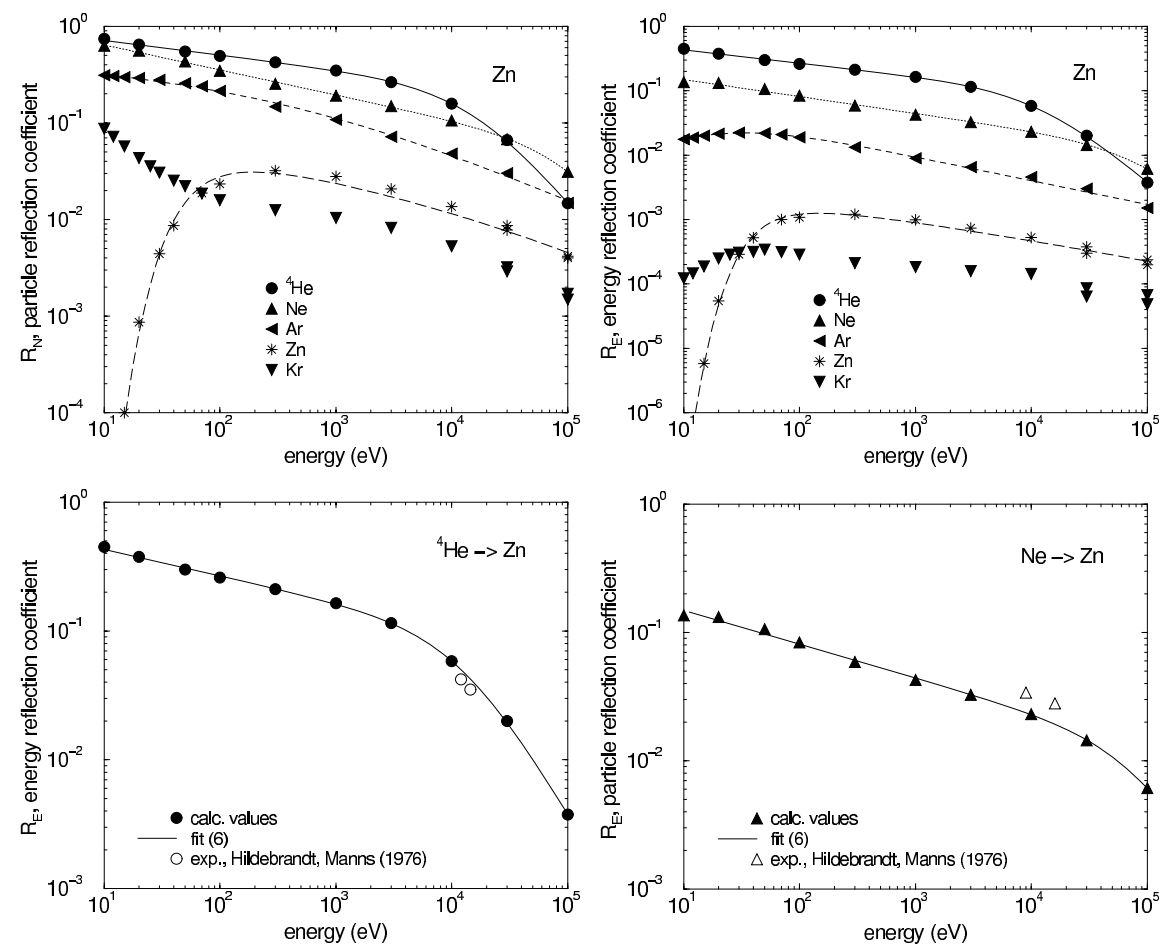

Figure 25: Calculated energy dependence of the particle and energy reflection coefficients at normal incidence for the bombardment of $\mathrm{Zn}$ with ${ }^{4} \mathrm{He}, \mathrm{Ne}, \mathrm{Ar}$, $\mathrm{Zn}, \mathrm{Kr}$ [30]. Lines are fits to the calculated values (see Tables 2 and 10). Experimental data of $R_{E}$ are given for ${ }^{4} \mathrm{He}$ [56] and Ne [56] 

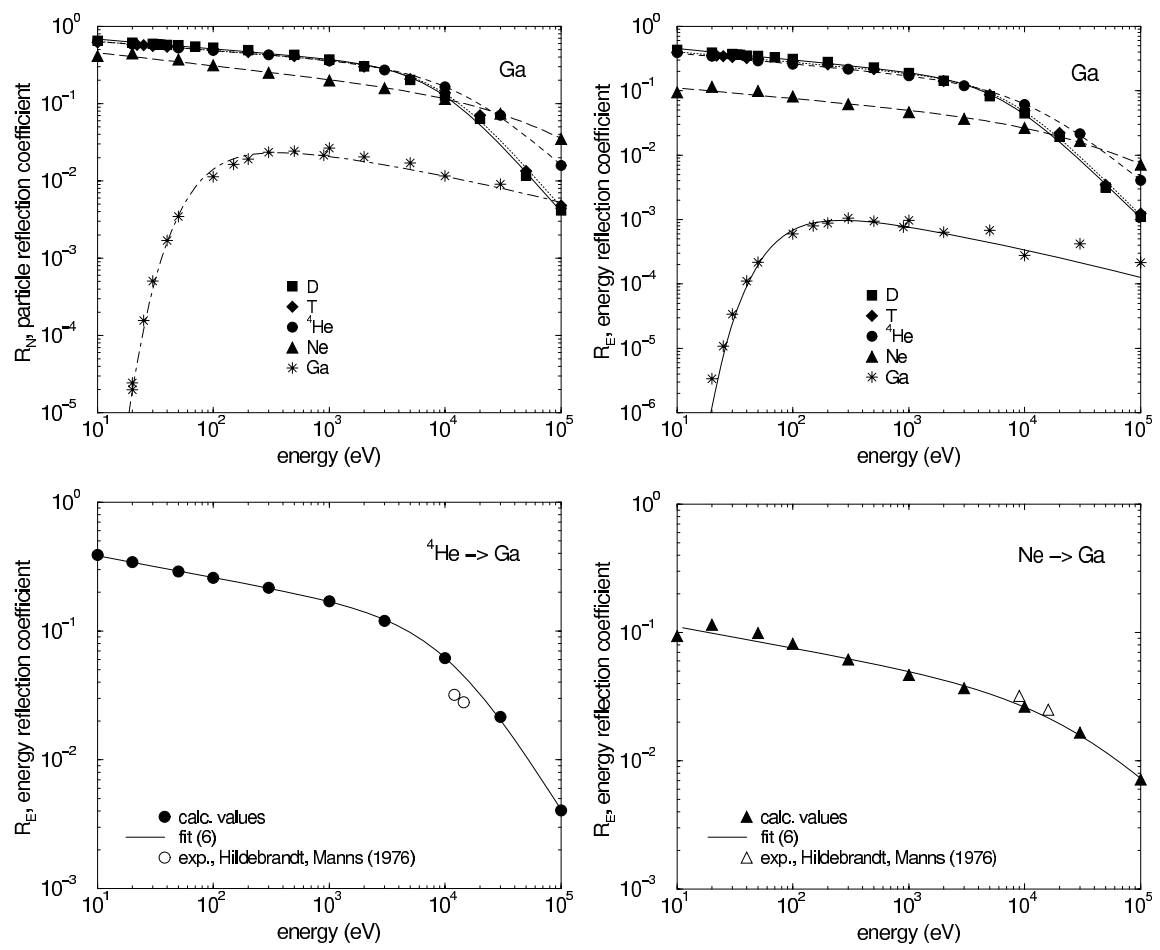

Figure 26: Calculated energy dependence of the particle and energy reflection coefficients at normal incidence for the bombardment of Ga with D, T, ${ }^{4} \mathrm{He}$, $\mathrm{Ne}, \mathrm{Ga}$ [30]. Lines are fits to the calculated values (see Tables 3 and 11). Experimental data of $R_{E}$ are given for ${ }^{4} \mathrm{He}$ [56] and Ne [56] 

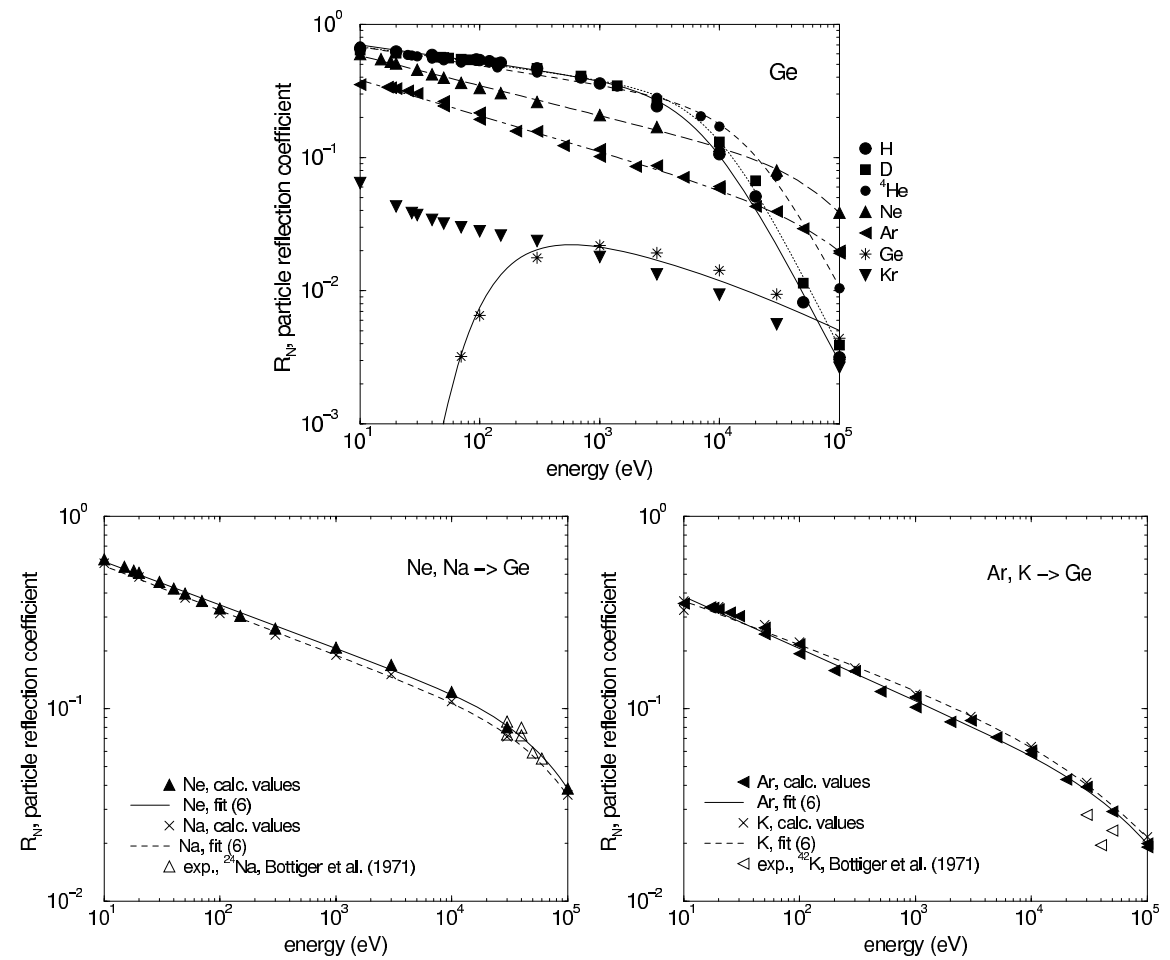

Figure 27: Calculated energy dependence of the particle reflection coefficient at normal incidence for the bombardment of Ge with $\mathrm{H}, \mathrm{D},{ }^{4} \mathrm{He}, \mathrm{Ne}, \mathrm{Ar}, \mathrm{Ge}, \mathrm{Kr}$ [30]. Lines are fits to the calculated values (see Table 3). Experimental data of $R_{N}$ are given for ${ }^{24} \mathrm{Na}$ [67] and ${ }^{42} \mathrm{~K}$ [67], theoretically determined values can be found in $[67,68]$ 

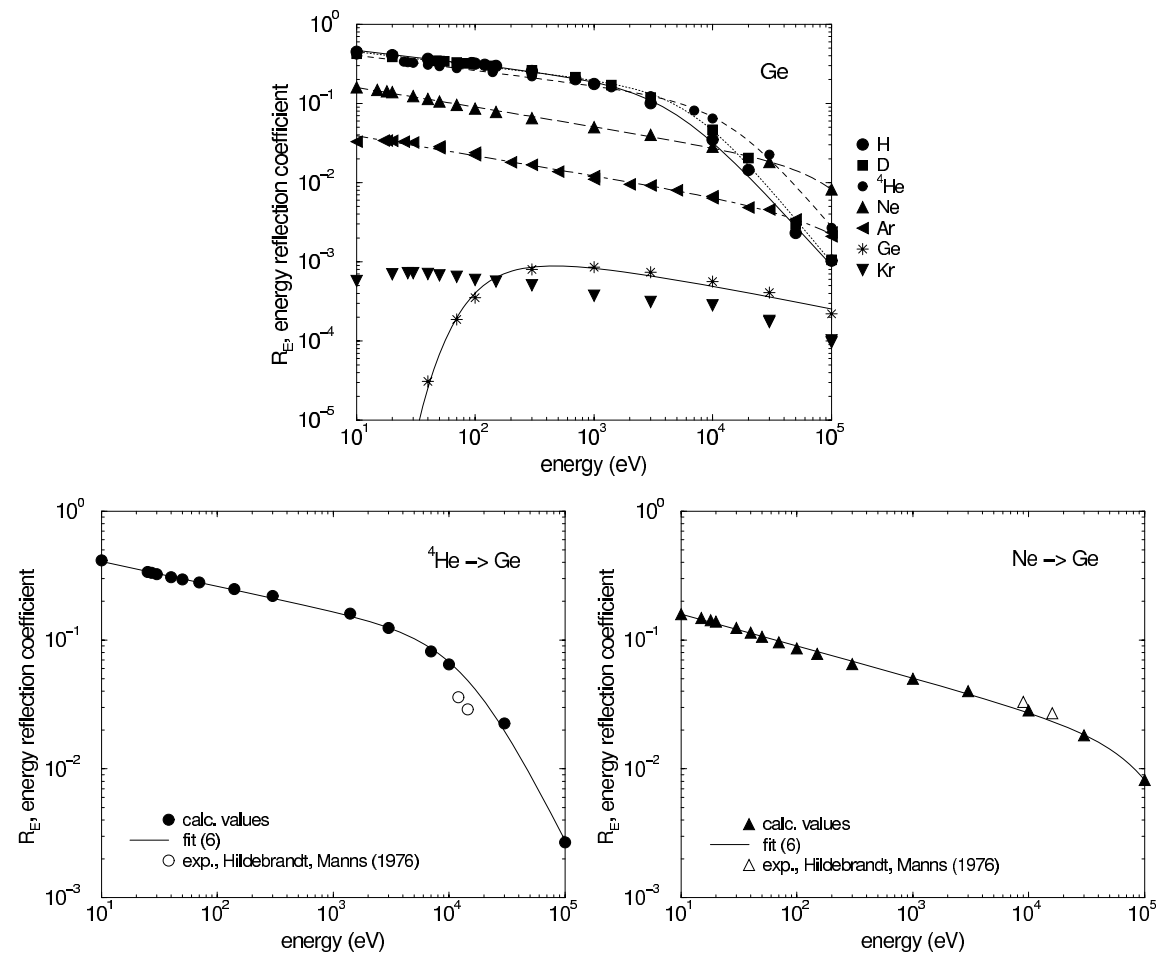

Figure 28: Calculated energy dependence of the energy reflection coefficient at normal incidence for the bombardment of Ge with $\mathrm{H}, \mathrm{D},{ }^{4} \mathrm{He}, \mathrm{Ne}, \mathrm{Ar}, \mathrm{Ge}, \mathrm{Kr}$ [30]. Lines are fits to the calculated values (see Table 11). Experimental data of $R_{E}$ are given for ${ }^{4} \mathrm{He}$ [56] and $\mathrm{Ne}$ [56] 

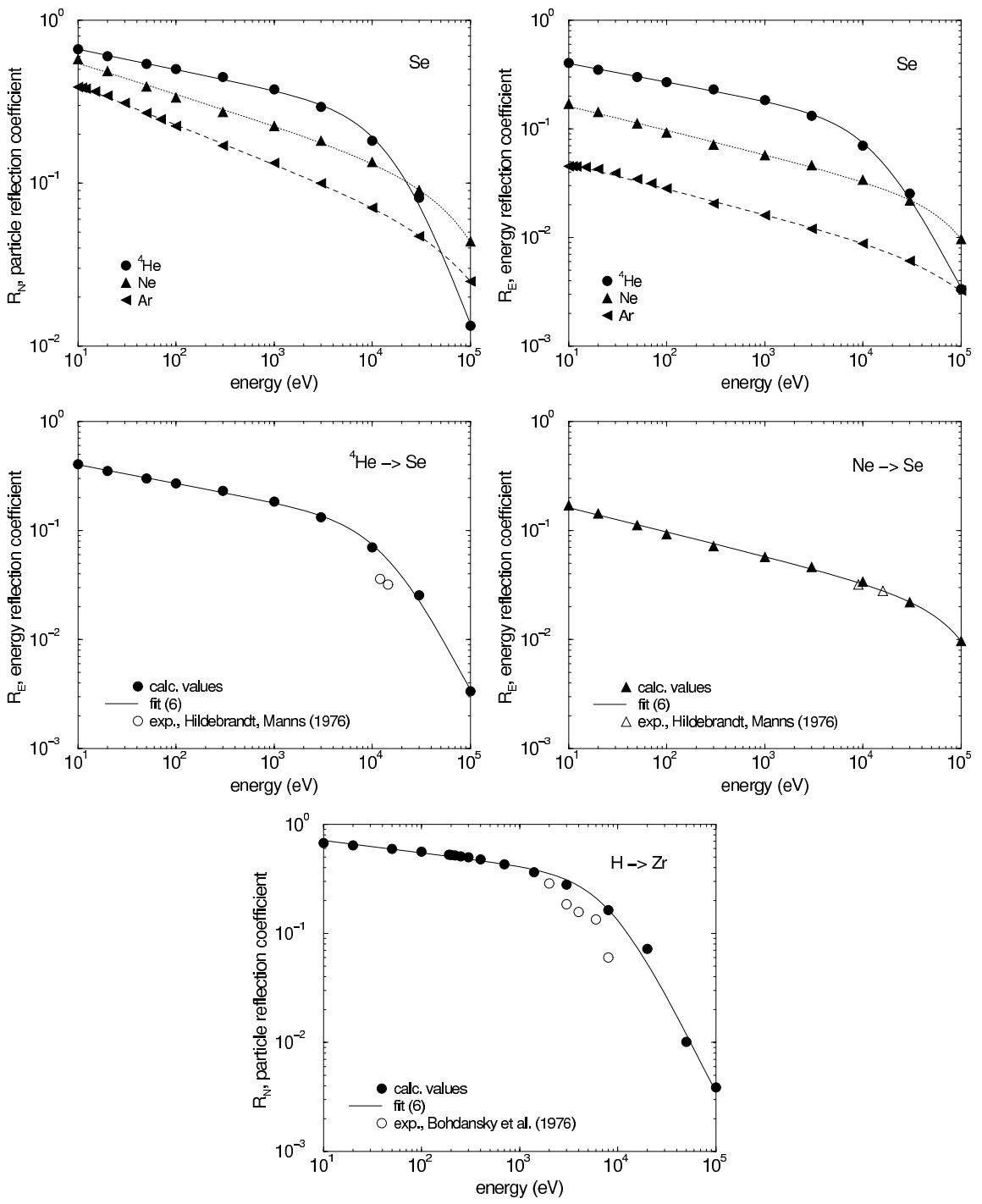

Figure 29: Calculated energy dependence of the particle and energy reflection coefficients at normal incidence for the bombardment of Se with ${ }^{4} \mathrm{He}, \mathrm{Ne}, \mathrm{Ar}$ [30]. Lines are fits to the calculated values (see Tables 33 and 11). Experimental data of $R_{E}$ are given for ${ }^{4} \mathrm{He}$ [56], Ne [56] on Se and of $R_{N}$ for $\mathrm{H}$ on $\mathrm{Zr}$ [60] 

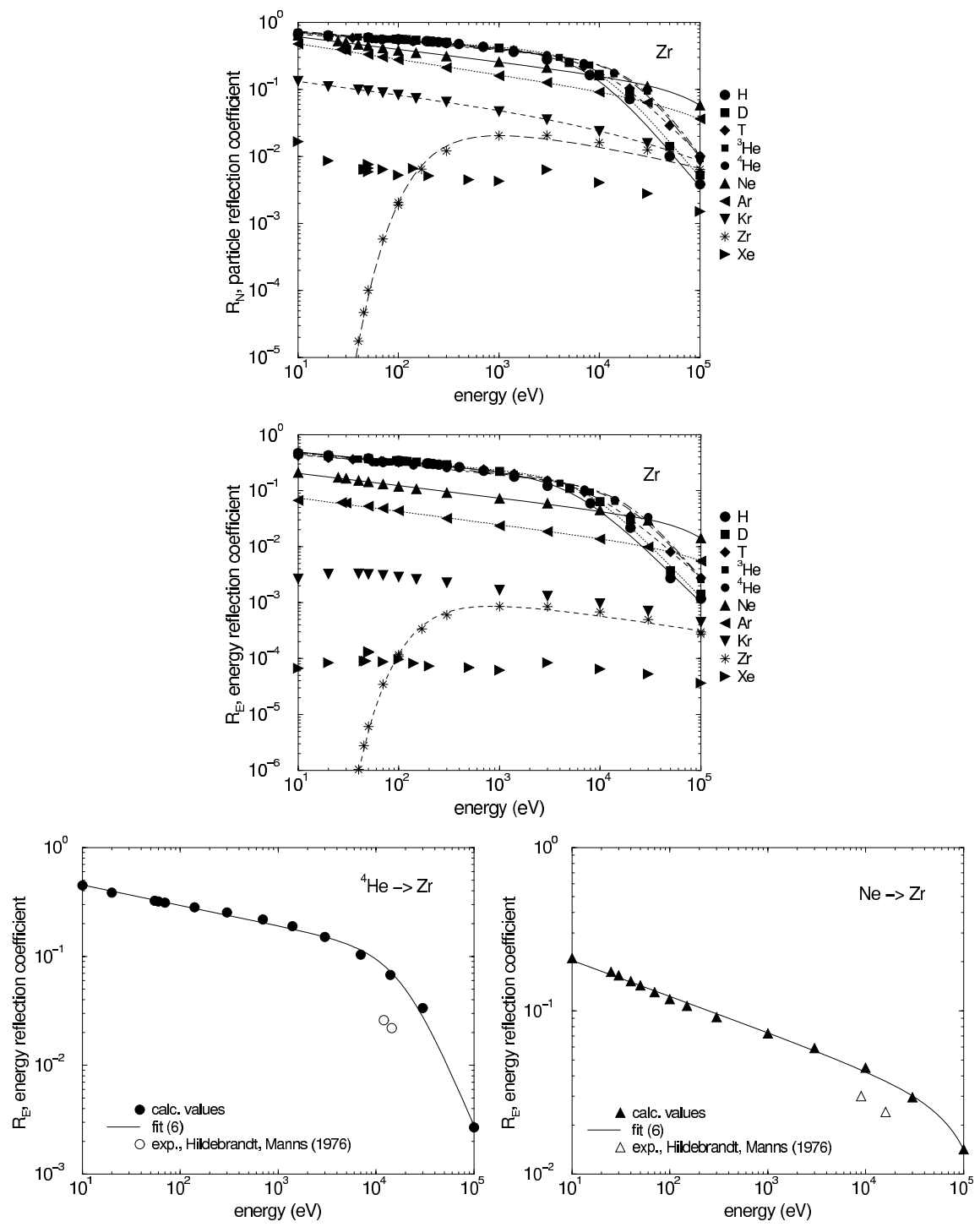

Figure 30: Calculated energy dependence of the particle and energy reflection coefficients at normal incidence for the bombardment of $\mathrm{Zr}$ with H, D, T, ${ }^{3} \mathrm{He}$, ${ }^{4} \mathrm{He}, \mathrm{Ne}, \mathrm{Ar}, \mathrm{Kr}, \mathrm{Zr}$, Xe [30]. Lines are fits to the calculated values (see Tables 3 and 11). Experimental data of $R_{E}$ are given for ${ }^{4} \mathrm{He}$ [56] and Ne [56] 

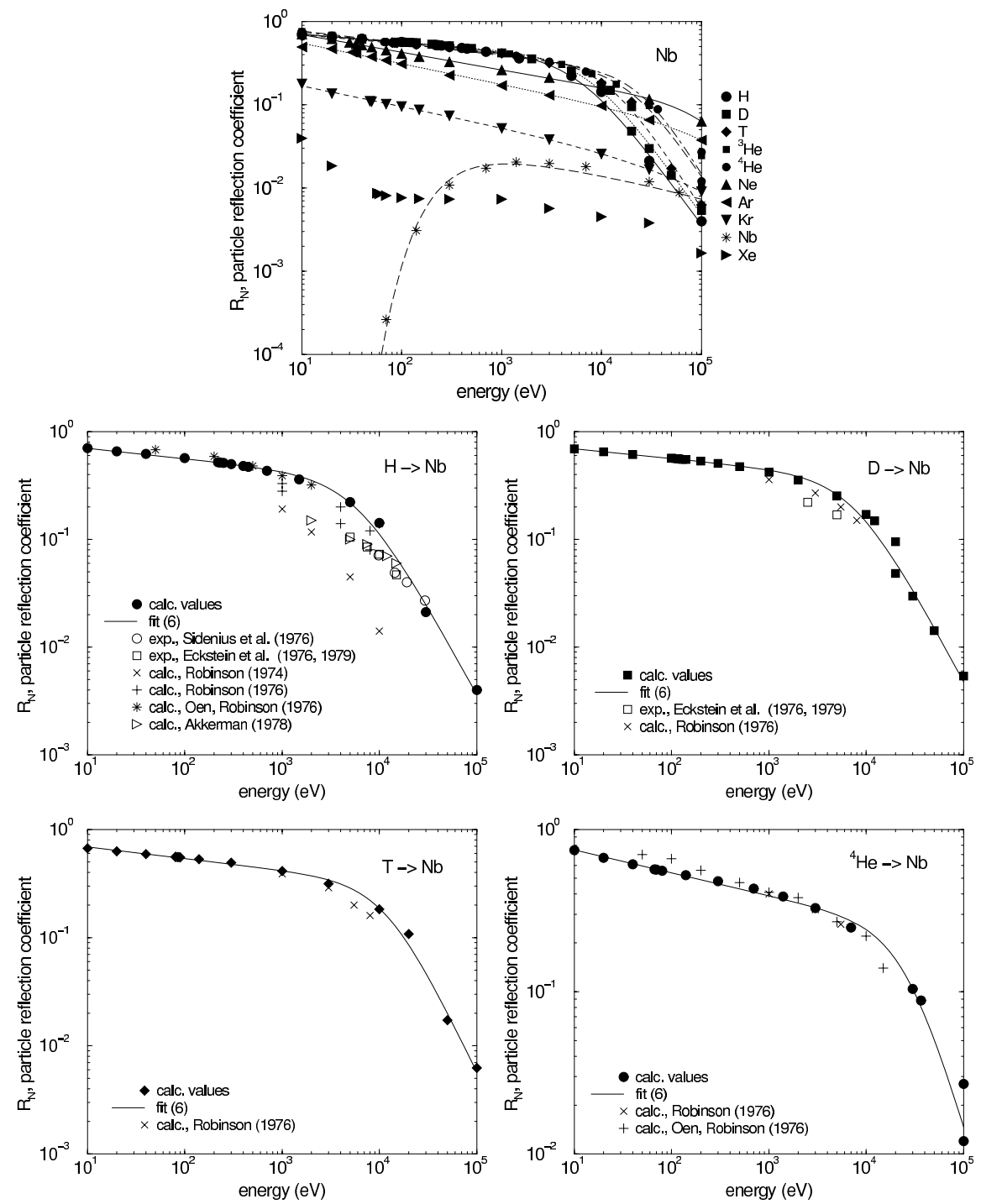

Figure 31: Calculated energy dependence of the particle reflection coefficient at normal incidence for the bombardment of $\mathrm{Nb}$ with $\mathrm{H}, \mathrm{D}, \mathrm{T},{ }^{3} \mathrm{He},{ }^{4} \mathrm{He}, \mathrm{Ne}$, $\mathrm{Ar}, \mathrm{Kr}, \mathrm{Nb}, \mathrm{Xe}[29,30]$. Lines are fits to the calculated values (see Table 3). Experimental data of $R_{N}$ are given for $\mathrm{H}$ [12, 16, 63], D [12, 63], calculated values for $\mathrm{H}[24,54,59,70], \mathrm{D}[54], \mathrm{T}[54],{ }^{4} \mathrm{He}[24,54]$ 

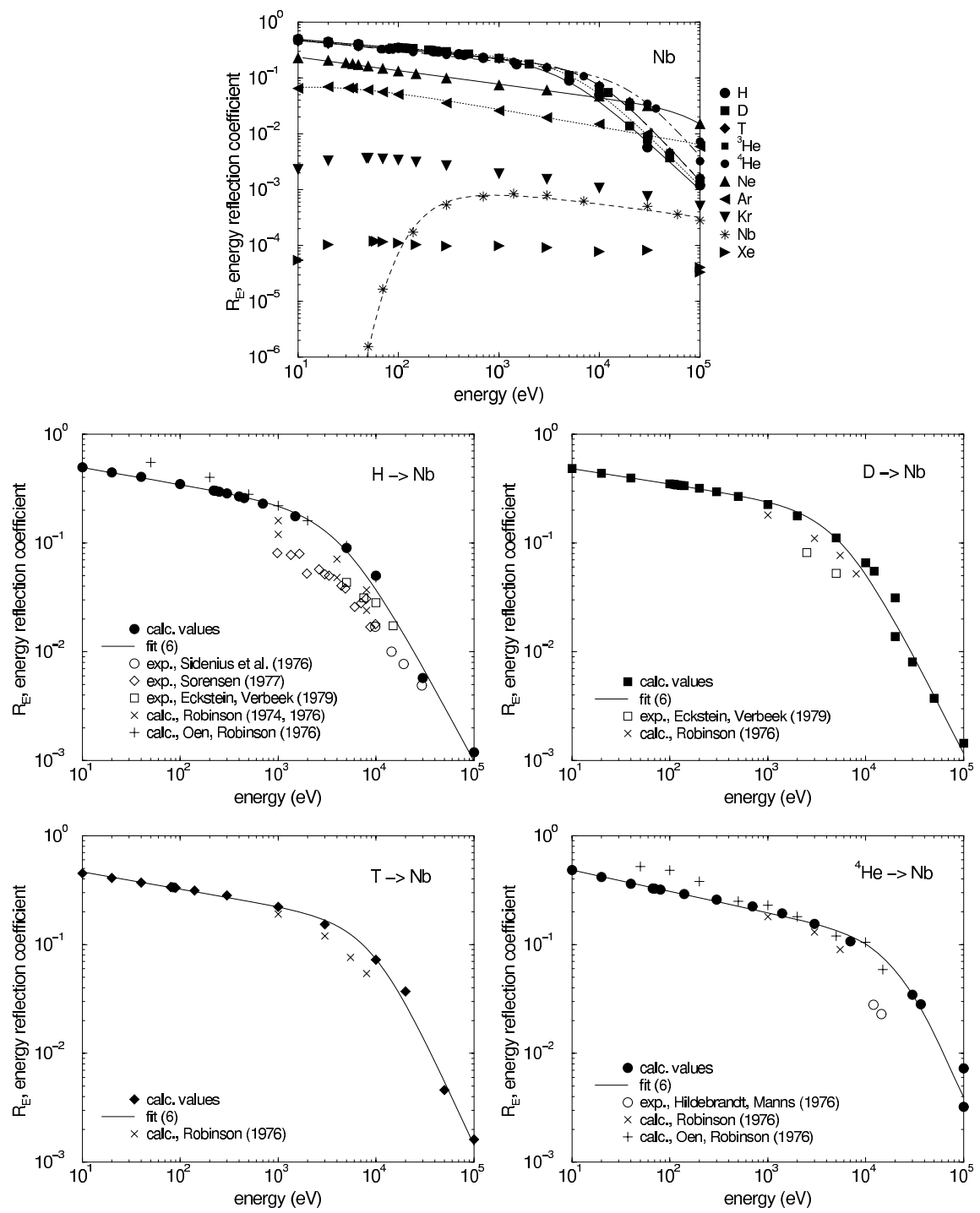

Figure 32: Calculated energy dependence of the energy reflection coefficient at normal incidence for the bombardment of $\mathrm{Nb}$ with $\mathrm{H}, \mathrm{D}, \mathrm{T},{ }^{3} \mathrm{He},{ }^{4} \mathrm{He}$, Ne, $\mathrm{Ar}, \mathrm{Kr}, \mathrm{Nb}, \mathrm{Xe}[29,30]$. Lines are fits to the calculated values (see Table 11). Experimental data of $R_{E}$ are given for $\mathrm{H}[12,16,69]$, D [12], ${ }^{4} \mathrm{He}$ [56], calculated values for $\mathrm{H}[24,54,70], \mathrm{D}[54], \mathrm{T}[54],{ }^{4} \mathrm{He}[24,54]$ 

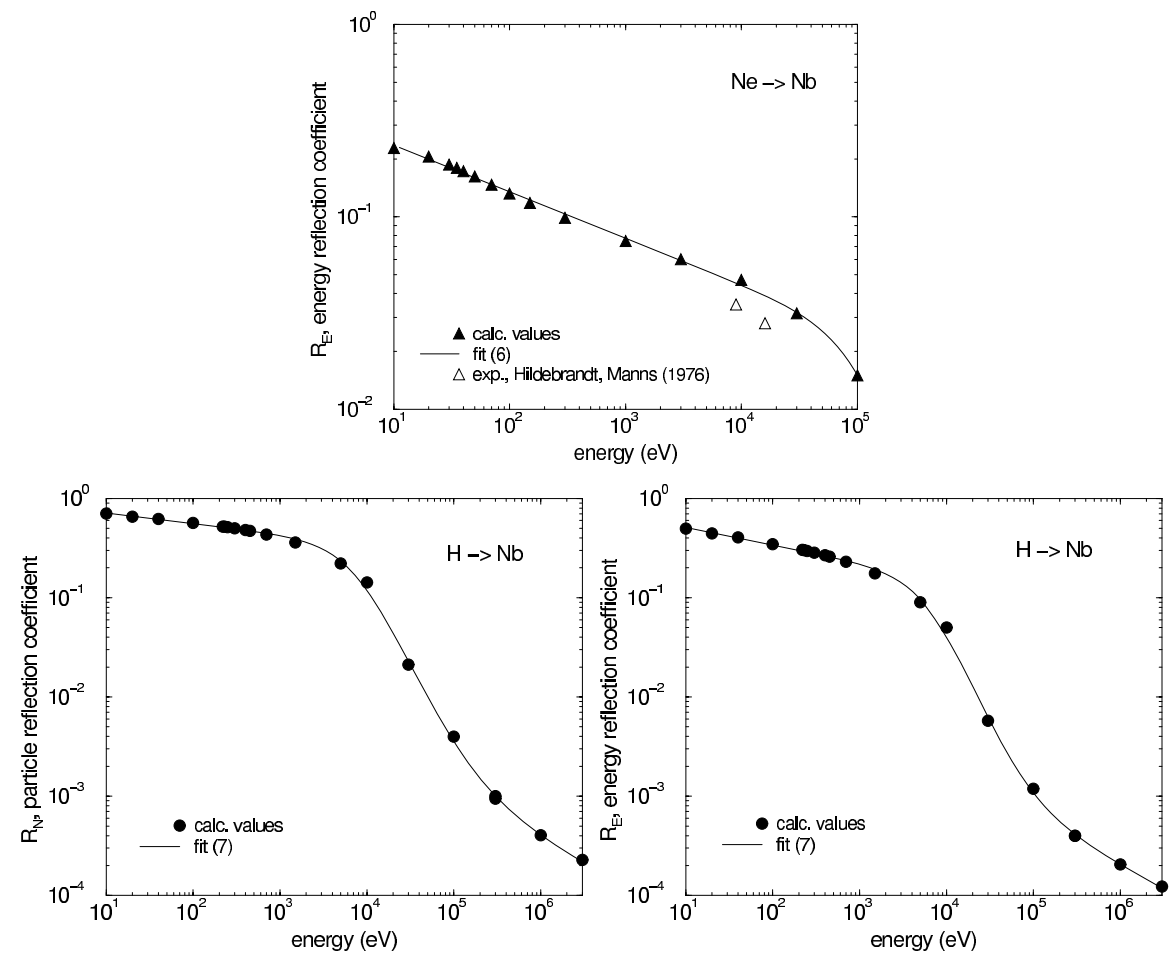

Figure 33: Calculated energy dependence of the energy reflection coefficient at normal incidence for the bombardment of $\mathrm{Nb}$ with $\mathrm{Ne}$. Experimental data of $R_{E}$ are given for $\mathrm{Ne}$ on $\mathrm{Nb}$ [56]. Calculated energy dependence of the particle and energy reflection coefficients at normal incidence for the bombardment of $\mathrm{Nb}$ with $\mathrm{H}$ up to high energies $(3 \mathrm{MeV})$ [30]. Lines are fits to the calculated values (see Tables 11, 7 and 15) 

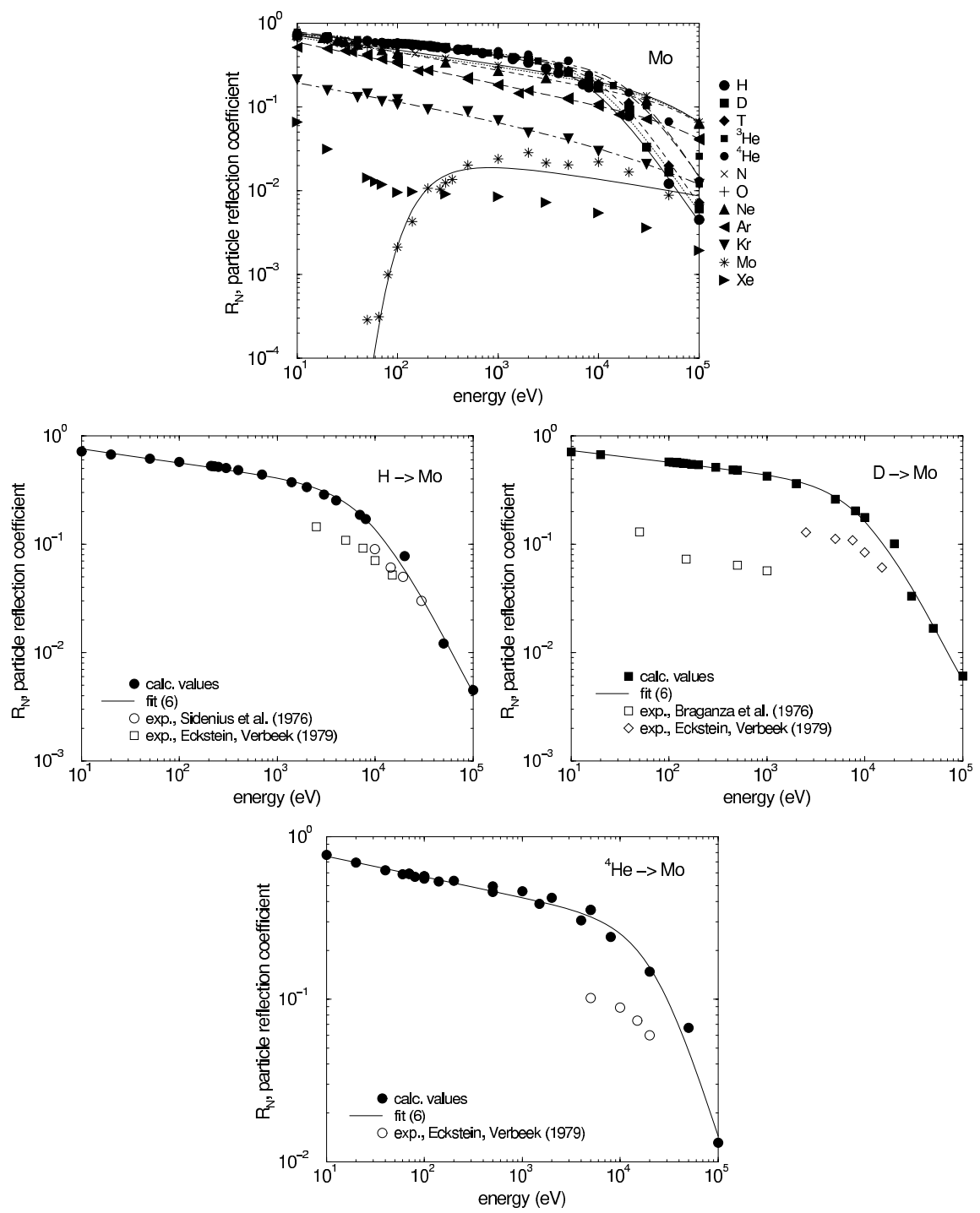

Figure 34: Calculated energy dependence of the particle reflection coefficient at normal incidence for the bombardment of Mo with $\mathrm{H}, \mathrm{D}, \mathrm{T},{ }^{3} \mathrm{He},{ }^{4} \mathrm{He}, \mathrm{N}, \mathrm{O}$, $\mathrm{Ne}, \mathrm{Ar}, \mathrm{Kr}, \mathrm{Mo}$, Xe [29, 30]. Lines are fits to the calculated values (see Table 3 ). Experimental data of $R_{N}$ are given for $\mathrm{H}[12,16]$, D [12, 71], ${ }^{4} \mathrm{He}$ [12] Older experimental data by [72] are not shown 

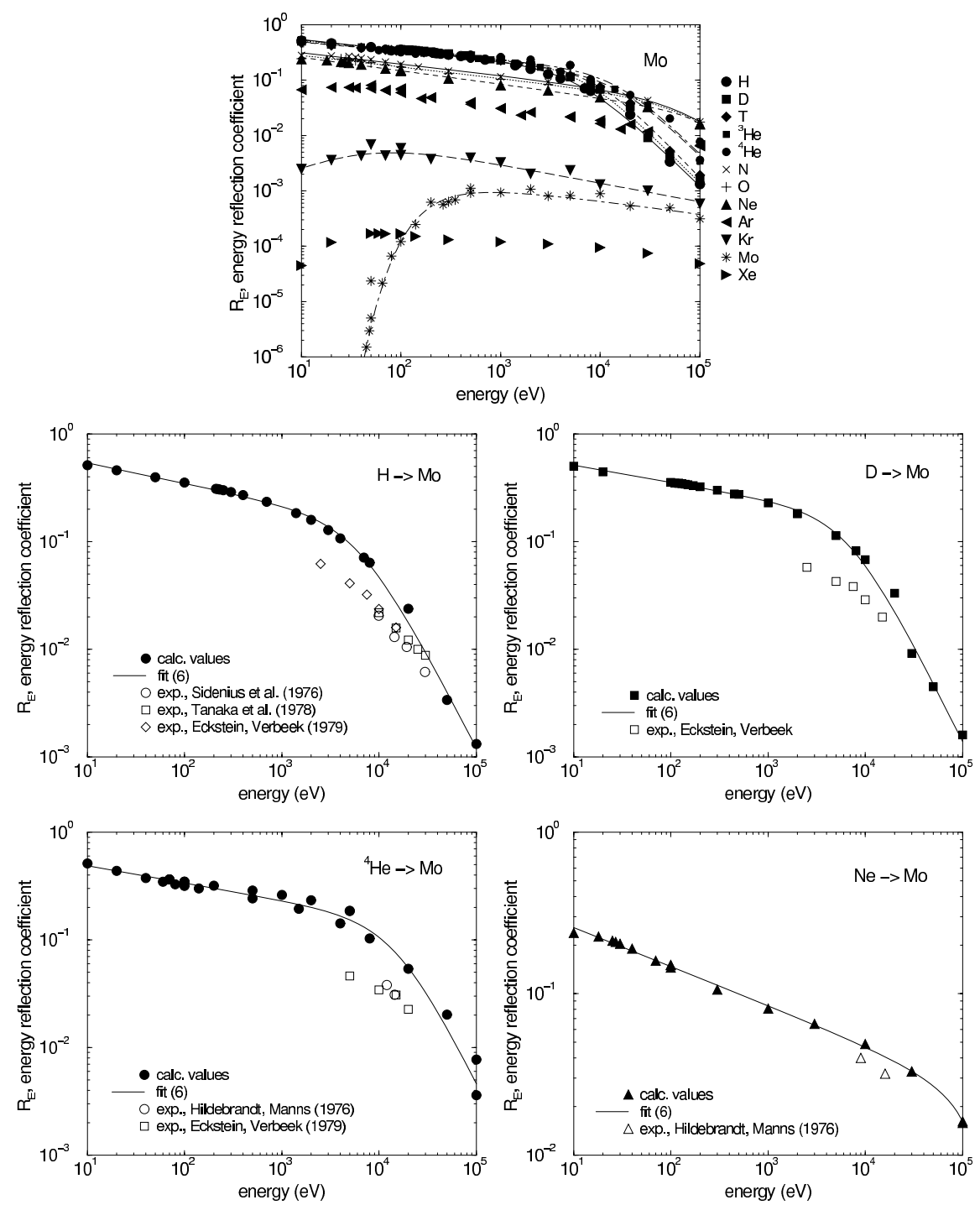

Figure 35: Calculated energy dependence of the energy reflection coefficient at normal incidence for the bombardment of Mo with $\mathrm{H}, \mathrm{D}, \mathrm{T},{ }^{3} \mathrm{He},{ }^{4} \mathrm{He}, \mathrm{N}, \mathrm{O}$, $\mathrm{Ne}, \mathrm{Ar}, \mathrm{Kr}, \mathrm{Mo}$, Xe [29, 30]. Lines are fits to the calculated values (see Table 11). Experimental data of $R_{E}$ are given for $\mathrm{H}[12,16,58]$, D [12], ${ }^{4} \mathrm{He}[12,56]$, Ne [56] 

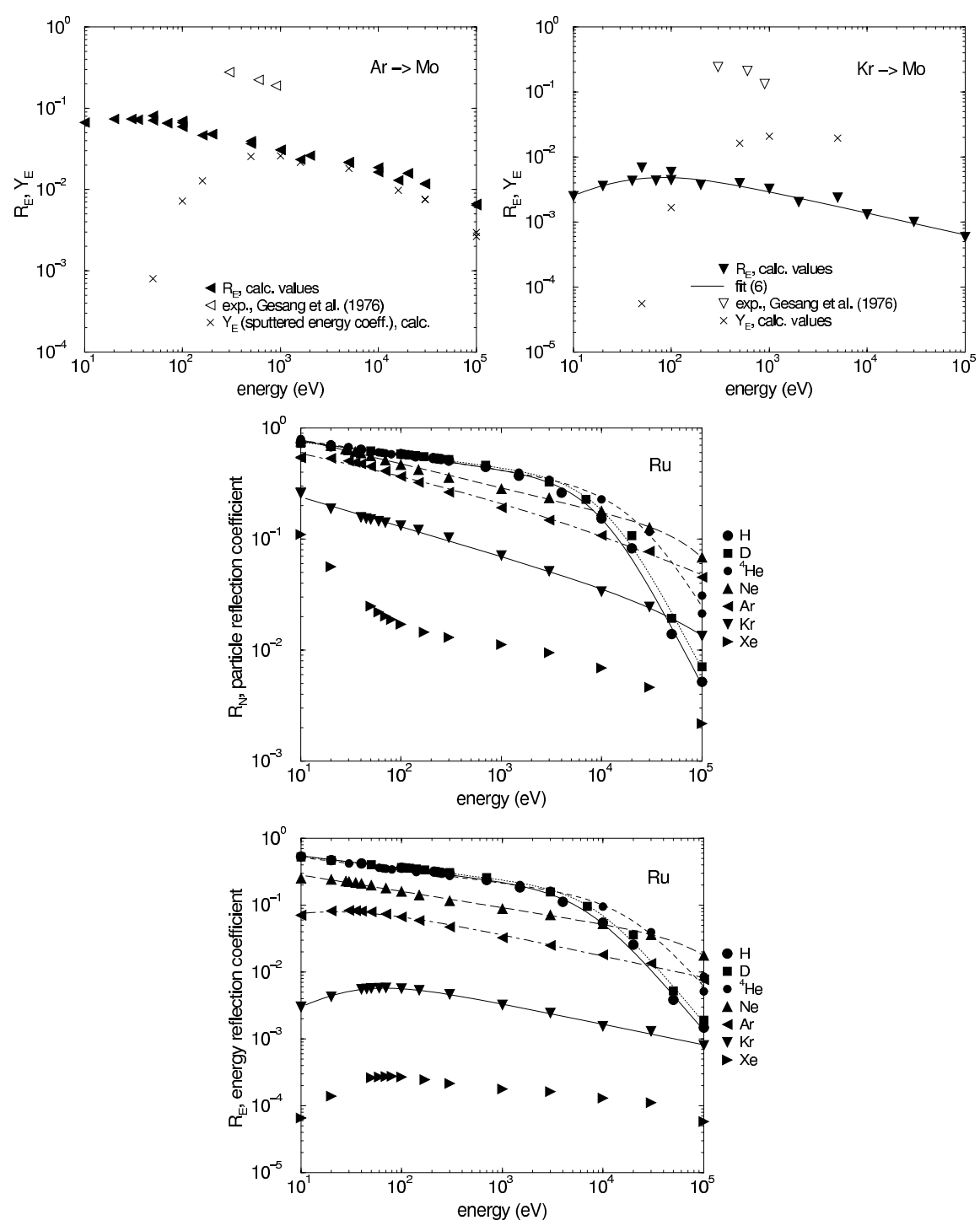

Figure 36: Calculated energy dependence of the energy reflection $R_{E}$ and sputtered energy coefficients, $R_{E}$ and $Y_{E}$, at normal incidence for the bombardment of Mo with Ar and Kr; experimental data by [62]. Calculated energy dependence of the particle and energy reflection coefficients at normal incidence for the bombardment of $\mathrm{Ru}$ with $\mathrm{H}, \mathrm{D},{ }^{4} \mathrm{He}, \mathrm{Ne}, \mathrm{Ar}, \mathrm{Kr}$, Xe [29, 30]. Lines are fits to the calculated values (see Table 3 and 11) 

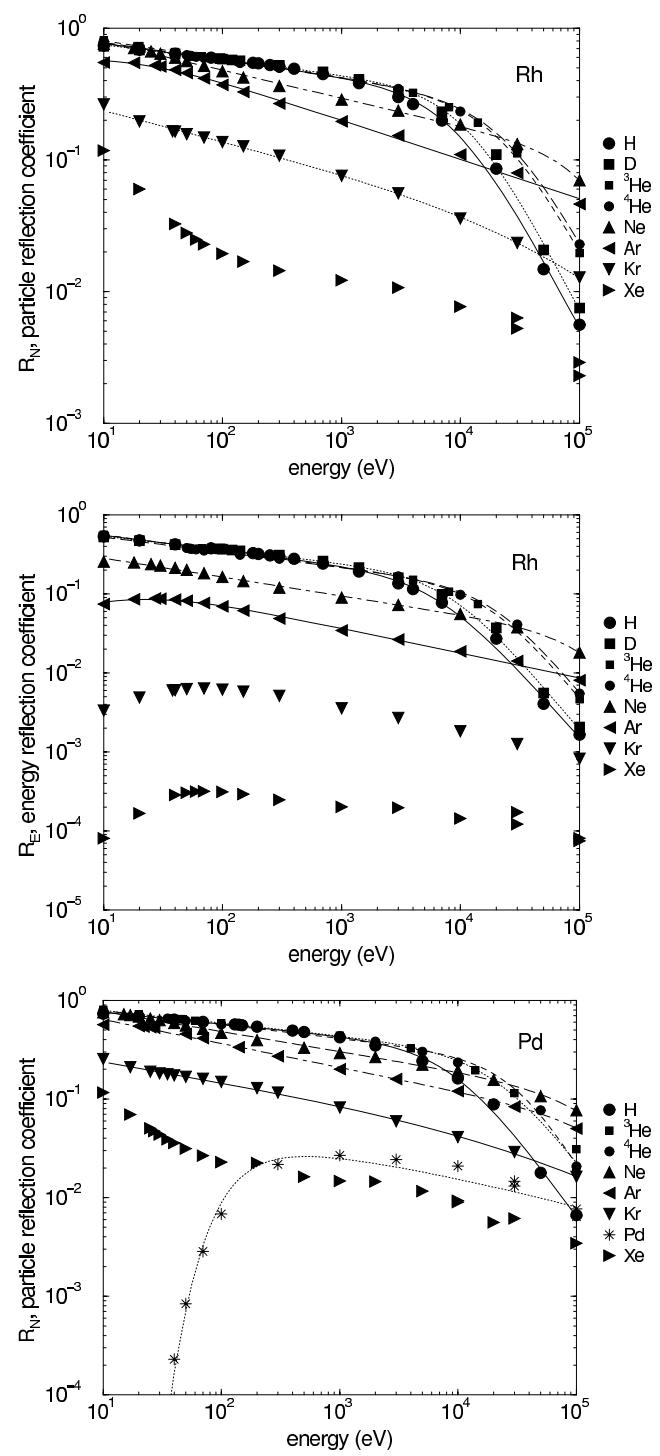

Figure 37: Calculated energy dependence of the particle and energy reflection coefficients at normal incidence for the bombardment of $\mathrm{Rh}$ and $\mathrm{Pd}$ with $\mathrm{H}, \mathrm{D}$, ${ }^{3} \mathrm{He},{ }^{4} \mathrm{He}, \mathrm{Ne}, \mathrm{Ar}, \mathrm{Kr}, \mathrm{Pd}, \mathrm{Xe}[29,30]$. Lines are fits to the calculated values (see Table 4 and 12) 

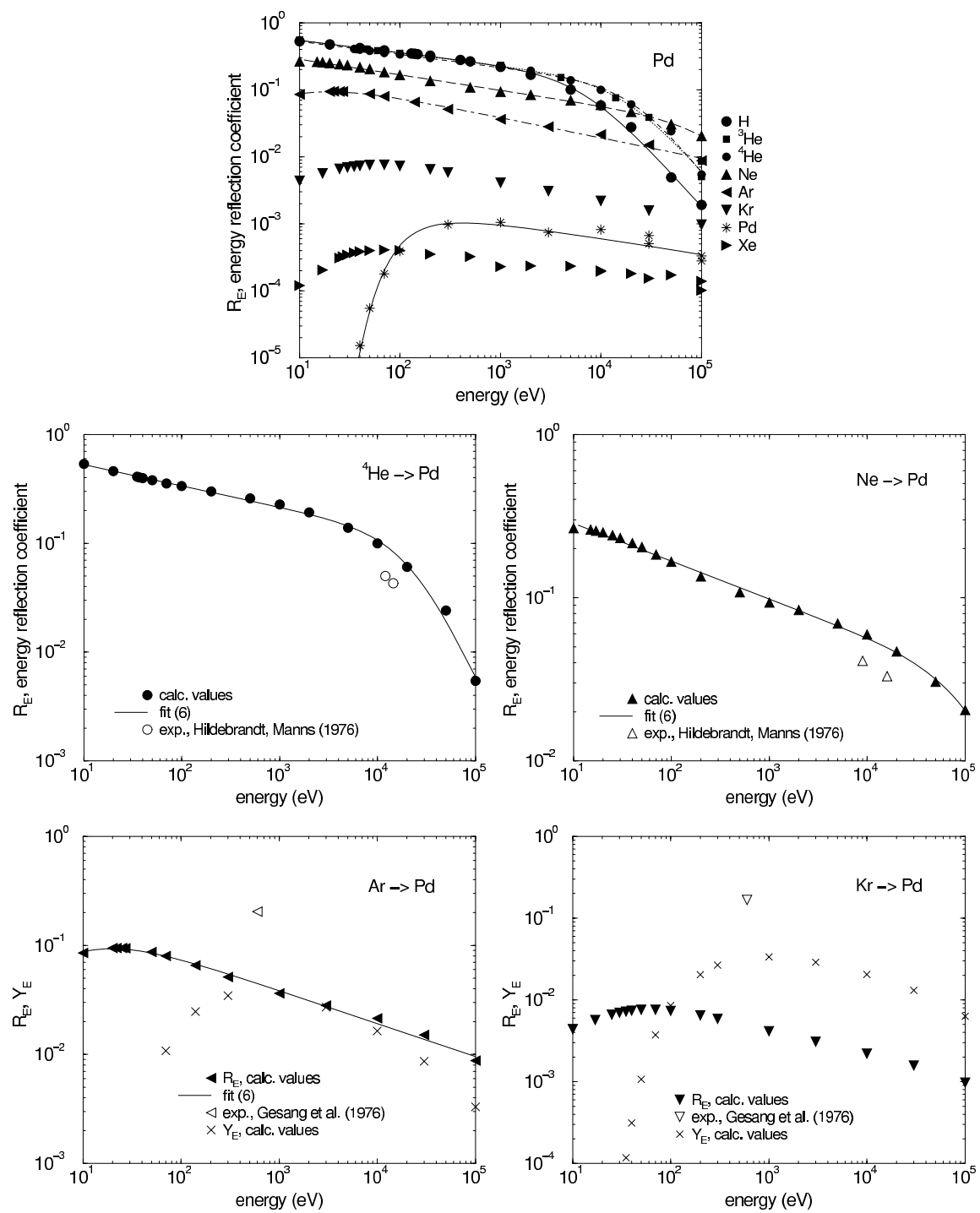

Figure 38: Calculated energy dependence of the energy reflection coefficient at normal incidence for the bombardment of $\mathrm{Pd}$ with $\mathrm{H},{ }^{3} \mathrm{He},{ }^{4} \mathrm{He}, \mathrm{Ne}, \mathrm{Ar}, \mathrm{Kr}, \mathrm{Pd}$, Xe $[29,30]$. Lines are fits to the calculated values (see Table 12). Experimental data of $R_{E}$ are given for ${ }^{4} \mathrm{He}$ [56], Ne [56], Ar and $\mathrm{Kr}$ [62]. For the last two cases the calculated sputtered energy, $Y_{E}$, is provided 

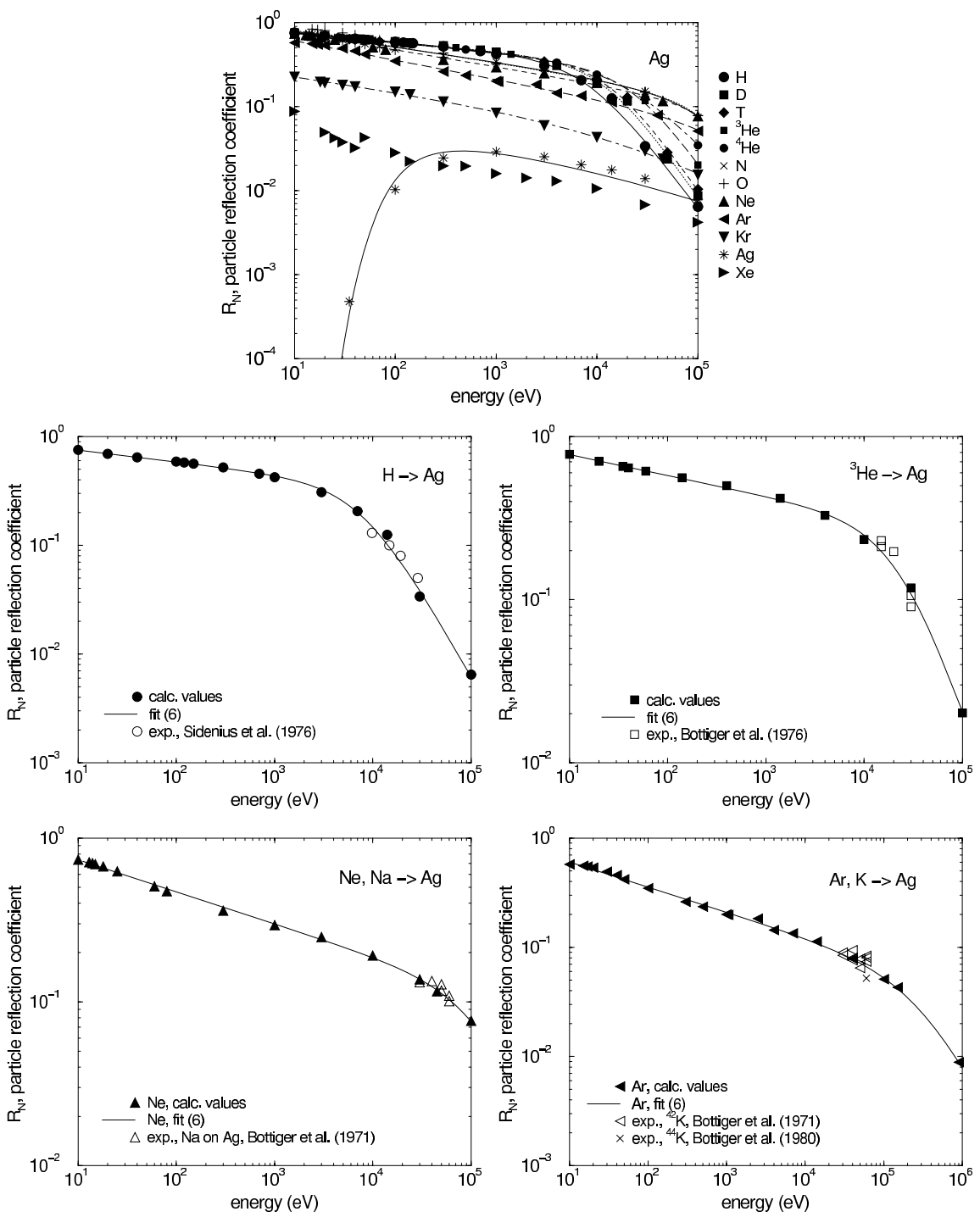

Figure 39: Calculated energy dependence of the particle reflection coefficient at normal incidence for the bombardment of $\mathrm{Ag}$ with $\mathrm{H}, \mathrm{D}, \mathrm{T},{ }^{3} \mathrm{He},{ }^{4} \mathrm{He}, \mathrm{N}, \mathrm{O}, \mathrm{Ne}$, $\mathrm{Ar}, \mathrm{Kr}, \mathrm{Ag}, \mathrm{Xe}[29,30]$. Lines are fits to the calculated values (see Table 4). Experimental data of $\mathrm{R}_{E}$ are given for $\mathrm{H}[16],{ }^{3} \mathrm{He}$ [73], Na [67], ${ }^{42} \mathrm{~K}$ [67], ${ }^{44} \mathrm{~K}$ [57], theoretically determined values can be found in $[67,68]$ 

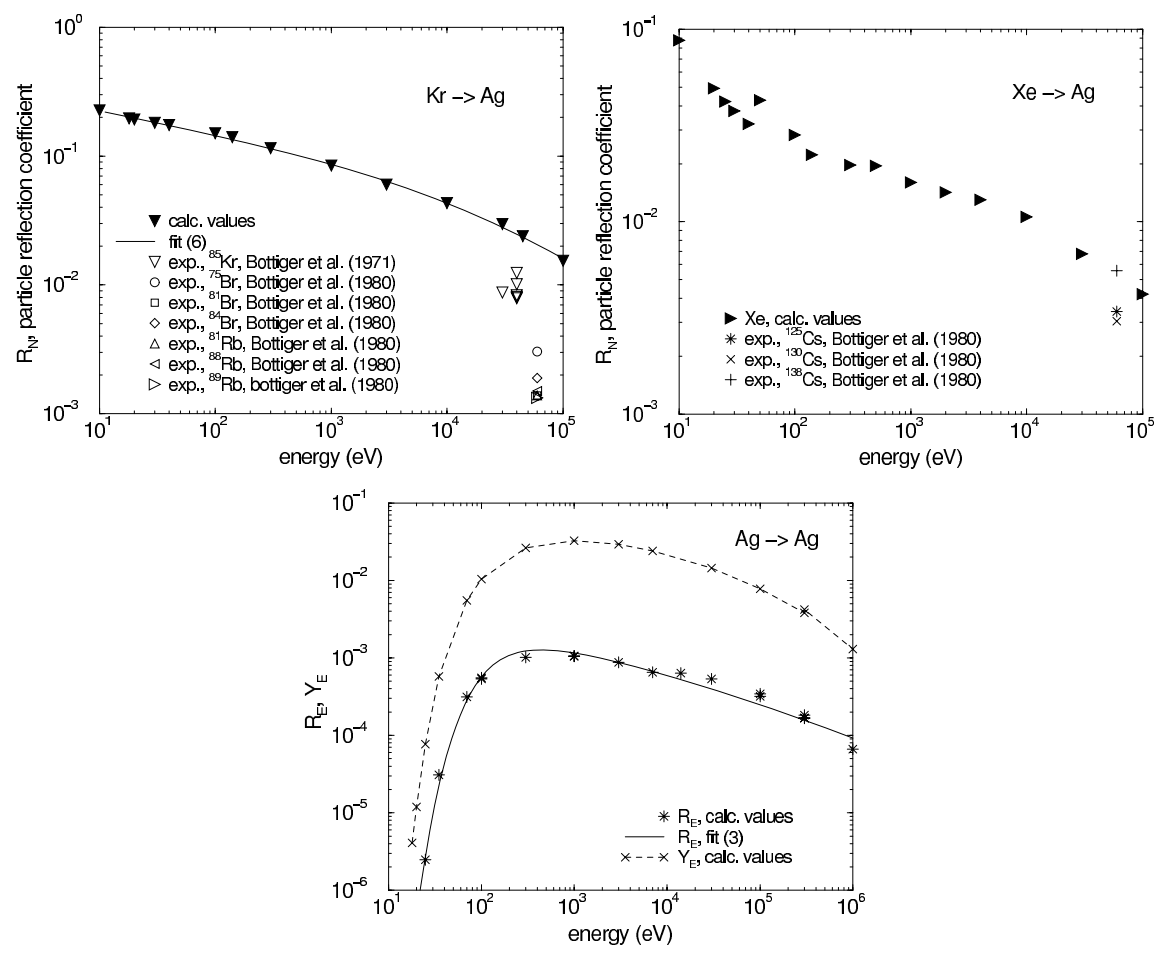

Figure 40: Calculated energy dependence of the particle and energy reflection coefficients at normal incidence for the bombardment of $\mathrm{Ag}$ with $\mathrm{Kr}$ and Xe. The line is a fit to the calculated values (see Table 4). Experimental data of $\mathrm{R}_{N}$ are given for ${ }^{85} \mathrm{Kr}$ [67], ${ }^{75} \mathrm{Br},{ }^{84} \mathrm{Br}[57],{ }^{81} \mathrm{Rb},{ }^{88} \mathrm{Rb},{ }^{89} \mathrm{Rb}{ }^{125} \mathrm{Cs},{ }^{130} \mathrm{Cs}$, ${ }^{138} \mathrm{Cs}$ [57], theoretically determined values can be found in [67, 68]. For $\mathrm{Ag}$ selfbombardment a comparison of calculated values of $R_{E}$ and $Y_{E}$ is provided; the lines are drawn to guide the eye 

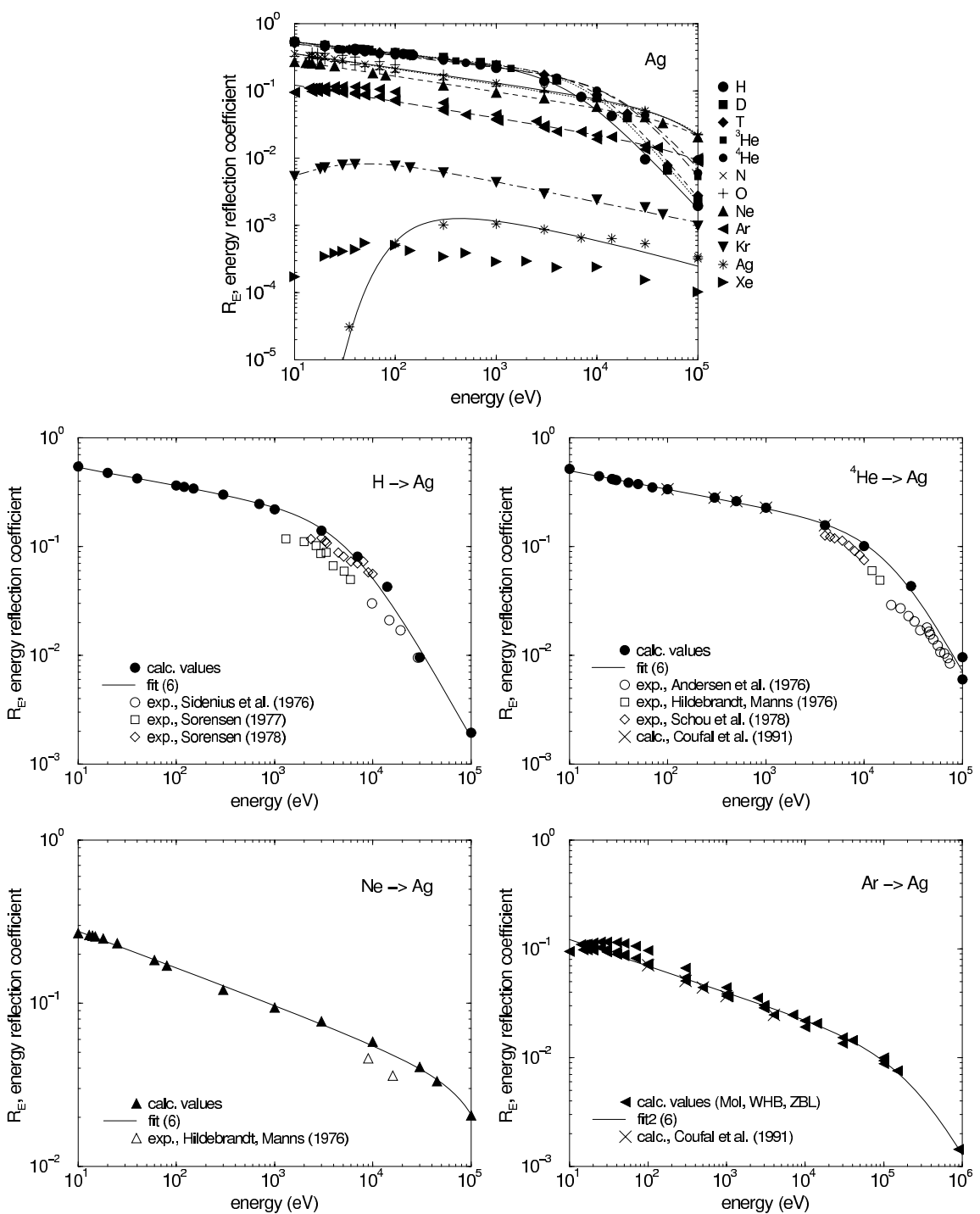

Figure 41: Calculated energy dependence of the energy reflection coefficient at normal incidence for the bombardment of $\mathrm{Ag}$ with $\mathrm{H}, \mathrm{D}, \mathrm{T},{ }^{3} \mathrm{He},{ }^{4} \mathrm{He}, \mathrm{N}, \mathrm{O}, \mathrm{Ne}$, $\mathrm{Ar}, \mathrm{Kr}, \mathrm{Ag}$, Xe [29, 30]. Lines are fits to the calculated values (see Table 12). Experimental data of $R_{E}$ are given for $\mathrm{H}[16,69,74],{ }^{4} \mathrm{He}[14,56,66]$, Ne [56]. Calculated values are published for Ne, Ar, Xe [38]. For Ar, calculated values for three interaction potentials are plotted 

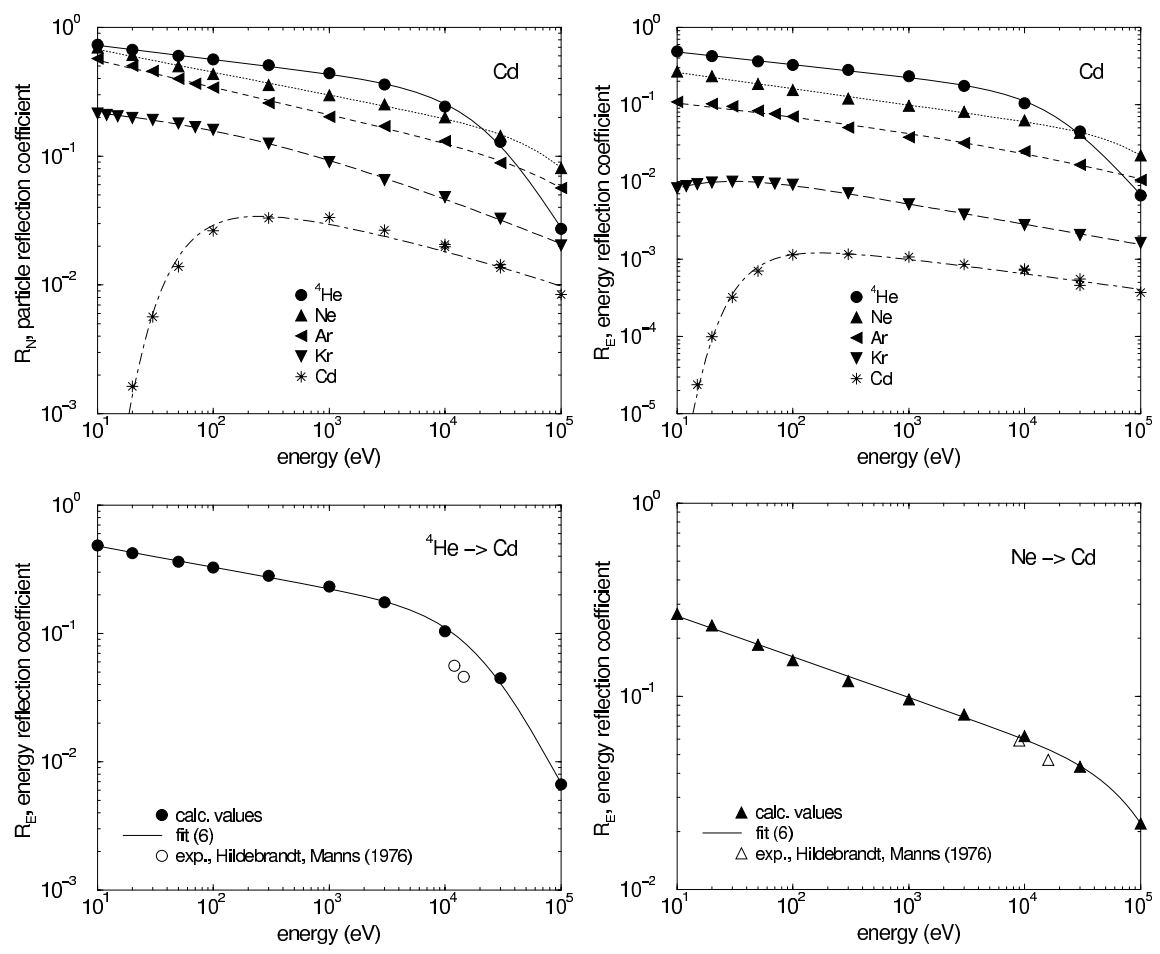

Figure 42: Calculated energy dependence of the particle and energy reflection coefficients at normal incidence for the bombardment of $\mathrm{Cd}$ with ${ }^{4} \mathrm{He}, \mathrm{Ne}, \mathrm{Ar}$, $\mathrm{Kr}, \mathrm{Cd}[30]$. Lines are fits to the calculated values (see Tables 4 and 12). Experimental data of $R_{E}$ are given for ${ }^{4} \mathrm{He}$ [56], Ne [56] 

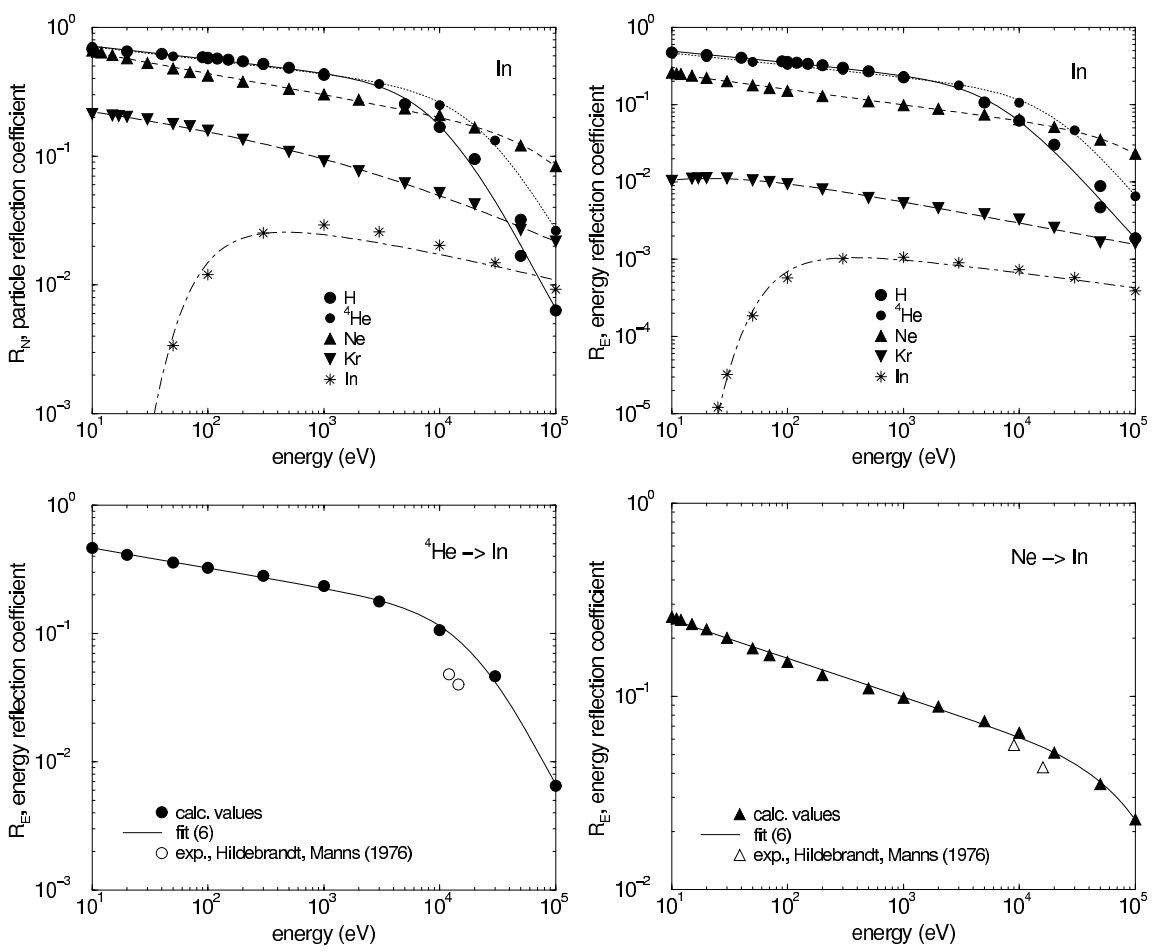

Figure 43: Calculated energy dependence of the particle and energy reflection coefficients at normal incidence for the bombardment of In with $\mathrm{H},{ }^{4} \mathrm{He}, \mathrm{Ne}$, $\mathrm{Kr}$, In [30]. Lines are fits to the calculated values (see Tables 4 and 12). Experimental data of $R_{E}$ are given for ${ }^{4} \mathrm{He}$ [56], Ne [56] 

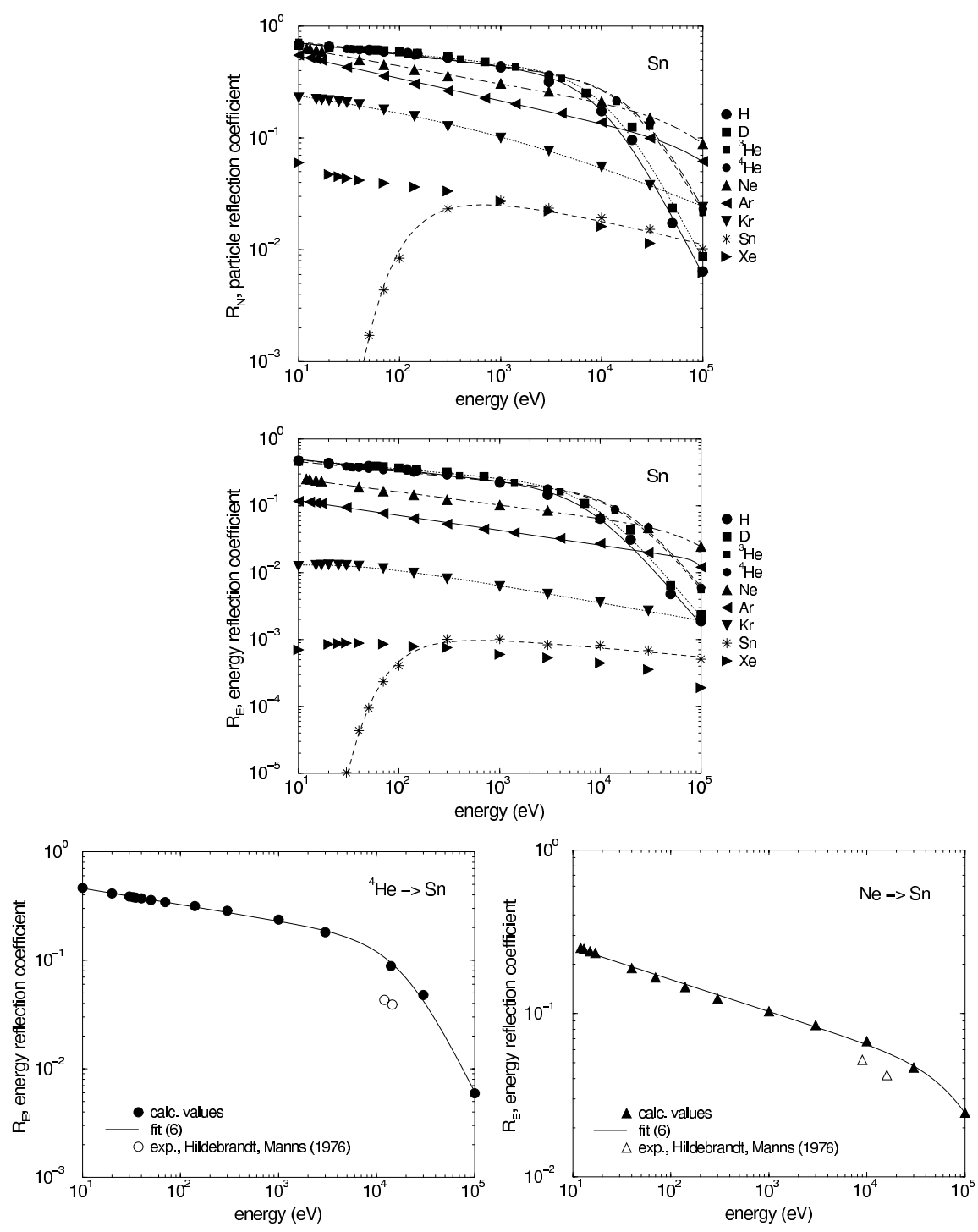

Figure 44: Calculated energy dependence of the particle and energy reflection coefficients at normal incidence for the bombardment of Sn with H, D, ${ }^{3} \mathrm{He}$, ${ }^{4} \mathrm{He}, \mathrm{Ne}, \mathrm{Ar}, \mathrm{Kr}, \mathrm{Sn}, \mathrm{Xe}[30]$. Lines are fits to the calculated values (see Tables 4 and 12). Experimental data of $R_{E}$ are given for ${ }^{4} \mathrm{He}$ [56], Ne [56] 

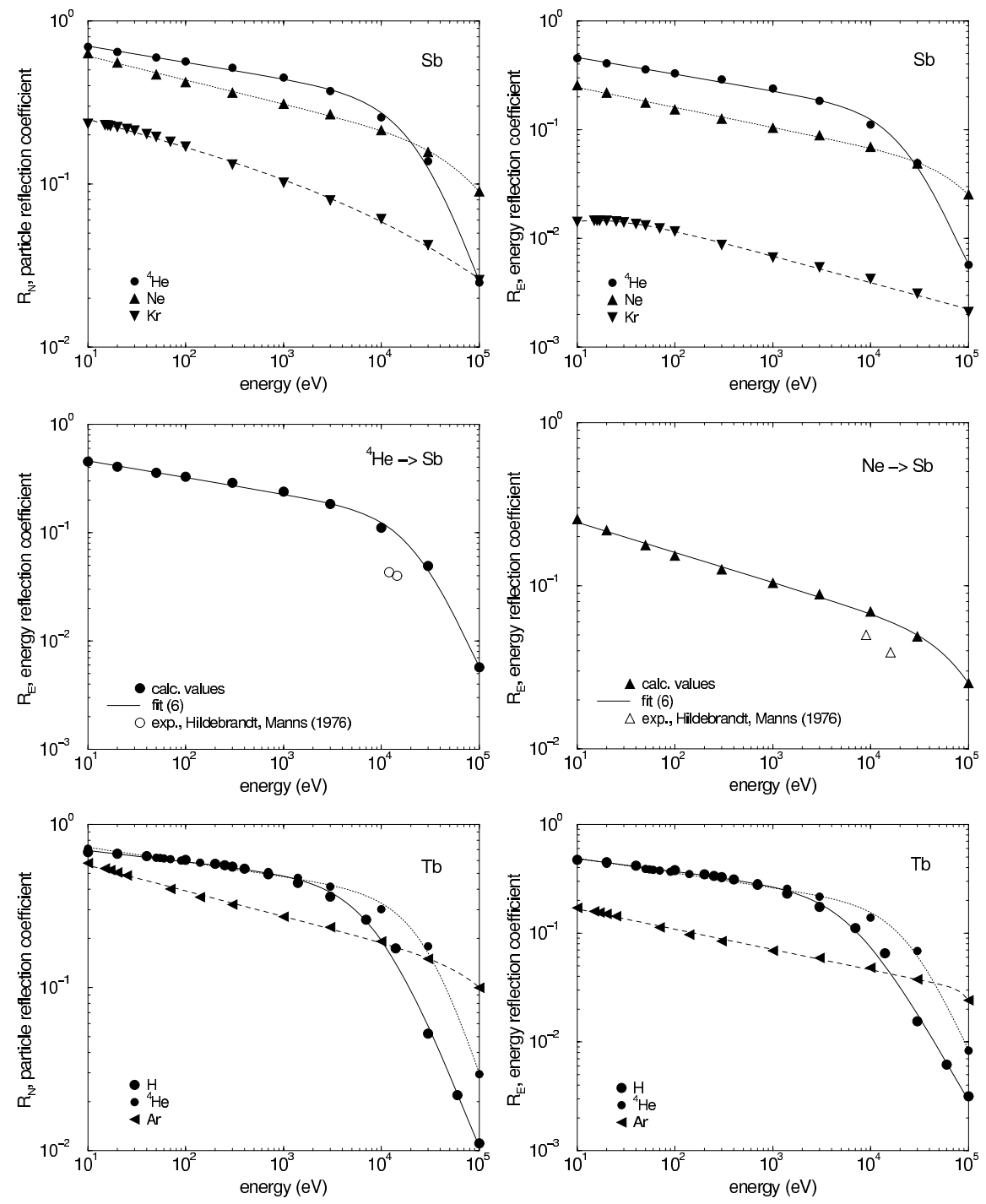

Figure 45: Calculated energy dependence of the particle and energy reflection coefficients at normal incidence for the bombardment of $\mathrm{Sb}$ with ${ }^{4} \mathrm{He}, \mathrm{Ne}, \mathrm{Kr}$, and $\mathrm{Tb}$ with $\mathrm{H},{ }^{4} \mathrm{He}, \mathrm{Ar}$ [30]. Lines are fits to the calculated values (see Table 4 and 10). (see Tables 4 and 12, 5 and 13). Experimental data of $R_{E}$ of $\mathrm{Sb}$ are given for ${ }^{4} \mathrm{He}[56]$, Ne [56] 

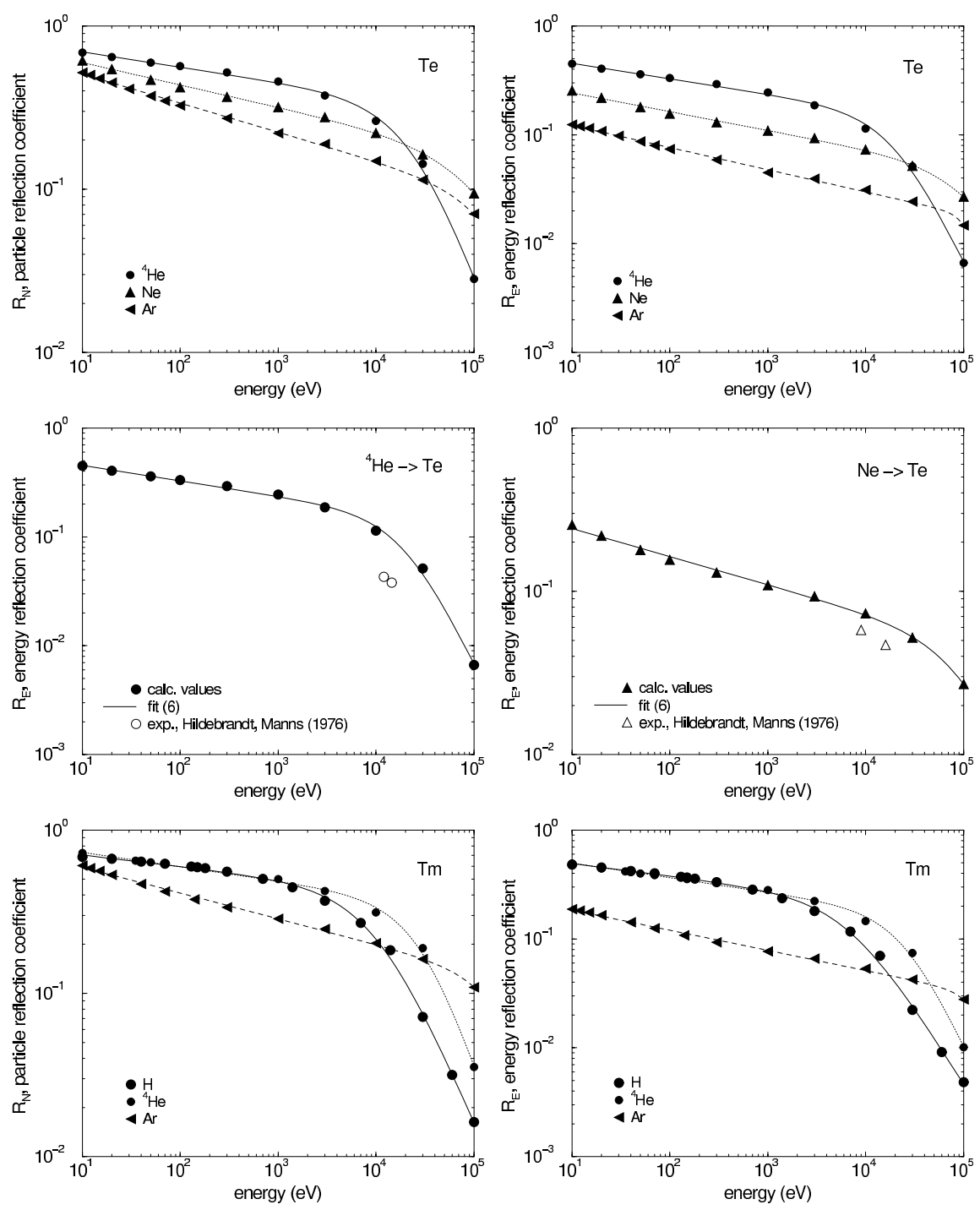

Figure 46: Calculated energy dependence of the particle and energy reflection coefficients at normal incidence for the bombardment of Te with ${ }^{4} \mathrm{He}, \mathrm{Ne}, \mathrm{Ar}$, and $\mathrm{Tm}$ with $\mathrm{H},{ }^{4} \mathrm{He}, \mathrm{Ar}[30]$. Lines are fits to the calculated values (see Tables 4 and 12, 5 and 13). Experimental data of $R_{E}$ of Te are given for ${ }^{4} \mathrm{He}$ [56], Ne [56] 

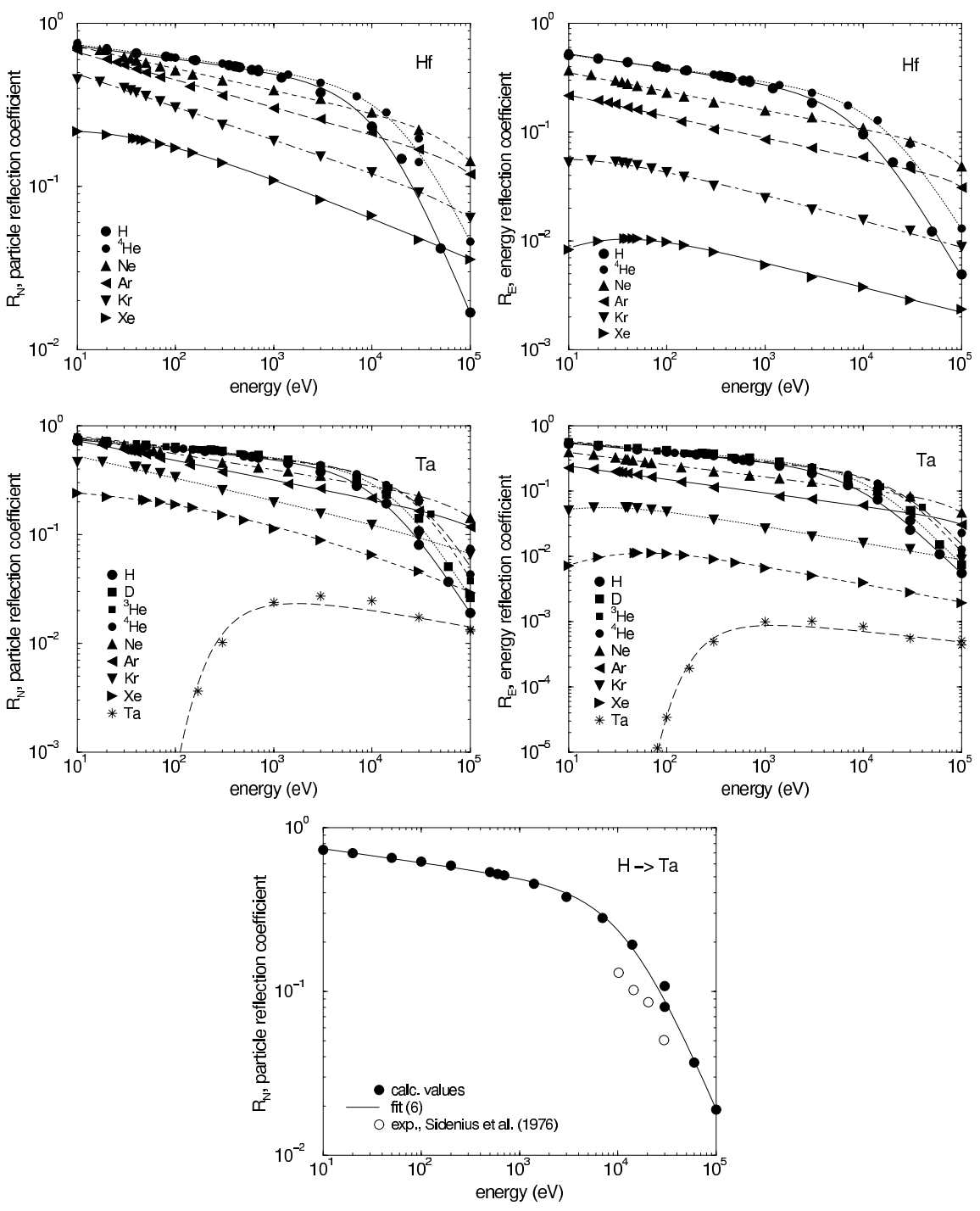

Figure 47: Calculated energy dependence of the particle and energy reflection coefficients at normal incidence for the bombardment of $\mathrm{Hf}$ with $\mathrm{H},{ }^{3} \mathrm{He},{ }^{4} \mathrm{He}$, $\mathrm{Ne}, \mathrm{Ar}, \mathrm{Kr}, \mathrm{Xe}$ [30], and of Ta with H, D, ${ }^{3} \mathrm{He},{ }^{4} \mathrm{He}, \mathrm{Ne}, \mathrm{Ar}, \mathrm{Kr}, \mathrm{Xe}$, Ta [30]. Lines are fits to the calculated values (see Tables 5 and 13). Experimental data of $R_{N}$ are given for $\mathrm{H}$ on Ta [16] 

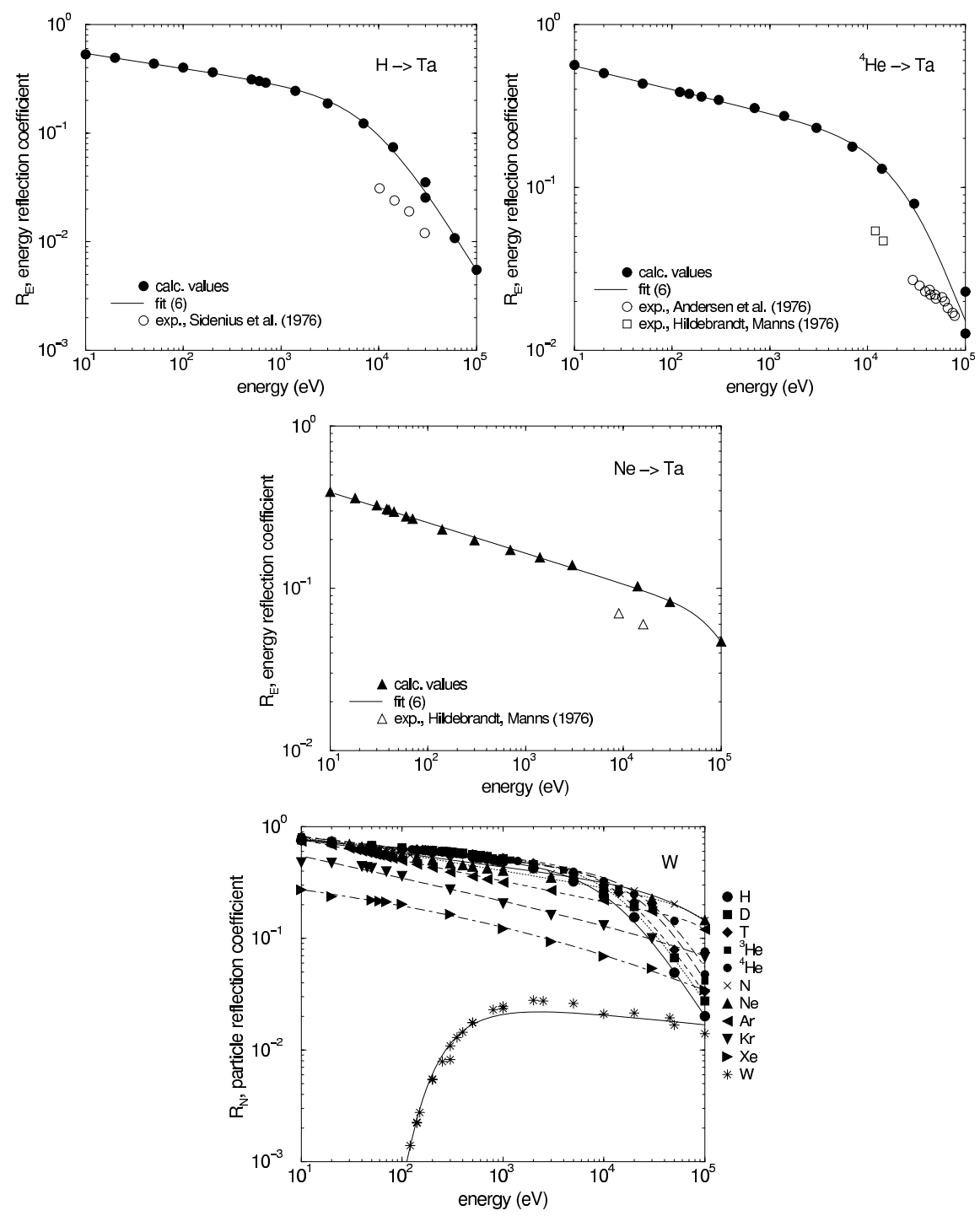

Figure 48: Calculated energy dependence of the particle and energy reflection coefficients at normal incidence for the bombardment of Ta with $\mathrm{H},{ }^{4} \mathrm{He}$, Ne, and $\mathrm{W}$ with $\mathrm{H}, \mathrm{D}, \mathrm{T},{ }^{3} \mathrm{He},{ }^{4} \mathrm{He}, \mathrm{N}, \mathrm{Ne}, \mathrm{Ar}, \mathrm{Kr}, \mathrm{Xe}, \mathrm{W}[8,30]$. Lines are fits to the calculated values (see Tables 5 and 13). Experimental data of $\mathrm{R}_{E}$ of Ta are given for $\mathrm{H}[16],{ }^{4} \mathrm{He}[14,56]$, Ne [56] 

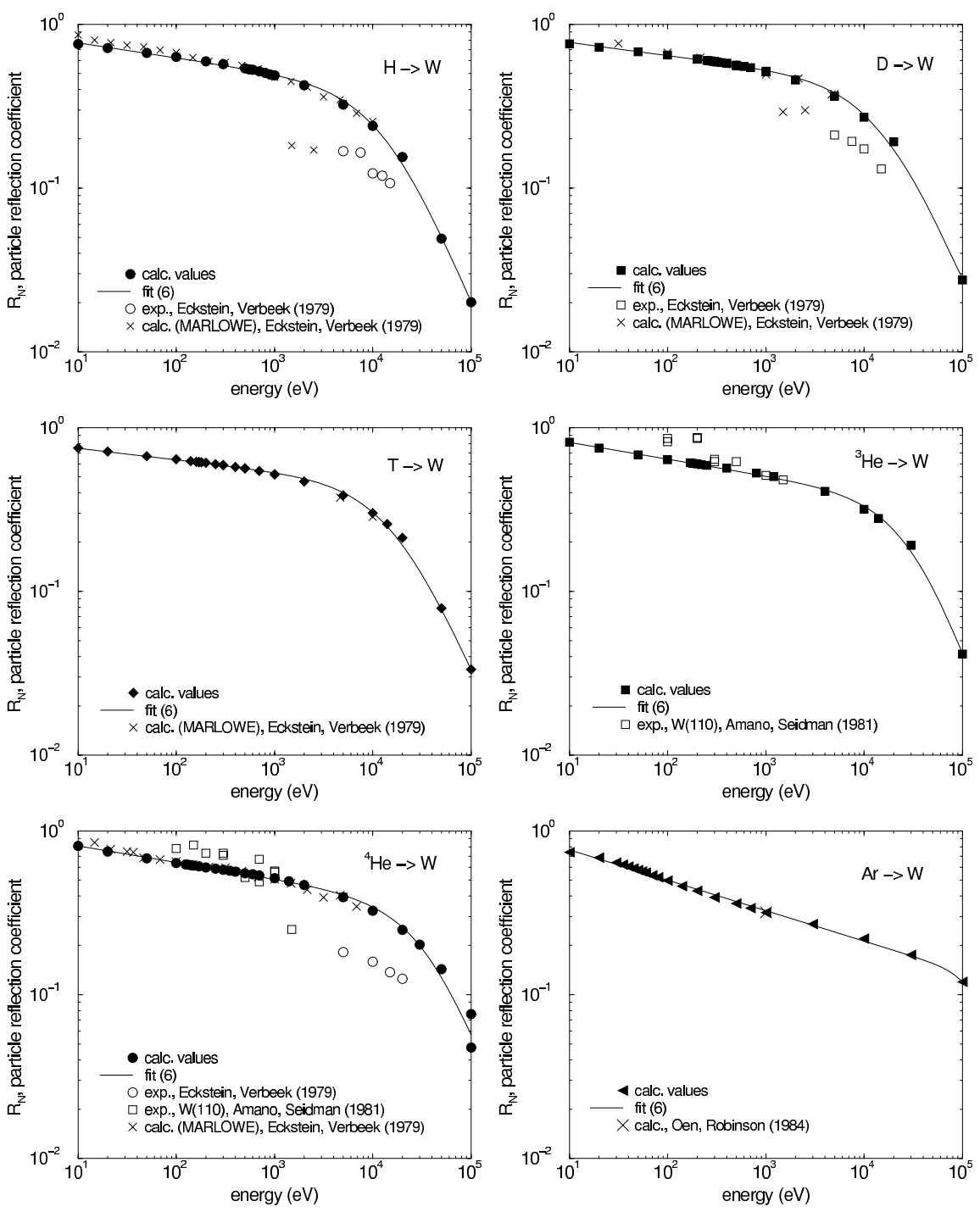

Figure 49: Calculated energy dependence of the particle reflection coefficient at normal incidence for the bombardment of $\mathrm{W}$ with $\mathrm{H}, \mathrm{D}, \mathrm{T},{ }^{3} \mathrm{He},{ }^{4} \mathrm{He}, \mathrm{Ar}[8,30]$. Lines are fits to the calculated values (see Table 5). Experimental data of $R_{N}$ are given for $\mathrm{H}$ [12], D [12], ${ }^{3} \mathrm{He}$ [13], ${ }^{4} \mathrm{He}$ [12, 13], calculated values for $\mathrm{H}$ [12], $\mathrm{D}[12], \mathrm{T}[12],{ }^{4} \mathrm{He}[12], \mathrm{Ar}[48]$ 

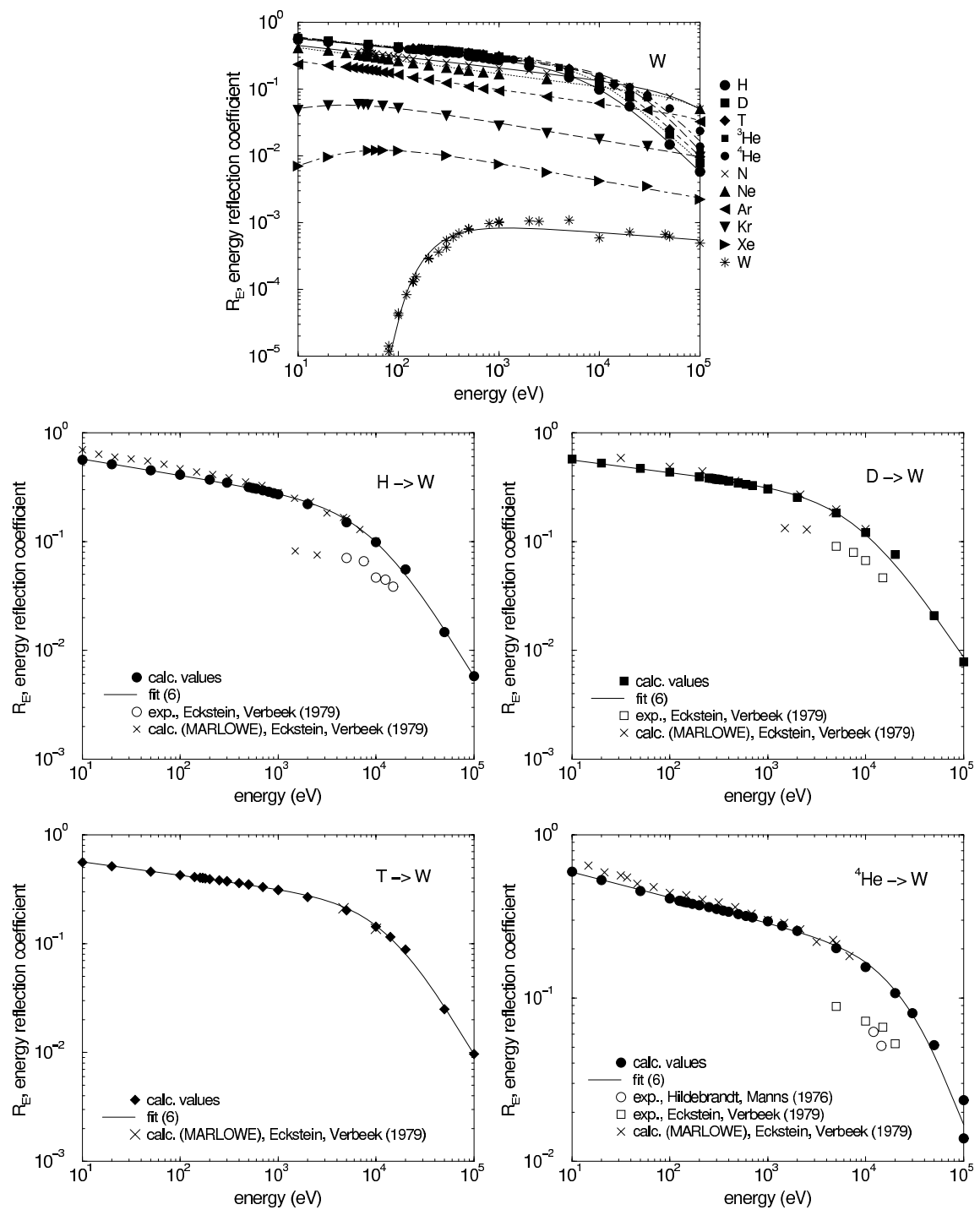

Figure 50: Calculated energy dependence of the energy reflection coefficient at normal incidence for the bombardment of $\mathrm{W}$ with $\mathrm{H}, \mathrm{D}, \mathrm{T},{ }^{3} \mathrm{He},{ }^{4} \mathrm{He}, \mathrm{N}, \mathrm{Ne}$, $\mathrm{Ar}, \mathrm{Kr}, \mathrm{Xe}, \mathrm{W}[8,30]$. Lines are fits to the calculated values (see Table 13). Experimental data of $R_{E}$ are given for $\mathrm{H}$ [12], $\mathrm{D}[12],{ }^{4} \mathrm{He}[56,12]$, Ne [56, 62], $\operatorname{Ar}[62], \mathrm{Kr}$ [62], calculated values for $\mathrm{H}$ [12], D [12], T [12], ${ }^{4} \mathrm{He}$ [12], Ar [48] 

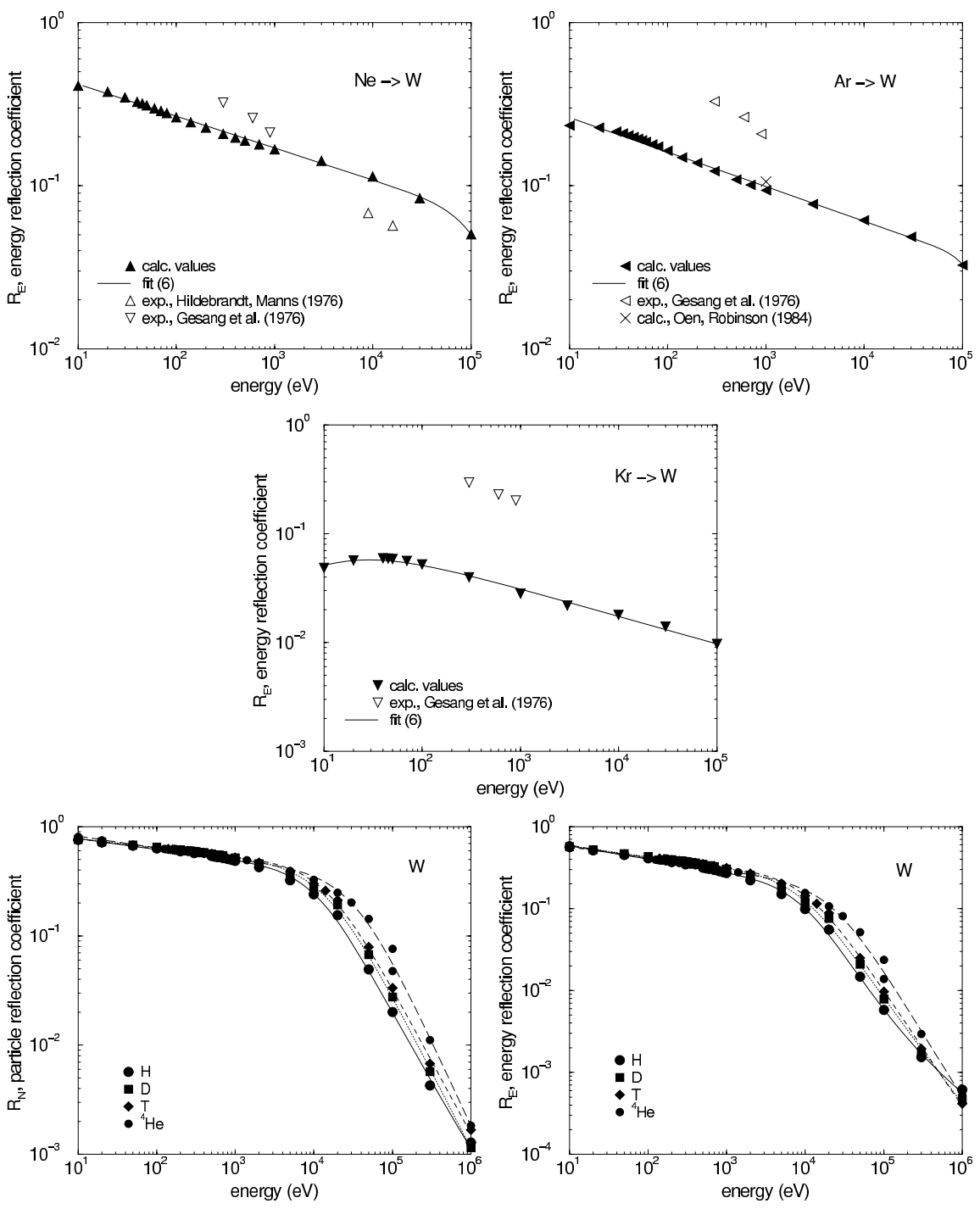

Figure 51: Calculated energy dependence of the energy reflection coefficient at normal incidence for the bombardment of $\mathrm{W}$ with $\mathrm{Ne}, \mathrm{Ar}, \mathrm{Kr}[8,30]$. Experimental data of $R_{E}$ are given Ne [56, 62], Ar [62], Kr [62]. Calculated energy dependence of the particle and energy reflection coefficients at normal incidence for the bombardment of $\mathrm{W}$ with $\mathrm{H}, \mathrm{D}, \mathrm{T},{ }^{4} \mathrm{He}$ up to high energies $(1 \mathrm{MeV})[30]$ Lines are fits to the calculated values (see Tables 13, 7 and 15) 

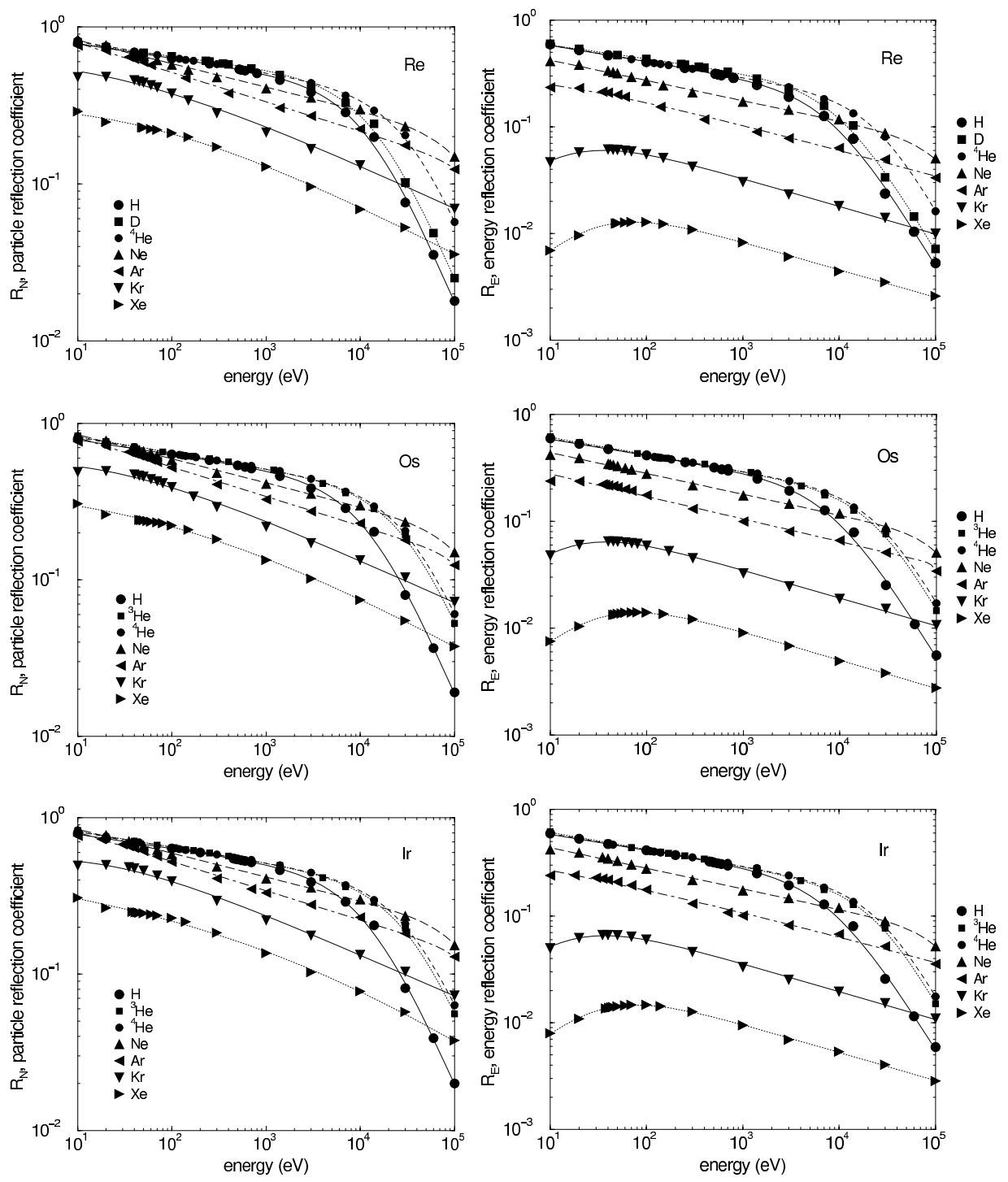

Figure 52: Calculated energy dependence of the particle and energy reflection coefficients at normal incidence for the bombardment of Re with $\mathrm{H}, \mathrm{D},{ }^{4} \mathrm{He}, \mathrm{Ne}$, Ar, $\mathrm{Kr}, \mathrm{Xe}$, and of Os, Ir with $\mathrm{H},{ }^{3} \mathrm{He},{ }^{4} \mathrm{He}, \mathrm{Ne}, \mathrm{Ar}, \mathrm{Kr}, \mathrm{Xe}$ [30]. Lines are fits to the calculated values (see Tables 5 and 13, 6 and 14) 

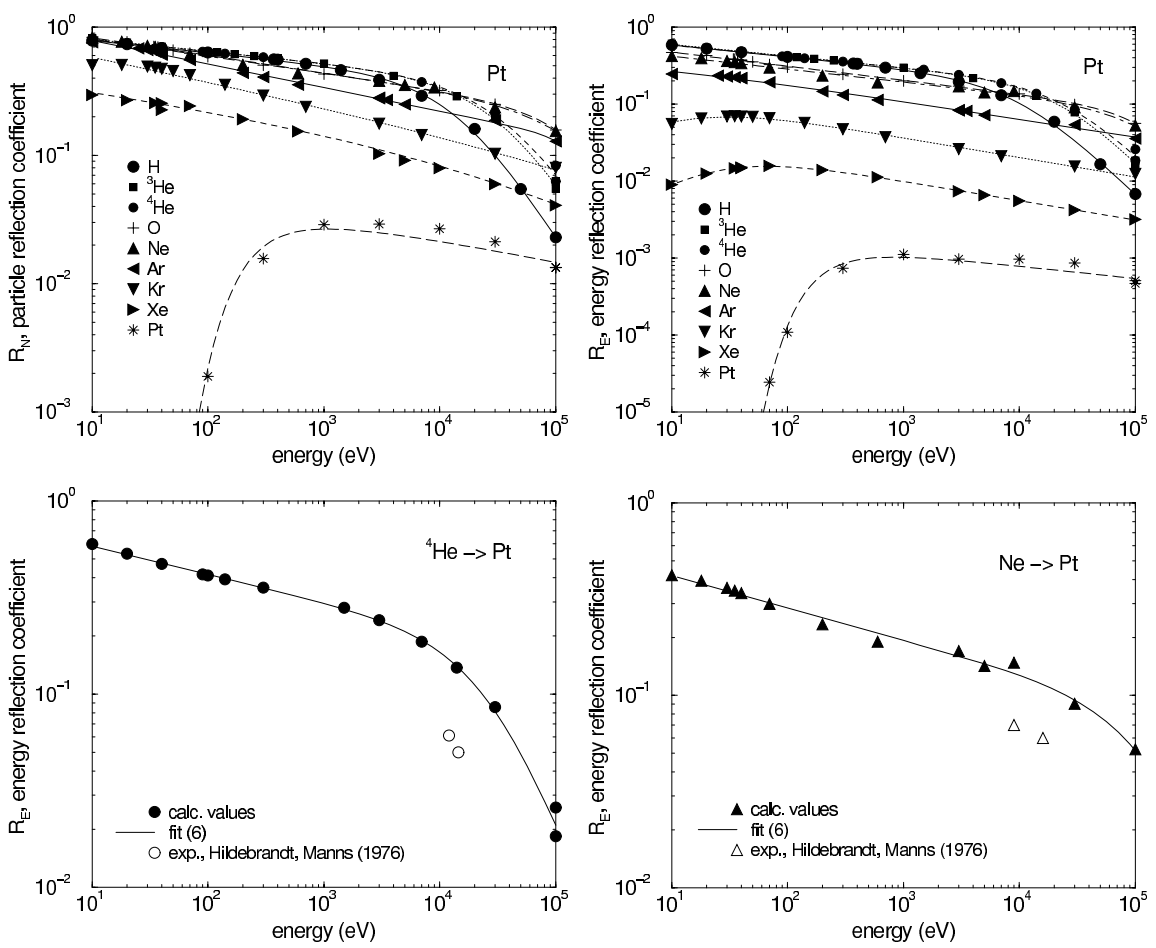

Figure 53: Calculated energy dependence of the particle and energy reflection coefficients at normal incidence for the bombardment of Pt with $\mathrm{H},{ }^{3} \mathrm{He},{ }^{4} \mathrm{He}$, $\mathrm{Ne}, \mathrm{Ar}, \mathrm{Kr}, \mathrm{Xe}, \mathrm{Pt}$ [30]. Lines are fits to the calculated values (see Tables 6 and 14). Experimental data of $R_{E}$ are given for ${ }^{4} \mathrm{He}$ [56] and Ne [56] 

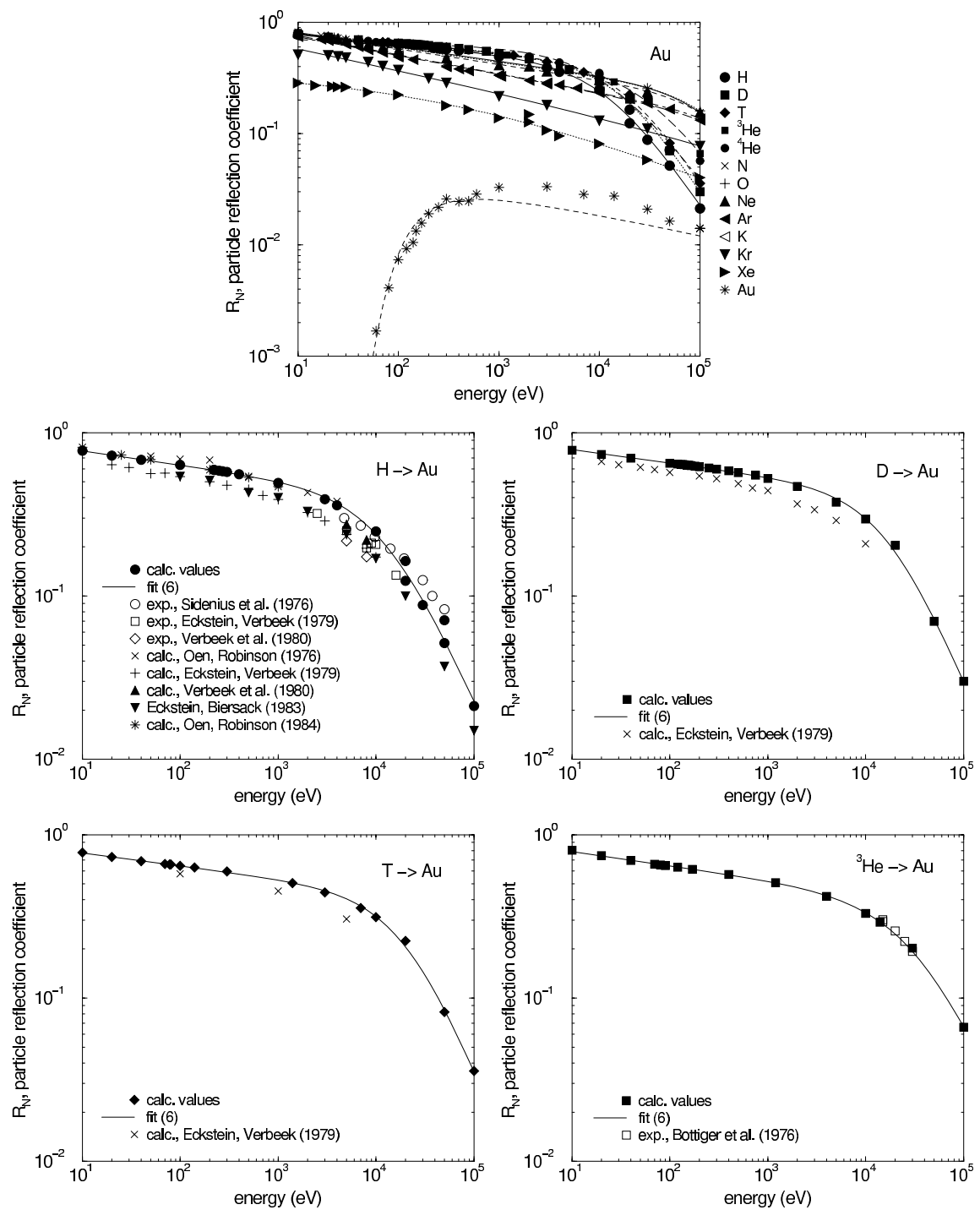

Figure 54: Calculated energy dependence of the particle reflection coefficient at normal incidence for the bombardment of Au with $\mathrm{H}, \mathrm{D}, \mathrm{T},{ }^{3} \mathrm{He},{ }^{4} \mathrm{He}, \mathrm{N}, \mathrm{O}$, $\mathrm{Ne}, \mathrm{Ar}, \mathrm{K}, \mathrm{Kr}, \mathrm{Xe}, \mathrm{Au}[30]$. Lines are fits to the calculated values (see Table 6). Experimental data of $R_{N}$ are given for $\mathrm{H}[12,16,75],{ }^{3} \mathrm{He}$ [73], calculated values for $\mathrm{H}[12,24,48,42,75], \mathrm{D}[12], \mathrm{T}$ [12] 

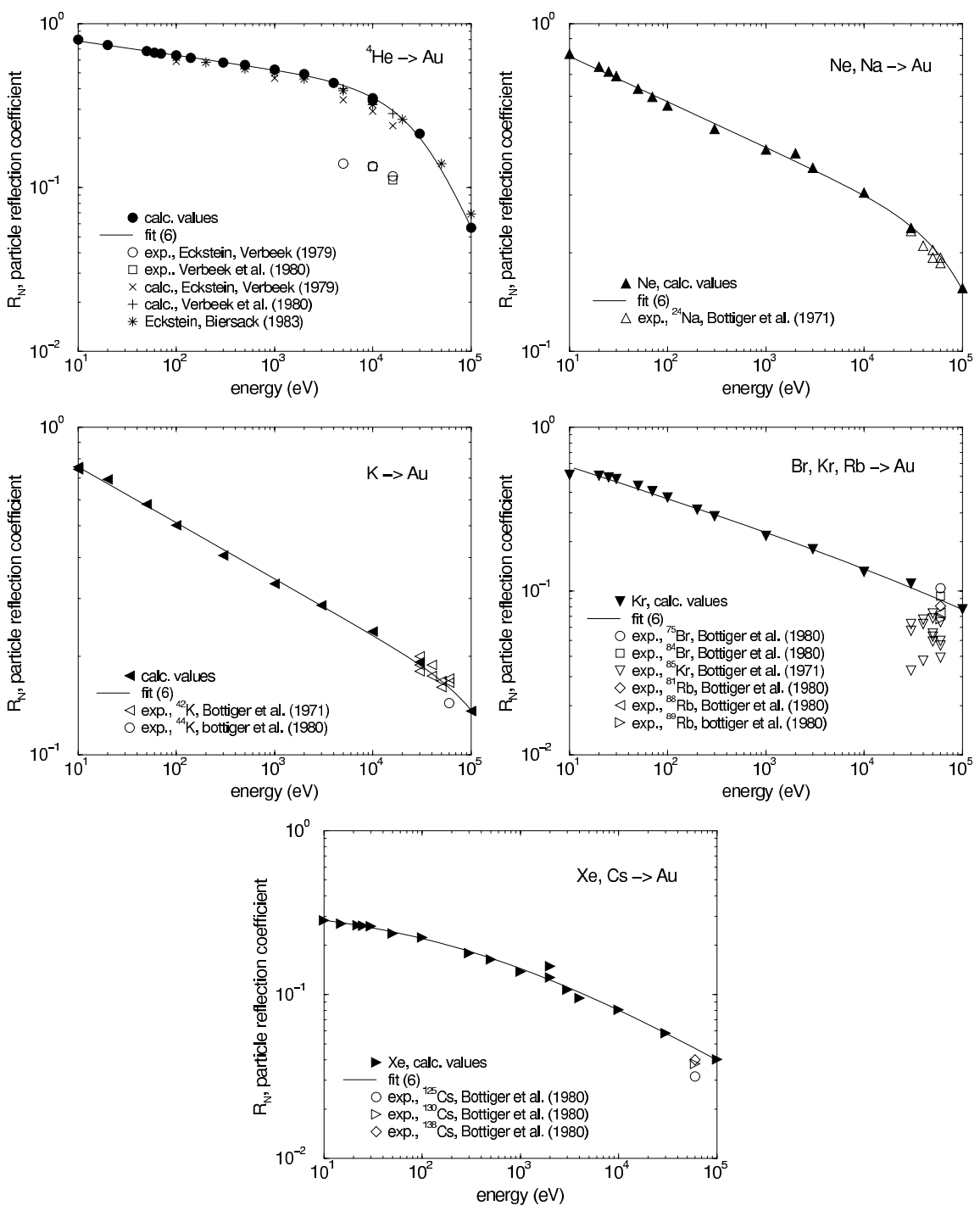

Figure 55: Calculated energy dependence of the particle reflection coefficient at normal incidence for the bombardment of $\mathrm{Au}$ with ${ }^{4} \mathrm{He}, \mathrm{Ne}, \mathrm{Na}, \mathrm{K}, \mathrm{Br}, \mathrm{Kr}, \mathrm{Rb}$, $\mathrm{Xe}$ Cs [30]. Lines are fits to the calculated values (see Table 6). Experimental data of $R_{N}$ are given for ${ }^{4} \mathrm{He}[12,75],{ }^{24} \mathrm{Na}[67],{ }^{42} \mathrm{~K}[67],{ }^{44} \mathrm{~K}[57],{ }^{75} \mathrm{Br},{ }^{84} \mathrm{Br}$ [57], ${ }^{85} \mathrm{Kr}[67],{ }^{81} \mathrm{Rb},{ }^{88} \mathrm{Rb},{ }^{89} \mathrm{Rb}[57],{ }^{125} \mathrm{Cs},{ }^{130} \mathrm{Cs},{ }^{138} \mathrm{Cs}$ [57], calculated values for ${ }^{4} \mathrm{He}[12,42,75]$, other theoretically determined values can be found in $[67,68]$ 

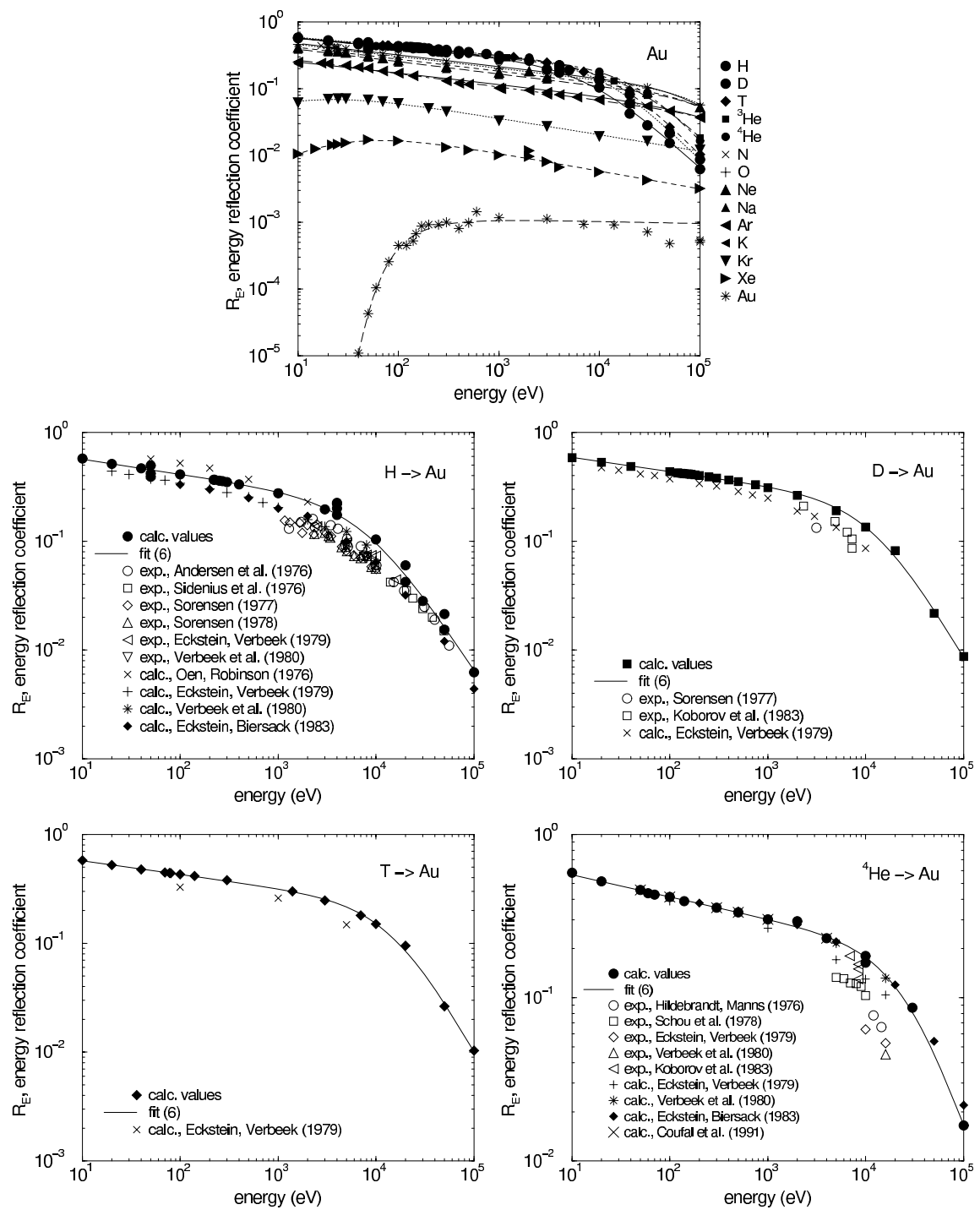

Figure 56: Calculated energy dependence of the energy reflection coefficient at normal incidence for the bombardment of Au with $\mathrm{H}, \mathrm{D}, \mathrm{T},{ }^{3} \mathrm{He},{ }^{4} \mathrm{He}, \mathrm{N}, \mathrm{O}, \mathrm{Ne}$, $\mathrm{Ar}, \mathrm{K}, \mathrm{Kr}, \mathrm{Xe}, \mathrm{Au}[30]$. Lines are fits to the calculated values (see Table 14). Experimental data of $R_{E}$ are given for $\mathrm{H}[14,16,69,74,12,75], \mathrm{D}[69,61]{ }^{4} \mathrm{He}$ $[12,56,61,66,75]$, calculated values for $\mathrm{H}[12,24,42,75], \mathrm{D}[12], \mathrm{T}[12],{ }^{4} \mathrm{He}$ $[12,38,42,75]$ 

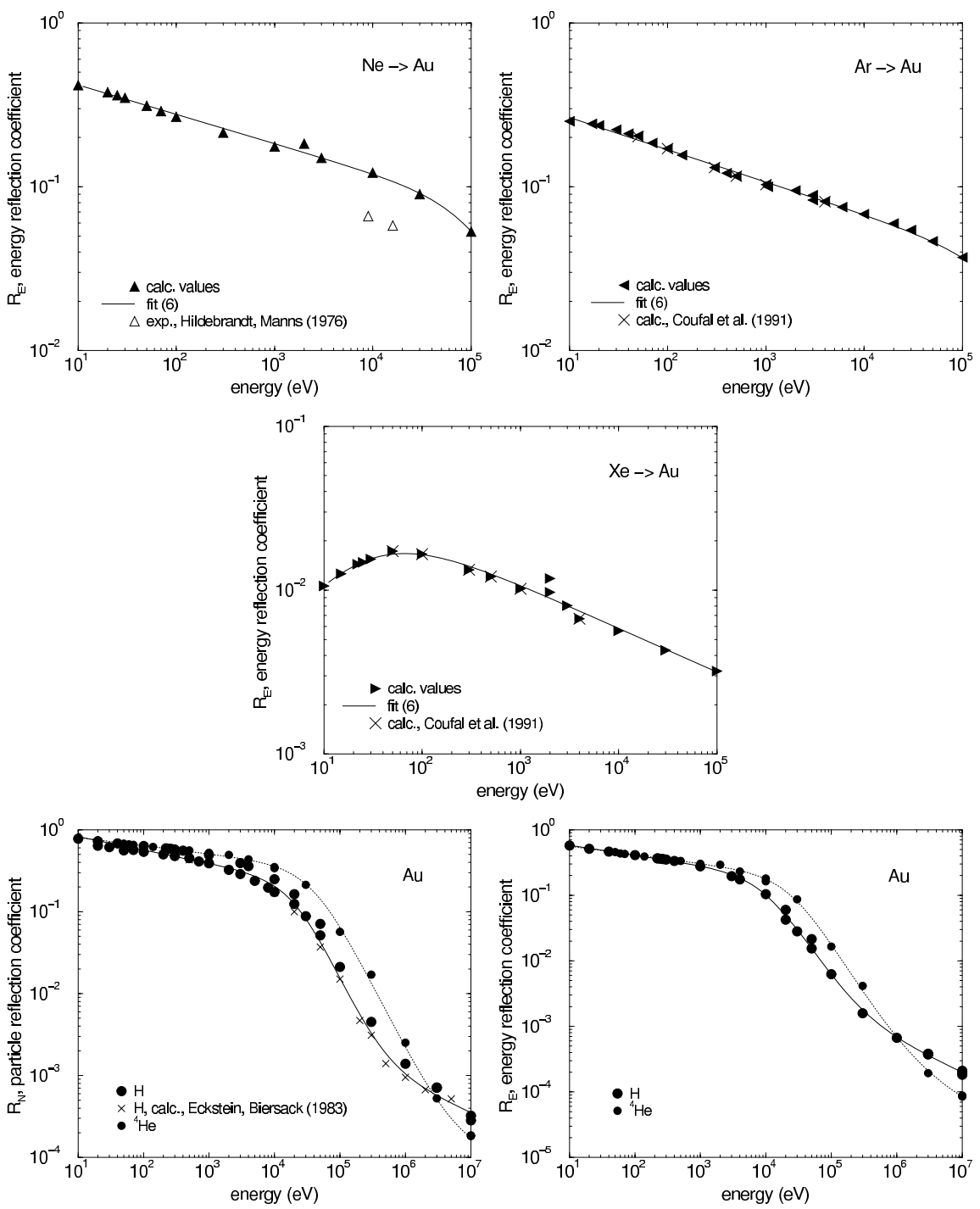

Figure 57: Calculated energy dependence of the energy reflection coefficient at normal incidence for the bombardment of Au with Ne, Ar, Xe [30]. Experimental data of $R_{E}$ are given for Ne [56], calculated values for Ar [38], Xe [38]. Calculated energy dependence of the particle and energy reflection coefficients of $\mathrm{Au}$ at normal incidence for the bombardment of $\mathrm{Au}$ with $\mathrm{H}$ and ${ }^{4} \mathrm{He}$ up to high energies $(10 \mathrm{MeV})$ [30]. Lines are fits to the calculated values (see Tables 14, 7 and 15) 


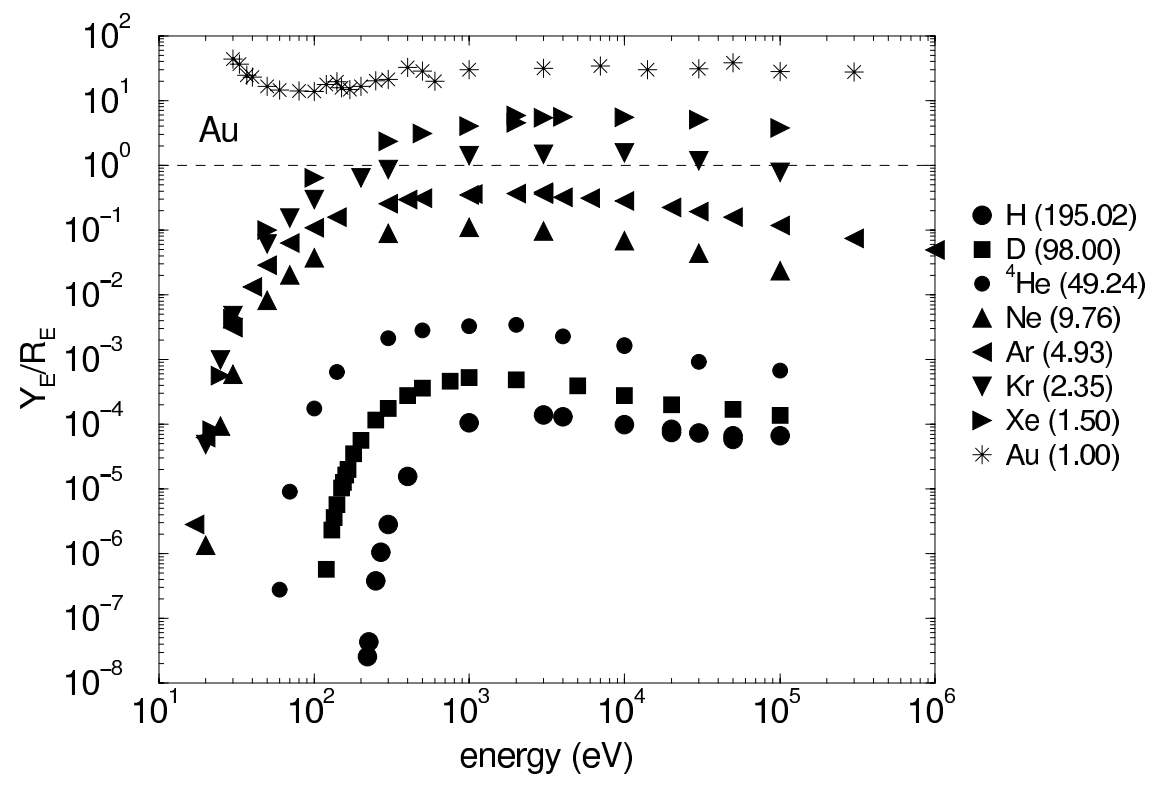

Figure 58: The ratio of the sputtered energy coefficient, $Y_{E}$, divided by the energy reflection coefficient, $R_{E}$, for bombardment of Au with different projectiles; the ratio of target mass to projectile mass in brackets 

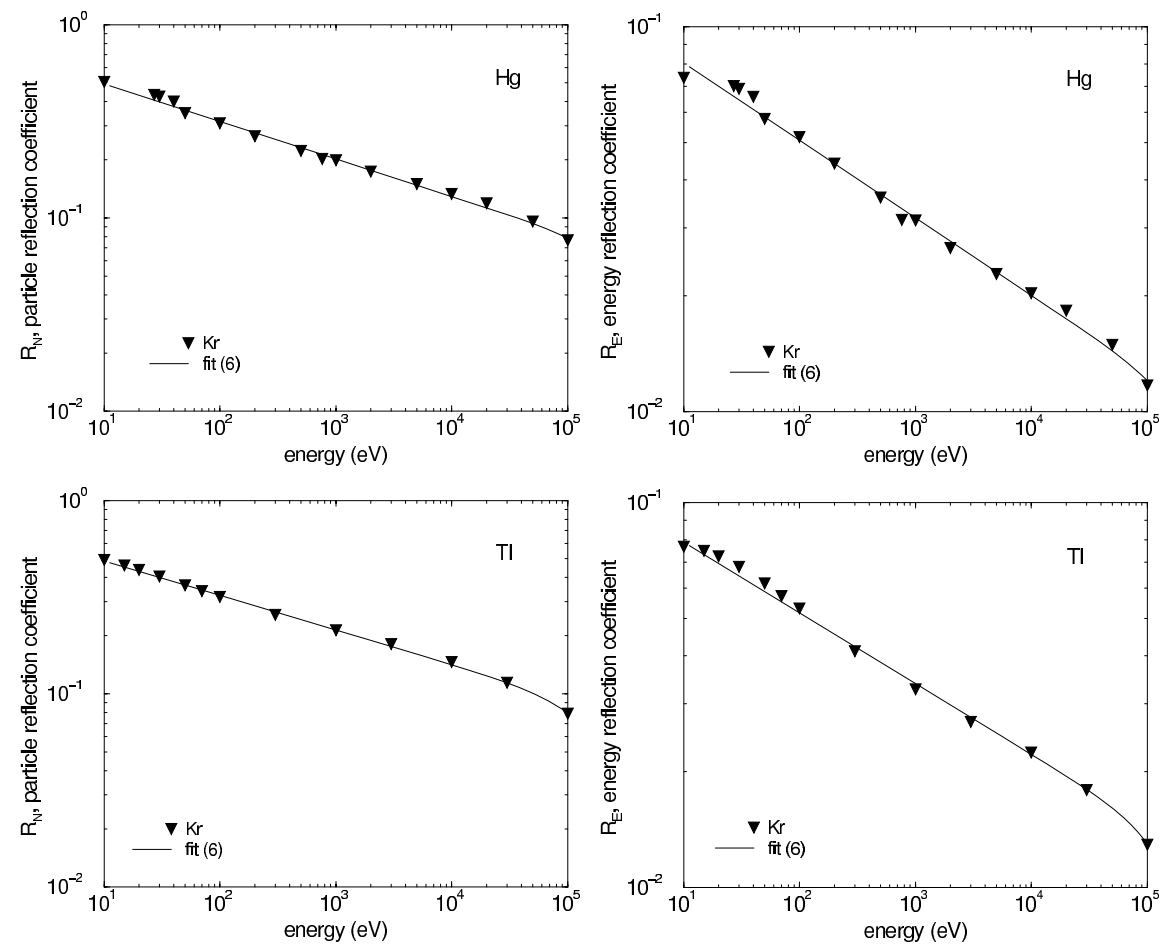

Figure 59: Calculated energy dependence of the particle and energy reflection coefficients at normal incidence for the bombardment of $\mathrm{Hg}$, $\mathrm{Tl}$ with $\mathrm{Kr}$ [30]. Lines are fits to the calculated values (see Tables 6 and 14) 

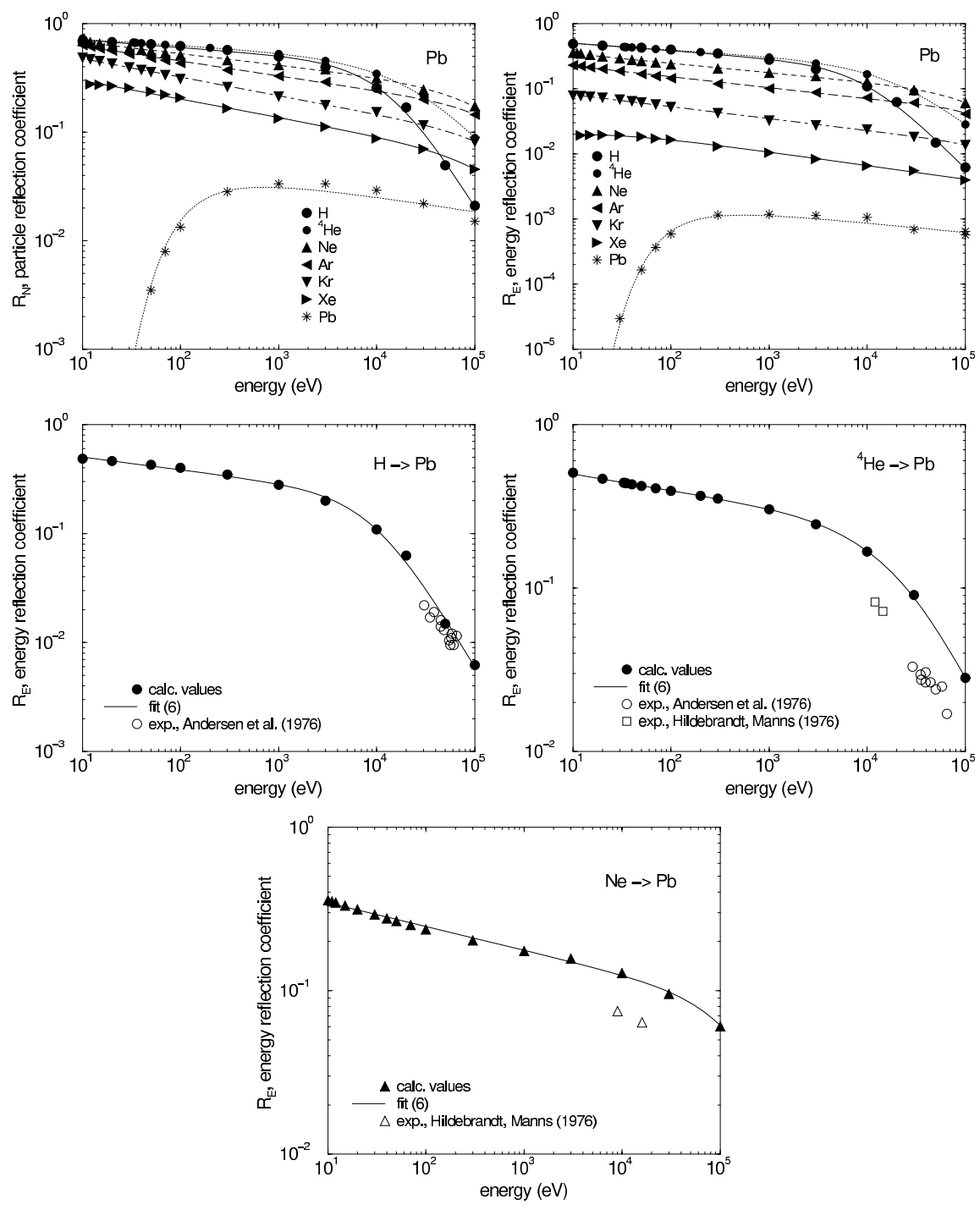

Figure 60: Calculated energy dependence of the particle and energy reflection coefficients at normal incidence for the bombardment of $\mathrm{Pb}$ with $\mathrm{H},{ }^{4} \mathrm{He}$, Ne, $\mathrm{Ar}, \mathrm{Kr}, \mathrm{Xe}, \mathrm{Pb}[30]$. Lines are fits to the calculated values (see Tables 6 and 14). Experimental data of $\mathrm{R}_{E}$ are given for $\mathrm{H}[14],{ }^{4} \mathrm{He}[14,56]$, Ne [56] 

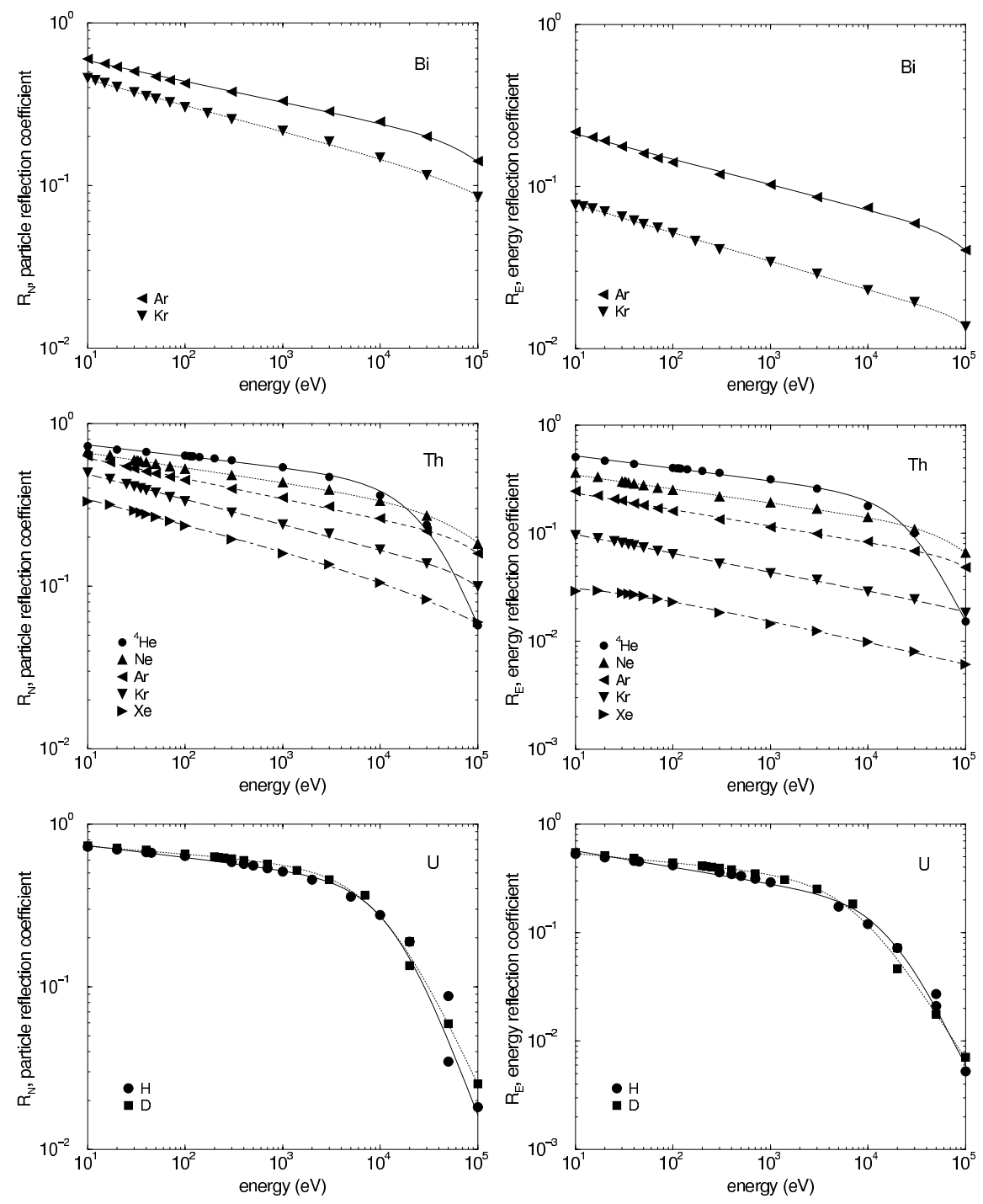

Figure 61: Calculated energy dependence of the particle and energy reflection coefficients at normal incidence for the bombardment of $\mathrm{Bi}$ with $\mathrm{Ar}, \mathrm{Kr}$, of Th with ${ }^{4} \mathrm{He}, \mathrm{Ne}, \mathrm{Ar}, \mathrm{Kr}, \mathrm{Xe}$, and of U with H, D [30]. Lines are fits to the calculated values (see Tables 6 and 14) 

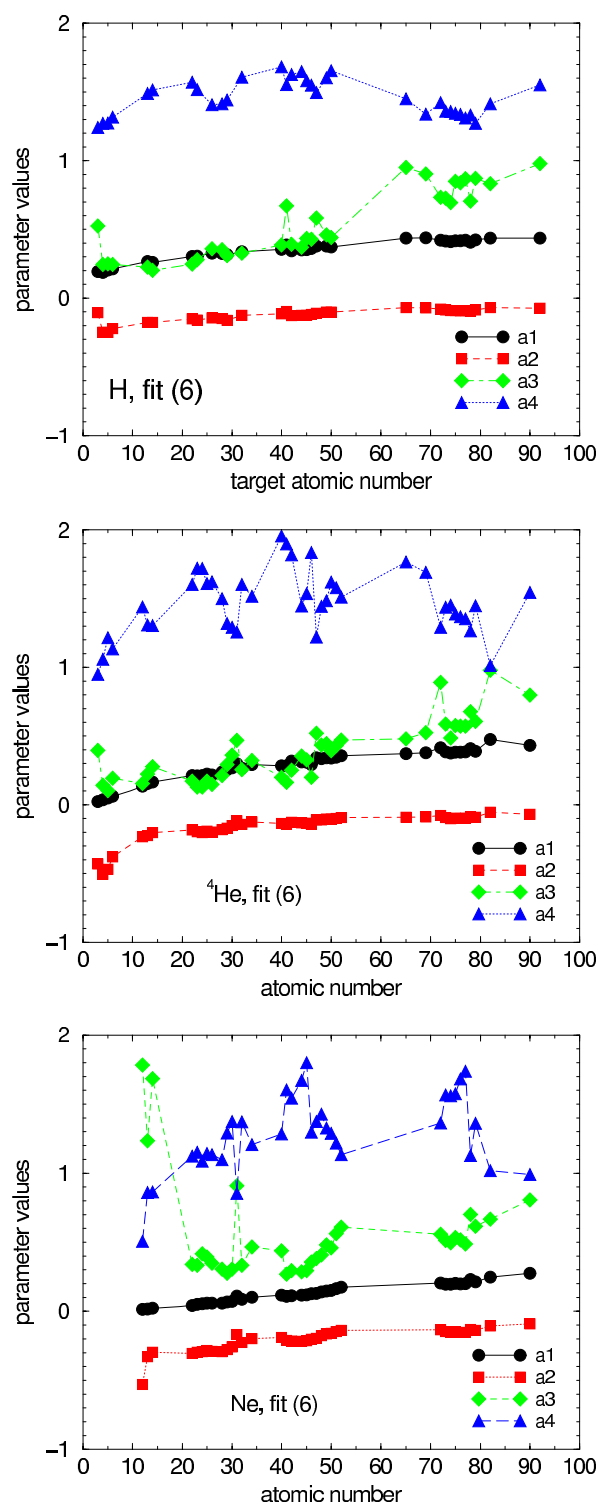

Figure 62: Fit parameters for fitting formula (6) for $\mathrm{H}$, He and Ne versus the atomic number of elemental targets using the fits to the calculated values 


\section{Angular Dependence of the Reflection Coeffi- cients}

Similarly as in the energy dependence, the angular dependence of the reflection coefficients are provided for each ion-target combination. In most cases formula (8) can very well describe the angular dependence. Discrepancies appear sometimes at grazing incidence, but this may not be important because surface roughness may strongly influence the behaviour in this angular range. The lower values of $R_{E}$ al large angles of incidence for $\mathrm{H}$ on $\mathrm{C}, \mathrm{Al}, \mathrm{Mo}$, and $\mathrm{W}$ compared to the calculated values (see figures) is an example for the influence of surface roughness and probably of oxide layers. As in sputtering, the calculated reflection coefficients may be larger than experimental values at normal incidence, and smaller at incident angles larger than $45^{\circ}$ due to surface roughness. The influence of a geometrically ordered surface structure on the reflection coefficients has been treated theoretically in [76].

The experimental basis is rather small, especially for the energy reflection coefficient.

Fitting parameters for $R_{N}$ are provided in tables 17 to 29 , and for $R_{E}$ in tables 30 to 42 .

For the use of reflection coefficients and energy distributions in plasma edge codes a special algorithm has been used to describe the backscattering from the plasma vessel walls [77]. A database for helium on several elemantal targets is provided in [78]. 

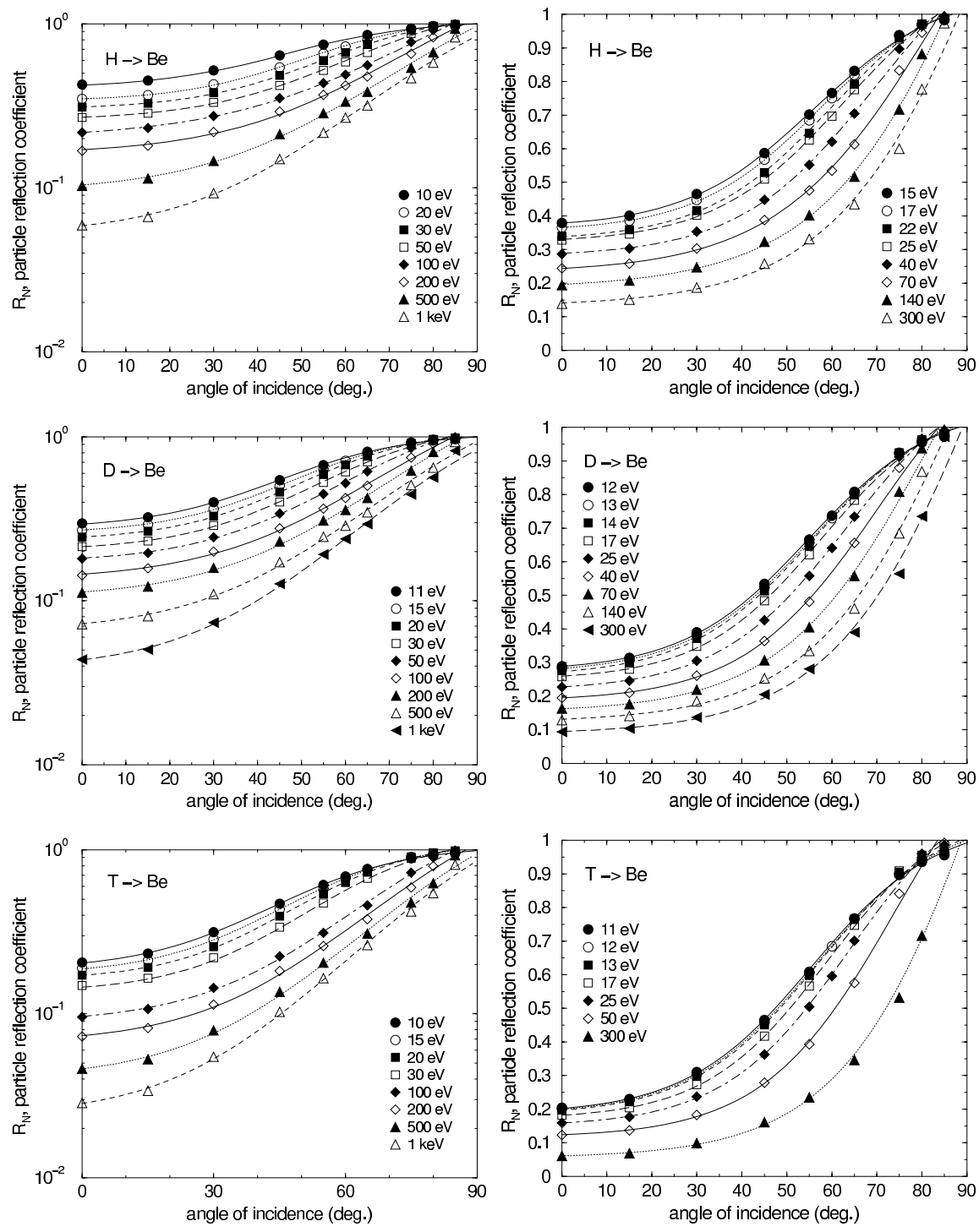

Figure 63: Calculated values for the angular dependence of the particle reflection coefficient at several incident energies for the bombardment of Be with $\mathrm{H}, \mathrm{D}, \mathrm{T}$ $[8,29]$. Lines are fits to the calculated values (see Tables 17 and 18) 

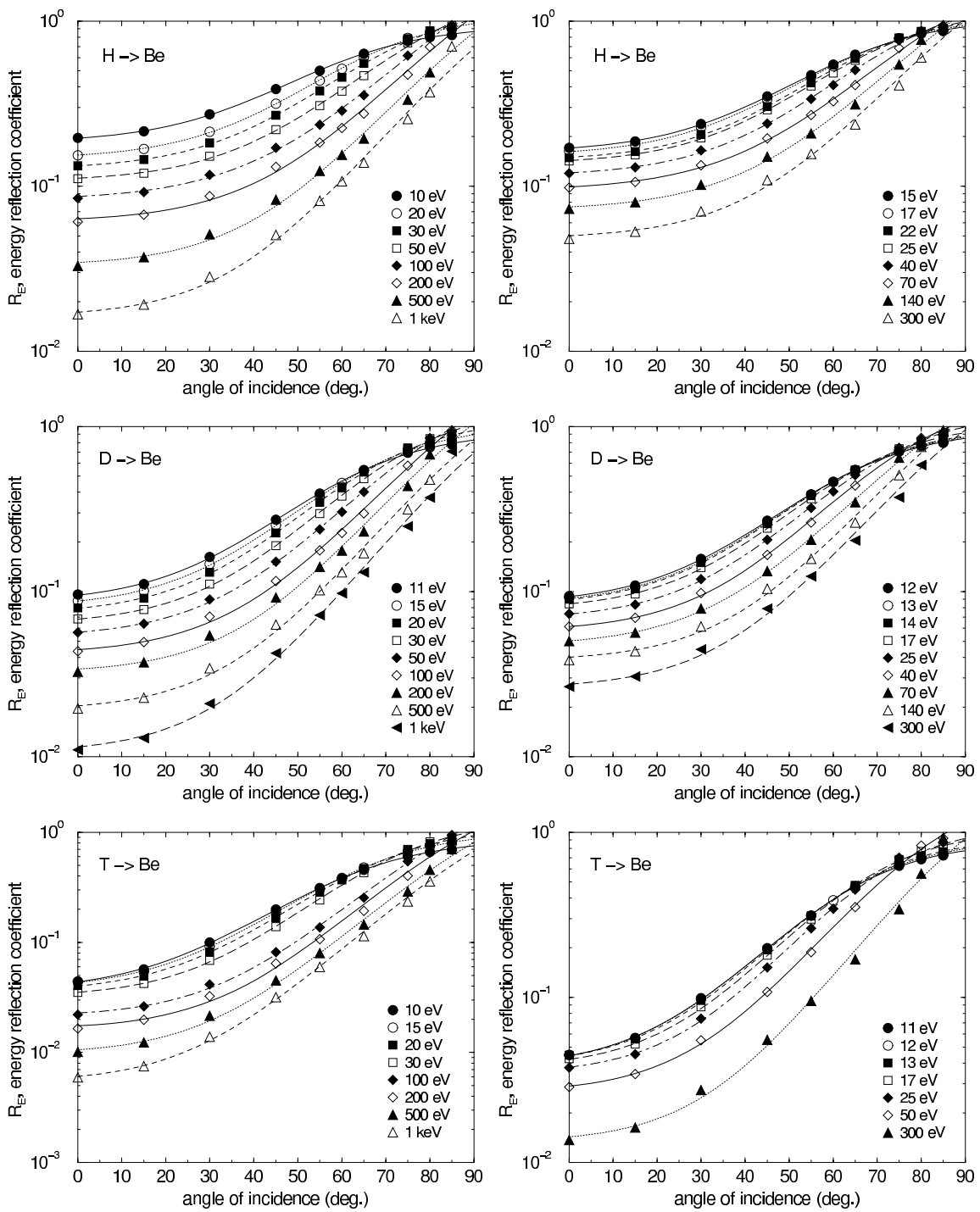

Figure 64: Calculated values for the angular dependence of the energy reflection coefficient at several incident energies for the bombardment of Be with $\mathrm{H}, \mathrm{D}, \mathrm{T}$ $[8,29]$. Lines are fits to the calculated values (see Tables 30 and 31) 

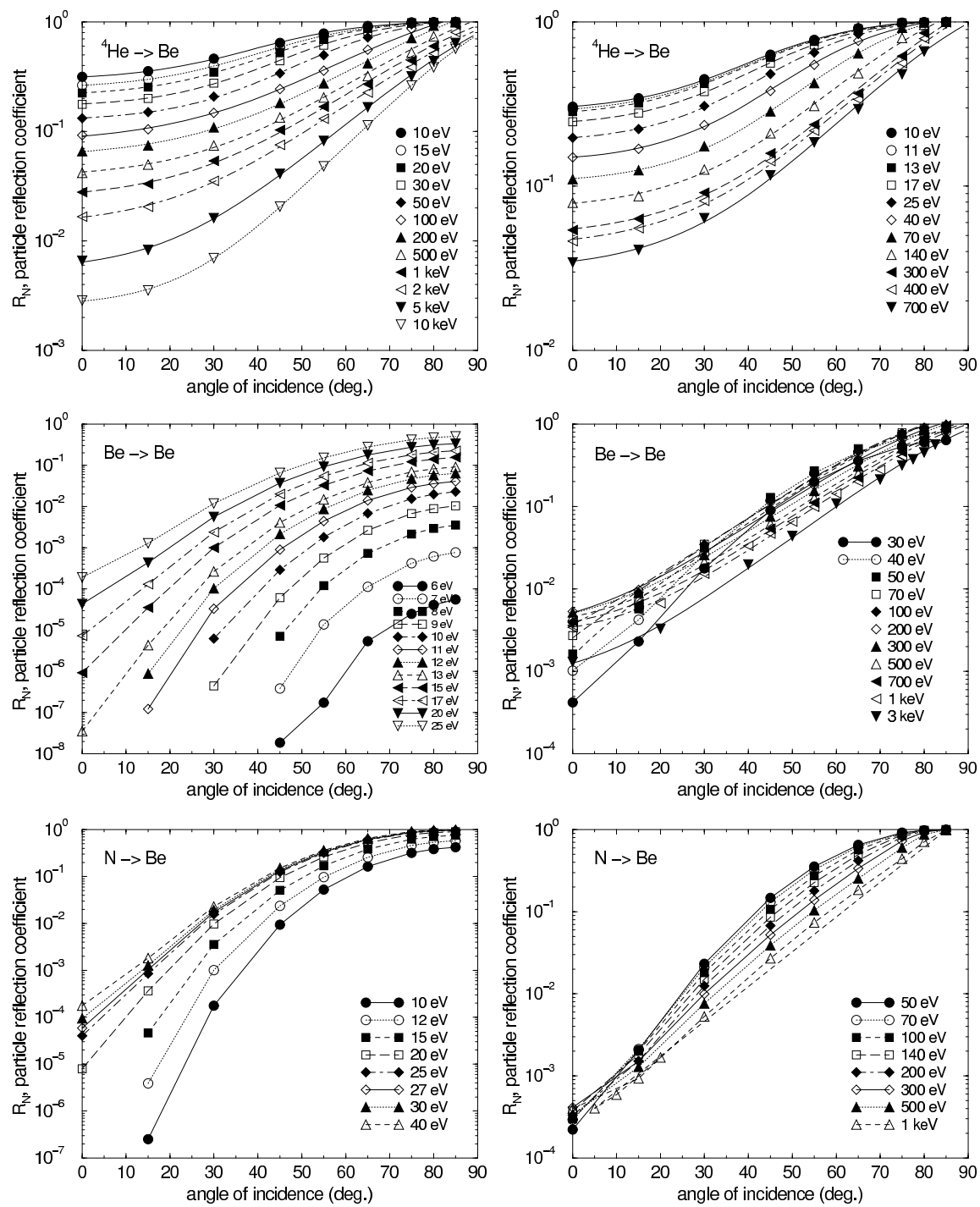

Figure 65: Calculated values for the angular dependence of the particle reflection coefficient at several incident energies for the bombardment of Be with ${ }^{4} \mathrm{He}, \mathrm{Be}$ $[8,29], N$ [29]. Lines are fits to the calculated values (see Table 18). Lines indicated in the legend for $\mathrm{Be}$ and $\mathrm{N}$ on $\mathrm{Be}$ are drawn to guide the eye 

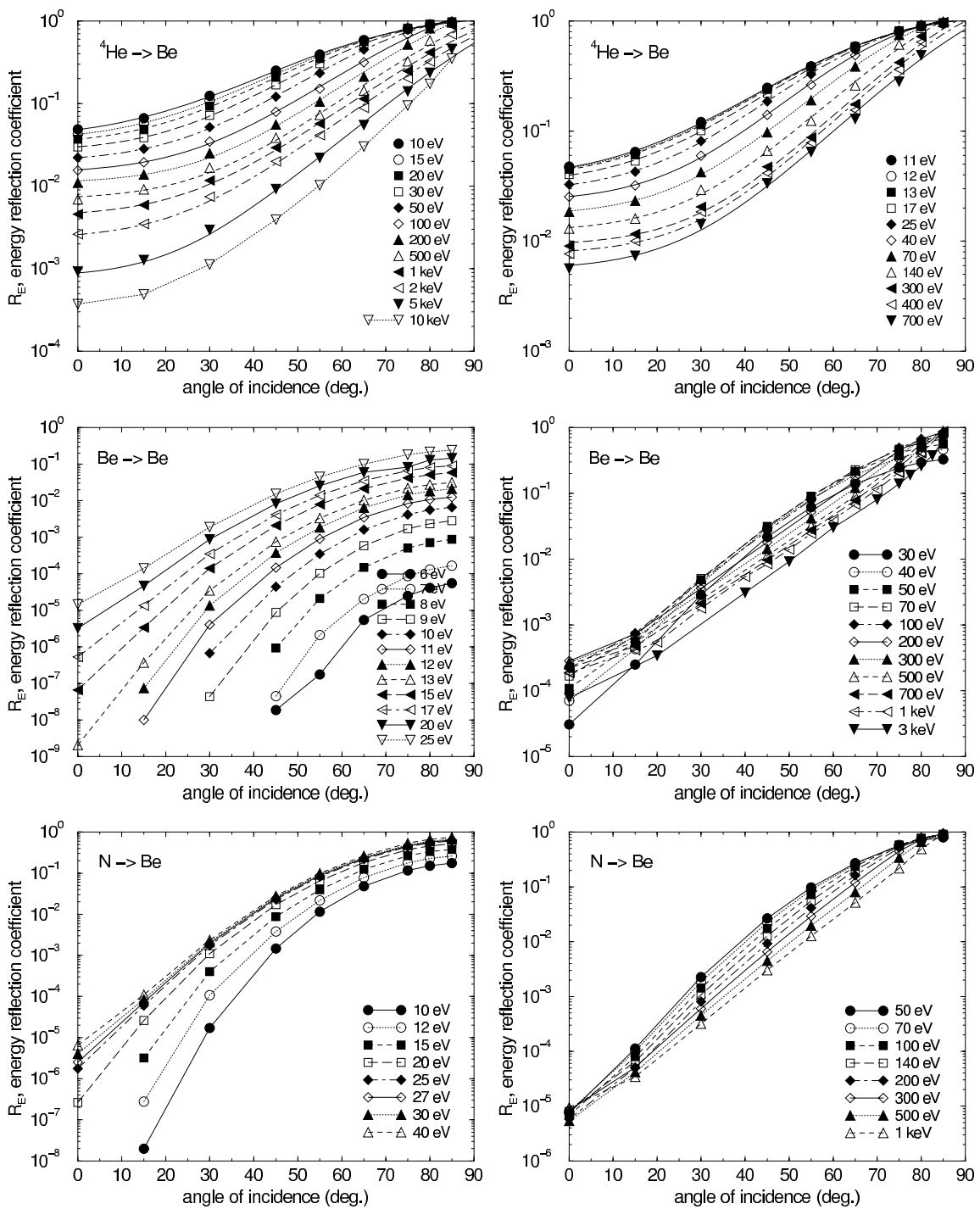

Figure 66: Calculated values for the angular dependence of the energy reflection coefficient at several incident energies for the bombardment of Be with ${ }^{4} \mathrm{He}, \mathrm{Be}$ [8, 29], N [29]. Lines are fits to the calculated values (see Table 31). Lines indicated in the legend for $\mathrm{Be}$ and $\mathrm{N}$ on Be are drawn to guide the eye 

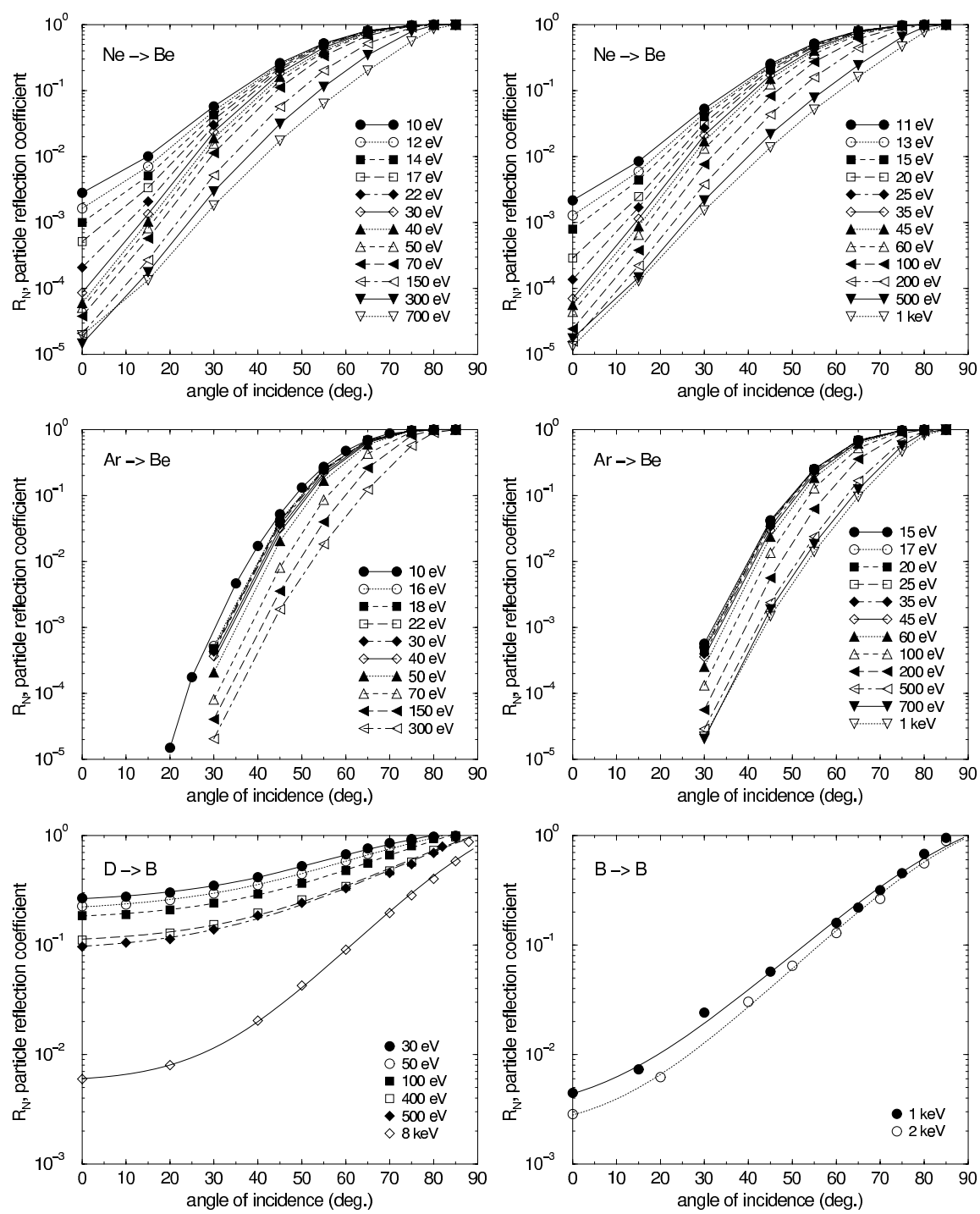

Figure 67: Calculated values for the angular dependence of the particle reflection coefficient at several incident energies for the bombardment of Be with $\mathrm{Ne}, \mathrm{Ar}$ $[8,29]$, and of B with D, B [29]. Lines indicated in the legend for Ne and Ar on $\mathrm{Be}$ are drawn to guide the eye. Lines for $\mathrm{D}$ an $\mathrm{B}$ on $\mathrm{B}$ are fits to the calculated values (see Table 18) 

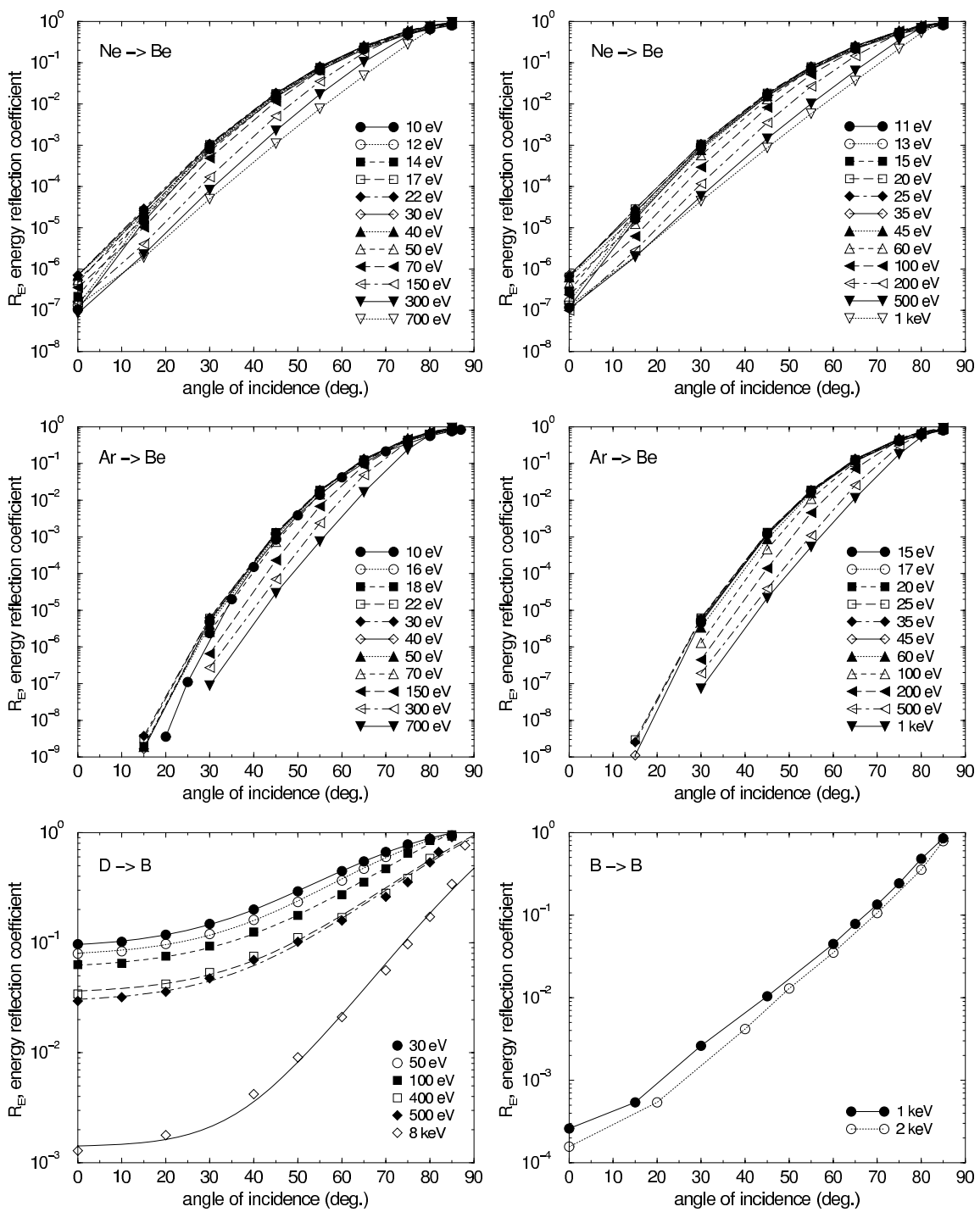

Figure 68: Calculated values for the angular dependence of the energy reflection coefficient at several incident energies for the bombardment of Be with $\mathrm{Ne}, \mathrm{Ar}$ $[8,29]$, and of $\mathrm{B}$ with $\mathrm{D}, \mathrm{B}[29]$. Lines indicated in the legend for $\mathrm{Ne}$ and $\mathrm{Ar}$ on $\mathrm{Be}$ and $\mathrm{B}$ on $\mathrm{B}$ are drawn to guide the eye. Lines for $\mathrm{D}$ on $\mathrm{B}$ are fits to the calculated values (see Table 31) 

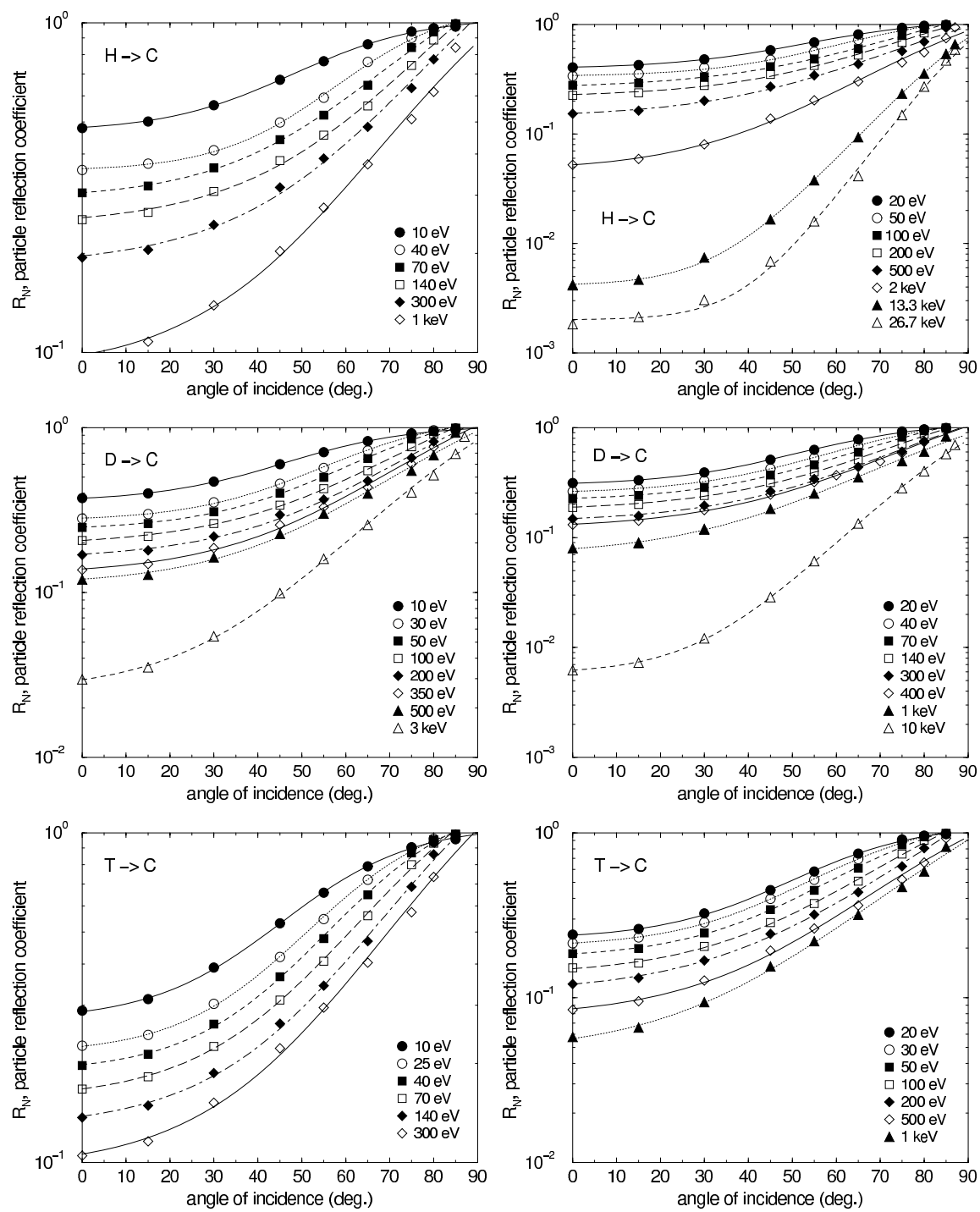

Figure 69: Calculated values for the angular dependence of the particle reflection coefficient at several incident energies for the bombardment of $\mathrm{C}$ with $\mathrm{H}, \mathrm{D}, \mathrm{T}$ $[8,29]$. Lines are fits to the calculated values (see Table 19) 

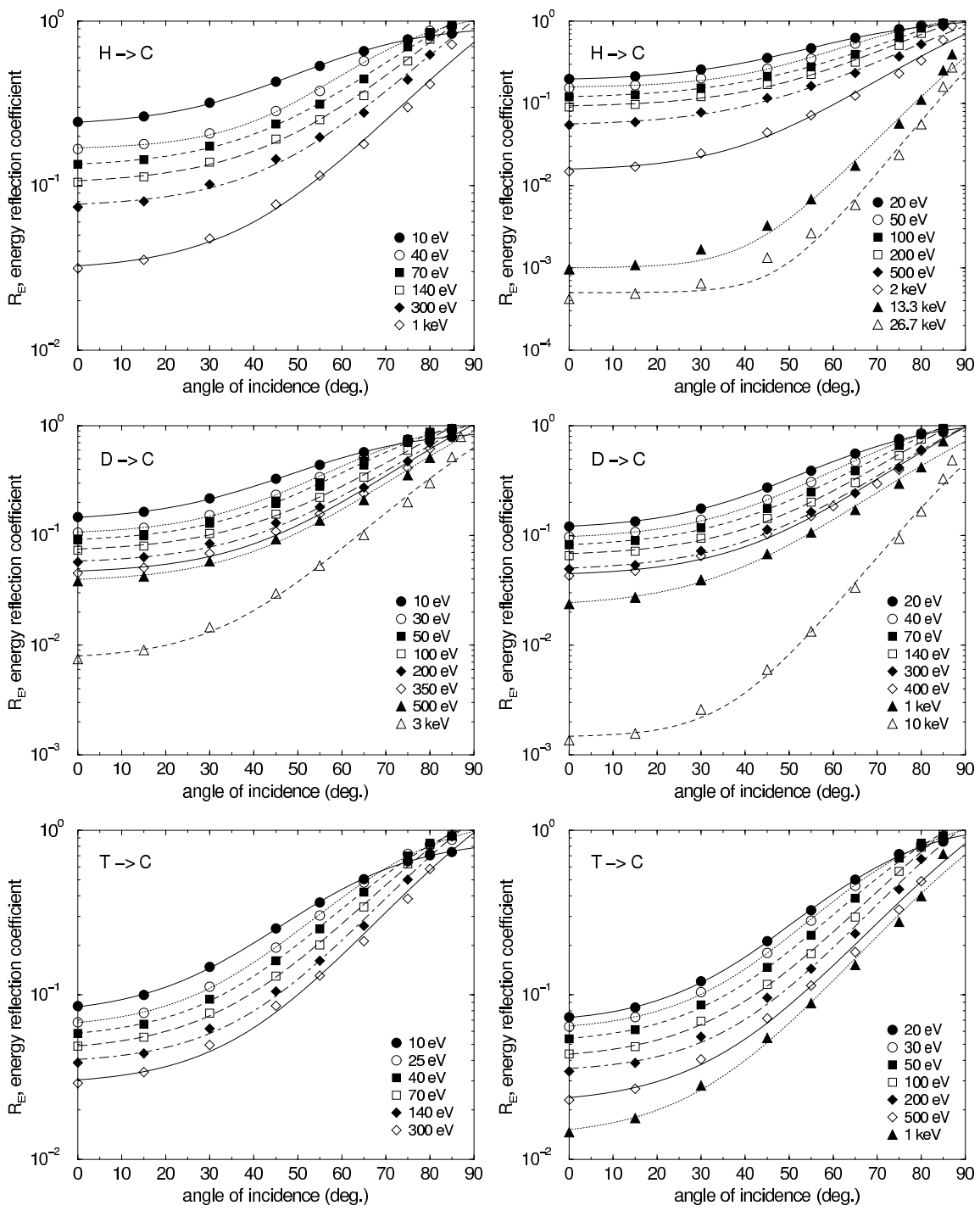

Figure 70: Calculated angular dependence of the energy reflection coefficient at several incident energies for the bombardment of $\mathrm{C}$ with $\mathrm{H}, \mathrm{D}, \mathrm{T}[8,29]$. Lines are fits to the calculated values (see Table 32) 

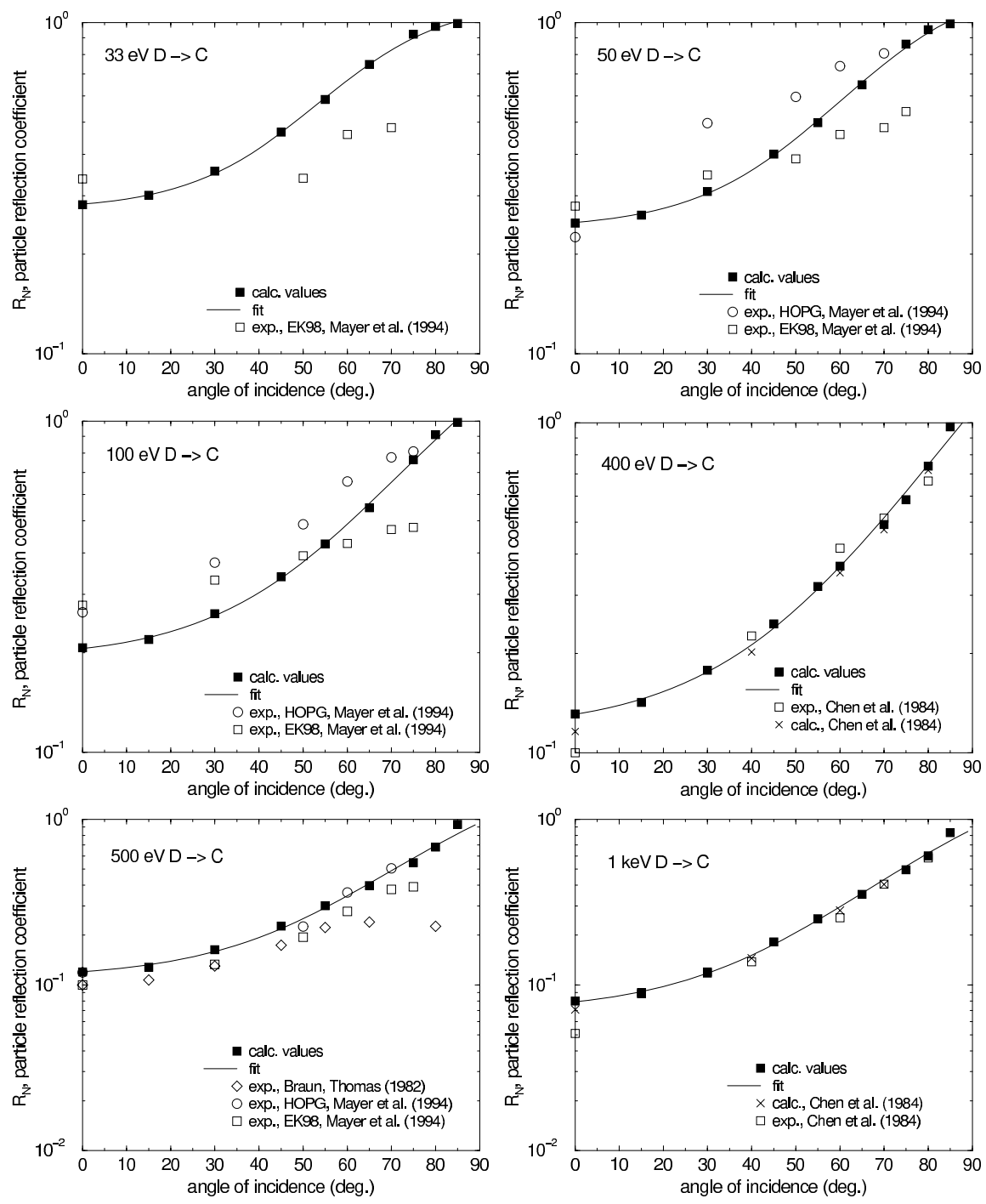

Figure 71: Calculated angular dependence of the particle reflection coefficient at four different incident energies for the bombardment of $\mathrm{C}$ with D. Experimental data of $R_{N}$ are given by $[11,53,79,80]$. Lines are fits to the calculated values (see Table 19) 

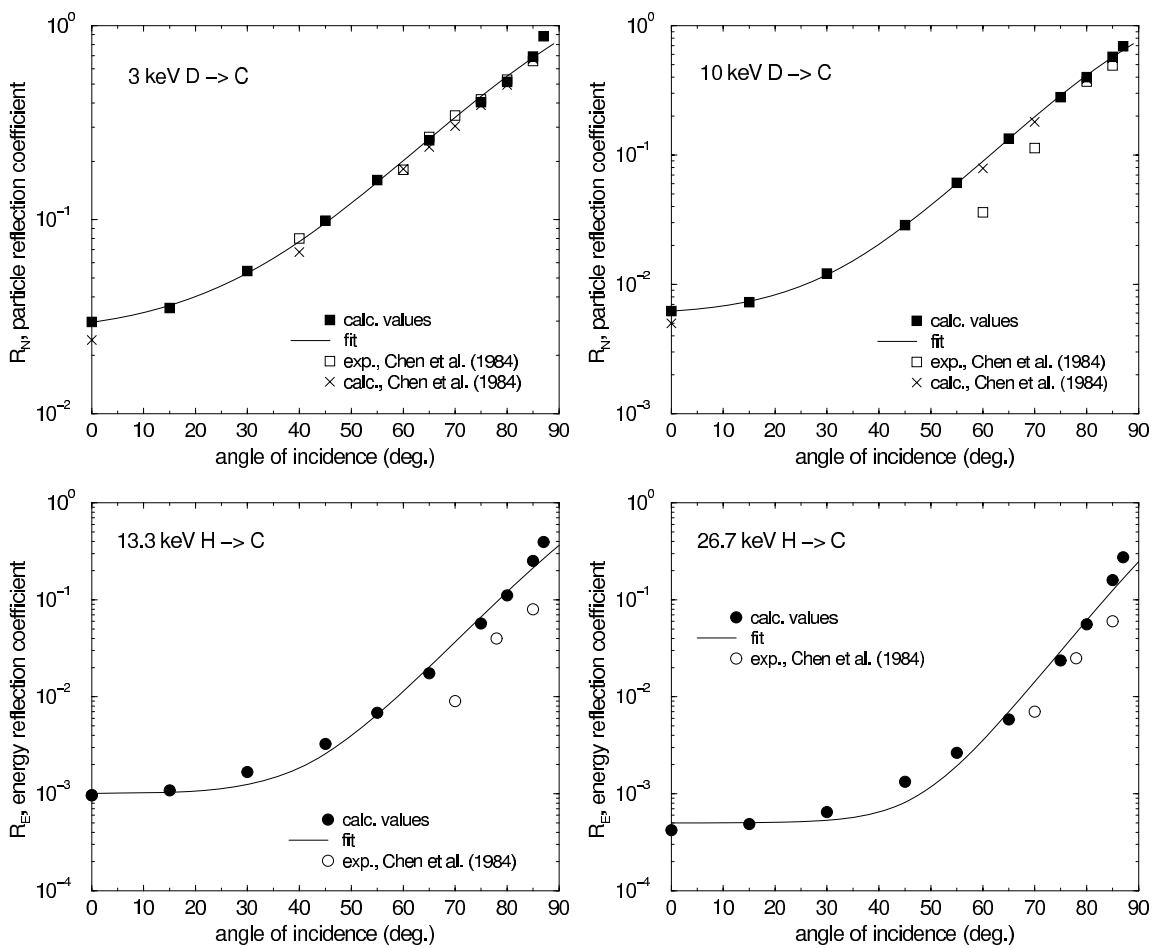

Figure 72: Calculated angular dependence of the particle reflection coefficient for the bombardment of $\mathrm{C}$ with $\mathrm{D}$, and of the energy reflection coefficient for the bombardment of $\mathrm{C}$ with $\mathrm{H}$. Experimental data of $R_{N}$ are given by [11], those of $R_{E}$ by [82]. Lines are fits to the calculated values (see Tables 19 and 32) 

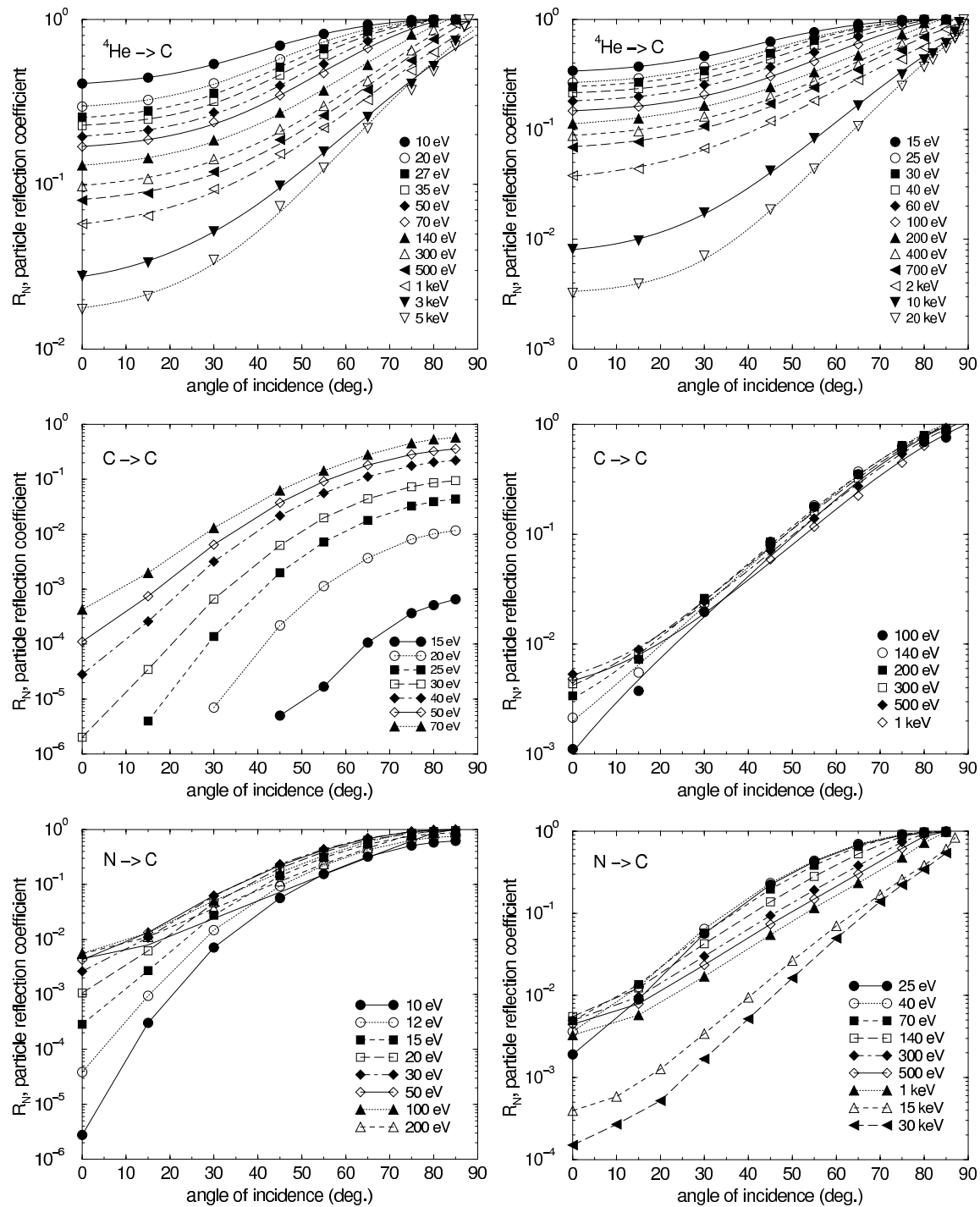

Figure 73: Calculated angular dependence of the particle reflection coefficient at several incident energies for the bombardment of $\mathrm{C}$ with ${ }^{4} \mathrm{He}, \mathrm{C}, \mathrm{N}[8,29]$. Lines are fits to the calculated values (see Table 20). Lines indicated in the legend for $\mathrm{C}$ and $\mathrm{N}$ on $\mathrm{C}$ are drawn to guide the eye 

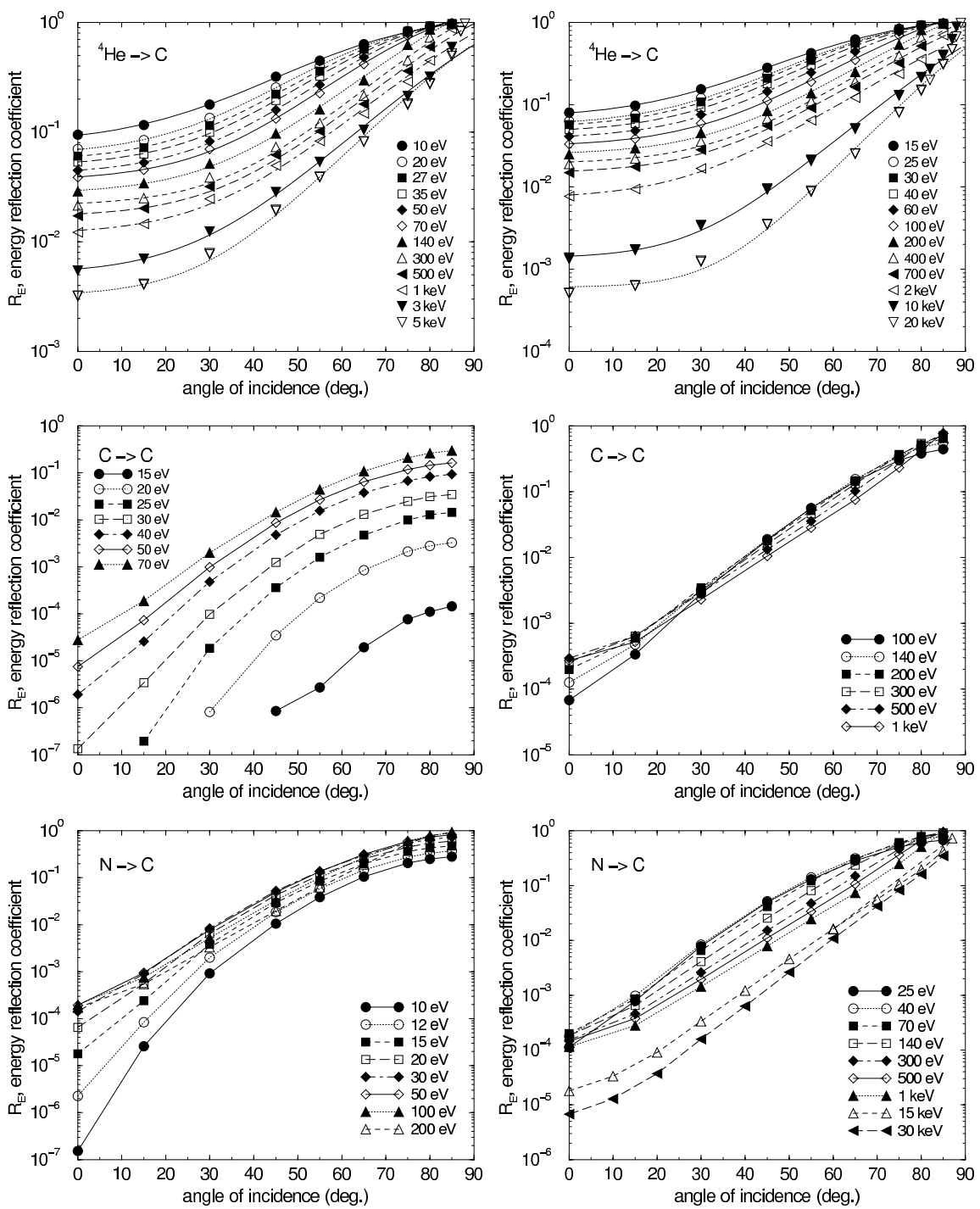

Figure 74: Calculated angular dependence of the energy reflection coefficient at several incident energies for the bombardment of $\mathrm{C}$ with ${ }^{4} \mathrm{He}, \mathrm{C}, \mathrm{N}[8,29]$. Lines are fits to the calculated values (see Table 33). Lines indicated in the legend for $\mathrm{C}$ and $\mathrm{N}$ on $\mathrm{C}$ are drawn to guide the eye 

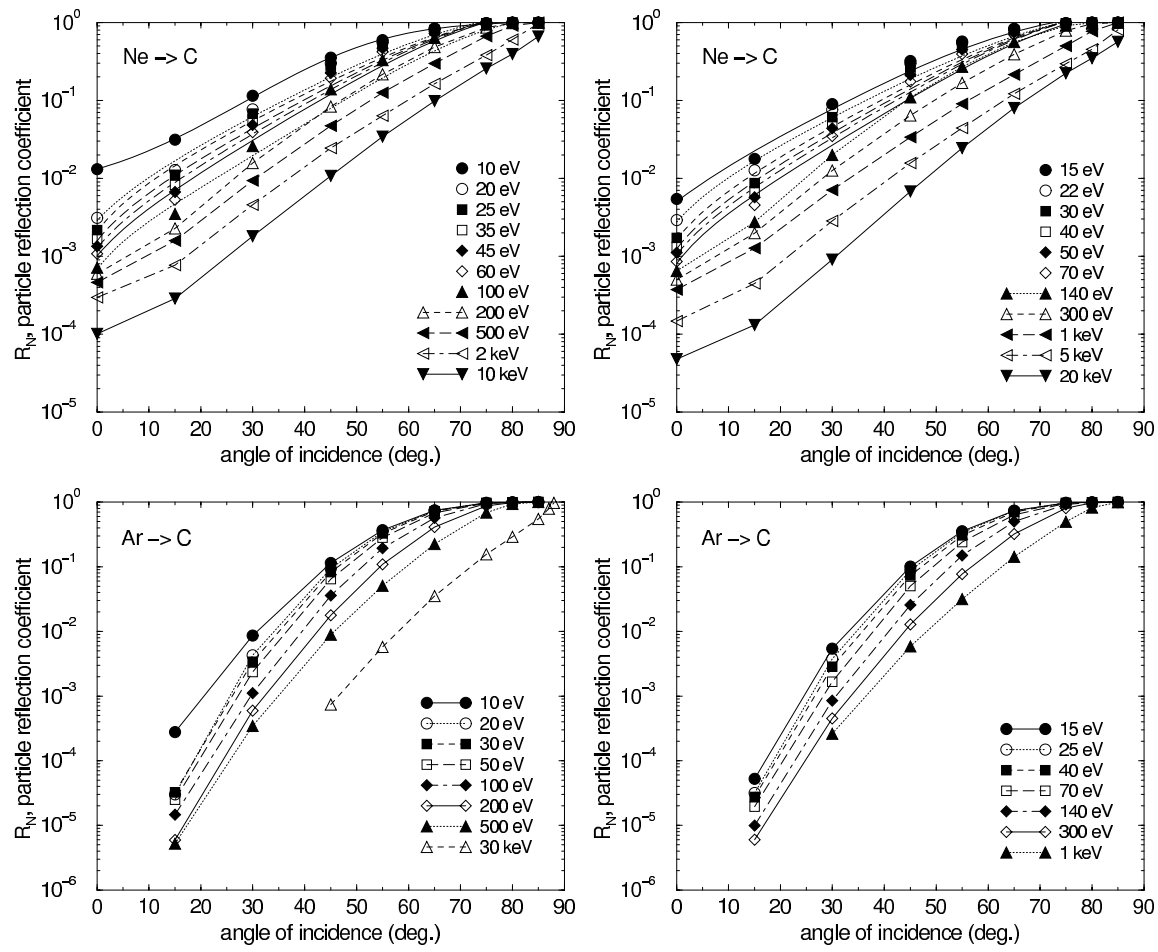

Figure 75: Calculated angular dependence of the particle reflection coefficient at several incident energies for the bombardment of $\mathrm{C}$ with $\mathrm{Ne}, \mathrm{Ar}[8,29]$. Lines are fits to the calculated values (see Table 20). Lines indicated in the legend for $\mathrm{Ne}$ and $\mathrm{Ar}$ on $\mathrm{C}$ are drawn to guide the eye 

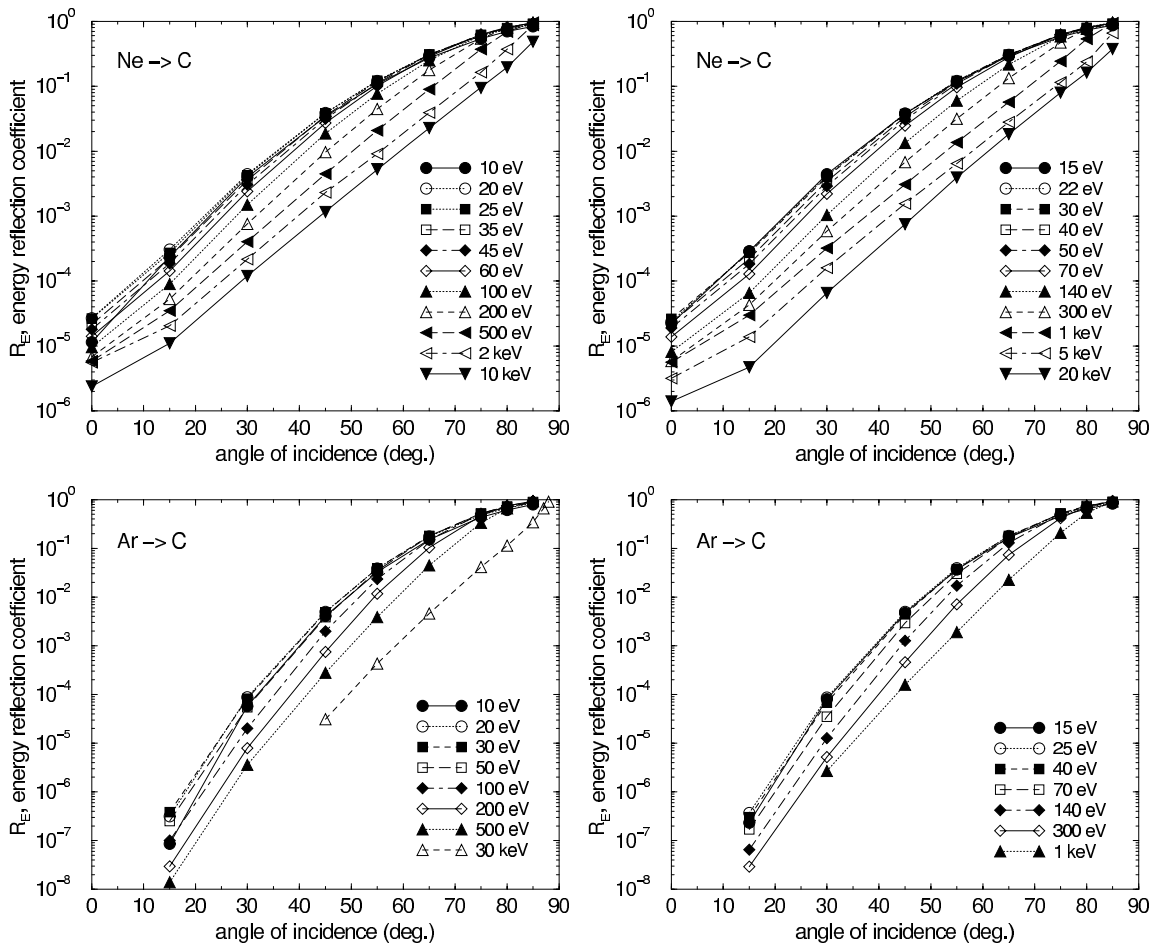

Figure 76: Calculated angular dependence of the particle reflection coefficient at several incident energies for the bombardment of $\mathrm{C}$ with $\mathrm{Ne}, \mathrm{Ar}[8,29]$. Lines indicated in the legend for $\mathrm{Ne}$ and $\mathrm{Ar}$ on $\mathrm{C}$ are drawn to guide the eye 

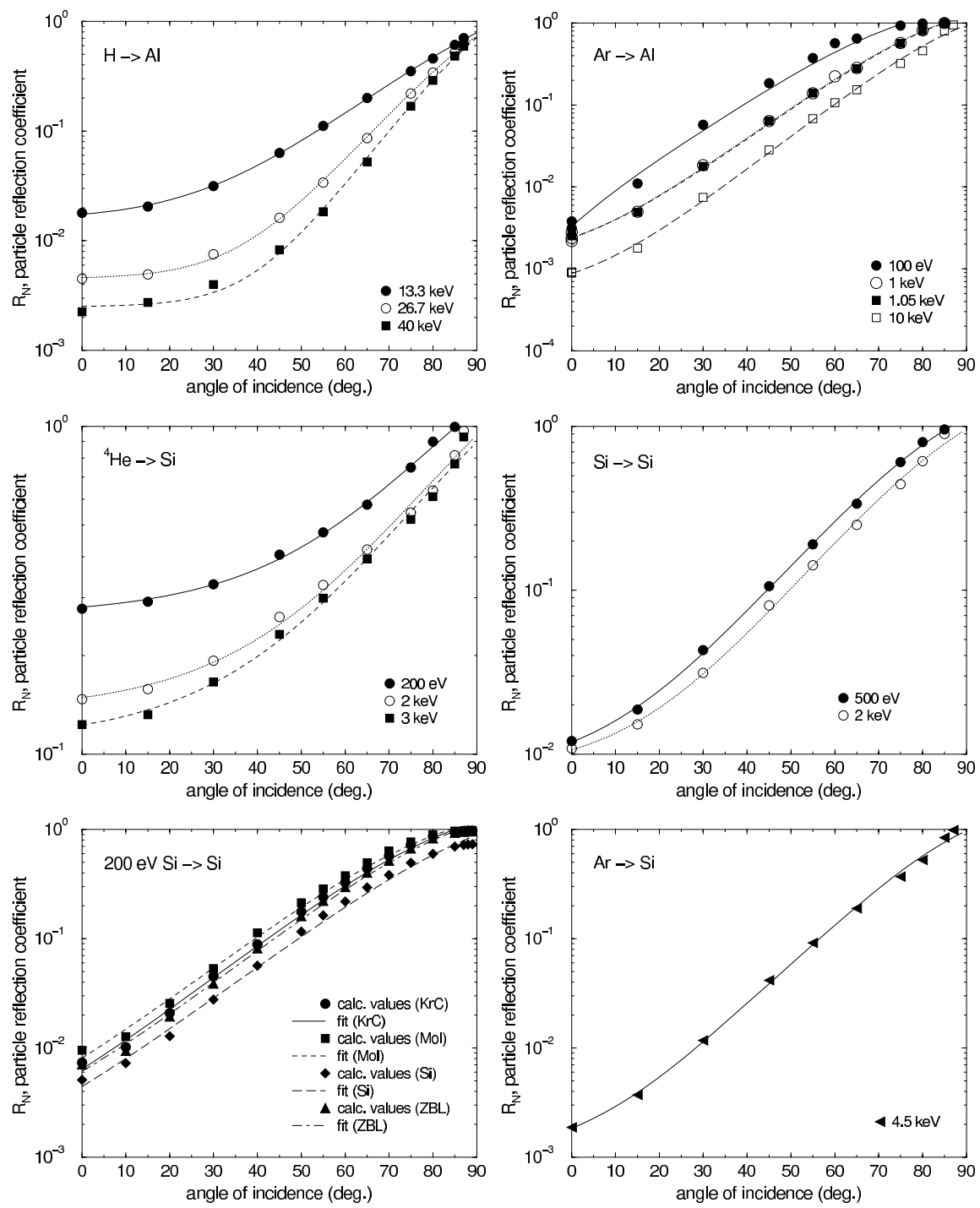

Figure 77: Calculated angular dependence of the particle reflection coefficient at several incident energies for the bombardment of $\mathrm{Al}$ with $\mathrm{H}$ and $\mathrm{Ar}$ and $\mathrm{Si}$ with ${ }^{4} \mathrm{He}, \mathrm{Si}$, Ar. One figure shows the dependence of $R_{N}$ for $200 \mathrm{eV} \mathrm{Si}$ selfbombardment on different interaction potentials: $\mathrm{KrC}$ (WHB) [20], Moliere [22], a specific Si-Si potential [23], ZBl [21]. Lines are fits to the calculated values (see Table 21) 

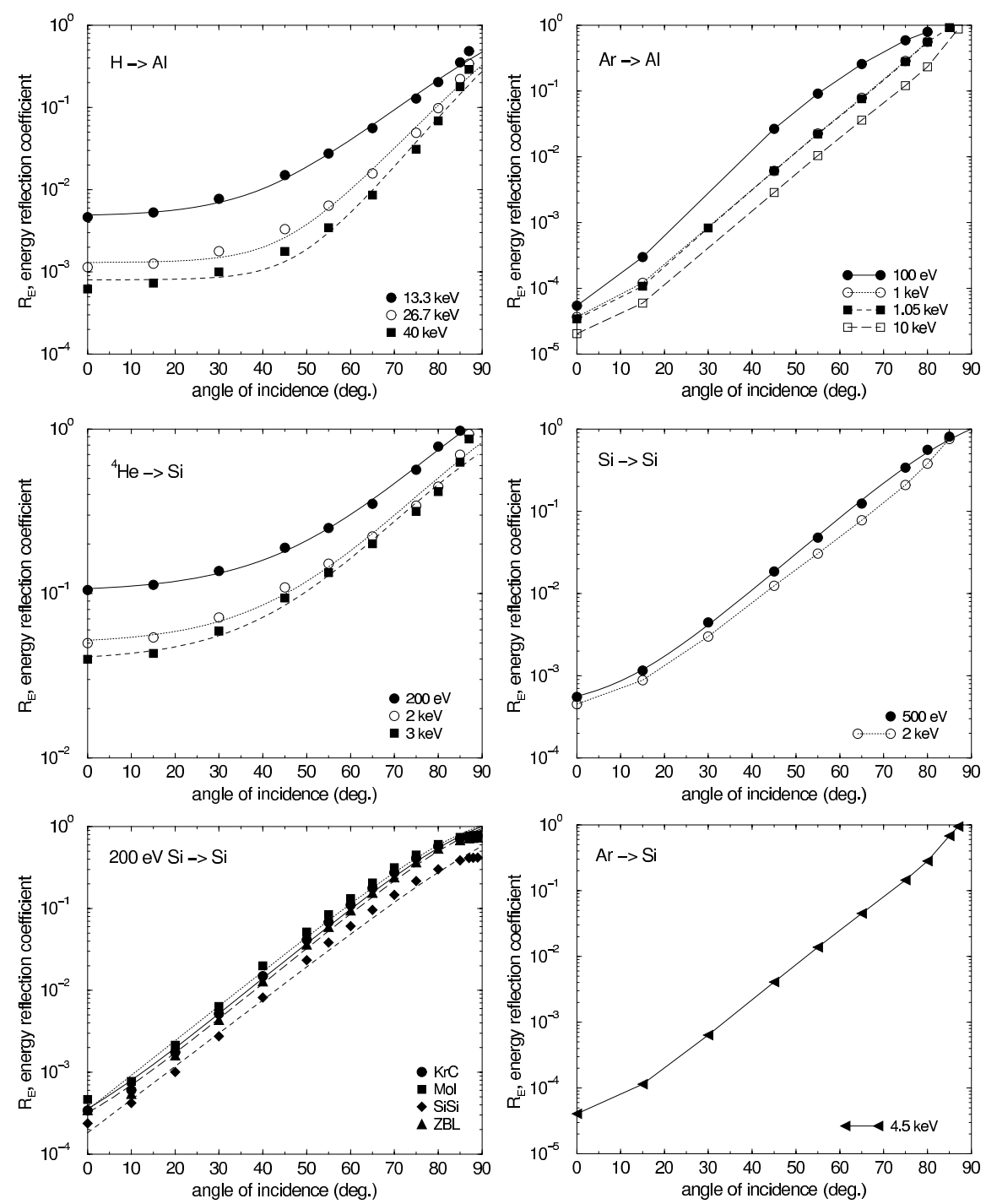

Figure 78: Calculated angular dependence of the energy reflection coefficient at several incident energies for the bombardment of $\mathrm{Al}$ with $\mathrm{H}$ and $\mathrm{Ar}$ and $\mathrm{Si}$ with ${ }^{4} \mathrm{He}, \mathrm{Si}, \mathrm{Ar}$. One figure shows the dependence of $R_{E}$ for $200 \mathrm{eV} \mathrm{Si}$ selfbombardment on different interaction potentials: $\mathrm{KrC}$ (WHB) [20], Moliere [22], a specific Si-Si potential [23], ZBl [21]. Lines are fits to the calculated values (see Table 34). Lines indicated in the legend for $\mathrm{Ar}$ on $\mathrm{Al}$ are drawn to guide the eye 

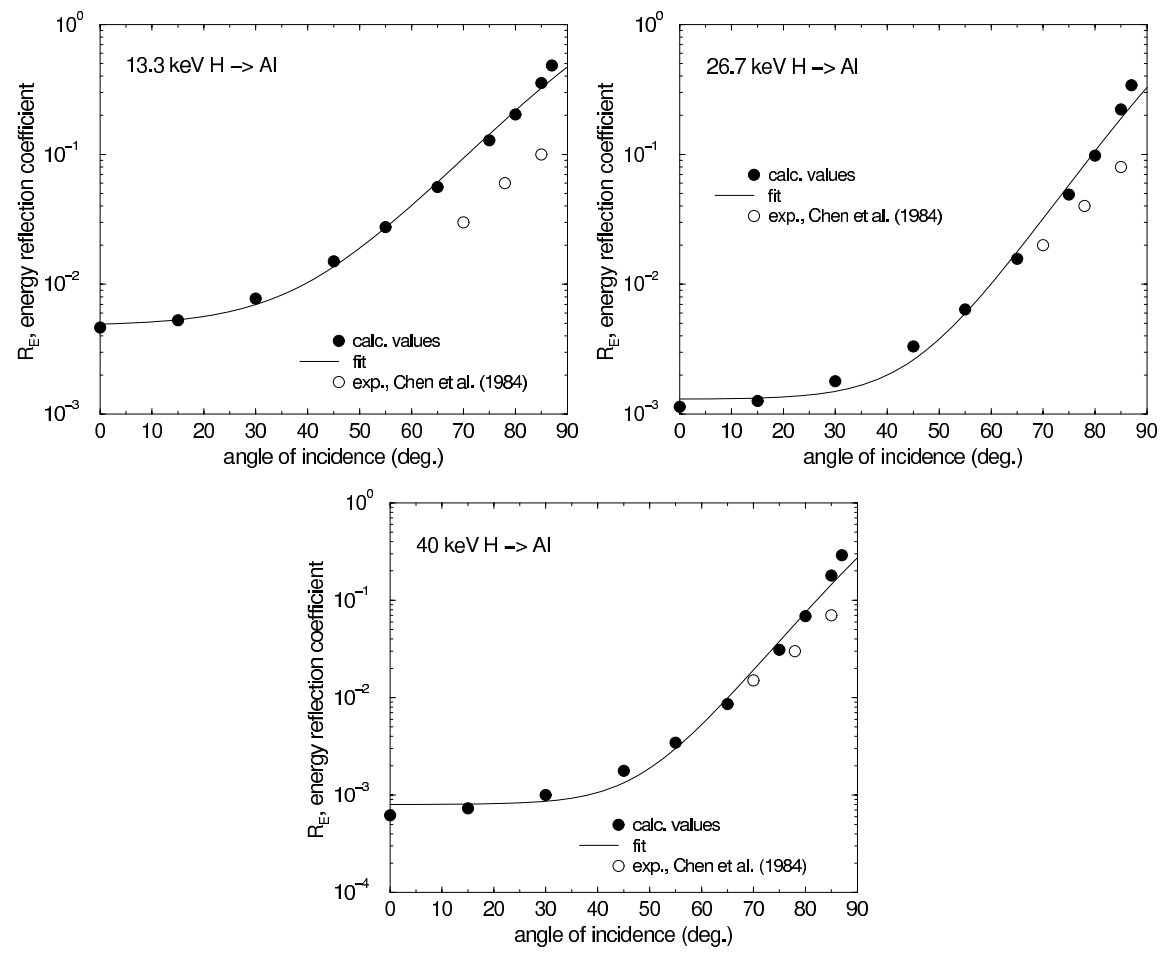

Figure 79: Calculated angular dependence of the energy reflection coefficient at several incident energies for the bombardment of $\mathrm{Al}$ with $\mathrm{H}$ [30]. Experimental values are reported in [82]. Lines are fits to the calculated values (see Table 34) 

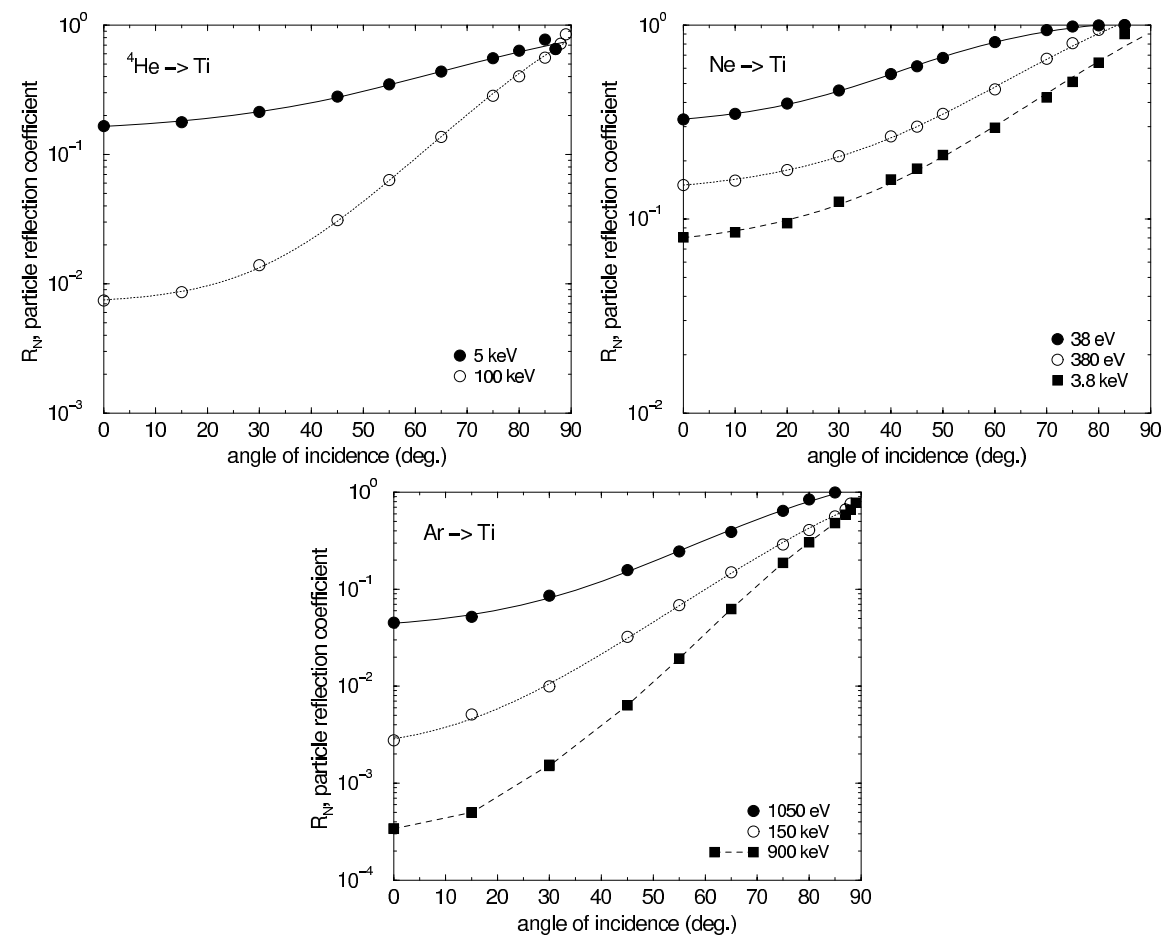

Figure 80: Calculated angular dependence of the particle reflection coefficient at several incident energies for the bombardment of $\mathrm{Ti}$ with ${ }^{4} \mathrm{He}, \mathrm{Ne}$ [29], $\mathrm{Ar}$ [30]. Lines are fits to the calculated values (see Table 21). The line indicated in the legend for $900 \mathrm{keV} \mathrm{Ar}$ on $\mathrm{Ti}$ is drawn to guide the eye 

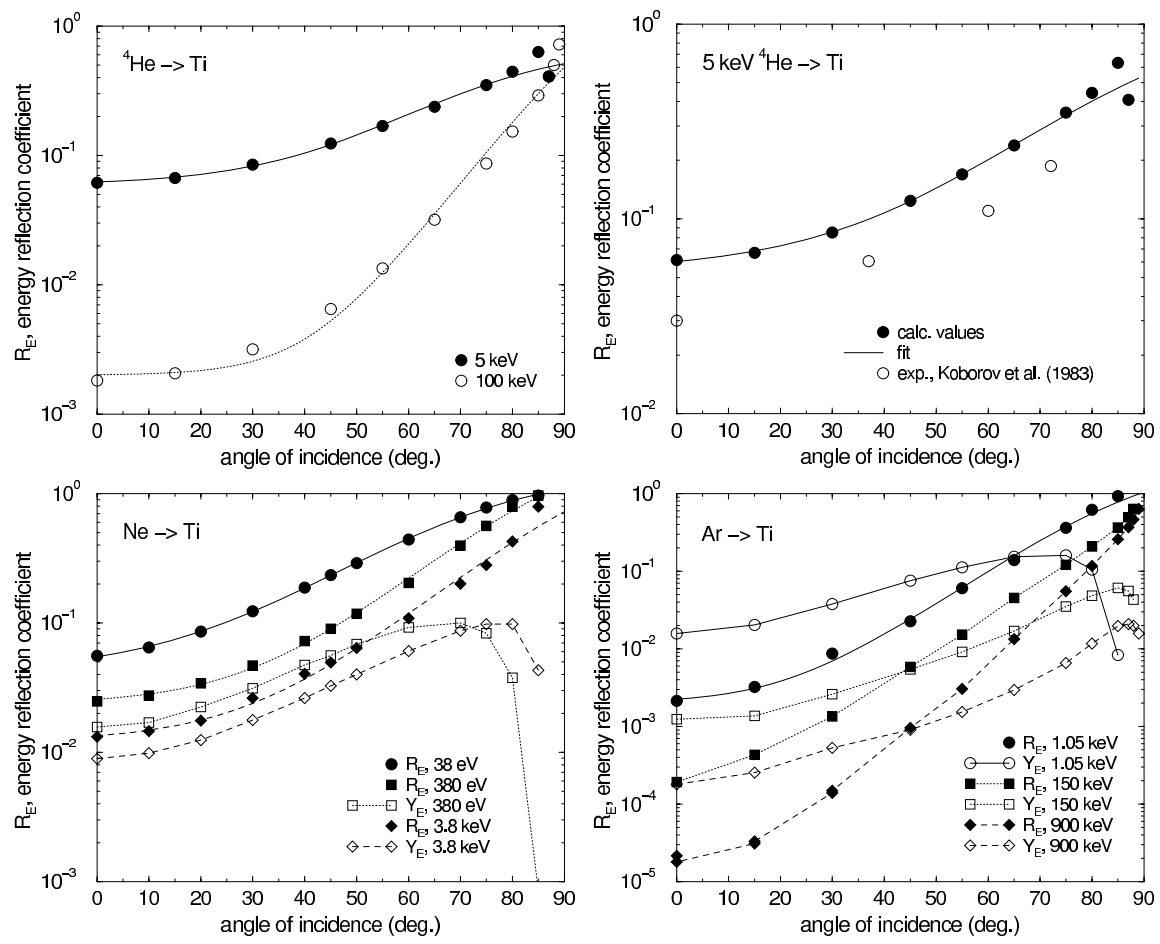

Figure 81: Calculated angular dependence of the energy reflection coefficient at several incident energies for the bombardment of $\mathrm{Ti}$ with ${ }^{4} \mathrm{He}, \mathrm{Ne}$ [29], $\mathrm{Ar}$ [30]. Experimental data for $5 \mathrm{keV}{ }^{4} \mathrm{He}$ are given by [61]. Lines are fits to the calculated values (see Table 34 ). Some lines for $R_{E}$ indicated in the legend for $\mathrm{Ar}$ on $\mathrm{Ti}$ and all lines for $Y_{E}$ are drawn to guide the eye 

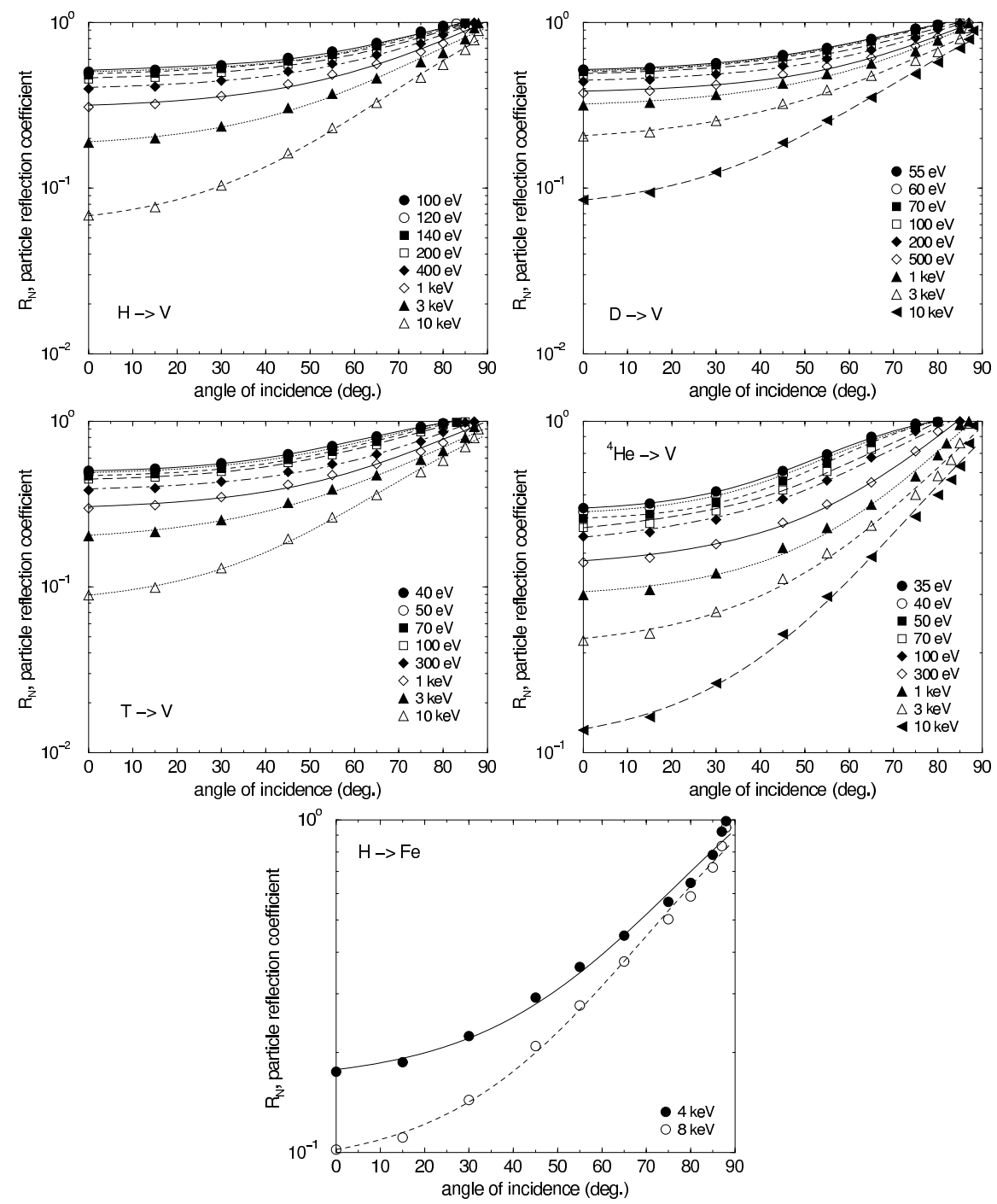

Figure 82: Calculated angular dependence of the particle reflection coefficient at several incident energies for the bombardment of $\mathrm{V}$ with $\mathrm{H}, \mathrm{D}, \mathrm{T},{ }^{4} \mathrm{He}$, and of Fe with $\mathrm{H}$ [30]. Lines are fits to the calculated values (see Tables 22 and 23) 

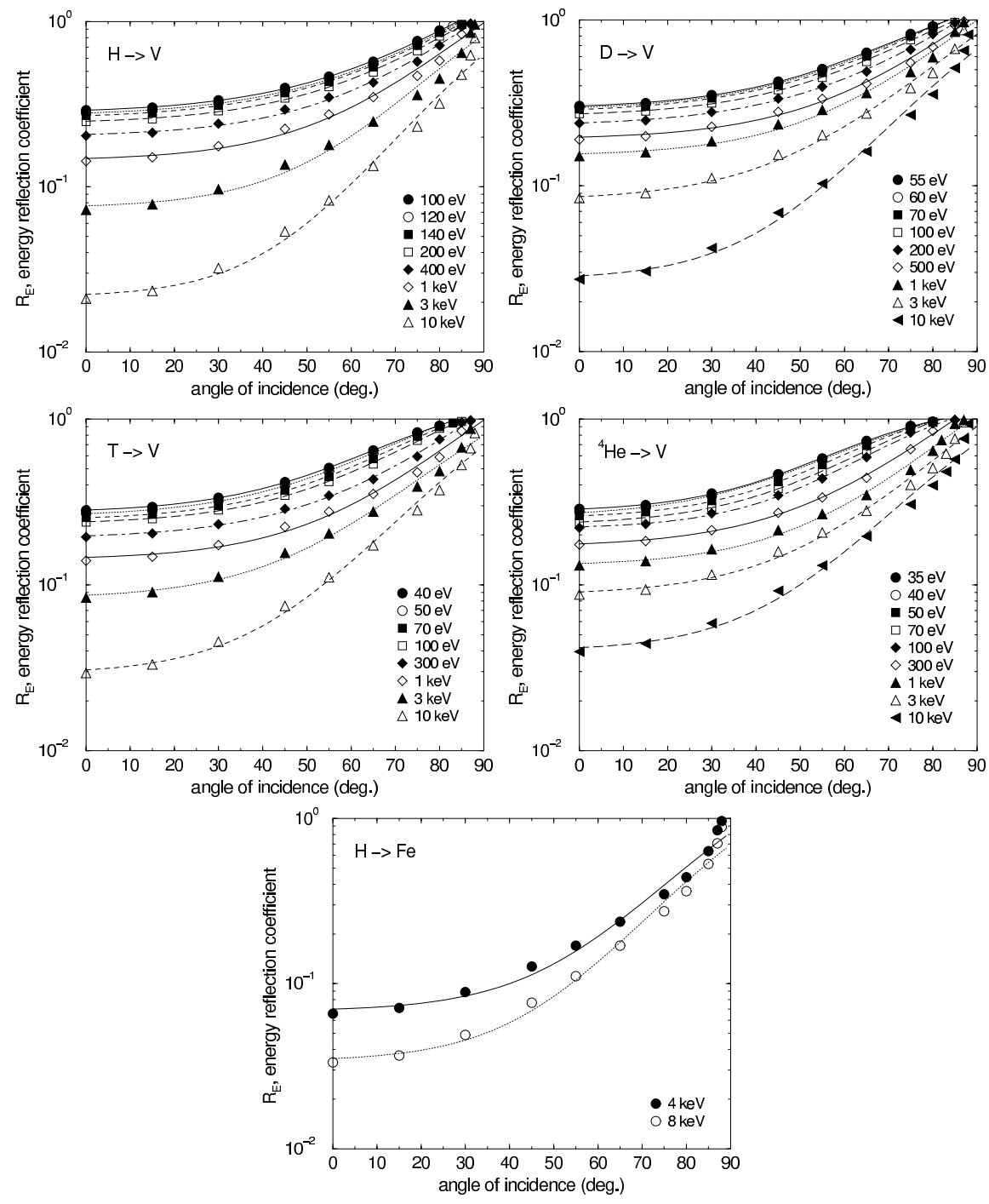

Figure 83: Calculated angular dependence of the energy reflection coefficient at several incident energies for the bombardment of $\mathrm{V}$ with $\mathrm{H}, \mathrm{D}, \mathrm{T},{ }^{4} \mathrm{He}$, and of Fe with $\mathrm{H}$ [30]. Lines are fits to the calculated values (see Tables 35 and 36) 

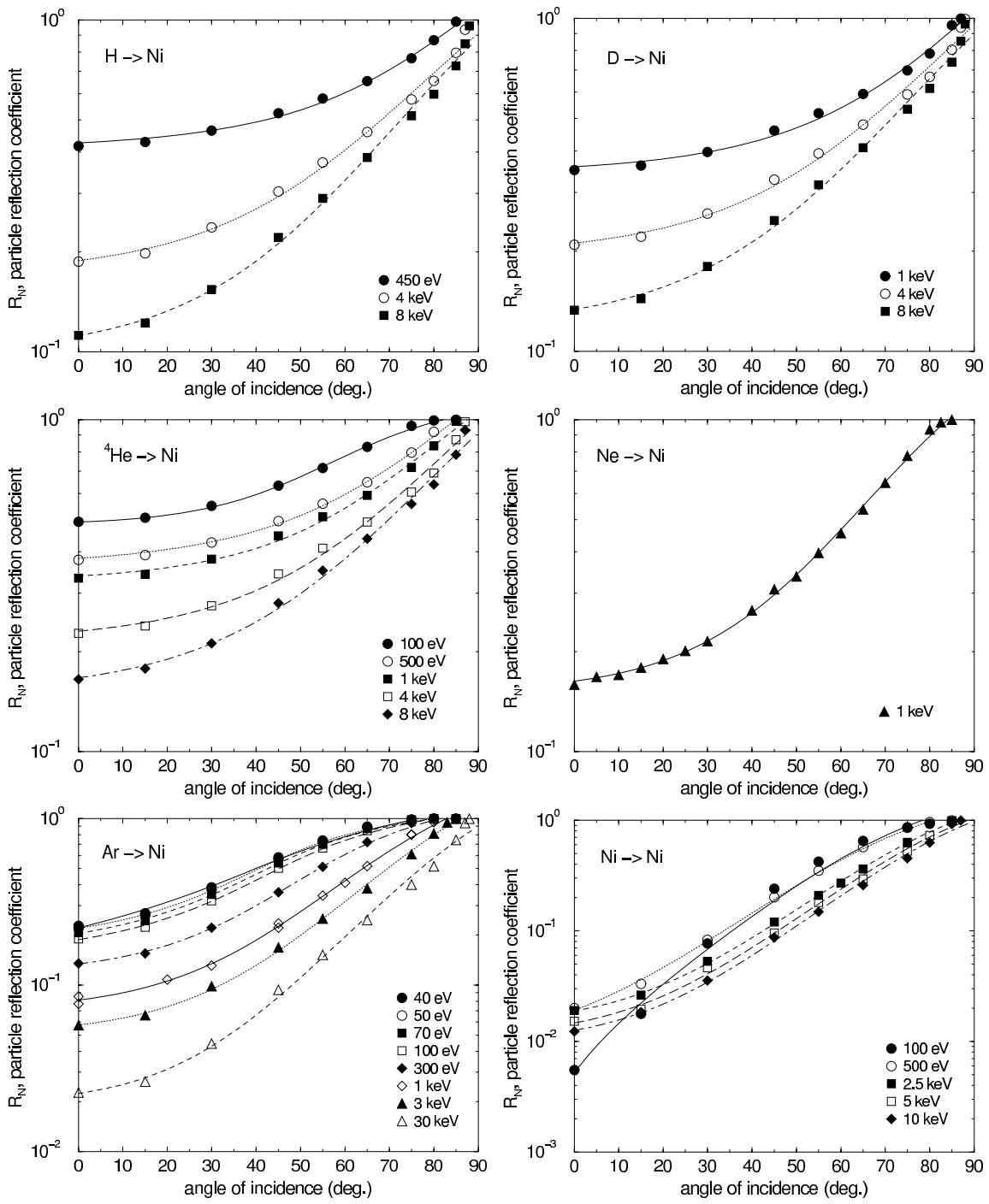

Figure 84: Calculated angular dependence of the particle reflection coefficient at several incident energies for the bombardment of $\mathrm{Ni}$ with $\mathrm{H}, \mathrm{D},{ }^{4} \mathrm{He}, \mathrm{Ar}, \mathrm{Ni}$ $[29,30]$. Lines are fits to the calculated values (see Table 23) 

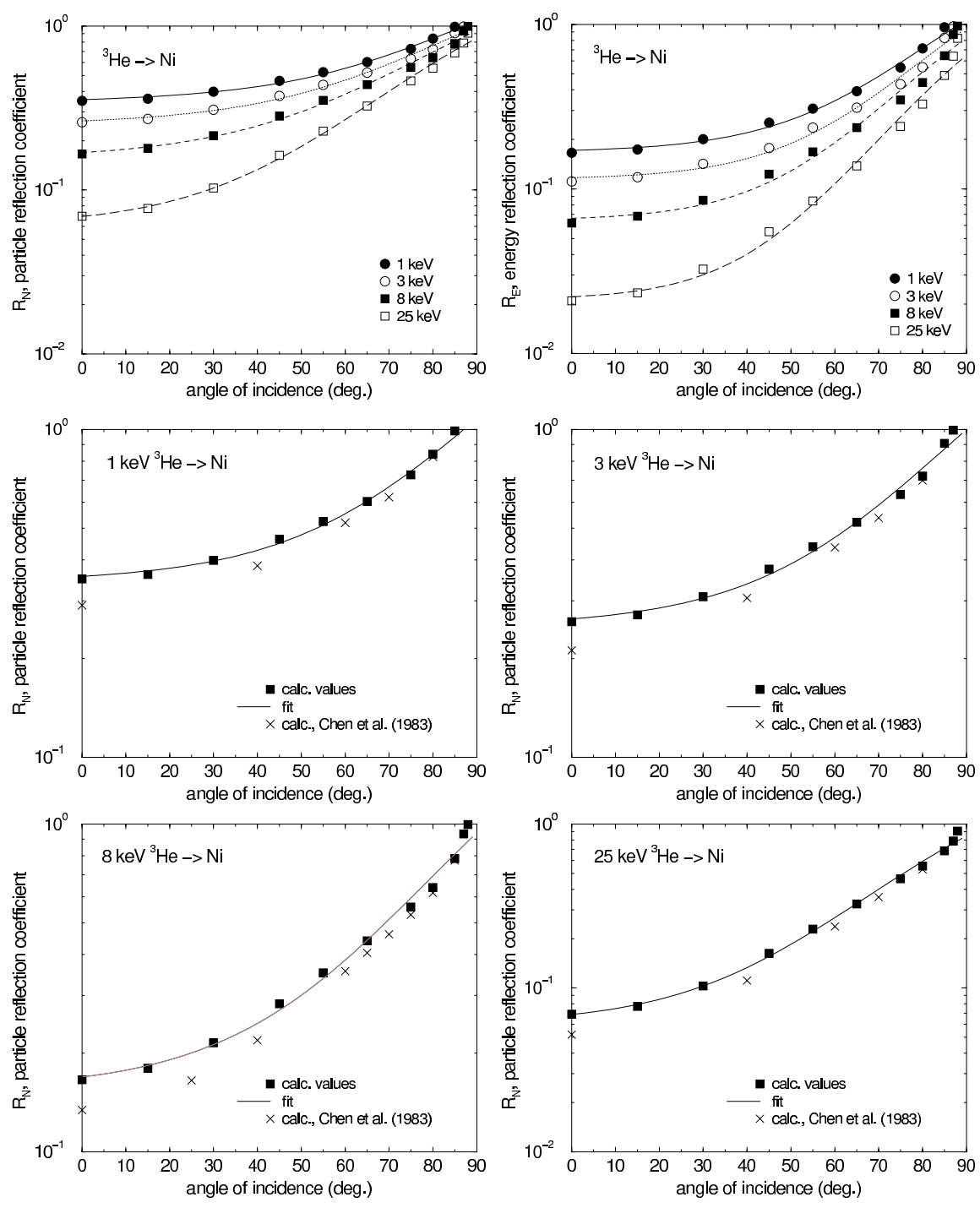

Figure 85: Calculated angular dependence of the particle reflection coefficient of ${ }^{3} \mathrm{He}$ at several incident energies for the bombardment of $\mathrm{Ni}$ with ${ }^{3} \mathrm{He}$ [30]. Earlier calculated values are given by [81]. Lines are fits to the calculated values (see Table 23) 

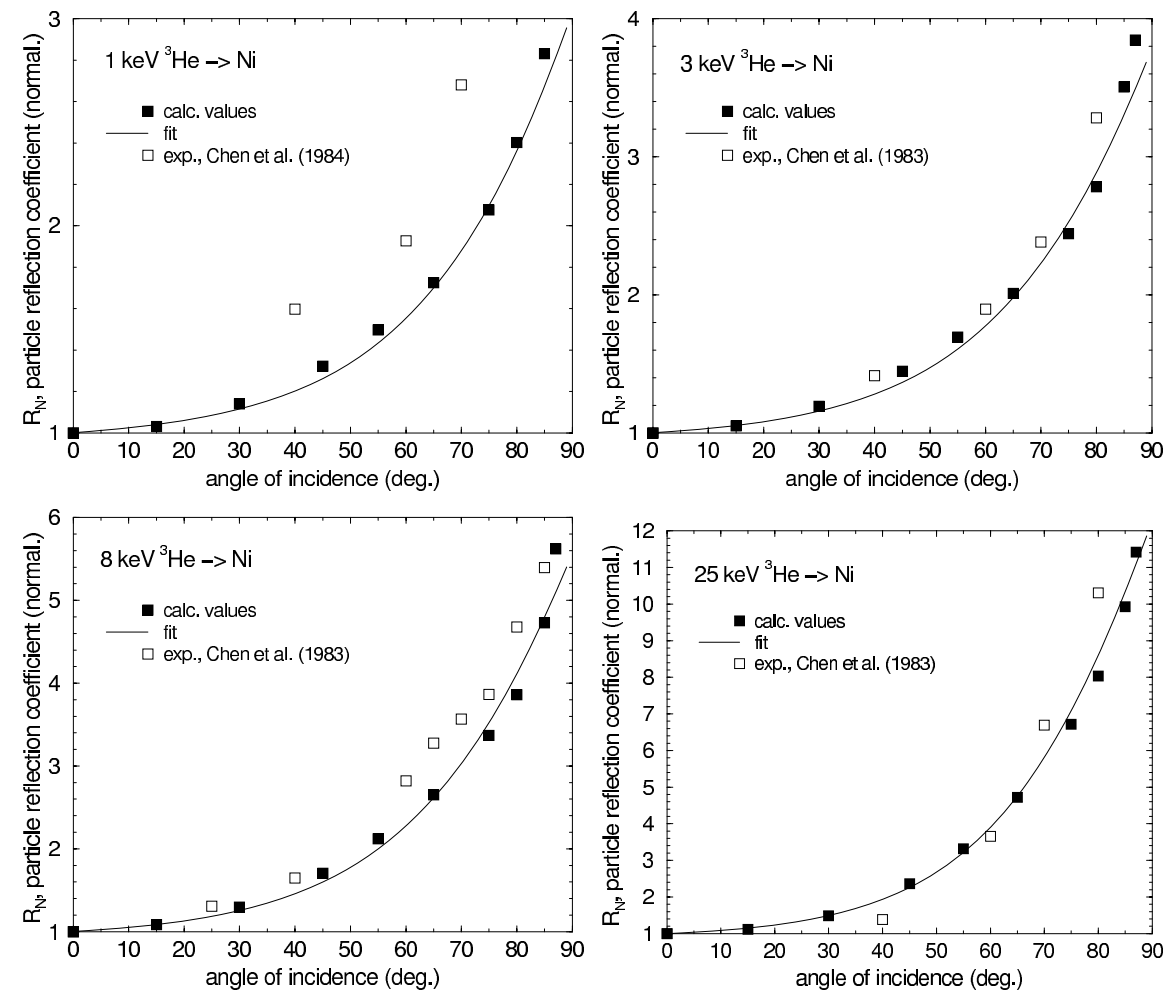

Figure 86: Calculated angular dependence of the normalized particle reflection coefficient, $R_{N}(\alpha) / R_{N}(0)$, for the bombardment of Ni with ${ }^{3} \mathrm{He}$ at several incident energies [30]. Experimental data are given by [81]. Lines are fits to the calculated values (see Table 23) 

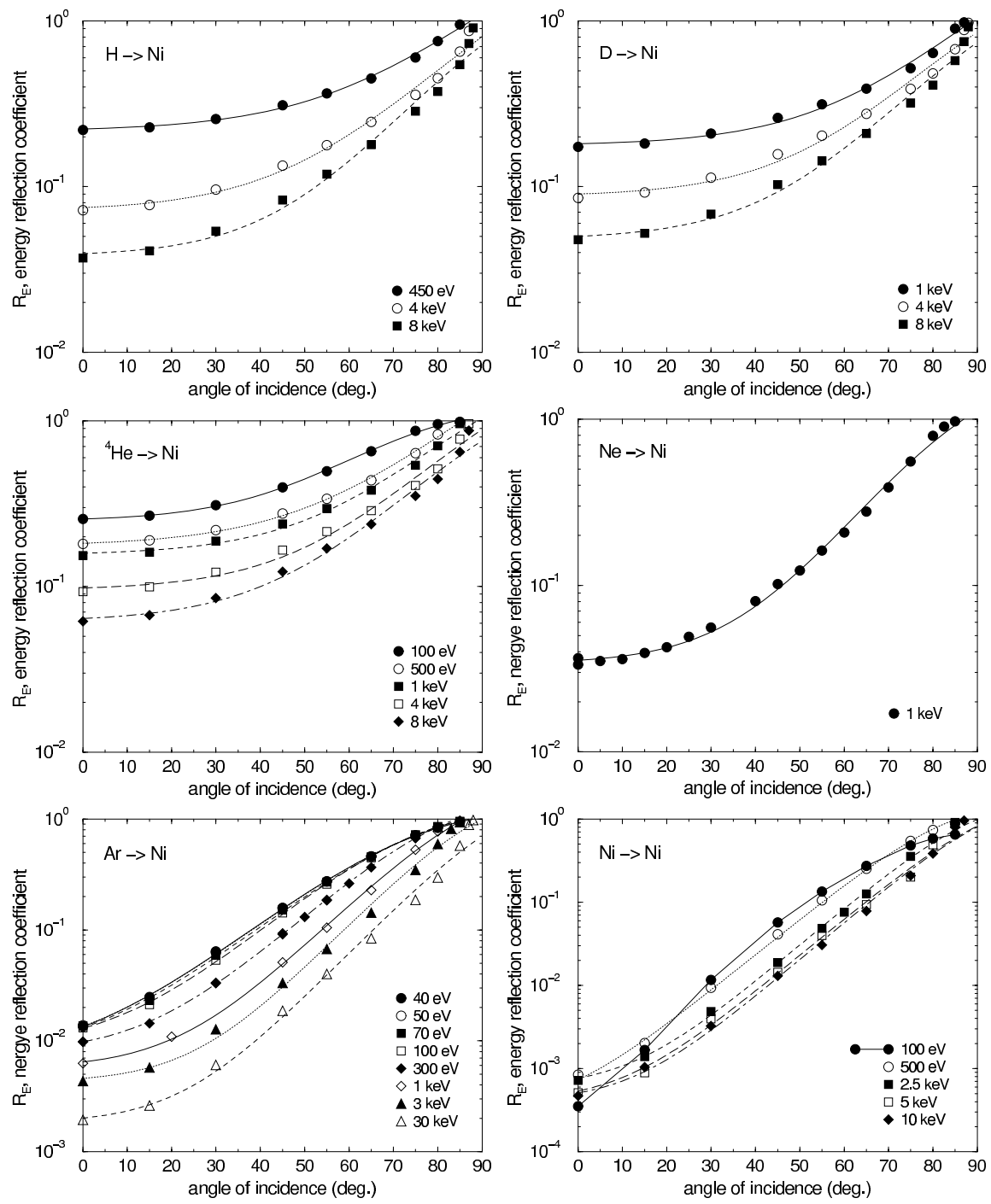

Figure 87: Calculated angular dependence of the energy reflection coefficient at several incident energies for the bombardment of $\mathrm{Ni}$ with $\mathrm{H}, \mathrm{D},{ }^{4} \mathrm{He}, \mathrm{Ar}, \mathrm{Ni}$ $[29,30]$. Lines are fits to the calculated values (see Table 36) 

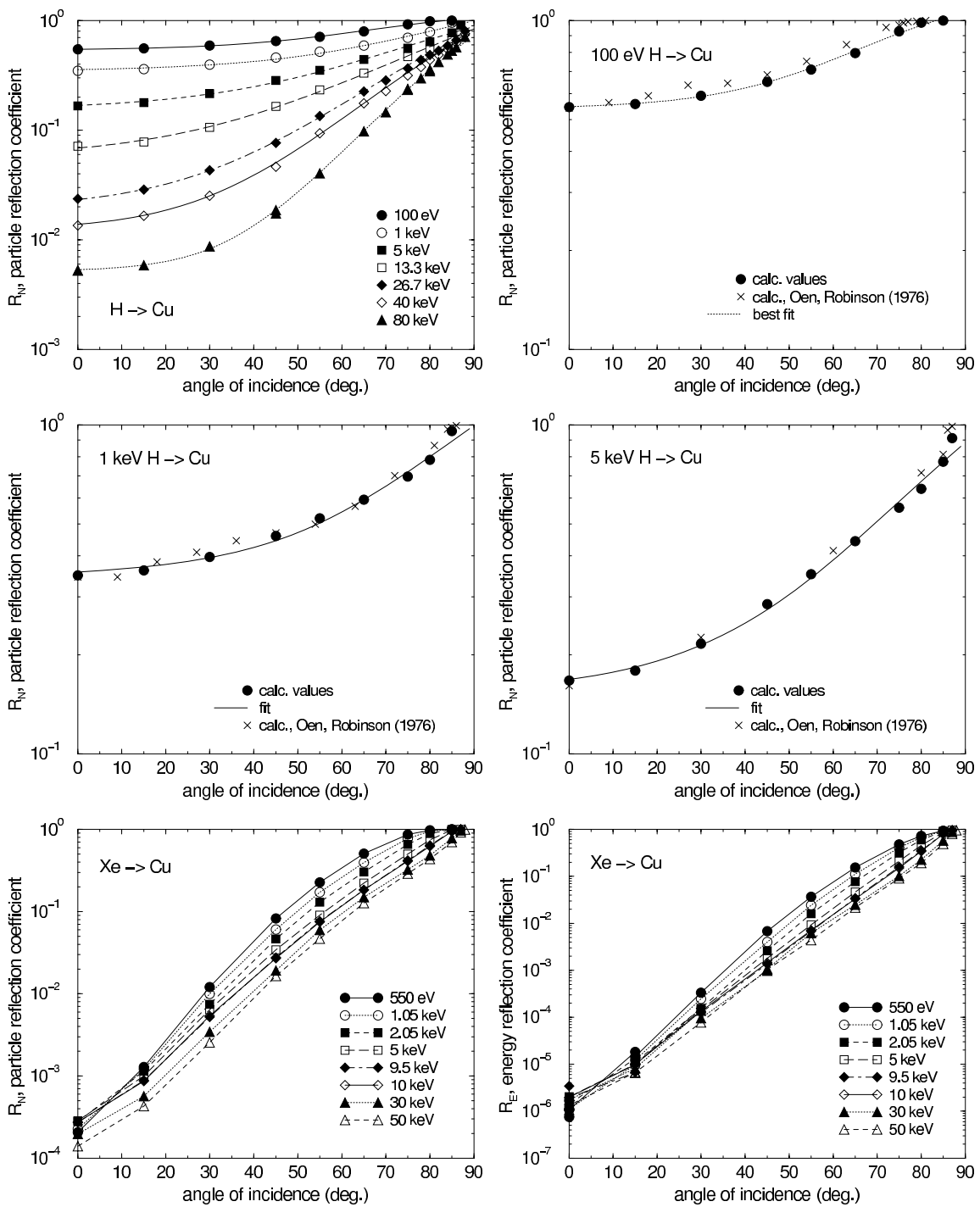

Figure 88: Calculated angular dependence of the particle reflection coefficient at several incident energies for the bombardment of $\mathrm{Cu}$ with $\mathrm{H}$ and $\mathrm{Xe}$ and of the energy reflection coefficient of $\mathrm{Xe}$ on $\mathrm{Cu}$ [30]. Earlier calculated values are given for $100 \mathrm{eV}, 1$ and $5 \mathrm{keV} \mathrm{H}$ [24]. Lines are fits to the calculated values (see Table 23). Lines indicated in the legend for $\mathrm{Xe}$ on $\mathrm{Cu}$ are drawn to guide the eye 

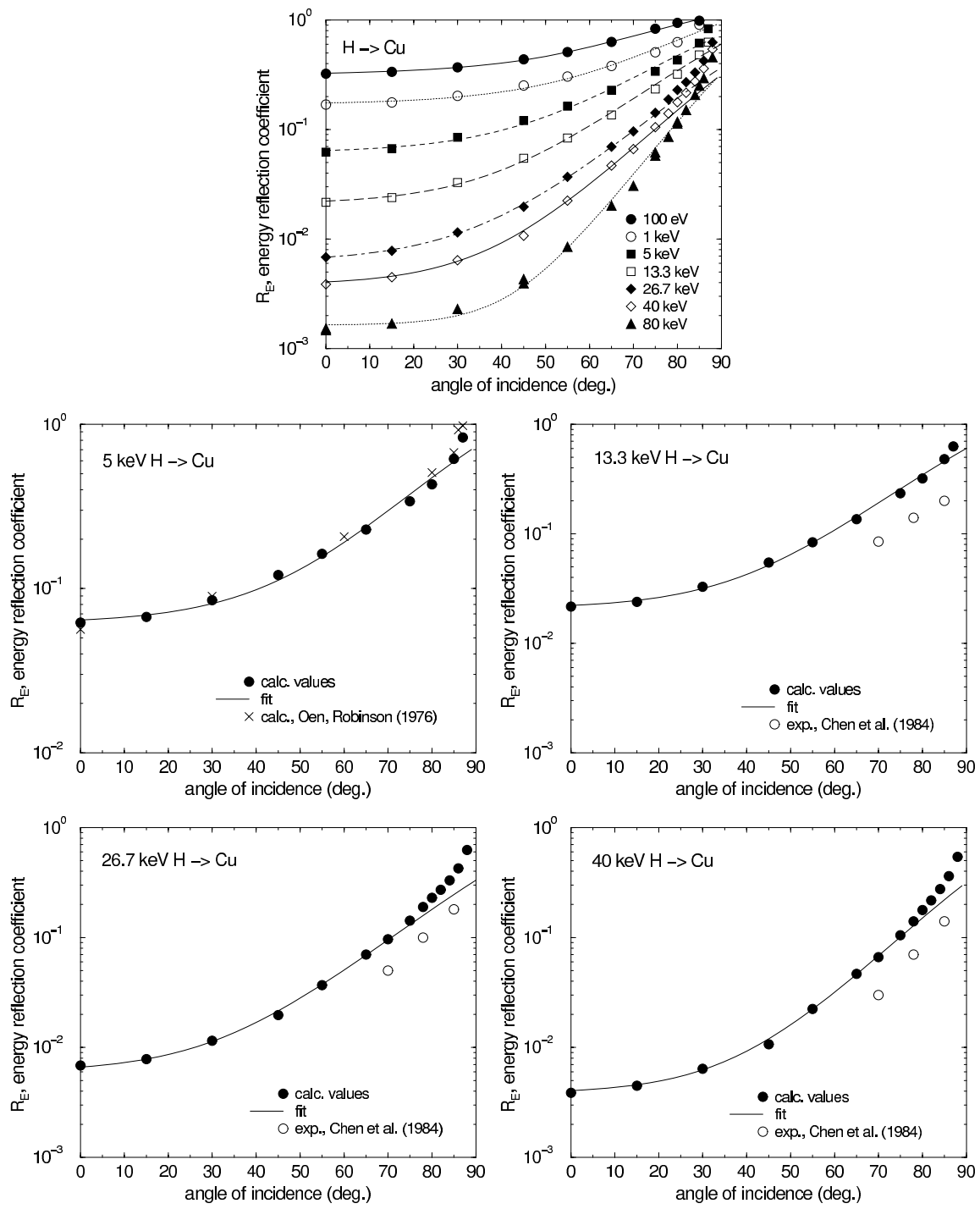

Figure 89: Calculated angular dependence of the energy reflection coefficient at several incident energies for the bombardment of $\mathrm{Cu}$ with $\mathrm{H}[30,83]$. Experimental values of $\mathrm{H}$ on $\mathrm{Cu}$ by [82]. Lines are fits to the calculated values (see Table 36) 

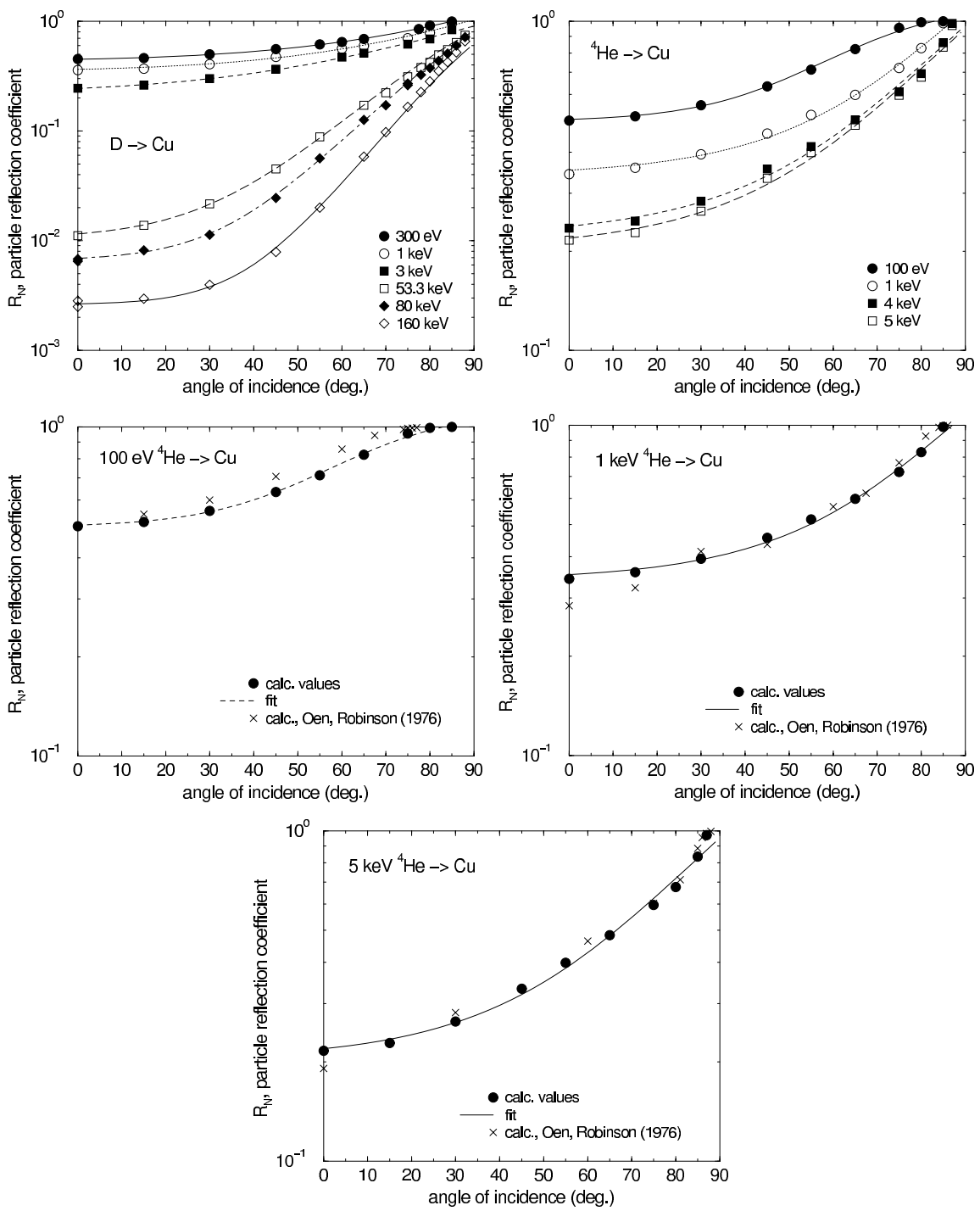

Figure 90: Calculated angular dependence of the particle reflection coefficient at several incident energies for the bombardment of $\mathrm{Cu}$ with $\mathrm{D}$ and ${ }^{4} \mathrm{He}$ [30]. Earlier calculated values are given for $100 \mathrm{eV}, 1$ and $5 \mathrm{keV}{ }^{4} \mathrm{He}$ [24]. Lines are fits to the calculated values (see Table 24) 

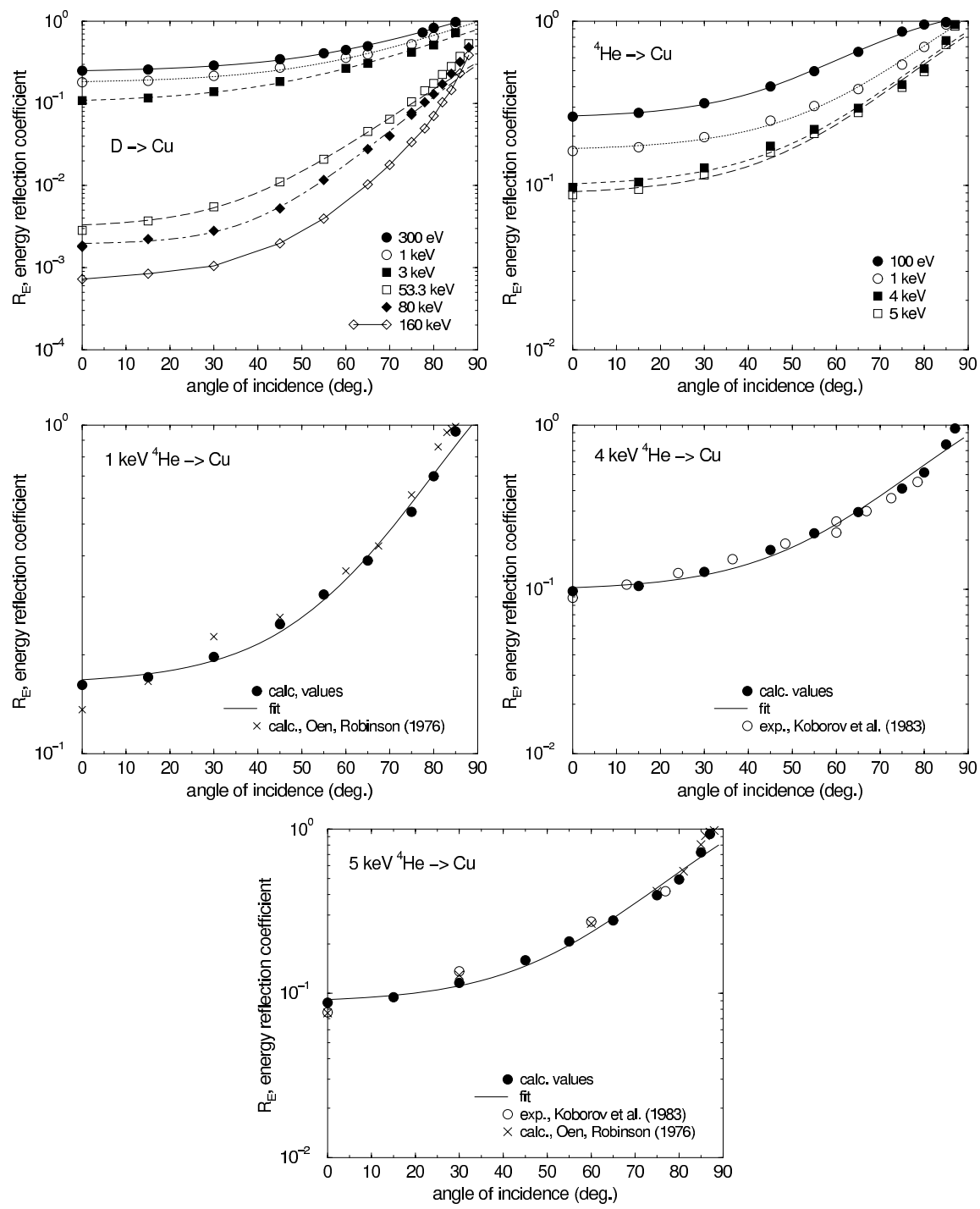

Figure 91: Calculated angular dependence of the energy reflection coefficient at several incident energies for the bombardment of $\mathrm{Cu}$ with ${ }^{4} \mathrm{He}$ [30]. Earlier calculated values for ${ }^{4} \mathrm{He}$ on $\mathrm{Cu}$ are given by [24], experimental values of ${ }^{4} \mathrm{He}$ on $\mathrm{Cu}$ by [61]. Lines are fits to the calculated values (see Table 37 ) 

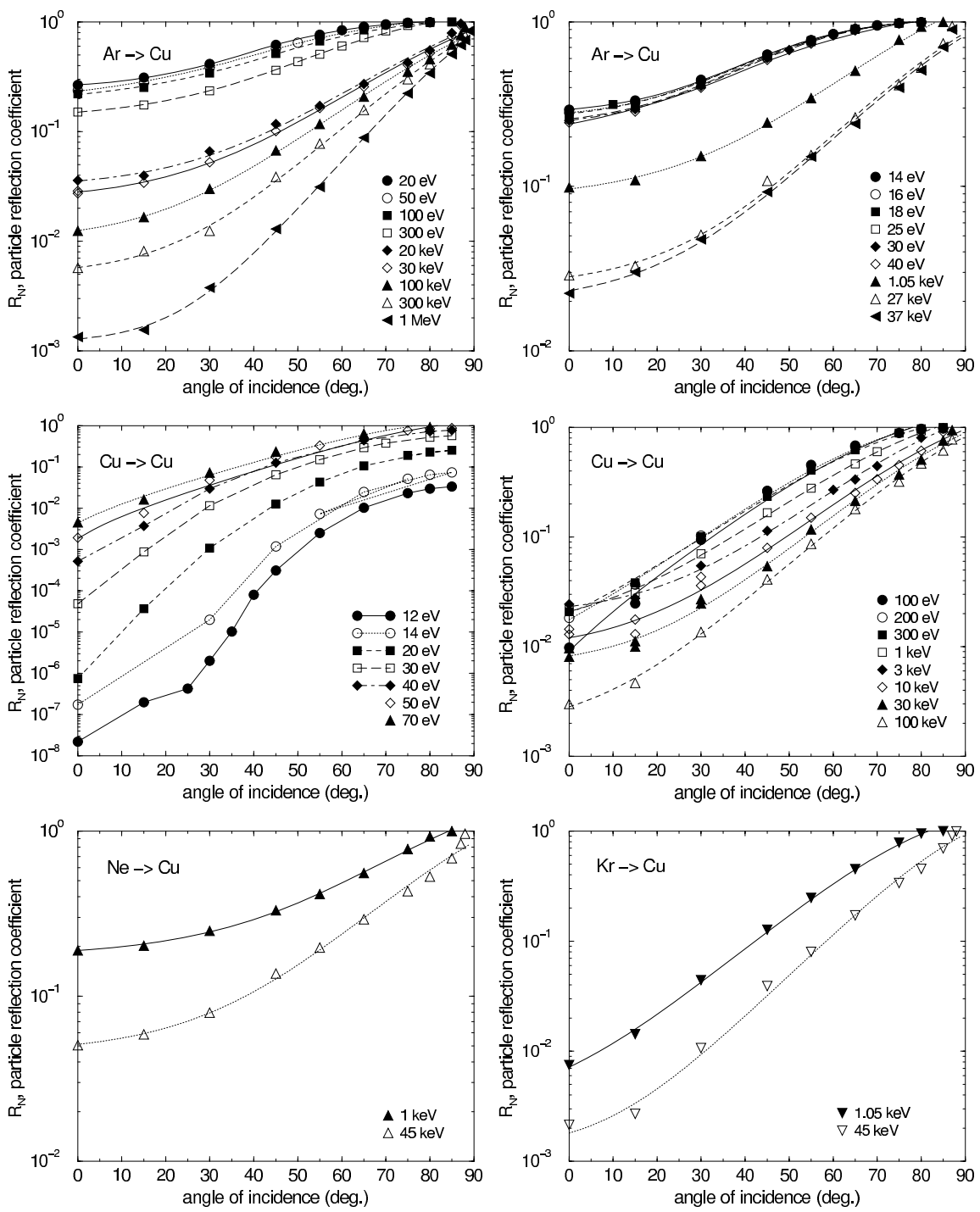

Figure 92: Calculated angular dependence of the particle reflection coefficient at several incident energies for the bombardment of $\mathrm{Cu}$ with $\mathrm{Ar}, \mathrm{Cu}, \mathrm{Ne}, \mathrm{Kr}$ $[29,30]$. Lines are fits to the calculated values (see Table 24. Some lines indicated in the legend for $\mathrm{Cu}$ on $\mathrm{Cu}$ are drawn to guide the eye 

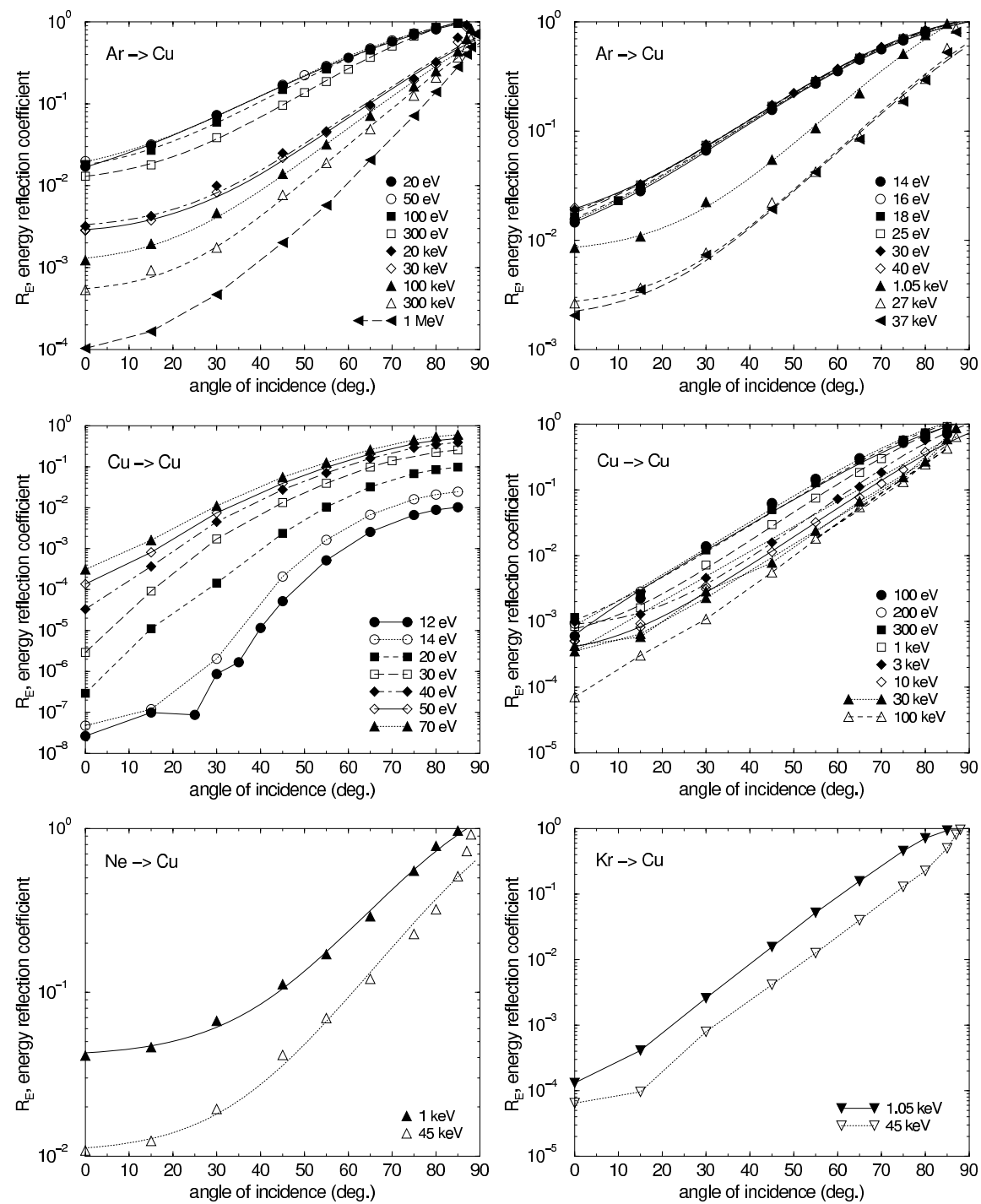

Figure 93: Calculated angular dependence of the particle reflection coefficient at several incident energies for the bombardment of $\mathrm{Cu}$ with $\mathrm{Ar}, \mathrm{Cu}, \mathrm{Ne}, \mathrm{Kr}$ $[29,30]$. Lines are fits to the calculated values (see Table 37). Some lines indicated in the legend for $\mathrm{Ar}, \mathrm{Cu}, \mathrm{Kr}$ on $\mathrm{Cu}$ are drawn to guide the eye 

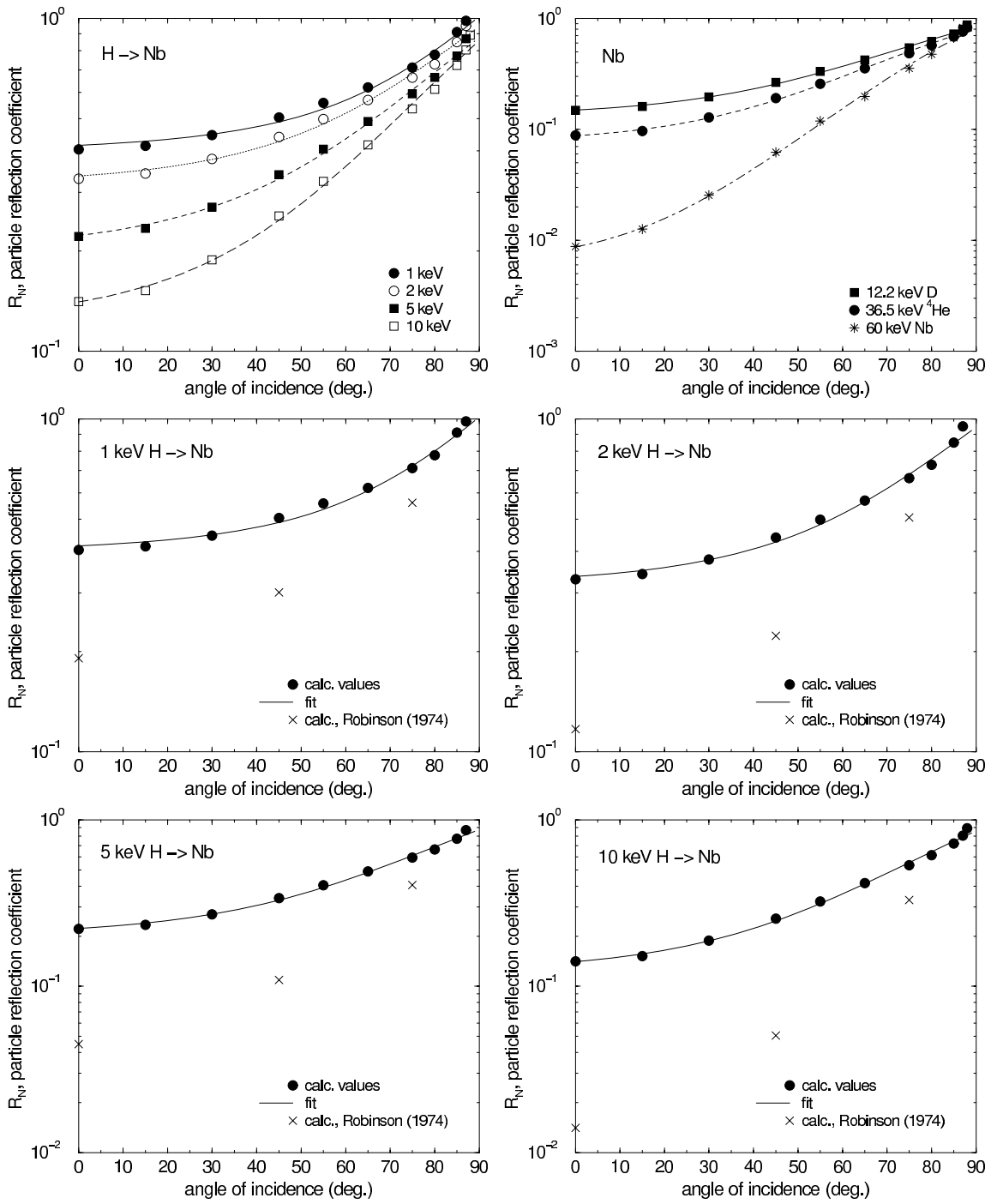

Figure 94: Calculated angular dependence of the particle reflection coefficient at several incident energies for the bombardment of $\mathrm{Nb}$ with $\mathrm{H}, \mathrm{D},{ }^{4} \mathrm{He}, \mathrm{Nb}$ [30]. Earlier calculated values are given for 1, 2, 5 and $10 \mathrm{keV}$ by [70]. Lines are fits to the calculated values (see Table 21) 

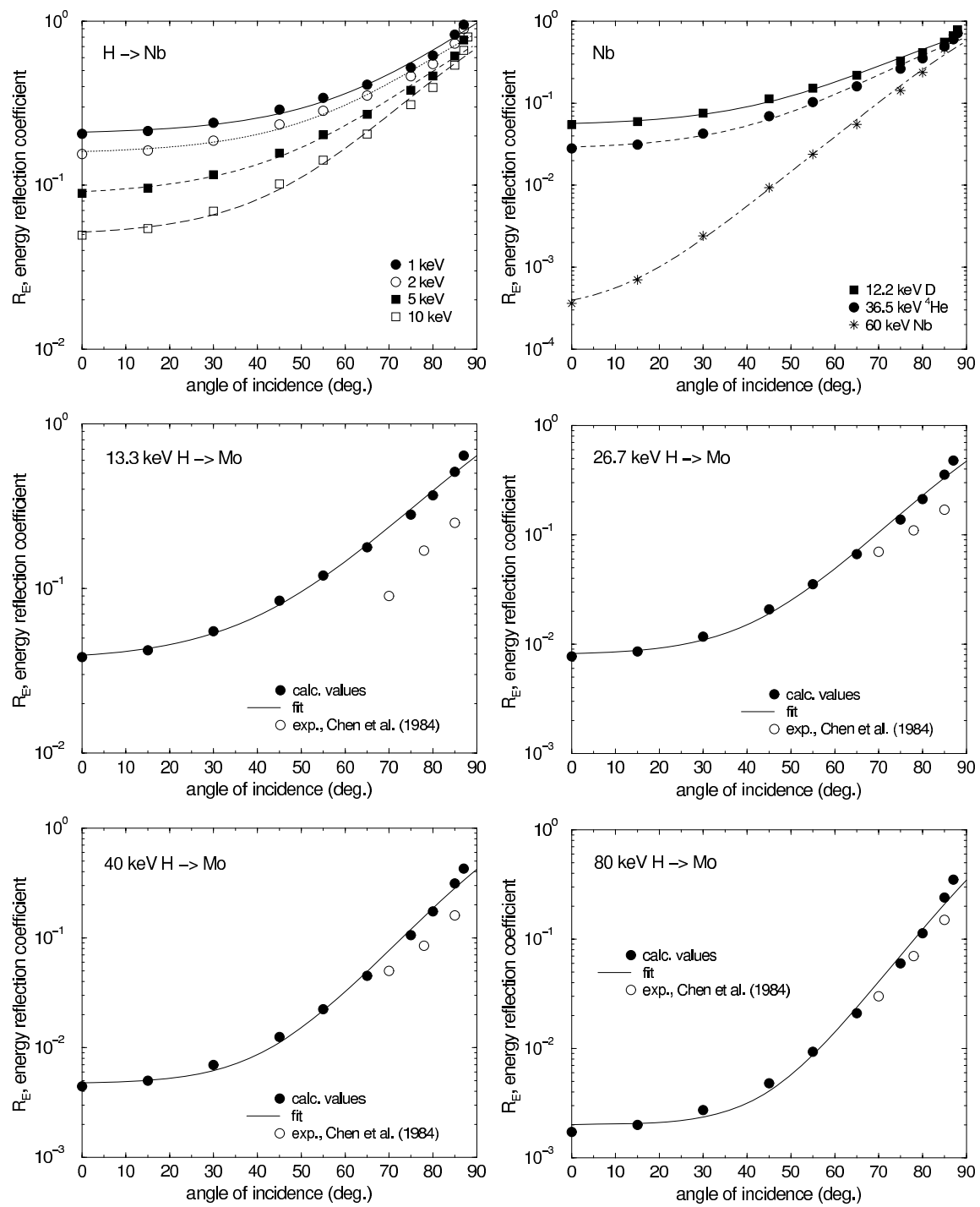

Figure 95: Calculated angular dependence of the energy reflection coefficient at several incident energies for the bombardment of $\mathrm{Nb}$ with $\mathrm{H}, \mathrm{D},{ }^{4} \mathrm{He}, \mathrm{Nb}[30]$ and of Mo with $\mathrm{H}$ [30]. Experimental values for $\mathrm{H}$ on Mo are given in [82]. Lines are fits to the calculated values (see Tables 34 and 38) 

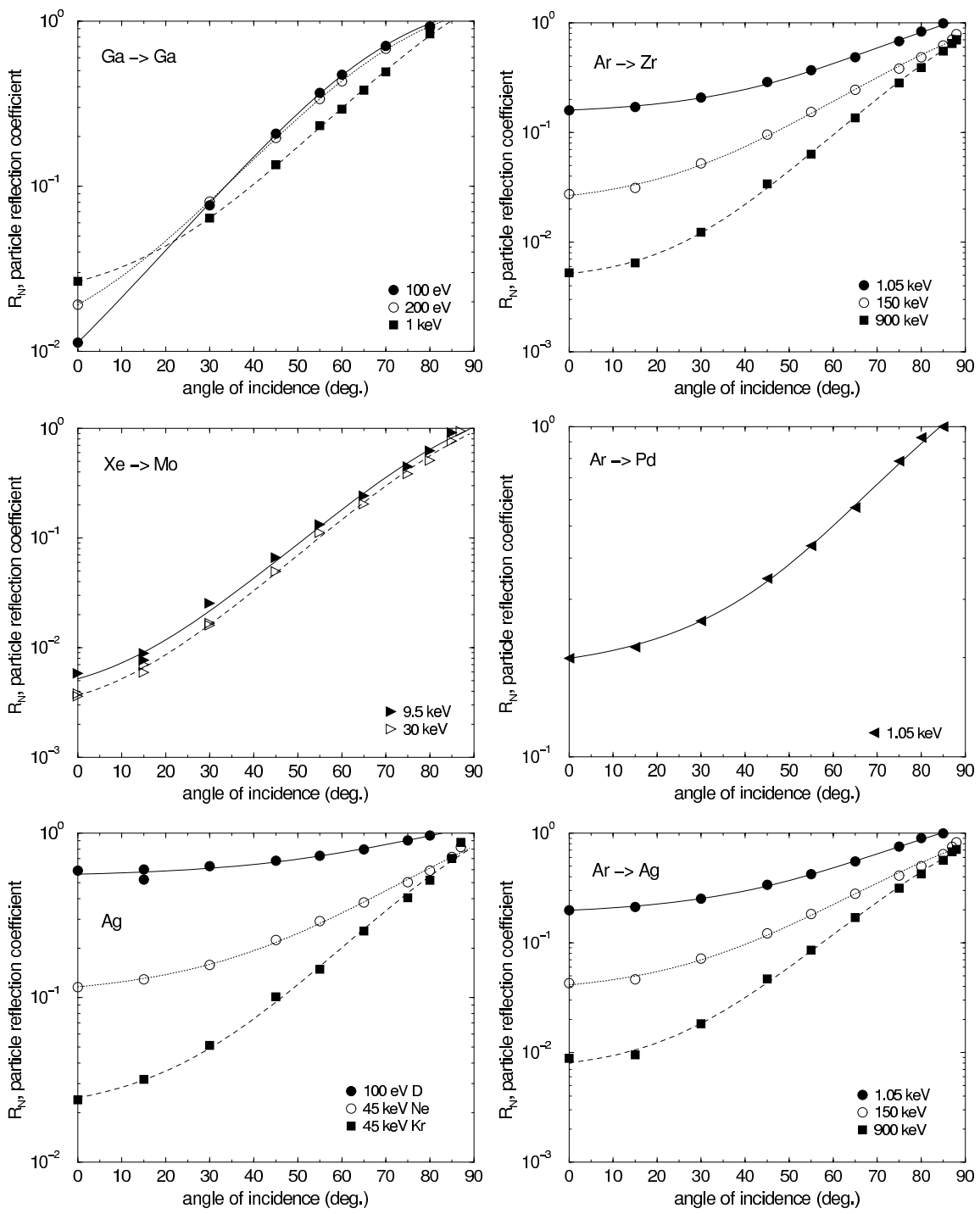

Figure 96: Calculated angular dependence of the particle reflection coefficient at several incident energies for the bombardment of $\mathrm{Ga}$ with $\mathrm{Ga}$, of $\mathrm{Zr}$ with $\mathrm{Ar}$, of Mo with $\mathrm{Xe}$, of $\mathrm{Pd}$ with $\mathrm{Ar}$, of $\mathrm{Ag}$ with $\mathrm{D}, \mathrm{Ne}, \mathrm{Ar}, \mathrm{Kr}$ [30]. Lines are fits to the calculated values (see Tables 21 and 22) 

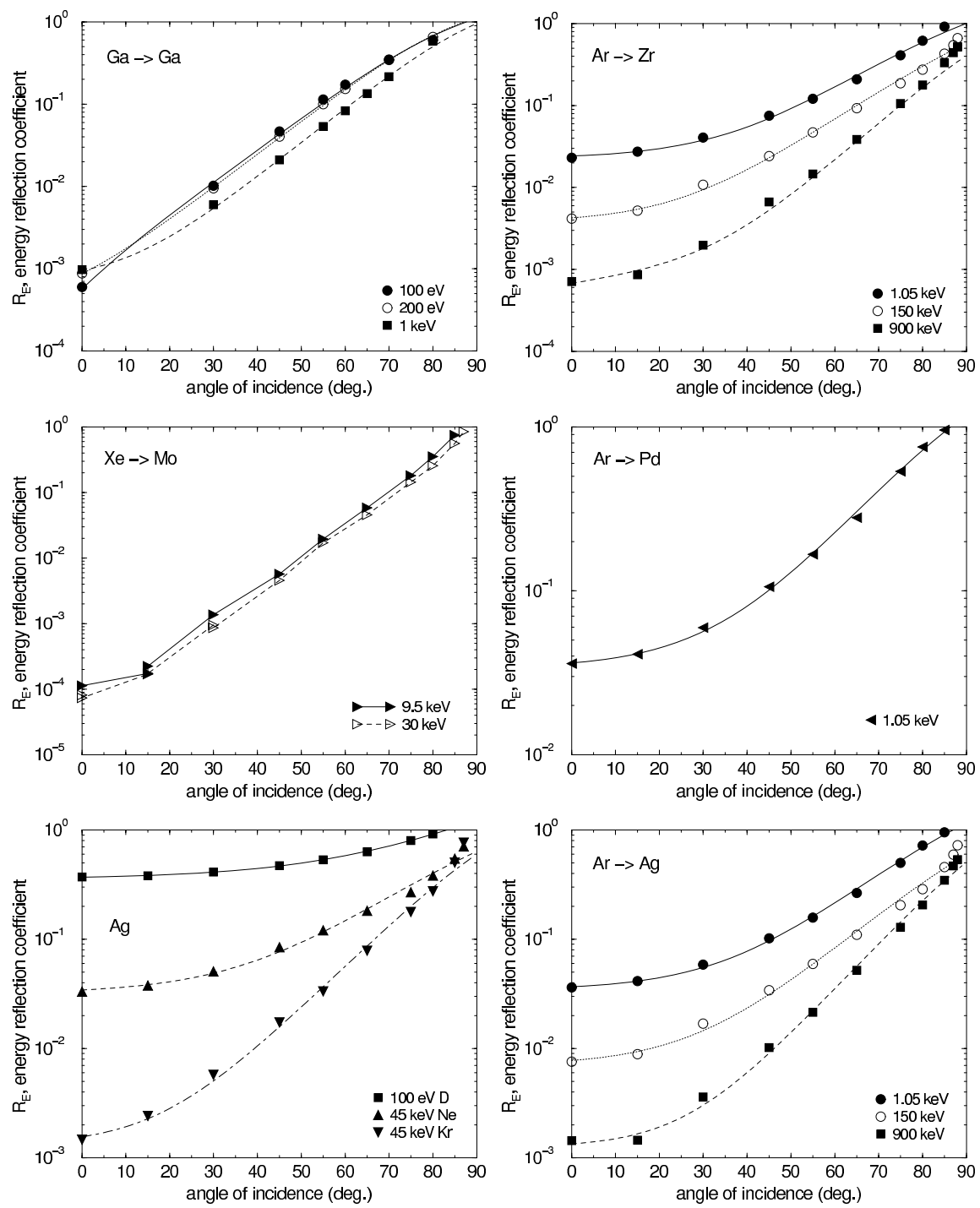

Figure 97: Calculated angular dependence of the energy reflection coefficient at several incident energies for the bombardment of $\mathrm{Ga}$ with $\mathrm{Ga}$, of $\mathrm{Zr}$ with $\mathrm{Ar}$, of Mo with $\mathrm{Xe}$, of $\mathrm{Pd}$ with $\mathrm{Ar}$, of $\mathrm{Ag}$ with $\mathrm{D}, \mathrm{Ne}, \mathrm{Ar}, \mathrm{Kr}$ [30]. Lines are fits to the calculated values (see Tables 34 and 35). Some lines indicated in the legend for Xe on Mo are drawn to guide the eye 

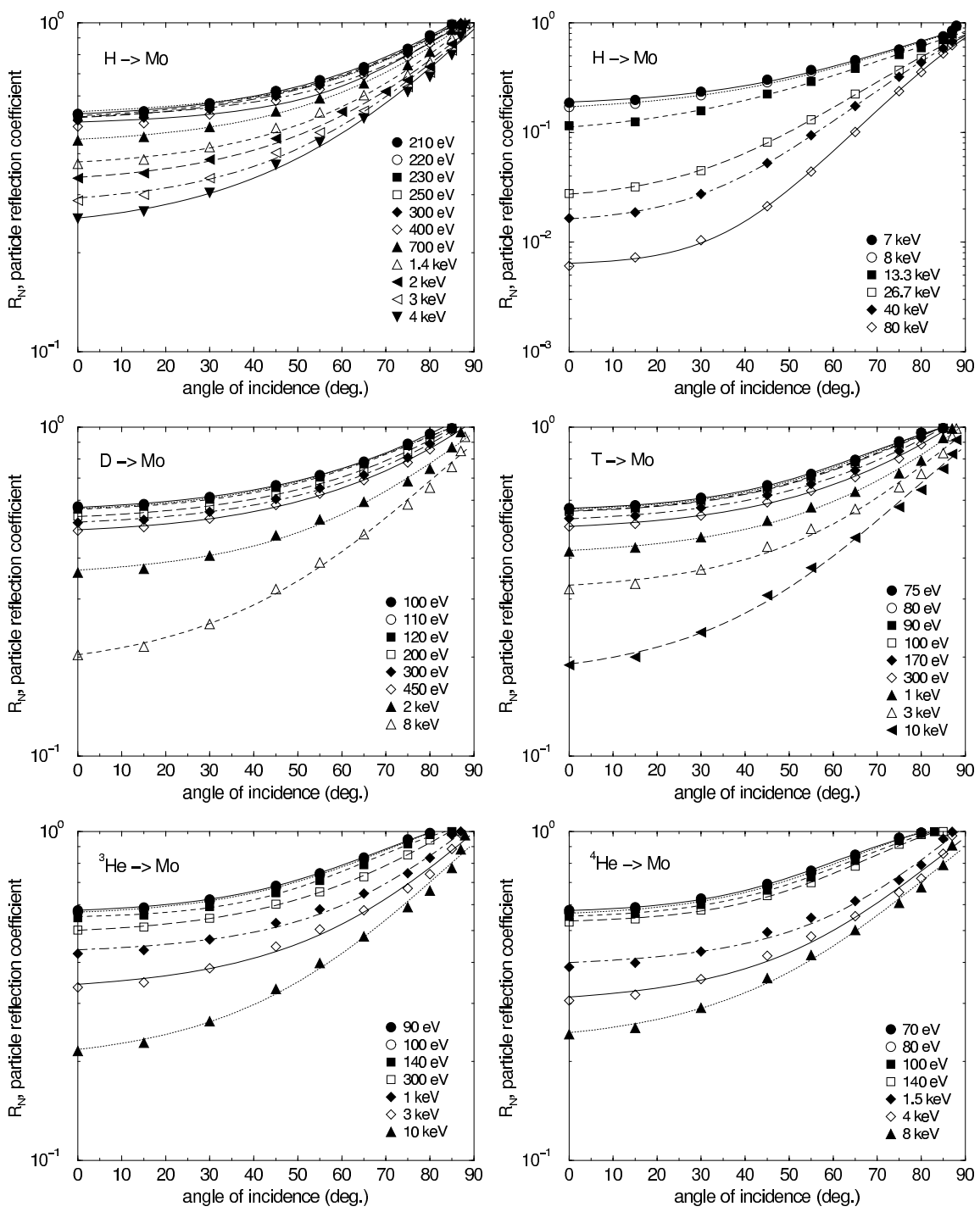

Figure 98: Calculated angular dependence of the particle reflection coefficient at several incident energies for the bombardment of Mo with $\mathrm{H}, \mathrm{D}, \mathrm{T},{ }^{3} \mathrm{He},{ }^{4} \mathrm{He}$ [30]. Lines are fits to the calculated values (see Tables 25) 

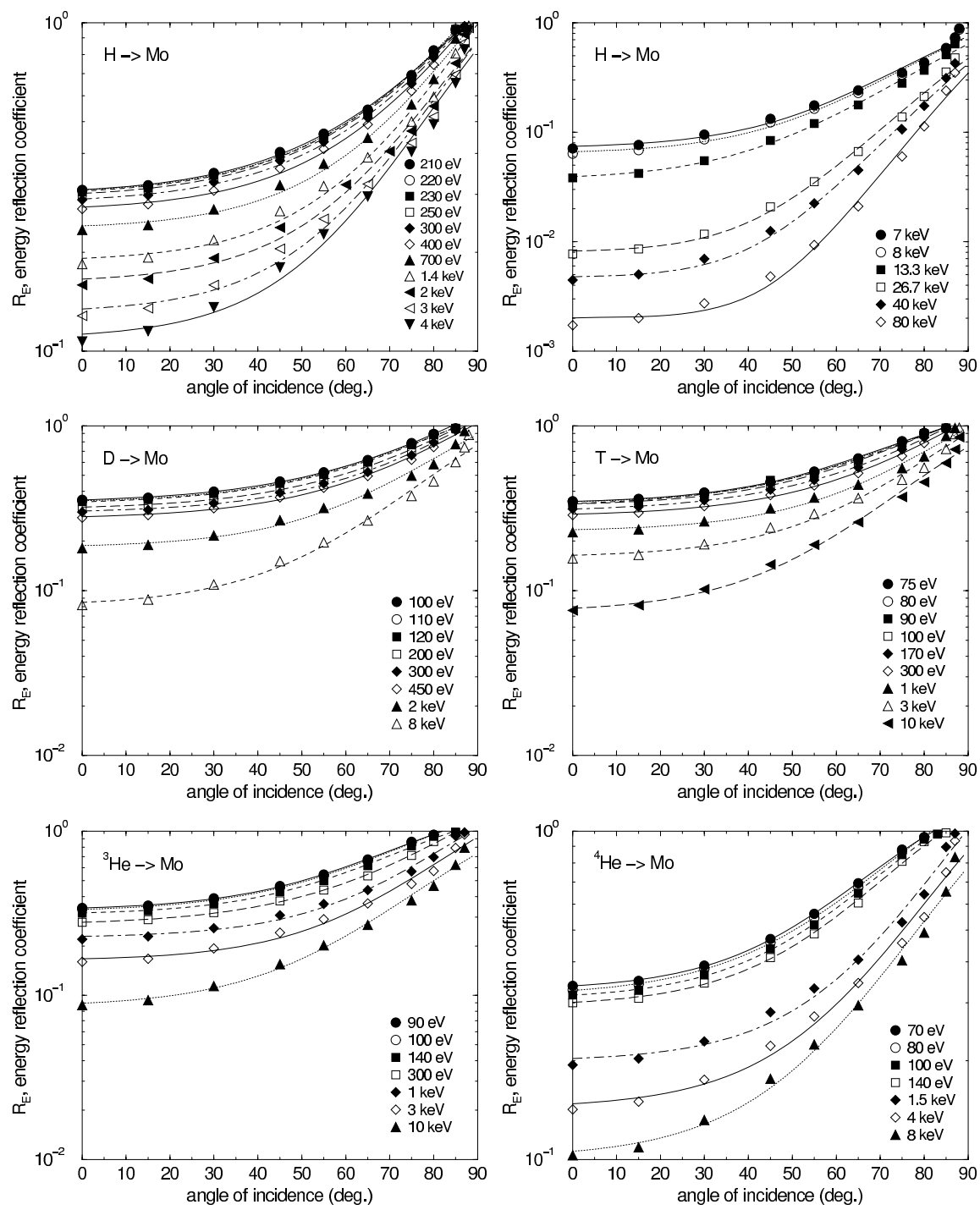

Figure 99: Calculated angular dependence of the energy reflection coefficient at several incident energies for the bombardment of Mo with $\mathrm{H}, \mathrm{D}, \mathrm{T},{ }^{3} \mathrm{He},{ }^{4} \mathrm{He}$ [30]. Lines are fits to the calculated values (see Tables 38) 

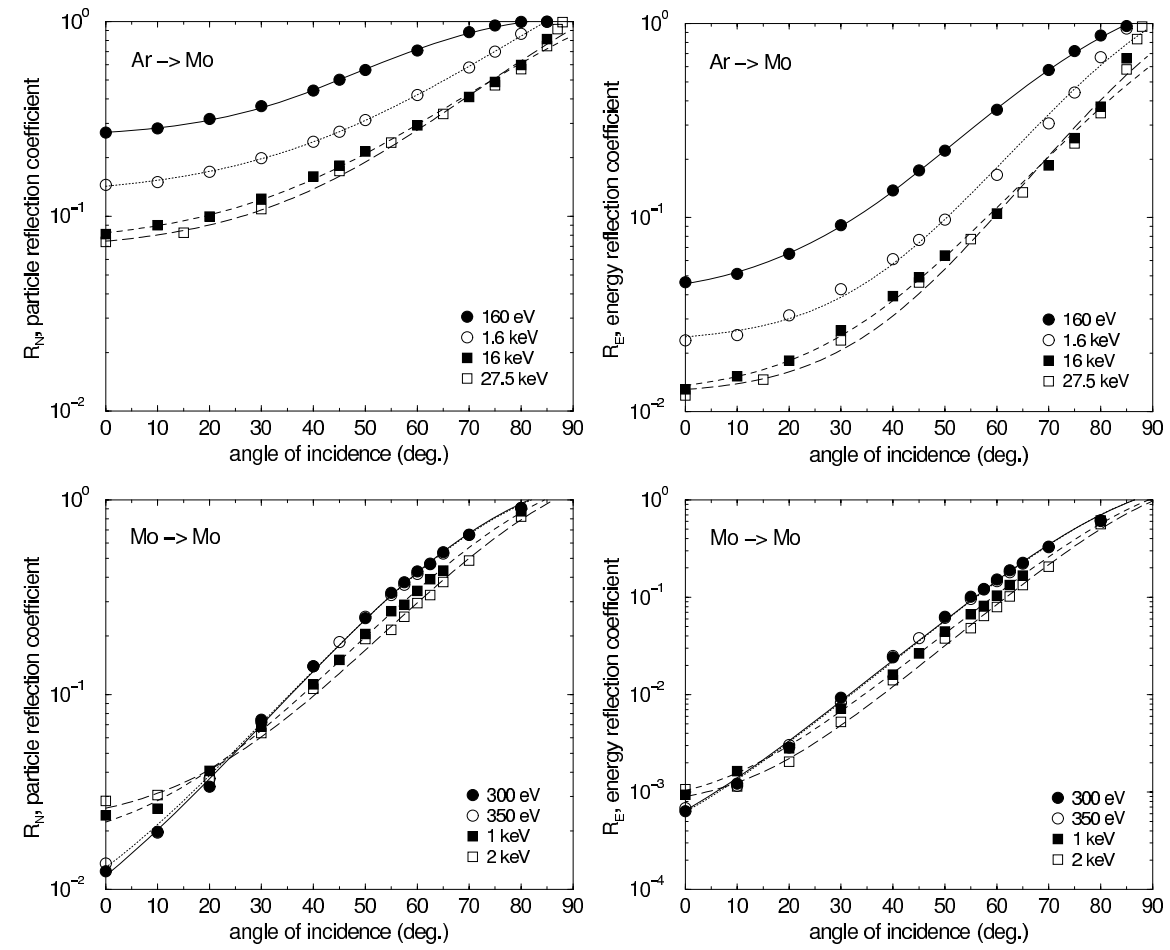

Figure 100: Calculated angular dependence of the particle and energy reflection coefficients at several incident energies for $\mathrm{Ar}$ on Mo and selfbombardment of Mo. Lines are fits to the calculated values (see Tables 22 and 35) 

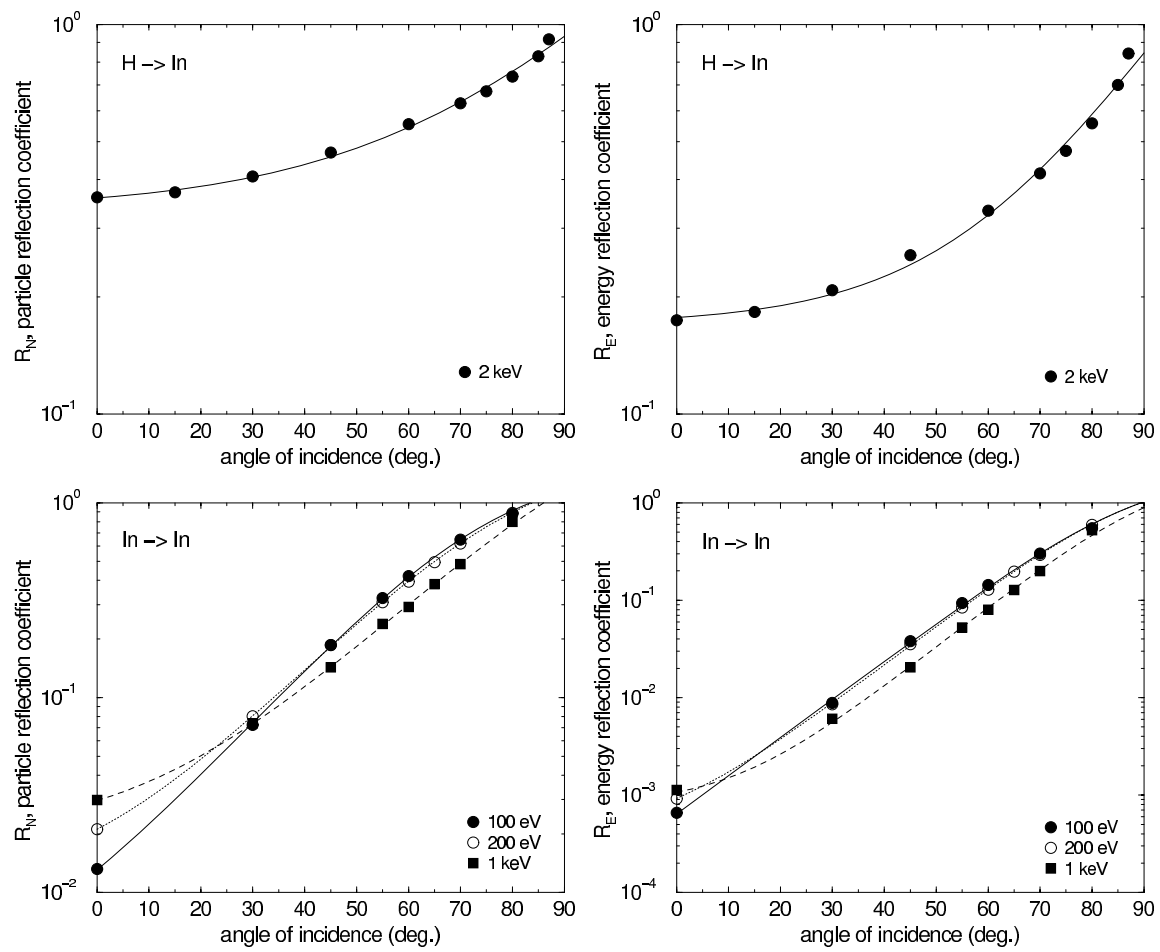

Figure 101: Calculated angular dependence of the particle and energy reflection coefficients at several incident energies for the bombardment of In with $\mathrm{H}$ and In [29]. Lines are fits to the calculated values (see Tables 21 and 34) 

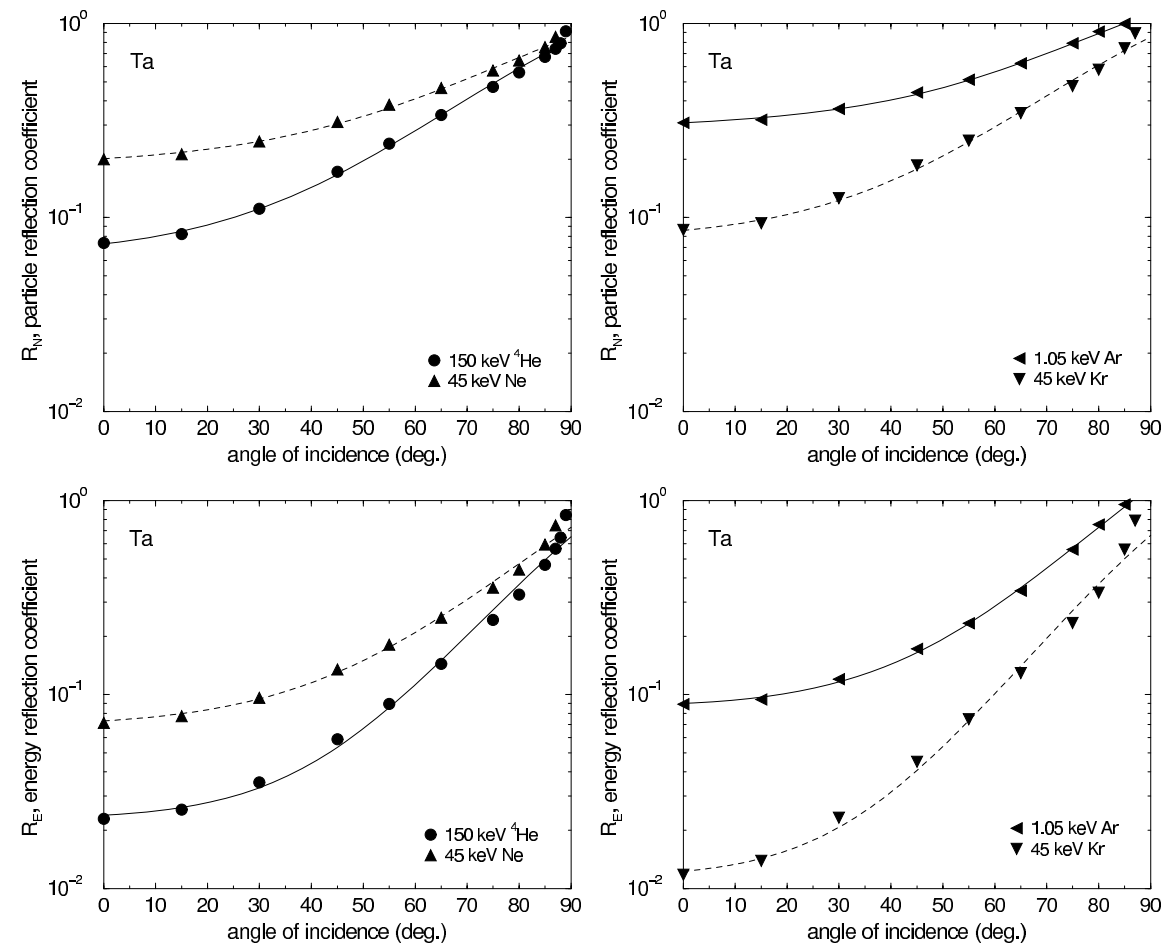

Figure 102: Calculated angular dependence of the particle and energy reflection coefficients at several incident energies for the bombardment of Ta with ${ }^{4} \mathrm{He}$, $\mathrm{Ne}, \mathrm{Ar}, \mathrm{Kr}$ [30]. Lines are fits to the calculated values (see Tables 29 and 42) 

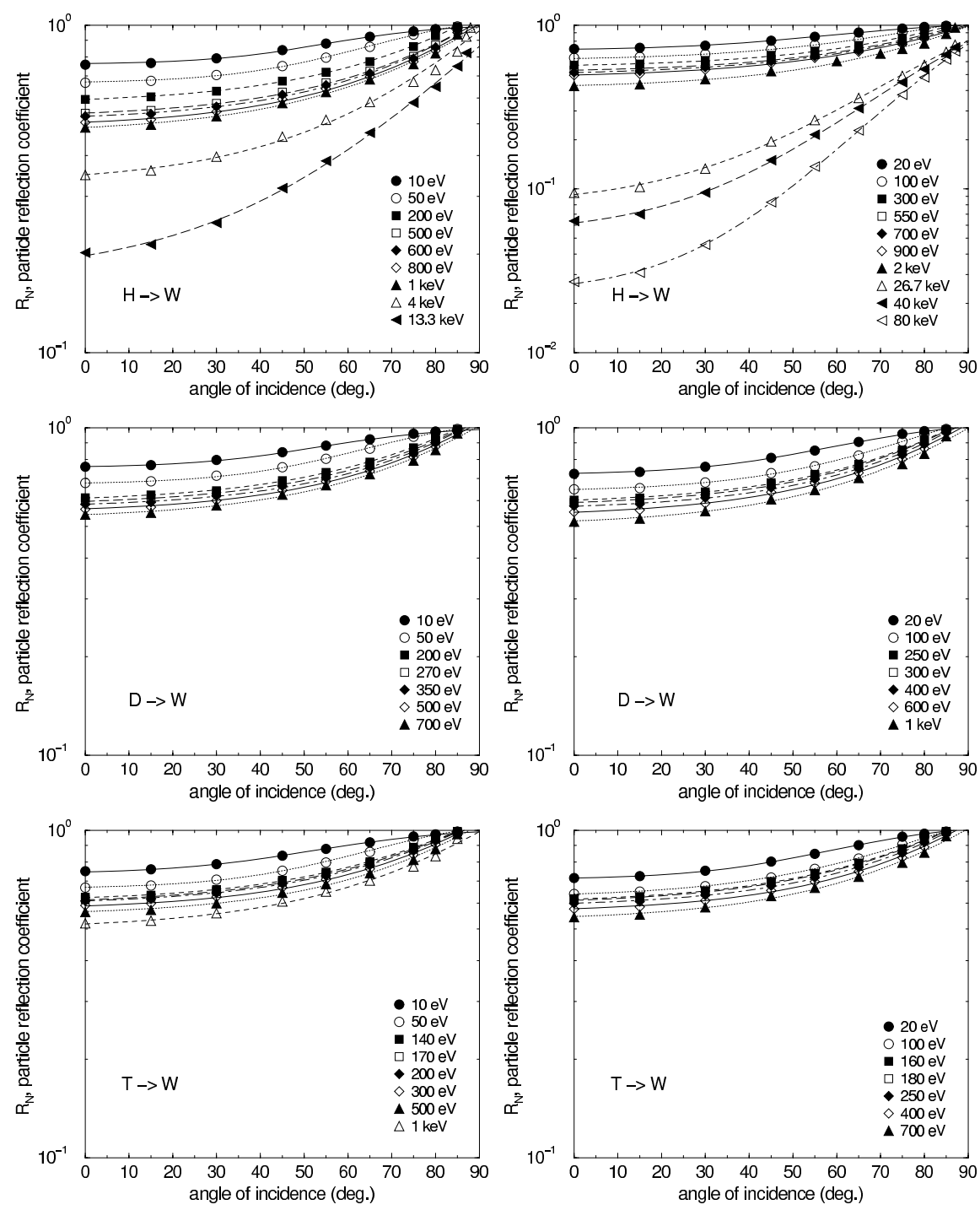

Figure 103: Calculated angular dependence of the particle reflection coefficient at several incident energies for the bombardment of $\mathrm{W}$ with $\mathrm{H}, \mathrm{D}, \mathrm{T}[8,30]$. Lines are fits to the calculated values (see Table 26) 

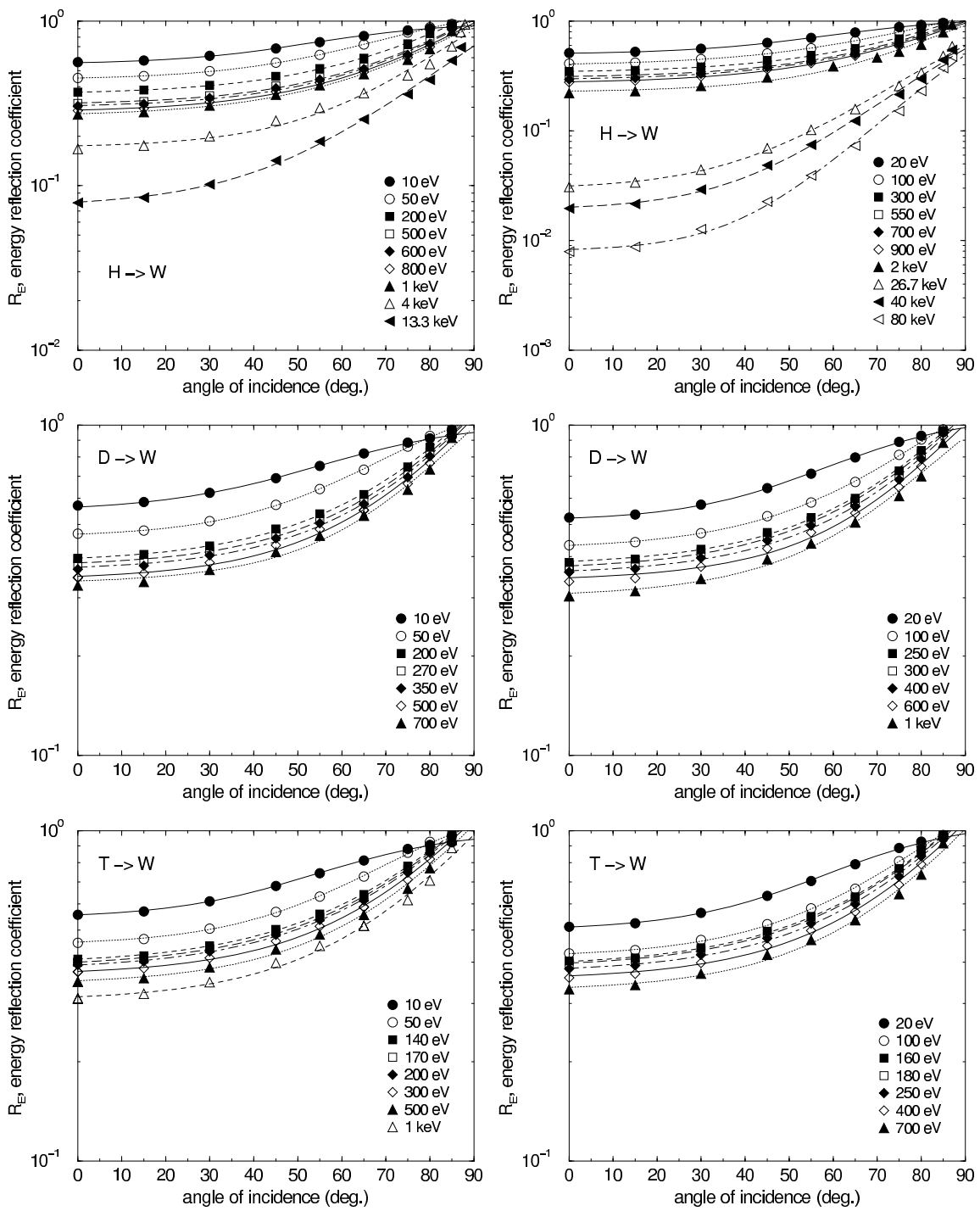

Figure 104: Calculated angular dependence of the energy reflection coefficient at several incident energies for the bombardment of $\mathrm{W}$ with $\mathrm{H}, \mathrm{D}, \mathrm{T}[8,30]$. Lines are fits to the calculated values (see Table 39) 

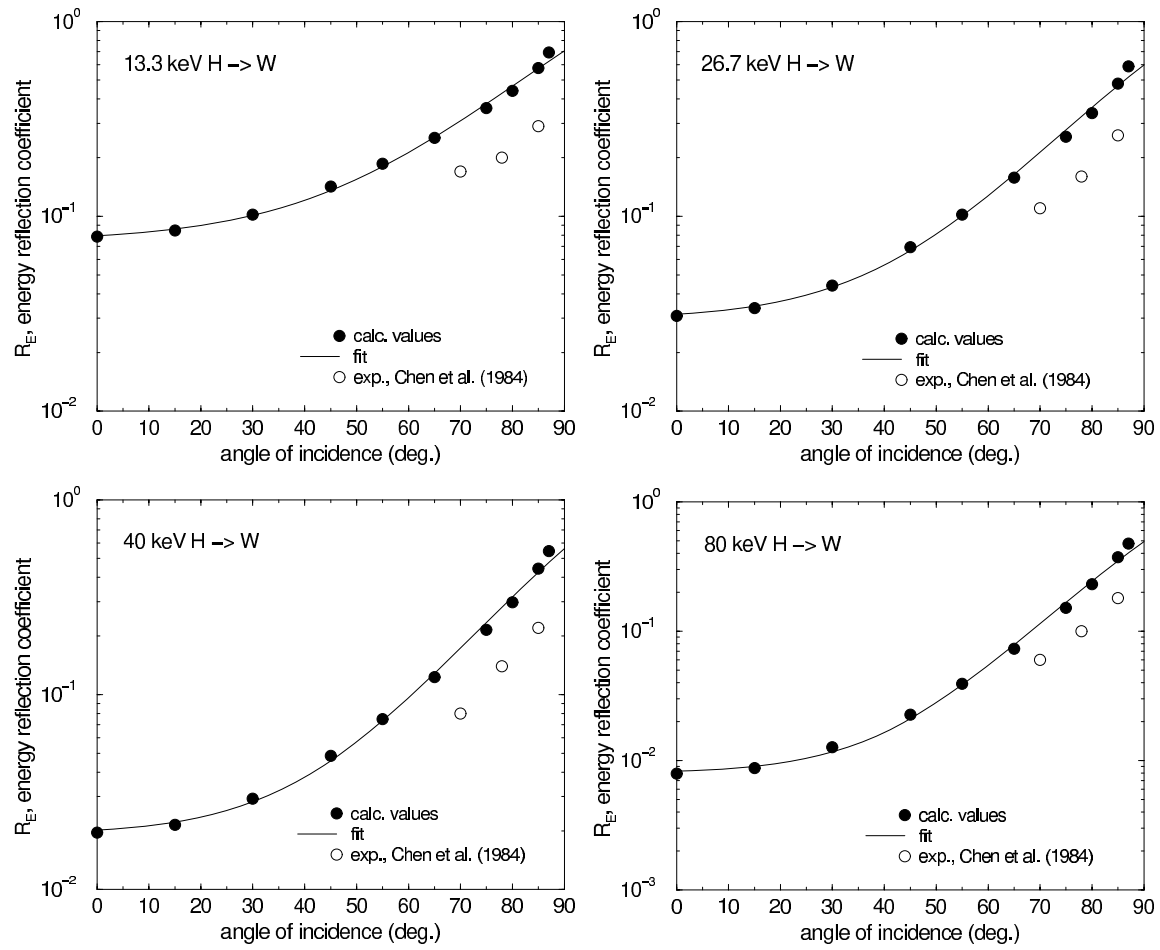

Figure 105: Calculated angular dependence of the energy reflection coefficient at several incident energies for the bombardment of $\mathrm{W}$ with $\mathrm{H}$ [30]. Experimental data $R_{E}$ are given by [82]. Lines are fits to the calculated values (see Table 39) 

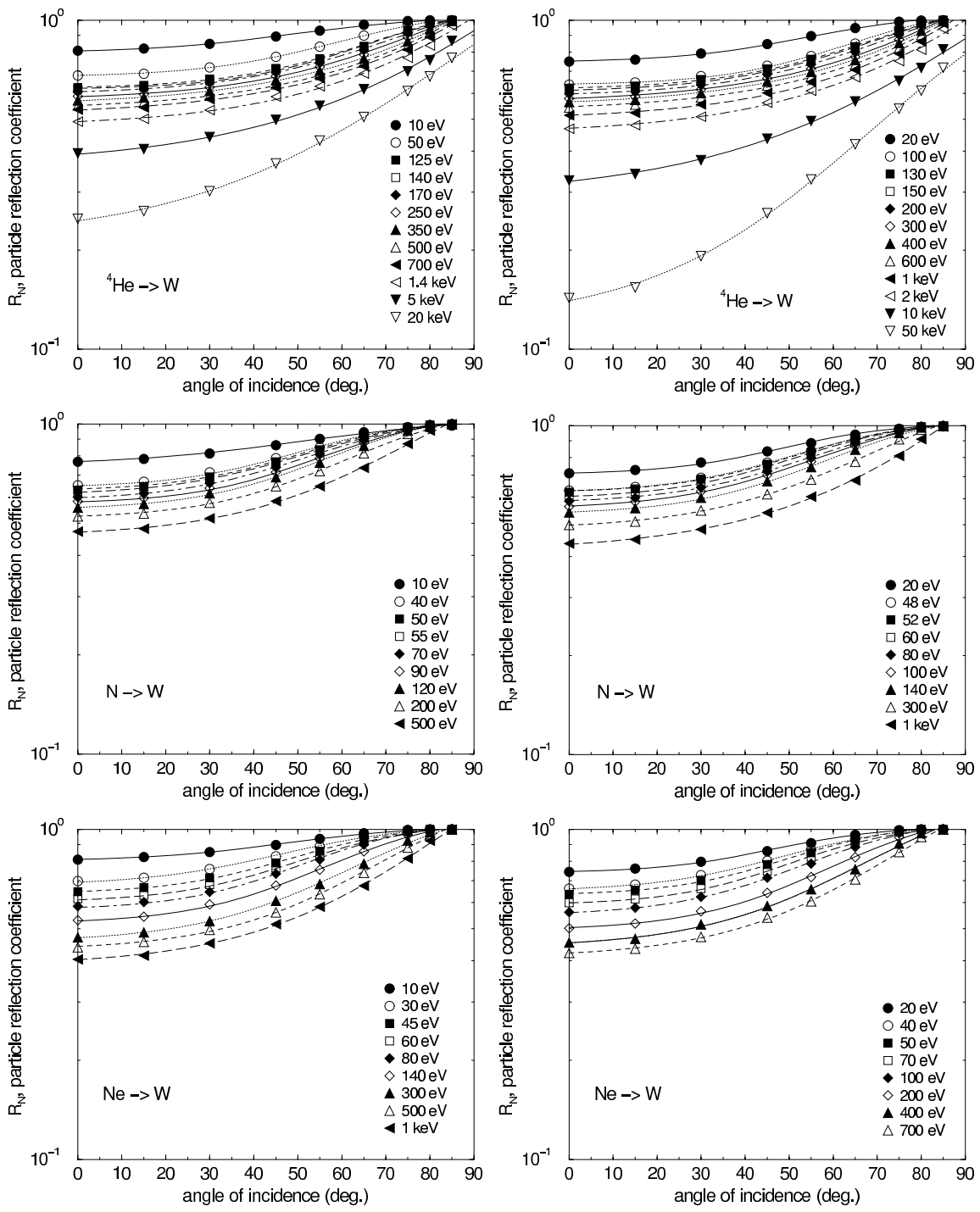

Figure 106: Calculated angular dependence of the particle reflection coefficient at several incident energies for the bombardment of $\mathrm{W}$ with ${ }^{4} \mathrm{He}, \mathrm{N}, \mathrm{Ne}$ [8]. Lines are fits to the calculated values (see Tables 27 and 28) 

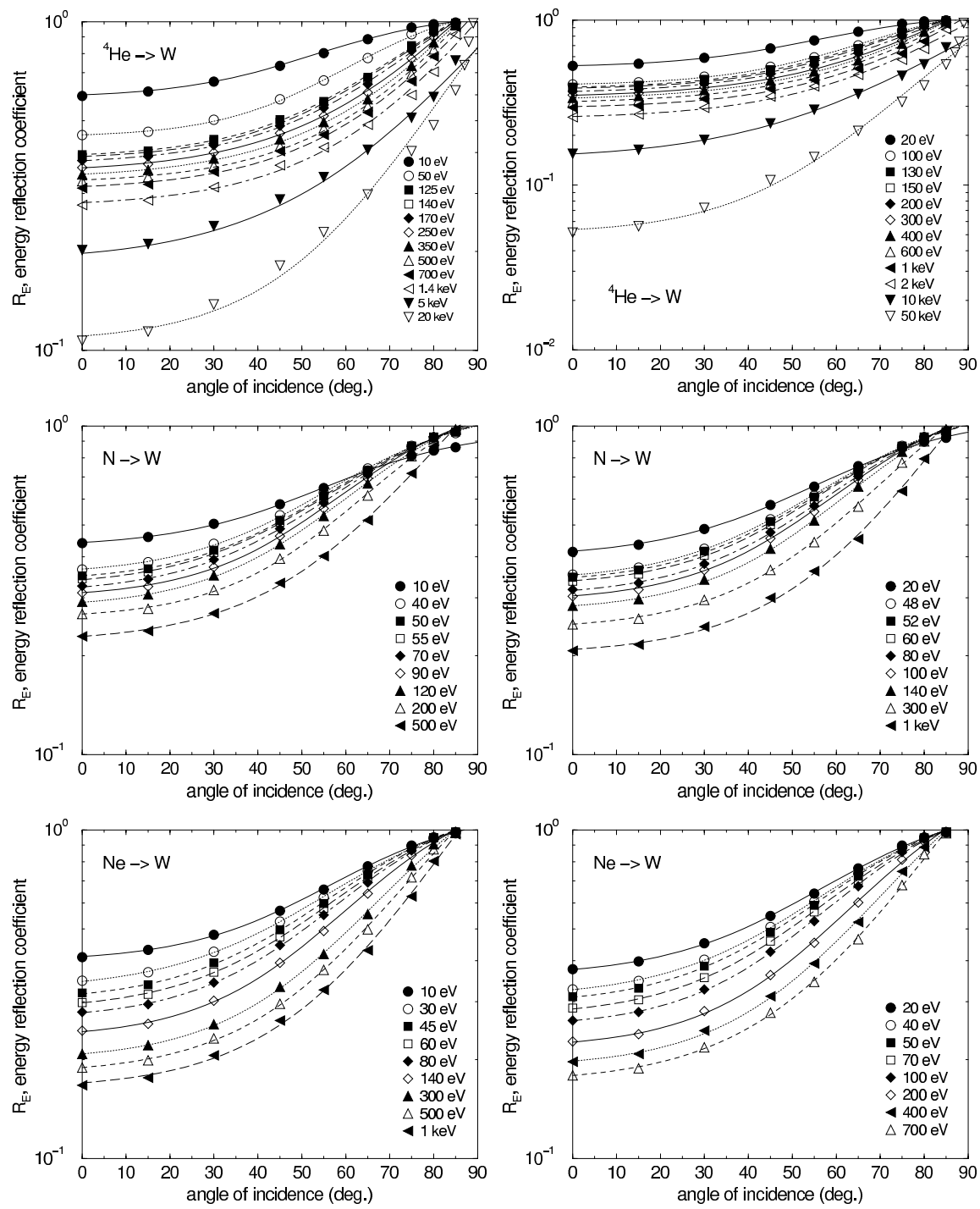

Figure 107: Calculated angular dependence of the energy reflection coefficient at several incident energies for the bombardment of $\mathrm{W}$ with ${ }^{4} \mathrm{He}, \mathrm{N}, \mathrm{Ne}[8]$. Lines are fits to the calculated values (see Tables 40 and 41) 

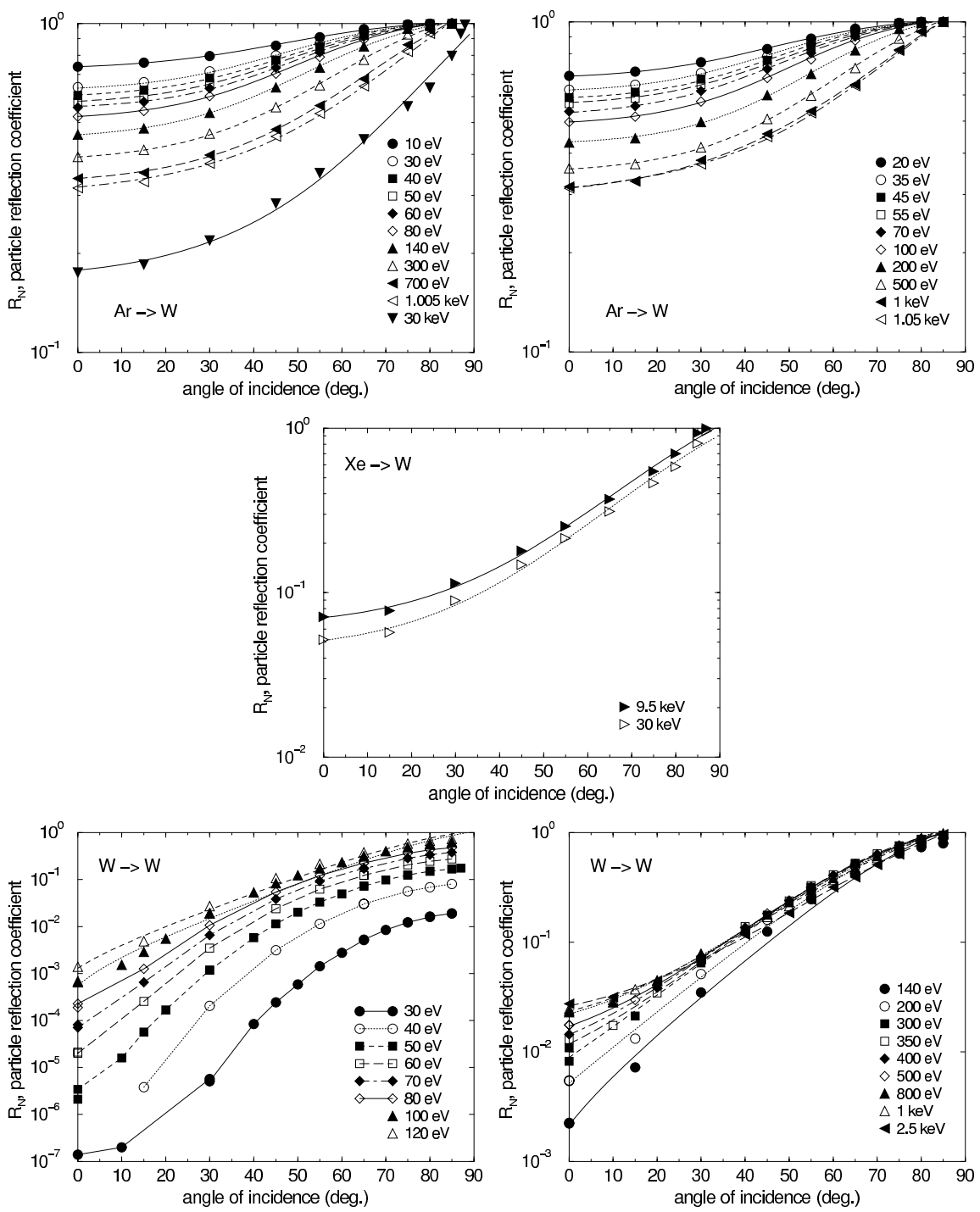

Figure 108: Calculated angular dependence of the particle reflection coefficient at several incident energies for the bombardment of W with Ar, Xe, W [8, 30]. Lines are fits to the calculated values (see Tables 27 and 28). Some lines indicated in the legend for $\mathrm{W}$ on $\mathrm{W}$ are drawn to guide the eye 

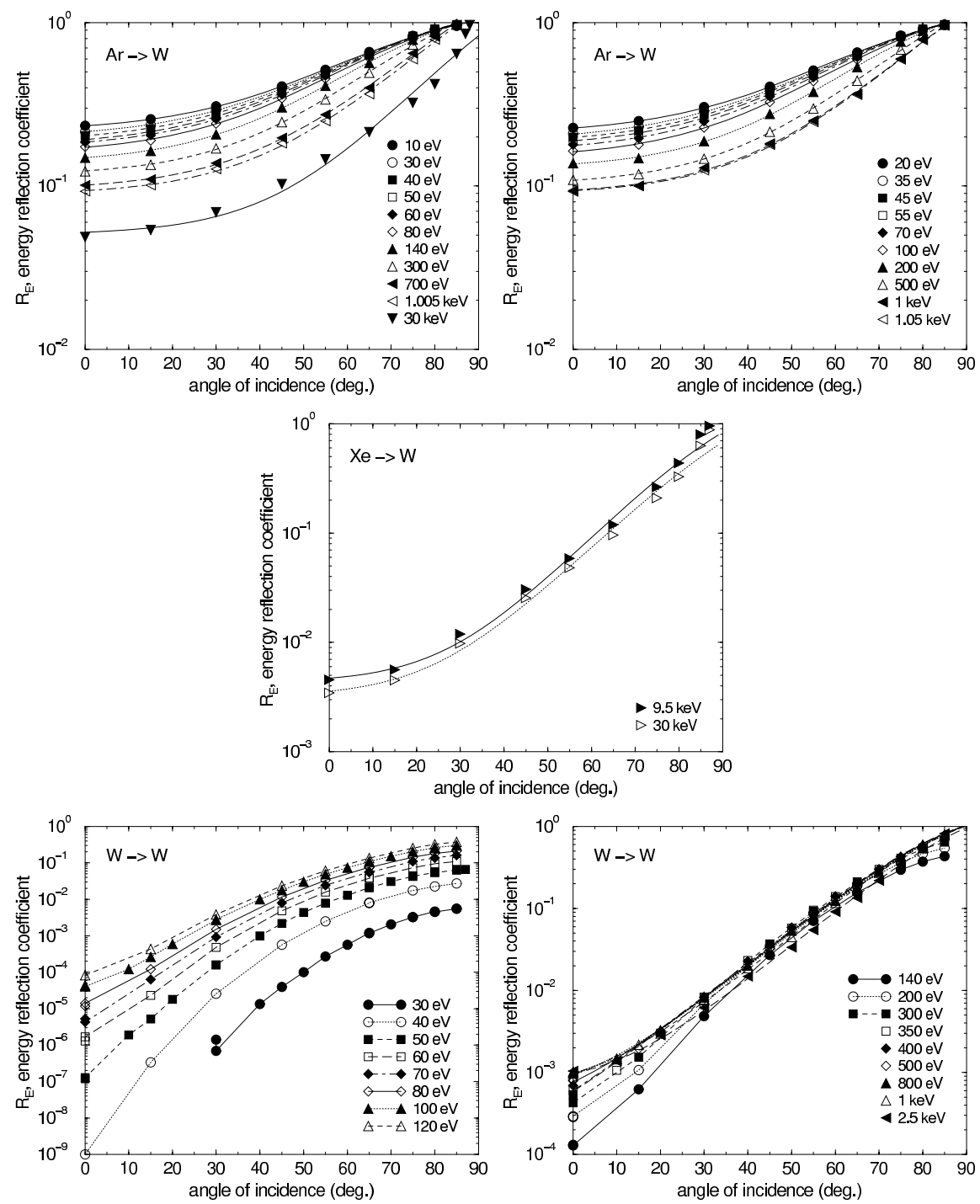

Figure 109: Calculated angular dependence of the energy reflection coefficient at several incident energies for the bombardment of $\mathrm{W}$ with Ar, Xe, W [8, 30]. Lines are fits to the calculated values (see Tables 40 and 41). Some lines indicated in the legend for $\mathrm{W}$ on $\mathrm{W}$ are drawn to guide the eye 

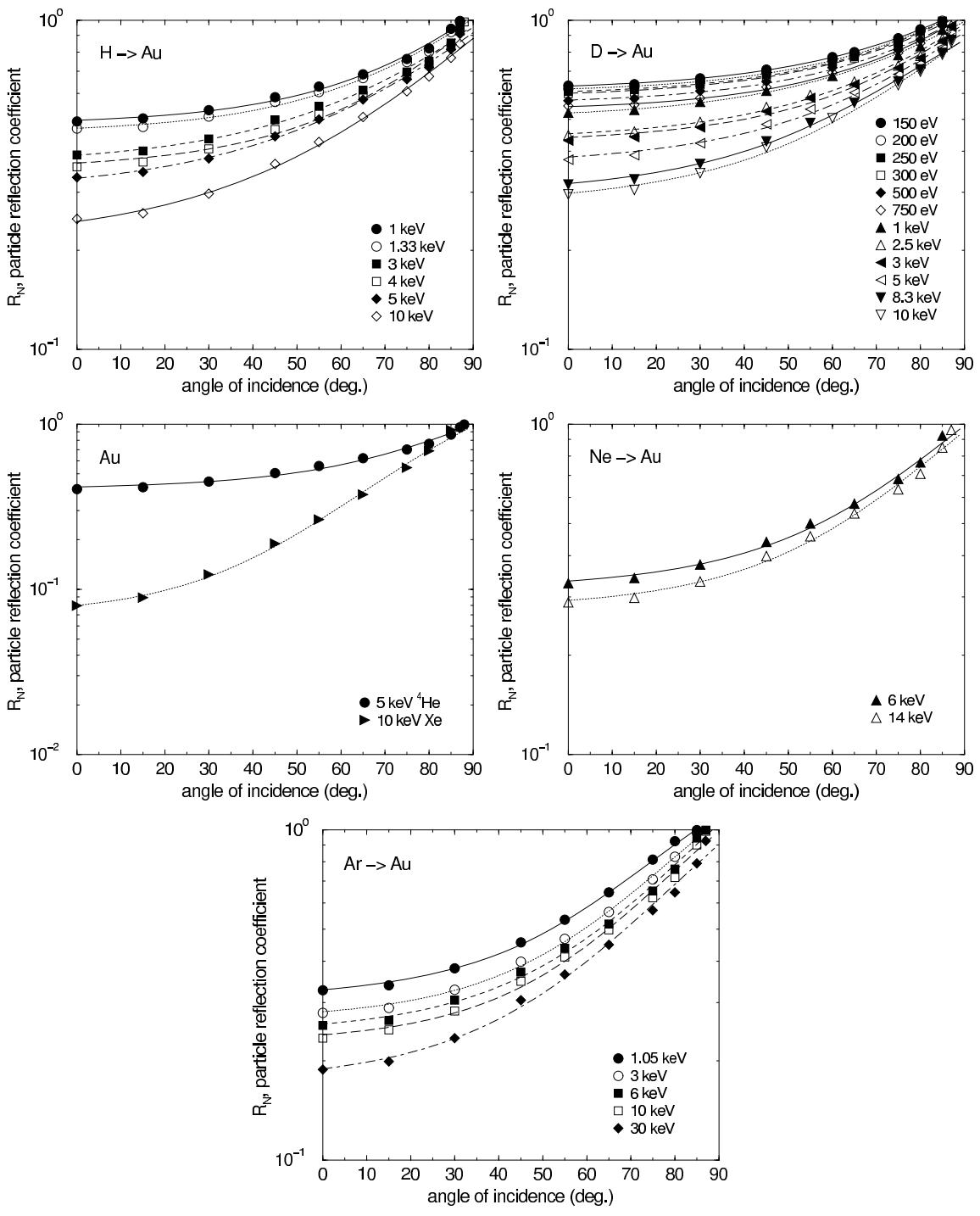

Figure 110: Calculated angular dependence of the particle reflection coefficient at several incident energies for the bombardment of Au with $\mathrm{H}, \mathrm{D},{ }^{4} \mathrm{He}, \mathrm{Xe}, \mathrm{Ne}$, $\operatorname{Ar}[8,30]$. Lines are fits to the calculated values (see Table 29) 

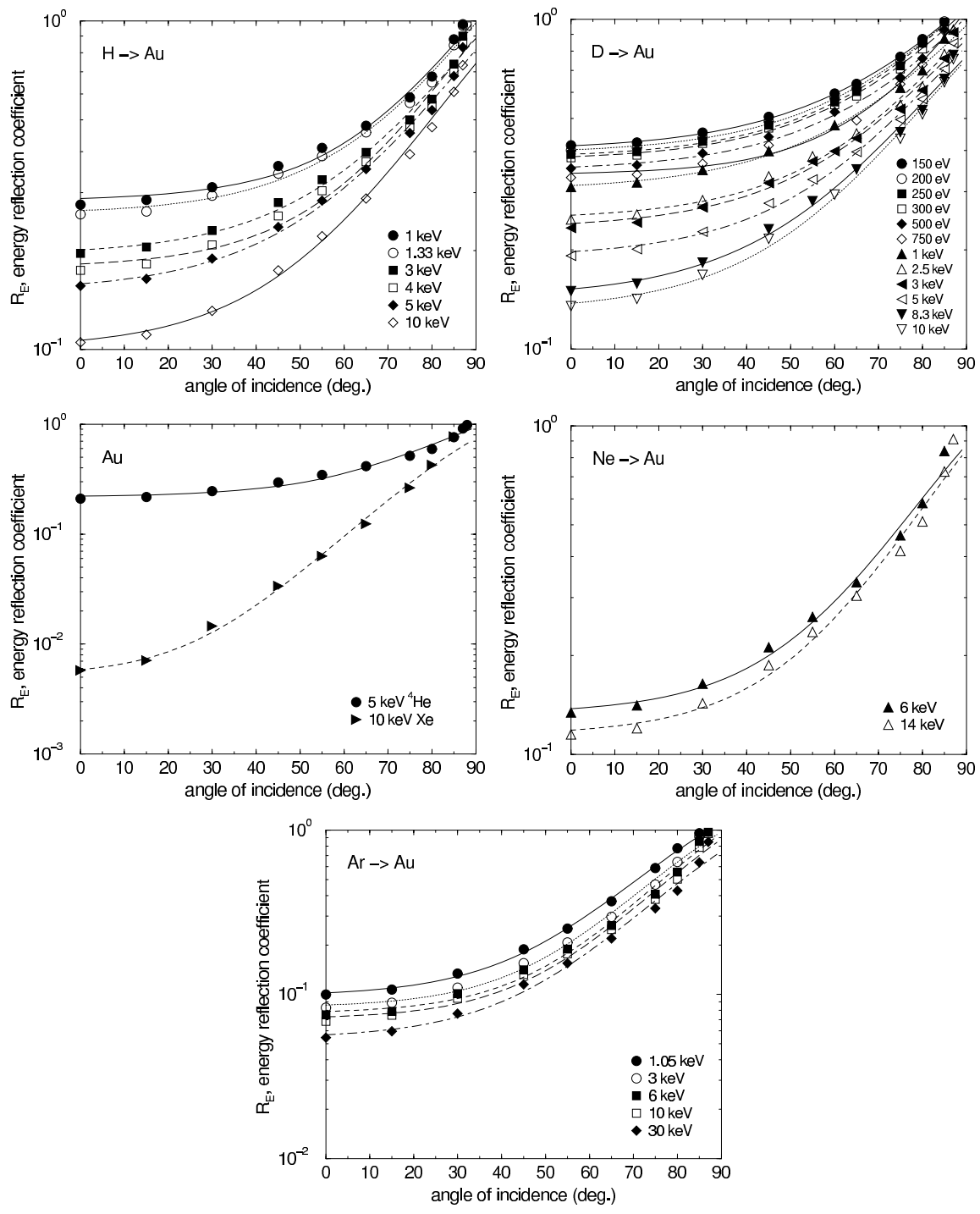

Figure 111: Calculated angular dependence of the energy reflection coefficient at several incident energies for the bombardment of $\mathrm{Au}$ with with $\mathrm{H}, \mathrm{D},{ }^{4} \mathrm{He}$, $\mathrm{Xe}, \mathrm{Ne}, \mathrm{Ar}[8,30]$. Lines are fits to the calculated values (see Table 42) 

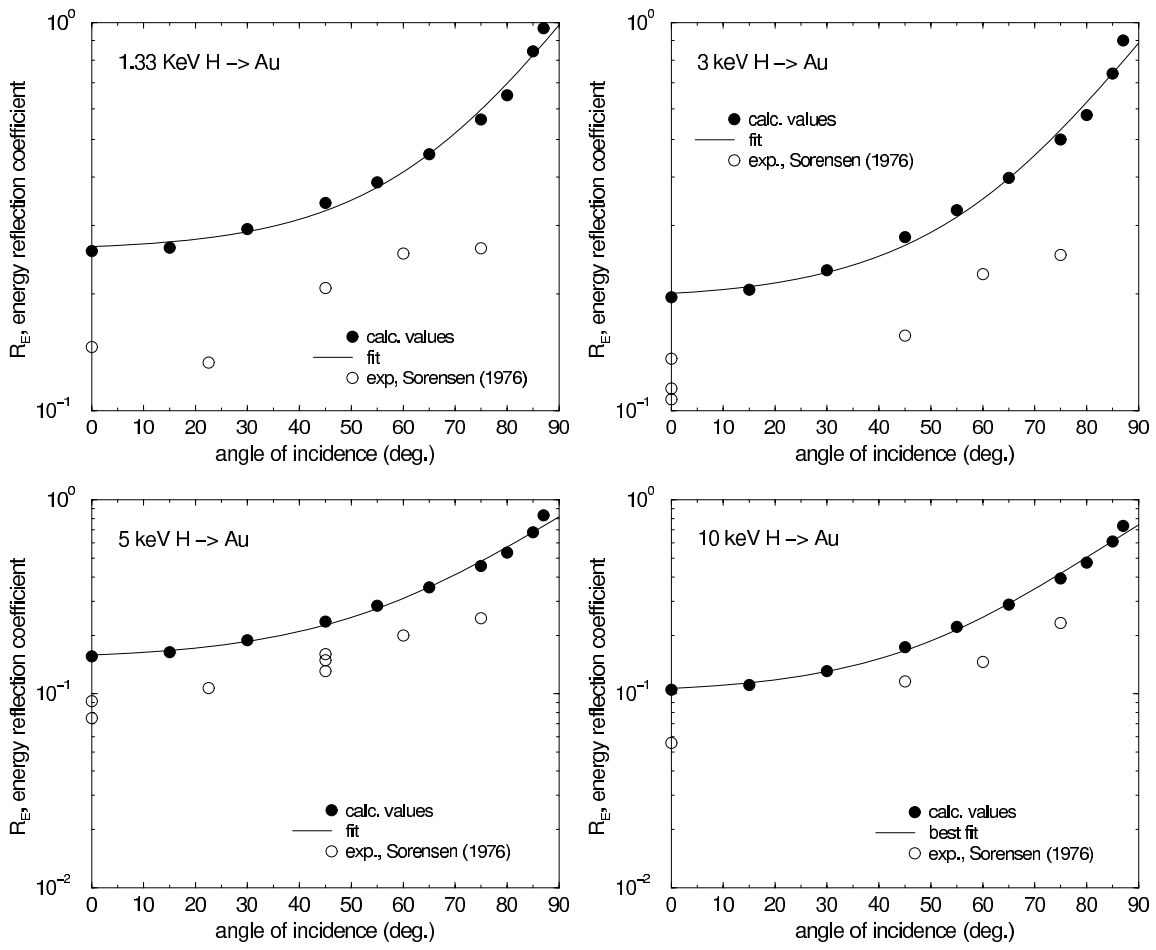

Figure 112: Calculated and measured angular dependences of the energy reflection coefficient at several incident energies for the bombardment of $\mathrm{Au}$ with $\mathrm{H}$ [30]. Lines are fits to the calculated values (see Table 42). Experimental data are given by [84] 

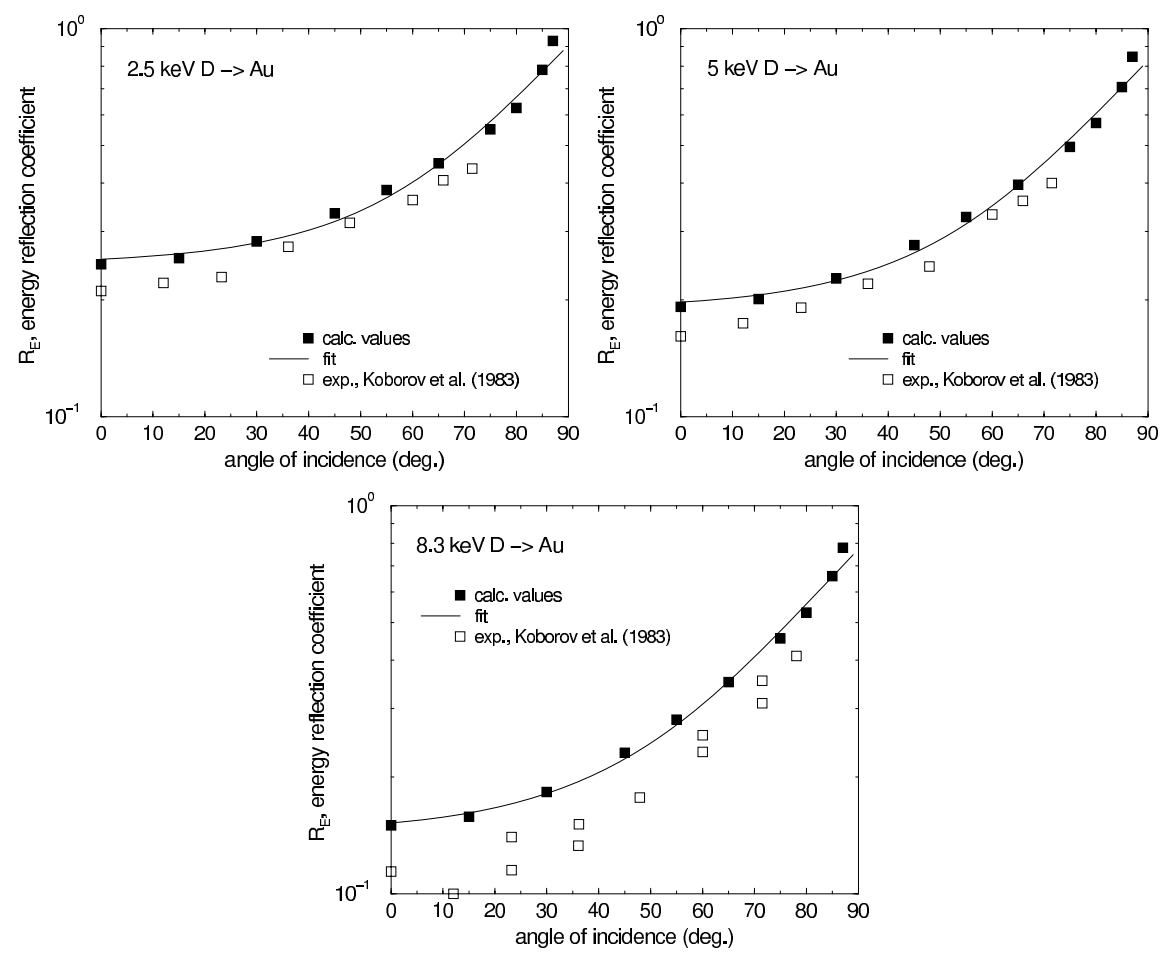

Figure 113: Calculated and measured angular dependences of the energy reflection coefficient at several incident energies for the bombardment of Au with D [30]. Lines are fits to the calculated values (see Table 42). Experimental data are given by [61] 

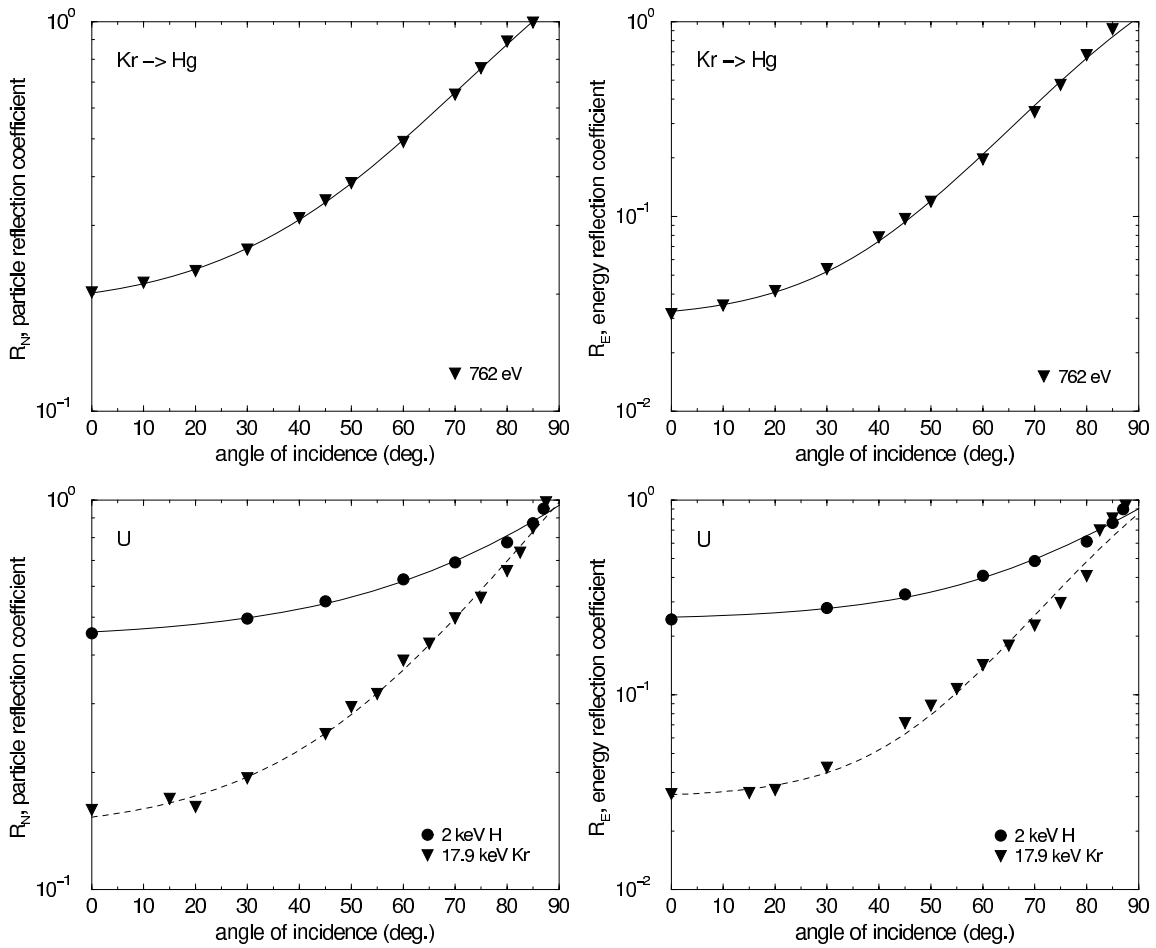

Figure 114: Calculated angular dependences of the particle and energy reflection coefficients at several incident energies for the bombardment of $\mathrm{Hg}$ with $\mathrm{Kr}$, and of $\mathrm{U}$ with $\mathrm{H}$ and $\mathrm{Kr}$ [29]. Lines are fits to the calculated values (see Tables 29 and 42) 


\section{References}

[1] T. Tabata, R. Ito, Y. Itikawa, N. Itoh, K. Morita, Report IPPJ-AM-18, Nagoya 1981

[2] R.A. Langley, J. BohdanskyW. Eckstein, P. Mioduszewski, J. Roth, E. Taglauer, E.W. Thomas, H. Verbeek, K.L. Wilson, Nucl. Fusion, Special Issue 1984, IAEA, Vienna, 1984 p. 12

[3] T. Tabata, R. Ito, Y. Itikawa, N. Itoh, K. Morita, H. Tawara, Report IPPJ-AM-34, Nagoya 1984

[4] R. Behrisch, W. Eckstein, Physics of Plasma-Wall-Interactions in Controlled Fusion, ed. by D.E. Post and R. Behrisch, (Plenum, New York 1986) p. 413

[5] W. Eckstein: Computer Simulation of Ion-Solid Interactions, Springer Series in Material Science, Vol.10, (Springer, Berlin Heidelberg New York 1991)

[6] E.W. Thomas, R.K. Janev, J.J. Smith INDC(NDS)-249, IAEA, Wien (1991)

[7] E.W. Thomas, R.K. Janev, J.J. Smith, Nucl. Instrum. Meth. B 69 (1992) 427

[8] W. Eckstein, IPP Report 9/117, Garching (1998)

[9] E.S. Mashkova, V.A. Molchanov, Medium-Energy Ion Reflection from Solids, Modern Problems in Condensed Matter Sciences, Vol. 11, (NorthHolland, Amsterdam, Oxford, New York, Tokyo 1985)

[10] J. Bøttiger, J.A. Davies, Radiat. Eff. 11 (1971) 61

[11] C.K. Chen, B.M.U. Scherzer, W. Eckstein, Appl. Phys. A 33 (1984) 265

[12] W. Eckstein, H. Verbeek, IPP Report 9/32, Garching (1979)

[13] J. Amano, D.N. Seidman, J. Appl. Phys. 52 (1981) 6934

[14] H.H. Andersen, T. Lenskjaer, G. Sidenius, H.Sørensen, J. Appl. Phys. 47 (1976) 13

[15] M.M. Jakas, M.M.R. Williams, J. Phys. D 13 (1980) 1169

[16] G. Sidenius, T. Lenskjaer, Nucl. Instrum. Meth. 132 (1976) 673

[17] J.P. Biersack, L.G. Haggmark, Nucl. Instrum. Meth. 174 (1980) 257

[18] J.P. Biersack, W. Eckstein, Appl. Phys. 34 (1984) 73

[19] W. Eckstein, R. Dohmen, A. Mutzke, R. Schneider, Report IPP 12/3, Garching 2007 
[20] W.D.Wilson, L.G.Haggmark, J.P. Biersack, Phys. Rev. B 15 (1977) 2458

[21] J.F. Ziegler, J.P. Biersack, U. Littmark, The Stopping and Range of Ions in Solids, The Stopping and Range of Ions in Matter, Vol. 1, ed. by J.F. Ziegler (Pergamon, New York 1985)

[22] G. Molière, Z. Naturf. A2 (1947) 133

[23] W. Eckstein, S. Hackel, D. Heinemann, B. Fricke Z. Phys. D 24 (1992) 171

[24] O.S. Oen, M.T. Robinson, Nucl. Instrum. Meth. 132 (1976) 647

[25] E. Fermi, E. Teller, Phys. Rev. 72 (1947) 399

[26] J. Lindhard, M. Scharff, Phys. Rev. 124 (1961) 128

[27] J.F. Ziegler, In Helium Stopping Powers and Ranges in All Elements, The Stopping and Range of Ions in Matter, Vol. 4, ed. by J.F. Ziegler (Pergamon, New York 1977)

[28] H.H. Andersen, J.F. Ziegler, In Hydrogen Stopping Powers and Ranges in All Elements, The Stopping and Range of Ions in Matter, Vol. 3, ed. by J.F. Ziegler (Pergamon, New York 1977)

[29] W. Eckstein, IPP Report 9/132, Garching (2002)

[30] W. Eckstein, newer values (also previous values) can be retrieved from ftp://ftp.rzg.mpg.de/ftp/pub/ipp/eckstein/rep05

[31] W. Möller, W. Eckstein, J.P. Biersack, Comp. Phys. Commun. 51 (1988) 355

[32] W. Eckstein, M. Hou, V.I. Shulga, Nucl. Instrum. Meth. B 119 (1996) 477

[33] W. Eckstein, J. Nucl. Mater. 281 (2000) 195

[34] K. Morita, T. Tabata, R. Ito, J. Nucl. Mater. 128 \& 129 (1984) 681

[35] M. Vicanek, H.M. Urbassek, Phys. Rev. B 44 (1991) 7234

[36] J.V. Vukanic, R.K. Janev, D. Heifetz, Nucl. Instrum. Meth. B 18 (1987) 131

[37] Y.-Y, Xia, C.-Y. Tan, L.-M. Mei, J. Appl. Phys. 69 (1991) 439

[38] H. Coufal, H.F. Winters, H.L. Bay, W. Eckstein, Phys. Rev. B 44 (1991) 4747

[39] W. Eckstein, J.P. Biersack, Appl. Phys. A 38 (1985) 123

[40] M.I. Baskes, J. Nucl. Mater. 128 \& 129 (1984) 676

[41] W. Eckstein, J.P. Biersack, Z. Phys. B 63 (1988) 109 
[42] W. Eckstein, J.P. Biersack, Z. Phys. A 310 (1983) 1

[43] D.P. Jackson, W. Eckstein, Nucl. Instrum. Meth. 194 (1982) 671

[44] O.S. Oen, M.T. Robinson, J. Nucl. Mater. 76 \& 77 (1978) 370

[45] W. Eckstein, H. Verbeek, J. Nucl. Mater. 76 \& 77 (1978) 365

[46] P.J. Schneider, H. Verbeek, J. Nucl. Mater. 97 (1981) 319

[47] M. Hou, W. Eckstein, Nucl. Instrum. Meth. B 13 (1986) 324

[48] O.S. Oen, M.T. Robinson, AIP Conf. Proc. No. 111 (1984) 171

[49] R. Becerra-Acevedo, B. Terreault, Nucl. Instrum. Meth. B 28 (1987) 1

[50] S.H. Overbury, P.F. Dittner, S. Datz, R,S, Thoe, J. Nucl. Mater. 93 \& 94 (1980) 529

[51] R. Aratari, W. Eckstein, J. Nucl. Mater. 162-164 (1989) 910

[52] R. Aratari, W. Eckstein, Nucl. Instrum. Meth. B 42 (1989) 11

[53] M. Braun, E.W. Thomas, J. Appl. Phys. 53 (1982) 6446

[54] J. Robinson, K.K.Kwok, D.A. Thompson, Nucl. Instrum. Meth. 132 (1976) 667

[55] G. Staudenmaier, J. Roth, R. Behrisch, J. Bohdansky, W. Eckstein, P. Staib, S. Matteson, S.K. Erents, J. Nucl. Mater. 84 (1979) 149

[56] D. Hildebrandt, R. Manns, phys. stat. sol. (a) 38 (1976) K155

[57] J. Bøttiger, O. Holck, G. Sidenius, and the Isolde Collaboration, Nucl. Instrum. Meth. 170 (1980) 499

[58] S. Tanaka, Y. Murakami, T. Shibata, Jpn. J. Appl. Phys. 17 (1978) 183

[59] A.F. Akkerman, phys. stat. sol. A 48 (1978) K47

[60] J. Bohdansky, J. Roth, M.K. Sinha, W. Ottenberger, J. Nucl. Mater. 63 (1976) 115

[61] N.N. Koborov, V.A. Kurnaev, V.G. Telkovsky, G.I. Zhabrev, Radiat. Eff. 69 (1983) 135

[62] W.R. Gesang, H. Oechsner, H. Schoof, Nucl. Instrum. Meth. 132 (1976) 687

[63] W. Eckstein, F.E.P. Matschke, H. Verbeek, J. Nucl. Mater. 63 (1976) 199

[64] E.W. Thomas, J. Appl. Phys. 51 (1980) 1176

[65] W. Takeuchi, Y. Yamamura, Radiat. Eff. 71 (1983) 53 
[66] J. Schou, H. Sørensen, U. Littmark, J. Nucl. Mater. 76 \& 77 (1978) 359

[67] J. Bøttiger, J.A. Davies, P. Sigmund, K.B. Winterbon, Radiat. Eff. 11 (1971) 69

[68] G. Betz, Z. Naturf. A 27 (1972) 1239

[69] H. Sørensen, Proc. Int. Symp. on Plasma-Wall Interaction, Jülich 1976 (Pergamon 1977)

[70] J.E. Robinson, Radiat. Eff. 23 (1974) 29

[71] C. Braganza, G. Carter, G. Farrell, Nucl. Instrum. Meth. 132 (1976) 679

[72] C. Brunnée, Z. Phys. 147 (1957) 161

[73] J. Bøttiger, N. Rud, Inst. Phys. Conf. Ser. No. 28 (1975) 224

[74] H. Sørensen,

[75] H. Verbeek, W. Eckstein, R.S. Bhattacharya, J. Appl. Phys. 51 (1980) 1783

[76] N.N. Koborov, V.A. Kurnaev, V.M. Sotnikov, J. Nucl. Mater. 128 (1984) 691

[77] W. Eckstein, Heifetz, J. Nucl. Mater. 145-147 (1987) 332

[78] D. Reiter, W. Eckstein, G. Giesen, H.J. Belitz, Report Jül-2605, Jülich, 1992

[79] M. Mayer, B.M.U. Scherzer, W. Eckstein Nucl. Instrum. Meth. B 85 (1994) 560

[80] M. Mayer, IPP Report 9/104, Garching 1995

[81] C.K. Chen, W. Eckstein, B.M.U. Scherzer, Appl. Phys. A 31 (1983) 37

[82] C.K. Chen, J. Bohdansky, W. Eckstein, M.T. Robinson, J. Nucl. Mater. 128 \& 129 (1984) 687

[83] W. Eckstein, IPP-JET-Report Nr.4, Garching 1981

[84] H. Sørensen, Appl. Phys. Lett. 29 (1976) 148 
Table 1: Constants, $a_{i}$, for the energy dependence of the particle reflection coefficient, $\mathrm{R}_{N}$, at normal incidence for different ions and targets. $\varepsilon_{L}$ is the scaling factor defined in (4), $\mathrm{E}_{\mathrm{sp}}$ the assumed surface binding energy of ions, and fit denotes the applied fitting formula.

\begin{tabular}{|c|c|c|c|c|c|c|c|c|}
\hline ion & solid & a1 & $\mathrm{a} 2$ & a3 & $\mathrm{a} 4$ & $\varepsilon_{L}$ & $\mathrm{E}_{\mathrm{sp}}$ & fit \\
\hline $\mathrm{H}$ & $\mathrm{Li}$ & $0.1936 \mathrm{e}+0$ & $-0.1051 \mathrm{e}+0$ & $0.5251 \mathrm{e}+0$ & $0.1242 \mathrm{e}+1$ & $1.85375 \mathrm{e}+2$ & 1.00 & $(6)$ \\
\hline $\mathrm{D}$ & $\mathrm{Li}$ & $0.1221 \mathrm{e}+0$ & $-0.3685 \mathrm{e}-1$ & $0.6331 \mathrm{e}+0$ & $0.1249 \mathrm{e}+1$ & $2.08692 \mathrm{e}+2$ & 1.00 & (6) \\
\hline $\mathrm{T}$ & $\mathrm{Li}$ & $0.6470 \mathrm{e}+0$ & $-0.7715 \mathrm{e}-1$ & $0.6705 \mathrm{e}+0$ & $0.1227 \mathrm{e}+1$ & $2.32243 e+2$ & 1.00 & (6) \\
\hline${ }^{4} \mathrm{He}$ & $\mathrm{Li}$ & $0.2445 \mathrm{e}-1$ & $-0.4312 e+0$ & $0.3950 e+0$ & $0.9480 e+0$ & $5.56715 \mathrm{e}+2$ & 0.00 & (6) \\
\hline $\mathrm{Li}$ & $\mathrm{Li}$ & $-0.1308 \mathrm{e}+0$ & $-0.8413 e+0$ & $0.6066 \mathrm{e}+1$ & $0.1613 \mathrm{e}+0$ & $1.12841 \mathrm{e}+3$ & 0.00 & (3) \\
\hline $\mathrm{H}$ & $\mathrm{Be}$ & $0.1878 \mathrm{e}+0$ & $-0.2469 \mathrm{e}+0$ & $0.2448 \mathrm{e}+0$ & $0.1271 \mathrm{e}+1$ & $2.56510 \mathrm{e}+2$ & 1.00 & (6) \\
\hline $\mathrm{D}$ & $\mathrm{Be}$ & $0.1169 \mathrm{e}+0$ & $-0.2841 \mathrm{e}+0$ & $0.2138 \mathrm{e}+0$ & $0.1205 \mathrm{e}+1$ & $2.82110 \mathrm{e}+2$ & 1.00 & (6) \\
\hline $\mathrm{T}$ & $\mathrm{Be}$ & $0.7312 \mathrm{e}-1$ & $-0.3123 e+0$ & $0.2143 \mathrm{e}+0$ & $0.1150 \mathrm{e}+1$ & $3.07966 \mathrm{e}+2$ & 1.00 & (6) \\
\hline${ }^{3} \mathrm{He}$ & $\mathrm{Be}$ & $0.5921 \mathrm{e}-1$ & $-0.4564 \mathrm{e}+0$ & $0.3277 \mathrm{e}+0$ & $0.9330 \mathrm{e}+0$ & $6.65344 \mathrm{e}+2$ & 0.00 & (6) \\
\hline${ }^{4} \mathrm{He}$ & $\mathrm{Be}$ & $0.3679 \mathrm{e}-1$ & $-0.5065 e+0$ & $0.1418 \mathrm{e}+0$ & $0.1058 \mathrm{e}+1$ & $7.19545 \mathrm{e}+2$ & 0.00 & (6) \\
\hline $\mathrm{Be}$ & $\mathrm{Be}$ & $-0.1861 \mathrm{e}-1$ & $-0.1270 e+1$ & $0.6540 \mathrm{e}+1$ & $0.1375 \mathrm{e}+0$ & $2.20796 \mathrm{e}+3$ & 3.38 & $(3)$ \\
\hline $\mathrm{H}$ & $\mathrm{B}$ & $0.2034 \mathrm{e}+0$ & $-0.2501 \mathrm{e}+0$ & $0.2484 \mathrm{e}+0$ & $0.1274 \mathrm{e}+1$ & $3.32864 \mathrm{e}+2$ & 1.00 & $(6)$ \\
\hline $\mathrm{D}$ & B & $0.1352 \mathrm{e}+0$ & $-0.2804 \mathrm{e}+0$ & $0.1714 \mathrm{e}+0$ & $0.1250 \mathrm{e}+1$ & $3.61025 \mathrm{e}+2$ & 1.00 & (6) \\
\hline $\mathrm{T}$ & B & $0.9250 \mathrm{e}-1$ & $-0.3045 \mathrm{e}+0$ & $0.1996 \mathrm{e}+0$ & $0.1181 \mathrm{e}+1$ & $3.89468 \mathrm{e}+2$ & 1.00 & (6) \\
\hline${ }^{4} \mathrm{He}$ & B & $0.4978 \mathrm{e}-1$ & $-0.4685 e+0$ & $0.1040 \mathrm{e}+0$ & $0.1216 \mathrm{e}+1$ & $8.94388 \mathrm{e}+2$ & 0.00 & (6) \\
\hline B & B & $-0.5023 \mathrm{e}-1$ & $-0.1041 e+1$ & $0.6329 \mathrm{e}+1$ & $0.1282 \mathrm{e}+0$ & $3.71634 \mathrm{e}+3$ & 5.73 & (3) \\
\hline $\mathrm{H}$ & $\mathrm{C}$ & $0.2119 \mathrm{e}+0$ & $-0.2217 \mathrm{e}+0$ & $0.2461 \mathrm{e}+0$ & $0.1316 \mathrm{e}+1$ & $4.14659 \mathrm{e}+2$ & 1.00 & (6) \\
\hline $\mathrm{D}$ & $\mathrm{C}$ & $0.1526 \mathrm{e}+0$ & $-0.2304 \mathrm{e}+0$ & $0.2113 \mathrm{e}+0$ & $0.1287 \mathrm{e}+1$ & $4.46507 \mathrm{e}+2$ & 1.00 & (6) \\
\hline $\mathrm{T}$ & $\mathrm{C}$ & $0.1044 \mathrm{e}+0$ & $-0.2618 \mathrm{e}+0$ & $0.2052 \mathrm{e}+0$ & $0.1228 \mathrm{e}+1$ & $4.78673 \mathrm{e}+2$ & 1.00 & (6) \\
\hline${ }^{3} \mathrm{He}$ & $\mathrm{C}$ & $0.8632 \mathrm{e}-1$ & $-0.3682 \mathrm{e}+0$ & $0.1487 \mathrm{e}+0$ & $0.1279 \mathrm{e}+1$ & $1.02061 \mathrm{e}+3$ & 0.00 & (6) \\
\hline${ }^{4} \mathrm{He}$ & $\mathrm{C}$ & $0.6302 \mathrm{e}-1$ & $-0.3796 e+0$ & $0.1931 \mathrm{e}+0$ & $0.1134 \mathrm{e}+1$ & $1.08716 \mathrm{e}+3$ & 0.00 & (6) \\
\hline C & $\mathrm{C}$ & $-0.3022 \mathrm{e}-1$ & $-0.1107 e+1$ & $0.6544 \mathrm{e}+1$ & $0.1256 \mathrm{e}+0$ & $5.68684 \mathrm{e}+3$ & 7.41 & (3) \\
\hline $\mathrm{N}$ & $\mathrm{C}$ & $-0.3902 \mathrm{e}-2$ & $-0.1180 e+1$ & $0.7520 \mathrm{e}+1$ & $0.1252 \mathrm{e}+0$ & $7.37899 \mathrm{e}+3$ & 1.00 & (3) \\
\hline${ }^{4} \mathrm{He}$ & $\mathrm{Mg}$ & $0.3324 \mathrm{e}+0$ & $-0.3925 \mathrm{e}+0$ & $0.1970 \mathrm{e}+1$ & $0.1111 \mathrm{e}+1$ & $2.24482 \mathrm{e}+3$ & 0.00 & $(6)$ \\
\hline $\mathrm{Ne}$ & $\mathrm{Mg}$ & $0.1332 \mathrm{e}-1$ & $-0.5294 \mathrm{e}+0$ & $0.1782 \mathrm{e}+1$ & $0.5057 \mathrm{e}+0$ & $2.12200 \mathrm{e}+4$ & 0.00 & (6) \\
\hline $\mathrm{Mg}$ & $\mathrm{Mg}$ & $-0.4489 \mathrm{e}-2$ & $-0.9497 \mathrm{e}+0$ & $0.6505 \mathrm{e}+1$ & $0.1274 \mathrm{e}+0$ & $2.86599 \mathrm{e}+4$ & 1.54 & (3) \\
\hline $\mathrm{H}$ & $\mathrm{Al}$ & $0.2658 \mathrm{e}+0$ & $-0.1763 e+0$ & $0.2251 \mathrm{e}+0$ & $0.1488 \mathrm{e}+1$ & $1.05916 \mathrm{e}+3$ & 1.00 & (6) \\
\hline $\mathrm{D}$ & $\mathrm{Al}$ & $0.2319 \mathrm{e}+0$ & $-0.1777 e+0$ & $0.1523 e+0$ & $0.1523 e+1$ & $1.09700 \mathrm{e}+3$ & 1.00 & (6) \\
\hline $\mathrm{T}$ & $\mathrm{Al}$ & $0.2037 \mathrm{e}+0$ & $-0.1749 e+0$ & $0.1516 \mathrm{e}+0$ & $0.1482 \mathrm{e}+1$ & $1.13522 \mathrm{e}+3$ & 1.00 & (6) \\
\hline${ }^{3} \mathrm{He}$ & $\mathrm{Al}$ & $0.1762 \mathrm{e}+0$ & $-0.2202 \mathrm{e}+0$ & $0.2245 \mathrm{e}+0$ & $0.1379 \mathrm{e}+1$ & $2.37037 \mathrm{e}+3$ & 0.00 & (6) \\
\hline${ }^{4} \mathrm{He}$ & $\mathrm{Al}$ & $0.1548 \mathrm{e}+0$ & $-0.2233 \mathrm{e}+0$ & $0.2240 \mathrm{e}+0$ & $0.1308 \mathrm{e}+1$ & $2.44780 \mathrm{e}+3$ & 0.00 & (6) \\
\hline $\mathrm{N}$ & $\mathrm{Al}$ & $-0.3070 \mathrm{e}-5$ & $-0.1794 e+1$ & $0.3976 \mathrm{e}+1$ & $0.1728 \mathrm{e}+0$ & $1.28804 \mathrm{e}+4$ & 1.00 & (3) \\
\hline $\mathrm{Ne}$ & $\mathrm{Al}$ & $0.1607 \mathrm{e}-1$ & $-0.3285 e+0$ & $0.1234 \mathrm{e}+1$ & $0.8608 \mathrm{e}+0$ & $2.22732 \mathrm{e}+4$ & 0.00 & (6) \\
\hline $\mathrm{Al}$ & $\mathrm{Al}$ & $-0.2355 \mathrm{e}-2$ & $-0.1098 e+1$ & $0.6569 \mathrm{e}+1$ & $0.1188 \mathrm{e}+0$ & $3.45451 \mathrm{e}+4$ & 2.70 & (3) \\
\hline $\mathrm{H}$ & $\mathrm{Si}$ & $0.2600 \mathrm{e}+0$ & $-0.1772 \mathrm{e}+0$ & $0.2015 \mathrm{e}+0$ & $0.1515 \mathrm{e}+1$ & $1.16317 \mathrm{e}+3$ & 1.00 & (6) \\
\hline $\mathrm{D}$ & $\mathrm{Si}$ & $0.2381 \mathrm{e}+0$ & $-0.1662 \mathrm{e}+0$ & $0.1552 \mathrm{e}+0$ & $0.1535 \mathrm{e}+1$ & $1.20314 \mathrm{e}+3$ & 1.00 & (6) \\
\hline $\mathrm{T}$ & $\mathrm{Si}$ & $0.2109 \mathrm{e}+0$ & $-0.1630 \mathrm{e}+0$ & $0.1601 \mathrm{e}+0$ & $0.1490 \mathrm{e}+1$ & $1.24352 \mathrm{e}+3$ & 1.00 & (6) \\
\hline${ }^{3} \mathrm{He}$ & $\mathrm{Si}$ & $0.1910 \mathrm{e}+0$ & $-0.1943 e+0$ & $0.2863 e+0$ & $0.1333 e+1$ & $2.59209 \mathrm{e}+3$ & 0.00 & (6) \\
\hline${ }^{4} \mathrm{He}$ & $\mathrm{Si}$ & $0.1664 \mathrm{e}+0$ & $-0.2028 \mathrm{e}+0$ & $0.2767 \mathrm{e}+0$ & $0.1303 e+1$ & $2.67374 \mathrm{e}+3$ & 0.00 & (6) \\
\hline $\mathrm{N}$ & $\mathrm{Si}$ & $-0.9449 e-4$ & $-0.1398 e+1$ & $0.3847 \mathrm{e}+1$ & $0.1785 \mathrm{e}+0$ & $1.38909 \mathrm{e}+4$ & 0.00 & (3) \\
\hline $\mathrm{Ne}$ & $\mathrm{Si}$ & $0.2138 \mathrm{e}-1$ & $-0.2975 e+0$ & $0.1684 \mathrm{e}+1$ & $0.8635 \mathrm{e}+0$ & $2.39034 \mathrm{e}+4$ & 0.00 & (6) \\
\hline $\mathrm{Si}$ & $\mathrm{Si}$ & $-0.3908 e+1$ & $-0.1721 \mathrm{e}+0$ & $0.1974 \mathrm{e}+1$ & $-0.5695 e+0$ & $4.10661 \mathrm{e}+4$ & 4.70 & (3) \\
\hline $\mathrm{Ca}$ & $\mathrm{Ca}$ & $-0.2276 \mathrm{e}-2$ & $-0.9076 \mathrm{e}+0$ & $0.6034 \mathrm{e}+1$ & $0.9998 \mathrm{e}-1$ & $9.43891 \mathrm{e}+4$ & 1.83 & $(3)$ \\
\hline $\mathrm{Sc}$ & $\mathrm{Sc}$ & $-0.4412 \mathrm{e}-2$ & $-0.8791 e+0$ & $0.6158 \mathrm{e}+1$ & $0.1084 \mathrm{e}+0$ & $1.05770 \mathrm{e}+5$ & 3.49 & $(3)$ \\
\hline
\end{tabular}


Table 2: Constants, $a_{i}$, for the energy dependence of the particle reflection coefficient, $\mathrm{R}_{N}$, at normal incidence for different ions and targets. $\varepsilon_{L}$ is the scaling factor defined in (4), $\mathrm{E}_{\mathrm{sp}}$ the assumed surface binding energy of ions, and fit denotes the applied fitting formula.

\begin{tabular}{|c|c|c|c|c|c|c|c|c|}
\hline ion & solid & a1 & a2 & $\mathrm{a} 3$ & $\mathrm{a} 4$ & $\varepsilon_{L}$ & $\mathrm{E}_{\mathrm{sp}}$ & fit \\
\hline $\mathrm{H}$ & $\mathrm{Ti}$ & $0.3040 \mathrm{e}+0$ & $-0.1516 \mathrm{e}+0$ & $0.2481 \mathrm{e}+0$ & $0.1571 \mathrm{e}+1$ & $2.05415 \mathrm{e}+3$ & 1.00 & $(6)$ \\
\hline $\mathrm{D}$ & $\mathrm{Ti}$ & $0.2821 \mathrm{e}+0$ & $-0.1578 \mathrm{e}+0$ & $0.1566 \mathrm{e}+0$ & $0.1593 e+1$ & $2.09615 \mathrm{e}+3$ & 1.00 & (6) \\
\hline $\mathrm{T}$ & $\mathrm{Ti}$ & $0.2716 \mathrm{e}+0$ & $-0.1440 \mathrm{e}+0$ & $0.1495 \mathrm{e}+0$ & $0.1603 \mathrm{e}+1$ & $2.13856 \mathrm{e}+3$ & 1.00 & (6) \\
\hline${ }^{3} \mathrm{He}$ & $\mathrm{Ti}$ & $0.2363 \mathrm{e}+0$ & $-0.1729 \mathrm{e}+0$ & $0.2200 \mathrm{e}+0$ & $0.1572 \mathrm{e}+1$ & $4.41677 \mathrm{e}+3$ & 0.00 & (6) \\
\hline${ }^{4} \mathrm{He}$ & $\mathrm{Ti}$ & $0.2130 \mathrm{e}+0$ & $-0.1831 \mathrm{e}+0$ & $0.1726 \mathrm{e}+0$ & $0.1603 e+1$ & $4.50177 \mathrm{e}+3$ & 0.00 & (6) \\
\hline $\mathrm{N}$ & $\mathrm{Ti}$ & $0.1091 \mathrm{e}+0$ & $-0.1903 e+0$ & $0.9592 \mathrm{e}+0$ & $0.9407 \mathrm{e}+0$ & $2.07557 \mathrm{e}+4$ & 1.00 & (6) \\
\hline $\mathrm{Ne}$ & $\mathrm{Ti}$ & $0.4160 \mathrm{e}-1$ & $-0.3055 \mathrm{e}+0$ & $0.3391 \mathrm{e}+0$ & $0.1124 \mathrm{e}+1$ & $3.39688 \mathrm{e}+4$ & 0.00 & (6) \\
\hline Ar & $\mathrm{Ti}$ & $0.2754 \mathrm{e}+0$ & $-0.1553 e+0$ & $0.4902 \mathrm{e}+2$ & $0.7071 \mathrm{e}+0$ & $8.56428 \mathrm{e}+4$ & 0.00 & (6) \\
\hline $\mathrm{Ti}$ & $\mathrm{Ti}$ & $-0.5395 e+1$ & $-0.1104 \mathrm{e}+0$ & $0.6992 \mathrm{e}-2$ & $0.8628 \mathrm{e}+0$ & $1.17898 \mathrm{e}+5$ & 4.89 & (3) \\
\hline $\mathrm{H}$ & $\mathrm{V}$ & $0.3059 \mathrm{e}+0$ & $-0.1585 \mathrm{e}+0$ & $0.2797 \mathrm{e}+0$ & $0.1516 \mathrm{e}+1$ & $2.17329 \mathrm{e}+3$ & 1.00 & $(6)$ \\
\hline D & $\mathrm{V}$ & $0.2968 \mathrm{e}+0$ & $-0.1536 \mathrm{e}+0$ & $0.1850 \mathrm{e}+0$ & $0.1549 \mathrm{e}+1$ & $2.21513 \mathrm{e}+3$ & 1.00 & (6) \\
\hline $\mathrm{T}$ & $\mathrm{V}$ & $0.2810 \mathrm{e}+0$ & $-0.1456 \mathrm{e}+0$ & $0.1640 \mathrm{e}+0$ & $0.1559 \mathrm{e}+1$ & $2.25738 \mathrm{e}+3$ & 1.00 & (6) \\
\hline${ }^{3} \mathrm{He}$ & $\mathrm{V}$ & $0.2303 \mathrm{e}+0$ & $-0.1902 \mathrm{e}+0$ & $0.1673 \mathrm{e}+0$ & $0.1674 \mathrm{e}+1$ & $4.65839 \mathrm{e}+3$ & 0.00 & (6) \\
\hline${ }^{4} \mathrm{He}$ & $\mathrm{V}$ & $0.2114 \mathrm{e}+0$ & $-0.1968 \mathrm{e}+0$ & $0.1287 \mathrm{e}+0$ & $0.1722 \mathrm{e}+1$ & $4.74299 \mathrm{e}+3$ & 0.00 & (6) \\
\hline $\mathrm{N}$ & $\mathrm{V}$ & $0.1024 \mathrm{e}+0$ & $-0.2135 \mathrm{e}+0$ & $0.6204 \mathrm{e}+0$ & $0.9894 \mathrm{e}+0$ & $2.16246 \mathrm{e}+4$ & 1.00 & (6) \\
\hline $\mathrm{Ne}$ & $\mathrm{V}$ & $0.5031 \mathrm{e}-1$ & $-0.2975 \mathrm{e}+0$ & $0.3320 \mathrm{e}+0$ & $0.1152 \mathrm{e}+1$ & $3.52128 \mathrm{e}+4$ & 0.00 & (6) \\
\hline Ar & $\mathrm{V}$ & $0.1817 \mathrm{e}-1$ & $-0.2798 \mathrm{e}+0$ & $0.2007 \mathrm{e}+1$ & $0.6752 \mathrm{e}+0$ & $8.78018 e+4$ & 0.00 & (6) \\
\hline V & $\mathrm{V}$ & $-0.4514 e-3$ & $-0.1185 e+1$ & $0.5993 e+1$ & $0.9121 \mathrm{e}-1$ & $1.30783 e+5$ & 6.10 & $(6)$ \\
\hline${ }^{4} \mathrm{He}$ & $\mathrm{Cr}$ & $0.2129 \mathrm{e}+0$ & $-0.2021 \mathrm{e}+0$ & $0.1283 \mathrm{e}+0$ & $0.1719 \mathrm{e}+1$ & $5.00096 \mathrm{e}+3$ & 0.00 & $(6)$ \\
\hline $\mathrm{Ne}$ & $\mathrm{Cr}$ & $0.5379 \mathrm{e}-1$ & $-0.2931 \mathrm{e}+0$ & $0.4160 \mathrm{e}+0$ & $0.1086 \mathrm{e}+1$ & $3.68638 \mathrm{e}+4$ & 0.00 & (6) \\
\hline Ar & $\mathrm{Cr}$ & $0.1819 \mathrm{e}-1$ & $-0.2926 \mathrm{e}+0$ & $0.1546 \mathrm{e}+1$ & $0.6081 \mathrm{e}+0$ & $9.15022 \mathrm{e}+4$ & 0.00 & (6) \\
\hline $\mathrm{Cr}$ & $\mathrm{Cr}$ & $-0.1578 \mathrm{e}-3$ & $-0.1279 e+1$ & $0.6023 \mathrm{e}+1$ & $0.8983 \mathrm{e}-1$ & $1.44437 \mathrm{e}+5$ & 4.12 & (3) \\
\hline${ }^{4} \mathrm{He}$ & $\mathrm{Mn}$ & $0.2240 \mathrm{e}+0$ & $-0.1919 \mathrm{e}+0$ & $0.1717 \mathrm{e}+0$ & $0.1610 \mathrm{e}+1$ & $5.24919 \mathrm{e}+3$ & 0.00 & $(6)$ \\
\hline $\mathrm{Ne}$ & $\mathrm{Mn}$ & $0.5883 \mathrm{e}-1$ & $-0.2834 \mathrm{e}+0$ & $0.3920 \mathrm{e}+0$ & $0.1143 e+1$ & $3.81587 \mathrm{e}+4$ & 0.00 & (6) \\
\hline $\mathrm{H}$ & $\mathrm{Fe}$ & $0.3276 \mathrm{e}+0$ & $-0.1448 \mathrm{e}+0$ & $0.3591 \mathrm{e}+0$ & $0.1407 \mathrm{e}+1$ & $2.54382 \mathrm{e}+3$ & 1.00 & (6) \\
\hline $\mathrm{D}$ & $\mathrm{Fe}$ & $0.3148 \mathrm{e}+0$ & $-0.1432 \mathrm{e}+0$ & $0.2396 \mathrm{e}+0$ & $0.1444 \mathrm{e}+1$ & $2.58856 \mathrm{e}+3$ & 1.00 & (6) \\
\hline $\mathrm{T}$ & $\mathrm{Fe}$ & $0.2991 \mathrm{e}+0$ & $-0.1393 e+0$ & $0.2100 \mathrm{e}+0$ & $0.1434 \mathrm{e}+1$ & $2.67374 \mathrm{e}+3$ & 1.00 & (6) \\
\hline${ }^{4} \mathrm{He}$ & $\mathrm{Fe}$ & $0.2179 \mathrm{e}+0$ & $-0.1976 \mathrm{e}+0$ & $0.1480 \mathrm{e}+0$ & $0.1621 \mathrm{e}+1$ & $5.51371 \mathrm{e}+3$ & 1.00 & (6) \\
\hline $\mathrm{Ne}$ & $\mathrm{Fe}$ & $0.5880 \mathrm{e}-1$ & $-0.2897 \mathrm{e}+0$ & $0.3481 \mathrm{e}+0$ & $0.1135 \mathrm{e}+1$ & $3.98491 \mathrm{e}+4$ & 0.00 & (6) \\
\hline $\mathrm{Ar}$ & $\mathrm{Fe}$ & $0.2875 \mathrm{e}-1$ & $-0.2534 \mathrm{e}+0$ & $0.2789 e+1$ & $0.6135 \mathrm{e}+0$ & $9.75914 \mathrm{e}+4$ & 0.00 & (6) \\
\hline $\mathrm{Fe}$ & $\mathrm{Fe}$ & $-0.5221 \mathrm{e}-4$ & $-0.1389 e+1$ & $0.5702 \mathrm{e}+1$ & $0.7674 \mathrm{e}-1$ & $1.74096 \mathrm{e}+5$ & 4.34 & (3) \\
\hline${ }^{4} \mathrm{He}$ & $\mathrm{Co}$ & $0.2269 \mathrm{e}+0$ & $-0.1931 \mathrm{e}+0$ & $0.1546 \mathrm{e}+0$ & $0.1558 \mathrm{e}+1$ & $5.76700 \mathrm{e}+3$ & 0.00 & $(6)$ \\
\hline $\mathrm{Ne}$ & Co & $0.6574 \mathrm{e}-1$ & $-0.2787 \mathrm{e}+0$ & $0.3967 \mathrm{e}+0$ & $0.1141 \mathrm{e}+1$ & $4.11463 \mathrm{e}+4$ & 0.00 & (6) \\
\hline Ar & $\mathrm{Co}$ & $0.3293 \mathrm{e}-1$ & $-0.2523 e+0$ & $0.2123 e+1$ & $0.5300 \mathrm{e}+0$ & $9.98420 \mathrm{e}+4$ & 0.00 & (6) \\
\hline $\mathrm{H}$ & $\mathrm{Ni}$ & $0.3248 \mathrm{e}+0$ & $-0.1476 \mathrm{e}+0$ & $0.3517 \mathrm{e}+0$ & $0.1413 \mathrm{e}+1$ & $2.79866 \mathrm{e}+3$ & 1.00 & (6) \\
\hline $\mathrm{D}$ & $\mathrm{Ni}$ & $0.3218 \mathrm{e}+0$ & $-0.1400 \mathrm{e}+0$ & $0.2567 \mathrm{e}+0$ & $0.1435 \mathrm{e}+1$ & $2.84552 \mathrm{e}+3$ & 1.00 & (6) \\
\hline $\mathrm{T}$ & $\mathrm{Ni}$ & $0.3009 \mathrm{e}+0$ & $-0.1418 \mathrm{e}+0$ & $0.2116 \mathrm{e}+0$ & $0.1431 \mathrm{e}+1$ & $2.89285 \mathrm{e}+3$ & 1.00 & (6) \\
\hline${ }^{4} \mathrm{He}$ & $\mathrm{Ni}$ & $0.2383 \mathrm{e}+0$ & $-0.1814 \mathrm{e}+0$ & $0.2133 e+0$ & $0.1500 \mathrm{e}+1$ & $6.04409 \mathrm{e}+3$ & 0.00 & (6) \\
\hline $\mathrm{N}$ & $\mathrm{Ni}$ & $0.1262 \mathrm{e}+0$ & $-0.1993 e+0$ & $0.7623 e+0$ & $0.8324 \mathrm{e}+0$ & $2.67793 e+4$ & 0.00 & (6) \\
\hline $\mathrm{O}$ & $\mathrm{Ni}$ & $0.8561 \mathrm{e}-1$ & $-0.2550 \mathrm{e}+0$ & $0.3365 \mathrm{e}+0$ & $0.1191 \mathrm{e}+1$ & $3.18555 \mathrm{e}+4$ & 0.00 & (6) \\
\hline $\mathrm{Ne}$ & $\mathrm{Ni}$ & $0.5921 \mathrm{e}-1$ & $-0.2930 \mathrm{e}+0$ & $0.3048 \mathrm{e}+0$ & $0.1097 \mathrm{e}+1$ & $4.30554 \mathrm{e}+4$ & 0.00 & (6) \\
\hline Ar & $\mathrm{Ni}$ & $0.3964 \mathrm{e}-1$ & $-0.2236 \mathrm{e}+0$ & $0.2830 \mathrm{e}+1$ & $0.5109 \mathrm{e}+0$ & $1.04416 \mathrm{e}+5$ & 0.00 & (6) \\
\hline $\mathrm{Ni}$ & $\mathrm{Ni}$ & $-0.3587 \mathrm{e}-4$ & $-0.1415 e+1$ & $0.5693 \mathrm{e}+1$ & $0.7770 \mathrm{e}-1$ & $2.06960 \mathrm{e}+5$ & 4.46 & (3) \\
\hline $\mathrm{H}$ & $\mathrm{Cu}$ & $0.3157 \mathrm{e}+0$ & $-0.1605 e+0$ & $0.3091 \mathrm{e}+0$ & $0.1442 \mathrm{e}+1$ & $2.92563 \mathrm{e}+3$ & 1.00 & $(6)$ \\
\hline $\mathrm{T}$ & $\mathrm{Cu}$ & $0.3063 e+0$ & $-0.1411 e+0$ & $0.1937 \mathrm{e}+0$ & $0.1479 \mathrm{e}+1$ & $3.01673 e+3$ & 1.00 & (6) \\
\hline${ }^{4} \mathrm{He}$ & $\mathrm{Cu}$ & $0.2551 \mathrm{e}+0$ & $-0.1703 e+0$ & $0.2930 \mathrm{e}+0$ & $0.1318 \mathrm{e}+1$ & $6.29218 \mathrm{e}+3$ & 0.00 & (6) \\
\hline $\mathrm{N}$ & $\mathrm{Cu}$ & $0.1133 \mathrm{e}+0$ & $-0.2318 \mathrm{e}+0$ & $0.4054 \mathrm{e}+0$ & $0.1041 \mathrm{e}+1$ & $2.75601 \mathrm{e}+4$ & 1.00 & (6) \\
\hline $\mathrm{Ne}$ & $\mathrm{Cu}$ & $0.6808 \mathrm{e}-1$ & $-0.2772 \mathrm{e}+0$ & $0.2758 \mathrm{e}+0$ & $0.1290 \mathrm{e}+1$ & $4.40689 \mathrm{e}+4$ & 0.00 & (6) \\
\hline $\mathrm{Ar}$ & $\mathrm{Cu}$ & $0.3629 \mathrm{e}-1$ & $-0.2411 \mathrm{e}+0$ & $0.1803 e+1$ & $0.6153 \mathrm{e}+0$ & $1.05525 \mathrm{e}+5$ & 0.00 & (6) \\
\hline $\mathrm{Cu}$ & $\mathrm{Cu}$ & $-0.2658 \mathrm{e}-4$ & $-0.1403 e+1$ & $0.5928 \mathrm{e}+1$ & $0.8322 \mathrm{e}-1$ & $2.24619 \mathrm{e}+5$ & 3.52 & (3) \\
\hline
\end{tabular}


Table 3: Constants, $a_{i}$, for the energy dependence of the particle reflection coefficient, $\mathrm{R}_{N}$, at normal incidence for different ions and targets. $\varepsilon_{L}$ is the scaling factor defined in (4), $\mathrm{E}_{\mathrm{sp}}$ the assumed surface binding energy of ions, and fit denotes the applied fitting formula.

\begin{tabular}{|c|c|c|c|c|c|c|c|c|}
\hline ion & solid & a1 & $\mathrm{a} 2$ & a3 & $\mathrm{a} 4$ & $\varepsilon_{L}$ & $E_{s p}$ & fit \\
\hline${ }^{4} \mathrm{He}$ & $\mathrm{Zn}$ & $0.2693 \mathrm{e}+0$ & $-0.1513 e+0$ & $0.3606 \mathrm{e}+0$ & $0.1291 \mathrm{e}+1$ & $6.56163 e+3$ & 0.00 & (6) \\
\hline $\mathrm{Ne}$ & $\mathrm{Zn}$ & $0.7229 \mathrm{e}-1$ & $-0.2591 \mathrm{e}+0$ & $0.3044 \mathrm{e}+0$ & $0.1374 \mathrm{e}+1$ & $4.56265 \mathrm{e}+4$ & 0.00 & (6) \\
\hline $\mathrm{Ar}$ & $\mathrm{Zn}$ & $0.1919 \mathrm{e}+0$ & $-0.7184 \mathrm{e}-1$ & $0.1172 \mathrm{e}+2$ & $0.4505 \mathrm{e}+0$ & $1.08696 \mathrm{e}+5$ & 0.00 & (6) \\
\hline $\mathrm{Zn}$ & $\mathrm{Zn}$ & $-0.5117 e+1$ & $0.9552 \mathrm{e}-1$ & $0.7560 \mathrm{e}-4$ & $-0.1185 e+1$ & $2.43109 \mathrm{e}+5$ & 1.35 & (3) \\
\hline $\mathrm{D}$ & $\mathrm{Ga}$ & $0.3324 \mathrm{e}+0$ & $-0.1245 \mathrm{e}+0$ & $0.2228 \mathrm{e}+0$ & $0.1610 \mathrm{e}+1$ & $3.23166 \mathrm{e}+3$ & 1.00 & (6) \\
\hline $\mathrm{T}$ & $\mathrm{Ga}$ & $0.3217 \mathrm{e}+0$ & $-0.1187 e+0$ & $0.1874 \mathrm{e}+0$ & $0.1624 \mathrm{e}+1$ & $3.27716 \mathrm{e}+3$ & 1.00 & (6) \\
\hline${ }^{4} \mathrm{He}$ & $\mathrm{Ga}$ & $0.2995 \mathrm{e}+0$ & $-0.1160 e+0$ & $0.4682 \mathrm{e}++$ & $0.1257 \mathrm{e}+1$ & $6.81977 \mathrm{e}+3$ & 0.00 & (6) \\
\hline $\mathrm{Ne}$ & $\mathrm{Ga}$ & $0.1102 \mathrm{e}+0$ & $-0.1678 \mathrm{e}+0$ & $0.9081 \mathrm{e}+0$ & $0.8525 \mathrm{e}+0$ & $4.67989 \mathrm{e}+4$ & 0.00 & (6) \\
\hline $\mathrm{Ga}$ & $\mathrm{Ga}$ & $-0.4923 e+1$ & $0.8137 \mathrm{e}-1$ & $0.2510 \mathrm{e}-3$ & $-0.1101 e+1$ & $2.62439 \mathrm{e}+5$ & 2.82 & (3) \\
\hline $\mathrm{H}$ & $\mathrm{Ge}$ & $0.3372 \mathrm{e}+0$ & $-0.1260 \mathrm{e}+0$ & $0.3259 \mathrm{e}+0$ & $0.1607 \mathrm{e}+1$ & $3.31932 \mathrm{e}+3$ & 1.00 & (6) \\
\hline D & $\mathrm{Ge}$ & $0.3353 \mathrm{e}+0$ & $-0.1213 e+0$ & $0.2040 \mathrm{e}+0$ & $0.1682 \mathrm{e}+1$ & $3.36441 \mathrm{e}+3$ & 1.00 & (6) \\
\hline${ }^{4} \mathrm{He}$ & $\mathrm{Ge}$ & $0.2673 e+0$ & $-0.1425 e+0$ & $0.2568 \mathrm{e}+0$ & $0.1602 \mathrm{e}+1$ & $7.08909 \mathrm{e}+3$ & 0.00 & (6) \\
\hline $\mathrm{Ne}$ & $\mathrm{Ge}$ & $0.8622 \mathrm{e}-1$ & $-0.2251 \mathrm{e}+0$ & $0.3339 \mathrm{e}+0$ & $0.1372 \mathrm{e}+1$ & $4.82268 \mathrm{e}+4$ & 0.00 & (6) \\
\hline $\mathrm{Na}$ & $\mathrm{Ge}$ & $0.7506 \mathrm{e}-1$ & $-0.2317 \mathrm{e}+0$ & $0.3948 \mathrm{e}+0$ & $0.1275 \mathrm{e}+1$ & $5.52188 \mathrm{e}+4$ & 0.00 & (6) \\
\hline Ar & $\mathrm{Ge}$ & $0.3072 \mathrm{e}-1$ & $-0.2704 \mathrm{e}+0$ & $0.6905 \mathrm{e}+0$ & $0.1085 \mathrm{e}+1$ & $1.12992 \mathrm{e}+5$ & 0.00 & (6) \\
\hline $\mathrm{K}$ & $\mathrm{Ge}$ & $0.4370 \mathrm{e}-1$ & $-0.2259 \mathrm{e}+0$ & $0.1281 \mathrm{e}+1$ & $0.7090 \mathrm{e}+0$ & $1.19246 \mathrm{e}+5$ & 0.00 & (6) \\
\hline $\mathrm{Ge}$ & $\mathrm{Ge}$ & $-0.5055 e+1$ & $0.9159 \mathrm{e}-1$ & $0.1291 \mathrm{e}-2$ & $-0.9454 \mathrm{e}+0$ & $2.82619 \mathrm{e}+5$ & 3.88 & (3) \\
\hline${ }^{4} \mathrm{He}$ & $\mathrm{Se}$ & $0.2915 \mathrm{e}+0$ & $-0.1245 \mathrm{e}+0$ & $0.3218 \mathrm{e}+0$ & $0.1517 \mathrm{e}+1$ & $7.08909 \mathrm{e}+3$ & 0.00 & (6) \\
\hline $\mathrm{Ne}$ & $\mathrm{Se}$ & $0.1023 e+0$ & $-0.1974 \mathrm{e}+0$ & $0.4648 \mathrm{e}+0$ & $0.1207 \mathrm{e}+1$ & $5.10477 \mathrm{e}+4$ & 0.00 & (6) \\
\hline $\mathrm{Ar}$ & $\mathrm{Se}$ & $0.4894 \mathrm{e}-1$ & $-0.1270 \mathrm{e}+1$ & $0.1190 \mathrm{e}+1$ & $-0.1028 \mathrm{e}+1$ & $1.18037 \mathrm{e}+5$ & 0.00 & (6) \\
\hline $\mathrm{H}$ & $\mathrm{Zr}$ & $0.3558 \mathrm{e}+0$ & $-0.1140 \mathrm{e}+0$ & $0.3864 \mathrm{e}+0$ & $0.1682 \mathrm{e}+1$ & $4.42211 \mathrm{e}+3$ & 1.00 & $(6)$ \\
\hline $\mathrm{D}$ & $\mathrm{Zr}$ & $0.3775 \mathrm{e}+0$ & $-0.9960 \mathrm{e}-1$ & $0.2941 \mathrm{e}+0$ & $0.1709 \mathrm{e}+1$ & $4.47714 \mathrm{e}+3$ & 1.00 & (6) \\
\hline $\mathrm{T}$ & $\mathrm{Zr}$ & $0.3638 \mathrm{e}+0$ & $-0.9849 \mathrm{e}-1$ & $0.2846 \mathrm{e}+0$ & $0.1466 \mathrm{e}+1$ & $4.52564 \mathrm{e}+3$ & 1.00 & (6) \\
\hline${ }^{3} \mathrm{He}$ & $\mathrm{Zr}$ & $0.2894 \mathrm{e}+0$ & $-0.1381 \mathrm{e}+0$ & $0.2353 \mathrm{e}+0$ & $0.1923 \mathrm{e}+1$ & $9.25829 \mathrm{e}+3$ & 0.00 & (6) \\
\hline${ }^{4} \mathrm{He}$ & $\mathrm{Zr}$ & $0.2847 \mathrm{e}+0$ & $-0.1352 \mathrm{e}+0$ & $0.2008 \mathrm{e}+0$ & $0.1956 \mathrm{e}+1$ & $9.35457 \mathrm{e}+3$ & 0.00 & (6) \\
\hline $\mathrm{Ne}$ & $\mathrm{Zr}$ & $0.1167 \mathrm{e}+0$ & $-0.1900 \mathrm{e}+0$ & $0.4382 \mathrm{e}+0$ & $0.1283 \mathrm{e}+1$ & $6.06862 \mathrm{e}+4$ & 0.00 & (6) \\
\hline $\mathrm{Ar}$ & $\mathrm{Zr}$ & $0.5075 \mathrm{e}-1$ & $-0.2365 \mathrm{e}+0$ & $0.7014 \mathrm{e}+0$ & $0.1068 \mathrm{e}+1$ & $1.37106 \mathrm{e}+5$ & 0.00 & (6) \\
\hline $\mathrm{Kr}$ & $\mathrm{Zr}$ & $0.1666 \mathrm{e}-1$ & $-0.1957 \mathrm{e}+0$ & $0.3355 \mathrm{e}+1$ & $0.5537 \mathrm{e}+0$ & $4.03685 \mathrm{e}+5$ & 0.00 & (6) \\
\hline $\mathrm{Zr}$ & $\mathrm{Zr}$ & $-0.4904 \mathrm{e}+1$ & $0.8187 \mathrm{e}-1$ & $0.1364 \mathrm{e}-2$ & $-0.9343 e+0$ & $4.75691 \mathrm{e}+5$ & 6.33 & $(3)$ \\
\hline $\mathrm{H}$ & $\mathrm{Nb}$ & $0.3876 \mathrm{e}+0$ & $-0.9724 \mathrm{e}-1$ & $0.6718 \mathrm{e}+0$ & $0.1553 e+1$ & $4.57351 \mathrm{e}+3$ & 1.00 & (6) \\
\hline D & $\mathrm{Nb}$ & $0.3976 \mathrm{e}+0$ & $-0.9134 \mathrm{e}-1$ & $0.4581 \mathrm{e}+0$ & $0.1604 \mathrm{e}+1$ & $4.62220 \mathrm{e}+3$ & 1.00 & (6) \\
\hline $\mathrm{T}$ & $\mathrm{Nb}$ & $0.3567 \mathrm{e}+0$ & $-0.1076 e+0$ & $0.2069 \mathrm{e}+0$ & $0.1770 \mathrm{e}+1$ & $4.67139 \mathrm{e}+3$ & 1.00 & (6) \\
\hline${ }^{3} \mathrm{He}$ & $\mathrm{Nb}$ & $0.2950 \mathrm{e}+0$ & $-0.1395 \mathrm{e}+0$ & $0.2884 \mathrm{e}+0$ & $0.1710 \mathrm{e}+1$ & $9.55327 \mathrm{e}+3$ & 0.00 & (6) \\
\hline${ }^{4} \mathrm{He}$ & $\mathrm{Nb}$ & $0.2847 \mathrm{e}+0$ & $-0.1408 \mathrm{e}+0$ & $0.1640 \mathrm{e}+0$ & $0.1898 \mathrm{e}+1$ & $9.65087 \mathrm{e}+3$ & 0.00 & (6) \\
\hline $\mathrm{Ne}$ & $\mathrm{Nb}$ & $0.1091 \mathrm{e}+0$ & $-0.2117 \mathrm{e}+0$ & $0.2688 \mathrm{e}+0$ & $0.1602 \mathrm{e}+1$ & $6.23657 \mathrm{e}+4$ & 0.00 & (6) \\
\hline $\mathrm{Ar}$ & $\mathrm{Nb}$ & $0.5180 \mathrm{e}-1$ & $-0.2470 \mathrm{e}+0$ & $0.7052 \mathrm{e}+0$ & $0.9589 \mathrm{e}+0$ & $1.40484 \mathrm{e}+5$ & 0.00 & (6) \\
\hline $\mathrm{Kr}$ & $\mathrm{Nb}$ & $0.1237 \mathrm{e}-1$ & $-0.2457 \mathrm{e}+0$ & $0.2360 \mathrm{e}+1$ & $0.6610 \mathrm{e}+0$ & $4.11932 \mathrm{e}+5$ & 0.00 & (6) \\
\hline $\mathrm{Nb}$ & $\mathrm{Nb}$ & $-0.4742 e+1$ & $0.7244 \mathrm{e}-1$ & $0.7923 \mathrm{e}-3$ & $-0.1006 e+1$ & $5.03904 \mathrm{e}+5$ & 7.59 & (3) \\
\hline $\mathrm{H}$ & Mo & $0.3449 \mathrm{e}+0$ & $-0.1278 e+0$ & $0.3920 \mathrm{e}+0$ & $0.1626 \mathrm{e}+1$ & $4.71832 \mathrm{e}+3$ & 1.00 & (6) \\
\hline $\mathrm{D}$ & Mo & $0.3776 \mathrm{e}+0$ & $-0.1079 e+0$ & $0.3523 \mathrm{e}+0$ & $0.1625 \mathrm{e}+1$ & $4.76698 \mathrm{e}+3$ & 1.00 & (6) \\
\hline $\mathrm{T}$ & Mo & $0.3595 \mathrm{e}+0$ & $-0.1093 e+0$ & $0.2297 \mathrm{e}+0$ & $0.1693 \mathrm{e}+1$ & $4.81614 \mathrm{e}+3$ & 1.00 & (6) \\
\hline${ }^{3} \mathrm{He}$ & Mo & $0.3039 \mathrm{e}+0$ & $-0.1382 e+0$ & $0.3173 \mathrm{e}+0$ & $0.1657 \mathrm{e}+1$ & $9.84614 \mathrm{e}+3$ & 0.00 & (6) \\
\hline${ }^{4} \mathrm{He}$ & Mo & $0.3173 \mathrm{e}+0$ & $-0.1266 \mathrm{e}+0$ & $0.2517 \mathrm{e}+0$ & $0.1817 \mathrm{e}+1$ & $9.94365 \mathrm{e}+3$ & 0.00 & (6) \\
\hline $\mathrm{N}$ & Mo & $0.1674 \mathrm{e}+0$ & $-0.1746 e+0$ & $0.4252 \mathrm{e}+0$ & $0.1156 \mathrm{e}+1$ & $4.10879 \mathrm{e}+4$ & 1.00 & (6) \\
\hline $\mathrm{O}$ & Mo & $0.1524 \mathrm{e}+0$ & $-0.1759 e+0$ & $0.4598 \mathrm{e}+0$ & $0.1130 \mathrm{e}+1$ & $4.83320 \mathrm{e}+4$ & 1.00 & (6) \\
\hline $\mathrm{Ne}$ & Mo & $0.1114 \mathrm{e}+0$ & $-0.2161 e+0$ & $0.2941 \mathrm{e}+0$ & $0.1543 e+1$ & $6.38956 \mathrm{e}+4$ & 0.00 & (6) \\
\hline $\mathrm{Ar}$ & Mo & $0.5353 \mathrm{e}-1$ & $-0.2509 \mathrm{e}+0$ & $0.6636 \mathrm{e}+0$ & $0.1310 \mathrm{e}+1$ & $1.43274 \mathrm{e}+5$ & 0.00 & (6) \\
\hline $\mathrm{Kr}$ & Mo & $0.1988 \mathrm{e}-1$ & $-0.2150 \mathrm{e}+0$ & $0.2584 \mathrm{e}+1$ & $0.4987 \mathrm{e}+0$ & $4.17411 \mathrm{e}+5$ & 0.00 & (6) \\
\hline Mo & Mo & $-0.4430 \mathrm{e}+1$ & $0.5276 \mathrm{e}-1$ & $0.8376 \mathrm{e}-4$ & $-0.1232 e+1$ & $5.33049 \mathrm{e}+5$ & 6.83 & (3) \\
\hline
\end{tabular}


Table 4: Constants, $a_{i}$, for the energy dependence of the particle reflection coefficient, $\mathrm{R}_{N}$, at normal incidence for different ions and targets. $\varepsilon_{L}$ is the scaling factor defined in (4), $\mathrm{E}_{\mathrm{sp}}$ the assumed surface binding energy of ions, and fit denotes the applied fitting formula.

\begin{tabular}{|c|c|c|c|c|c|c|c|c|}
\hline ion & solid & a1 & $\mathrm{a} 2$ & a3 & $\mathrm{a} 4$ & $\varepsilon_{L}$ & $\mathrm{E}_{\mathrm{sp}}$ & fit \\
\hline $\mathrm{H}$ & $\mathrm{Ru}$ & $0.3507 \mathrm{e}+0$ & $-0.1248 \mathrm{e}+0$ & $0.3699 \mathrm{e}+0$ & $0.1647 \mathrm{e}+1$ & $5.01173 \mathrm{e}+3$ & 1.00 & $(6)$ \\
\hline $\mathrm{D}$ & $\mathrm{Ru}$ & $0.3720 \mathrm{e}+0$ & $-0.1126 \mathrm{e}+0$ & $0.3027 \mathrm{e}+0$ & $0.1647 \mathrm{e}+1$ & $5.06083 e+3$ & 1.00 & (6) \\
\hline${ }^{4} \mathrm{He}$ & $\mathrm{Ru}$ & $0.3108 \mathrm{e}+0$ & $-0.1321 \mathrm{e}+0$ & $0.3556 \mathrm{e}+0$ & $0.1446 \mathrm{e}+1$ & $1.05397 \mathrm{e}+4$ & 0.00 & (6) \\
\hline $\mathrm{Ne}$ & $\mathrm{Ru}$ & $0.1160 \mathrm{e}+0$ & $-0.2172 \mathrm{e}+0$ & $0.2851 \mathrm{e}+0$ & $0.1672 \mathrm{e}+1$ & $6.70985 \mathrm{e}+4$ & 0.00 & (6) \\
\hline $\mathrm{Ar}$ & $\mathrm{Ru}$ & $0.5661 \mathrm{e}-1$ & $-0.4633 e+0$ & $0.3824 \mathrm{e}+0$ & $-0.3059 e+0$ & $1.49334 \mathrm{e}+5$ & 0.00 & (6) \\
\hline $\mathrm{Kr}$ & $\mathrm{Ru}$ & $0.1380 \mathrm{e}-1$ & $-0.2673 e+0$ & $0.1835 \mathrm{e}+1$ & $0.8801 \mathrm{e}+0$ & $4.30460 \mathrm{e}+5$ & 0.00 & (6) \\
\hline $\mathrm{H}$ & $\mathrm{Rh}$ & $0.3533 \mathrm{e}+0$ & $-0.1241 \mathrm{e}+0$ & $0.4349 \mathrm{e}+0$ & $0.1582 \mathrm{e}+1$ & $5.16041 \mathrm{e}+3$ & 1.00 & (6) \\
\hline $\mathrm{D}$ & $\mathrm{Rh}$ & $0.3697 \mathrm{e}+0$ & $-0.1131 \mathrm{e}+0$ & $0.2966 \mathrm{e}+0$ & $0.1645 \mathrm{e}+1$ & $5.21007 \mathrm{e}+3$ & 1.00 & (6) \\
\hline${ }^{3} \mathrm{He}$ & Rh & $0.3098 \mathrm{e}+0$ & $-0.1372 \mathrm{e}+0$ & $0.3595 \mathrm{e}+0$ & $0.1549 \mathrm{e}+1$ & $1.07444 \mathrm{e}+4$ & 0.00 & (6) \\
\hline${ }^{4} \mathrm{He}$ & $\mathrm{Rh}$ & $0.3093 \mathrm{e}+0$ & $-0.1329 e+0$ & $0.3269 \mathrm{e}+0$ & $0.1536 \mathrm{e}+1$ & $1.08438 \mathrm{e}+4$ & 0.00 & (6) \\
\hline $\mathrm{Ne}$ & $\mathrm{Rh}$ & $0.1198 \mathrm{e}+0$ & $-0.2125 \mathrm{e}+0$ & $0.2950 \mathrm{e}+0$ & $0.1801 \mathrm{e}+1$ & $6.87945 \mathrm{e}+4$ & 0.00 & (6) \\
\hline $\mathrm{Ar}$ & Rh & $0.8428 \mathrm{e}+3$ & $0.6300 \mathrm{e}+0$ & $0.1885 e+5$ & $0.9289 \mathrm{e}+0$ & $1.52692 \mathrm{e}+5$ & 0.00 & (6) \\
\hline $\mathrm{Kr}$ & $\mathrm{Rh}$ & $0.1983 \mathrm{e}-1$ & $-0.2324 \mathrm{e}+0$ & $0.2928 \mathrm{e}+1$ & $0.6034 \mathrm{e}+0$ & $4.38429 \mathrm{e}+5$ & 0.00 & (6) \\
\hline $\mathrm{H}$ & $\mathrm{Pd}$ & $0.3602 \mathrm{e}+0$ & $-0.1184 \mathrm{e}+0$ & $0.4274 \mathrm{e}+0$ & $0.1548 \mathrm{e}+1$ & $5.30935 \mathrm{e}+3$ & 1.00 & $(6)$ \\
\hline${ }^{3} \mathrm{He}$ & $\mathrm{Pd}$ & $0.3232 \mathrm{e}+0$ & $-0.1281 e+0$ & $0.4075 \mathrm{e}+0$ & $0.1479 \mathrm{e}+1$ & $1.10446 \mathrm{e}+4$ & 0.00 & (6) \\
\hline${ }^{4} \mathrm{He}$ & $\mathrm{Pd}$ & $0.2932 \mathrm{e}+0$ & $-0.1416 \mathrm{e}+0$ & $0.2008 \mathrm{e}+0$ & $0.1834 \mathrm{e}+1$ & $1.11435 \mathrm{e}+4$ & 0.00 & (6) \\
\hline $\mathrm{Ne}$ & $\mathrm{Pd}$ & $0.1272 \mathrm{e}+0$ & $-0.2033 e+0$ & $0.3555 \mathrm{e}+0$ & $0.1296 \mathrm{e}+1$ & $7.03218 \mathrm{e}+4$ & 0.00 & (6) \\
\hline Ar & $\mathrm{Pd}$ & $0.6128 \mathrm{e}-1$ & $-0.2428 \mathrm{e}+0$ & $0.5979 \mathrm{e}+0$ & $0.1247 \mathrm{e}+1$ & $1.55391 \mathrm{e}+5$ & 0.00 & (6) \\
\hline $\mathrm{Kr}$ & $\mathrm{Pd}$ & $0.2691 \mathrm{e}-1$ & $-0.2048 \mathrm{e}+0$ & $0.2574 \mathrm{e}+1$ & $0.4935 \mathrm{e}+0$ & $4.43321 \mathrm{e}+5$ & 0.00 & (6) \\
\hline $\mathrm{Pd}$ & $\mathrm{Pd}$ & $-0.4757 e+1$ & $0.7461 \mathrm{e}-1$ & $0.6187 \mathrm{e}-4$ & $-0.1189 e+1$ & $6.59103 \mathrm{e}+5$ & 3.91 & (3) \\
\hline $\mathrm{H}$ & $\mathrm{Ag}$ & $0.3784 \mathrm{e}+0$ & $-0.1093 e+0$ & $0.5837 \mathrm{e}+0$ & $0.1496 \mathrm{e}+1$ & $5.46029 \mathrm{e}+3$ & 1.00 & (6) \\
\hline $\mathrm{D}$ & $\mathrm{Ag}$ & $0.3738 \mathrm{e}+0$ & $-0.1122 \mathrm{e}+0$ & $0.2918 \mathrm{e}+0$ & $0.1636 \mathrm{e}+1$ & $5.51044 \mathrm{e}+3$ & 1.00 & (6) \\
\hline $\mathrm{T}$ & $\mathrm{Ag}$ & $0.3723 e+0$ & $-0.1067 e+0$ & $0.2528 \mathrm{e}+0$ & $0.1631 \mathrm{e}+1$ & $5.56109 \mathrm{e}+3$ & 1.00 & (6) \\
\hline${ }^{3} \mathrm{He}$ & $\mathrm{Ag}$ & $0.3172 \mathrm{e}+0$ & $-0.1275 e+0$ & $0.3671 \mathrm{e}+0$ & $0.1597 \mathrm{e}+1$ & $1.13527 \mathrm{e}+4$ & 0.00 & (6) \\
\hline${ }^{4} \mathrm{He}$ & $\mathrm{Ag}$ & $0.3420 \mathrm{e}+0$ & $-0.1108 \mathrm{e}+0$ & $0.5202 \mathrm{e}+0$ & $0.1219 \mathrm{e}+1$ & $1.14531 \mathrm{e}+4$ & 0.00 & (6) \\
\hline $\mathrm{N}$ & $\mathrm{Ag}$ & $0.1698 \mathrm{e}+0$ & $-0.1776 \mathrm{e}+0$ & $0.3673 \mathrm{e}+0$ & $0.1335 \mathrm{e}+1$ & $4.66664 \mathrm{e}+4$ & 0.00 & (6) \\
\hline $\mathrm{O}$ & $\mathrm{Ag}$ & $0.1513 \mathrm{e}+0$ & $-0.1943 e+0$ & $0.3177 \mathrm{e}+0$ & $0.1357 \mathrm{e}+1$ & $5.47544 \mathrm{e}+4$ & 0.00 & (6) \\
\hline $\mathrm{Ne}$ & $\mathrm{Ag}$ & $0.1292 \mathrm{e}+0$ & $-0.1961 e+0$ & $0.3832 \mathrm{e}+0$ & $0.1373 \mathrm{e}+1$ & $7.20736 \mathrm{e}+4$ & 0.00 & (6) \\
\hline $\mathrm{Ar}$ & $\mathrm{Ag}$ & $0.6381 \mathrm{e}-1$ & $-0.2329 \mathrm{e}+0$ & $0.5690 \mathrm{e}+0$ & $0.1096 \mathrm{e}+1$ & $1.58921 \mathrm{e}+5$ & 0.00 & (6) \\
\hline $\mathrm{Kr}$ & $\mathrm{Ag}$ & $0.3432 \mathrm{e}-1$ & $-0.1768 e+0$ & $0.3846 \mathrm{e}+1$ & $0.5034 \mathrm{e}+0$ & $4.51993 e+5$ & 0.00 & (6) \\
\hline $\mathrm{Ag}$ & $\mathrm{Ag}$ & $-0.4981 e+1$ & $0.8713 \mathrm{e}-1$ & $0.1220 \mathrm{e}-3$ & $0.1090 \mathrm{e}+1$ & $6.93021 \mathrm{e}+5$ & 2.97 & (3) \\
\hline${ }^{4} \mathrm{He}$ & $\mathrm{Cd}$ & $0.3356 \mathrm{e}+0$ & $-0.1096 \mathrm{e}+0$ & $0.4336 \mathrm{e}+0$ & $0.1443 \mathrm{e}+1$ & $1.17532 \mathrm{e}+4$ & 0.00 & $(6)$ \\
\hline $\mathrm{Ne}$ & $\mathrm{Cd}$ & $0.1397 \mathrm{e}+0$ & $-0.1765 e+0$ & $0.4086 \mathrm{e}+0$ & $0.1428 \mathrm{e}+1$ & $7.35198 \mathrm{e}+4$ & 0.00 & (6) \\
\hline $\mathrm{Ar}$ & $\mathrm{Cd}$ & $0.7188 \mathrm{e}-1$ & $-0.2110 \mathrm{e}+0$ & $0.6731 \mathrm{e}+0$ & $0.1038 \mathrm{e}+1$ & $1.61276 \mathrm{e}+5$ & 0.00 & (6) \\
\hline $\mathrm{Kr}$ & $\mathrm{Cd}$ & $0.3854 \mathrm{e}+0$ & $0.2120 \mathrm{e}-1$ & $0.3098 \mathrm{e}+2$ & $0.4011 \mathrm{e}+0$ & $4.55218 \mathrm{e}+5$ & 0.00 & (6) \\
\hline $\mathrm{Cd}$ & $\mathrm{Cd}$ & $-0.4553 e+1$ & $0.7404 \mathrm{e}-1$ & $0.6364 \mathrm{e}-4$ & $-0.1063 e+1$ & $7.27916 \mathrm{e}+5$ & 1.16 & (3) \\
\hline $\mathrm{H}$ & In & $0.3791 \mathrm{e}+0$ & $-0.1003 e+0$ & $0.4605 \mathrm{e}+0$ & $0.1602 \mathrm{e}+1$ & $5.76339 \mathrm{e}+3$ & 1.00 & $(6)$ \\
\hline${ }^{4} \mathrm{He}$ & In & $0.3419 \mathrm{e}+0$ & $-0.1029 e+0$ & $0.4449 \mathrm{e}+0$ & $0.1485 \mathrm{e}+1$ & $1.20632 \mathrm{e}+4$ & 0.00 & (6) \\
\hline $\mathrm{Ne}$ & In & $0.1475 \mathrm{e}+0$ & $-0.1644 \mathrm{e}+0$ & $0.4808 \mathrm{e}+1$ & $0.1326 \mathrm{e}+1$ & $7.51927 \mathrm{e}+4$ & 0.00 & (6) \\
\hline $\mathrm{Kr}$ & In & $0.9177 \mathrm{e}-1$ & $-0.9345 \mathrm{e}-1$ & $0.6790 \mathrm{e}+1$ & $0.3656 \mathrm{e}+0$ & $4.62260 \mathrm{e}+5$ & 0.00 & (6) \\
\hline In & In & $-0.4284 e+1$ & $0.5576 \mathrm{e}-1$ & $0.7298 \mathrm{e}-4$ & $-0.1101 e+1$ & $7.63793 e+5$ & 2.49 & (3) \\
\hline $\mathrm{H}$ & $\mathrm{Sn}$ & $0.3720 \mathrm{e}+0$ & $-0.1016 \mathrm{e}+0$ & $0.4411 \mathrm{e}+0$ & $0.1655 \mathrm{e}+1$ & $5.91631 \mathrm{e}+3$ & 1.00 & $(6)$ \\
\hline D & $\mathrm{Sn}$ & $0.3993 \mathrm{e}+0$ & $-0.8973 \mathrm{e}-1$ & $0.3808 \mathrm{e}+0$ & $0.1641 \mathrm{e}+1$ & $5.96574 \mathrm{e}+3$ & 1.00 & (6) \\
\hline${ }^{3} \mathrm{He}$ & $\mathrm{Sn}$ & $0.3418 \mathrm{e}+0$ & $-0.1065 e+0$ & $0.4365 \mathrm{e}+0$ & $0.1599 \mathrm{e}+1$ & $1.22714 \mathrm{e}+4$ & 0.00 & (6) \\
\hline${ }^{4} \mathrm{He}$ & $\mathrm{Sn}$ & $0.3403 \mathrm{e}+0$ & $-0.1034 \mathrm{e}+0$ & $0.3933 \mathrm{e}+0$ & $0.1620 \mathrm{e}+1$ & $1.23702 \mathrm{e}+4$ & 0.00 & (6) \\
\hline $\mathrm{Ne}$ & $\mathrm{Sn}$ & $0.1515 \mathrm{e}+0$ & $-0.1604 \mathrm{e}+0$ & $0.4591 \mathrm{e}+0$ & $0.1288 \mathrm{e}+1$ & $7.67367 \mathrm{e}+4$ & 0.00 & (6) \\
\hline $\mathrm{Ar}$ & $\mathrm{Sn}$ & $0.7702 \mathrm{e}-1$ & $-0.2002 \mathrm{e}+0$ & $0.7862 \mathrm{e}+0$ & $0.1340 \mathrm{e}+1$ & $1.67157 \mathrm{e}+5$ & 0.00 & (6) \\
\hline $\mathrm{Kr}$ & $\mathrm{Sn}$ & $0.1156 \mathrm{e}+0$ & $-0.8240 \mathrm{e}-1$ & $0.7455 \mathrm{e}+1$ & $0.3466 \mathrm{e}+0$ & $4.66941 \mathrm{e}+5$ & 0.00 & (6) \\
\hline $\mathrm{Sn}$ & $\mathrm{Sn}$ & $-0.4302 \mathrm{e}+1$ & $0.5898 \mathrm{e}-1$ & $0.2231 \mathrm{e}-3$ & $-0.1010 \mathrm{e}+0$ & $8.00660 \mathrm{e}+5$ & 3.12 & (3) \\
\hline
\end{tabular}


Table 5: Constants, $a_{i}$, for the energy dependence of the particle reflection coefficient, $\mathrm{R}_{N}$, at normal incidence for different ions and targets. $\varepsilon_{L}$ is the scaling factor defined in (4), $\mathrm{E}_{\mathrm{sp}}$ the assumed surface binding energy of ions, and fit denotes the applied fitting formula.

\begin{tabular}{|c|c|c|c|c|c|c|c|c|}
\hline ion & solid & a1 & $\mathrm{a} 2$ & a3 & $\mathrm{a} 4$ & $\varepsilon_{L}$ & $\mathrm{E}_{\mathrm{sp}}$ & fit \\
\hline${ }^{4} \mathrm{He}$ & $\mathrm{Sb}$ & $0.3454 \mathrm{e}+0$ & $-0.9946 \mathrm{e}-1$ & $0.4299 \mathrm{e}+0$ & $0.1578 \mathrm{e}+1$ & $1.26821 \mathrm{e}+4$ & 0.00 & $\overline{(6)}$ \\
\hline $\mathrm{Ne}$ & $\mathrm{Sb}$ & $0.1632 \mathrm{e}+0$ & $-0.1467 \mathrm{e}+0$ & $0.5617 \mathrm{e}+0$ & $0.1218 \mathrm{e}+1$ & $7.83708 \mathrm{e}+4$ & 0.00 & (6) \\
\hline $\mathrm{Kr}$ & $\mathrm{Sb}$ & $0.4971 \mathrm{e}-1$ & $-0.1521 \mathrm{e}+0$ & $0.2691 \mathrm{e}+1$ & $0.4242 \mathrm{e}+0$ & $4.73059 \mathrm{e}+5$ & 0.00 & (6) \\
\hline${ }^{4} \mathrm{He}$ & $\mathrm{Te}$ & $0.3573 \mathrm{e}+0$ & $-0.9304 \mathrm{e}-1$ & $0.4711 \mathrm{e}+0$ & $0.1510 \mathrm{e}+1$ & $1.29871 \mathrm{e}+4$ & 0.00 & (6) \\
\hline $\mathrm{Ne}$ & $\mathrm{Te}$ & $0.1739 e+0$ & $-0.1372 \mathrm{e}-1$ & $0.6083 \mathrm{e}+0$ & $0.1133 \mathrm{e}+1$ & $7.97723 \mathrm{e}+4$ & 0.00 & (6) \\
\hline $\mathrm{Ar}$ & $\mathrm{Te}$ & $0.8910 \mathrm{e}-1$ & $-0.1785 e+0$ & $0.9047 \mathrm{e}+0$ & $0.1508 \mathrm{e}+1$ & $1.72282 \mathrm{e}+5$ & 0.00 & (6) \\
\hline $\mathrm{H}$ & $\mathrm{Tb}$ & $0.4373 \mathrm{e}+0$ & $-0.6849 \mathrm{e}-1$ & $0.9509 \mathrm{e}+0$ & $0.1449 \mathrm{e}+1$ & $8.32995 \mathrm{e}+3$ & 1.00 & $(6)$ \\
\hline${ }^{4} \mathrm{He}$ & $\mathrm{Tb}$ & $0.3729 \mathrm{e}+0$ & $-0.8952 e+0$ & $0.4794 \mathrm{e}+0$ & $0.1766 \mathrm{e}+1$ & $1.72593 \mathrm{e}+4$ & 0.00 & (6) \\
\hline Ar & $\mathrm{Tb}$ & $0.1172 \mathrm{e}+0$ & $-0.1570 \mathrm{e}+0$ & $0.9077 \mathrm{e}+0$ & $0.1369 \mathrm{e}+1$ & $2.15976 \mathrm{e}+5$ & 0.00 & (6) \\
\hline $\mathrm{H}$ & $\mathrm{Tm}$ & $0.4379 \mathrm{e}+0$ & $-0.7029 e-1$ & $0.9035 \mathrm{e}+0$ & $0.1339 \mathrm{e}+1$ & $9.00672 \mathrm{e}+3$ & 1.00 & $\overline{(6)}$ \\
\hline${ }^{4} \mathrm{He}$ & $\mathrm{Tm}$ & $0.3788 \mathrm{e}+0$ & $-0.8766 \mathrm{e}-1$ & $0.5249 \mathrm{e}+0$ & $0.1691 \mathrm{e}+1$ & $1.86300 \mathrm{e}+4$ & 0.00 & (6) \\
\hline $\mathrm{Ar}$ & $\mathrm{Tm}$ & $0.1210 \mathrm{e}+0$ & $-0.1582 \mathrm{e}+0$ & $0.1134 \mathrm{e}+1$ & $0.1765 \mathrm{e}+1$ & $2.29744 \mathrm{e}+5$ & 0.00 & (6) \\
\hline $\mathrm{H}$ & Hf & $0.4225 \mathrm{e}+0$ & $-0.7936 \mathrm{e}-1$ & $0.7331 \mathrm{e}+0$ & $0.1422 \mathrm{e}+1$ & $9.52208 \mathrm{e}+3$ & 1.00 & (6) \\
\hline${ }^{4} \mathrm{He}$ & $\mathrm{Hf}$ & $0.4143 e+0$ & $-0.7715 \mathrm{e}-1$ & $0.8910 \mathrm{e}+0$ & $0.1291 \mathrm{e}+1$ & $1.96695 \mathrm{e}+4$ & 0.00 & (6) \\
\hline $\mathrm{Ne}$ & $\mathrm{Hf}$ & $0.2044 \mathrm{e}+0$ & $-0.1348 \mathrm{e}+0$ & $0.5583 e+0$ & $0.1363 \mathrm{e}+1$ & $1.15397 \mathrm{e}+5$ & 0.00 & (6) \\
\hline $\mathrm{Ar}$ & $\mathrm{Hf}$ & $0.1208 \mathrm{e}+0$ & $-0.1692 \mathrm{e}+0$ & $0.8371 \mathrm{e}+0$ & $0.1779 \mathrm{e}+1$ & $2.39690 \mathrm{e}+5$ & 0.00 & (6) \\
\hline $\mathrm{Kr}$ & $\mathrm{Hf}$ & $0.5246 \mathrm{e}-1$ & $-0.2022 \mathrm{e}+0$ & $0.1818 \mathrm{e}+1$ & $0.1246 \mathrm{e}+1$ & $6.21788 \mathrm{e}+5$ & 0.00 & (6) \\
\hline $\mathrm{Xe}$ & $\mathrm{Hf}$ & $0.1982 \mathrm{e}+2$ & $0.3086 \mathrm{e}+0$ & $0.1019 \mathrm{e}+4$ & $0.5599 \mathrm{e}+0$ & $1.16579 \mathrm{e}+6$ & 0.00 & (6) \\
\hline $\mathrm{H}$ & $\mathrm{Ta}$ & $0.4144 \mathrm{e}+0$ & $-0.8540 \mathrm{e}-1$ & $0.7264 \mathrm{e}+0$ & $0.1360 \mathrm{e}+1$ & $9.69565 \mathrm{e}+3$ & 1.00 & (6) \\
\hline D & $\mathrm{Ta}$ & $0.4297 \mathrm{e}+0$ & $-0.8343 \mathrm{e}-1$ & $0.4641 \mathrm{e}+0$ & $0.1439 \mathrm{e}+1$ & $9.74893 \mathrm{e}+3$ & 1.00 & (6) \\
\hline${ }^{3} \mathrm{He}$ & $\mathrm{Ta}$ & $0.3661 \mathrm{e}+0$ & $-0.1042 \mathrm{e}+0$ & $0.5353 \mathrm{e}+0$ & $0.1640 \mathrm{e}+1$ & $1.99149 \mathrm{e}+4$ & 0.00 & (6) \\
\hline${ }^{4} \mathrm{He}$ & $\mathrm{Ta}$ & $0.3846 \mathrm{e}+0$ & $-0.9361 \mathrm{e}-1$ & $0.5882 \mathrm{e}+0$ & $0.1437 \mathrm{e}+1$ & $2.00210 \mathrm{e}+4$ & 0.00 & (6) \\
\hline $\mathrm{Ne}$ & $\mathrm{Ta}$ & $0.1960 \mathrm{e}+0$ & $-0.1467 \mathrm{e}+0$ & $0.5129 \mathrm{e}+0$ & $0.1566 \mathrm{e}+1$ & $1.17263 \mathrm{e}+5$ & 0.00 & (6) \\
\hline $\mathrm{Ar}$ & $\mathrm{Ta}$ & $0.1174 \mathrm{e}+0$ & $-0.1808 \mathrm{e}+0$ & $0.6117 \mathrm{e}+0$ & $0.1429 \mathrm{e}+1$ & $2.43225 \mathrm{e}+5$ & 0.00 & (6) \\
\hline $\mathrm{Kr}$ & $\mathrm{Ta}$ & $0.5706 \mathrm{e}-1$ & $-0.2013 e+0$ & $0.6372 \mathrm{e}+0$ & $0.5238 \mathrm{e}+0$ & $6.29462 \mathrm{e}+5$ & 0.00 & (6) \\
\hline $\mathrm{Xe}$ & $\mathrm{Ta}$ & $0.3601 \mathrm{e}+0$ & $0.1204 \mathrm{e}-1$ & $0.2805 \mathrm{e}+2$ & $0.3893 e+0$ & $1.17814 \mathrm{e}+6$ & 0.00 & (6) \\
\hline $\mathrm{Ta}$ & $\mathrm{Ta}$ & $-0.4091 \mathrm{e}+1$ & $0.4645 \mathrm{e}-1$ & $0.1126 \mathrm{e}-3$ & $-0.1081 \mathrm{e}+1$ & $1.93615 \mathrm{e}+6$ & 8.10 & (3) \\
\hline $\mathrm{H}$ & W & $0.4116 \mathrm{e}+0$ & $-0.9122 \mathrm{e}-1$ & $0.6956 \mathrm{e}+0$ & $0.1357 \mathrm{e}+1$ & $9.86986 \mathrm{e}+3$ & 1.00 & (6) \\
\hline $\mathrm{D}$ & W & $0.4453 \mathrm{e}+0$ & $-0.8079 \mathrm{e}-1$ & $0.5964 \mathrm{e}+0$ & $0.1344 \mathrm{e}+1$ & $9.92326 \mathrm{e}+3$ & 1.00 & (6) \\
\hline $\mathrm{T}$ & W & $0.4632 \mathrm{e}+0$ & $-0.6965 \mathrm{e}-1$ & $0.5252 \mathrm{e}+0$ & $0.1322 \mathrm{e}+1$ & $9.97718 \mathrm{e}+3$ & 1.00 & (6) \\
\hline${ }^{3} \mathrm{He}$ & W & $0.3744 \mathrm{e}+0$ & $-0.1023 e+0$ & $0.6106 \mathrm{e}+0$ & $0.1538 \mathrm{e}+1$ & $2.02666 \mathrm{e}+4$ & 0.00 & (6) \\
\hline${ }^{4} \mathrm{He}$ & W & $0.3769 \mathrm{e}+0$ & $-0.1006 e+0$ & $0.4862 \mathrm{e}+0$ & $0.1452 \mathrm{e}+1$ & $2.03728 \mathrm{e}+4$ & 0.00 & (6) \\
\hline $\mathrm{N}$ & W & $0.2595 \mathrm{e}+0$ & $-0.1199 \mathrm{e}+0$ & $0.5825 \mathrm{e}+0$ & $0.1119 \mathrm{e}+1$ & $7.90505 \mathrm{e}+4$ & 1.00 & (6) \\
\hline $\mathrm{Ne}$ & W & $0.1963 e+0$ & $-0.1504 \mathrm{e}+0$ & $0.4963 \mathrm{e}+0$ & $0.1560 \mathrm{e}+1$ & $1.19107 \mathrm{e}+5$ & 0.00 & (6) \\
\hline $\mathrm{Ar}$ & W & $0.1169 \mathrm{e}+0$ & $-0.1861 \mathrm{e}+0$ & $0.4054 \mathrm{e}+1$ & $0.3557 \mathrm{e}+1$ & $2.46646 \mathrm{e}+5$ & 0.00 & (6) \\
\hline $\mathrm{Kr}$ & W & $0.6283 \mathrm{e}-1$ & $-0.1960 \mathrm{e}+0$ & $0.7022 \mathrm{e}+0$ & $0.4645 \mathrm{e}+0$ & $6.36677 \mathrm{e}+5$ & 0.00 & (6) \\
\hline $\mathrm{Xe}$ & W & $0.1070 \mathrm{e}+0$ & $-0.9107 \mathrm{e}-1$ & $0.6633 e+1$ & $0.3337 \mathrm{e}+0$ & $1.18932 \mathrm{e}+6$ & 0.00 & (6) \\
\hline W & $\mathrm{W}$ & $-0.3685 e+1$ & $0.2780 \mathrm{e}-1$ & $0.7825 \mathrm{e}-4$ & $-0.1109 e+1$ & $1.99860 \mathrm{e}+6$ & 8.68 & (3) \\
\hline $\mathrm{H}$ & $\mathrm{Re}$ & $0.4170 \mathrm{e}+0$ & $-0.9100 \mathrm{e}-1$ & $0.8500 \mathrm{e}+0$ & $0.1345 \mathrm{e}+1$ & $1.00450 \mathrm{e}+4$ & 1.00 & (6) \\
\hline${ }^{4} \mathrm{He}$ & $\operatorname{Re}$ & $0.3836 \mathrm{e}+0$ & $-0.9754 \mathrm{e}-1$ & $0.5774 \mathrm{e}+0$ & $0.1387 \mathrm{e}+1$ & $2.07275 \mathrm{e}+4$ & 0.00 & (6) \\
\hline $\mathrm{Ne}$ & $\operatorname{Re}$ & $0.2017 \mathrm{e}+0$ & $-0.1495 \mathrm{e}+0$ & $0.5350 \mathrm{e}+0$ & $0.1576 \mathrm{e}+1$ & $1.20995 \mathrm{e}+5$ & 0.00 & (6) \\
\hline $\mathrm{Ar}$ & $\operatorname{Re}$ & $0.1184 \mathrm{e}+0$ & $-0.1873 e+0$ & $0.7853 e+0$ & $0.2021 \mathrm{e}+1$ & $2.50221 \mathrm{e}+5$ & 0.00 & (6) \\
\hline $\mathrm{Kr}$ & $\operatorname{Re}$ & $0.4376 \mathrm{e}+1$ & $0.1194 \mathrm{e}+0$ & $0.1041 \mathrm{e}+3$ & $0.4007 \mathrm{e}+0$ & $6.44513 \mathrm{e}+5$ & 0.00 & (6) \\
\hline $\mathrm{Xe}$ & $\mathrm{Re}$ & $0.1187 \mathrm{e}+1$ & $0.8035 \mathrm{e}-1$ & $0.7016 \mathrm{e}+2$ & $0.3971 \mathrm{e}+0$ & $1.20203 \mathrm{e}+6$ & 0.00 & (6) \\
\hline
\end{tabular}


Table 6: Constants, $a_{i}$, for the energy dependence of the particle reflection coefficient, $\mathrm{R}_{N}$, at normal incidence for different ions and targets. $\varepsilon_{L}$ is the scaling factor defined in (4), $\mathrm{E}_{\mathrm{sp}}$ the assumed surface binding energy of ions, and fit denotes the applied fitting formula.

\begin{tabular}{|c|c|c|c|c|c|c|c|c|}
\hline ion & solid & a1 & $\mathrm{a} 2$ & $\mathrm{a} 3$ & $\mathrm{a} 4$ & $\varepsilon_{L}$ & $\mathrm{E}_{\mathrm{sp}}$ & fir \\
\hline $\mathrm{H}$ & Os & $0.4162 \mathrm{e}+0$ & $-0.9212 \mathrm{e}-1$ & $0.8381 \mathrm{e}+0$ & $0.1336 \mathrm{e}+1$ & $1.02204 \mathrm{e}+4$ & 1.00 & $(6)$ \\
\hline${ }^{3} \mathrm{He}$ & Os & $0.3813 e+0$ & $-0.1012 \mathrm{e}+0$ & $0.6460 \mathrm{e}+0$ & $0.1384 \mathrm{e}+1$ & $2.09735 \mathrm{e}+4$ & 0.00 & $(6)$ \\
\hline${ }^{4} \mathrm{He}$ & Os & $0.3835 \mathrm{e}+0$ & $-0.9874 \mathrm{e}-1$ & $0.5731 \mathrm{e}+0$ & $0.1368 \mathrm{e}+1$ & $2.10799 \mathrm{e}+4$ & 0.00 & $(6)$ \\
\hline $\mathrm{Ne}$ & Os & $0.1988 \mathrm{e}+0$ & $-0.1541 \mathrm{e}+0$ & $0.5165 \mathrm{e}+0$ & $0.1682 \mathrm{e}+1$ & $1.22785 \mathrm{e}+5$ & 0.00 & $(6)$ \\
\hline $\mathrm{Ar}$ & Os & $0.1202 \mathrm{e}+0$ & $-0.1885 e+0$ & $0.2274 \mathrm{e}+1$ & $0.2910 \mathrm{e}+1$ & $2.53422 \mathrm{e}+5$ & 0.00 & $(6)$ \\
\hline $\mathrm{Kr}$ & Os & $0.1412 \mathrm{e}+2$ & $0.2126 \mathrm{e}+0$ & $0.3256 \mathrm{e}+3$ & $0.4844 \mathrm{e}+0$ & $6.50625 \mathrm{e}+5$ & 0.00 & (6) \\
\hline $\mathrm{Xe}$ & Os & $0.5161 \mathrm{e}+0$ & $0.1413 \mathrm{e}-1$ & $0.2977 \mathrm{e}+2$ & $0.3536 \mathrm{e}+0$ & $1.21043 \mathrm{e}+6$ & 0.00 & $(6)$ \\
\hline $\mathrm{H}$ & Ir & $0.4211 \mathrm{e}+0$ & $-0.8968 \mathrm{e}-1$ & $0.8681 \mathrm{e}+0$ & $0.1311 \mathrm{e}+1$ & $1.03972 \mathrm{e}+4$ & 1.00 & $(6)$ \\
\hline${ }^{3} \mathrm{He}$ & $\mathrm{Ir}$ & $0.3850 \mathrm{e}+0$ & $-0.9896 \mathrm{e}-1$ & $0.6598 \mathrm{e}+0$ & $0.1354 \mathrm{e}+1$ & $2.13313 \mathrm{e}+4$ & 0.00 & $(6)$ \\
\hline${ }^{4} \mathrm{He}$ & Ir & $0.3857 \mathrm{e}+0$ & $-0.9762 \mathrm{e}-1$ & $0.5725 \mathrm{e}+0$ & $0.1354 \mathrm{e}+1$ & $2.14384 \mathrm{e}+4$ & 0.00 & $(6)$ \\
\hline $\mathrm{Ne}$ & $\mathrm{Ir}$ & $0.1997 \mathrm{e}+0$ & $-0.1529 e+0$ & $0.4874 \mathrm{e}+0$ & $0.1738 \mathrm{e}+1$ & $1.24708 \mathrm{e}+5$ & 0.00 & $(6)$ \\
\hline $\mathrm{Ar}$ & $\mathrm{Ir}$ & $0.1207 \mathrm{e}+0$ & $-0.1873 e+0$ & $0.1018 \mathrm{e}+1$ & $0.2323 e+1$ & $2.57103 \mathrm{e}+5$ & 0.00 & $(6)$ \\
\hline $\mathrm{Kr}$ & Ir & $0.4040 \mathrm{e}+2$ & $0.3015 \mathrm{e}+0$ & $0.9167 \mathrm{e}+3$ & $0.5663 \mathrm{e}+0$ & $6.58875 \mathrm{e}+5$ & 0.00 & $(6)$ \\
\hline $\mathrm{Xe}$ & Ir & $0.1434 \mathrm{e}+0$ & $-0.7734 \mathrm{e}-1$ & $0.8545 \mathrm{e}+1$ & $0.3361 \mathrm{e}+0$ & $1.22412 \mathrm{e}+5$ & 0.00 & $(6)$ \\
\hline $\mathrm{H}$ & $\mathrm{Pt}$ & $0.4072 \mathrm{e}+0$ & $-0.9516 \mathrm{e}-1$ & $0.7048 \mathrm{e}+0$ & $0.1332 \mathrm{e}+1$ & $1.05744 \mathrm{e}+4$ & 1.00 & $(6)$ \\
\hline${ }^{3} \mathrm{He}$ & $\mathrm{Pt}$ & $0.3962 \mathrm{e}+0$ & $-0.9330 \mathrm{e}-1$ & $0.7038 \mathrm{e}+0$ & $0.1313 \mathrm{e}+1$ & $2.16890 \mathrm{e}+4$ & 0.00 & $(6)$ \\
\hline${ }^{4} \mathrm{He}$ & $\mathrm{Pt}$ & $0.4028 \mathrm{e}+0$ & $-0.8960 \mathrm{e}-1$ & $0.6573 \mathrm{e}+0$ & $0.1251 \mathrm{e}+1$ & $2.17963 \mathrm{e}+4$ & 0.00 & $(6)$ \\
\hline $\mathrm{O}$ & $\mathrm{Pt}$ & $0.2326 \mathrm{e}+0$ & $-0.1382 \mathrm{e}+0$ & $0.4461 \mathrm{e}+0$ & $0.1384 \mathrm{e}+1$ & $9.78987 \mathrm{e}+4$ & 0.00 & $(6)$ \\
\hline $\mathrm{Ne}$ & $\mathrm{Pt}$ & $0.2304 \mathrm{e}+0$ & $-0.1324 \mathrm{e}+0$ & $0.7012 \mathrm{e}+0$ & $0.1127 \mathrm{e}+1$ & $1.26583 \mathrm{e}+5$ & 0.00 & $(6)$ \\
\hline $\mathrm{Ar}$ & $\mathrm{Pt}$ & $0.1213 \mathrm{e}+0$ & $-0.1841 \mathrm{e}+0$ & $0.2170 \mathrm{e}+1$ & $0.3014 \mathrm{e}+1$ & $2.60590 \mathrm{e}+5$ & 0.00 & $(6)$ \\
\hline $\mathrm{Kr}$ & $\mathrm{Pt}$ & $0.7893 \mathrm{e}-1$ & $-0.1829 \mathrm{e}+0$ & $0.7645 \mathrm{e}+0$ & $0.2769 \mathrm{e}+0$ & $6.66218 \mathrm{e}+5$ & 0.00 & $(6)$ \\
\hline $\mathrm{Xe}$ & $\mathrm{Pt}$ & $0.7591 \mathrm{e}-1$ & $-0.1249 e+0$ & $0.3548 \mathrm{e}+1$ & $0.3242 \mathrm{e}+0$ & $1.23553 \mathrm{e}+6$ & 0.00 & $(6)$ \\
\hline $\mathrm{Pt}$ & $\mathrm{Pt}$ & $-0.4123 e+1$ & $0.4971 \mathrm{e}-1$ & $0.2489 \mathrm{e}-4$ & $-0.1185 e+1$ & $2.25981 \mathrm{e}+6$ & 5.86 & $(3)$ \\
\hline $\mathrm{H}$ & $\mathrm{Au}$ & $0.3124 \mathrm{e}+0$ & $-0.1165 e+0$ & $0.9679 \mathrm{e}+0$ & $0.1231 \mathrm{e}+1$ & $1.07527 \mathrm{e}+4$ & 0.00 & $(6)$ \\
\hline $\mathrm{D}$ & $\mathrm{Au}$ & $0.4476 \mathrm{e}+0$ & $-0.8110 \mathrm{e}-1$ & $0.5771 \mathrm{e}+0$ & $0.1367 \mathrm{e}+1$ & $1.08070 \mathrm{e}+4$ & 0.00 & $(6)$ \\
\hline $\mathrm{T}$ & $\mathrm{Au}$ & $0.4466 \mathrm{e}+0$ & $-0.7924 \mathrm{e}-1$ & $0.4805 \mathrm{e}+0$ & $0.1380 \mathrm{e}+1$ & $1.08618 \mathrm{e}+4$ & 0.00 & $(6)$ \\
\hline${ }^{3} \mathrm{He}$ & $\mathrm{Au}$ & $0.4088 \mathrm{e}+0$ & $-0.8632 \mathrm{e}-1$ & $0.7669 \mathrm{e}+0$ & $0.1204 \mathrm{e}+1$ & $2.20499 \mathrm{e}+4$ & 0.00 & $(6)$ \\
\hline${ }^{4} \mathrm{He}$ & $\mathrm{Au}$ & $0.4028 \mathrm{e}+0$ & $-0.8695 \mathrm{e}-1$ & $0.6487 \mathrm{e}+0$ & $0.1433 e+1$ & $2.21579 \mathrm{e}+4$ & 0.00 & $(6)$ \\
\hline $\mathrm{N}$ & $\mathrm{Au}$ & $0.2524 \mathrm{e}+0$ & $-0.1285 \mathrm{e}+0$ & $0.4891 \mathrm{e}+0$ & $0.1353 \mathrm{e}+1$ & $8.55290 \mathrm{e}+4$ & 0.00 & $(6)$ \\
\hline $\mathrm{O}$ & $\mathrm{Au}$ & $0.2403 \mathrm{e}+0$ & $-0.1306 \mathrm{e}+0$ & $0.5002 \mathrm{e}+2$ & $0.1411 \mathrm{e}+1$ & $9.94281 \mathrm{e}+4$ & 0.00 & $(6)$ \\
\hline $\mathrm{Ne}$ & $\mathrm{Au}$ & $0.2133 \mathrm{e}+0$ & $-0.1391 \mathrm{e}+0$ & $0.6149 \mathrm{e}+0$ & $0.1362 \mathrm{e}+1$ & $1.28526 \mathrm{e}+5$ & 0.00 & $(6)$ \\
\hline $\mathrm{Ar}$ & $\mathrm{Au}$ & $0.1337 \mathrm{e}+0$ & $-0.1681 \mathrm{e}+0$ & $0.5565 \mathrm{e}+0$ & $0.1060 \mathrm{e}+1$ & $2.64318 \mathrm{e}+5$ & 0.00 & $(6)$ \\
\hline $\mathrm{K}$ & $\mathrm{Au}$ & $0.1314 \mathrm{e}+0$ & $-0.1711 \mathrm{e}+0$ & $0.7177 \mathrm{e}+0$ & $0.1562 \mathrm{e}+1$ & $2.79384 \mathrm{e}+5$ & 0.00 & $(6)$ \\
\hline $\mathrm{Kr}$ & $\mathrm{Au}$ & $0.7483 \mathrm{e}-1$ & $-0.1860 e+0$ & $0.6550 \mathrm{e}+0$ & $0.2804 \mathrm{e}+0$ & $6.74618 \mathrm{e}+5$ & 0.00 & $(6)$ \\
\hline $\mathrm{Xe}$ & $\mathrm{Au}$ & $0.4026 \mathrm{e}+0$ & $0.6730 \mathrm{e}-2$ & $0.2244 \mathrm{e}+2$ & $0.3649 \mathrm{e}+1$ & $1.24955 \mathrm{e}+6$ & 0.00 & $(6)$ \\
\hline $\mathrm{Au}$ & $\mathrm{Au}$ & $-0.4390 e+1$ & $0.5187 \mathrm{e}-1$ & $0.5571 \mathrm{e}-5$ & $-0.1276 \mathrm{e}+1$ & $2.32799 \mathrm{e}+6$ & 3.80 & $(3)$ \\
\hline $\mathrm{Kr}$ & $\mathrm{Hg}$ & $0.5677 \mathrm{e}-1$ & $-0.1944 \mathrm{e}+0$ & $0.8225 \mathrm{e}+1$ & $0.2650 \mathrm{e}+1$ & $6.81273 \mathrm{e}+5$ & 0.00 & $(6)$ \\
\hline $\mathrm{Kr}$ & $\mathrm{Tl}$ & $0.6669 \mathrm{e}-1$ & $-0.1785 e+0$ & $0.4831 \mathrm{e}+1$ & $0.1695 \mathrm{e}+1$ & $6.87824 \mathrm{e}+5$ & 0.00 & $(6)$ \\
\hline $\mathrm{H}$ & $\mathrm{Pb}$ & $0.4371 \mathrm{e}+0$ & $-0.6936 \mathrm{e}-1$ & $0.8320 \mathrm{e}+0$ & $0.1414 \mathrm{e}+1$ & $1.12905 \mathrm{e}+4$ & 0.00 & $(6)$ \\
\hline${ }^{4} \mathrm{He}$ & $\mathrm{Pb}$ & $0.4748 \mathrm{e}+0$ & $-0.5502 \mathrm{e}-1$ & $0.9790 \mathrm{e}+0$ & $0.1014 \mathrm{e}+1$ & $2.32414 \mathrm{e}+4$ & 0.00 & $(6)$ \\
\hline $\mathrm{Ne}$ & $\mathrm{Pb}$ & $0.2479 \mathrm{e}+0$ & $-0.1054 \mathrm{e}+0$ & $0.6668 \mathrm{e}+0$ & $0.1018 \mathrm{e}+1$ & $1.34122 \mathrm{e}+5$ & 0.00 & $(6)$ \\
\hline $\mathrm{Ar}$ & $\mathrm{Pb}$ & $0.1529 \mathrm{e}+0$ & $-0.1369 \mathrm{e}+0$ & $0.8643 \mathrm{e}+0$ & $0.1421 \mathrm{e}+1$ & $2.74555 \mathrm{e}+5$ & 0.00 & $(6)$ \\
\hline $\mathrm{Kr}$ & $\mathrm{Pb}$ & $0.7082 \mathrm{e}-1$ & $-0.1710 \mathrm{e}+0$ & $0.2686 \mathrm{e}+1$ & $0.1328 \mathrm{e}+1$ & $6.95357 \mathrm{e}+5$ & 0.00 & $(6)$ \\
\hline $\mathrm{Xe}$ & $\mathrm{Pb}$ & $0.4056 \mathrm{e}-1$ & $-0.1698 \mathrm{e}+0$ & $0.6117 \mathrm{e}+1$ & $0.1095 \mathrm{e}+1$ & $1.28038 \mathrm{e}+6$ & 0.00 & $(6)$ \\
\hline $\mathrm{Pb}$ & $\mathrm{Pb}$ & $-0.3788 \mathrm{e}+1$ & $0.4264 \mathrm{e}-1$ & $0.4577 \mathrm{e}-4$ & $-0.1027 e+1$ & $2.53951 \mathrm{e}+5$ & 2.03 & $(3)$ \\
\hline
\end{tabular}


Table 7: Constants, $a_{i}$, for the energy dependence of the particle reflection coefficient, $\mathrm{R}_{N}$, at normal incidence for different ions and targets. $\varepsilon_{L}$ is the scaling factor defined in (4), $\mathrm{E}_{\mathrm{sp}}$ the assumed surface binding energy of ions, and fit denotes the applied fitting formula.

\begin{tabular}{|r|r|r|r|r|r|r|r|r|}
\hline ion & solid & $\mathrm{a} 1$ & $\mathrm{a} 2$ & $\mathrm{a} 3$ & $\mathrm{a} 4$ & $\varepsilon_{L}$ & $\mathrm{E}_{\mathrm{sp}}$ & fit \\
\hline $\mathrm{Ar}$ & $\mathrm{Bi}$ & $0.1580 \mathrm{e}+0$ & $-0.1281 \mathrm{e}+0$ & $0.1260 \mathrm{e}+1$ & $0.1429 \mathrm{e}+1$ & $2.78342 \mathrm{e}+5$ & 0.00 & $(6)$ \\
$\mathrm{Kr}$ & $\mathrm{Bi}$ & $0.7558 \mathrm{e}-1$ & $-0.1594 \mathrm{e}+0$ & $0.9118 \mathrm{e}+0$ & $0.8210 \mathrm{e}+1$ & $7.03904 \mathrm{e}+5$ & 0.00 & $(6)$ \\
\hline${ }^{4} \mathrm{He}$ & $\mathrm{Th}$ & $0.4315 \mathrm{e}+0$ & $-0.6896 \mathrm{e}-1$ & $0.7987 \mathrm{e}+0$ & $0.1544 \mathrm{e}+1$ & $2.61980 \mathrm{e}+4$ & 0.00 & $(6)$ \\
$\mathrm{Ne}$ & $\mathrm{Th}$ & $0.2744 \mathrm{e}+0$ & $-0.9120 \mathrm{e}-1$ & $0.8056 \mathrm{e}+0$ & $0.9902 \mathrm{e}+0$ & $1.49508 \mathrm{e}+5$ & 0.00 & $(6)$ \\
$\mathrm{Ar}$ & $\mathrm{Th}$ & $0.1681 \mathrm{e}+0$ & $-0.1252 \mathrm{e}+0$ & $0.1729 \mathrm{e}+1$ & $0.1867 \mathrm{e}+1$ & $3.02995 \mathrm{e}+5$ & 0.00 & $(6)$ \\
$\mathrm{Kr}$ & $\mathrm{Th}$ & $0.8508 \mathrm{e}-1$ & $-0.1556 \mathrm{e}+0$ & $0.6158 \mathrm{e}+1$ & $0.1763 \mathrm{e}+1$ & $7.54519 \mathrm{e}+5$ & 0.00 & $(6)$ \\
$\mathrm{Xe}$ & $\mathrm{Th}$ & $0.5468 \mathrm{e}-1$ & $-0.1552 \mathrm{e}+0$ & $0.1428 \mathrm{e}+1$ & $0.5013 \mathrm{e}+0$ & $1.37124 \mathrm{e}+6$ & 0.00 & $(6)$ \\
\hline $\mathrm{H}$ & $\mathrm{U}$ & $0.4361 \mathrm{e}+0$ & $-0.7379 \mathrm{e}-1$ & $0.9786 \mathrm{e}+0$ & $0.1550 \mathrm{e}+1$ & $1.31300 \mathrm{e}+4$ & 1.00 & $(6)$ \\
$\mathrm{D}$ & $\mathrm{U}$ & $0.5214 \mathrm{e}+0$ & $-0.4744 \mathrm{e}-1$ & $0.1357 \mathrm{e}+1$ & $0.1284 \mathrm{e}+1$ & $1.31849 \mathrm{e}+4$ & 1.00 & $(6)$ \\
\hline
\end{tabular}

Table 8: Constants for the energy dependence of the particle reflection coefficient at normal incidence up to high energies.

\begin{tabular}{|r|r|r|r|r|r|r|r|r|}
\hline ion & solid & $\mathrm{b} 1$ & $\mathrm{~b} 2$ & $\mathrm{~b} 3$ & $\mathrm{~b} 4$ & $\mathrm{~b} 5$ & $\mathrm{~b} 6$ & fit \\
\hline $\mathrm{H}$ & $\mathrm{Be}$ & $0.1652 \mathrm{e}+0$ & $-0.2991 \mathrm{e}+0$ & $0.1196 \mathrm{e}+0$ & $0.1374 \mathrm{e}+1$ & $0.4694 \mathrm{e}-4$ & $0.1476 \mathrm{e}+1$ & $(7)$ \\
$\mathrm{D}$ & $\mathrm{Be}$ & $0.9725 \mathrm{e}-1$ & $-0.2597 \mathrm{e}+0$ & $0.1623 \mathrm{e}+0$ & $0.1446 \mathrm{e}+1$ & $0.2505 \mathrm{e}+0$ & $0.4941 \mathrm{e}+0$ & $(7)$ \\
$\mathrm{T}$ & $\mathrm{Be}$ & $0.5283 \mathrm{e}-1$ & $-0.3846 \mathrm{e}+0$ & $0.1825 \mathrm{e}+0$ & $0.1171 \mathrm{e}+1$ & $0.6160 \mathrm{e}+2$ & $0.3601 \mathrm{e}-1$ & $(7)$ \\
${ }^{4} \mathrm{He}$ & $\mathrm{Be}$ & $0.3841 \mathrm{e}-1$ & $-0.4949 \mathrm{e}+0$ & $0.1886 \mathrm{e}+0$ & $0.1008 \mathrm{e}+1$ & & & $(6)$ \\
\hline $\mathrm{H}$ & $\mathrm{C}$ & $0.2016 \mathrm{e}+0$ & $-0.2404 \mathrm{e}+0$ & $0.1888 \mathrm{e}+0$ & $0.1392 \mathrm{e}+1$ & $0.2136 \mathrm{e}-2$ & $0.1082 \mathrm{e}+1$ & $(7)$ \\
$\mathrm{D}$ & $\mathrm{C}$ & $0.1375 \mathrm{e}+0$ & $-0.2648 \mathrm{e}+0$ & $0.1494 \mathrm{e}+0$ & $0.1467 \mathrm{e}+1$ & $0.6183 \mathrm{e}-1$ & $0.7212 \mathrm{e}+0$ & $(7)$ \\
$\mathrm{T}$ & $\mathrm{C}$ & $0.4854 \mathrm{e}-1$ & $-0.1796 \mathrm{e}+0$ & $0.2203 \mathrm{e}+0$ & $0.1280 \mathrm{e}+1$ & $0.4869 \mathrm{e}+2$ & $-0.3260 \mathrm{e}-1$ & $(7)$ \\
${ }^{4} \mathrm{He}$ & $\mathrm{C}$ & $0.2766 \mathrm{e}-1$ & $-0.3912 \mathrm{e}+0$ & $0.2510 \mathrm{e}+0$ & $0.1282 \mathrm{e}+1$ & $0.3560 \mathrm{e}+1$ & $0.6313 \mathrm{e}-1$ & $(7)$ \\
\hline $\mathrm{H}$ & $\mathrm{Nb}$ & $0.3702 \mathrm{e}+0$ & $-0.1076 \mathrm{e}+0$ & $0.5098 \mathrm{e}+0$ & $0.1771 \mathrm{e}+1$ & $0.8464 \mathrm{e}-2$ & $0.1375 \mathrm{e}+1$ & $(7)$ \\
\hline $\mathrm{H}$ & $\mathrm{W}$ & $0.2393 \mathrm{e}+0$ & $0.7644 \mathrm{e}-1$ & $0.8777 \mathrm{e}+0$ & $0.1444 \mathrm{e}+1$ & $0.7384 \mathrm{e}+1$ & $0.1545 \mathrm{e}+0$ & $(7)$ \\
$\mathrm{D}$ & $\mathrm{W}$ & $0.3246 \mathrm{e}+0$ & $-0.8766 \mathrm{e}-1$ & $0.5956 \mathrm{e}+0$ & $0.1356 \mathrm{e}+1$ & $0.3579 \mathrm{e}+1$ & $0.6475 \mathrm{e}-1$ & $(7)$ \\
$\mathrm{T}$ & $\mathrm{W}$ & $0.2408 \mathrm{e}+0$ & $0.4945 \mathrm{e}-1$ & $0.5626 \mathrm{e}+0$ & $0.1402 \mathrm{e}+1$ & $0.9493 \mathrm{e}+1$ & $-0.3282 \mathrm{e}-1$ & $(7)$ \\
${ }^{4} \mathrm{He}$ & $\mathrm{W}$ & $0.2970 \mathrm{e}+0$ & $-0.1087 \mathrm{e}+0$ & $0.4774 \mathrm{e}+0$ & $0.1477 \mathrm{e}+1$ & $0.9263 \mathrm{e}+1$ & $0.3485 \mathrm{e}-1$ & $(7)$ \\
\hline $\mathrm{H}$ & $\mathrm{Au}$ & $0.2654 \mathrm{e}+0$ & $-0.1620 \mathrm{e}+0$ & $0.3585 \mathrm{e}+0$ & $0.1600 \mathrm{e}+1$ & $0.5248 \mathrm{e}-2$ & $0.1410 \mathrm{e}+1$ & $(7)$ \\
${ }^{4} \mathrm{He}$ & $\mathrm{Au}$ & $0.3728 \mathrm{e}+0$ & $-0.1001 \mathrm{e}+0$ & $0.4304 \mathrm{e}+0$ & $0.1494 \mathrm{e}+1$ & $0.6009 \mathrm{e}-3$ & $0.1361 \mathrm{e}+1$ & $(7)$ \\
\hline
\end{tabular}


Table 9: Constants, $a_{i}$, for the energy dependence of the energy reflection coefficient, $\mathrm{R}_{E}$, at normal incidence for different ions and targets. $\varepsilon_{L}$ is the scaling factor defined in (4), $\mathrm{E}_{\mathrm{sp}}$ the assumed surface binding energy of ions, and fit denotes the applied fitting formula.

\begin{tabular}{|c|c|c|c|c|c|c|c|c|}
\hline ion & solid & a1 & $\mathrm{a} 2$ & a3 & $\mathrm{a} 4$ & $\varepsilon_{L}$ & $\mathrm{E}_{\mathrm{sp}}$ & fit \\
\hline $\mathrm{H}$ & $\mathrm{Li}$ & $0.7236 \mathrm{e}-1$ & $-0.1137 \mathrm{e}+0$ & $0.7950 \mathrm{e}+0$ & $0.1270 \mathrm{e}+1$ & $1.85375 \mathrm{e}+2$ & 1.00 & (6) \\
\hline $\mathrm{D}$ & $\mathrm{Li}$ & $0.2376 \mathrm{e}-1$ & $-0.1716 \mathrm{e}+0$ & $0.3565 \mathrm{e}+0$ & $0.1361 \mathrm{e}+1$ & $2.08692 \mathrm{e}+2$ & 1.00 & (6) \\
\hline $\mathrm{T}$ & $\mathrm{Li}$ & $0.1197 \mathrm{e}-1$ & $-0.1006 \mathrm{e}-1$ & $0.6932 e+0$ & $0.1358 \mathrm{e}+1$ & $2.32243 \mathrm{e}+2$ & 1.00 & (6) \\
\hline${ }^{4} \mathrm{He}$ & $\mathrm{Li}$ & $0.2949 \mathrm{e}-2$ & $-0.4038 e+0$ & $0.4047 \mathrm{e}+0$ & $0.1087 e+1$ & $5.56715 \mathrm{e}+2$ & 0.00 & (6) \\
\hline $\mathrm{Li}$ & $\mathrm{Li}$ & $-0.2977 \mathrm{e}+0$ & $-0.6949 e+0$ & $0.8814 \mathrm{e}+1$ & $0.1356 \mathrm{e}+0$ & $1.12841 \mathrm{e}+3$ & 1.67 & (3) \\
\hline $\mathrm{H}$ & $\mathrm{Be}$ & $0.7524 \mathrm{e}-1$ & $-0.2871 \mathrm{e}+0$ & $0.4301 \mathrm{e}+0$ & $0.1233 \mathrm{e}+1$ & $2.56510 \mathrm{e}+2$ & 1.00 & (6) \\
\hline $\mathrm{D}$ & $\mathrm{Be}$ & $0.3521 \mathrm{e}-1$ & $-0.3095 \mathrm{e}+0$ & $0.2794 \mathrm{e}+0$ & $0.1243 \mathrm{e}+1$ & $2.82110 \mathrm{e}+2$ & 1.00 & (6) \\
\hline $\mathrm{T}$ & $\mathrm{Be}$ & $0.1725 \mathrm{e}-1$ & $-0.2993 e+0$ & $0.2554 \mathrm{e}+0$ & $0.1250 \mathrm{e}+1$ & $3.07966 \mathrm{e}+2$ & 1.00 & (6) \\
\hline${ }^{3} \mathrm{He}$ & $\mathrm{Be}$ & $0.1364 \mathrm{e}-1$ & $-0.4511 \mathrm{e}+0$ & $0.4356 \mathrm{e}+0$ & $0.1003 \mathrm{e}+1$ & $6.65344 \mathrm{e}+2$ & 0.00 & (6) \\
\hline${ }^{4} \mathrm{He}$ & $\mathrm{Be}$ & $0.6307 \mathrm{e}-2$ & $-0.4862 e+0$ & $0.1618 \mathrm{e}+0$ & $0.1196 \mathrm{e}+1$ & $7.19545 \mathrm{e}+2$ & 0.00 & (6) \\
\hline $\mathrm{Be}$ & $\mathrm{Be}$ & $-0.2380 \mathrm{e}-1$ & $-0.1227 e+1$ & $0.9516 e+1$ & $0.9733 \mathrm{e}-1$ & $2.20796 \mathrm{e}+3$ & 3.38 & $(3)$ \\
\hline $\mathrm{H}$ & $\mathrm{B}$ & $0.8626 \mathrm{e}-1$ & $-0.2925 \mathrm{e}+0$ & $0.4545 \mathrm{e}+0$ & $0.1231 \mathrm{e}+1$ & $3.32864 \mathrm{e}+2$ & 1.00 & (6) \\
\hline $\mathrm{D}$ & B & $0.4416 \mathrm{e}-1$ & $-0.3108 \mathrm{e}+0$ & $0.2211 \mathrm{e}+0$ & $0.1279 \mathrm{e}+1$ & $3.61025 \mathrm{e}+2$ & 1.00 & (6) \\
\hline $\mathrm{T}$ & B & $0.2558 \mathrm{e}-1$ & $-0.3008 e+0$ & $0.2414 \mathrm{e}+0$ & $0.1272 \mathrm{e}+1$ & $3.89468 \mathrm{e}+2$ & 1.00 & (6) \\
\hline${ }^{4} \mathrm{He}$ & B & $0.1028 \mathrm{e}-1$ & $-0.4579 e+0$ & $0.1401 \mathrm{e}+0$ & $0.1274 \mathrm{e}+1$ & $8.94388 \mathrm{e}+2$ & 0.00 & (6) \\
\hline B & B & $-0.2087 e+0$ & $-0.7726 e+0$ & $0.9059 \mathrm{e}+1$ & $0.1246 \mathrm{e}+0$ & $3.71634 \mathrm{e}+3$ & 5.73 & (3) \\
\hline $\mathrm{H}$ & $\mathrm{C}$ & $0.8723 \mathrm{e}-1$ & $-0.2768 \mathrm{e}+0$ & $0.3988 \mathrm{e}+0$ & $0.1282 \mathrm{e}+1$ & $4.14659 \mathrm{e}+2$ & 1.00 & (6) \\
\hline $\mathrm{D}$ & $\mathrm{C}$ & $0.5142 \mathrm{e}-1$ & $-0.2714 \mathrm{e}+0$ & $0.2668 \mathrm{e}+0$ & $0.1316 \mathrm{e}+1$ & $4.46507 \mathrm{e}+2$ & 1.00 & (6) \\
\hline $\mathrm{T}$ & C & $0.2889 \mathrm{e}-1$ & $-0.2868 \mathrm{e}+0$ & $0.2312 \mathrm{e}+0$ & $0.1293 \mathrm{e}+1$ & $4.78673 \mathrm{e}+2$ & 1.00 & (6) \\
\hline${ }^{3} \mathrm{He}$ & $\mathrm{C}$ & $0.2273 \mathrm{e}-1$ & $-0.3971 \mathrm{e}+0$ & $0.2023 \mathrm{e}+0$ & $0.1279 \mathrm{e}+1$ & $1.02061 \mathrm{e}+2$ & 0.00 & (6) \\
\hline${ }^{4} \mathrm{He}$ & C & $0.1366 \mathrm{e}-1$ & $-0.3997 e+0$ & $0.2269 \mathrm{e}+0$ & $0.1192 \mathrm{e}+1$ & $1.08716 \mathrm{e}+2$ & 0.00 & (6) \\
\hline $\mathrm{C}$ & C & $-0.4218 \mathrm{e}-1$ & $-0.1036 e+1$ & $0.9620 \mathrm{e}+1$ & $0.9158 \mathrm{e}-1$ & $5.68684 \mathrm{e}+3$ & 7.41 & (3) \\
\hline $\mathrm{N}$ & $\mathrm{C}$ & $-0.1006 \mathrm{e}-2$ & $-0.1374 e+1$ & $0.1104 \mathrm{e}+2$ & $0.7621 \mathrm{e}-1$ & $7.37899 \mathrm{e}+3$ & 1.00 & (3) \\
\hline${ }^{4} \mathrm{He}$ & $\mathrm{Mg}$ & $0.4521 \mathrm{e}-1$ & $-0.2648 \mathrm{e}+0$ & $0.2346 \mathrm{e}+0$ & $0.1455 \mathrm{e}+1$ & $2.24482 \mathrm{e}+3$ & 0.00 & (6) \\
\hline $\mathrm{Ne}$ & $\mathrm{Mg}$ & $0.6791 \mathrm{e}-3$ & $-0.2856 \mathrm{e}+0$ & $0.1061 \mathrm{e}+1$ & $0.1158 \mathrm{e}+1$ & $2.12200 \mathrm{e}+4$ & 0.00 & (6) \\
\hline $\mathrm{Mg}$ & $\mathrm{Mg}$ & $-0.1054 \mathrm{e}-1$ & $-0.8353 e+0$ & $0.1018 \mathrm{e}+2$ & $0.9603 \mathrm{e}-1$ & $2.86599 \mathrm{e}+4$ & 1.54 & (3) \\
\hline $\mathrm{H}$ & $\mathrm{Al}$ & $1256 \mathrm{e}+0$ & $-0.2264 \mathrm{e}+0$ & $0.4590 \mathrm{e}+0$ & $0.1388 \mathrm{e}+1$ & $1.05916 \mathrm{e}+3$ & 1.00 & (6) \\
\hline $\mathrm{D}$ & $\mathrm{Al}$ & $0.1013 \mathrm{e}+0$ & $-0.2226 \mathrm{e}+0$ & $0.2563 \mathrm{e}+0$ & $0.1493 \mathrm{e}+1$ & $1.09700 \mathrm{e}+3$ & 1.00 & (6) \\
\hline $\mathrm{T}$ & $\mathrm{Al}$ & $0.7990 \mathrm{e}-1$ & $-0.2188 \mathrm{e}+0$ & $0.2031 \mathrm{e}+0$ & $0.1505 \mathrm{e}+1$ & $1.13522 \mathrm{e}+3$ & 1.00 & (6) \\
\hline${ }^{3} \mathrm{He}$ & $\mathrm{Al}$ & $0.6284 \mathrm{e}-1$ & $-0.2751 \mathrm{e}+0$ & $0.2744 \mathrm{e}+0$ & $0.1424 \mathrm{e}+1$ & $2.37037 \mathrm{e}+3$ & 0.00 & (6) \\
\hline${ }^{4} \mathrm{He}$ & $\mathrm{Al}$ & $0.5131 \mathrm{e}-1$ & $-0.2755 \mathrm{e}+0$ & $0.2707 \mathrm{e}+0$ & $0.1323 \mathrm{e}+1$ & $2.44780 \mathrm{e}+3$ & 0.00 & (6) \\
\hline $\mathrm{N}$ & $\mathrm{Al}$ & $-0.2723 \mathrm{e}-2$ & $-0.8990 \mathrm{e}+0$ & $0.6208 \mathrm{e}+1$ & $0.1111 \mathrm{e}+0$ & $1.28804 \mathrm{e}+4$ & 1.00 & (3) \\
\hline $\mathrm{Ne}$ & $\mathrm{Al}$ & $0.1567 \mathrm{e}-2$ & $-0.2403 e+0$ & $0.2060 \mathrm{e}+1$ & $0.9378 \mathrm{e}+0$ & $2.22732 \mathrm{e}+4$ & 1.00 & (6) \\
\hline $\mathrm{Al}$ & $\mathrm{Al}$ & $-0.8594 e+1$ & $0.5963 \mathrm{e}-1$ & $0.1455 \mathrm{e}-2$ & $-0.1161 e+1$ & $3.45451 \mathrm{e}+4$ & 2.70 & (3) \\
\hline $\mathrm{H}$ & $\mathrm{Si}$ & $0.1220 \mathrm{e}+0$ & $-0.2272 \mathrm{e}+0$ & $0.4110 \mathrm{e}+0$ & $0.1425 \mathrm{e}+1$ & $1.16317 \mathrm{e}+3$ & 1.00 & (6) \\
\hline $\mathrm{D}$ & $\mathrm{Si}$ & $0.1038 \mathrm{e}+0$ & $-0.2114 \mathrm{e}+0$ & $0.2525 \mathrm{e}+0$ & $0.1526 \mathrm{e}+1$ & $1.20314 \mathrm{e}+3$ & 1.00 & (6) \\
\hline $\mathrm{T}$ & $\mathrm{Si}$ & $0.8495 \mathrm{e}-1$ & $-0.2004 \mathrm{e}+0$ & $0.2207 \mathrm{e}+0$ & $0.1525 \mathrm{e}+1$ & $1.24352 \mathrm{e}+3$ & 1.00 & (6) \\
\hline${ }^{3} \mathrm{He}$ & $\mathrm{Si}$ & $0.6682 \mathrm{e}-1$ & $-0.2544 e+0$ & $0.3073 e+0$ & $0.1416 \mathrm{e}+1$ & $2.59209 \mathrm{e}+3$ & 0.00 & (6) \\
\hline${ }^{4} \mathrm{He}$ & $\mathrm{Si}$ & $0.5765 \mathrm{e}-1$ & $-0.2420 \mathrm{e}+0$ & $0.3618 \mathrm{e}+0$ & $0.1319 \mathrm{e}+1$ & $2.67374 \mathrm{e}+3$ & 0.00 & (6) \\
\hline $\mathrm{N}$ & $\mathrm{Si}$ & $-0.5303 e-1$ & $-0.5410 \mathrm{e}+1$ & $0.5943 e+1$ & $0.1234 \mathrm{e}+0$ & $1.38909 \mathrm{e}+4$ & 0.00 & (3) \\
\hline $\mathrm{Ne}$ & $\mathrm{Si}$ & $0.1461 \mathrm{e}-2$ & $-0.2798 e+0$ & $0.1187 \mathrm{e}+1$ & $0.1065 \mathrm{e}+1$ & $2.39034 \mathrm{e}+4$ & 0.00 & (6) \\
\hline $\mathrm{Si}$ & $\mathrm{Si}$ & $-0.8631 e+1$ & $0.6888 \mathrm{e}-1$ & $0.1808 \mathrm{e}-1$ & $-0.8577 \mathrm{e}-0$ & $4.10661 \mathrm{e}+4$ & 4.70 & (3) \\
\hline $\mathrm{Ca}$ & $\mathrm{Ca}$ & $-0.8313 e+1$ & $0.5317 \mathrm{e}-1$ & $0.23732-2$ & $-0.9080 \mathrm{e}+0$ & $9.43891 \mathrm{e}+4$ & 1.83 & (3) \\
\hline $\mathrm{Sc}$ & $\mathrm{Sc}$ & $-0.8427 e+1$ & $0.6222 \mathrm{e}-1$ & $0.77062-2$ & $-0.8173 e+0$ & $1.05770 \mathrm{e}+5$ & 3.49 & (3) \\
\hline
\end{tabular}


Table 10: Constants, $a_{i}$, for the energy dependence of the energy reflection coefficient, $\mathrm{R}_{E}$, at normal incidence for different ions and targets. $\varepsilon_{L}$ is the scaling factor defined in (4), $\mathrm{E}_{\mathrm{sp}}$ the assumed surface binding energy of ions, and fit denotes the applied fitting formula.

\begin{tabular}{|c|c|c|c|c|c|c|c|c|}
\hline ion & solid & a1 & $\mathrm{a} 2$ & a3 & $\mathrm{a} 4$ & $\varepsilon$ & $\mathrm{E}_{\mathrm{sp}}$ & fit \\
\hline $\mathrm{H}$ & $\mathrm{Ti}$ & $0.1414 \mathrm{e}+0$ & $-0.2176 \mathrm{e}+0$ & $0.4518 \mathrm{e}+0$ & $0.1477 \mathrm{e}+1$ & $2.05415 \mathrm{e}+3$ & 1.00 & (6) \\
\hline $\mathrm{D}$ & $\mathrm{Ti}$ & $0.1333 \mathrm{e}+0$ & $-0.2097 \mathrm{e}+0$ & $0.2613 \mathrm{e}+0$ & $0.1565 \mathrm{e}+1$ & $2.09615 \mathrm{e}+3$ & 1.00 & (6) \\
\hline $\mathrm{T}$ & $\mathrm{Ti}$ & $0.1223 \mathrm{e}+0$ & $-0.1921 e+0$ & $0.2107 \mathrm{e}+0$ & $0.1634 \mathrm{e}+1$ & $2.13856 \mathrm{e}+3$ & 1.00 & (6) \\
\hline${ }^{3} \mathrm{He}$ & $\mathrm{Ti}$ & $0.9974 \mathrm{e}-1$ & $-0.2204 \mathrm{e}+0$ & $0.3307 \mathrm{e}+0$ & $0.1575 \mathrm{e}+1$ & $4.41677 \mathrm{e}+3$ & 0.00 & (6) \\
\hline${ }^{4} \mathrm{He}$ & $\mathrm{Ti}$ & $0.8430 \mathrm{e}-1$ & $-0.2355 \mathrm{e}+0$ & $0.2301 \mathrm{e}+0$ & $0.1626 \mathrm{e}+1$ & $4.50177 \mathrm{e}+3$ & 0.00 & (6) \\
\hline $\mathrm{N}$ & $\mathrm{Ti}$ & $-0.3464 e+1$ & $0.1110 \mathrm{e}+0$ & $0.3487 \mathrm{e}+0$ & $0.8846 \mathrm{e}+0$ & $2.07557 \mathrm{e}+4$ & 1.00 & (3) \\
\hline $\mathrm{Ne}$ & $\mathrm{Ti}$ & $0.6908 \mathrm{e}-2$ & $-0.3015 e+0$ & $0.3614 \mathrm{e}+0$ & $0.1163 e+1$ & $3.39688 \mathrm{e}+4$ & 0.00 & (6) \\
\hline $\mathrm{Ar}$ & $\mathrm{Ti}$ & $0.1823 e+0$ & $0.3667 \mathrm{e}+0$ & $0.5288 \mathrm{e}+3$ & $0.8020 \mathrm{e}+0$ & $8.56428 \mathrm{e}+4$ & 0.00 & (6) \\
\hline $\mathrm{Ti}$ & $\mathrm{Ti}$ & $-0.8274 \mathrm{e}+1$ & $0.5806 \mathrm{e}-1$ & $0.1474 \mathrm{e}-1$ & $-0.7557 \mathrm{e}+0$ & $1.17898 \mathrm{e}+5$ & 4.52 & (3) \\
\hline $\mathrm{H}$ & $\mathrm{V}$ & $0.1448 \mathrm{e}+0$ & $-0.2239 \mathrm{e}+0$ & $0.4940 \mathrm{e}+0$ & $0.1439 \mathrm{e}+1$ & $2.17329 \mathrm{e}+3$ & 1.00 & (6) \\
\hline $\mathrm{D}$ & $\mathrm{V}$ & $0.1399 \mathrm{e}+0$ & $-0.2144 e+0$ & $0.2935 \mathrm{e}+0$ & $0.1535 \mathrm{e}+1$ & $2.21513 e+3$ & 1.00 & (6) \\
\hline $\mathrm{T}$ & V & $0.1286 \mathrm{e}+0$ & $-0.1960 e+0$ & $0.2356 \mathrm{e}+0$ & $0.1580 \mathrm{e}+1$ & $2.25738 \mathrm{e}+3$ & 1.00 & (6) \\
\hline${ }^{3} \mathrm{He}$ & $\mathrm{V}$ & $0.9741 \mathrm{e}-1$ & $-0.2463 e+0$ & $0.2711 \mathrm{e}+0$ & $0.1625 \mathrm{e}+1$ & $4.65839 \mathrm{e}+3$ & 0.00 & (6) \\
\hline${ }^{4} \mathrm{He}$ & $\mathrm{V}$ & $0.8590 \mathrm{e}-1$ & $-0.2486 e+0$ & $0.2007 \mathrm{e}+0$ & $0.1682 \mathrm{e}+1$ & $4.74299 \mathrm{e}+3$ & 0.00 & (6) \\
\hline $\mathrm{N}$ & $\mathrm{V}$ & $0.2776 \mathrm{e}-1$ & $-0.2080 e+0$ & $0.1013 \mathrm{e}+1$ & $0.9532 \mathrm{e}+0$ & $2.16246 \mathrm{e}+4$ & 1.00 & (6) \\
\hline $\mathrm{Ne}$ & $\mathrm{V}$ & $0.1090 \mathrm{e}-1$ & $-0.2601 e+0$ & $0.7915 \mathrm{e}+0$ & $0.8454 \mathrm{e}+0$ & $3.52128 \mathrm{e}+4$ & 0.00 & (6) \\
\hline Ar & $\mathrm{V}$ & $0.4145 \mathrm{e}+1$ & $0.6620 \mathrm{e}+0$ & $0.8285 \mathrm{e}+4$ & $0.1081 \mathrm{e}+1$ & $8.78018 e+4$ & 0.00 & (6) \\
\hline $\mathrm{V}$ & $\mathrm{V}$ & $-0.7523 e+1$ & $0.2900 \mathrm{e}-1$ & $0.3610 \mathrm{e}-3$ & $-0.1196 e+1$ & $1.30783 \mathrm{e}+5$ & 5.33 & (3) \\
\hline${ }^{4} \mathrm{He}$ & $\mathrm{Cr}$ & $0.8632 \mathrm{e}-1$ & $-0.2572 \mathrm{e}+0$ & $0.1938 \mathrm{e}+0$ & $0.1682 \mathrm{e}+1$ & $5.00096 \mathrm{e}+3$ & 0.00 & (6) \\
\hline $\mathrm{Ne}$ & $\mathrm{Cr}$ & $0.1448 \mathrm{e}-1$ & $-0.2218 \mathrm{e}+0$ & $0.1393 \mathrm{e}+1$ & $0.7045 \mathrm{e}+0$ & $3.68638 \mathrm{e}+4$ & 0.00 & (6) \\
\hline $\mathrm{Ar}$ & $\mathrm{Cr}$ & $0.1186 \mathrm{e}+2$ & $0.8283 e+0$ & $0.1993 \mathrm{e}+5$ & $0.1230 \mathrm{e}+1$ & $9.15022 \mathrm{e}+4$ & 0.00 & (6) \\
\hline $\mathrm{Cr}$ & $\mathrm{Cr}$ & $-0.8251 \mathrm{e}+1$ & $0.4711 \mathrm{e}-1$ & $0.4295 \mathrm{e}-4$ & $-0.1448 e+1$ & $1.44437 \mathrm{e}+5$ & 4.12 & (3) \\
\hline${ }^{4} \mathrm{He}$ & $\mathrm{Mn}$ & $0.9222 \mathrm{e}-1$ & $-0.2463 e+0$ & $0.2481 \mathrm{e}+0$ & $0.1584 \mathrm{e}+1$ & $5.24919 \mathrm{e}+3$ & 0.00 & (6) \\
\hline $\mathrm{Ne}$ & $\mathrm{Mn}$ & $0.1427 \mathrm{e}-1$ & $-0.2371 \mathrm{e}+0$ & $0.9776 \mathrm{e}+0$ & $0.8487 \mathrm{e}+0$ & $3.81587 \mathrm{e}+4$ & 0.00 & (6) \\
\hline $\mathrm{H}$ & $\mathrm{Fe}$ & $0.1600 \mathrm{e}+0$ & $-0.2061 \mathrm{e}+0$ & $0.6614 \mathrm{e}+0$ & $0.1323 \mathrm{e}+1$ & $2.54382 \mathrm{e}+3$ & 1.00 & (6) \\
\hline $\mathrm{D}$ & $\mathrm{Fe}$ & $0.1536 \mathrm{e}+0$ & $-0.1972 \mathrm{e}+0$ & $0.4025 \mathrm{e}+0$ & $0.1419 \mathrm{e}+1$ & $2.58856 \mathrm{e}+3$ & 1.00 & (6) \\
\hline $\mathrm{T}$ & $\mathrm{Fe}$ & $0.1392 \mathrm{e}+0$ & $-0.1922 \mathrm{e}+0$ & $0.3008 \mathrm{e}+0$ & $0.1451 \mathrm{e}+1$ & $2.67374 \mathrm{e}+3$ & 1.00 & (6) \\
\hline${ }^{4} \mathrm{He}$ & $\mathrm{Fe}$ & $0.9012 \mathrm{e}-1$ & $-0.2536 e+0$ & $0.2304 \mathrm{e}+0$ & $0.1589 \mathrm{e}+1$ & $5.51371 \mathrm{e}+3$ & 1.00 & (6) \\
\hline $\mathrm{Ne}$ & $\mathrm{Fe}$ & $0.1921 \mathrm{e}+1$ & $0.3156 \mathrm{e}+0$ & $0.2839 \mathrm{e}+3$ & $0.7636 \mathrm{e}+0$ & $3.98491 \mathrm{e}+4$ & 0.00 & (6) \\
\hline Ar & $\mathrm{Fe}$ & $0.6061 \mathrm{e}+1$ & $0.7176 \mathrm{e}+0$ & $0.8568 \mathrm{e}+4$ & $0.1151 \mathrm{e}+1$ & $9.75914 \mathrm{e}+4$ & 0.00 & (6) \\
\hline $\mathrm{Fe}$ & $\mathrm{Fe}$ & $-0.7670 e+1$ & $0.2862 \mathrm{e}-1$ & $0.5824 \mathrm{e}-5$ & $-0.1642 e+1$ & $1.74096 \mathrm{e}+5$ & 4.34 & (3) \\
\hline${ }^{4} \mathrm{He}$ & $\mathrm{Co}$ & $0.9483 \mathrm{e}-1$ & $-0.2480 \mathrm{e}+0$ & $0.2261 \mathrm{e}+0$ & $0.1562 \mathrm{e}+1$ & $5.76700 \mathrm{e}+3$ & 0.00 & (6) \\
\hline $\mathrm{Ne}$ & $\mathrm{Co}$ & $0.4225 \mathrm{e}+0$ & $0.1330 \mathrm{e}+0$ & $0.5318 \mathrm{e}+2$ & $0.6079 \mathrm{e}+0$ & $4.11463 \mathrm{e}+4$ & 0.00 & (6) \\
\hline $\mathrm{Ar}$ & $\mathrm{Co}$ & $0.2715 \mathrm{e}+2$ & $0.8647 \mathrm{e}+0$ & $0.2593 \mathrm{e}+5$ & $0.1267 \mathrm{e}+1$ & $9.98420 \mathrm{e}+4$ & 0.00 & (6) \\
\hline $\mathrm{H}$ & $\mathrm{Ni}$ & $0.1583 \mathrm{e}+0$ & $-0.2109 e+0$ & $0.6354 \mathrm{e}+0$ & $0.1334 \mathrm{e}+1$ & $2.79866 \mathrm{e}+3$ & 1.00 & (6) \\
\hline $\mathrm{D}$ & $\mathrm{Ni}$ & $0.1575 \mathrm{e}+0$ & $-0.1958 e+0$ & $0.4189 \mathrm{e}+0$ & $0.1411 \mathrm{e}+1$ & $2.84552 \mathrm{e}+3$ & 1.00 & (6) \\
\hline $\mathrm{T}$ & $\mathrm{Ni}$ & $0.1420 \mathrm{e}+0$ & $-0.1946 e+0$ & $0.3094 \mathrm{e}+0$ & $0.1445 \mathrm{e}+1$ & $2.89285 \mathrm{e}+3$ & 1.00 & (6) \\
\hline${ }^{4} \mathrm{He}$ & $\mathrm{Ni}$ & $0.9909 \mathrm{e}-1$ & $-0.2411 \mathrm{e}+0$ & $0.2935 \mathrm{e}+0$ & $0.1489 \mathrm{e}+1$ & $6.04409 e+3$ & 0.00 & (6) \\
\hline $\mathrm{N}$ & $\mathrm{Ni}$ & $0.5707 \mathrm{e}-1$ & $-0.1374 e+0$ & $0.2561 \mathrm{e}+1$ & $0.6596 \mathrm{e}+0$ & $2.67793 \mathrm{e}+4$ & 0.00 & (6) \\
\hline $\mathrm{O}$ & $\mathrm{Ni}$ & $0.2126 \mathrm{e}-1$ & $-0.2661 e+0$ & $0.4616 \mathrm{e}+1$ & $0.1179 \mathrm{e}+1$ & $3.18555 \mathrm{e}+4$ & 0.00 & (6) \\
\hline $\mathrm{Ne}$ & $\mathrm{Ni}$ & $0.8377 \mathrm{e}+2$ & $0.7376 \mathrm{e}+0$ & $0.1090 \mathrm{e}+5$ & $0.1144 \mathrm{e}+1$ & $4.30554 \mathrm{e}+4$ & 0.00 & (6) \\
\hline $\mathrm{Ar}$ & $\mathrm{Ni}$ & $0.4472 \mathrm{e}+2$ & $0.9162 \mathrm{e}+0$ & $0.4521 \mathrm{e}+5$ & $0.1320 \mathrm{e}+1$ & $1.04416 \mathrm{e}+5$ & 0.00 & (6) \\
\hline $\mathrm{Ni}$ & $\mathrm{Ni}$ & $-0.7885 e+1$ & $0.3614 \mathrm{e}-1$ & $0.7097 \mathrm{e}-5$ & $-0.1601 e+1$ & $2.06960 \mathrm{e}+5$ & 4.46 & (3) \\
\hline $\mathrm{H}$ & $\mathrm{Cu}$ & $0.1561 \mathrm{e}+0$ & $-0.2199 \mathrm{e}+0$ & $0.6065 \mathrm{e}+0$ & $0.1338 \mathrm{e}+1$ & $2.92563 \mathrm{e}+3$ & 1.00 & (6) \\
\hline $\mathrm{T}$ & $\mathrm{Cu}$ & $0.1448 \mathrm{e}+0$ & $-0.1952 e+0$ & $0.2790 \mathrm{e}+0$ & $0.1495 \mathrm{e}+1$ & $3.01673 e+3$ & 1.00 & (6) \\
\hline${ }^{4} \mathrm{He}$ & $\mathrm{Cu}$ & $0.1092 \mathrm{e}+0$ & $-0.2251 \mathrm{e}+0$ & $0.4144 \mathrm{e}+0$ & $0.1338 \mathrm{e}+1$ & $6.29218 \mathrm{e}+3$ & 0.00 & (6) \\
\hline $\mathrm{N}$ & $\mathrm{Cu}$ & $0.3587 \mathrm{e}-1$ & $-0.2295 \mathrm{e}+0$ & $0.8060 \mathrm{e}+0$ & $0.9223 \mathrm{e}+0$ & $2.75601 \mathrm{e}+4$ & 1.00 & (6) \\
\hline $\mathrm{Ne}$ & $\mathrm{Cu}$ & $0.1585 \mathrm{e}-1$ & $-0.2661 e+0$ & $0.4612 \mathrm{e}+0$ & $0.1258 \mathrm{e}+1$ & $4.40689 \mathrm{e}+4$ & 0.00 & (6) \\
\hline $\mathrm{Ar}$ & $\mathrm{Cu}$ & $0.1465 \mathrm{e}-2$ & $-0.3890 e+0$ & $0.2444 \mathrm{e}-4$ & $-0.1283 e+1$ & $1.05525 \mathrm{e}+5$ & 0.00 & (6) \\
\hline $\mathrm{Cu}$ & $\mathrm{Cu}$ & $-0.9043 e+1$ & $0.6704 \mathrm{e}-1$ & $0.1451 \mathrm{e}-3$ & $-0.1221 \mathrm{e}+1$ & $2.24619 \mathrm{e}+5$ & 3.52 & (3) \\
\hline
\end{tabular}


Table 11: Constants, $a_{i}$, for the energy dependence of the energy reflection coefficient, $\mathrm{R}_{E}$, at normal incidence for different ions and targets. $\varepsilon_{L}$ is the scaling factor defined in (4), $\mathrm{E}_{\mathrm{sp}}$ the assumed surface binding energy of ions, and fit denotes the applied fitting formula.

\begin{tabular}{|c|c|c|c|c|c|c|c|c|}
\hline ion & solid & a1 & a2 & a3 & $\mathrm{a} 4$ & $\varepsilon$ & $\mathrm{E}_{\mathrm{sp}}$ & fit \\
\hline${ }^{4} \mathrm{He}$ & $\mathrm{Zn}$ & $0.1150 \mathrm{e}+0$ & $-0.2039 \mathrm{e}+0$ & $0.4768 \mathrm{e}+0$ & $0.1326 \mathrm{e}+1$ & $6.56163 \mathrm{e}+3$ & 0.00 & (6) \\
\hline $\mathrm{Ne}$ & $\mathrm{Zn}$ & $0.1622 \mathrm{e}-1$ & $-0.2629 \mathrm{e}+0$ & $0.4247 \mathrm{e}+0$ & $0.1293 \mathrm{e}+1$ & $4.56265 \mathrm{e}+4$ & 0.00 & (6) \\
\hline Ar & $\mathrm{Zn}$ & $0.3259 \mathrm{e}+2$ & $0.7647 \mathrm{e}+0$ & $0.1960 \mathrm{e}+5$ & $0.1134 \mathrm{e}+1$ & $1.08696 \mathrm{e}+5$ & 0.00 & (6) \\
\hline $\mathrm{Zn}$ & $\mathrm{Zn}$ & $-0.8006 e+1$ & $0.4300 \mathrm{e}-1$ & $0.1508 \mathrm{e}-4$ & $-0.1343 e+1$ & $2.43109 \mathrm{e}+5$ & 1.35 & (3) \\
\hline $\mathrm{D}$ & $\mathrm{Ga}$ & $0.1635 \mathrm{e}+0$ & $-0.1760 \mathrm{e}+0$ & $0.3674 \mathrm{e}+0$ & $0.1591 \mathrm{e}+1$ & $3.23166 \mathrm{e}+3$ & 1.00 & (6) \\
\hline $\mathrm{T}$ & $\mathrm{Ga}$ & $0.1572 \mathrm{e}+0$ & $-0.1625 e+0$ & $0.2962 \mathrm{e}+0$ & $0.1636 \mathrm{e}+1$ & $3.27716 \mathrm{e}+3$ & 1.00 & (6) \\
\hline${ }^{4} \mathrm{He}$ & $\mathrm{Ga}$ & $0.1294 \mathrm{e}+0$ & $-0.1673 e+0$ & $0.5998 \mathrm{e}+0$ & $0.1312 \mathrm{e}+1$ & $6.81977 \mathrm{e}+3$ & 0.00 & (6) \\
\hline $\mathrm{Ne}$ & $\mathrm{Ga}$ & $0.2815 \mathrm{e}-1$ & $-0.1622 \mathrm{e}+0$ & $0.1315 \mathrm{e}+1$ & $0.8018 \mathrm{e}+0$ & $4.67989 \mathrm{e}+4$ & 0.00 & (6) \\
\hline $\mathrm{Ga}$ & $\mathrm{Ga}$ & $-0.8731 e+1$ & $0.5480 \mathrm{e}-1$ & $0.2129 \mathrm{e}-3$ & $-0.1116 e+1$ & $2.62439 \mathrm{e}+5$ & 2.82 & (3) \\
\hline $\mathrm{H}$ & $\mathrm{Ge}$ & $0.1666 \mathrm{e}+0$ & $-0.1790 \mathrm{e}+0$ & $0.6550 \mathrm{e}+0$ & $0.1487 \mathrm{e}+1$ & $3.31932 \mathrm{e}+3$ & 1.00 & (6) \\
\hline D & $\mathrm{Ge}$ & $0.1649 \mathrm{e}+0$ & $-0.1724 \mathrm{e}+0$ & $0.3384 \mathrm{e}+0$ & $0.1667 \mathrm{e}+1$ & $3.36441 \mathrm{e}+3$ & 1.00 & (6) \\
\hline${ }^{4} \mathrm{He}$ & $\mathrm{Ge}$ & $0.1155 \mathrm{e}+0$ & $-0.1923 e+0$ & $0.3563 e+0$ & $0.1620 \mathrm{e}+1$ & $7.08909 \mathrm{e}+3$ & 0.00 & (6) \\
\hline $\mathrm{Ne}$ & $\mathrm{Ge}$ & $0.1907 \mathrm{e}-1$ & $-0.2507 \mathrm{e}+0$ & $0.3212 \mathrm{e}+0$ & $0.1483 e+1$ & $4.82268 \mathrm{e}+4$ & 0.00 & (6) \\
\hline Ar & $\mathrm{Ge}$ & $0.3631 \mathrm{e}-2$ & $-0.2551 \mathrm{e}+0$ & $0.7686 \mathrm{e}+0$ & $0.1124 \mathrm{e}+1$ & $1.12992 \mathrm{e}+5$ & 0.00 & (6) \\
\hline $\mathrm{Ge}$ & $\mathrm{Ge}$ & $-0.7908 e+1$ & $0.3985 \mathrm{e}-1$ & $0.4678 \mathrm{e}-3$ & $-0.1047 e+1$ & $2.82619 \mathrm{e}+5$ & 3.88 & (3) \\
\hline${ }^{4} \mathrm{He}$ & $\mathrm{Se}$ & $0.1284 \mathrm{e}+0$ & $-0.1722 \mathrm{e}+0$ & $0.4381 \mathrm{e}+0$ & $0.1564 \mathrm{e}+1$ & $7.63288 \mathrm{e}+3$ & 0.00 & $(6)$ \\
\hline $\mathrm{Ne}$ & $\mathrm{Se}$ & $0.2346 \mathrm{e}-1$ & $-0.2271 \mathrm{e}+0$ & $0.4065 \mathrm{e}+0$ & $0.1448 \mathrm{e}+1$ & $5.10477 \mathrm{e}+4$ & 0.00 & (6) \\
\hline $\mathrm{Ar}$ & $\mathrm{Se}$ & $0.5260 \mathrm{e}-2$ & $-0.2359 e+0$ & $0.8284 \mathrm{e}+0$ & $0.9700 \mathrm{e}+0$ & $1.18037 \mathrm{e}+5$ & 0.00 & (6) \\
\hline $\mathrm{H}$ & $\mathrm{Zr}$ & $0.1757 \mathrm{e}+0$ & $-0.1679 e+0$ & $0.7282 \mathrm{e}+0$ & $0.1598 \mathrm{e}+1$ & $4.42211 \mathrm{e}+3$ & 1.00 & (6) \\
\hline $\mathrm{D}$ & $\mathrm{Zr}$ & $0.1905 \mathrm{e}+0$ & $-0.1488 e+0$ & $0.4577 \mathrm{e}+0$ & $0.1733 e+1$ & $4.47714 \mathrm{e}+3$ & 1.00 & (6) \\
\hline $\mathrm{T}$ & $\mathrm{Zr}$ & $0.1864 \mathrm{e}+0$ & $-0.1381 e+0$ & $0.4456 \mathrm{e}+0$ & $0.1490 \mathrm{e}+1$ & $4.52564 \mathrm{e}+3$ & 1.00 & (6) \\
\hline${ }^{3} \mathrm{He}$ & $\mathrm{Zr}$ & $0.1311 \mathrm{e}+0$ & $-0.1913 e+0$ & $0.3851 \mathrm{e}+0$ & $0.1851 \mathrm{e}+1$ & $9.25829 \mathrm{e}+3$ & 0.00 & (6) \\
\hline${ }^{4} \mathrm{He}$ & $\mathrm{Zr}$ & $0.1258 \mathrm{e}+0$ & $-0.1877 e+0$ & $0.2824 \mathrm{e}+0$ & $0.1970 \mathrm{e}+1$ & $9.35457 \mathrm{e}+3$ & 0.00 & (6) \\
\hline $\mathrm{Ne}$ & $\mathrm{Zr}$ & $0.2945 \mathrm{e}-1$ & $-0.2227 \mathrm{e}+0$ & $0.4035 \mathrm{e}+0$ & $0.1520 \mathrm{e}+1$ & $6.06862 \mathrm{e}+4$ & 0.00 & (6) \\
\hline $\mathrm{Ar}$ & $\mathrm{Zr}$ & $0.7375 \mathrm{e}-2$ & $-0.2427 \mathrm{e}+0$ & $0.6152 \mathrm{e}+0$ & $0.1224 \mathrm{e}+1$ & $1.37106 \mathrm{e}+5$ & 0.00 & (6) \\
\hline $\mathrm{Zr}$ & $\mathrm{Zr}$ & $-0.7828 e+1$ & $0.3797 \mathrm{e}-1$ & $0.6764 \mathrm{e}-3$ & $-0.1004 \mathrm{e}+1$ & $4.75691 \mathrm{e}+5$ & 6.33 & (3) \\
\hline $\mathrm{H}$ & $\mathrm{Nb}$ & $0.1896 \mathrm{e}+0$ & $-0.1565 e+0$ & $0.1089 \mathrm{e}+1$ & $0.1526 \mathrm{e}+1$ & $4.57351 \mathrm{e}+3$ & 1.00 & (6) \\
\hline $\mathrm{D}$ & $\mathrm{Nb}$ & $0.2022 \mathrm{e}+0$ & $-0.1418 \mathrm{e}+0$ & $0.7294 \mathrm{e}+0$ & $0.1643 \mathrm{e}+1$ & $4.62220 \mathrm{e}+3$ & 1.00 & (6) \\
\hline $\mathrm{T}$ & $\mathrm{Nb}$ & $0.1764 \mathrm{e}+0$ & $-0.1580 \mathrm{e}+0$ & $0.2924 \mathrm{e}+0$ & $0.1827 \mathrm{e}+1$ & $4.67139 \mathrm{e}+3$ & 1.00 & (6) \\
\hline${ }^{3} \mathrm{He}$ & $\mathrm{Nb}$ & $0.1583 \mathrm{e}+0$ & $-0.1573 e+0$ & $0.1067 \mathrm{e}+1$ & $0.1830 \mathrm{e}+1$ & $9.55327 \mathrm{e}+3$ & 0.00 & (6) \\
\hline${ }^{4} \mathrm{He}$ & $\mathrm{Nb}$ & $0.1264 \mathrm{e}+0$ & $-0.1952 \mathrm{e}+0$ & $0.2308 \mathrm{e}+0$ & $0.1915 \mathrm{e}+1$ & $9.65087 \mathrm{e}+3$ & 0.00 & (6) \\
\hline $\mathrm{Ne}$ & $\mathrm{Nb}$ & $0.2851 \mathrm{e}-1$ & $-0.2417 \mathrm{e}+0$ & $0.2775 \mathrm{e}+0$ & $0.1954 \mathrm{e}+1$ & $6.23657 \mathrm{e}+4$ & 0.00 & (6) \\
\hline Ar & $\mathrm{Nb}$ & $0.2110 \mathrm{e}+2$ & $0.5140 \mathrm{e}+0$ & $0.3666 \mathrm{e}+4$ & $0.8323 e+0$ & $1.40484 \mathrm{e}+5$ & 0.00 & (6) \\
\hline $\mathrm{Nb}$ & $\mathrm{Nb}$ & $-0.7775 e+1$ & $0.3412 \mathrm{e}-1$ & $0.3236 \mathrm{e}-3$ & $-0.1096 \mathrm{e}+1$ & $5.03904 \mathrm{e}+5$ & 7.59 & (3) \\
\hline $\mathrm{H}$ & Mo & $0.1671 \mathrm{e}+0$ & $-0.1904 e+0$ & $0.6634 \mathrm{e}+0$ & $0.1562 \mathrm{e}+1$ & $4.71832 \mathrm{e}+3$ & 1.00 & $(6)$ \\
\hline D & Mo & $0.1918 \mathrm{e}+0$ & $-0.1596 \mathrm{e}+0$ & $0.5579 \mathrm{e}+0$ & $0.1656 \mathrm{e}+1$ & $4.76698 \mathrm{e}+3$ & 1.00 & (6) \\
\hline $\mathrm{T}$ & Mo & $0.1790 \mathrm{e}+0$ & $-0.1607 \mathrm{e}+0$ & $0.3340 \mathrm{e}+0$ & $0.1743 \mathrm{e}+1$ & $4.81614 \mathrm{e}+3$ & 1.00 & (6) \\
\hline${ }^{3} \mathrm{He}$ & Mo & $0.1379 \mathrm{e}+0$ & $-0.1962 \mathrm{e}+0$ & $0.4854 \mathrm{e}+0$ & $0.1615 \mathrm{e}+1$ & $9.84614 \mathrm{e}+3$ & 0.00 & (6) \\
\hline${ }^{4} \mathrm{He}$ & Mo & $0.1607 \mathrm{e}+0$ & $-0.1616 \mathrm{e}+0$ & $0.5242 \mathrm{e}+0$ & $0.1659 \mathrm{e}+1$ & $9.94365 \mathrm{e}+3$ & 0.00 & (6) \\
\hline $\mathrm{N}$ & Mo & $0.5442 \mathrm{e}-1$ & $-0.2105 e+0$ & $0.5109 \mathrm{e}+0$ & $0.1239 \mathrm{e}+1$ & $4.10879 \mathrm{e}+4$ & 0.00 & (6) \\
\hline $\mathrm{O}$ & Mo & $0.4740 \mathrm{e}-1$ & $-0.2089 e+0$ & $0.5737 \mathrm{e}+0$ & $0.1158 \mathrm{e}+1$ & $4.83320 \mathrm{e}+4$ & 0.00 & (6) \\
\hline $\mathrm{Ne}$ & Mo & $0.3045 \mathrm{e}-1$ & $-0.2440 \mathrm{e}+0$ & $0.3450 \mathrm{e}+0$ & $0.1608 \mathrm{e}+1$ & $6.38956 \mathrm{e}+4$ & 0.00 & (6) \\
\hline $\mathrm{Kr}$ & Mo & $0.1279 \mathrm{e}+2$ & $0.7824 \mathrm{e}+0$ & $0.3265 \mathrm{e}+5$ & $0.1119 \mathrm{e}+1$ & $4.17411 \mathrm{e}+5$ & 0.00 & (6) \\
\hline Mo & Mo & $-0.7622 e+1$ & $0.3443 \mathrm{e}-1$ & $0.3014 \mathrm{e}-3$ & $-0.1084 e+1$ & $5.33049 \mathrm{e}+5$ & 6.83 & (3) \\
\hline
\end{tabular}


Table 12: Constants, $a_{i}$, for the energy dependence of the energy reflection coefficient, $\mathrm{R}_{E}$, at normal incidence for different ions and targets. $\varepsilon_{L}$ is the scaling factor defined in (4), $\mathrm{E}_{\mathrm{sp}}$ the assumed surface binding energy of ions, and fit denotes the applied fitting formula.

\begin{tabular}{|c|c|c|c|c|c|c|c|c|}
\hline ion & solid & a1 & $\mathrm{a} 2$ & a3 & a4 & $\varepsilon_{L}$ & $\mathrm{E}_{\mathrm{sp}}$ & fit \\
\hline $\mathrm{H}$ & $\mathrm{Ru}$ & $0.1689 \mathrm{e}+0$ & $-0.1904 \mathrm{e}+0$ & $0.6222 \mathrm{e}+0$ & $0.1591 \mathrm{e}+1$ & $5.01173 e+3$ & 1.00 & (6) \\
\hline D & $\mathrm{Ru}$ & $0.1863 \mathrm{e}+0$ & $-0.1706 \mathrm{e}+0$ & $0.4635 \mathrm{e}+0$ & $0.1663 \mathrm{e}+1$ & $5.06083 \mathrm{e}+3$ & 1.00 & (6) \\
\hline${ }^{4} \mathrm{He}$ & $\mathrm{Ru}$ & $0.1388 \mathrm{e}+0$ & $-0.1901 e+0$ & $0.4360 \mathrm{e}+0$ & $0.1566 \mathrm{e}+1$ & $1.05397 \mathrm{e}+4$ & 0.00 & (6) \\
\hline $\mathrm{Ne}$ & $\mathrm{Ru}$ & $0.3292 \mathrm{e}-1$ & $-0.2437 \mathrm{e}+0$ & $0.3686 \mathrm{e}+0$ & $0.1561 \mathrm{e}+1$ & $6.70985 \mathrm{e}+4$ & 0.00 & (6) \\
\hline $\mathrm{Ar}$ & $\mathrm{Ru}$ & $0.7251 \mathrm{e}-2$ & $-0.3228 \mathrm{e}+0$ & $0.2584 \mathrm{e}-3$ & $-0.8738 \mathrm{e}+0$ & $1.49334 \mathrm{e}+5$ & 0.00 & (6) \\
\hline $\mathrm{Kr}$ & $\mathrm{Ru}$ & $0.9207 \mathrm{e}+2$ & $0.9456 \mathrm{e}+0$ & $0.1765 \mathrm{e}+6$ & $0.1252 \mathrm{e}+1$ & $4.30460 \mathrm{e}+5$ & 0.00 & (6) \\
\hline $\mathrm{H}$ & $\mathrm{Rh}$ & $0.1724 \mathrm{e}+0$ & $-0.1867 e+0$ & $0.7345 \mathrm{e}+0$ & $0.1523 \mathrm{e}+1$ & $5.16041 \mathrm{e}+3$ & 1.00 & (6) \\
\hline $\mathrm{D}$ & $\mathrm{Rh}$ & $0.1862 \mathrm{e}+0$ & $-0.1698 \mathrm{e}+0$ & $0.4734 \mathrm{e}+0$ & $0.1645 \mathrm{e}+1$ & $5.21007 \mathrm{e}+3$ & 1.00 & (6) \\
\hline${ }^{3} \mathrm{He}$ & $\mathrm{Rh}$ & $0.1364 \mathrm{e}+0$ & $-0.2031 \mathrm{e}+0$ & $0.4453 \mathrm{e}+0$ & $0.1670 \mathrm{e}+1$ & $1.07444 \mathrm{e}+4$ & 0.00 & (6) \\
\hline${ }^{4} \mathrm{He}$ & Rh & $0.1377 \mathrm{e}+0$ & $-0.10918+0$ & $0.3876 \mathrm{e}+0$ & $0.1686 \mathrm{e}+1$ & $1.08438 \mathrm{e}+4$ & 0.00 & (6) \\
\hline $\mathrm{Ne}$ & $\mathrm{Rh}$ & $0.3430 \mathrm{e}-1$ & $-0.2391 \mathrm{e}+0$ & $0.3980 \mathrm{e}+0$ & $0.1620 \mathrm{e}+1$ & $6.87945 \mathrm{e}+4$ & 0.00 & (6) \\
\hline $\mathrm{Ar}$ & $\mathrm{Rh}$ & $0.2471 \mathrm{e}+3$ & $0.7654 \mathrm{e}+0$ & $0.3270 \mathrm{e}+5$ & $0.1080 \mathrm{e}+1$ & $1.52692 \mathrm{e}+5$ & 0.00 & (6) \\
\hline $\mathrm{H}$ & $\mathrm{Pd}$ & $0.1749 \mathrm{e}+0$ & $-0.1816 \mathrm{e}+0$ & $0.7100 \mathrm{e}+0$ & $0.1497 \mathrm{e}+1$ & $5.30935 \mathrm{e}+3$ & 1.00 & (6) \\
\hline${ }^{3} \mathrm{He}$ & $\mathrm{Pd}$ & $0.1472 \mathrm{e}+0$ & $-0.1874 \mathrm{e}+0$ & $0.5570 \mathrm{e}+0$ & $0.1525 \mathrm{e}+1$ & $1.10446 \mathrm{e}+4$ & 0.00 & (6) \\
\hline${ }^{4} \mathrm{He}$ & $\mathrm{Pd}$ & $0.1349 \mathrm{e}+0$ & $-0.1947 \mathrm{e}+0$ & $0.3547 \mathrm{e}+0$ & $0.1710 \mathrm{e}+1$ & $1.11435 \mathrm{e}+4$ & 0.00 & (6) \\
\hline $\mathrm{Ne}$ & $\mathrm{Pd}$ & $0.3669 \mathrm{e}-1$ & $-0.2316 \mathrm{e}+0$ & $0.4077 \mathrm{e}+0$ & $0.1419 \mathrm{e}+1$ & $7.03218 \mathrm{e}+4$ & 0.00 & (6) \\
\hline Ar & $\mathrm{Pd}$ & $0.7649 \mathrm{e}+3$ & $0.8559 \mathrm{e}+0$ & $0.9181 \mathrm{e}+5$ & $0.1159 \mathrm{e}+1$ & $1.55391 \mathrm{e}+5$ & 0.00 & (6) \\
\hline $\mathrm{Pd}$ & $\mathrm{Pd}$ & $-0.7750 \mathrm{e}+1$ & $0.3384 \mathrm{e}-1$ & $0.1903 \mathrm{e}-4$ & $-0.1299 e+1$ & $6.59103 \mathrm{e}+5$ & 3.91 & (3) \\
\hline $\mathrm{H}$ & $\mathrm{Ag}$ & $0.1870 \mathrm{e}+0$ & $-0.1674 \mathrm{e}+0$ & $0.9797 \mathrm{e}+0$ & $0.1449 \mathrm{e}+1$ & $5.46029 \mathrm{e}+3$ & 1.00 & (6) \\
\hline D & $\mathrm{Ag}$ & $0.1890 \mathrm{e}+0$ & $-0.1662 \mathrm{e}+0$ & $0.4586 \mathrm{e}+0$ & $0.1644 \mathrm{e}+1$ & $5.51044 e+3$ & 1.00 & (6) \\
\hline $\mathrm{T}$ & $\mathrm{Ag}$ & $0.1881 \mathrm{e}+0$ & $-0.1560 \mathrm{e}+0$ & $0.3604 \mathrm{e}+0$ & $0.1692 \mathrm{e}+1$ & $5.56109 \mathrm{e}+3$ & 1.00 & (6) \\
\hline${ }^{3} \mathrm{He}$ & $\mathrm{Ag}$ & $0.1440 \mathrm{e}+0$ & $-0.1866 \mathrm{e}+0$ & $0.5089 \mathrm{e}+0$ & $0.1641 e+1$ & $1.13527 \mathrm{e}+4$ & 0.00 & (6) \\
\hline${ }^{4} \mathrm{He}$ & $\mathrm{Ag}$ & $0.1497 \mathrm{e}+0$ & $-0.1714 \mathrm{e}+0$ & $0.5476 \mathrm{e}+0$ & $0.1509 \mathrm{e}+1$ & $1.14531 \mathrm{e}+4$ & 0.00 & (6) \\
\hline $\mathrm{N}$ & $\mathrm{Ag}$ & $0.5593 \mathrm{e}-1$ & $-0.2199 \mathrm{e}+0$ & $0.3905 \mathrm{e}+0$ & $0.1455 \mathrm{e}+1$ & $4.66664 \mathrm{e}+4$ & 0.00 & (6) \\
\hline $\mathrm{O}$ & $\mathrm{Ag}$ & $0.5010 \mathrm{e}-1$ & $-0.2280 \mathrm{e}+0$ & $0.3964 \mathrm{e}+0$ & $0.1436 \mathrm{e}+1$ & $5.47544 \mathrm{e}+5$ & 0.00 & (6) \\
\hline $\mathrm{Ne}$ & $\mathrm{Ag}$ & $0.3663 \mathrm{e}-1$ & $-0.2276 \mathrm{e}+0$ & $0.4074 \mathrm{e}+0$ & $0.1477 \mathrm{e}+1$ & $7.20736 \mathrm{e}+4$ & 0.00 & (6) \\
\hline Ar & $\mathrm{Ag}$ & $0.1148 \mathrm{e}-1$ & $-0.2442 \mathrm{e}+0$ & $0.6374 \mathrm{e}+0$ & $0.1088 \mathrm{e}+1$ & $1.58921 \mathrm{e}+5$ & 0.00 & (6) \\
\hline $\mathrm{Kr}$ & $\mathrm{Ag}$ & $0.1081 \mathrm{e}+3$ & $0.8904 \mathrm{e}+0$ & $0.1542 \mathrm{e}+6$ & $0.1193 e+1$ & $4.51993 \mathrm{e}+5$ & 0.00 & (6) \\
\hline $\mathrm{Ag}$ & $\mathrm{Ag}$ & $-0.8435 e+1$ & $0.5341 \mathrm{e}-1$ & $0.4405 \mathrm{e}-3$ & $-0.9576 e+0$ & $6.93021 \mathrm{e}+5$ & 2.97 & (3) \\
\hline${ }^{4} \mathrm{He}$ & $\mathrm{Cd}$ & $0.1513 \mathrm{e}+0$ & $-0.1639 e+0$ & $0.5221 \mathrm{e}+0$ & $0.1595 \mathrm{e}+1$ & $1.17532 \mathrm{e}+4$ & 0.00 & (6) \\
\hline $\mathrm{Ne}$ & $\mathrm{Cd}$ & $0.3976 \mathrm{e}-1$ & $-0.2113 e+0$ & $0.4235 \mathrm{e}+0$ & $0.1623 e+1$ & $7.35197 \mathrm{e}+4$ & 0.00 & (6) \\
\hline $\mathrm{Ar}$ & $\mathrm{Cd}$ & $0.2598 \mathrm{e}-1$ & $-0.1507 e+0$ & $0.1828 \mathrm{e}+1$ & $0.3361 \mathrm{e}+0$ & $1.61276 \mathrm{e}+5$ & 0.00 & (6) \\
\hline $\mathrm{Kr}$ & $\mathrm{Cd}$ & $0.5584 \mathrm{e}+3$ & $0.9757 \mathrm{e}+0$ & $0.5366 \mathrm{e}+6$ & $0.1238 \mathrm{e}+1$ & $4.55218 \mathrm{e}+5$ & 0.00 & (6) \\
\hline $\mathrm{Cd}$ & $\mathrm{Cd}$ & $-0.7551 e+1$ & $0.2968 \mathrm{e}-1$ & $0.7381 \mathrm{e}-5$ & $-0.1251 e+1$ & $7.27916 \mathrm{e}+5$ & 1.16 & (3) \\
\hline $\mathrm{H}$ & In & $0.1918 \mathrm{e}+0$ & $-0.1492 \mathrm{e}+0$ & $0.8232 \mathrm{e}+0$ & $0.1549 \mathrm{e}+1$ & $5.76339 e+3$ & 1.00 & (6) \\
\hline${ }^{4} \mathrm{He}$ & In & $0.1543 \mathrm{e}+0$ & $-0.1556 \mathrm{e}+0$ & $0.5316 \mathrm{e}+0$ & $0.1637 \mathrm{e}+1$ & $1.20632 \mathrm{e}+4$ & 0.00 & (6) \\
\hline $\mathrm{Ne}$ & In & $0.4170 \mathrm{e}-1$ & $-0.2006 \mathrm{e}+0$ & $0.4717 \mathrm{e}+0$ & $0.1504 \mathrm{e}+1$ & $7.51927 \mathrm{e}+4$ & 0.00 & (6) \\
\hline $\mathrm{Kr}$ & In & $0.1203 e+2$ & $0.5858 \mathrm{e}+0$ & $0.1190 \mathrm{e}+5$ & $0.8639 \mathrm{e}+0$ & $4.62260 \mathrm{e}+5$ & 0.00 & (6) \\
\hline In & In & $-0.7489 e+1$ & $0.2846 \mathrm{e}-1$ & $0.3945 \mathrm{e}-4$ & $-0.1155 e+1$ & $7.63793 e+5$ & 2.49 & (3) \\
\hline $\mathrm{H}$ & $\mathrm{Sn}$ & $0.1818 \mathrm{e}+0$ & $-0.1577 \mathrm{e}+0$ & $0.7366 \mathrm{e}+0$ & $0.1613 e+1$ & $5.91631 \mathrm{e}+3$ & 1.00 & (6) \\
\hline D & $\mathrm{Sn}$ & $0.2084 \mathrm{e}+0$ & $-0.1343 e+0$ & $0.6323 \mathrm{e}+0$ & $0.1656 \mathrm{e}+1$ & $5.96574 \mathrm{e}+3$ & 1.00 & (6) \\
\hline${ }^{3} \mathrm{He}$ & $\mathrm{Sn}$ & $0.1562 \mathrm{e}+0$ & $-0.1611 e+0$ & $0.5871 \mathrm{e}+0$ & $0.1676 \mathrm{e}+1$ & $1.22714 \mathrm{e}+4$ & 0.00 & (6) \\
\hline${ }^{4} \mathrm{He}$ & $\mathrm{Sn}$ & $0.1585 \mathrm{e}+0$ & $-0.1504 e+0$ & $0.5265 \mathrm{e}+0$ & $0.1720 \mathrm{e}+1$ & $1.23702 \mathrm{e}+4$ & 0.00 & (6) \\
\hline $\mathrm{Ne}$ & $\mathrm{Sn}$ & $0.4395 \mathrm{e}-1$ & $-0.1957 e+0$ & $0.4624 \mathrm{e}+0$ & $0.1565 e+1$ & $7.67367 \mathrm{e}+4$ & 0.00 & (6) \\
\hline Ar & $\mathrm{Sn}$ & $0.1378 \mathrm{e}-1$ & $-0.2225 \mathrm{e}+0$ & $0.1180 \mathrm{e}+2$ & $0.7301 \mathrm{e}+1$ & $1.67157 \mathrm{e}+5$ & 0.00 & (6) \\
\hline $\mathrm{Kr}$ & $\mathrm{Sn}$ & $0.5113 e+0$ & $0.4753 e+0$ & $0.3990 \mathrm{e}+4$ & $0.7408 \mathrm{e}+0$ & $4.66941 \mathrm{e}+5$ & 0.00 & (6) \\
\hline $\mathrm{Sn}$ & $\mathrm{Sn}$ & $-0.7115 e+1$ & $0.2065 \mathrm{e}-1$ & $0.7433 \mathrm{e}-4$ & $-0.1108 e+1$ & $8.00660 \mathrm{e}+5$ & 3.12 & $(3)$ \\
\hline
\end{tabular}


Table 13: Constants, $a_{i}$, for the energy dependence of the energy reflection coefficient, $\mathrm{R}_{E}$, at normal incidence for different ions and targets. $\varepsilon_{L}$ is the scaling factor defined in (4), $\mathrm{E}_{\mathrm{sb}}$ the assumed surface binding energy of ions, and fit denotes the applied fitting formula.

\begin{tabular}{|c|c|c|c|c|c|c|c|c|}
\hline ion & solid & a1 & $\mathrm{a} 2$ & a3 & $\mathrm{a} 4$ & $\varepsilon_{L}$ & $\mathrm{E}_{\mathrm{sp}}$ & fit \\
\hline${ }^{4} \mathrm{He}$ & $\mathrm{Sb}$ & $0.1165 \mathrm{e}+0$ & $-0.1544 \mathrm{e}+0$ & $0.1190 \mathrm{e}+2$ & $0.1846 \mathrm{e}+1$ & $1.26821 \mathrm{e}+4$ & 0.00 & $(6)$ \\
\hline $\mathrm{Ne}$ & $\mathrm{Sb}$ & $0.4676 \mathrm{e}-1$ & $-0.1846 e+0$ & $0.5282 \mathrm{e}+0$ & $0.1552 \mathrm{e}+1$ & $7.83708 \mathrm{e}+4$ & 0.00 & (6) \\
\hline $\mathrm{Kr}$ & $\mathrm{Sb}$ & $0.1527 \mathrm{e}-2$ & $-0.2443 e+0$ & $0.6524 \mathrm{e}-5$ & $-0.1041 \mathrm{e}+1$ & $4.73059 \mathrm{e}+5$ & 0.00 & (6) \\
\hline${ }^{4} \mathrm{He}$ & $\mathrm{Te}$ & $0.1644 \mathrm{e}+0$ & $-0.1422 \mathrm{e}+0$ & $0.5804 \mathrm{e}+0$ & $0.1695 \mathrm{e}+1$ & $1.29871 \mathrm{e}+4$ & 0.00 & (6) \\
\hline $\mathrm{Ne}$ & $\mathrm{Te}$ & $0.5156 \mathrm{e}-1$ & $-0.1723 e+0$ & $0.6169 \mathrm{e}+0$ & $0.1373 \mathrm{e}+1$ & $7.97723 \mathrm{e}+4$ & 0.00 & (6) \\
\hline $\mathrm{Ar}$ & $\mathrm{Te}$ & $0.1669 \mathrm{e}-1$ & $-0.2040 \mathrm{e}+0$ & $0.4892 \mathrm{e}+1$ & $0.5326 \mathrm{e}+1$ & $1.72282 \mathrm{e}+5$ & 0.00 & (6) \\
\hline $\mathrm{H}$ & $\mathrm{Tb}$ & $0.2217 \mathrm{e}+0$ & $-0.1165 e+0$ & $0.1499 \mathrm{e}+1$ & $0.1469 \mathrm{e}+1$ & $8.32995 \mathrm{e}+3$ & 1.00 & (6) \\
\hline${ }^{4} \mathrm{He}$ & $\mathrm{Tb}$ & $0.1786 \mathrm{e}+0$ & $-0.1354 \mathrm{e}+0$ & $0.6498 \mathrm{e}+0$ & $0.1853 \mathrm{e}+1$ & $1.72593 \mathrm{e}+4$ & 0.00 & (6) \\
\hline Ar & $\mathrm{Tb}$ & $0.2595 \mathrm{e}-1$ & $-0.1865 e+0$ & $0.2059 \mathrm{e}+2$ & $0.5736 \mathrm{e}+1$ & $2.15976 \mathrm{e}+5$ & 0.00 & (6) \\
\hline $\mathrm{H}$ & $\mathrm{Tm}$ & $0.2267 \mathrm{e}+0$ & $-0.1149 e+0$ & $0.1478 \mathrm{e}+1$ & $0.1340 \mathrm{e}+1$ & $9.00672 \mathrm{e}+3$ & 1.00 & (6) \\
\hline${ }^{4} \mathrm{He}$ & $\mathrm{Tm}$ & $0.1818 \mathrm{e}+0$ & $-0.1343 e+0$ & $0.6982 \mathrm{e}+0$ & $0.1791 \mathrm{e}+1$ & $1.86300 \mathrm{e}+4$ & 0.00 & (6) \\
\hline $\mathrm{Ar}$ & $\mathrm{Tm}$ & $0.2863 \mathrm{e}-1$ & $-0.1854 \mathrm{e}+0$ & $0.3607 \mathrm{e}+1$ & $0.3447 \mathrm{e}+1$ & $2.29744 \mathrm{e}+5$ & 0.00 & (6) \\
\hline $\mathrm{H}$ & $\mathrm{Hf}$ & $0.2118 \mathrm{e}+0$ & $-0.1322 \mathrm{e}+0$ & $0.1146 \mathrm{e}+1$ & $0.1423 \mathrm{e}+1$ & $9.52208 \mathrm{e}+3$ & 1.00 & (6) \\
\hline${ }^{4} \mathrm{He}$ & Hf & $0.2068 \mathrm{e}+0$ & $-0.1207 \mathrm{e}+0$ & $0.1326 \mathrm{e}+1$ & $0.1387 \mathrm{e}+1$ & $1.96695 \mathrm{e}+4$ & 0.00 & (6) \\
\hline $\mathrm{Ne}$ & Hf & $0.6998 \mathrm{e}-1$ & $-0.1726 \mathrm{e}+0$ & $0.6271 \mathrm{e}+0$ & $0.1932 \mathrm{e}+1$ & $1.15397 \mathrm{e}+5$ & 0.00 & (6) \\
\hline $\mathrm{Ar}$ & $\mathrm{Hf}$ & $0.3001 \mathrm{e}-1$ & $-0.1972 \mathrm{e}+0$ & $0.2832 \mathrm{e}+1$ & $0.3387 \mathrm{e}+1$ & $2.39690 \mathrm{e}+5$ & 0.00 & (6) \\
\hline $\mathrm{Kr}$ & $\mathrm{Hf}$ & $0.1859 \mathrm{e}+2$ & $0.4188 \mathrm{e}+0$ & $0.3305 \mathrm{e}+4$ & $0.6612 \mathrm{e}+0$ & $6.21788 \mathrm{e}+5$ & 0.00 & (6) \\
\hline $\mathrm{Xe}$ & Hf & $0.1712 \mathrm{e}+3$ & $0.7950 \mathrm{e}+0$ & $0.1365 \mathrm{e}+6$ & $0.1024 \mathrm{e}+1$ & $1.16579 \mathrm{e}+6$ & 0.00 & (6) \\
\hline$\overline{\mathrm{H}}$ & $\mathrm{Ta}$ & $0.2096 \mathrm{e}+0$ & $-0.1385 e+0$ & $0.1168 \mathrm{e}+1$ & $0.1355 \mathrm{e}+1$ & $9.69565 \mathrm{e}+3$ & 1.00 & (6) \\
\hline $\mathrm{D}$ & $\mathrm{Ta}$ & $0.2282 \mathrm{e}+0$ & $-0.1314 e+0$ & $0.7287 \mathrm{e}+0$ & $0.1472 \mathrm{e}+1$ & $9.74893 e+3$ & 1.00 & (6) \\
\hline${ }^{3} \mathrm{He}$ & $\mathrm{Ta}$ & $0.1752 \mathrm{e}+0$ & $-0.1592 \mathrm{e}+0$ & $0.7811 \mathrm{e}+0$ & $0.1656 \mathrm{e}+1$ & $1.99149 \mathrm{e}+4$ & 0.00 & (6) \\
\hline${ }^{4} \mathrm{He}$ & $\mathrm{Ta}$ & $0.1859 \mathrm{e}+0$ & $-0.1447 \mathrm{e}+0$ & $0.7856 \mathrm{e}+0$ & $0.1528 \mathrm{e}+1$ & $2.00210 \mathrm{e}+4$ & 0.00 & (6) \\
\hline $\mathrm{Ne}$ & $\mathrm{Ta}$ & $0.6691 \mathrm{e}-1$ & $-0.1886 \mathrm{e}+0$ & $0.6717 \mathrm{e}+0$ & $0.2129 \mathrm{e}+1$ & $1.17263 \mathrm{e}+5$ & 0.00 & (6) \\
\hline Ar & $\mathrm{Ta}$ & $0.3001 \mathrm{e}-1$ & $-0.2071 \mathrm{e}+0$ & $0.1138 \mathrm{e}+1$ & $0.2134 \mathrm{e}+1$ & $2.43225 \mathrm{e}+5$ & 0.00 & (6) \\
\hline $\mathrm{Kr}$ & $\mathrm{Ta}$ & $0.3290 \mathrm{e}+3$ & $0.7163 e+0$ & $0.5928 \mathrm{e}+5$ & $0.9713 e+0$ & $6.29462 \mathrm{e}+5$ & 0.00 & (6) \\
\hline $\mathrm{Xe}$ & $\mathrm{Ta}$ & $0.1267 \mathrm{e}+3$ & $0.8058 \mathrm{e}+0$ & $0.1246 \mathrm{e}+6$ & $0.1078 \mathrm{e}+1$ & $1.17814 \mathrm{e}+6$ & 0.00 & (6) \\
\hline $\mathrm{Ta}$ & $\mathrm{Ta}$ & $-0.7454 e+1$ & $0.2439 \mathrm{e}-1$ & $0.4053 \mathrm{e}-4$ & $-0.1174 \mathrm{e}+1$ & $1.93615 \mathrm{e}+6$ & 8.10 & (3) \\
\hline $\mathrm{H}$ & W & $0.2039 \mathrm{e}+0$ & $-0.1501 e+0$ & $0.1064 \mathrm{e}+1$ & $0.1359 \mathrm{e}+1$ & $9.86986 \mathrm{e}+3$ & 1.00 & (6) \\
\hline $\mathrm{D}$ & W & $0.2547 \mathrm{e}+0$ & $-0.1146 \mathrm{e}+0$ & $0.1195 \mathrm{e}+1$ & $0.1259 \mathrm{e}+1$ & $9.92326 \mathrm{e}+3$ & 1.00 & (6) \\
\hline $\mathrm{T}$ & $\mathrm{W}$ & $0.2547 \mathrm{e}+0$ & $-0.1113 e+0$ & $0.8170 \mathrm{e}+0$ & $0.1354 \mathrm{e}+1$ & $9.97718 \mathrm{e}+3$ & 1.00 & (6) \\
\hline${ }^{3} \mathrm{He}$ & W & $0.1768 \mathrm{e}+0$ & $-0.1609 e+0$ & $0.8362 \mathrm{e}+0$ & $0.1618 \mathrm{e}+1$ & $2.02666 \mathrm{e}+4$ & 0.00 & (6) \\
\hline${ }^{4} \mathrm{He}$ & W & $0.1813 e+0$ & $-0.1553 e+0$ & $0.6580 \mathrm{e}+0$ & $0.1549 \mathrm{e}+1$ & $2.03728 \mathrm{e}+4$ & 0.00 & (6) \\
\hline $\mathrm{N}$ & W & $0.9951 \mathrm{e}-1$ & $-0.1688 \mathrm{e}+0$ & $0.6816 \mathrm{e}+0$ & $0.1215 \mathrm{e}+1$ & $7.90505 \mathrm{e}+4$ & 1.00 & (6) \\
\hline $\mathrm{Ne}$ & W & $0.6680 \mathrm{e}-1$ & $-0.1957 \mathrm{e}+0$ & $0.5718 \mathrm{e}+0$ & $0.2308 \mathrm{e}+1$ & $1.19107 \mathrm{e}+5$ & 0.00 & (6) \\
\hline Ar & W & $0.3071 \mathrm{e}-1$ & $-0.2121 \mathrm{e}+0$ & $0.9017 \mathrm{e}+1$ & $0.4659 \mathrm{e}+1$ & $2.46646 \mathrm{e}+5$ & 0.00 & (6) \\
\hline $\mathrm{Kr}$ & $\mathrm{W}$ & $0.4409 \mathrm{e}+3$ & $0.7560 \mathrm{e}+0$ & $0.7283 e+5$ & $0.1010 \mathrm{e}+1$ & $6.36677 \mathrm{e}+5$ & 0.00 & (6) \\
\hline $\mathrm{Xe}$ & W & $0.3725 \mathrm{e}+3$ & $0.9036 \mathrm{e}+0$ & $0.3022 \mathrm{e}+6$ & $0.1163 e+0$ & $1.18932 \mathrm{e}+6$ & 0.00 & (6) \\
\hline W & W & $-0.7168 e+1$ & $0.1685 \mathrm{e}-1$ & $0.7005 e-5$ & $-0.1343 e+1$ & $1.99860 \mathrm{e}+6$ & 8.68 & (3) \\
\hline $\mathrm{H}$ & $\mathrm{Re}$ & $0.2050 \mathrm{e}+0$ & $-0.1508 \mathrm{e}+0$ & $0.1249 \mathrm{e}+1$ & $0.1356 \mathrm{e}+1$ & $1.00450 \mathrm{e}+4$ & 1.00 & (6) \\
\hline${ }^{4} \mathrm{He}$ & $\operatorname{Re}$ & $0.1843 \mathrm{e}+0$ & $-0.1519 e+0$ & $0.7652 \mathrm{e}+0$ & $0.1522 \mathrm{e}+1$ & $2.07275 \mathrm{e}+4$ & 0.00 & (6) \\
\hline $\mathrm{Ne}$ & $\operatorname{Re}$ & $0.7057 \mathrm{e}-1$ & $-0.1903 e+0$ & $0.6729 \mathrm{e}+0$ & $0.1774 \mathrm{e}+1$ & $1.20995 \mathrm{e}+5$ & 0.00 & (6) \\
\hline $\mathrm{Ar}$ & Re & $0.8472 \mathrm{e}+1$ & $0.2070 \mathrm{e}+0$ & $0.3053 e+3$ & $0.4505 \mathrm{e}+0$ & $2.50221 \mathrm{e}+5$ & 0.00 & (6) \\
\hline $\mathrm{Kr}$ & $\mathrm{Re}$ & $0.1576 \mathrm{e}+4$ & $0.8869 e+0$ & $0.2592 \mathrm{e}+6$ & $0.1147 \mathrm{e}+1$ & $6.44513 \mathrm{e}+5$ & 0.00 & (6) \\
\hline $\mathrm{Xe}$ & $\mathrm{Re}$ & $0.4408 \mathrm{e}+3$ & $0.9221 \mathrm{e}+0$ & $0.3344 \mathrm{e}+6$ & $0.1182 \mathrm{e}+1$ & $1.20203 \mathrm{e}+6$ & 0.00 & (6) \\
\hline
\end{tabular}


Table 14: Constants, $a_{i}$, for the energy dependence of the energy reflection coefficient, $\mathrm{R}_{E}$, at normal incidence for different ions and targets. $\varepsilon_{L}$ is the scaling factor defined in (4), $\mathrm{E}_{\mathrm{sb}}$ the assumed surface binding energy of ions, and fit denotes the applied fitting formula.

\begin{tabular}{|c|c|c|c|c|c|c|c|c|}
\hline ion & solid & a1 & $\mathrm{a} 2$ & a3 & $\mathrm{a} 4$ & $\varepsilon_{L}$ & $E_{s p}$ & fit \\
\hline $\mathrm{H}$ & Os & $0.2084 \mathrm{e}+0$ & $-0.1498 \mathrm{e}+0$ & $0.1296 \mathrm{e}+1$ & $0.1339 \mathrm{e}+1$ & $1.02204 \mathrm{e}+4$ & 1.00 & (6) \\
\hline${ }^{3} \mathrm{He}$ & Os & $0.1795 \mathrm{e}+0$ & $-0.1609 e+0$ & $0.8528 \mathrm{e}+0$ & $0.1505 \mathrm{e}+1$ & $2.09735 \mathrm{e}+4$ & 0.00 & (6) \\
\hline${ }^{4} \mathrm{He}$ & Os & $0.1847 \mathrm{e}+0$ & $-0.1533 \mathrm{e}+0$ & $0.7631 \mathrm{e}+0$ & $0.1501 \mathrm{e}+1$ & $2.10799 \mathrm{e}+4$ & 0.00 & (6) \\
\hline $\mathrm{Ne}$ & Os & $0.6984 \mathrm{e}-1$ & $-0.1954 \mathrm{e}+0$ & $0.6666 \mathrm{e}+0$ & $0.2137 \mathrm{e}+1$ & $1.22785 \mathrm{e}+5$ & 0.00 & (6) \\
\hline $\mathrm{Ar}$ & Os & $0.3265 \mathrm{e}-1$ & $-0.2111 \mathrm{e}+0$ & $0.1458 \mathrm{e}+5$ & $0.1224 \mathrm{e}+2$ & $2.53422 \mathrm{e}+5$ & 0.00 & (6) \\
\hline $\mathrm{Kr}$ & Os & $0.2679 \mathrm{e}+4$ & $0.9342 \mathrm{e}+0$ & $0.4164 \mathrm{e}+6$ & $0.1195 \mathrm{e}+1$ & $6.50625 e+5$ & 0.00 & (6) \\
\hline $\mathrm{Xe}$ & Os & $0.2782 \mathrm{e}+3$ & $0.8766 \mathrm{e}+0$ & $0.1979 e+6$ & $0.1142 \mathrm{e}+1$ & $1.21043 \mathrm{e}+6$ & 0.00 & (6) \\
\hline $\mathrm{H}$ & Ir & $0.2101 \mathrm{e}+0$ & $-0.1476 \mathrm{e}+0$ & $0.1322 \mathrm{e}+1$ & $0.1321 \mathrm{e}+1$ & $1.03972 \mathrm{e}+4$ & 1.00 & (6) \\
\hline${ }^{3} \mathrm{He}$ & Ir & $0.1797 \mathrm{e}+0$ & $-0.1604 e+0$ & $0.8565 \mathrm{e}+0$ & $0.1506 \mathrm{e}+1$ & $2.13313 e+4$ & 0.00 & (6) \\
\hline${ }^{4} \mathrm{He}$ & Ir & $0.1858 \mathrm{e}+0$ & $-0.1522 \mathrm{e}+0$ & $0.7712 \mathrm{e}+0$ & $0.1499 \mathrm{e}+1$ & $2.14384 \mathrm{e}+4$ & 0.00 & (6) \\
\hline $\mathrm{Ne}$ & Ir & $0.7062 \mathrm{e}-1$ & $-0.1935 \mathrm{e}+0$ & $0.6368 \mathrm{e}+0$ & $0.1941 \mathrm{e}+1$ & $1.24708 \mathrm{e}+5$ & 0.00 & (6) \\
\hline $\mathrm{Ar}$ & $\mathrm{Ir}$ & $0.7594 \mathrm{e}+0$ & $-0.9636 \mathrm{e}-2$ & $0.2506 \mathrm{e}+2$ & $0.2431 \mathrm{e}+0$ & $2.57103 e+5$ & 0.00 & (6) \\
\hline $\mathrm{Kr}$ & Ir & $0.1739 \mathrm{e}+4$ & $0.8893 \mathrm{e}+0$ & $0.2660 \mathrm{e}+6$ & $0.1151 \mathrm{e}+1$ & $6.58875 e+5$ & 0.00 & (6) \\
\hline $\mathrm{Xe}$ & Ir & $0.3129 \mathrm{e}+3$ & $0.8814 \mathrm{e}+0$ & $0.2118 \mathrm{e}+6$ & $0.1146 \mathrm{e}+1$ & $1.22412 \mathrm{e}+5$ & 0.00 & (6) \\
\hline $\mathrm{H}$ & $\mathrm{Pt}$ & $0.2035 \mathrm{e}+0$ & $-0.1523 e+0$ & $0.1100 \mathrm{e}+1$ & $0.1321 \mathrm{e}+1$ & $1.05744 \mathrm{e}+4$ & 1.00 & (6) \\
\hline${ }^{3} \mathrm{He}$ & $\mathrm{Pt}$ & $0.1886 \mathrm{e}+0$ & $-0.1507 \mathrm{e}+0$ & $0.9444 \mathrm{e}+0$ & $0.1417 \mathrm{e}+1$ & $2.16890 \mathrm{e}+4$ & 0.00 & (6) \\
\hline${ }^{4} \mathrm{He}$ & $\mathrm{Pt}$ & $0.1926 \mathrm{e}+0$ & $-0.1447 \mathrm{e}+0$ & $0.8496 \mathrm{e}+0$ & $0.1360 \mathrm{e}+1$ & $2.17963 e+4$ & 0.00 & (6) \\
\hline $\mathrm{O}$ & $\mathrm{Pt}$ & $0.8799 \mathrm{e}-1$ & $-0.1833 e+0$ & $0.5431 \mathrm{e}+0$ & $0.1649 \mathrm{e}+1$ & $9.78987 \mathrm{e}+4$ & 0.00 & (6) \\
\hline $\mathrm{Ne}$ & $\mathrm{Pt}$ & $0.8584 \mathrm{e}-1$ & $-0.1679 e+0$ & $0.9949 \mathrm{e}+0$ & $0.1304 \mathrm{e}+1$ & $1.26583 \mathrm{e}+5$ & 0.00 & (6) \\
\hline $\mathrm{Ar}$ & $\mathrm{Pt}$ & $0.3510 \mathrm{e}+2$ & $0.2952 \mathrm{e}+0$ & $0.1177 \mathrm{e}+4$ & $0.5267 \mathrm{e}+0$ & $2.60590 \mathrm{e}+5$ & 0.00 & (6) \\
\hline $\mathrm{Kr}$ & $\mathrm{Pt}$ & $0.1193 e+4$ & $0.8333 \mathrm{e}+0$ & $0.1716 \mathrm{e}+6$ & $0.1088 \mathrm{e}+1$ & $6.66218 \mathrm{e}+5$ & 0.00 & (6) \\
\hline $\mathrm{Xe}$ & $\mathrm{Pt}$ & $0.6961 \mathrm{e}+3$ & $0.9325 \mathrm{e}+0$ & $0.4222 \mathrm{e}+6$ & $0.1185 e+1$ & $1.23553 \mathrm{e}+6$ & 0.00 & (6) \\
\hline $\mathrm{Pt}$ & $\mathrm{Pt}$ & $-0.7364 e+1$ & $0.2412 \mathrm{e}-1$ & $0.7618 \mathrm{e}-5$ & $-0.1289 e+1$ & $2.25981 \mathrm{e}+6$ & 5.86 & (3) \\
\hline $\mathrm{H}$ & $\mathrm{Au}$ & $0.2188 \mathrm{e}+0$ & $-0.1366 \mathrm{e}+0$ & $0.1448 \mathrm{e}+1$ & $0.1264 \mathrm{e}+1$ & $1.07527 \mathrm{e}+4$ & 1.00 & (6) \\
\hline $\mathrm{D}$ & $\mathrm{Au}$ & $0.2386 \mathrm{e}+0$ & $-0.1296 \mathrm{e}+0$ & $0.8904 \mathrm{e}+0$ & $0.1411 \mathrm{e}+1$ & $1.08070 \mathrm{e}+4$ & 0.00 & (6) \\
\hline $\mathrm{T}$ & $\mathrm{Au}$ & $0.2386 \mathrm{e}+0$ & $-0.1259 \mathrm{e}+0$ & $0.6745 \mathrm{e}+0$ & $0.1471 \mathrm{e}+1$ & $1.08618 \mathrm{e}+4$ & 0.00 & (6) \\
\hline${ }^{3} \mathrm{He}$ & $\mathrm{Au}$ & $0.1931 \mathrm{e}+0$ & $-0.1436 e+0$ & $0.1008 \mathrm{e}+1$ & $0.1350 \mathrm{e}+1$ & $2.20499 \mathrm{e}+4$ & 0.00 & (6) \\
\hline${ }^{4} \mathrm{He}$ & $\mathrm{Au}$ & $0.2011 \mathrm{e}+0$ & $-0.1345 \mathrm{e}+0$ & $0.8914 \mathrm{e}+0$ & $0.1562 \mathrm{e}+1$ & $2.21579 \mathrm{e}+4$ & 0.00 & (6) \\
\hline $\mathrm{N}$ & $\mathrm{Au}$ & $0.9902 \mathrm{e}-1$ & $-0.1739 e+0$ & $0.6064 \mathrm{e}+0$ & $0.1533 e+1$ & $8.55290 \mathrm{e}+4$ & 0.00 & (6) \\
\hline $\mathrm{O}$ & $\mathrm{Au}$ & $0.8944 \mathrm{e}-1$ & $-0.1778 e+0$ & $0.5435 \mathrm{e}+0$ & $0.1735 \mathrm{e}+1$ & $9.94281 \mathrm{e}+4$ & 0.00 & (6) \\
\hline $\mathrm{Ne}$ & $\mathrm{Au}$ & $0.7686 \mathrm{e}-1$ & $-0.1789 e+0$ & $0.7223 \mathrm{e}+0$ & $0.1410 \mathrm{e}+1$ & $1.28526 \mathrm{e}+5$ & 0.00 & (6) \\
\hline $\mathrm{Na}$ & $\mathrm{Au}$ & $0.6640 \mathrm{e}-1$ & $-0.1850 \mathrm{e}+0$ & $0.7257 \mathrm{e}+0$ & $0.1957 \mathrm{e}+1$ & $1.44150 \mathrm{e}+5$ & 0.00 & (6) \\
\hline $\mathrm{Ar}$ & $\mathrm{Au}$ & $0.3473 \mathrm{e}-1$ & $-0.2006 e+0$ & $0.7277 \mathrm{e}+0$ & $0.1730 \mathrm{e}+1$ & $2.64318 \mathrm{e}+5$ & 0.00 & (6) \\
\hline $\mathrm{K}$ & $\mathrm{Au}$ & $0.4581 \mathrm{e}-1$ & $-0.1663 e+0$ & $0.1027 \mathrm{e}+1$ & $0.8168 \mathrm{e}+1$ & $2.79384 \mathrm{e}+5$ & 0.00 & (6) \\
\hline $\mathrm{Kr}$ & $\mathrm{Au}$ & $0.5287 \mathrm{e}+3$ & $0.7344 \mathrm{e}+0$ & $0.7297 \mathrm{e}+5$ & $0.9811 \mathrm{e}+0$ & $6.74618 e+5$ & 0.00 & (6) \\
\hline $\mathrm{Xe}$ & $\mathrm{Au}$ & $0.1575 e+3$ & $0.7903 e+0$ & $0.9797 \mathrm{e}+5$ & $0.1058 \mathrm{e}+1$ & $1.24955 \mathrm{e}+6$ & 0.00 & (6) \\
\hline $\mathrm{Au}$ & $\mathrm{Au}$ & $-0.6351 e+1$ & $0.4689 \mathrm{e}-2$ & $0.2066 \mathrm{e}-5$ & $-0.1347 e+1$ & $2.32799 \mathrm{e}+6$ & 3.80 & (3) \\
\hline $\mathrm{Kr}$ & $\mathrm{Hg}$ & $0.8561 \mathrm{e}-2$ & $-0.2015 e+0$ & $0.5436 \mathrm{e}+1$ & $0.2461 \mathrm{e}+1$ & $6.81273 e+5$ & 0.00 & $(6)$ \\
\hline $\mathrm{Kr}$ & $\mathrm{Tl}$ & $0.1020 \mathrm{e}-1$ & $-0.1837 e+0$ & $0.7786 \mathrm{e}+1$ & $0.2164 \mathrm{e}+1$ & $6.87824 \mathrm{e}+5$ & 0.00 & $(6)$ \\
\hline $\mathrm{H}$ & $\mathrm{Pb}$ & $0.2237 \mathrm{e}+0$ & $-0.1160 e+0$ & $0.1313 e+1$ & $0.1423 e+1$ & $1.12905 \mathrm{e}+4$ & 1.00 & (6) \\
\hline${ }^{4} \mathrm{He}$ & $\mathrm{Pb}$ & $0.2334 \mathrm{e}+0$ & $-0.9889 \mathrm{e}-1$ & $0.1258 \mathrm{e}+1$ & $0.1124 \mathrm{e}+1$ & $2.32414 \mathrm{e}+4$ & 0.00 & (6) \\
\hline $\mathrm{Ne}$ & $\mathrm{Pb}$ & $0.8664 \mathrm{e}-1$ & $-0.1453 \mathrm{e}+0$ & $0.7067 \mathrm{e}+0$ & $0.1329 \mathrm{e}+1$ & $1.34122 \mathrm{e}+5$ & 0.00 & (6) \\
\hline $\mathrm{Ar}$ & $\mathrm{Pb}$ & $0.4052 \mathrm{e}-1$ & $-0.1677 \mathrm{e}+0$ & $0.2386 \mathrm{e}+1$ & $0.2786 \mathrm{e}+1$ & $2.74555 \mathrm{e}+5$ & 0.00 & (6) \\
\hline $\mathrm{Kr}$ & $\mathrm{Pb}$ & $0.1045 \mathrm{e}-1$ & $-0.1838 e+0$ & $0.2243 \mathrm{e}+1$ & $0.1658 \mathrm{e}+1$ & $6.95357 \mathrm{e}+5$ & 0.00 & (6) \\
\hline $\mathrm{Xe}$ & $\mathrm{Pb}$ & $0.3023 e+2$ & $0.5228 \mathrm{e}+0$ & $0.1279 \mathrm{e}+5$ & $0.7351 \mathrm{e}+0$ & $1.28038 \mathrm{e}+6$ & 0.00 & (6) \\
\hline $\mathrm{Pb}$ & $\mathrm{Pb}$ & $-0.7188 e+1$ & $0.2213 \mathrm{e}-1$ & $0.2404 \mathrm{e}-4$ & $-0.1080 e+1$ & $2.53951 \mathrm{e}+5$ & 2.03 & (3) \\
\hline
\end{tabular}


Table 15: Constants, $a_{i}$, for the energy dependence of the energy reflection coefficient, $\mathrm{R}_{E}$, at normal incidence for different ions and targets. $\varepsilon_{L}$ is the scaling factor defined in (4), $\mathrm{E}_{\mathrm{sb}}$ the assumed surface binding energy of ions, and fit denotes the applied fitting formula.

\begin{tabular}{|r|r|r|r|r|r|r|r|r|}
\hline ion & solid & $\mathrm{a} 1$ & $\mathrm{a} 2$ & $\mathrm{a} 3$ & $\mathrm{a} 4$ & $\varepsilon_{L}$ & $\mathrm{E}_{\mathrm{sp}}$ & fit \\
\hline $\mathrm{Ar}$ & $\mathrm{Bi}$ & $0.4252 \mathrm{e}-1$ & $-0.1568 \mathrm{e}+0$ & $0.2062 \mathrm{e}+1$ & $0.2077 \mathrm{e}+1$ & $2.78342 \mathrm{e}+5$ & 0.00 & $(6)$ \\
$\mathrm{Kr}$ & $\mathrm{Bi}$ & $0.1092 \mathrm{e}-1$ & $-0.1759 \mathrm{e}+0$ & $0.3666 \mathrm{e}+2$ & $0.2939 \mathrm{e}+1$ & $7.03904 \mathrm{e}+5$ & 0.00 & $(6)$ \\
\hline${ }^{4} \mathrm{He}$ & $\mathrm{Th}$ & $0.2113 \mathrm{e}+0$ & $-0.1152 \mathrm{e}+0$ & $0.1047 \mathrm{e}+1$ & $0.1787 \mathrm{e}+1$ & $2.61980 \mathrm{e}+4$ & 0.00 & $(6)$ \\
$\mathrm{Ne}$ & $\mathrm{Th}$ & $0.9932 \mathrm{e}-1$ & $-0.1299 \mathrm{e}+0$ & $0.1076 \mathrm{e}+1$ & $0.1471 \mathrm{e}+1$ & $1.49508 \mathrm{e}+5$ & 0.00 & $(6)$ \\
$\mathrm{Ar}$ & $\mathrm{Th}$ & $0.4865 \mathrm{e}-1$ & $-0.1527 \mathrm{e}+0$ & $0.2644 \mathrm{e}+1$ & $0.2310 \mathrm{e}+1$ & $3.02995 \mathrm{e}+5$ & 0.00 & $(6)$ \\
$\mathrm{Kr}$ & $\mathrm{Th}$ & $0.1372 \mathrm{e}-1$ & $-0.1745 \mathrm{e}+0$ & $0.1038 \mathrm{e}+1$ & $0.1474 \mathrm{e}+1$ & $7.54519 \mathrm{e}+5$ & 0.00 & $(6)$ \\
$\mathrm{Xe}$ & $\mathrm{Th}$ & $0.7521 \mathrm{e}+1$ & $0.3397 \mathrm{e}+0$ & $0.2093 \mathrm{e}+4$ & $0.5431 \mathrm{e}+0$ & $1.37124 \mathrm{e}+6$ & 0.00 & $(6)$ \\
\hline $\mathrm{H}$ & $\mathrm{U}$ & $0.1920 \mathrm{e}+0$ & $-0.1513 \mathrm{e}+0$ & $0.7672 \mathrm{e}+0$ & $0.1684 \mathrm{e}+1$ & $1.31300 \mathrm{e}+4$ & 1.00 & $(6)$ \\
$\mathrm{D}$ & $\mathrm{U}$ & $0.2953 \mathrm{e}+0$ & $-0.8327 \mathrm{e}-1$ & $0.2337 \mathrm{e}+1$ & $0.1352 \mathrm{e}+1$ & $1.31849 \mathrm{e}+4$ & 1.00 & $(6)$ \\
\hline
\end{tabular}

Table 16: Constants for the energy dependence of the energy reflection coefficient at normal incidence up to high energies.

\begin{tabular}{|r|r|r|r|r|r|r|r|r|}
\hline ion & solid & $\mathrm{b} 1$ & $\mathrm{~b} 2$ & $\mathrm{~b} 3$ & $\mathrm{~b} 4$ & $\mathrm{~b} 5$ & $\mathrm{~b} 6$ & fit \\
\hline $\mathrm{H}$ & $\mathrm{Be}$ & $0.6656 \mathrm{e}-1$ & $-0.3327 \mathrm{e}+0$ & $0.2542 \mathrm{e}+0$ & $0.1342 \mathrm{e}+1$ & $0.1402 \mathrm{e}-2$ & $0.1149 \mathrm{e}+1$ & $(7)$ \\
$\mathrm{D}$ & $\mathrm{Be}$ & $0.3305 \mathrm{e}-1$ & $-0.3314 \mathrm{e}+0$ & $0.1982 \mathrm{e}+0$ & $0.1304 \mathrm{e}+1$ & $0.4603 \mathrm{e}-3$ & $0.1178 \mathrm{e}+1$ & $(7)$ \\
$\mathrm{T}$ & $\mathrm{Be}$ & $0.7229 \mathrm{e}-2$ & $-0.2502 \mathrm{e}+0$ & $0.3048 \mathrm{e}+0$ & $0.1415 \mathrm{e}+1$ & $0.3736 \mathrm{e}+1$ & $0.1321 \mathrm{e}+0$ & $(7)$ \\
${ }^{4} \mathrm{He}$ & $\mathrm{Be}$ & $0.3549 \mathrm{e}-2$ & $-0.5020 \mathrm{e}+0$ & $0.2203 \mathrm{e}+0$ & $0.1289 \mathrm{e}+1$ & $0.3012 \mathrm{e}+1$ & $0.1712 \mathrm{e}+0$ & $(7)$ \\
\hline $\mathrm{H}$ & $\mathrm{C}$ & $0.7983 \mathrm{e}-1$ & $-0.3088 \mathrm{e}+0$ & $0.2743 \mathrm{e}+0$ & $0.1368 \mathrm{e}+1$ & $0.8600 \mathrm{e}-3$ & $0.1261 \mathrm{e}+1$ & $(7)$ \\
$\mathrm{D}$ & $\mathrm{C}$ & $0.4806 \mathrm{e}-1$ & $-0.2963 \mathrm{e}+0$ & $0.1866 \mathrm{e}+0$ & $0.1410 \mathrm{e}+1$ & $0.1778 \mathrm{e}-2$ & $0.1109 \mathrm{e}+1$ & $(7)$ \\
$\mathrm{T}$ & $\mathrm{C}$ & $0.1738 \mathrm{e}-1$ & $-0.1534 \mathrm{e}+0$ & $0.2513 \mathrm{e}+0$ & $0.1446 \mathrm{e}+1$ & $0.2318 \mathrm{e}+1$ & $0.9630 \mathrm{e}-1$ & $(7)$ \\
${ }^{4} \mathrm{He}$ & $\mathrm{C}$ & $0.7452 \mathrm{e}-2$ & $-0.4039 \mathrm{e}+0$ & $0.2403 \mathrm{e}+0$ & $0.1444 \mathrm{e}+1$ & $0.1287 \mathrm{e}+1$ & $0.3144 \mathrm{e}+0$ & $(7)$ \\
\hline $\mathrm{H}$ & $\mathrm{Nb}$ & $0.1723 \mathrm{e}+0$ & $-0.1776 \mathrm{e}+0$ & $0.6878 \mathrm{e}+0$ & $0.1886 \mathrm{e}+1$ & $0.1138 \mathrm{e}-1$ & $0.1575 \mathrm{e}+1$ & $(7)$ \\
\hline $\mathrm{H}$ & $\mathrm{W}$ & $0.1923 \mathrm{e}+0$ & $-0.1605 \mathrm{e}+0$ & $0.1187 \mathrm{e}+1$ & $0.1582 \mathrm{e}+1$ & $0.1424 \mathrm{e}+0$ & $0.1044 \mathrm{e}+1$ & $(7)$ \\
$\mathrm{D}$ & $\mathrm{W}$ & $0.2040 \mathrm{e}+0$ & $-0.1114 \mathrm{e}+0$ & $0.1204 \mathrm{e}+1$ & $0.1420 \mathrm{e}+1$ & $0.4466 \mathrm{e}+0$ & $0.3570 \mathrm{e}+0$ & $(7)$ \\
$\mathrm{T}$ & $\mathrm{W}$ & $0.1253 \mathrm{e}+0$ & $-0.1479 \mathrm{e}+0$ & $0.8593 \mathrm{e}+0$ & $0.1429 \mathrm{e}+1$ & $0.9369 \mathrm{e}+1$ & $0.2049 \mathrm{e}+0$ & $(7)$ \\
$\mathrm{He}$ & $\mathrm{W}$ & $0.1081 \mathrm{e}+0$ & $-0.1252 \mathrm{e}+0$ & $0.8423 \mathrm{e}+0$ & $0.1503 \mathrm{e}+1$ & $0.1776 \mathrm{e}+1$ & $0.1077 \mathrm{e}+0$ & $(7)$ \\
\hline $\mathrm{H}$ & $\mathrm{Au}$ & $0.2006 \mathrm{e}+0$ & $-0.1529 \mathrm{e}+0$ & $0.1192 \mathrm{e}+1$ & $0.1459 \mathrm{e}+1$ & $0.2752 \mathrm{e}-1$ & $0.1154 \mathrm{e}+1$ & $(7)$ \\
$\mathrm{He}$ & $\mathrm{Au}$ & $0.2038 \mathrm{e}+0$ & $-0.1319 \mathrm{e}+0$ & $0.9541 \mathrm{e}+0$ & $0.1510 \mathrm{e}+1$ & $0.1112 \mathrm{e}-1$ & $0.1164 \mathrm{e}+1$ & $(7)$ \\
\hline
\end{tabular}


Table 17: Constants for the angular dependence of the particle reflection coefficient at several energies.

\begin{tabular}{|c|c|c|c|c|c|c|c|}
\hline ion & solid & energy & $\mathrm{c} 1$ & $\mathrm{c} 2$ & c3 & $\mathrm{c} 4$ & fit \\
\hline $\mathrm{H}$ & $\mathrm{Be}$ & 10 & $0.7266 \mathrm{e}+0$ & $0.3173 \mathrm{e}+0$ & $0.2003 e+1$ & $-0.1847 e+1$ & 1.8 \\
\hline $\mathrm{H}$ & $\mathrm{Be}$ & 15 & $0.7188 \mathrm{e}+0$ & $0.3530 \mathrm{e}+0$ & $0.2029 \mathrm{e}+1$ & $-0.1983 e+1$ & 1.8 \\
\hline $\mathrm{H}$ & $\mathrm{Be}$ & 17 & $0.7177 \mathrm{e}+0$ & $0.3634 \mathrm{e}+0$ & $0.2077 \mathrm{e}+1$ & $-0.2074 e+1$ & 1.8 \\
\hline $\mathrm{H}$ & $\mathrm{Be}$ & 20 & $0.7176 \mathrm{e}+0$ & $0.3789 e+0$ & $0.2075 \mathrm{e}+1$ & $-0.2119 e+1$ & 1.8 \\
\hline $\mathrm{H}$ & $\mathrm{Be}$ & 22 & $0.7279 e+0$ & $0.4008 \mathrm{e}+0$ & $0.2019 \mathrm{e}+1$ & $-0.2122 \mathrm{e}+1$ & 1.8 \\
\hline $\mathrm{H}$ & $\mathrm{Be}$ & 25 & $0.7394 \mathrm{e}+0$ & $0.4195 \mathrm{e}+0$ & $0.1994 \mathrm{e}+1$ & $-0.2183 e+1$ & 1.8 \\
\hline $\mathrm{H}$ & $\mathrm{Be}$ & 30 & $0.7403 \mathrm{e}+0$ & $0.4381 \mathrm{e}+0$ & $0.2002 \mathrm{e}+1$ & $-0.2244 \mathrm{e}+1$ & 1.8 \\
\hline $\mathrm{H}$ & $\mathrm{Be}$ & 40 & $0.7839 \mathrm{e}+0$ & $0.5050 \mathrm{e}+0$ & $0.1897 \mathrm{e}+1$ & $-0.2306 e+1$ & 1.8 \\
\hline $\mathrm{H}$ & $\mathrm{Be}$ & 50 & $0.8313 \mathrm{e}+0$ & $0.5726 \mathrm{e}+0$ & $0.1797 \mathrm{e}+1$ & $-0.2317 e+1$ & 1.8 \\
\hline $\mathrm{H}$ & $\mathrm{Be}$ & 70 & $0.9920 \mathrm{e}+0$ & $0.7609 \mathrm{e}+0$ & $0.1635 e+1$ & $-0.2387 e+1$ & 1.8 \\
\hline $\mathrm{H}$ & $\mathrm{Be}$ & 100 & $0.1532 \mathrm{e}+1$ & $0.1330 \mathrm{e}+1$ & $0.1464 \mathrm{e}+1$ & $-0.2774 \mathrm{e}+1$ & 1.8 \\
\hline $\mathrm{H}$ & $\mathrm{Be}$ & 140 & $0.3034 \mathrm{e}+1$ & $0.2859 \mathrm{e}+1$ & $0.1347 \mathrm{e}+1$ & $-0.2887 e+1$ & 1.8 \\
\hline $\mathrm{H}$ & $\mathrm{Be}$ & 200 & $0.3098 \mathrm{e}+1$ & $0.2945 e+1$ & $0.1392 \mathrm{e}+1$ & $-0.2978 \mathrm{e}+1$ & 1.8 \\
\hline $\mathrm{H}$ & $\mathrm{Be}$ & 300 & $0.2946 \mathrm{e}+1$ & $0.2821 \mathrm{e}+1$ & $0.1415 e+1$ & $-0.3003 e+1$ & 1.8 \\
\hline $\mathrm{H}$ & $\mathrm{Be}$ & 500 & $0.2297 \mathrm{e}+1$ & $0.2206 \mathrm{e}+1$ & $0.1461 \mathrm{e}+1$ & $-0.2961 \mathrm{e}+1$ & 1.8 \\
\hline $\mathrm{H}$ & $\mathrm{Be}$ & 1000 & $0.2275 \mathrm{e}+1$ & $0.2227 \mathrm{e}+1$ & $0.1521 \mathrm{e}+1$ & $-0.3090 e+1$ & 1.8 \\
\hline $\mathrm{D}$ & $\mathrm{Be}$ & 11 & $0.6676 \mathrm{e}+0$ & $0.3904 \mathrm{e}+0$ & $0.2012 \mathrm{e}+1$ & $-0.1900 e+1$ & 1.8 \\
\hline D & $\mathrm{Be}$ & 12 & $0.6696 \mathrm{e}+0$ & $0.3957 \mathrm{e}+0$ & $0.2070 \mathrm{e}+1$ & $-0.1986 e+1$ & 1.8 \\
\hline D & $\mathrm{Be}$ & 13 & $0.6701 \mathrm{e}+0$ & $0.4020 \mathrm{e}+0$ & $0.2059 \mathrm{e}+1$ & $-0.1999 e+1$ & 1.8 \\
\hline $\mathrm{D}$ & $\mathrm{Be}$ & 14 & $0.6701 \mathrm{e}+0$ & $0.4112 \mathrm{e}+0$ & $0.2057 \mathrm{e}+1$ & $-0.2013 e+1$ & 1.8 \\
\hline $\mathrm{D}$ & $\mathrm{Be}$ & 15 & $0.6710 \mathrm{e}+0$ & $0.4132 \mathrm{e}+0$ & $0.2086 \mathrm{e}+1$ & $-0.2068 e+1$ & 1.8 \\
\hline $\mathrm{D}$ & $\mathrm{Be}$ & 17 & $0.6694 \mathrm{e}+0$ & $0.4214 \mathrm{e}+0$ & $0.2124 \mathrm{e}+1$ & $-0.2141 e+1$ & 1.8 \\
\hline $\mathrm{D}$ & $\mathrm{Be}$ & 20 & $0.6823 \mathrm{e}+0$ & $0.4486 \mathrm{e}+0$ & $0.2070 \mathrm{e}+1$ & $-0.2168 \mathrm{e}+1$ & 1.8 \\
\hline D & $\mathrm{Be}$ & 25 & $0.7030 \mathrm{e}+0$ & $0.4867 \mathrm{e}+0$ & $0.2004 \mathrm{e}+1$ & $-0.2216 e+1$ & 1.8 \\
\hline $\mathrm{D}$ & $\mathrm{Be}$ & 30 & $0.7146 \mathrm{e}+0$ & $0.5117 \mathrm{e}+0$ & $0.1966 \mathrm{e}+1$ & $-0.2254 \mathrm{e}+1$ & 1.8 \\
\hline $\mathrm{D}$ & $\mathrm{Be}$ & 40 & $0.7530 \mathrm{e}+0$ & $0.5684 \mathrm{e}+0$ & $0.1929 \mathrm{e}+1$ & $-0.2354 \mathrm{e}+1$ & 1.8 \\
\hline D & $\mathrm{Be}$ & 50 & $0.8272 \mathrm{e}+0$ & $0.6554 \mathrm{e}+0$ & $0.1841 \mathrm{e}+1$ & $-0.2413 e+1$ & 1.8 \\
\hline D & $\mathrm{Be}$ & 70 & $0.9430 \mathrm{e}+0$ & $0.7911 \mathrm{e}+0$ & $0.1759 \mathrm{e}+1$ & $-0.2505 e+1$ & 1.8 \\
\hline D & $\mathrm{Be}$ & 100 & $0.1652 \mathrm{e}+1$ & $0.1523 \mathrm{e}+1$ & $0.1526 \mathrm{e}+1$ & $-0.2698 \mathrm{e}+1$ & 1.8 \\
\hline D & $\mathrm{Be}$ & 140 & $0.2509 \mathrm{e}+1$ & $0.2395 \mathrm{e}+1$ & $0.1476 \mathrm{e}+1$ & $-0.2928 \mathrm{e}+1$ & 1.8 \\
\hline D & $\mathrm{Be}$ & 200 & $0.3013 e+1$ & $0.2913 \mathrm{e}+1$ & $0.1497 \mathrm{e}+1$ & $-0.3095 e+1$ & 1.8 \\
\hline D & $\mathrm{Be}$ & 300 & $0.2586 \mathrm{e}+1$ & $0.2504 \mathrm{e}+1$ & $0.1501 \mathrm{e}+1$ & $-0.3043 e+1$ & 1.8 \\
\hline D & $\mathrm{Be}$ & 500 & $0.1839 \mathrm{e}+1$ & $0.1776 \mathrm{e}+1$ & $0.1590 \mathrm{e}+1$ & $-0.3007 e+1$ & 1.8 \\
\hline $\mathrm{D}$ & $\mathrm{Be}$ & 1000 & $0.1482 \mathrm{e}+1$ & $0.1453 \mathrm{e}+1$ & $0.1671 \mathrm{e}+1$ & $-0.3053 e+1$ & 1.8 \\
\hline $\mathrm{Be}$ & $\mathrm{Be}$ & 50 & $0.9095 \mathrm{e}+0$ & $0.9102 \mathrm{e}+0$ & $0.2434 \mathrm{e}+1$ & $-0.3343 e+1$ & 1.8 \\
\hline $\mathrm{Be}$ & $\mathrm{Be}$ & 70 & $0.8716 \mathrm{e}+0$ & $0.8717 \mathrm{e}+0$ & $0.2407 \mathrm{e}+1$ & $-0.3244 \mathrm{e}+1$ & 1.8 \\
\hline $\mathrm{Be}$ & $\mathrm{Be}$ & 100 & $0.8737 \mathrm{e}+0$ & $0.8728 \mathrm{e}+0$ & $0.2376 \mathrm{e}+1$ & $-0.3236 e+1$ & 1.8 \\
\hline $\mathrm{Be}$ & $\mathrm{Be}$ & 200 & $0.8559 \mathrm{e}+0$ & $0.8528 \mathrm{e}+0$ & $0.2395 \mathrm{e}+1$ & $-0.3359 e+1$ & 1.8 \\
\hline $\mathrm{Be}$ & $\mathrm{Be}$ & 300 & $0.1079 \mathrm{e}+1$ & $0.1076 \mathrm{e}+1$ & $0.2336 \mathrm{e}+1$ & $-0.3529 e+1$ & 1.8 \\
\hline $\mathrm{Be}$ & $\mathrm{Be}$ & 500 & $0.1093 e+1$ & $0.1090 \mathrm{e}+1$ & $0.2363 e+1$ & $-0.3667 e+1$ & 1.8 \\
\hline $\mathrm{Be}$ & $\mathrm{Be}$ & 700 & $0.1098 \mathrm{e}+1$ & $0.1095 \mathrm{e}+1$ & $0.2396 \mathrm{e}+1$ & $-0.3772 e+1$ & 1.8 \\
\hline $\mathrm{Be}$ & $\mathrm{Be}$ & 1000 & $0.1068 \mathrm{e}+1$ & $0.1065 \mathrm{e}+1$ & $0.2442 \mathrm{e}+1$ & $-0.3877 e+1$ & 1.8 \\
\hline $\mathrm{Be}$ & $\mathrm{Be}$ & 3000 & $0.1043 e+1$ & $0.1042 \mathrm{e}+0$ & $0.2560 \mathrm{e}+1$ & $-0.4184 e+1$ & 1.8 \\
\hline
\end{tabular}


Table 18: Constants for the angular dependence of the particle reflection coefficient at several energies.

\begin{tabular}{|c|c|c|c|c|c|c|c|}
\hline ion & solid & energy & $\mathrm{c} 1$ & $\mathrm{c} 2$ & c3 & $\mathrm{c} 4$ & fit \\
\hline $\mathrm{T}$ & $\mathrm{Be}$ & 10 & $0.6248 \mathrm{e}+0$ & $0.4389 e+0$ & $0.1987 e+1$ & $-0.1928 \mathrm{e}+1$ & (8) \\
\hline $\mathrm{T}$ & $\mathrm{Be}$ & 11 & $0.6225 \mathrm{e}+0$ & $0.4363 e+0$ & $0.2063 \mathrm{e}+1$ & $-0.1994 e+1$ & (8) \\
\hline $\mathrm{T}$ & $\mathrm{Be}$ & 12 & $0.6295 \mathrm{e}+0$ & $0.4460 \mathrm{e}+0$ & $0.2037 \mathrm{e}+1$ & $-0.2005 e+1$ & (8) \\
\hline $\mathrm{T}$ & $\mathrm{Be}$ & 13 & $0.6336 \mathrm{e}+0$ & $0.4523 \mathrm{e}+0$ & $0.2043 \mathrm{e}+1$ & $-0.2031 e+1$ & (8) \\
\hline $\mathrm{T}$ & $\mathrm{Be}$ & 15 & $0.6446 \mathrm{e}+0$ & $0.4708 \mathrm{e}+0$ & $0.2046 \mathrm{e}+1$ & $-0.2087 e+1$ & (8) \\
\hline $\mathrm{T}$ & $\mathrm{Be}$ & 17 & $0.6406 \mathrm{e}+0$ & $0.4709 \mathrm{e}+0$ & $0.2116 \mathrm{e}+1$ & $-0.2179 \mathrm{e}+1$ & (8) \\
\hline $\mathrm{T}$ & $\mathrm{Be}$ & 20 & $0.6525 \mathrm{e}+0$ & $0.4916 \mathrm{e}+0$ & $0.2117 \mathrm{e}+1$ & $-0.2248 \mathrm{e}+1$ & (8) \\
\hline $\mathrm{T}$ & $\mathrm{Be}$ & 25 & $0.6722 \mathrm{e}+0$ & $0.5239 \mathrm{e}+0$ & $0.2058 \mathrm{e}+1$ & $-0.2292 \mathrm{e}+1$ & (8) \\
\hline $\mathrm{T}$ & $\mathrm{Be}$ & 30 & $0.6962 \mathrm{e}+0$ & $0.5562 \mathrm{e}+0$ & $0.2057 \mathrm{e}+1$ & $-0.2383 e+1$ & (8) \\
\hline $\mathrm{T}$ & $\mathrm{Be}$ & 50 & $0.7915 \mathrm{e}+0$ & $0.6763 \mathrm{e}+0$ & $0.1962 \mathrm{e}+1$ & $-0.2540 \mathrm{e}+1$ & (8) \\
\hline $\mathrm{T}$ & $\mathrm{Be}$ & 100 & $0.1439 \mathrm{e}+1$ & $0.1354 \mathrm{e}+1$ & $0.1663 \mathrm{e}+1$ & $-0.2780 \mathrm{e}+1$ & (8) \\
\hline $\mathrm{T}$ & $\mathrm{Be}$ & 200 & $0.2098 \mathrm{e}+1$ & $0.2034 \mathrm{e}+1$ & $0.1640 \mathrm{e}+1$ & $-0.3072 e+1$ & (8) \\
\hline $\mathrm{T}$ & $\mathrm{Be}$ & 300 & $0.1857 \mathrm{e}+1$ & $0.1804 \mathrm{e}+1$ & $0.1666 \mathrm{e}+1$ & $-0.3074 \mathrm{e}+1$ & (8) \\
\hline $\mathrm{T}$ & $\mathrm{Be}$ & 500 & $0.1714 \mathrm{e}+1$ & $0.1675 \mathrm{e}+1$ & $0.1687 \mathrm{e}+1$ & $-0.3112 \mathrm{e}+1$ & (8) \\
\hline $\mathrm{T}$ & $\mathrm{Be}$ & 1000 & $0.1435 \mathrm{e}+1$ & $0.1412 \mathrm{e}+1$ & $0.1778 \mathrm{e}+1$ & $-0.3192 e+1$ & (8) \\
\hline${ }^{4} \mathrm{He}$ & $\mathrm{Be}$ & 10 & $0.6732 \mathrm{e}+0$ & $0.3763 \mathrm{e}+0$ & $0.2265 \mathrm{e}+1$ & $-0.1836 e+1$ & (8) \\
\hline${ }^{4} \mathrm{He}$ & $\mathrm{Be}$ & 11 & $0.6740 \mathrm{e}+0$ & $0.3868 \mathrm{e}+0$ & $0.2231 \mathrm{e}+1$ & $-0.1851 \mathrm{e}+1$ & (8) \\
\hline${ }^{4} \mathrm{He}$ & $\mathrm{Be}$ & 12 & $0.6685 \mathrm{e}+0$ & $0.3908 \mathrm{e}+0$ & $0.2254 \mathrm{e}+1$ & $-0.1883 e+1$ & (8) \\
\hline${ }^{4} \mathrm{He}$ & $\mathrm{Be}$ & 13 & $0.6603 \mathrm{e}+0$ & $0.3931 \mathrm{e}+0$ & $0.2260 \mathrm{e}+1$ & $-0.1892 \mathrm{e}+1$ & (8) \\
\hline${ }^{4} \mathrm{He}$ & $\mathrm{Be}$ & 15 & $0.6574 \mathrm{e}+0$ & $0.4097 \mathrm{e}+0$ & $0.2253 \mathrm{e}+1$ & $-0.1936 e+1$ & (8) \\
\hline${ }^{4} \mathrm{He}$ & $\mathrm{Be}$ & 17 & $0.6528 \mathrm{e}+0$ & $0.4206 \mathrm{e}+0$ & $0.2259 \mathrm{e}+1$ & $-0.1987 e+1$ & (8) \\
\hline${ }^{4} \mathrm{He}$ & $\mathrm{Be}$ & 20 & $0.6435 \mathrm{e}+0$ & $0.4315 \mathrm{e}+0$ & $0.2322 \mathrm{e}+1$ & $-0.2076 \mathrm{e}+1$ & (8) \\
\hline${ }^{4} \mathrm{He}$ & $\mathrm{Be}$ & 25 & $0.6409 \mathrm{e}+0$ & $0.4553 \mathrm{e}+0$ & $0.2307 \mathrm{e}+1$ & $-0.2165 e+1$ & (8) \\
\hline${ }^{4} \mathrm{He}$ & $\mathrm{Be}$ & 30 & $0.6371 \mathrm{e}+0$ & $0.4687 \mathrm{e}+0$ & $0.2322 \mathrm{e}+1$ & $-0.2256 \mathrm{e}+1$ & (8) \\
\hline${ }^{4} \mathrm{He}$ & $\mathrm{Be}$ & 40 & $0.6472 \mathrm{e}+0$ & $0.5061 \mathrm{e}+0$ & $0.2266 \mathrm{e}+1$ & $-0.2348 \mathrm{e}+1$ & (8) \\
\hline${ }^{4} \mathrm{He}$ & $\mathrm{Be}$ & 50 & $0.6520 \mathrm{e}+0$ & $0.5266 \mathrm{e}+0$ & $0.2287 \mathrm{e}+1$ & $-0.2466 \mathrm{e}+1$ & (8) \\
\hline${ }^{4} \mathrm{He}$ & $\mathrm{Be}$ & 70 & $0.7004 \mathrm{e}+0$ & $0.5960 \mathrm{e}+0$ & $0.2195 \mathrm{e}+1$ & $-0.2580 \mathrm{e}+1$ & (8) \\
\hline${ }^{4} \mathrm{He}$ & $\mathrm{Be}$ & 100 & $0.7850 \mathrm{e}+0$ & $0.6997 \mathrm{e}+0$ & $0.2058 \mathrm{e}+1$ & $-0.2650 \mathrm{e}+1$ & (8) \\
\hline${ }^{4} \mathrm{He}$ & $\mathrm{Be}$ & 140 & $0.9275 \mathrm{e}+0$ & $0.8553 \mathrm{e}+0$ & $0.1991 \mathrm{e}+1$ & $-0.2803 e+1$ & (8) \\
\hline${ }^{4} \mathrm{He}$ & $\mathrm{Be}$ & 200 & $0.1425 \mathrm{e}+1$ & $0.1367 \mathrm{e}+1$ & $0.1794 \mathrm{e}+1$ & $-0.2944 e+1$ & (8) \\
\hline${ }^{4} \mathrm{He}$ & $\mathrm{Be}$ & 300 & $0.2274 \mathrm{e}+1$ & $0.2228 \mathrm{e}+1$ & $0.1730 \mathrm{e}+1$ & $-0.3206 e+1$ & (8) \\
\hline${ }^{4} \mathrm{He}$ & $\mathrm{Be}$ & 400 & $0.1719 \mathrm{e}+1$ & $0.1678 \mathrm{e}+1$ & $0.1816 \mathrm{e}+1$ & $-0.3188 e+1$ & (8) \\
\hline${ }^{4} \mathrm{He}$ & $\mathrm{Be}$ & 500 & $0.1695 \mathrm{e}+1$ & $0.1658 \mathrm{e}+1$ & $0.1827 \mathrm{e}+1$ & $-0.3223 e+1$ & (8) \\
\hline${ }^{4} \mathrm{He}$ & $\mathrm{Be}$ & 700 & $0.1520 \mathrm{e}+1$ & $0.1490 \mathrm{e}+1$ & $0.1855 \mathrm{e}+1$ & $-0.3239 e+1$ & (8) \\
\hline${ }^{4} \mathrm{He}$ & $\mathrm{Be}$ & 1000 & $0.1342 \mathrm{e}+1$ & $0.1318 \mathrm{e}+1$ & $0.1886 \mathrm{e}+1$ & $-0.3246 e+1$ & (8) \\
\hline${ }^{4} \mathrm{He}$ & $\mathrm{Be}$ & 2000 & $0.1222 \mathrm{e}+1$ & $0.1209 \mathrm{e}+1$ & $0.1973 \mathrm{e}+1$ & $-0.3392 e+1$ & (8) \\
\hline${ }^{4} \mathrm{He}$ & $\mathrm{Be}$ & 5000 & $0.1115 \mathrm{e}+1$ & $0.1109 e+1$ & $0.2264 \mathrm{e}+1$ & $-0.3847 e+1$ & (8) \\
\hline${ }^{4} \mathrm{He}$ & $\mathrm{Be}$ & 10000 & $0.1024 \mathrm{e}+1$ & $0.1021 \mathrm{e}+1$ & $0.2688 \mathrm{e}+1$ & $-0.4476 \mathrm{e}+1$ & $(8)$ \\
\hline $\mathrm{D}$ & B & 30 & $0.7468 \mathrm{e}+0$ & $0.4923 \mathrm{e}+0$ & $0.1924 \mathrm{e}+1$ & $-0.2152 \mathrm{e}+1$ & (8) \\
\hline D & B & 50 & $0.7726 \mathrm{e}+0$ & $0.5550 \mathrm{e}+0$ & $0.1897 \mathrm{e}+1$ & $-0.2312 \mathrm{e}+1$ & (8) \\
\hline $\mathrm{D}$ & B & 100 & $0.1105 \mathrm{e}+1$ & $0.9323 \mathrm{e}+0$ & $0.1634 \mathrm{e}+1$ & $-0.2505 e+1$ & (8) \\
\hline D & B & 400 & $0.2702 \mathrm{e}+1$ & $0.2602 \mathrm{e}+1$ & $0.1479 \mathrm{e}+1$ & $-0.3052 e+1$ & (8) \\
\hline D & B & 500 & $0.2163 \mathrm{e}+1$ & $0.2079 \mathrm{e}+1$ & $0.1482 \mathrm{e}+1$ & $-0.2931 e+1$ & (8) \\
\hline $\mathrm{D}$ & B & 8000 & $0.1036 \mathrm{e}+1$ & $0.1031 \mathrm{e}+1$ & $0.2566 \mathrm{e}+1$ & $-0.4262 \mathrm{e}+1$ & (8) \\
\hline B & B & 1000 & $0.1066 \mathrm{e}+1$ & $0.1064 \mathrm{e}+1$ & $0.2269 \mathrm{e}+1$ & $-0.3610 e+1$ & (8) \\
\hline B & B & 2000 & $0.1085 \mathrm{e}+1$ & $0.1083 e+1$ & $0.2438 \mathrm{e}+1$ & $-0.3913 e+1$ & (8) \\
\hline
\end{tabular}


Table 19: Constants for the angular dependence of the particle reflection coefficient at several energies.

\begin{tabular}{|c|c|c|c|c|c|c|c|}
\hline ion & solid & energy & c1 & c2 & c3 & c4 & fit \\
\hline $\mathrm{H}$ & $\mathrm{C}$ & 10 & $0.7546 \mathrm{e}+0$ & $0.2869 \mathrm{e}+0$ & $0.1979 e+1$ & $-0.1854 e+1$ & (8) \\
\hline $\mathrm{H}$ & $\mathrm{C}$ & 20 & $0.7595 \mathrm{e}+0$ & $0.3637 \mathrm{e}+0$ & $0.1915 \mathrm{e}+1$ & $-0.2018 e+1$ & (8) \\
\hline $\mathrm{H}$ & $\mathrm{C}$ & 40 & $0.7526 \mathrm{e}+0$ & $0.3975 \mathrm{e}+0$ & $0.2164 \mathrm{e}+1$ & $-0.2467 \mathrm{e}+1$ & (8) \\
\hline $\mathrm{H}$ & $\mathrm{C}$ & 50 & $0.7964 e+0$ & $0.4606 \mathrm{e}+0$ & $0.1996 \mathrm{e}+1$ & $-0.2422 \mathrm{e}+1$ & (8) \\
\hline $\mathrm{H}$ & $\mathrm{C}$ & 70 & $0.9768 \mathrm{e}+0$ & $0.6845 \mathrm{e}+0$ & $0.1604 e+1$ & $-0.2322 \mathrm{e}+1$ & (8) \\
\hline $\mathrm{H}$ & $\mathrm{C}$ & 100 & $0.1322 \mathrm{e}+1$ & $0.1059 \mathrm{e}+1$ & $0.1457 \mathrm{e}+1$ & $-0.2463 e+1$ & (8) \\
\hline $\mathrm{H}$ & $\mathrm{C}$ & 140 & $0.3355 \mathrm{e}+1$ & $0.3125 \mathrm{e}+1$ & $0.1268 \mathrm{e}+1$ & $-0.2863 e+1$ & (8) \\
\hline $\mathrm{H}$ & $\mathrm{C}$ & 200 & $0.2935 \mathrm{e}+1$ & $0.2727 \mathrm{e}+1$ & $0.1323 e+1$ & $-0.2878 e+1$ & (8) \\
\hline $\mathrm{H}$ & $\mathrm{C}$ & 300 & $0.3093 e+1$ & $0.2916 \mathrm{e}+1$ & $0.1335 e+1$ & $-0.2944 e+1$ & (8) \\
\hline $\mathrm{H}$ & $\mathrm{C}$ & 500 & $0.2755 \mathrm{e}+1$ & $0.2622 \mathrm{e}+1$ & $0.1309 \mathrm{e}+1$ & $-0.2870 \mathrm{e}+1$ & (8) \\
\hline $\mathrm{H}$ & $\mathrm{C}$ & 1000 & $0.2489 \mathrm{e}+1$ & $0.2405 \mathrm{e}+1$ & $0.1383 e+1$ & $-0.2942 \mathrm{e}+1$ & (8) \\
\hline $\mathrm{H}$ & $\mathrm{C}$ & 2000 & $0.1784 \mathrm{e}+1$ & $0.1739 \mathrm{e}+1$ & $0.1632 \mathrm{e}+1$ & $-0.3108 \mathrm{e}+1$ & (8) \\
\hline $\mathrm{H}$ & $\mathrm{C}$ & 13333 & $0.1043 e+1$ & $0.1039 \mathrm{e}+1$ & $0.2893 \mathrm{e}+1$ & $-0.4823 e+1$ & (8) \\
\hline $\mathrm{H}$ & $\mathrm{C}$ & 26667 & $0.1015 \mathrm{e}+1$ & $0.1013 \mathrm{e}+1$ & $0.3525 \mathrm{e}+1$ & $-0.5874 \mathrm{e}+1$ & (8) \\
\hline D & $\mathrm{C}$ & 10 & $0.6983 e+0$ & $0.3396 \mathrm{e}+0$ & $0.2020 \mathrm{e}+1$ & $-0.1887 e+1$ & (8) \\
\hline D & $\mathrm{C}$ & 20 & $0.7129 \mathrm{e}+0$ & $0.4135 \mathrm{e}+0$ & $0.1982 \mathrm{e}+1$ & $-0.2089 e+1$ & (8) \\
\hline $\mathrm{D}$ & $\mathrm{C}$ & 30 & $0.7349 \mathrm{e}+0$ & $0.4624 \mathrm{e}+0$ & $0.1983 \mathrm{e}+1$ & $-0.2256 \mathrm{e}+1$ & (8) \\
\hline D & $\mathrm{C}$ & 33 & $0.7211 \mathrm{e}+0$ & $0.4475 \mathrm{e}+0$ & $0.2035 \mathrm{e}+1$ & $-0.2248 \mathrm{e}+1$ & (8) \\
\hline D & $\mathrm{C}$ & 40 & $0.7832 \mathrm{e}+0$ & $0.5310 \mathrm{e}+0$ & $0.1852 \mathrm{e}+1$ & $-0.2282 \mathrm{e}+1$ & (8) \\
\hline $\mathrm{D}$ & $\mathrm{C}$ & 50 & $0.8113 \mathrm{e}+0$ & $0.5720 \mathrm{e}+0$ & $0.1846 \mathrm{e}+1$ & $-0.2367 \mathrm{e}+1$ & (8) \\
\hline D & $\mathrm{C}$ & 70 & $0.9492 \mathrm{e}+0$ & $0.7331 \mathrm{e}+0$ & $0.1692 \mathrm{e}+1$ & $-0.2416 \mathrm{e}+1$ & (8) \\
\hline D & $\mathrm{C}$ & 100 & $0.1311 \mathrm{e}+1$ & $0.1119 \mathrm{e}+1$ & $0.1532 \mathrm{e}+1$ & $-0.2545 e+1$ & (8) \\
\hline $\mathrm{D}$ & $\mathrm{C}$ & 140 & $0.2893 \mathrm{e}+1$ & $0.2725 \mathrm{e}+1$ & $0.1359 \mathrm{e}+1$ & $-0.2873 e+1$ & (8) \\
\hline D & $\mathrm{C}$ & 200 & $0.2858 \mathrm{e}+1$ & $0.2705 \mathrm{e}+1$ & $0.1388 \mathrm{e}+1$ & $-0.2931 e+1$ & (8) \\
\hline D & $\mathrm{C}$ & 300 & $0.2884 \mathrm{e}+1$ & $0.2750 \mathrm{e}+1$ & $0.1426 \mathrm{e}+1$ & $-0.3016 e+1$ & (8) \\
\hline D & $\mathrm{C}$ & 350 & $0.3048 \mathrm{e}+1$ & $0.2926 \mathrm{e}+1$ & $0.1412 \mathrm{e}+1$ & $-0.3019 e+1$ & (8) \\
\hline $\mathrm{D}$ & $\mathrm{C}$ & 400 & $0.3422 \mathrm{e}+1$ & $0.3305 \mathrm{e}+1$ & $0.1413 e+1$ & $-0.3099 e+1$ & (8) \\
\hline D & $\mathrm{C}$ & 500 & $0.2943 \mathrm{e}+1$ & $0.2838 \mathrm{e}+1$ & $0.1412 \mathrm{e}+1$ & $-0.3043 e+1$ & (8) \\
\hline D & $\mathrm{C}$ & 1000 & $0.1979 \mathrm{e}+1$ & $0.1912 \mathrm{e}+1$ & $0.1452 \mathrm{e}+1$ & $-0.2905 e+1$ & (8) \\
\hline $\mathrm{D}$ & C & 3000 & $0.1529 \mathrm{e}+1$ & $0.1505 \mathrm{e}+1$ & $0.1750 \mathrm{e}+1$ & $-0.3221 e+1$ & (8) \\
\hline D & $\mathrm{C}$ & 10000 & $0.1090 \mathrm{e}+1$ & $0.1084 \mathrm{e}+1$ & $0.2514 \mathrm{e}+1$ & $-0.4240 e+1$ & (8) \\
\hline $\mathrm{T}$ & $\mathrm{C}$ & 10 & $0.6614 \mathrm{e}+0$ & $0.3907 \mathrm{e}+0$ & $0.2008 \mathrm{e}+1$ & $-0.1926 e+1$ & (8) \\
\hline $\mathrm{T}$ & $\mathrm{C}$ & 20 & $0.6794 \mathrm{e}+0$ & $0.4513 \mathrm{e}+0$ & $0.2048 \mathrm{e}+1$ & $-0.2167 \mathrm{e}+1$ & (8) \\
\hline $\mathrm{T}$ & $\mathrm{C}$ & 25 & $0.6940 \mathrm{e}+0$ & $0.4799 \mathrm{e}+0$ & $0.2014 \mathrm{e}+1$ & $-0.2226 e+1$ & $(8)$ \\
\hline $\mathrm{T}$ & $\mathrm{C}$ & 30 & $0.7262 \mathrm{e}+0$ & $0.5225 \mathrm{e}+0$ & $0.1956 \mathrm{e}+1$ & $-0.2280 e+1$ & (8) \\
\hline $\mathrm{T}$ & $\mathrm{C}$ & 40 & $0.7491 \mathrm{e}+0$ & $0.5606 \mathrm{e}+0$ & $0.1943 e+1$ & $-0.2372 \mathrm{e}+1$ & (8) \\
\hline $\mathrm{T}$ & $\mathrm{C}$ & 50 & $0.8132 \mathrm{e}+0$ & $0.6394 \mathrm{e}+0$ & $0.1831 \mathrm{e}+1$ & $-0.2386 e+1$ & (8) \\
\hline $\mathrm{T}$ & $\mathrm{C}$ & 70 & $0.9731 \mathrm{e}+0$ & $0.8172 \mathrm{e}+0$ & $0.1710 \mathrm{e}+1$ & $-0.2478 \mathrm{e}+1$ & (8) \\
\hline $\mathrm{T}$ & $\mathrm{C}$ & 100 & $0.1290 \mathrm{e}+1$ & $0.1153 e+1$ & $0.1597 \mathrm{e}+1$ & $-0.2614 \mathrm{e}+1$ & (8) \\
\hline $\mathrm{T}$ & C & 140 & $0.2341 \mathrm{e}+1$ & $0.2219 \mathrm{e}+1$ & $0.1465 \mathrm{e}+1$ & $-0.2876 e+1$ & $(8)$ \\
\hline $\mathrm{T}$ & $\mathrm{C}$ & 200 & $0.2726 \mathrm{e}+1$ & $0.2619 \mathrm{e}+1$ & $0.1470 \mathrm{e}+1$ & $-0.3001 e+1$ & (8) \\
\hline $\mathrm{T}$ & $\mathrm{C}$ & 300 & $0.2835 \mathrm{e}+1$ & $0.2743 \mathrm{e}+1$ & $0.1450 \mathrm{e}+1$ & $-0.3032 \mathrm{e}+1$ & (8) \\
\hline $\mathrm{T}$ & $\mathrm{C}$ & 500 & $0.2102 \mathrm{e}+1$ & $0.2028 \mathrm{e}+1$ & $0.1501 \mathrm{e}+1$ & $-0.2965 e+1$ & (8) \\
\hline $\mathrm{T}$ & $\mathrm{C}$ & 1000 & $0.1778 \mathrm{e}+1$ & $0.1731 \mathrm{e}+1$ & $0.1525 \mathrm{e}+1$ & $-0.2950 \mathrm{e}+1$ & $(8)$ \\
\hline
\end{tabular}


Table 20: Constants for the angular dependence of the particle reflection coefficient at several energies.

\begin{tabular}{|c|c|c|c|c|c|c|c|}
\hline ion & solid & energy & $\mathrm{c} 1$ & $\mathrm{c} 2$ & c3 & c4 & fit \\
\hline${ }^{4} \mathrm{He}$ & $\mathrm{C}$ & 10 & $0.7210 \mathrm{e}+0$ & $0.3304 \mathrm{e}+0$ & $0.2162 \mathrm{e}+1$ & $-0.1776 \mathrm{e}+1$ & $(8)$ \\
\hline${ }^{4} \mathrm{He}$ & $\mathrm{C}$ & 15 & $0.6935 \mathrm{e}+0$ & $0.3701 \mathrm{e}+0$ & $0.2222 \mathrm{e}+1$ & $-0.1909 e+1$ & (8) \\
\hline${ }^{4} \mathrm{He}$ & $\mathrm{C}$ & 20 & $0.6803 \mathrm{e}+0$ & $0.3957 \mathrm{e}+0$ & $0.2258 \mathrm{e}+1$ & $-0.2043 e+1$ & (8) \\
\hline${ }^{4} \mathrm{He}$ & $\mathrm{C}$ & 25 & $0.6719 \mathrm{e}+0$ & $0.4167 \mathrm{e}+0$ & $0.2240 \mathrm{e}+1$ & $-0.2114 \mathrm{e}+1$ & (8) \\
\hline${ }^{4} \mathrm{He}$ & C & 27 & $0.6722 \mathrm{e}+0$ & $0.4305 \mathrm{e}+0$ & $0.2208 \mathrm{e}+1$ & $-0.2116 \mathrm{e}+1$ & (8) \\
\hline${ }^{4} \mathrm{He}$ & $\mathrm{C}$ & 30 & $0.6698 \mathrm{e}+0$ & $0.4347 \mathrm{e}+0$ & $0.2279 \mathrm{e}+1$ & $-0.2217 \mathrm{e}+1$ & (8) \\
\hline${ }^{4} \mathrm{He}$ & $\mathrm{C}$ & 35 & $0.6693 \mathrm{e}+0$ & $0.4504 \mathrm{e}+0$ & $0.2273 \mathrm{e}+1$ & $-0.2284 \mathrm{e}+1$ & (8) \\
\hline${ }^{4} \mathrm{He}$ & C & 40 & $0.6730 \mathrm{e}+0$ & $0.4663 \mathrm{e}+0$ & $0.2251 \mathrm{e}+1$ & $-0.2330 \mathrm{e}+1$ & (8) \\
\hline${ }^{4} \mathrm{He}$ & C & 50 & $0.6929 \mathrm{e}+0$ & $0.5054 \mathrm{e}+0$ & $0.2169 \mathrm{e}+1$ & $-0.2374 \mathrm{e}+1$ & (8) \\
\hline${ }^{4} \mathrm{He}$ & $\mathrm{C}$ & 60 & $0.7083 \mathrm{e}+0$ & $0.5357 \mathrm{e}+0$ & $0.2097 \mathrm{e}+1$ & $-0.2394 \mathrm{e}+1$ & (8) \\
\hline${ }^{4} \mathrm{He}$ & C & 70 & $0.7393 \mathrm{e}+0$ & $0.5782 \mathrm{e}+0$ & $0.2041 \mathrm{e}+1$ & $-0.2433 e+1$ & (8) \\
\hline${ }^{4} \mathrm{He}$ & $\mathrm{C}$ & 100 & $0.8013 e+0$ & $0.6619 e+0$ & $0.1970 \mathrm{e}+1$ & $-0.2537 \mathrm{e}+1$ & (8) \\
\hline${ }^{4} \mathrm{He}$ & $\mathrm{C}$ & 140 & $0.9759 \mathrm{e}+0$ & $0.8550 \mathrm{e}+0$ & $0.1802 \mathrm{e}+1$ & $-0.2596 \mathrm{e}+1$ & (8) \\
\hline${ }^{4} \mathrm{He}$ & $\mathrm{C}$ & 200 & $0.1525 \mathrm{e}+1$ & $0.1423 e+1$ & $0.1635 \mathrm{e}+1$ & $-0.2780 e+1$ & (8) \\
\hline${ }^{4} \mathrm{He}$ & C & 300 & $0.2708 \mathrm{e}+1$ & $0.2623 \mathrm{e}+1$ & $0.1547 \mathrm{e}+1$ & $-0.3066 \mathrm{e}+1$ & (8) \\
\hline${ }^{4} \mathrm{He}$ & $\mathrm{C}$ & 400 & $0.2369 \mathrm{e}+1$ & $0.2292 \mathrm{e}+1$ & $0.1599 \mathrm{e}+1$ & $-0.3088 e+1$ & (8) \\
\hline${ }^{4} \mathrm{He}$ & $\mathrm{C}$ & 500 & $0.2008 \mathrm{e}+1$ & $0.1936 \mathrm{e}+1$ & $0.1660 \mathrm{e}+1$ & $-0.3090 \mathrm{e}+1$ & (8) \\
\hline${ }^{4} \mathrm{He}$ & $\mathrm{C}$ & 700 & $0.1839 e+1$ & $0.1778 e+1$ & $0.1633 e+1$ & $-0.3039 e+1$ & (8) \\
\hline${ }^{4} \mathrm{He}$ & $\mathrm{C}$ & 1000 & $0.1935 \mathrm{e}+1$ & $0.1885 e+1$ & $0.1635 \mathrm{e}+1$ & $-0.3107 e+1$ & (8) \\
\hline${ }^{4} \mathrm{He}$ & $\mathrm{C}$ & 2000 & $0.1619 \mathrm{e}+1$ & $0.1588 \mathrm{e}+1$ & $0.1672 \mathrm{e}+1$ & $-0.3118 e+1$ & (8) \\
\hline${ }^{4} \mathrm{He}$ & $\mathrm{C}$ & 3000 & $0.1485 \mathrm{e}+1$ & $0.1463 \mathrm{e}+1$ & $0.1738 \mathrm{e}+1$ & $-0.3196 e+1$ & (8) \\
\hline${ }^{4} \mathrm{He}$ & $\mathrm{C}$ & 5000 & $0.1250 \mathrm{e}+1$ & $0.1235 \mathrm{e}+1$ & $0.2082 \mathrm{e}+1$ & $-0.3543 e+1$ & (8) \\
\hline${ }^{4} \mathrm{He}$ & $\mathrm{C}$ & 10000 & $0.1125 \mathrm{e}+1$ & $0.1118 \mathrm{e}+1$ & $0.2306 \mathrm{e}+1$ & $-0.3902 e+1$ & (8) \\
\hline${ }^{4} \mathrm{He}$ & $\mathrm{C}$ & 20000 & $0.1050 \mathrm{e}+1$ & $0.1047 \mathrm{e}+1$ & $0.2827 \mathrm{e}+1$ & $-0.4677 \mathrm{e}+1$ & (8) \\
\hline $\mathrm{C}$ & $\mathrm{C}$ & 100 & $0.9463 \mathrm{e}+0$ & $0.9467 \mathrm{e}+0$ & $0.2431 \mathrm{e}+1$ & $-0.3591 e+1$ & (8) \\
\hline $\mathrm{C}$ & $\mathrm{C}$ & 140 & $0.9218 \mathrm{e}+0$ & $0.9216 \mathrm{e}+0$ & $0.2402 \mathrm{e}+1$ & $-0.3468 \mathrm{e}+1$ & (8) \\
\hline $\mathrm{C}$ & $\mathrm{C}$ & 200 & $0.8600 \mathrm{e}+0$ & $0.8588 \mathrm{e}+0$ & $0.2368 \mathrm{e}+1$ & $-0.3379 e+1$ & (8) \\
\hline $\mathrm{C}$ & $\mathrm{C}$ & 300 & $0.8906 \mathrm{e}+0$ & $0.8882 \mathrm{e}+0$ & $0.2356 \mathrm{e}+1$ & $-0.3417 e+1$ & (8) \\
\hline $\mathrm{C}$ & $\mathrm{C}$ & 500 & $0.1077 \mathrm{e}+1$ & $0.1074 \mathrm{e}+1$ & $0.2267 \mathrm{e}+1$ & $-0.3518 e+1$ & (8) \\
\hline $\mathrm{C}$ & $\mathrm{C}$ & 1000 & $0.1108 \mathrm{e}+1$ & $0.1105 \mathrm{e}+1$ & $0.2281 \mathrm{e}+1$ & $-0.3649 e+1$ & (8) \\
\hline $\mathrm{Ne}$ & $\mathrm{C}$ & 10 & $0.5301 \mathrm{e}+0$ & $0.5223 \mathrm{e}+0$ & $0.2891 \mathrm{e}+1$ & $-0.2624 \mathrm{e}+1$ & (8) \\
\hline $\mathrm{Ne}$ & C & 15 & $0.8172 \mathrm{e}+0$ & $0.8197 \mathrm{e}+0$ & $0.2303 \mathrm{e}+1$ & $-0.2689 e+1$ & (8) \\
\hline $\mathrm{Ne}$ & $\mathrm{C}$ & 20 & $0.8983 \mathrm{e}+0$ & $0.9014 \mathrm{e}+0$ & $0.2313 \mathrm{e}+1$ & $-0.2854 \mathrm{e}+1$ & (8) \\
\hline $\mathrm{Ne}$ & $\mathrm{C}$ & 22 & $0.8858 \mathrm{e}+0$ & $0.8888 \mathrm{e}+0$ & $0.2351 \mathrm{e}+1$ & $-0.2864 \mathrm{e}+1$ & (8) \\
\hline $\mathrm{Ne}$ & C & 25 & $0.9441 \mathrm{e}+0$ & $0.9471 \mathrm{e}+0$ & $0.2344 \mathrm{e}+1$ & $-0.2954 \mathrm{e}+1$ & (8) \\
\hline $\mathrm{Ne}$ & $\mathrm{C}$ & 30 & $0.9533 \mathrm{e}+0$ & $0.9556 \mathrm{e}+0$ & $0.2435 \mathrm{e}+1$ & $-0.3089 e+1$ & (8) \\
\hline $\mathrm{Ne}$ & $\mathrm{C}$ & 35 & $0.9479 \mathrm{e}+0$ & $0.9499 \mathrm{e}+0$ & $0.2478 \mathrm{e}+1$ & $-0.3141 \mathrm{e}+1$ & (8) \\
\hline $\mathrm{Ne}$ & $\mathrm{C}$ & 40 & $0.9492 \mathrm{e}+0$ & $0.9508 \mathrm{e}+0$ & $0.2563 \mathrm{e}+1$ & $-0.3250 \mathrm{e}+1$ & (8) \\
\hline $\mathrm{Ne}$ & C & 45 & $0.9330 \mathrm{e}+0$ & $0.9343 \mathrm{e}+0$ & $0.2623 \mathrm{e}+1$ & $-0.3299 e+1$ & (8) \\
\hline $\mathrm{Ne}$ & $\mathrm{C}$ & 50 & $0.9462 \mathrm{e}+0$ & $0.9473 \mathrm{e}+0$ & $0.2656 \mathrm{e}+1$ & $-0.3383 e+1$ & (8) \\
\hline $\mathrm{Ne}$ & C & 60 & $0.9616 \mathrm{e}+0$ & $0.9625 \mathrm{e}+0$ & $0.2699 \mathrm{e}+1$ & $-0.3459 e+1$ & (8) \\
\hline $\mathrm{Ne}$ & $\mathrm{C}$ & 70 & $0.9629 \mathrm{e}+0$ & $0.9638 \mathrm{e}+0$ & $0.2738 \mathrm{e}+1$ & $-0.3536 \mathrm{e}+1$ & (8) \\
\hline
\end{tabular}


Table 21: Constants for the angular dependence of the particle reflection coefficient at several energies.

\begin{tabular}{|c|c|c|c|c|c|c|c|}
\hline ion & solid & energy & $\mathrm{c} 1$ & c2 & c3 & $\mathrm{c} 4$ & fit \\
\hline $\mathrm{H}$ & $\mathrm{Al}$ & 13333 & $0.1177 \mathrm{e}+1$ & $0.1162 \mathrm{e}+1$ & $0.2026 \mathrm{e}+1$ & $-0.3532 \mathrm{e}+1$ & (8) \\
\hline $\mathrm{H}$ & $\mathrm{Al}$ & 26667 & $0.1016 \mathrm{e}+1$ & $0.1012 \mathrm{e}+1$ & $0.2934 \mathrm{e}+1$ & $-0.4897 e+1$ & (8) \\
\hline $\mathrm{H}$ & $\mathrm{Al}$ & 40000 & $0.1003 e+1$ & $0.1001 \mathrm{e}+1$ & $0.3401 \mathrm{e}+1$ & $-0.5644 e+1$ & (8) \\
\hline Ar & $\mathrm{Al}$ & 100 & $0.9423 e+0$ & $0.9434 \mathrm{e}+0$ & $0.2342 \mathrm{e}+1$ & $-0.3026 e+1$ & (8) \\
\hline $\mathrm{Ar}$ & $\mathrm{Al}$ & 1000 & $0.9620 \mathrm{e}+0$ & $0.9608 \mathrm{e}+0$ & $0.2542 \mathrm{e}+1$ & $-0.3720 \mathrm{e}+1$ & (8) \\
\hline Ar & $\mathrm{Al}$ & 1050 & $0.9458 \mathrm{e}+0$ & $0.9445 \mathrm{e}+0$ & $0.2551 \mathrm{e}+1$ & $-0.3735 e+1$ & (8) \\
\hline $\mathrm{Ar}$ & $\mathrm{Al}$ & 10000 & $0.1046 \mathrm{e}+1$ & $0.1046 \mathrm{e}+1$ & $0.2686 \mathrm{e}+1$ & $-0.4301 e+1$ & (8) \\
\hline${ }^{4} \mathrm{He}$ & $\mathrm{Si}$ & 200 & $0.5273 \mathrm{e}+1$ & $0.5018 \mathrm{e}+1$ & $0.1238 \mathrm{e}+1$ & $-0.3091 e+1$ & $(8)$ \\
\hline${ }^{4} \mathrm{He}$ & $\mathrm{Si}$ & 2000 & $0.2907 \mathrm{e}+1$ & $0.2776 \mathrm{e}+1$ & $0.1315 \mathrm{e}+1$ & $-0.2937 e+1$ & (8) \\
\hline${ }^{4} \mathrm{He}$ & $\mathrm{Si}$ & 3000 & $0.2576 \mathrm{e}+1$ & $0.2468 \mathrm{e}+1$ & $0.1346 \mathrm{e}+1$ & $-0.2923 e+1$ & (8) \\
\hline $\mathrm{Si}(\mathrm{KrC})$ & $\mathrm{Si}$ & 200 & $0.8545 \mathrm{e}+0$ & $0.8531 \mathrm{e}+0$ & $0.2073 \mathrm{e}+1$ & $-0.2926 e+1$ & (8) \\
\hline $\mathrm{Si}(\mathrm{Mol})$ & $\mathrm{Si}$ & 200 & $0.6563 e+0$ & $0.6520 \mathrm{e}+0$ & $0.2279 \mathrm{e}+1$ & $-0.2831 e+1$ & (8) \\
\hline $\mathrm{Si}(\mathrm{Si})$ & $\mathrm{Si}$ & 200 & $0.9021 \mathrm{e}+0$ & $0.9013 e+0$ & $0.1965 \mathrm{e}+1$ & $-0.3109 e+1$ & (8) \\
\hline $\mathrm{Si}(\mathrm{ZBL})$ & $\mathrm{Si}$ & 200 & $0.8430 \mathrm{e}+0$ & $0.8410 \mathrm{e}+0$ & $0.2103 e+1$ & $-0.2993 e+1$ & (8) \\
\hline $\mathrm{Si}$ & $\mathrm{Si}$ & 500 & $0.1194 \mathrm{e}+1$ & $0.1187 \mathrm{e}+1$ & $0.1992 \mathrm{e}+1$ & $-0.3149 e+1$ & (8) \\
\hline $\mathrm{Si}$ & $\mathrm{Si}$ & 2000 & $0.1241 \mathrm{e}+1$ & $0.1234 \mathrm{e}+1$ & $0.2032 \mathrm{e}+1$ & $-0.3383 e+1$ & (8) \\
\hline Ar & $\mathrm{Si}$ & 4500 & $0.1044 \mathrm{e}+1$ & $0.1042 \mathrm{e}+0$ & $0.2470 \mathrm{e}+1$ & $-0.3938 e+1$ & (8) \\
\hline${ }^{4} \mathrm{He}$ & $\mathrm{Ti}$ & 5000 & $0.6067 \mathrm{e}+0$ & $0.4524 \mathrm{e}+0$ & $0.1580 \mathrm{e}+1$ & $-0.2164 \mathrm{e}+1$ & $(8)$ \\
\hline${ }^{4} \mathrm{He}$ & $\mathrm{Ti}$ & 100000 & $0.1057 \mathrm{e}+1$ & $0.1050 \mathrm{e}+1$ & $0.2499 \mathrm{e}+1$ & $-0.4193 e+1$ & (8) \\
\hline $\mathrm{Ne}$ & $\mathrm{Ti}$ & 38 & $0.7014 \mathrm{e}+0$ & $0.3976 \mathrm{e}+0$ & $0.1936 \mathrm{e}+1$ & $-0.1727 e+1$ & (8) \\
\hline $\mathrm{Ne}$ & $\mathrm{Ti}$ & 380 & $0.1035 \mathrm{e}+1$ & $0.8997 \mathrm{e}+0$ & $0.1637 \mathrm{e}+1$ & $-0.2430 \mathrm{e}+1$ & (8) \\
\hline $\mathrm{Ne}$ & $\mathrm{Ti}$ & 3800 & $0.1820 \mathrm{e}+1$ & $0.1751 \mathrm{e}+1$ & $0.1495 \mathrm{e}+1$ & $-0.2889 e+1$ & (8) \\
\hline Ar & $\mathrm{Ti}$ & 1050 & $0.1762 \mathrm{e}+1$ & $0.1727 \mathrm{e}+1$ & $0.1725 \mathrm{e}+1$ & $-0.3026 e+1$ & (8) \\
\hline $\mathrm{Ar}$ & $\mathrm{Ti}$ & 150000 & $0.1056 \mathrm{e}+1$ & $0.1054 \mathrm{e}+1$ & $0.2357 \mathrm{e}+1$ & $-0.3981 e+1$ & $(8)$ \\
\hline $\mathrm{Ga}$ & $\mathrm{Ga}$ & 100 & $0.7389 \mathrm{e}+0$ & $0.7374 \mathrm{e}+0$ & $0.2022 \mathrm{e}+1$ & $-0.2503 e+1$ & (8) \\
\hline $\mathrm{Ga}$ & $\mathrm{Ga}$ & 200 & & $0.7634 \mathrm{e}+0$ & $0.1959 \mathrm{e}+1$ & $-0.2529 e+1$ & (8) \\
\hline $\mathrm{Ga}$ & $\mathrm{Ga}$ & 1000 & $0.1452 \mathrm{e}+1$ & $0.1432 \mathrm{e}+1$ & $0.1829 \mathrm{e}+1$ & $-0.3032 e+1$ & $(8)$ \\
\hline $\mathrm{Ar}$ & $\mathrm{Zr}$ & 1050 & $0.2740 \mathrm{e}+1$ & $0.2599 \mathrm{e}+1$ & $0.1388 \mathrm{e}+1$ & $-0.2880 e+1$ & (8) \\
\hline Ar & $\mathrm{Zr}$ & 150000 & $0.1336 \mathrm{e}+1$ & $0.1315 \mathrm{e}+1$ & $0.1710 \mathrm{e}+1$ & $-0.3127 e+1$ & (8) \\
\hline $\mathrm{Ar}$ & $\mathrm{Zr}$ & 900000 & $0.1070 \mathrm{e}+1$ & $0.1066 e+1$ & $0.2378 \mathrm{e}+1$ & $-0.4047 e+1$ & (8) \\
\hline $\mathrm{H}$ & $\mathrm{Nb}$ & 1000 & $0.5987 \mathrm{e}+1$ & $0.5596 \mathrm{e}+1$ & $0.1185 \mathrm{e}+1$ & $-0.3279 e+1$ & (8) \\
\hline $\mathrm{H}$ & $\mathrm{Nb}$ & 2000 & $0.5809 \mathrm{e}+1$ & $0.5501 \mathrm{e}+1$ & $0.1098 \mathrm{e}+1$ & $-0.3103 e+1$ & (8) \\
\hline $\mathrm{H}$ & $\mathrm{Nb}$ & 5000 & $0.3644 \mathrm{e}+1$ & $0.3451 \mathrm{e}+1$ & $0.1072 \mathrm{e}+1$ & $-0.2776 e+1$ & (8) \\
\hline $\mathrm{H}$ & $\mathrm{Nb}$ & 10000 & $0.2441 \mathrm{e}+1$ & $0.2321 \mathrm{e}+1$ & $0.1218 \mathrm{e}+1$ & $-0.2732 e+1$ & (8) \\
\hline D & $\mathrm{Nb}$ & 12200 & $0.3139 \mathrm{e}+1$ & $0.3016 \mathrm{e}+1$ & $0.1152 \mathrm{e}+1$ & $-0.2787 e+1$ & (8) \\
\hline${ }^{4} \mathrm{He}$ & $\mathrm{Nb}$ & 36500 & $0.2023 e+1$ & $0.1951 \mathrm{e}+1$ & $0.1329 \mathrm{e}+1$ & $-0.2791 e+1$ & (8) \\
\hline $\mathrm{Nb}$ & $\mathrm{Nb}$ & 60000 & $0.1165 e+1$ & $0.1158 \mathrm{e}+1$ & $0.1997 \mathrm{e}+1$ & $-0.3436 e+1$ & $(8)$ \\
\hline $\mathrm{Ar}$ & $\mathrm{Pd}$ & 1050 & $0.1213 e+1$ & $0.1031 \mathrm{e}+1$ & $0.1514 \mathrm{e}+1$ & $-0.2432 \mathrm{e}+1$ & $(8)$ \\
\hline $\mathrm{D}$ & $\mathrm{Ag}$ & 100 & $0.1152 \mathrm{e}+1$ & $0.6071 \mathrm{e}+0$ & $0.1261 \mathrm{e}+1$ & $-0.2078 e+1$ & (8) \\
\hline $\mathrm{Ne}$ & $\mathrm{Ag}$ & 45000 & $0.2772 \mathrm{e}+1$ & $0.2675 \mathrm{e}+1$ & $0.1244 \mathrm{e}+1$ & $-0.2857 e+1$ & (8) \\
\hline Ar & $\mathrm{Ag}$ & 1050 & $0.1319 \mathrm{e}+1$ & $0.1138 \mathrm{e}+1$ & $0.1492 \mathrm{e}+1$ & $-0.2492 \mathrm{e}+1$ & (8) \\
\hline Ar & $\mathrm{Ag}$ & 150000 & $0.1584 \mathrm{e}+1$ & $0.1550 \mathrm{e}+1$ & $0.1582 \mathrm{e}+1$ & $-0.3029 e+1$ & (8) \\
\hline $\mathrm{Ar}$ & $\mathrm{Ag}$ & 900000 & $0.9460 \mathrm{e}+0$ & $0.9390 \mathrm{e}+0$ & $0.2235 \mathrm{e}+1$ & $-0.3718 e+1$ & (8) \\
\hline $\mathrm{Kr}$ & $\mathrm{Ag}$ & 45000 & $0.1433 \mathrm{e}+1$ & $0.1414 \mathrm{e}+1$ & $0.1731 \mathrm{e}+1$ & $-0.3152 e+1$ & $(8)$ \\
\hline $\mathrm{H}$ & In & 2000 & $0.4127 \mathrm{e}+1$ & $0.3791 \mathrm{e}+1$ & $0.1057 \mathrm{e}+1$ & $-0.2890 e+1$ & (8) \\
\hline In & In & 100 & $0.7961 \mathrm{e}+0$ & $0.7929 \mathrm{e}+0$ & $0.1923 e+1$ & $-0.2536 e+1$ & (8) \\
\hline In & In & 200 & $0.8956 \mathrm{e}+0$ & $0.8854 \mathrm{e}+0$ & $0.1817 \mathrm{e}+1$ & $-0.2543 e+1$ & (8) \\
\hline In & In & 1000 & $0.1808 \mathrm{e}+1$ & $0.1788 \mathrm{e}+1$ & $0.1641 \mathrm{e}+1$ & $-0.2951 \mathrm{e}+1$ & (8) \\
\hline
\end{tabular}


Table 22: Constants for the angular dependence of the particle reflection coefficient at several energies.

\begin{tabular}{|c|c|c|c|c|c|c|c|}
\hline ion & solid & energy & c1 & c2 & c3 & c4 & fit \\
\hline $\mathrm{H}$ & $\mathrm{V}$ & 100 & $0.2615 \mathrm{e}+1$ & $0.2131 \mathrm{e}+1$ & $0.1169 \mathrm{e}+1$ & $-0.2674 \mathrm{e}+1$ & (8) \\
\hline $\mathrm{H}$ & $\mathrm{V}$ & 120 & $0.1499 \mathrm{e}+1$ & $0.1025 e+1$ & $0.1257 \mathrm{e}+1$ & $-0.2367 e+1$ & (8) \\
\hline $\mathrm{H}$ & $\mathrm{V}$ & 140 & $0.1624 \mathrm{e}+1$ & $0.1161 \mathrm{e}+1$ & $0.1238 \mathrm{e}+1$ & $-0.2425 \mathrm{e}+1$ & (8) \\
\hline $\mathrm{H}$ & $\mathrm{V}$ & 200 & $0.4116 \mathrm{e}+1$ & $0.3682 \mathrm{e}+1$ & $0.1136 \mathrm{e}+1$ & $-0.2937 e+1$ & (8) \\
\hline $\mathrm{H}$ & $\mathrm{V}$ & 400 & $0.5190 \mathrm{e}+1$ & $0.4808 \mathrm{e}+1$ & $0.1197 \mathrm{e}+1$ & $154 e+1$ & (8) \\
\hline $\mathrm{H}$ & $\mathrm{V}$ & 1000 & $0.4799 \mathrm{e}+1$ & $0.4506 \mathrm{e}+1$ & $0.1184 \mathrm{e}+1$ & $-0.3076 \mathrm{e}+1$ & (8) \\
\hline $\mathrm{H}$ & $\mathrm{V}$ & 3000 & $0.3331 \mathrm{e}+1$ & $0.3159 \mathrm{e}+1$ & $0.1269 \mathrm{e}+1$ & $-0.2962 \mathrm{e}+1$ & (8) \\
\hline $\mathrm{H}$ & $\mathrm{V}$ & 10000 & $0.1787 \mathrm{e}+1$ & $0.1731 \mathrm{e}+1$ & $0.1452 \mathrm{e}+1$ & $-0.2880 \mathrm{e}+1$ & (8) \\
\hline $\mathrm{D}$ & $\mathrm{V}$ & 55 & $0.9863 \mathrm{e}+0$ & $0.4805 \mathrm{e}+0$ & $0.1485 \mathrm{e}+1$ & $-0.2096 e+1$ & (8) \\
\hline $\mathrm{D}$ & $\mathrm{V}$ & 60 & $0.9827 \mathrm{e}+0$ & $0.4837 \mathrm{e}+0$ & $0.1514 \mathrm{e}+1$ & $30 e+1$ & (8) \\
\hline $\mathrm{D}$ & $\mathrm{V}$ & 70 & $0.1058 \mathrm{e}+1$ & $0.5700 \mathrm{e}+0$ & $0.1413 \mathrm{e}+1$ & $37 e+1$ & (8) \\
\hline $\mathrm{D}$ & $\mathrm{V}$ & 100 & $0.9835 \mathrm{e}+0$ & $0.5098 \mathrm{e}+0$ & $0.1549 \mathrm{e}+1$ & $-0.2254 \mathrm{e}+1$ & (8) \\
\hline $\mathrm{D}$ & $\mathrm{V}$ & 200 & $0.4686 \mathrm{e}+1$ & $0.4270 \mathrm{e}+1$ & $0.1133 \mathrm{e}+1$ & $-0.2990 \mathrm{e}+1$ & (8) \\
\hline $\mathrm{D}$ & $\mathrm{V}$ & 500 & $0.4728 \mathrm{e}+1$ & $0.4366 \mathrm{e}+1$ & $0.1213 \mathrm{e}+1$ & $-0.3123 e+1$ & (8) \\
\hline $\mathrm{D}$ & $\mathrm{V}$ & 1000 & 0.4 & & & +1 & (8) \\
\hline $\mathrm{D}$ & $\mathrm{V}$ & 3000 & $0.3362 \mathrm{e}+1$ & $0.3180 \mathrm{e}+1$ & $0.1151 \mathrm{e}+1$ & $18 e+1$ & (8) \\
\hline $\mathrm{D}$ & $\mathrm{V}$ & 10000 & $0.2164 \mathrm{e}+1$ & $0.2095 \mathrm{e}+1$ & $0.1357 \mathrm{e}+1$ & $54 e+1$ & (8) \\
\hline $\mathrm{T}$ & $\mathrm{V}$ & 40 & $17 \mathrm{e}+0$ & $7 e+0$ & $0.1705 \mathrm{e}+1$ & $4 e+1$ & (8) \\
\hline $\mathrm{T}$ & $\mathrm{V}$ & 50 & $0.9345 \mathrm{e}+0$ & $0.4559 \mathrm{e}+0$ & $0.1597 \mathrm{e}+1$ & $36 e+1$ & (8) \\
\hline $\mathrm{T}$ & $\mathrm{V}$ & 70 & $00 \mathrm{e}+0$ & $0.4598 \mathrm{e}+0$ & $0.1659 \mathrm{e}+1$ & $34 e+1$ & (8) \\
\hline $\mathrm{T}$ & $\mathrm{V}$ & 100 & & & $e+1$ & $\mathrm{e}+1$ & (8) \\
\hline $\mathrm{T}$ & $\mathrm{V}$ & 300 & $91 e+1$ & $0.4326 \mathrm{e}+1$ & $0.1186 \mathrm{e}+1$ & $59 e+1$ & (8) \\
\hline $\mathrm{T}$ & $\mathrm{V}$ & 1000 & $00 e+1$ & $0.4014 \mathrm{e}+1$ & $0.1232 \mathrm{e}+1$ & $82 e+1$ & (8) \\
\hline $\mathrm{T}$ & $\mathrm{V}$ & 000 & $79 e+1$ & $8 e+1$ & $9 e+1$ & $7 e+1$ & (8) \\
\hline $\mathrm{T}$ & $\mathrm{V}$ & 10000 & $0.1915 \mathrm{e}+1$ & $0.1841 \mathrm{e}+1$ & $0.1370 \mathrm{e}+1$ & $-0.2796 \mathrm{e}+1$ & (8) \\
\hline${ }^{4} \mathrm{He}$ & $\mathrm{V}$ & 35 & $0.8262 \mathrm{e}+0$ & $0.2907 \mathrm{e}+0$ & $0.1900 \mathrm{e}+1$ & $30 e+1$ & (8) \\
\hline${ }^{4} \mathrm{He}$ & $\mathrm{V}$ & 40 & $51 e+0$ & $2 e+0$ & $0.1884 \mathrm{e}+1$ & -0.1 & (8) \\
\hline${ }^{4} \mathrm{He}$ & $\mathrm{V}$ & 50 & $0.8179 \mathrm{e}+0$ & $0.3159 \mathrm{e}+0$ & $0.2023 e+1$ & $53 e+1$ & (8) \\
\hline${ }^{4} \mathrm{He}$ & $\mathrm{V}$ & 70 & $0.8729 \mathrm{e}+0$ & $0.4054 \mathrm{e}+0$ & $0.1752 \mathrm{e}+1$ & $-0.2111 e+1$ & (8) \\
\hline${ }^{4} \mathrm{He}$ & V & 100 & $0.8146 \mathrm{e}+0$ & $2 e+0$ & $0.1974 \mathrm{e}+1$ & $304 e+1$ & (8) \\
\hline${ }^{4} \mathrm{He}$ & $\mathrm{V}$ & 300 & $0.1722 \mathrm{e}+1$ & $0.1367 \mathrm{e}+1$ & $0.1302 \mathrm{e}+1$ & $-0.2506 \mathrm{e}+1$ & (8) \\
\hline${ }^{4} \mathrm{He}$ & $\mathrm{V}$ & 1000 & $0.4600 \mathrm{e}+1$ & $0.4320 \mathrm{e}+1$ & $0.1231 \mathrm{e}+1$ & $-0.3081 e+1$ & (8) \\
\hline${ }^{4} \mathrm{He}$ & $\mathrm{V}$ & 3000 & $0.4812 \mathrm{e}+1$ & $0.4615 \mathrm{e}+1$ & $0.1201 \mathrm{e}+1$ & $-0.3075 e+1$ & (8) \\
\hline${ }^{4} \mathrm{He}$ & $\mathrm{V}$ & 10000 & $0.2620 \mathrm{e}+1$ & $0.2519 \mathrm{e}+1$ & $0.1297 \mathrm{e}+1$ & $-0.2873 e+1$ & (8) \\
\hline $\mathrm{Ar}$ & Mo & & & $0.4511 \mathrm{e}+0$ & & $-0.2058 \mathrm{e}+1$ & $(8)$ \\
\hline Ar & Mo & 1601 & $0.3092 \mathrm{e}+1$ & $0.2966 \mathrm{e}+1$ & $0.1386 \mathrm{e}+1$ & -0.2 & (8) \\
\hline Ar & Mo & 16010 & $0.1842 \mathrm{e}+1$ & $0.1773 e+1$ & $0.1408 \mathrm{e}+1$ & $19 e+1$ & (8) \\
\hline Ar & Mo & 27500 & $0.1928 \mathrm{e}+1$ & $0.1863 e+1$ & $0.1549 \mathrm{e}+1$ & $24 e+1$ & (8) \\
\hline Mo & Mo & 300 & & $0.8173 e+0$ & $0.2004 \mathrm{e}+1$ & $629 e+1$ & (8) \\
\hline Mo & Mo & 350 & $0.8165 \mathrm{e}+0$ & $0.8122 \mathrm{e}+0$ & $0.1987 \mathrm{e}+1$ & $-0.2614 \mathrm{e}+1$ & (8) \\
\hline Mo & Mo & 1000 & $0.9238 \mathrm{e}+0$ & $0.9082 \mathrm{e}+0$ & $0.1961 \mathrm{e}+1$ & $-0.2809 e+1$ & (8) \\
\hline Mo & Mo & 2000 & $0.1000 \mathrm{e}+1$ & $0.9794 \mathrm{e}+0$ & $0.1971 \mathrm{e}+1$ & $-0.2971 \mathrm{e}+1$ & (8) \\
\hline $\mathrm{Xe}$ & Mo & 9500 & $0.1045 \mathrm{e}+1$ & $0.1042 \mathrm{e}+1$ & $0.2243 \mathrm{e}+1$ & $-0.3536 e+1$ & (8) \\
\hline $\mathrm{Xe}$ & Mo & 30000 & $0.1060 \mathrm{e}+1$ & $0.1058 \mathrm{e}+1$ & $0.2290 \mathrm{e}+1$ & $-0.3708 \mathrm{e}+1$ & (8) \\
\hline
\end{tabular}


Table 23: Constants for the angular dependence of the particle reflection coefficient at several energies.

\begin{tabular}{|c|c|c|c|c|c|c|c|}
\hline ion & solid & energy & c1 & c2 & c3 & c4 & fit \\
\hline $\mathrm{H}$ & $\mathrm{Fe}$ & 4000 & $0.2774 \mathrm{e}+1$ & $0.2615 \mathrm{e}+1$ & $0.1282 \mathrm{e}+1$ & $-0.2873 e+1$ & (8) \\
\hline $\mathrm{H}$ & $\mathrm{Fe}$ & 8000 & $0.2372 \mathrm{e}+1$ & $0.2286 \mathrm{e}+1$ & $0.1352 \mathrm{e}+1$ & $-0.2889 e+1$ & (8) \\
\hline $\mathrm{H}$ & $\mathrm{Ni}$ & 450 & $0.4375 \mathrm{e}+1$ & $0.3976 \mathrm{e}+1$ & $0.1191 \mathrm{e}+1$ & $-0.3045 e+1$ & (8) \\
\hline $\mathrm{H}$ & $\mathrm{Ni}$ & 4000 & $0.2916 \mathrm{e}+1$ & $0.2750 \mathrm{e}+1$ & $0.1209 \mathrm{e}+1$ & $-0.2811 e+1$ & (8) \\
\hline $\mathrm{H}$ & $\mathrm{Ni}$ & 8000 & $0.2801 \mathrm{e}+1$ & $0.2704 \mathrm{e}+1$ & $0.1332 \mathrm{e}+1$ & $-0.2955 e+1$ & (8) \\
\hline D & $\mathrm{Ni}$ & 1000 & $0.3778 \mathrm{e}+1$ & $0.3444 \mathrm{e}+1$ & $0.1231 \mathrm{e}+1$ & $-0.3013 e+1$ & (8) \\
\hline D & $\mathrm{Ni}$ & 4000 & $0.3223 \mathrm{e}+1$ & $0.3030 \mathrm{e}+1$ & $0.1245 \mathrm{e}+1$ & $-0.2913 e+1$ & (8) \\
\hline $\mathrm{D}$ & $\mathrm{Ni}$ & 8000 & $0.2858 \mathrm{e}+1$ & $0.2744 \mathrm{e}+1$ & $0.1268 \mathrm{e}+1$ & $-0.2876 \mathrm{e}+1$ & (8) \\
\hline${ }^{3} \mathrm{He}$ & $\mathrm{Ni}$ & 1000 & $0.5557 \mathrm{e}+1$ & $0.5225 \mathrm{e}+1$ & $0.1220 \mathrm{e}+1$ & $-0.3185 e+1$ & (8) \\
\hline${ }^{3} \mathrm{He}$ & $\mathrm{Ni}$ & 3000 & $0.5126 \mathrm{e}+1$ & $0.4884 \mathrm{e}+1$ & $0.1230 \mathrm{e}+1$ & $-0.3153 e+1$ & (8) \\
\hline${ }^{3} \mathrm{He}$ & $\mathrm{Ni}$ & 8000 & $0.3088 \mathrm{e}+1$ & $0.2937 \mathrm{e}+1$ & $0.1294 \mathrm{e}+1$ & $-0.2951 \mathrm{e}+1$ & (8) \\
\hline${ }^{3} \mathrm{He}$ & $\mathrm{Ni}$ & 25000 & $0.1817 \mathrm{e}+1$ & $0.1760 \mathrm{e}+1$ & $0.1471 \mathrm{e}+1$ & $-0.2917 \mathrm{e}+1$ & (8) \\
\hline${ }^{4} \mathrm{He}$ & $\mathrm{Ni}$ & 100 & $0.8133 \mathrm{e}+0$ & $0.3304 \mathrm{e}+0$ & $0.1949 \mathrm{e}+1$ & $-0.2152 \mathrm{e}+1$ & (8) \\
\hline${ }^{4} \mathrm{He}$ & $\mathrm{Ni}$ & 500 & $0.3287 \mathrm{e}+1$ & $0.2932 \mathrm{e}+1$ & $0.1205 \mathrm{e}+1$ & $-0.2825 \mathrm{e}+1$ & (8) \\
\hline${ }^{4} \mathrm{He}$ & $\mathrm{Ni}$ & 1000 & $0.5113 \mathrm{e}+1$ & $0.4801 \mathrm{e}+1$ & $0.1204 \mathrm{e}+1$ & $-0.3104 \mathrm{e}+1$ & (8) \\
\hline${ }^{4} \mathrm{He}$ & $\mathrm{Ni}$ & 4000 & $0.3876 \mathrm{e}+1$ & $0.3667 \mathrm{e}+1$ & $0.1232 \mathrm{e}+1$ & $-0.3001 e+1$ & (8) \\
\hline${ }^{4} \mathrm{He}$ & $\mathrm{Ni}$ & 8000 & $0.3879 \mathrm{e}+1$ & $0.3737 \mathrm{e}+1$ & $0.1192 \mathrm{e}+1$ & $-0.2945 \mathrm{e}+1$ & (8) \\
\hline $\mathrm{Ne}$ & $\mathrm{Ni}$ & 1000 & $0.1344 \mathrm{e}+1$ & $0.1196 \mathrm{e}+1$ & $0.1557 \mathrm{e}+1$ & $-0.2569 e+1$ & (8) \\
\hline $\mathrm{Ar}$ & $\mathrm{Ni}$ & 40 & $0.6478 \mathrm{e}+0$ & $0.4470 \mathrm{e}+0$ & $0.2068 \mathrm{e}+1$ & $-0.1761 e+1$ & (8) \\
\hline $\mathrm{Ar}$ & $\mathrm{Ni}$ & 50 & $0.6403 e+0$ & $0.4448 \mathrm{e}+0$ & $0.2100 \mathrm{e}+1$ & $-0.1805 e+1$ & (8) \\
\hline Ar & $\mathrm{Ni}$ & 70 & $0.6340 \mathrm{e}+0$ & $0.4479 \mathrm{e}+0$ & $0.2149 \mathrm{e}+1$ & $-0.1889 e+1$ & (8) \\
\hline Ar & $\mathrm{Ni}$ & 100 & $0.6376 \mathrm{e}+0$ & $0.4655 \mathrm{e}+0$ & $0.2154 \mathrm{e}+1$ & $-0.1985 e+1$ & (8) \\
\hline $\mathrm{Ar}$ & $\mathrm{Ni}$ & 300 & $0.6662 \mathrm{e}+0$ & $0.5421 \mathrm{e}+0$ & $0.2117 \mathrm{e}+1$ & $-0.2301 e+1$ & (8) \\
\hline Ar & $\mathrm{Ni}$ & 1000 & $0.9254 \mathrm{e}+0$ & $0.8525 \mathrm{e}+0$ & $0.1875 \mathrm{e}+1$ & $-0.2632 e+1$ & (8) \\
\hline Ar & $\mathrm{Ni}$ & 3000 & $0.1815 \mathrm{e}+1$ & $0.1766 \mathrm{e}+1$ & $0.1717 \mathrm{e}+1$ & $-0.3056 e+1$ & (8) \\
\hline $\mathrm{Ar}$ & $\mathrm{Ni}$ & 30000 & $0.1351 \mathrm{e}+1$ & $0.1332 \mathrm{e}+1$ & $0.1926 \mathrm{e}+1$ & $-0.3350 e+1$ & (8) \\
\hline $\mathrm{Ni}$ & $\mathrm{Ni}$ & 100 & $0.8494 \mathrm{e}+0$ & $0.8528 \mathrm{e}+0$ & $0.2063 \mathrm{e}+1$ & $-0.2638 \mathrm{e}+1$ & (8) \\
\hline $\mathrm{Ni}$ & $\mathrm{Ni}$ & 500 & $0.6502 \mathrm{e}+0$ & $0.6373 e+0$ & $0.2210 \mathrm{e}+1$ & $-0.2612 e+1$ & (8) \\
\hline $\mathrm{Ni}$ & $\mathrm{Ni}$ & 2500 & $0.1283 e+1$ & $0.1269 \mathrm{e}+1$ & $0.1932 \mathrm{e}+1$ & $-0.3100 e+1$ & (8) \\
\hline $\mathrm{Ni}$ & $\mathrm{Ni}$ & 5000 & $0.1223 \mathrm{e}+1$ & $0.1213 e+1$ & $0.1980 \mathrm{e}+1$ & $-0.3214 \mathrm{e}+1$ & (8) \\
\hline $\mathrm{Ni}$ & $\mathrm{Ni}$ & 10000 & $0.1250 \mathrm{e}+1$ & $0.1240 \mathrm{e}+1$ & $0.2006 \mathrm{e}+1$ & $-0.3335 e+1$ & $(8)$ \\
\hline $\mathrm{H}$ & $\mathrm{Cu}$ & 100 & $0.8877 \mathrm{e}+0$ & $0.3481 \mathrm{e}+0$ & $0.1751 \mathrm{e}+1$ & $-0.2213 e+1$ & (8) \\
\hline $\mathrm{H}$ & $\mathrm{Cu}$ & 1000 & $0.4281 \mathrm{e}+1$ & $0.3952 \mathrm{e}+1$ & $0.1170 \mathrm{e}+1$ & $-0.3007 e+1$ & (8) \\
\hline $\mathrm{H}$ & $\mathrm{Cu}$ & 5000 & $0.3874 \mathrm{e}+1$ & $0.3732 \mathrm{e}+1$ & $0.1158 \mathrm{e}+1$ & $-0.2903 e+1$ & (8) \\
\hline $\mathrm{H}$ & $\mathrm{Cu}$ & 13333 & $0.1904 \mathrm{e}+1$ & $0.1847 \mathrm{e}+1$ & $0.1410 \mathrm{e}+1$ & $-0.2873 e+1$ & (8) \\
\hline $\mathrm{H}$ & $\mathrm{Cu}$ & 26667 & $0.7444 \mathrm{e}+0$ & $0.7244 \mathrm{e}+0$ & $0.1904 \mathrm{e}+1$ & $-0.3056 e+1$ & (8) \\
\hline $\mathrm{H}$ & $\mathrm{Cu}$ & 40000 & $0.9668 \mathrm{e}+0$ & $0.9544 \mathrm{e}+0$ & $0.2153 \mathrm{e}+1$ & $-0.3625 e+1$ & (8) \\
\hline $\mathrm{H}$ & $\mathrm{Cu}$ & 80000 & $0.1023 e+1$ & $0.1018 e+1$ & $0.2803 e+1$ & $-0.4697 e+1$ & (8) \\
\hline
\end{tabular}


Table 24: Constants for the angular dependence of the particle reflection coefficient at several energies.

\begin{tabular}{|c|c|c|c|c|c|c|c|}
\hline ion & solid & energy & c1 & c2 & c3 & c4 & fit \\
\hline $\mathrm{D}$ & $\mathrm{Cu}$ & 300 & $0.5214 \mathrm{e}+1$ & $0.4785 \mathrm{e}+1$ & $0.1131 \mathrm{e}+1$ & $-0.3056 e+1$ & (8) \\
\hline $\mathrm{D}$ & $\mathrm{Cu}$ & 1000 & $0.5610 \mathrm{e}+1$ & $0.5270 \mathrm{e}+1$ & $0.1157 \mathrm{e}+1$ & $-0.3146 e+1$ & (8) \\
\hline $\mathrm{D}$ & $\mathrm{Cu}$ & 3000 & $0.2573 \mathrm{e}+1$ & $0.2357 \mathrm{e}+1$ & $0.1072 \mathrm{e}+1$ & $-0.2568 \mathrm{e}+1$ & (8) \\
\hline $\mathrm{D}$ & $\mathrm{Cu}$ & 53333 & $0.9631 \mathrm{e}+0$ & $0.9530 \mathrm{e}+0$ & $0.2226 \mathrm{e}+1$ & $-0.3714 e+1$ & (8) \\
\hline $\mathrm{D}$ & $\mathrm{Cu}$ & 80000 & $0.8485 e+0$ & $0.8419 \mathrm{e}+0$ & $0.2622 \mathrm{e}+1$ & $-0.4270 e+1$ & (8) \\
\hline D & $\mathrm{Cu}$ & 160000 & $0.1023 e+1$ & $0.1020 \mathrm{e}+1$ & $0.3100 \mathrm{e}+1$ & $-0.5324 \mathrm{e}+1$ & (8) \\
\hline${ }^{4} \mathrm{He}$ & $\mathrm{Cu}$ & 100 & $0.8118 \mathrm{e}+0$ & $0.3162 \mathrm{e}+0$ & $0.1994 \mathrm{e}+1$ & $-0.2201 \mathrm{e}+1$ & (8) \\
\hline${ }^{4} \mathrm{He}$ & $\mathrm{Cu}$ & 1000 & $0.3965 \mathrm{e}+1$ & $0.3637 \mathrm{e}+1$ & $0.1253 \mathrm{e}+1$ & $-0.3039 e+1$ & (8) \\
\hline${ }^{4} \mathrm{He}$ & $\mathrm{Cu}$ & 4000 & $0.3994 \mathrm{e}+1$ & $0.3777 \mathrm{e}+1$ & $0.1211 \mathrm{e}+1$ & $-0.2988 \mathrm{e}+1$ & (8) \\
\hline${ }^{4} \mathrm{He}$ & $\mathrm{Cu}$ & 5000 & $0.4203 \mathrm{e}+1$ & $0.4006 \mathrm{e}+1$ & $0.1213 \mathrm{e}+1$ & $-0.3026 e+1$ & (8) \\
\hline $\mathrm{Ne}$ & $\mathrm{Cu}$ & 1000 & $0.1275 \mathrm{e}+1$ & $0.1102 \mathrm{e}+1$ & $0.1521 \mathrm{e}+1$ & $-0.2487 e+1$ & (8) \\
\hline $\mathrm{Ne}$ & $\mathrm{Cu}$ & 45000 & $0.1840 \mathrm{e}+1$ & $0.1797 \mathrm{e}+1$ & $0.1599 \mathrm{e}+1$ & $-0.3106 e+1$ & (8) \\
\hline Ar & $\mathrm{Cu}$ & 14 & $0.6689 \mathrm{e}+0$ & $0.3951 \mathrm{e}+0$ & $0.2195 \mathrm{e}+1$ & $-0.1814 e+1$ & (8) \\
\hline $\mathrm{Ar}$ & $\mathrm{Cu}$ & 16 & $0.6634 \mathrm{e}+0$ & $0.4064 \mathrm{e}+0$ & $0.2135 \mathrm{e}+1$ & $-0.1758 \mathrm{e}+1$ & (8) \\
\hline Ar & $\mathrm{Cu}$ & 18 & $0.6678 \mathrm{e}+0$ & $0.4169 \mathrm{e}+0$ & $0.2055 \mathrm{e}+1$ & $-0.1718 e+1$ & (8) \\
\hline Ar & $\mathrm{Cu}$ & 20 & $0.6569 \mathrm{e}+0$ & $0.4075 \mathrm{e}+0$ & $0.2250 \mathrm{e}+1$ & $-0.1862 e+1$ & (8) \\
\hline Ar & $\mathrm{Cu}$ & 25 & $0.6569 \mathrm{e}+0$ & $0.4242 \mathrm{e}+0$ & $0.2111 \mathrm{e}+1$ & $-0.1757 \mathrm{e}+1$ & (8) \\
\hline $\mathrm{Ar}$ & $\mathrm{Cu}$ & 30 & $0.6609 \mathrm{e}+0$ & $0.4345 \mathrm{e}+0$ & $0.2050 \mathrm{e}+1$ & $-0.1739 e+1$ & (8) \\
\hline Ar & $\mathrm{Cu}$ & 40 & $0.6554 \mathrm{e}+0$ & $0.4370 \mathrm{e}+0$ & $0.2052 \mathrm{e}+1$ & $-0.1764 e+1$ & (8) \\
\hline $\mathrm{Ar}$ & $\mathrm{Cu}$ & 50 & $0.6656 \mathrm{e}+0$ & $0.4533 \mathrm{e}+0$ & $0.2020 \mathrm{e}+1$ & $-0.1792 e+1$ & (8) \\
\hline Ar & $\mathrm{Cu}$ & 100 & $0.6527 \mathrm{e}+0$ & $0.4466 \mathrm{e}+0$ & $0.2163 e+1$ & $-0.2010 \mathrm{e}+1$ & (8) \\
\hline $\mathrm{Ar}$ & $\mathrm{Cu}$ & 300 & $0.6899 \mathrm{e}+0$ & $0.5482 \mathrm{e}+0$ & $0.2069 \mathrm{e}+1$ & $-0.2304 e+1$ & (8) \\
\hline $\mathrm{Ar}$ & $\mathrm{Cu}$ & 1050 & $0.9813 \mathrm{e}+0$ & $0.8937 \mathrm{e}+0$ & $0.1794 \mathrm{e}+1$ & $-0.2602 e+1$ & (8) \\
\hline $\mathrm{Ar}$ & $\mathrm{Cu}$ & 20000 & $0.1629 \mathrm{e}+1$ & $0.1600 \mathrm{e}+1$ & $0.1737 \mathrm{e}+1$ & $-0.3194 e+1$ & (8) \\
\hline $\mathrm{Ar}$ & $\mathrm{Cu}$ & 27000 & $0.1318 \mathrm{e}+1$ & $0.1294 \mathrm{e}+1$ & $0.1850 \mathrm{e}+1$ & $-0.3235 e+1$ & (8) \\
\hline $\mathrm{Ar}$ & $\mathrm{Cu}$ & 30000 & $0.1354 \mathrm{e}+1$ & $0.1331 \mathrm{e}+1$ & $0.1794 \mathrm{e}+1$ & $-0.3183 e+1$ & (8) \\
\hline $\mathrm{Ar}$ & $\mathrm{Cu}$ & 37000 & $0.1263 \mathrm{e}+1$ & $0.1244 \mathrm{e}+1$ & $0.1797 \mathrm{e}+1$ & $-0.3168 e+1$ & (8) \\
\hline $\mathrm{Ar}$ & $\mathrm{Cu}$ & 100000 & $0.1264 \mathrm{e}+1$ & $0.1254 \mathrm{e}+1$ & $0.1967 \mathrm{e}+1$ & $-0.3455 e+1$ & (8) \\
\hline $\mathrm{Ar}$ & $\mathrm{Cu}$ & 300000 & $0.1022 \mathrm{e}+1$ & $0.1017 \mathrm{e}+1$ & $0.2349 \mathrm{e}+1$ & $-0.3934 e+1$ & (8) \\
\hline Ar & $\mathrm{Cu}$ & 1000000 & $0.1035 \mathrm{e}+1$ & $0.1034 \mathrm{e}+1$ & $0.2980 \mathrm{e}+1$ & $-0.4947 e+1$ & (8) \\
\hline $\mathrm{Cu}$ & $\mathrm{Cu}$ & 50 & $0.9515 \mathrm{e}+0$ & $0.9535 \mathrm{e}+0$ & $0.2221 \mathrm{e}+1$ & $-0.3110 e+1$ & (8) \\
\hline $\mathrm{Cu}$ & $\mathrm{Cu}$ & 70 & $0.8992 \mathrm{e}+0$ & $0.9018 \mathrm{e}+0$ & $0.2169 \mathrm{e}+1$ & $-0.2774 e+1$ & (8) \\
\hline $\mathrm{Cu}$ & $\mathrm{Cu}$ & 100 & $0.6949 \mathrm{e}+0$ & $0.6951 \mathrm{e}+0$ & $0.2209 \mathrm{e}+1$ & $-0.2514 \mathrm{e}+1$ & (8) \\
\hline $\mathrm{Cu}$ & $\mathrm{Cu}$ & 200 & $0.5609 \mathrm{e}+0$ & $0.5504 \mathrm{e}+0$ & $0.2360 \mathrm{e}+1$ & $-0.2460 \mathrm{e}+1$ & (8) \\
\hline $\mathrm{Cu}$ & $\mathrm{Cu}$ & 300 & $0.6323 e+0$ & $0.6215 \mathrm{e}+0$ & $0.2145 \mathrm{e}+1$ & $-0.2430 e+1$ & (8) \\
\hline $\mathrm{Cu}$ & $\mathrm{Cu}$ & 1000 & $0.8183 \mathrm{e}+0$ & $0.8039 \mathrm{e}+0$ & $0.2025 \mathrm{e}+1$ & $-0.2744 \mathrm{e}+1$ & (8) \\
\hline $\mathrm{Cu}$ & $\mathrm{Cu}$ & 3000 & $0.1361 \mathrm{e}+1$ & $0.1342 \mathrm{e}+1$ & $0.1984 \mathrm{e}+1$ & $-0.3231 e+1$ & (8) \\
\hline $\mathrm{Cu}$ & $\mathrm{Cu}$ & 10000 & $0.1137 \mathrm{e}+1$ & $0.1128 \mathrm{e}+1$ & $0.2047 \mathrm{e}+1$ & $-0.3349 e+1$ & (8) \\
\hline $\mathrm{Cu}$ & $\mathrm{Cu}$ & 30000 & $0.1118 \mathrm{e}+1$ & $0.1111 \mathrm{e}+1$ & $0.2171 \mathrm{e}+1$ & $-0.3595 e+1$ & (8) \\
\hline $\mathrm{Cu}$ & $\mathrm{Cu}$ & 100000 & $0.9386 \mathrm{e}+0$ & $0.9368 e+0$ & $0.2316 \mathrm{e}+1$ & $-0.3770 e+1$ & (8) \\
\hline $\mathrm{Kr}$ & $\mathrm{Cu}$ & 1050 & $0.8710 \mathrm{e}+0$ & $0.8673 e+0$ & $0.2263 e+1$ & $-0.3087 e+1$ & (8) \\
\hline $\mathrm{Kr}$ & $\mathrm{Cu}$ & 45000 & $0.1033 e+1$ & $0.1032 \mathrm{e}+1$ & $0.2552 \mathrm{e}+1$ & $-0.4097 \mathrm{e}+1$ & (8) \\
\hline
\end{tabular}


Table 25: Constants for the angular dependence of the particle reflection coefficient at several energies.

\begin{tabular}{|c|c|c|c|c|c|c|c|}
\hline ion & solid & energy & $\mathrm{c} 1$ & c2 & c3 & $\mathrm{c} 4$ & fit \\
\hline $\mathrm{H}$ & Mo & 210 & $0.4025 \mathrm{e}+1$ & $0.3515 \mathrm{e}+1$ & $0.1117 \mathrm{e}+1$ & $-0.2959 e+1$ & (8) \\
\hline $\mathrm{H}$ & Mo & 220 & $0.4981 \mathrm{e}+1$ & $0.4476 \mathrm{e}+1$ & $0.1104 \mathrm{e}+1$ & $-0.3070 e+1$ & (8) \\
\hline $\mathrm{H}$ & Mo & 230 & $0.3783 e+1$ & $0.3279 \mathrm{e}+1$ & $0.1113 e+1$ & $-0.2921 e+1$ & (8) \\
\hline $\mathrm{H}$ & Mo & 250 & $0.6630 \mathrm{e}+1$ & $0.6135 \mathrm{e}+1$ & $0.1088 \mathrm{e}+1$ & $-0.3209 e+1$ & (8) \\
\hline $\mathrm{H}$ & Mo & 300 & $0.4431 \mathrm{e}+1$ & $0.3945 \mathrm{e}+1$ & $0.1112 \mathrm{e}+1$ & $-0.3015 e+1$ & (8) \\
\hline $\mathrm{H}$ & Mo & 400 & $0.5974 \mathrm{e}+1$ & $0.5508 \mathrm{e}+1$ & $0.1133 e+1$ & $-0.3210 e+1$ & (8) \\
\hline $\mathrm{H}$ & Mo & 700 & $0.4421 \mathrm{e}+1$ & $0.3996 \mathrm{e}+1$ & $0.1177 \mathrm{e}+1$ & $-0.3092 e+1$ & (8) \\
\hline $\mathrm{H}$ & Mo & 1400 & $0.4599 \mathrm{e}+1$ & $0.4236 \mathrm{e}+1$ & $0.1229 \mathrm{e}+1$ & $-0.3166 e+1$ & (8) \\
\hline $\mathrm{H}$ & Mo & 2000 & $0.3743 e+1$ & $0.3421 \mathrm{e}+1$ & $0.1208 \mathrm{e}+1$ & $-0.3017 e+1$ & (8) \\
\hline $\mathrm{H}$ & Mo & 3000 & $0.4419 \mathrm{e}+1$ & $0.4147 \mathrm{e}+1$ & $0.1194 \mathrm{e}+1$ & $-0.3064 e+1$ & (8) \\
\hline $\mathrm{H}$ & Mo & 4000 & $0.4749 \mathrm{e}+1$ & $0.4515 \mathrm{e}+1$ & $0.1160 \mathrm{e}+1$ & $-0.3043 e+1$ & (8) \\
\hline $\mathrm{H}$ & Mo & 7000 & $0.2616 \mathrm{e}+1$ & $0.2450 \mathrm{e}+1$ & $0.1197 \mathrm{e}+1$ & $-0.2741 e+1$ & (8) \\
\hline $\mathrm{H}$ & Mo & 8000 & $0.2973 \mathrm{e}+1$ & $0.2826 \mathrm{e}+1$ & $0.1166 \mathrm{e}+1$ & $-0.2769 e+1$ & (8) \\
\hline $\mathrm{H}$ & Mo & 13333 & $0.1915 \mathrm{e}+1$ & $0.1822 \mathrm{e}+1$ & $0.1243 \mathrm{e}+1$ & $-0.2636 e+1$ & (8) \\
\hline $\mathrm{H}$ & Mo & 26667 & $0.1215 \mathrm{e}+1$ & $0.1191 \mathrm{e}+1$ & $0.1890 \mathrm{e}+1$ & $-0.3349 e+1$ & (8) \\
\hline $\mathrm{H}$ & Mo & 40000 & $0.1129 \mathrm{e}+1$ & $0.1114 \mathrm{e}+1$ & $0.2156 \mathrm{e}+1$ & $-0.3728 e+1$ & (8) \\
\hline $\mathrm{H}$ & Mo & 80000 & $0.1057 \mathrm{e}+1$ & $0.1051 \mathrm{e}+1$ & $0.2734 \mathrm{e}+1$ & $-0.4615 e+1$ & (8) \\
\hline $\mathrm{D}$ & Mo & 100 & $0.2498 \mathrm{e}+1$ & $0.1948 \mathrm{e}+1$ & $0.1100 \mathrm{e}+1$ & $-0.2612 \mathrm{e}+1$ & (8) \\
\hline D & Mo & 110 & $0.1154 \mathrm{e}+1$ & $0.6035 \mathrm{e}+0$ & $0.1281 \mathrm{e}+1$ & $-0.2161 e+1$ & (8) \\
\hline $\mathrm{D}$ & Mo & 120 & $0.1112 \mathrm{e}+1$ & $0.5621 \mathrm{e}+0$ & $0.1356 \mathrm{e}+1$ & $-0.2215 e+1$ & (8) \\
\hline $\mathrm{D}$ & Mo & 200 & $0.6292 \mathrm{e}+1$ & $0.5777 \mathrm{e}+1$ & $0.1084 \mathrm{e}+1$ & $-0.3180 e+1$ & (8) \\
\hline D & Mo & 300 & $0.6567 \mathrm{e}+1$ & $0.6069 \mathrm{e}+1$ & $0.1137 \mathrm{e}+1$ & $-0.3287 e+1$ & (8) \\
\hline D & Mo & 450 & $0.4748 \mathrm{e}+1$ & $0.4277 \mathrm{e}+1$ & $0.1142 \mathrm{e}+1$ & $-0.3112 e+1$ & (8) \\
\hline $\mathrm{D}$ & Mo & 2000 & $0.5056 \mathrm{e}+1$ & $0.4715 \mathrm{e}+1$ & $0.1121 \mathrm{e}+1$ & $-0.3074 e+1$ & (8) \\
\hline D & Mo & 8000 & $0.3422 \mathrm{e}+1$ & $0.3244 \mathrm{e}+1$ & $0.1122 \mathrm{e}+1$ & $-0.2799 e+1$ & (8) \\
\hline $\mathrm{T}$ & Mo & 75 & $0.9109 \mathrm{e}+0$ & $0.3539 \mathrm{e}+0$ & $0.1595 \mathrm{e}+1$ & $-0.2114 \mathrm{e}+1$ & (8) \\
\hline $\mathrm{T}$ & Mo & 80 & $0.9333 \mathrm{e}+0$ & $0.3790 \mathrm{e}+0$ & $0.1582 \mathrm{e}+1$ & $-0.2166 \mathrm{e}+1$ & (8) \\
\hline $\mathrm{T}$ & Mo & 90 & $0.9789 \mathrm{e}+0$ & $0.4328 \mathrm{e}+0$ & $0.1496 \mathrm{e}+1$ & $-0.2169 e+1$ & (8) \\
\hline $\mathrm{T}$ & Mo & 100 & $0.9960 \mathrm{e}+0$ & $0.4519 \mathrm{e}+0$ & $0.1483 e+1$ & $-0.2194 \mathrm{e}+1$ & (8) \\
\hline $\mathrm{T}$ & Mo & 170 & $0.1955 \mathrm{e}+1$ & $0.1445 \mathrm{e}+1$ & $0.1183 e+1$ & $-0.2553 e+1$ & (8) \\
\hline $\mathrm{T}$ & Mo & 300 & $0.6145 \mathrm{e}+1$ & $0.5664 \mathrm{e}+1$ & $0.1136 \mathrm{e}+1$ & $-0.3231 e+1$ & (8) \\
\hline $\mathrm{T}$ & Mo & 1000 & $0.4942 \mathrm{e}+1$ & $0.4537 \mathrm{e}+1$ & $0.1178 \mathrm{e}+1$ & $-0.3159 e+1$ & (8) \\
\hline $\mathrm{T}$ & Mo & 3000 & $0.3886 \mathrm{e}+1$ & $0.3579 \mathrm{e}+1$ & $0.1192 \mathrm{e}+1$ & $-0.3007 e+1$ & (8) \\
\hline $\mathrm{T}$ & Mo & 10000 & $0.3205 \mathrm{e}+1$ & $0.3043 e+1$ & $0.1122 \mathrm{e}+1$ & $-0.2760 \mathrm{e}+1$ & (8) \\
\hline${ }^{3} \mathrm{He}$ & Mo & 90 & $0.9763 e+0$ & $0.4122 \mathrm{e}+0$ & $0.1520 \mathrm{e}+1$ & $-0.2080 e+1$ & (8) \\
\hline${ }^{3} \mathrm{He}$ & Mo & 100 & $0.1020 \mathrm{e}+1$ & $0.4651 \mathrm{e}+0$ & $0.1455 \mathrm{e}+1$ & $-0.2093 e+1$ & (8) \\
\hline${ }^{3} \mathrm{He}$ & Mo & 140 & $0.8868 \mathrm{e}+0$ & $0.3421 \mathrm{e}+0$ & $0.1821 \mathrm{e}+1$ & $-0.2310 \mathrm{e}+1$ & (8) \\
\hline${ }^{3} \mathrm{He}$ & Mo & 300 & $0.1716 \mathrm{e}+1$ & $0.1232 \mathrm{e}+1$ & $0.1237 \mathrm{e}+1$ & $-0.2490 e+1$ & (8) \\
\hline${ }^{3} \mathrm{He}$ & Mo & 1000 & $0.5294 \mathrm{e}+1$ & $0.4885 \mathrm{e}+1$ & $0.1174 \mathrm{e}+1$ & $-0.3162 \mathrm{e}+1$ & (8) \\
\hline${ }^{3} \mathrm{He}$ & Mo & 3000 & $0.5327 \mathrm{e}+1$ & $0.5004 \mathrm{e}+1$ & $0.1176 \mathrm{e}+1$ & $-0.3160 e+1$ & (8) \\
\hline${ }^{3} \mathrm{He}$ & Mo & 10000 & $0.4037 \mathrm{e}+1$ & $0.3844 \mathrm{e}+1$ & $0.1167 \mathrm{e}+1$ & $-0.2951 \mathrm{e}+1$ & (8) \\
\hline${ }^{4} \mathrm{He}$ & Mo & 70 & $0.8697 \mathrm{e}+0$ & $0.3034 \mathrm{e}+0$ & $0.1801 \mathrm{e}+1$ & $-0.2092 \mathrm{e}+1$ & (8) \\
\hline${ }^{4} \mathrm{He}$ & Mo & 80 & $0.9200 \mathrm{e}+0$ & $0.3668 \mathrm{e}+0$ & $0.1631 \mathrm{e}+1$ & $-0.2061 e+1$ & (8) \\
\hline${ }^{4} \mathrm{He}$ & Mo & 100 & $0.8902 \mathrm{e}+0$ & $0.3466 \mathrm{e}+0$ & $0.1721 \mathrm{e}+1$ & $-0.2149 \mathrm{e}+1$ & (8) \\
\hline${ }^{4} \mathrm{He}$ & Mo & 140 & $0.9017 \mathrm{e}+0$ & $0.3751 \mathrm{e}+0$ & $0.1743 \mathrm{e}+1$ & $-0.2269 e+1$ & (8) \\
\hline${ }^{4} \mathrm{He}$ & Mo & 1500 & $0.5585 \mathrm{e}+1$ & $0.5211 \mathrm{e}+1$ & $0.1210 \mathrm{e}+1$ & $-0.3240 \mathrm{e}+1$ & (8) \\
\hline${ }^{4} \mathrm{He}$ & Mo & 4000 & $0.4202 \mathrm{e}+1$ & $0.3913 e+1$ & $0.1168 \mathrm{e}+1$ & $-0.3005 e+1$ & (8) \\
\hline${ }^{4} \mathrm{He}$ & Mo & 8000 & $0.3347 \mathrm{e}+1$ & $0.3132 \mathrm{e}+1$ & $0.1093 \mathrm{e}+1$ & $-0.2757 e+1$ & (8) \\
\hline
\end{tabular}


Table 26: Constants for the angular dependence of the particle reflection coefficient at several energies.

\begin{tabular}{|c|c|c|c|c|c|c|c|}
\hline ion & solid & energy & $\mathrm{c} 1$ & $\mathrm{c} 2$ & c3 & $\mathrm{c} 4$ & fit \\
\hline $\mathrm{H}$ & $\mathrm{W}$ & 10 & $0.8844 \mathrm{e}+0$ & $0.1239 \mathrm{e}+0$ & $0.2116 \mathrm{e}+1$ & $-0.2075 e+1$ & (8) \\
\hline $\mathrm{H}$ & $\mathrm{W}$ & 20 & $0.8818 \mathrm{e}+0$ & $0.1780 \mathrm{e}+0$ & $0.1653 \mathrm{e}+1$ & $-0.1752 \mathrm{e}+1$ & (8) \\
\hline $\mathrm{H}$ & W & 50 & $0.8867 \mathrm{e}+0$ & $0.2213 \mathrm{e}+0$ & $0.1809 \mathrm{e}+1$ & $-0.2151 \mathrm{e}+1$ & (8) \\
\hline $\mathrm{H}$ & W & 100 & $0.1123 e+1$ & $0.5127 \mathrm{e}+0$ & $0.1225 \mathrm{e}+1$ & $-0.2062 e+1$ & (8) \\
\hline $\mathrm{H}$ & W & 200 & $0.4322 \mathrm{e}+1$ & $0.3746 \mathrm{e}+1$ & $0.1075 \mathrm{e}+1$ & $-0.3011 e+1$ & (8) \\
\hline $\mathrm{H}$ & W & 300 & $0.4685 \mathrm{e}+1$ & $0.4131 \mathrm{e}+1$ & $0.1108 \mathrm{e}+1$ & $-0.3102 e+1$ & (8) \\
\hline $\mathrm{H}$ & W & 500 & $0.4319 \mathrm{e}+1$ & $0.3797 \mathrm{e}+1$ & $0.1101 \mathrm{e}+1$ & $-0.3046 e+1$ & (8) \\
\hline $\mathrm{H}$ & W & 550 & $0.4519 \mathrm{e}+1$ & $0.4005 \mathrm{e}+1$ & $0.1097 \mathrm{e}+1$ & $-0.3059 e+1$ & (8) \\
\hline $\mathrm{H}$ & $\mathrm{W}$ & 600 & $0.5454 \mathrm{e}+1$ & $0.4944 \mathrm{e}+1$ & $0.1109 \mathrm{e}+1$ & $-0.3186 e+1$ & (8) \\
\hline $\mathrm{H}$ & W & 700 & $0.6237 \mathrm{e}+1$ & $0.5743 \mathrm{e}+1$ & $0.1044 \mathrm{e}+1$ & $-0.3171 e+1$ & (8) \\
\hline $\mathrm{H}$ & W & 800 & $0.4291 \mathrm{e}+1$ & $0.3804 \mathrm{e}+1$ & $0.1093 \mathrm{e}+1$ & $-0.3021 e+1$ & (8) \\
\hline $\mathrm{H}$ & $\mathrm{W}$ & 900 & $0.5567 \mathrm{e}+1$ & $0.5088 \mathrm{e}+1$ & $0.1064 \mathrm{e}+1$ & $-0.3134 e+1$ & (8) \\
\hline $\mathrm{H}$ & W & 1000 & $0.3963 \mathrm{e}+1$ & $0.3494 \mathrm{e}+1$ & $0.1076 \mathrm{e}+1$ & $-0.2944 e+1$ & (8) \\
\hline $\mathrm{H}$ & W & 2000 & $0.5501 \mathrm{e}+1$ & $0.5088 \mathrm{e}+1$ & $0.1159 \mathrm{e}+1$ & $-0.3233 e+1$ & (8) \\
\hline $\mathrm{H}$ & $\mathrm{W}$ & 4000 & $0.4497 \mathrm{e}+1$ & $0.4168 \mathrm{e}+1$ & $0.1134 \mathrm{e}+1$ & $-0.2999 e+1$ & (8) \\
\hline $\mathrm{H}$ & W & 13333 & $0.2981 \mathrm{e}+1$ & $0.2811 \mathrm{e}+1$ & $0.1066 \mathrm{e}+1$ & $-0.2656 \mathrm{e}+1$ & (8) \\
\hline $\mathrm{H}$ & W & 26667 & $0.1150 \mathrm{e}+1$ & $0.1071 \mathrm{e}+1$ & $0.1410 \mathrm{e}+1$ & $-0.2550 \mathrm{e}+1$ & (8) \\
\hline $\mathrm{H}$ & $\mathrm{W}$ & 40000 & $0.1112 \mathrm{e}+1$ & $0.1059 \mathrm{e}+1$ & $0.1551 \mathrm{e}+1$ & $-0.2752 \mathrm{e}+1$ & (8) \\
\hline $\mathrm{H}$ & W & 80000 & & & $0.1865 \mathrm{e}+1$ & $-0.3240 e+1$ & (8) \\
\hline D & W & 10 & $0.8830 \mathrm{e}+0$ & $0.1272 \mathrm{e}+0$ & $0.1958 \mathrm{e}+1$ & $-0.1893 e+1$ & (8) \\
\hline $\mathrm{D}$ & $\mathrm{W}$ & 20 & $0.8856 \mathrm{e}+0$ & $0.1660 \mathrm{e}+0$ & $0.1808 \mathrm{e}+1$ & $-0.1924 e+1$ & (8) \\
\hline D & W & 50 & & & & & (8) \\
\hline $\mathrm{D}$ & W & 100 & $0.1017 \mathrm{e}+1$ & $0.3781 \mathrm{e}+0$ & $0.1425 \mathrm{e}+1$ & $-0.2159 e+1$ & (8) \\
\hline $\mathrm{D}$ & W & 200 & $0.2437 \mathrm{e}+1$ & $0.1843 \mathrm{e}+1$ & $0.1097 \mathrm{e}+1$ & $-0.2685 e+1$ & (8) \\
\hline $\mathrm{D}$ & W & 250 & $0.5971 \mathrm{e}+1$ & $0.5386 \mathrm{e}+1$ & $0.1080 \mathrm{e}+1$ & $-0.3232 e+1$ & (8) \\
\hline D & W & 270 & $0.6068 \mathrm{e}+1$ & $0.5489 \mathrm{e}+1$ & $0.1069 \mathrm{e}+1$ & $-0.3220 e+1$ & (8) \\
\hline $\mathrm{D}$ & $\mathrm{W}$ & 300 & $0.4791 \mathrm{e}+1$ & $0.4215 \mathrm{e}+1$ & $0.1099 \mathrm{e}+1$ & $-0.3125 e+1$ & (8) \\
\hline $\mathrm{D}$ & W & 350 & $0.4577 \mathrm{e}+1$ & $0.4008 \mathrm{e}+1$ & $0.1129 \mathrm{e}+1$ & $-0.3144 e+1$ & (8) \\
\hline $\mathrm{D}$ & W & 400 & $0.5096 \mathrm{e}+1$ & $0.4535 \mathrm{e}+1$ & $0.1122 \mathrm{e}+1$ & $-0.3195 e+1$ & (8) \\
\hline $\mathrm{D}$ & W & 500 & $0.5417 \mathrm{e}+1$ & $0.4867 \mathrm{e}+1$ & $0.1105 \mathrm{e}+1$ & $-0.3207 e+1$ & (8) \\
\hline $\mathrm{D}$ & W & 600 & $0.6877 \mathrm{e}+1$ & $0.6342 \mathrm{e}+1$ & $0.1074 \mathrm{e}+1$ & $-0.3293 e+1$ & (8) \\
\hline D & W & 700 & $0.4009 \mathrm{e}+1$ & $0.3482 \mathrm{e}+1$ & $0.1101 \mathrm{e}+1$ & $-0.3023 e+1$ & (8) \\
\hline D & W & 1000 & $0.3545 \mathrm{e}+1$ & $0.3043 \mathrm{e}+1$ & $0.1100 \mathrm{e}+1$ & $-0.2939 e+1$ & (8) \\
\hline $\mathrm{T}$ & W & 10 & $0.8776 \mathrm{e}+0$ & $0.1407 \mathrm{e}+0$ & $0.1759 \mathrm{e}+1$ & $-0.1670 e+1$ & (8) \\
\hline $\mathrm{T}$ & W & 20 & $0.8826 \mathrm{e}+0$ & $0.1749 \mathrm{e}+0$ & $0.1807 \mathrm{e}+1$ & $-0.1927 e+1$ & (8) \\
\hline $\mathrm{T}$ & $\mathrm{W}$ & 50 & $0.8900 \mathrm{e}+0$ & $0.2261 \mathrm{e}+0$ & $0.1749 \mathrm{e}+1$ & $-0.2079 e+1$ & (8) \\
\hline $\mathrm{T}$ & W & 100 & $0.1151 \mathrm{e}+1$ & $0.5277 \mathrm{e}+0$ & $0.1218 \mathrm{e}+1$ & $-0.2106 \mathrm{e}+1$ & (8) \\
\hline $\mathrm{T}$ & W & 140 & $0.1428 \mathrm{e}+1$ & $0.8205 \mathrm{e}+0$ & $0.1160 \mathrm{e}+1$ & $-0.2311 e+1$ & (8) \\
\hline $\mathrm{T}$ & W & 160 & $0.2767 \mathrm{e}+1$ & $0.2168 \mathrm{e}+1$ & $0.1078 \mathrm{e}+1$ & $-0.2745 e+1$ & (8) \\
\hline $\mathrm{T}$ & $\mathrm{W}$ & 170 & $0.2622 \mathrm{e}+1$ & $0.2028 \mathrm{e}+1$ & $0.1065 \mathrm{e}+1$ & $-0.2687 e+1$ & (8) \\
\hline $\mathrm{T}$ & W & 180 & $0.4379 \mathrm{e}+1$ & $0.3786 \mathrm{e}+1$ & $0.1054 \mathrm{e}+1$ & $-0.3006 e+1$ & (8) \\
\hline $\mathrm{T}$ & W & 200 & $0.3442 \mathrm{e}+1$ & $0.2851 \mathrm{e}+1$ & $0.1069 \mathrm{e}+1$ & $-0.2881 \mathrm{e}+1$ & (8) \\
\hline $\mathrm{T}$ & $\mathrm{W}$ & 250 & $0.4213 \mathrm{e}+1$ & $0.3630 \mathrm{e}+1$ & $0.1106 \mathrm{e}+1$ & $-0.3062 \mathrm{e}+1$ & (8) \\
\hline $\mathrm{T}$ & W & 300 & $0.5056 \mathrm{e}+1$ & $0.4485 \mathrm{e}+1$ & $0.1084 \mathrm{e}+1$ & $-0.3132 e+1$ & (8) \\
\hline $\mathrm{T}$ & W & 400 & $0.5191 \mathrm{e}+1$ & $0.4629 \mathrm{e}+1$ & $0.1119 \mathrm{e}+1$ & $-0.3206 e+1$ & (8) \\
\hline $\mathrm{T}$ & W & 500 & $0.4843 \mathrm{e}+1$ & $0.4293 \mathrm{e}+1$ & $0.1124 \mathrm{e}+1$ & $-0.3168 \mathrm{e}+1$ & (8) \\
\hline $\mathrm{T}$ & W & 700 & $0.5206 \mathrm{e}+1$ & $0.4677 \mathrm{e}+1$ & $0.1090 \mathrm{e}+1$ & $-0.3166 e+1$ & (8) \\
\hline $\mathrm{T}$ & $\mathrm{W}$ & 1000 & $0.3644 \mathrm{e}+1$ & $0.3145 \mathrm{e}+1$ & $0.1053 \mathrm{e}+1$ & $-0.2887 e+1$ & (8) \\
\hline
\end{tabular}


Table 27: Constants for the angular dependence of the particle reflection coefficient at several energies.

\begin{tabular}{|c|c|c|c|c|c|c|c|}
\hline ion & solid & energy & $\mathrm{c} 1$ & c2 & $\mathrm{c} 3$ & $\mathrm{c} 4$ & fit \\
\hline${ }^{4} \mathrm{He}$ & W & 10 & $0.9138 e+0$ & $-0.1161 e+0$ & $-0.1793 e+1$ & $0.1574 \mathrm{e}+1$ & (8) \\
\hline${ }^{4} \mathrm{He}$ & W & 20 & $0.8996 \mathrm{e}+0$ & $0.1537 \mathrm{e}+0$ & $0.1963 e+1$ & $-0.1923 e+1$ & (8) \\
\hline${ }^{4} \mathrm{He}$ & W & 50 & $0.8856 \mathrm{e}+0$ & $0.2130 \mathrm{e}+0$ & $0.1822 \mathrm{e}+1$ & $-0.2007 \mathrm{e}+1$ & (8) \\
\hline${ }^{4} \mathrm{He}$ & W & 100 & $0.8814 \mathrm{e}+0$ & $0.2457 \mathrm{e}+0$ & $0.1967 \mathrm{e}+1$ & $-0.2326 e+1$ & (8) \\
\hline${ }^{4} \mathrm{He}$ & W & 125 & $0.9071 \mathrm{e}+0$ & $0.2878 \mathrm{e}+0$ & $0.1733 \mathrm{e}+1$ & $-0.2190 \mathrm{e}+1$ & (8) \\
\hline${ }^{4} \mathrm{He}$ & W & 130 & $0.9291 \mathrm{e}+0$ & $0.3139 \mathrm{e}+0$ & $0.1645 \mathrm{e}+1$ & $-0.2170 \mathrm{e}+1$ & (8) \\
\hline${ }^{4} \mathrm{He}$ & W & 140 & $0.8873 e+0$ & $0.2719 \mathrm{e}+0$ & $0.1852 \mathrm{e}+1$ & $-0.2281 e+1$ & (8) \\
\hline${ }^{4} \mathrm{He}$ & W & 150 & $0.9489 \mathrm{e}+0$ & $0.3452 \mathrm{e}+0$ & $0.1573 \mathrm{e}+1$ & $-0.2147 \mathrm{e}+1$ & (8) \\
\hline${ }^{4} \mathrm{He}$ & W & 170 & $0.9831 \mathrm{e}+0$ & $0.3877 \mathrm{e}+0$ & $0.1470 \mathrm{e}+1$ & $-0.2113 e+1$ & (8) \\
\hline${ }^{4} \mathrm{He}$ & W & 200 & $0.1008 \mathrm{e}+1$ & $0.4212 \mathrm{e}+0$ & $0.1470 \mathrm{e}+1$ & $-0.2179 e+1$ & (8) \\
\hline${ }^{4} \mathrm{He}$ & W & 250 & $0.1106 \mathrm{e}+1$ & $0.5334 \mathrm{e}+0$ & $0.1347 \mathrm{e}+1$ & $-0.2193 e+1$ & (8) \\
\hline${ }^{4} \mathrm{He}$ & W & 300 & $0.1864 \mathrm{e}+1$ & $0.1303 \mathrm{e}+1$ & $0.1163 e+1$ & $-0.2515 \mathrm{e}+1$ & (8) \\
\hline${ }^{4} \mathrm{He}$ & W & 350 & $0.3043 e+1$ & $0.2493 \mathrm{e}+1$ & $0.1102 \mathrm{e}+1$ & $-0.2788 \mathrm{e}+1$ & (8) \\
\hline${ }^{4} \mathrm{He}$ & W & 400 & $0.4279 \mathrm{e}+1$ & $0.3731 \mathrm{e}+1$ & $0.1114 \mathrm{e}+1$ & $-0.3026 e+1$ & (8) \\
\hline${ }^{4} \mathrm{He}$ & W & 500 & $0.4615 \mathrm{e}+1$ & $0.4081 \mathrm{e}+1$ & $0.1124 \mathrm{e}+1$ & $-0.3080 e+1$ & (8) \\
\hline${ }^{4} \mathrm{He}$ & W & 600 & $0.6346 \mathrm{e}+1$ & $0.5817 \mathrm{e}+1$ & $0.1122 \mathrm{e}+1$ & $-0.3272 \mathrm{e}+1$ & (8) \\
\hline${ }^{4} \mathrm{He}$ & $\mathrm{W}$ & 700 & $0.6186 \mathrm{e}+1$ & $0.5670 \mathrm{e}+1$ & $0.1103 e+1$ & $-0.3225 e+1$ & (8) \\
\hline${ }^{4} \mathrm{He}$ & W & 1000 & $0.7157 \mathrm{e}+1$ & $0.6659 \mathrm{e}+1$ & $0.1144 \mathrm{e}+1$ & $-0.3362 \mathrm{e}+1$ & (8) \\
\hline${ }^{4} \mathrm{He}$ & W & 1400 & $0.3666 \mathrm{e}+1$ & $0.3191 \mathrm{e}+1$ & $0.1129 \mathrm{e}+1$ & $-0.2955 \mathrm{e}+1$ & (8) \\
\hline${ }^{4} \mathrm{He}$ & $\mathrm{W}$ & 2000 & $0.4463 \mathrm{e}+1$ & $0.4012 \mathrm{e}+1$ & $0.1092 \mathrm{e}+1$ & $-0.3020 e+1$ & (8) \\
\hline${ }^{4} \mathrm{He}$ & W & 5000 & $0.5508 \mathrm{e}+1$ & $0.5145 \mathrm{e}+1$ & $0.9659 \mathrm{e}+0$ & $-0.2937 \mathrm{e}+1$ & (8) \\
\hline${ }^{4} \mathrm{He}$ & W & 10000 & $0.2448 \mathrm{e}+1$ & $0.2155 \mathrm{e}+1$ & $0.9757 \mathrm{e}+0$ & $-0.2455 \mathrm{e}+1$ & (8) \\
\hline${ }^{4} \mathrm{He}$ & W & 20000 & $0.1595 \mathrm{e}+1$ & $0.1379 \mathrm{e}+1$ & $0.1048 \mathrm{e}+1$ & $-0.2256 e+1$ & (8) \\
\hline${ }^{4} \mathrm{He}$ & W & 50000 & $0.9215 \mathrm{e}+0$ & $0.7997 \mathrm{e}+0$ & $0.1308 \mathrm{e}+1$ & $-0.2221 \mathrm{e}+1$ & (8) \\
\hline $\mathrm{N}$ & W & 10 & $0.8999 e+0$ & $0.1494 \mathrm{e}+0$ & $0.1417 \mathrm{e}+1$ & $-0.1349 e+1$ & (8) \\
\hline $\mathrm{N}$ & W & 20 & $0.8686 \mathrm{e}+0$ & $0.1573 e+0$ & $0.2154 \mathrm{e}+1$ & $-0.1908 \mathrm{e}+1$ & (8) \\
\hline $\mathrm{N}$ & W & 40 & $0.8410 \mathrm{e}+0$ & $0.1997 \mathrm{e}+0$ & $0.2050 \mathrm{e}+1$ & $-0.18953+1$ & (8) \\
\hline $\mathrm{N}$ & W & 48 & $0.8336 \mathrm{e}+0$ & $0.2077 \mathrm{e}+0$ & $0.2062 \mathrm{e}+1$ & $-0.1934 e+1$ & (8) \\
\hline $\mathrm{N}$ & W & 50 & $0.8394 \mathrm{e}+0$ & $0.2135 \mathrm{e}+0$ & $0.1993 e+1$ & $-0.1892 e+1$ & (8) \\
\hline $\mathrm{N}$ & W & 52 & $0.8377 \mathrm{e}+0$ & $0.2111 \mathrm{e}+0$ & $0.2095 \mathrm{e}+1$ & $-0.2005 e+1$ & (8) \\
\hline $\mathrm{N}$ & W & 55 & $0.8367 \mathrm{e}+0$ & $0.2284 \mathrm{e}+0$ & $0.1850 \mathrm{e}+1$ & $-0.1788 e+1$ & (8) \\
\hline $\mathrm{N}$ & W & 60 & $0.8304 \mathrm{e}+0$ & $0.2305 \mathrm{e}+0$ & $0.1962 \mathrm{e}+1$ & $-0.1897 e+1$ & (8) \\
\hline $\mathrm{N}$ & $\mathrm{W}$ & 70 & $0.8254 \mathrm{e}+0$ & $0.2383 \mathrm{e}+0$ & $0.1906 \mathrm{e}+1$ & $-0.1868 \mathrm{e}+1$ & (8) \\
\hline $\mathrm{N}$ & W & 80 & $0.8305 \mathrm{e}+0$ & $0.2488 \mathrm{e}+0$ & $0.1887 \mathrm{e}+1$ & $-0.1921 e+1$ & (8) \\
\hline $\mathrm{N}$ & W & 90 & $0.8237 \mathrm{e}+0$ & $0.2506 \mathrm{e}+0$ & $0.2016 \mathrm{e}+1$ & $-0.2066 e+1$ & (8) \\
\hline $\mathrm{N}$ & W & 100 & $0.8342 \mathrm{e}+0$ & $0.2759 \mathrm{e}+0$ & $0.1804 \mathrm{e}+1$ & $-0.1911 e+1$ & (8) \\
\hline $\mathrm{N}$ & W & 120 & $0.8273 e+0$ & $0.2771 \mathrm{e}+0$ & $0.1930 \mathrm{e}+1$ & $-0.2055 \mathrm{e}+1$ & (8) \\
\hline $\mathrm{N}$ & W & 140 & $0.8317 \mathrm{e}+0$ & $0.2953 \mathrm{e}+0$ & $0.1831 \mathrm{e}+1$ & $-0.2030 \mathrm{e}+1$ & (8) \\
\hline $\mathrm{N}$ & W & 200 & $0.8429 e+0$ & $0.3267 \mathrm{e}+0$ & $0.1799 e+1$ & $-0.2113 e+1$ & (8) \\
\hline $\mathrm{N}$ & W & 300 & $0.9301 \mathrm{e}+0$ & $0.4465 \mathrm{e}+0$ & $0.1511 \mathrm{e}+1$ & $-0.2052 e+1$ & (8) \\
\hline $\mathrm{N}$ & W & 500 & $0.1046 \mathrm{e}+1$ & $0.5898 \mathrm{e}+0$ & $0.1465 \mathrm{e}+1$ & $-0.2230 e+1$ & (8) \\
\hline $\mathrm{N}$ & W & 1000 & $0.4913 e+1$ & $0.4499 \mathrm{e}+1$ & $0.1123 e+1$ & $-0.2999 e+1$ & (8) \\
\hline $\mathrm{Xe}$ & W & 9500 & $0.2287 \mathrm{e}+1$ & $0.2228 \mathrm{e}+1$ & $0.1557 \mathrm{e}+1$ & $-0.3047 e+1$ & (8) \\
\hline $\mathrm{Xe}$ & W & 30000 & $0.1619 \mathrm{e}+1$ & $0.1577 \mathrm{e}+1$ & $0.1607 \mathrm{e}+1$ & $-0.2983 e+1$ & (8) \\
\hline
\end{tabular}


Table 28: Constants for the angular dependence of the particle reflection coefficient at several energies.

\begin{tabular}{|c|c|c|c|c|c|c|c|}
\hline ion & solid & energy & $\mathrm{c} 1$ & $\overline{c 2}$ & $\overline{c 3}$ & $\overline{c 4}$ & fit \\
\hline $\mathrm{Ne}$ & $\bar{W}$ & 10 & $0.9133 \mathrm{e}+0$ & $0.1105 \mathrm{e}+0$ & $0.1894 \mathrm{e}+1$ & $-0.1600 e+1$ & (8) \\
\hline $\mathrm{Ne}$ & W & 20 & $0.8807 \mathrm{e}+0$ & $0.1419 \mathrm{e}+0$ & $0.2229 \mathrm{e}+1$ & $-0.1869 e+1$ & (8) \\
\hline $\mathrm{Ne}$ & W & 30 & $0.8589 \mathrm{e}+0$ & $0.1816 \mathrm{e}+0$ & $0.1854 \mathrm{e}+1$ & $-0.1587 \mathrm{e}+1$ & (8) \\
\hline $\mathrm{Ne}$ & W & 40 & $0.8502 \mathrm{e}+0$ & $0.2010 \mathrm{e}+0$ & $0.1937 \mathrm{e}+1$ & $-0.1751 e+1$ & (8) \\
\hline $\mathrm{Ne}$ & W & 45 & $0.8401 \mathrm{e}+0$ & $0.2010 \mathrm{e}+0$ & $0.2068 \mathrm{e}+1$ & $-0.1863 e+1$ & (8) \\
\hline $\mathrm{Ne}$ & W & 50 & $0.8397 \mathrm{e}+0$ & $0.2087 \mathrm{e}+0$ & $0.2146 \mathrm{e}+1$ & $-0.1979 e+1$ & (8) \\
\hline $\mathrm{Ne}$ & W & 60 & $0.8282 \mathrm{e}+0$ & $0.2269 \mathrm{e}+0$ & $0.2005 \mathrm{e}+1$ & $-0.1880 e+1$ & (8) \\
\hline $\mathrm{Ne}$ & $\mathrm{W}$ & 70 & $0.8182 \mathrm{e}+0$ & $0.2279 \mathrm{e}+0$ & $0.2124 \mathrm{e}+1$ & $-0.1988 e+1$ & (8) \\
\hline $\mathrm{Ne}$ & W & 80 & $0.8173 \mathrm{e}+0$ & $0.2462 \mathrm{e}+0$ & $0.2075 \mathrm{e}+1$ & $-0.1985 e+1$ & (8) \\
\hline $\mathrm{Ne}$ & W & 100 & $0.8203 \mathrm{e}+0$ & $0.2717 \mathrm{e}+0$ & $0.1914 \mathrm{e}+1$ & $-0.1927 e+1$ & (8) \\
\hline $\mathrm{Ne}$ & W & 140 & $0.8187 \mathrm{e}+0$ & $0.3009 \mathrm{e}+0$ & $0.1894 \mathrm{e}+1$ & $-0.2022 e+1$ & (8) \\
\hline $\mathrm{Ne}$ & W & 200 & $0.8320 \mathrm{e}+0$ & $0.3380 \mathrm{e}+0$ & $0.1837 \mathrm{e}+1$ & $-0.2099 e+1$ & (8) \\
\hline $\mathrm{Ne}$ & W & 300 & $0.8643 \mathrm{e}+0$ & $0.4091 \mathrm{e}+0$ & $0.1620 \mathrm{e}+1$ & $-0.2013 e+1$ & (8) \\
\hline $\mathrm{Ne}$ & W & 400 & $0.9356 \mathrm{e}+0$ & $0.4969 \mathrm{e}+0$ & $0.1534 \mathrm{e}+1$ & $-0.2096 \mathrm{e}+1$ & (8) \\
\hline $\mathrm{Ne}$ & W & 500 & $0.9358 \mathrm{e}+0$ & $0.5051 \mathrm{e}+0$ & $0.1613 \mathrm{e}+1$ & $-0.2226 e+1$ & (8) \\
\hline $\mathrm{Ne}$ & W & 700 & $0.1060 \mathrm{e}+1$ & $0.6510 \mathrm{e}+0$ & $0.1501 \mathrm{e}+1$ & $-0.2298 \mathrm{e}+1$ & (8) \\
\hline $\mathrm{Ne}$ & W & 1000 & $0.1506 \mathrm{e}+1$ & $0.1119 \mathrm{e}+1$ & $0.1327 \mathrm{e}+1$ & $150 \mathrm{e}+1$ & (8) \\
\hline $\mathrm{Ar}$ & W & 10 & $0.8840 \mathrm{e}+0$ & $0.1589 \mathrm{e}+0$ & $0.1774 \mathrm{e}+1$ & $-0.1523 e+1$ & (8) \\
\hline $\mathrm{Ar}$ & W & 20 & $0.8558 \mathrm{e}+0$ & $0.1852 \mathrm{e}+0$ & $0.1909 \mathrm{e}+1$ & $-0.1629 e+1$ & (8) \\
\hline Ar & W & 30 & $0.8356 \mathrm{e}+0$ & $0.2134 \mathrm{e}+0$ & $0.1943 \mathrm{e}+1$ & $36 e+1$ & (8) \\
\hline $\mathrm{Ar}$ & $\mathrm{W}$ & 35 & $0.8230 \mathrm{e}+0$ & $0.2143 \mathrm{e}+0$ & $0.2044 \mathrm{e}+1$ & $-0.1751 \mathrm{e}+1$ & (8) \\
\hline $\mathrm{Ar}$ & W & 40 & $0.8260 \mathrm{e}+0$ & $0.2317 \mathrm{e}+0$ & $0.1989 \mathrm{e}+1$ & $-0.1783 e+1$ & (8) \\
\hline Ar & W & 45 & & & $6 e+1$ & $0 e+1$ & (8) \\
\hline $\mathrm{Ar}$ & W & 50 & $0.8091 \mathrm{e}+0$ & $0.2409 \mathrm{e}+0$ & $0.2021 \mathrm{e}+1$ & $-0.1813 e+1$ & (8) \\
\hline $\mathrm{Ar}$ & W & 55 & $0.8094 \mathrm{e}+0$ & $0.2526 \mathrm{e}+0$ & $0.1993 \mathrm{e}+1$ & $-0.1824 \mathrm{e}+1$ & (8) \\
\hline Ar & W & 60 & $19 e+0$ & $0.2530 \mathrm{e}+0$ & $0.2062 \mathrm{e}+1$ & $-0.1896 \mathrm{e}+1$ & (8) \\
\hline Ar & W & 70 & $0.7938 \mathrm{e}+0$ & $0.2776 \mathrm{e}+0$ & $0.1928 \mathrm{e}+1$ & $-0.1778 e+1$ & (8) \\
\hline $\mathrm{Ar}$ & W & 80 & $0.7955 \mathrm{e}+0$ & $0.2877 \mathrm{e}+0$ & $0.1938 \mathrm{e}+1$ & $-0.1845 e+1$ & (8) \\
\hline $\mathrm{Ar}$ & W & 100 & $0.7826 \mathrm{e}+0$ & $0.2966 \mathrm{e}+0$ & $0.2039 \mathrm{e}+1$ & $-0.1968 \mathrm{e}+1$ & (8) \\
\hline Ar & W & 140 & $0.7804 \mathrm{e}+0$ & $0.3358 \mathrm{e}+0$ & $0.1872 \mathrm{e}+1$ & $-0.1908 \mathrm{e}+1$ & (8) \\
\hline $\mathrm{Ar}$ & $\mathrm{W}$ & 200 & $0.7719 \mathrm{e}+0$ & $0.3481 \mathrm{e}+0$ & $0.2046 \mathrm{e}+1$ & $-0.2170 \mathrm{e}+1$ & (8) \\
\hline Ar & W & 300 & $0.8027 \mathrm{e}+0$ & $0.4235 \mathrm{e}+0$ & $0.1777 \mathrm{e}+1$ & $-0.2065 e+1$ & (8) \\
\hline Ar & W & 500 & $0.8059 \mathrm{e}+0$ & $0.4583 e+0$ & $0.1830 \mathrm{e}+1$ & $-0.2231 \mathrm{e}+1$ & (8) \\
\hline Ar & $\mathrm{W}$ & 700 & $0.9361 \mathrm{e}+0$ & $0.6123 e+0$ & $0.1588 \mathrm{e}+1$ & $-0.2226 \mathrm{e}+1$ & (8) \\
\hline $\mathrm{Ar}$ & W & 1000 & $0.1243 \mathrm{e}+1$ & $0.9497 \mathrm{e}+0$ & $0.1359 \mathrm{e}+1$ & $-0.2262 \mathrm{e}+1$ & (8) \\
\hline W & W & 100 & $0.9282 \mathrm{e}+0$ & $0.9289 \mathrm{e}+0$ & $0.2416 \mathrm{e}+1$ & $-0.3653 e+1$ & (8) \\
\hline W & W & 120 & $0.9073 e+0$ & $0.9082 \mathrm{e}+0$ & $0.2287 \mathrm{e}+1$ & $-0.3356 e+1$ & (8) \\
\hline W & W & 140 & $0.9017 \mathrm{e}+0$ & $0.9028 \mathrm{e}+0$ & $0.2212 \mathrm{e}+1$ & $-0.3159 e+1$ & (8) \\
\hline W & W & 200 & $0.8435 \mathrm{e}+0$ & $0.8437 \mathrm{e}+0$ & $0.2117 \mathrm{e}+1$ & $-0.2874 \mathrm{e}+1$ & (8) \\
\hline W & W & 300 & $0.7965 \mathrm{e}+0$ & $0.7958 \mathrm{e}+0$ & $0.1985 \mathrm{e}+1$ & $-0.2633 e+1$ & (8) \\
\hline W & $\mathrm{W}$ & 350 & $0.7080 \mathrm{e}+0$ & $0.7034 \mathrm{e}+0$ & $0.2085 \mathrm{e}+1$ & $-0.2633 e+1$ & (8) \\
\hline W & W & 400 & $0.6799 \mathrm{e}+0$ & $0.6734 \mathrm{e}+0$ & $0.2050 \mathrm{e}+1$ & $-0.2571 \mathrm{e}+1$ & (8) \\
\hline W & W & 500 & $0.7028 \mathrm{e}+0$ & $0.6934 \mathrm{e}+0$ & $0.2040 \mathrm{e}+1$ & $-0.2592 \mathrm{e}+1$ & (8) \\
\hline W & W & 800 & $0.7637 \mathrm{e}+0$ & $0.7497 \mathrm{e}+0$ & $0.1961 \mathrm{e}+1$ & $-0.2603 e+1$ & (8) \\
\hline W & W & 1000 & $0.8366 \mathrm{e}+0$ & $0.8215 \mathrm{e}+0$ & $0.1905 \mathrm{e}+1$ & $-0.2641 e+1$ & (8) \\
\hline W & W & 2500 & $0.1195 \mathrm{e}+1$ & $0.1177 \mathrm{e}+0$ & $0.1777 \mathrm{e}+1$ & $-0.2833 e+1$ & (8) \\
\hline
\end{tabular}


Table 29: Constants for the angular dependence of the particle reflection coefficient at several energies.

\begin{tabular}{|c|c|c|c|c|c|c|c|}
\hline ion & solid & energy & c1 & c2 & c3 & $\mathrm{c} 4$ & fit \\
\hline${ }^{4} \mathrm{He}$ & $\mathrm{Ta}$ & 150000 & $0.1705 \mathrm{e}+1$ & $0.1645 \mathrm{e}+1$ & $0.1423 \mathrm{e}+1$ & $-0.2799 e+1$ & (8) \\
\hline $\mathrm{Ne}$ & $\mathrm{Ta}$ & 45000 & $0.3087 \mathrm{e}+1$ & $0.2914 \mathrm{e}+1$ & $0.1117 \mathrm{e}+1$ & $-0.2746 e+1$ & (8) \\
\hline Ar & $\mathrm{Ta}$ & 1050 & $0.1780 \mathrm{e}+1$ & $0.1496 \mathrm{e}+1$ & $0.1289 \mathrm{e}+1$ & $-0.2479 e+1$ & (8) \\
\hline $\mathrm{Kr}$ & $\mathrm{Ta}$ & 45000 & $0.2144 \mathrm{e}+1$ & $0.2071 \mathrm{e}+1$ & $0.1433 e+1$ & $-0.2950 e+1$ & (8) \\
\hline $\mathrm{H}$ & $\mathrm{Au}$ & 1000 & $0.6395 \mathrm{e}+1$ & $0.5911 \mathrm{e}+1$ & $0.1199 \mathrm{e}+1$ & $-0.3392 e+1$ & (8) \\
\hline $\mathrm{H}$ & $\mathrm{Au}$ & 1333 & $0.4506 \mathrm{e}+1$ & $0.4051 \mathrm{e}+1$ & $0.1173 e+1$ & $-0.3143 e+1$ & (8) \\
\hline $\mathrm{H}$ & $\mathrm{Au}$ & 3000 & $0.4679 \mathrm{e}+1$ & $0.4314 \mathrm{e}+1$ & $0.1047 \mathrm{e}+1$ & $-0.2954 \mathrm{e}+1$ & (8) \\
\hline $\mathrm{H}$ & $\mathrm{Au}$ & 4000 & $0.2591 \mathrm{e}+1$ & $0.2246 \mathrm{e}+1$ & $0.1384 \mathrm{e}+1$ & $-0.2946 e+1$ & (8) \\
\hline $\mathrm{H}$ & $\mathrm{Au}$ & 5000 & $0.3810 \mathrm{e}+1$ & $0.3508 \mathrm{e}+1$ & $0.1007 \mathrm{e}+1$ & $-0.2755 \mathrm{e}+1$ & (8) \\
\hline $\mathrm{H}$ & $\mathrm{Au}$ & 10000 & $0.3673 \mathrm{e}+1$ & $0.3457 \mathrm{e}+1$ & $0.1033 e+1$ & $-0.2743 e+1$ & (8) \\
\hline $\mathrm{D}$ & $\mathrm{Au}$ & 150 & $0.4578 \mathrm{e}+1$ & $0.3964 \mathrm{e}+1$ & $0.1062 \mathrm{e}+1$ & $-0.3058 e+1$ & (8) \\
\hline $\mathrm{D}$ & $\mathrm{Au}$ & 200 & $0.7234 \mathrm{e}+1$ & $0.6630 \mathrm{e}+1$ & $0.1101 \mathrm{e}+1$ & $-0.3382 e+1$ & (8) \\
\hline $\mathrm{D}$ & $\mathrm{Au}$ & 250 & $0.5630 \mathrm{e}+1$ & $0.5037 \mathrm{e}+1$ & $0.1119 \mathrm{e}+1$ & $-0.3261 e+1$ & (8) \\
\hline $\mathrm{D}$ & $\mathrm{Au}$ & 300 & $0.6736 \mathrm{e}+1$ & $0.6152 \mathrm{e}+1$ & $0.1079 \mathrm{e}+1$ & $-0.3311 e+1$ & (8) \\
\hline $\mathrm{D}$ & $\mathrm{Au}$ & 500 & $0.5982 \mathrm{e}+1$ & $0.5425 \mathrm{e}+1$ & $0.1101 \mathrm{e}+1$ & $-0.3277 \mathrm{e}+1$ & (8) \\
\hline $\mathrm{D}$ & $\mathrm{Au}$ & 750 & $0.4573 \mathrm{e}+1$ & $0.4041 \mathrm{e}+1$ & $0.1159 \mathrm{e}+1$ & $-0.3187 e+1$ & (8) \\
\hline $\mathrm{D}$ & $\mathrm{Au}$ & 1000 & $0.5033 \mathrm{e}+1$ & $0.4531 \mathrm{e}+1$ & $0.1025 \mathrm{e}+1$ & $-0.3042 e+1$ & (8) \\
\hline $\mathrm{D}$ & $\mathrm{Au}$ & 2500 & $0.4866 \mathrm{e}+1$ & $0.4431 \mathrm{e}+1$ & $0.1113 e+1$ & $-0.3115 e+1$ & (8) \\
\hline $\mathrm{D}$ & $\mathrm{Au}$ & 3000 & $0.3512 \mathrm{e}+1$ & $0.3101 \mathrm{e}+1$ & $0.1075 \mathrm{e}+1$ & $-0.2855 e+1$ & (8) \\
\hline $\mathrm{D}$ & $\mathrm{Au}$ & 5000 & $0.4666 \mathrm{e}+1$ & $0.4320 \mathrm{e}+1$ & $0.9854 \mathrm{e}+0$ & $-0.2865 \mathrm{e}+1$ & (8) \\
\hline $\mathrm{D}$ & $\mathrm{Au}$ & 8300 & $0.4730 \mathrm{e}+1$ & $0.4447 \mathrm{e}+1$ & $0.9724 \mathrm{e}+0$ & $-0.2827 e+1$ & (8) \\
\hline $\mathrm{D}$ & $\mathrm{Au}$ & 10000 & $0.2791 \mathrm{e}+1$ & $0.2527 \mathrm{e}+1$ & $0.1016 \mathrm{e}+1$ & $-0.2581 e+1$ & (8) \\
\hline${ }^{4} \mathrm{He}$ & $\mathrm{Au}$ & 5000 & $0.4771 \mathrm{e}+1$ & $0.4382 \mathrm{e}+1$ & $0.1145 \mathrm{e}+1$ & $-0.3102 e+1$ & (8) \\
\hline $\mathrm{Ne}$ & $\mathrm{Au}$ & 6000 & $0.4458 \mathrm{e}+1$ & $0.4149 \mathrm{e}+1$ & $0.1141 \mathrm{e}+1$ & $-0.2991 \mathrm{e}+1$ & (8) \\
\hline $\mathrm{Ne}$ & $\mathrm{Au}$ & 14000 & $0.4288 \mathrm{e}+1$ & $0.4019 \mathrm{e}+1$ & $0.1177 \mathrm{e}+1$ & $-0.3022 e+1$ & (8) \\
\hline Ar & $\mathrm{Au}$ & 1050 & $0.1180 \mathrm{e}+1$ & $0.8702 \mathrm{e}+0$ & $0.1446 \mathrm{e}+1$ & $-0.2343 e+1$ & (8) \\
\hline Ar & $\mathrm{Au}$ & 3000 & $0.4166 \mathrm{e}+1$ & $0.3907 \mathrm{e}+1$ & $0.1254 \mathrm{e}+1$ & $-0.3018 e+1$ & (8) \\
\hline Ar & $\mathrm{Au}$ & 6000 & $0.4187 \mathrm{e}+1$ & $0.3949 \mathrm{e}+1$ & $0.1276 \mathrm{e}+1$ & $-0.3079 e+1$ & (8) \\
\hline Ar & $\mathrm{Au}$ & 10000 & $0.3802 \mathrm{e}+1$ & $0.3582 \mathrm{e}+1$ & $0.1287 e+1$ & $-0.3057 e+1$ & (8) \\
\hline Ar & $\mathrm{Au}$ & 30000 & $0.4171 \mathrm{e}+1$ & $0.4002 \mathrm{e}+1$ & $0.1212 \mathrm{e}+1$ & $-0.3031 e+1$ & (8) \\
\hline $\mathrm{Xe}$ & $\mathrm{Au}$ & 10000 & $0.2077 \mathrm{e}+1$ & $0.2008 \mathrm{e}+1$ & $0.1513 \mathrm{e}+1$ & $-0.2947 \mathrm{e}+1$ & (8) \\
\hline $\mathrm{Kr}$ & $\mathrm{Hg}$ & 762 & $0.1380 \mathrm{e}+1$ & $0.1197 \mathrm{e}+1$ & $0.1417 \mathrm{e}+1$ & $-0.2428 \mathrm{e}+1$ & $(8)$ \\
\hline $\mathrm{H}$ & $\mathrm{U}$ & 2000 & $0.5500 \mathrm{e}+1$ & $0.5061 \mathrm{e}+1$ & $0.1064 \mathrm{e}+1$ & $-0.3119 e+1$ & (8) \\
\hline $\mathrm{Kr}$ & $\mathrm{U}$ & 17900 & $0.2526 \mathrm{e}+1$ & $0.2385 \mathrm{e}+1$ & $0.1404 \mathrm{e}+1$ & $-0.2973 e+1$ & (8) \\
\hline
\end{tabular}


Table 30: Constants for the angular dependence of the energy reflection coefficient at several energies.

\begin{tabular}{|c|c|c|c|c|c|c|c|}
\hline ion & solid & energy & c1 & c2 & c3 & c4 & fit \\
\hline $\mathrm{H}$ & $\mathrm{Be}$ & 10 & $0.5642 \mathrm{e}+0$ & $0.3808 \mathrm{e}+0$ & $0.1992 \mathrm{e}+1$ & $-0.2069 e+1$ & (8) \\
\hline $\mathrm{H}$ & $\mathrm{Be}$ & 15 & $0.6015 \mathrm{e}+0$ & $0.4411 \mathrm{e}+0$ & $0.2010 \mathrm{e}+1$ & $-0.2230 \mathrm{e}+1$ & (8) \\
\hline $\mathrm{H}$ & $\mathrm{Be}$ & 17 & $0.6151 \mathrm{e}+0$ & $0.4625 \mathrm{e}+0$ & $0.2027 \mathrm{e}+1$ & $-0.2293 e+1$ & (8) \\
\hline $\mathrm{H}$ & $\mathrm{Be}$ & 20 & $0.6336 \mathrm{e}+0$ & $0.4870 \mathrm{e}+0$ & $0.2061 \mathrm{e}+1$ & $-0.2400 \mathrm{e}+1$ & (8) \\
\hline $\mathrm{H}$ & $\mathrm{Be}$ & 22 & $0.6344 \mathrm{e}+0$ & $0.4916 \mathrm{e}+0$ & $0.2106 \mathrm{e}+1$ & $-0.2465 e+1$ & (8) \\
\hline $\mathrm{H}$ & $\mathrm{Be}$ & 25 & $0.6594 \mathrm{e}+0$ & $0.5230 \mathrm{e}+0$ & $0.2080 \mathrm{e}+1$ & $-0.2513 e+1$ & (8) \\
\hline $\mathrm{H}$ & $\mathrm{Be}$ & 30 & $0.7091 \mathrm{e}+0$ & $0.5827 \mathrm{e}+0$ & $0.2033 e+1$ & $-0.2583 e+1$ & (8) \\
\hline $\mathrm{H}$ & $\mathrm{Be}$ & 40 & $0.7937 \mathrm{e}+0$ & $0.6788 \mathrm{e}+0$ & $0.2026 \mathrm{e}+1$ & $-0.2743 e+1$ & (8) \\
\hline $\mathrm{H}$ & $\mathrm{Be}$ & 50 & $0.9247 \mathrm{e}+0$ & $0.8182 \mathrm{e}+0$ & $0.1973 e+1$ & $-0.2860 e+1$ & (8) \\
\hline $\mathrm{H}$ & $\mathrm{Be}$ & 70 & $0.1305 \mathrm{e}+1$ & $0.1211 \mathrm{e}+1$ & $0.1904 \mathrm{e}+1$ & $-0.3091 e+1$ & (8) \\
\hline $\mathrm{H}$ & $\mathrm{Be}$ & 100 & $0.2010 \mathrm{e}+1$ & $0.1928 \mathrm{e}+1$ & $0.1884 \mathrm{e}+1$ & $-0.3392 \mathrm{e}+1$ & (8) \\
\hline $\mathrm{H}$ & $\mathrm{Be}$ & 140 & $0.1749 \mathrm{e}+1$ & $0.1678 \mathrm{e}+1$ & $0.1953 \mathrm{e}+1$ & $-0.3445 e+1$ & (8) \\
\hline $\mathrm{H}$ & $\mathrm{Be}$ & 200 & $0.1766 \mathrm{e}+1$ & $0.1705 \mathrm{e}+1$ & $0.2003 e+1$ & $-0.3574 e+1$ & (8) \\
\hline $\mathrm{H}$ & $\mathrm{Be}$ & 300 & $0.1901 \mathrm{e}+1$ & $0.1853 \mathrm{e}+1$ & $0.2054 \mathrm{e}+1$ & $-0.3737 e+1$ & (8) \\
\hline $\mathrm{H}$ & $\mathrm{Be}$ & 500 & $0.1357 \mathrm{e}+1$ & $0.1326 \mathrm{e}+1$ & $0.2104 \mathrm{e}+1$ & $-0.3705 e+1$ & (8) \\
\hline $\mathrm{H}$ & $\mathrm{Be}$ & 1000 & $0.1245 \mathrm{e}+1$ & $0.1229 \mathrm{e}+1$ & $0.2149 \mathrm{e}+1$ & $-0.3869 e+1$ & (8) \\
\hline $\mathrm{D}$ & $\mathrm{Be}$ & 11 & $0.5096 \mathrm{e}+0$ & $0.4238 \mathrm{e}+0$ & $0.2051 \mathrm{e}+1$ & $-0.2246 \mathrm{e}+1$ & (8) \\
\hline D & $\mathrm{Be}$ & 12 & & $\mathrm{le}+0$ & $0.2044 \mathrm{e}+1$ & $-0.2275 e+1$ & (8) \\
\hline $\mathrm{D}$ & $\mathrm{Be}$ & 13 & $0.5319 \mathrm{e}+0$ & $0.4493 \mathrm{e}+0$ & $0.2084 \mathrm{e}+1$ & $-0.2332 \mathrm{e}+1$ & (8) \\
\hline $\mathrm{D}$ & $\mathrm{Be}$ & 14 & $0.5388 \mathrm{e}+0$ & $0.4573 \mathrm{e}+0$ & $0.2102 \mathrm{e}+1$ & $-0.2367 \mathrm{e}+1$ & (8) \\
\hline $\mathrm{D}$ & $\mathrm{Be}$ & 15 & $38 \mathrm{e}+0$ & $38 e+0$ & $0.2103 e+1$ & $-0.2408 \mathrm{e}+1$ & (8) \\
\hline $\mathrm{D}$ & $\mathrm{Be}$ & 17 & $0.5712 \mathrm{e}+0$ & $0.4936 \mathrm{e}+0$ & $0.2145 \mathrm{e}+1$ & $-0.2494 \mathrm{e}+1$ & (8) \\
\hline $\mathrm{D}$ & $\mathrm{Be}$ & 20 & $0.5990 \mathrm{e}+0$ & $0.5260 \mathrm{e}+0$ & $0.2134 \mathrm{e}+1$ & $-0.2558 \mathrm{e}+1$ & (8) \\
\hline D & $\mathrm{Be}$ & 25 & $0.6502 \mathrm{e}+0$ & $0.5825 \mathrm{e}+0$ & $0.2140 \mathrm{e}+1$ & $-0.2682 \mathrm{e}+1$ & (8) \\
\hline $\mathrm{D}$ & $\mathrm{Be}$ & 30 & $0.7089 \mathrm{e}+0$ & $0.6458 \mathrm{e}+0$ & $0.2115 \mathrm{e}+1$ & $-0.2769 \mathrm{e}+1$ & (8) \\
\hline $\mathrm{D}$ & $\mathrm{Be}$ & 40 & $0.7833 \mathrm{e}+0$ & $0.7259 \mathrm{e}+0$ & $0.2150 \mathrm{e}+1$ & $-0.2941 \mathrm{e}+1$ & (8) \\
\hline $\mathrm{D}$ & $\mathrm{Be}$ & 50 & $0.9649 \mathrm{e}+0$ & $0.9120 \mathrm{e}+0$ & $0.2082 \mathrm{e}+1$ & $-0.3076 e+1$ & (8) \\
\hline D & $\mathrm{Be}$ & 70 & $0.1314 \mathrm{e}+1$ & $0.1267 \mathrm{e}+1$ & $0.2039 \mathrm{e}+1$ & $-0.3288 \mathrm{e}+1$ & (8) \\
\hline $\mathrm{D}$ & $\mathrm{Be}$ & 100 & $0.1722 \mathrm{e}+1$ & $0.1680 \mathrm{e}+1$ & $0.2075 \mathrm{e}+1$ & $-0.3563 e+1$ & (8) \\
\hline $\mathrm{D}$ & $\mathrm{Be}$ & 140 & $0.1448 \mathrm{e}+1$ & $0.1410 \mathrm{e}+1$ & $0.2163 \mathrm{e}+1$ & $-0.3620 \mathrm{e}+1$ & (8) \\
\hline $\mathrm{D}$ & $\mathrm{Be}$ & 200 & $0.1404 \mathrm{e}+1$ & $0.1372 \mathrm{e}+1$ & $0.2167 \mathrm{e}+1$ & $-0.3676 \mathrm{e}+1$ & (8) \\
\hline $\mathrm{D}$ & $\mathrm{Be}$ & 300 & $0.1326 \mathrm{e}+1$ & $0.1300 \mathrm{e}+1$ & $0.2163 \mathrm{e}+1$ & $-0.3693 e+1$ & (8) \\
\hline $\mathrm{D}$ & $\mathrm{Be}$ & 500 & $0.1250 \mathrm{e}+1$ & $0.1231 \mathrm{e}+1$ & $0.2185 \mathrm{e}+1$ & $-0.3787 e+1$ & (8) \\
\hline D & $\mathrm{Be}$ & 1000 & $0.1179 \mathrm{e}+1$ & $0.1169 \mathrm{e}+1$ & $0.2229 \mathrm{e}+1$ & $-0.3944 e+1$ & (8) \\
\hline
\end{tabular}


Table 31: Constants for the angular dependence of the energy reflection coefficient at several energies.

\begin{tabular}{|c|c|c|c|c|c|c|c|}
\hline ion & solid & energy & $\mathrm{c} 1$ & $\mathrm{c} 2$ & $\mathrm{c3}$ & $\mathrm{c} 4$ & fit \\
\hline $\mathrm{T}$ & $\mathrm{Be}$ & 10 & $0.4506 \mathrm{e}+0$ & $0.4143 \mathrm{e}+0$ & $0.2083 \mathrm{e}+1$ & $-0.2347 \mathrm{e}+1$ & (8) \\
\hline $\mathrm{T}$ & $\mathrm{Be}$ & 11 & $0.4593 \mathrm{e}+0$ & $0.4216 \mathrm{e}+0$ & $0.2152 \mathrm{e}+1$ & $-0.2417 e+1$ & (8) \\
\hline $\mathrm{T}$ & $\mathrm{Be}$ & 12 & $0.4760 \mathrm{e}+0$ & $0.4386 \mathrm{e}+0$ & $0.2135 \mathrm{e}+1$ & $-0.2430 \mathrm{e}+1$ & (8) \\
\hline $\mathrm{T}$ & $\mathrm{Be}$ & 13 & $0.4848 \mathrm{e}+0$ & $0.4464 \mathrm{e}+0$ & $0.2196 \mathrm{e}+1$ & $-0.2509 e+1$ & (8) \\
\hline $\mathrm{T}$ & $\mathrm{Be}$ & 15 & $0.5285 \mathrm{e}+0$ & $0.4911 \mathrm{e}+0$ & $0.2165 \mathrm{e}+1$ & $-0.2559 \mathrm{e}+1$ & (8) \\
\hline $\mathrm{T}$ & $\mathrm{Be}$ & 20 & $0.5809 \mathrm{e}+0$ & $0.5454 \mathrm{e}+0$ & $0.2218 \mathrm{e}+1$ & $-0.2727 \mathrm{e}+1$ & (8) \\
\hline $\mathrm{T}$ & $\mathrm{Be}$ & 25 & $0.5696 \mathrm{e}+0$ & $0.5353 \mathrm{e}+0$ & $0.2316 \mathrm{e}+1$ & $-0.2864 \mathrm{e}+1$ & (8) \\
\hline $\mathrm{T}$ & $\mathrm{Be}$ & 30 & $0.6714 \mathrm{e}+0$ & $0.6394 \mathrm{e}+0$ & $0.2292 \mathrm{e}+1$ & $-0.2994 \mathrm{e}+1$ & (8) \\
\hline $\mathrm{T}$ & $\mathrm{Be}$ & 50 & $0.9383 \mathrm{e}+0$ & $0.9118 \mathrm{e}+0$ & $0.2243 \mathrm{e}+1$ & $-0.3294 e+1$ & (8) \\
\hline $\mathrm{T}$ & $\mathrm{Be}$ & 100 & $0.1367 \mathrm{e}+1$ & $0.1346 \mathrm{e}+1$ & $0.2281 \mathrm{e}+1$ & $-0.3715 e+1$ & (8) \\
\hline $\mathrm{T}$ & $\mathrm{Be}$ & 200 & $0.1230 \mathrm{e}+1$ & $0.1214 \mathrm{e}+1$ & $0.2349 \mathrm{e}+1$ & $-0.3856 e+1$ & (8) \\
\hline $\mathrm{T}$ & $\mathrm{Be}$ & 300 & $0.1169 \mathrm{e}+1$ & $0.1156 \mathrm{e}+1$ & $0.2321 \mathrm{e}+1$ & $-0.3870 e+1$ & (8) \\
\hline $\mathrm{T}$ & $\mathrm{Be}$ & 500 & $0.1190 \mathrm{e}+1$ & $0.1180 \mathrm{e}+1$ & $0.2302 \mathrm{e}+1$ & $-0.3951 e+1$ & (8) \\
\hline $\mathrm{T}$ & $\mathrm{Be}$ & 1000 & $0.1090 \mathrm{e}+1$ & $0.1085 e+1$ & $0.2322 \mathrm{e}+1$ & $-0.4052 \mathrm{e}+1$ & (8) \\
\hline${ }^{4} \mathrm{He}$ & $\mathrm{Be}$ & 10 & $0.6224 \mathrm{e}+0$ & $0.5846 \mathrm{e}+0$ & $0.2014 \mathrm{e}+1$ & $-0.2338 \mathrm{e}+1$ & (8) \\
\hline${ }^{4} \mathrm{He}$ & $\mathrm{Be}$ & 11 & $0.6297 \mathrm{e}+0$ & $0.5933 \mathrm{e}+0$ & $0.2019 \mathrm{e}+1$ & $-0.2356 \mathrm{e}+1$ & (8) \\
\hline${ }^{4} \mathrm{He}$ & $\mathrm{Be}$ & 12 & $0.6234 \mathrm{e}+0$ & $0.5871 \mathrm{e}+0$ & $0.2066 \mathrm{e}+1$ & $-0.2398 \mathrm{e}+1$ & (8) \\
\hline${ }^{4} \mathrm{He}$ & $\mathrm{Be}$ & 13 & $0.6326 \mathrm{e}+0$ & $0.5971 \mathrm{e}+0$ & $0.2074 \mathrm{e}+1$ & $-0.2425 \mathrm{e}+1$ & (8) \\
\hline${ }^{4} \mathrm{He}$ & $\mathrm{Be}$ & 15 & $0.6254 \mathrm{e}+0$ & $0.5910 \mathrm{e}+0$ & $0.2143 \mathrm{e}+1$ & $-0.2499 e+1$ & (8) \\
\hline${ }^{4} \mathrm{He}$ & $\mathrm{Be}$ & 17 & $0.6307 \mathrm{e}+0$ & $0.5983 \mathrm{e}+0$ & $0.2172 \mathrm{e}+1$ & $-0.2549 e+1$ & (8) \\
\hline${ }^{4} \mathrm{He}$ & $\mathrm{Be}$ & 20 & $0.6256 \mathrm{e}+0$ & $0.5946 \mathrm{e}+0$ & $0.2260 \mathrm{e}+1$ & $-0.2653 e+1$ & (8) \\
\hline${ }^{4} \mathrm{He}$ & $\mathrm{Be}$ & 25 & $0.6389 \mathrm{e}+0$ & $0.6112 \mathrm{e}+0$ & $0.2317 \mathrm{e}+1$ & $-0.2771 \mathrm{e}+1$ & (8) \\
\hline${ }^{4} \mathrm{He}$ & $\mathrm{Be}$ & 30 & $0.6456 \mathrm{e}+0$ & $0.6196 \mathrm{e}+0$ & $0.2383 \mathrm{e}+1$ & $-0.2892 \mathrm{e}+1$ & (8) \\
\hline${ }^{4} \mathrm{He}$ & $\mathrm{Be}$ & 40 & $0.6735 \mathrm{e}+0$ & $0.6508 e+0$ & $0.2466 \mathrm{e}+1$ & $-0.3086 e+1$ & (8) \\
\hline${ }^{4} \mathrm{He}$ & $\mathrm{Be}$ & 50 & $0.7428 \mathrm{e}+0$ & $0.7231 \mathrm{e}+0$ & $0.2437 \mathrm{e}+1$ & $-0.3196 e+1$ & (8) \\
\hline${ }^{4} \mathrm{He}$ & $\mathrm{Be}$ & 70 & $0.8488 \mathrm{e}+0$ & $0.8318 \mathrm{e}+0$ & $0.2464 \mathrm{e}+1$ & $-0.3415 e+1$ & (8) \\
\hline${ }^{4} \mathrm{He}$ & $\mathrm{Be}$ & 100 & $0.1104 \mathrm{e}+1$ & $0.1089 \mathrm{e}+1$ & $0.2428 \mathrm{e}+1$ & $-0.3649 e+1$ & (8) \\
\hline${ }^{4} \mathrm{He}$ & $\mathrm{Be}$ & 140 & $0.1215 \mathrm{e}+1$ & $0.1203 e+1$ & $0.2476 \mathrm{e}+1$ & $-0.3845 e+1$ & (8) \\
\hline${ }^{4} \mathrm{He}$ & $\mathrm{Be}$ & 200 & $0.1240 \mathrm{e}+1$ & $0.1229 \mathrm{e}+1$ & $0.2542 \mathrm{e}+1$ & $-0.4017 e+1$ & (8) \\
\hline${ }^{4} \mathrm{He}$ & $\mathrm{Be}$ & 300 & $0.1201 \mathrm{e}+1$ & $0.1192 \mathrm{e}+1$ & $0.2576 \mathrm{e}+1$ & $-0.4128 \mathrm{e}+1$ & (8) \\
\hline${ }^{4} \mathrm{He}$ & $\mathrm{Be}$ & 400 & $0.1117 \mathrm{e}+1$ & $0.1110 \mathrm{e}+1$ & $0.2568 \mathrm{e}+1$ & $-0.4144 \mathrm{e}+1$ & (8) \\
\hline${ }^{4} \mathrm{He}$ & $\mathrm{Be}$ & 500 & $0.1122 \mathrm{e}+1$ & $0.1115 \mathrm{e}+1$ & $0.2545 \mathrm{e}+1$ & $-0.4164 e+1$ & (8) \\
\hline${ }^{4} \mathrm{He}$ & $\mathrm{Be}$ & 700 & $0.1121 \mathrm{e}+1$ & $0.1115 \mathrm{e}+1$ & $0.2496 \mathrm{e}+1$ & $-0.4177 \mathrm{e}+1$ & (8) \\
\hline${ }^{4} \mathrm{He}$ & $\mathrm{Be}$ & 1000 & $0.1084 \mathrm{e}+1$ & $0.1080 \mathrm{e}+1$ & $0.2496 \mathrm{e}+1$ & $-0.4226 e+1$ & (8) \\
\hline${ }^{4} \mathrm{He}$ & $\mathrm{Be}$ & 2000 & $0.1052 \mathrm{e}+1$ & $0.1049 \mathrm{e}+1$ & $0.2568 \mathrm{e}+1$ & $-0.4435 e+1$ & (8) \\
\hline${ }^{4} \mathrm{He}$ & $\mathrm{Be}$ & 5000 & $0.1032 \mathrm{e}+1$ & $0.1031 \mathrm{e}+1$ & $0.2858 \mathrm{e}+1$ & $-0.5010 \mathrm{e}+1$ & (8) \\
\hline $\mathrm{D}$ & B & 30 & $0.7477 \mathrm{e}+0$ & $0.6587 \mathrm{e}+0$ & $0.2018 \mathrm{e}+1$ & $-0.2603 e+1$ & $(8)$ \\
\hline $\mathrm{D}$ & B & 50 & $0.8080 \mathrm{e}+0$ & $0.7322 \mathrm{e}+0$ & $0.2098 \mathrm{e}+1$ & $-0.2868 \mathrm{e}+1$ & (8) \\
\hline $\mathrm{D}$ & B & 100 & $0.1649 \mathrm{e}+1$ & $0.1591 \mathrm{e}+1$ & $0.1935 \mathrm{e}+1$ & $-0.3307 \mathrm{e}+1$ & (8) \\
\hline $\mathrm{D}$ & B & 400 & $0.1459 \mathrm{e}+1$ & $0.1424 \mathrm{e}+1$ & $0.2090 \mathrm{e}+1$ & $-0.3660 e+1$ & (8) \\
\hline $\mathrm{D}$ & B & 500 & $0.1356 \mathrm{e}+1$ & $0.1327 \mathrm{e}+1$ & $0.2098 \mathrm{e}+1$ & $-0.3650 e+1$ & (8) \\
\hline $\mathrm{D}$ & B & 8000 & $0.9680 \mathrm{e}+0$ & $0.9666 \mathrm{e}+0$ & $0.3236 \mathrm{e}+1$ & $-0.5648 e+1$ & (8) \\
\hline
\end{tabular}


Table 32: Constants for the angular dependence of the energy reflection coefficient at several energies.

\begin{tabular}{|c|c|c|c|c|c|c|c|}
\hline ion & solid & energy & c1 & c2 & c3 & c4 & fit \\
\hline $\mathrm{H}$ & $\mathrm{C}$ & 10 & $0.5915 \mathrm{e}+0$ & $0.3602 \mathrm{e}+0$ & $0.1980 \mathrm{e}+1$ & $-0.2047 e+1$ & (8) \\
\hline $\mathrm{H}$ & $\mathrm{C}$ & 20 & $0.6546 \mathrm{e}+0$ & $0.4654 \mathrm{e}+0$ & $0.1995 \mathrm{e}+1$ & $-0.2323 e+1$ & (8) \\
\hline $\mathrm{H}$ & $\mathrm{C}$ & 40 & $0.6795 \mathrm{e}+0$ & $0.5131 \mathrm{e}+0$ & $0.2296 \mathrm{e}+1$ & $-0.2837 e+1$ & (8) \\
\hline $\mathrm{H}$ & $\mathrm{C}$ & 50 & $0.7432 \mathrm{e}+0$ & $0.5876 \mathrm{e}+0$ & $0.2247 \mathrm{e}+1$ & $-0.2919 e+1$ & (8) \\
\hline $\mathrm{H}$ & $\mathrm{C}$ & 70 & $0.1186 \mathrm{e}+1$ & $0.1057 \mathrm{e}+1$ & $0.1829 \mathrm{e}+1$ & $-0.2917 e+1$ & (8) \\
\hline $\mathrm{H}$ & $\mathrm{C}$ & 100 & $0.2327 \mathrm{e}+1$ & $0.2212 \mathrm{e}+1$ & $0.1720 \mathrm{e}+1$ & $-0.3266 e+1$ & (8) \\
\hline $\mathrm{H}$ & $\mathrm{C}$ & 140 & $0.2456 \mathrm{e}+1$ & $0.2354 \mathrm{e}+1$ & $0.1783 e+1$ & $-0.3416 e+1$ & (8) \\
\hline $\mathrm{H}$ & $\mathrm{C}$ & 200 & $0.1861 \mathrm{e}+1$ & $0.1771 \mathrm{e}+1$ & $0.1898 e+1$ & $-0.3444 e+1$ & (8) \\
\hline $\mathrm{H}$ & $\mathrm{C}$ & 300 & $0.1960 \mathrm{e}+1$ & $0.1886 \mathrm{e}+1$ & $0.1908 \mathrm{e}+1$ & $-0.3553 e+1$ & (8) \\
\hline $\mathrm{H}$ & $\mathrm{C}$ & 500 & $0.1668 \mathrm{e}+1$ & $0.1615 \mathrm{e}+1$ & $0.1904 \mathrm{e}+1$ & $-0.3530 e+1$ & (8) \\
\hline $\mathrm{H}$ & $\mathrm{C}$ & 1000 & $0.1496 \mathrm{e}+1$ & $0.1465 \mathrm{e}+1$ & $0.1976 \mathrm{e}+1$ & $-0.3671 e+1$ & (8) \\
\hline $\mathrm{H}$ & $\mathrm{C}$ & 2000 & $0.1216 \mathrm{e}+1$ & $0.1201 \mathrm{e}+1$ & $0.2318 \mathrm{e}+1$ & $-0.4105 e+1$ & (8) \\
\hline $\mathrm{H}$ & $\mathrm{C}$ & 13333 & $0.9842 \mathrm{e}+0$ & $0.9832 \mathrm{e}+0$ & $0.3586 \mathrm{e}+1$ & $-0.6375 e+1$ & (8) \\
\hline $\mathrm{H}$ & $\mathrm{C}$ & 26667 & $0.8928 \mathrm{e}+0$ & $0.8923 \mathrm{e}+0$ & $0.4331 \mathrm{e}+1$ & $-0.7719 e+1$ & (8) \\
\hline $\mathrm{D}$ & $\mathrm{C}$ & 10 & $0.5330 \mathrm{e}+0$ & $0.3979 e+0$ & $0.1981 \mathrm{e}+1$ & $-0.2133 e+1$ & (8) \\
\hline $\mathrm{D}$ & $\mathrm{C}$ & 20 & $0.6298 \mathrm{e}+0$ & $0.5159 \mathrm{e}+0$ & $0.2052 \mathrm{e}+1$ & $-0.2465 e+1$ & (8) \\
\hline $\mathrm{D}$ & $\mathrm{C}$ & 30 & $0.6918 \mathrm{e}+0$ & $0.5901 \mathrm{e}+0$ & $0.2093 e+1$ & $-0.2675 e+1$ & (8) \\
\hline $\mathrm{D}$ & $\mathrm{C}$ & 33 & $0.7437 \mathrm{e}+0$ & $0.6456 \mathrm{e}+0$ & $0.1978 \mathrm{e}+1$ & $92 \mathrm{e}+1$ & (8) \\
\hline D & $\mathrm{C}$ & 40 & $0.8061 \mathrm{e}+0$ & $0.7136 \mathrm{e}+0$ & $0.2027 \mathrm{e}+1$ & $-0.2794 e+1$ & (8) \\
\hline D & $\mathrm{C}$ & 50 & $0.8668 \mathrm{e}+0$ & $0.7794 \mathrm{e}+0$ & $0.2029 e+1$ & $-0.2874 \mathrm{e}+1$ & (8) \\
\hline $\mathrm{D}$ & $\mathrm{C}$ & 70 & $0.1331 \mathrm{e}+1$ & $0.1254 \mathrm{e}+1$ & $0.1900 \mathrm{e}+1$ & $08 e+1$ & (8) \\
\hline $\mathrm{D}$ & $\mathrm{C}$ & 100 & $0.2148 \mathrm{e}+1$ & $0.2077 \mathrm{e}+1$ & $0.1886 \mathrm{e}+1$ & $-0.3441 e+1$ & (8) \\
\hline $\mathrm{D}$ & $\mathrm{C}$ & 140 & $0.1867 \mathrm{e}+1$ & $0.1802 \mathrm{e}+1$ & $0.1965 \mathrm{e}+1$ & $-0.3502 e+1$ & (8) \\
\hline $\mathrm{D}$ & $\mathrm{C}$ & 200 & $0.1980 \mathrm{e}+1$ & $0.1925 \mathrm{e}+1$ & $0.1975 \mathrm{e}+1$ & $95 e+1$ & (8) \\
\hline D & $\mathrm{C}$ & 300 & $0.1678 \mathrm{e}+1$ & $0.1630 \mathrm{e}+1$ & $0.1985 \mathrm{e}+1$ & $-0.3569 e+1$ & (8) \\
\hline $\mathrm{D}$ & $\mathrm{C}$ & 350 & $0.1692 \mathrm{e}+1$ & $0.1647 \mathrm{e}+1$ & $0.2046 \mathrm{e}+1$ & $-0.3642 e+1$ & (8) \\
\hline $\mathrm{D}$ & $\mathrm{C}$ & 400 & $0.1706 \mathrm{e}+1$ & $0.1664 \mathrm{e}+1$ & $0.2035 \mathrm{e}+1$ & $-0.3671 \mathrm{e}+1$ & (8) \\
\hline D & $\mathrm{C}$ & 500 & $0.1602 \mathrm{e}+1$ & $0.1564 \mathrm{e}+1$ & $0.2023 e+1$ & $-0.3668 e+1$ & (8) \\
\hline $\mathrm{D}$ & $\mathrm{C}$ & 1000 & $0.1188 \mathrm{e}+1$ & $0.1166 \mathrm{e}+1$ & $0.1997 \mathrm{e}+1$ & $-0.3562 e+1$ & (8) \\
\hline $\mathrm{D}$ & $\mathrm{C}$ & 3000 & $0.1062 \mathrm{e}+1$ & $0.1054 \mathrm{e}+1$ & $0.2406 \mathrm{e}+1$ & $-0.4214 e+1$ & (8) \\
\hline D & $\mathrm{C}$ & 10000 & $0.1029 \mathrm{e}+1$ & $0.1027 \mathrm{e}+1$ & $0.3186 \mathrm{e}+1$ & $-0.5633 e+1$ & (8) \\
\hline $\mathrm{T}$ & $\mathrm{C}$ & 10 & $0.4800 \mathrm{e}+0$ & $0.4041 \mathrm{e}+0$ & $0.2057 \mathrm{e}+1$ & $-0.2258 \mathrm{e}+1$ & (8) \\
\hline $\mathrm{T}$ & $\mathrm{C}$ & 20 & $0.6000 \mathrm{e}+0$ & $0.5329 \mathrm{e}+0$ & $0.2136 \mathrm{e}+1$ & $-0.2607 \mathrm{e}+1$ & (8) \\
\hline $\mathrm{T}$ & $\mathrm{C}$ & 25 & $0.6618 \mathrm{e}+0$ & $0.5993 e+0$ & $0.2108 \mathrm{e}+1$ & $-0.2698 \mathrm{e}+1$ & (8) \\
\hline $\mathrm{T}$ & $\mathrm{C}$ & 30 & $0.6802 \mathrm{e}+0$ & $0.6198 \mathrm{e}+0$ & $0.2183 e+1$ & $-0.2838 \mathrm{e}+1$ & (8) \\
\hline $\mathrm{T}$ & $\mathrm{C}$ & 40 & $0.8494 \mathrm{e}+0$ & $0.7952 \mathrm{e}+0$ & $0.2075 \mathrm{e}+1$ & $-0.2946 \mathrm{e}+1$ & (8) \\
\hline $\mathrm{T}$ & $\mathrm{C}$ & 50 & $0.9702 \mathrm{e}+0$ & $0.9197 \mathrm{e}+0$ & $0.2076 \mathrm{e}+1$ & $-0.3085 e+1$ & (8) \\
\hline $\mathrm{T}$ & $\mathrm{C}$ & 70 & $0.1410 \mathrm{e}+1$ & $0.1364 \mathrm{e}+1$ & $0.2014 \mathrm{e}+1$ & $-0.3314 \mathrm{e}+1$ & (8) \\
\hline $\mathrm{T}$ & $\mathrm{C}$ & 100 & $0.1802 \mathrm{e}+1$ & $0.1762 \mathrm{e}+1$ & $0.2017 \mathrm{e}+1$ & $-0.3514 \mathrm{e}+1$ & (8) \\
\hline $\mathrm{T}$ & $\mathrm{C}$ & 140 & $0.1618 \mathrm{e}+1$ & $0.1580 \mathrm{e}+1$ & $0.2130 \mathrm{e}+1$ & $-0.3645 e+1$ & (8) \\
\hline $\mathrm{T}$ & $\mathrm{C}$ & 200 & $0.1449 \mathrm{e}+1$ & $0.1415 \mathrm{e}+1$ & $0.2157 \mathrm{e}+1$ & $-0.3666 \mathrm{e}+1$ & (8) \\
\hline $\mathrm{T}$ & C & 300 & $0.1426 \mathrm{e}+1$ & $0.1397 \mathrm{e}+1$ & $0.2118 \mathrm{e}+1$ & $-0.3668 \mathrm{e}+1$ & (8) \\
\hline $\mathrm{T}$ & $\mathrm{C}$ & 500 & $0.1396 \mathrm{e}+1$ & $0.1374 \mathrm{e}+1$ & $0.2044 \mathrm{e}+1$ & $-0.3647 e+1$ & (8) \\
\hline $\mathrm{T}$ & $\mathrm{C}$ & 1000 & $0.1144 \mathrm{e}+1$ & $0.1130 \mathrm{e}+1$ & $0.2082 \mathrm{e}+1$ & $-0.3675 e+1$ & (8) \\
\hline
\end{tabular}


Table 33: Constants for the angular dependence of the energy reflection coefficient at several energies.

\begin{tabular}{|r|r|r|r|r|r|r|r|}
\hline ion & solid & energy & $\mathrm{c} 1$ & $\mathrm{c} 2$ & $\mathrm{c} 3$ & $\mathrm{c} 4$ & fit \\
\hline${ }^{4} \mathrm{He}$ & $\mathrm{C}$ & 10 & $0.6477 \mathrm{e}+0$ & $0.5694 \mathrm{e}+0$ & $0.1876 \mathrm{e}+1$ & $-0.2146 \mathrm{e}+1$ & $(8)$ \\
${ }^{4} \mathrm{He}$ & $\mathrm{C}$ & 15 & $0.6414 \mathrm{e}+0$ & $0.5721 \mathrm{e}+0$ & $0.2042 \mathrm{e}+1$ & $-0.2343 \mathrm{e}+1$ & $(8)$ \\
${ }^{4} \mathrm{He}$ & $\mathrm{C}$ & 20 & $0.6474 \mathrm{e}+0$ & $0.5861 \mathrm{e}+0$ & $0.2130 \mathrm{e}+1$ & $-0.2486 \mathrm{e}+1$ & $(8)$ \\
${ }^{4} \mathrm{He}$ & $\mathrm{C}$ & 25 & $0.6667 \mathrm{e}+0$ & $0.6111 \mathrm{e}+0$ & $0.2171 \mathrm{e}+1$ & $-0.2601 \mathrm{e}+1$ & $(8)$ \\
${ }^{4} \mathrm{He}$ & $\mathrm{C}$ & 27 & $0.6714 \mathrm{e}+0$ & $0.6175 \mathrm{e}+0$ & $0.2186 \mathrm{e}+1$ & $-0.2641 \mathrm{e}+1$ & $(8)$ \\
${ }^{4} \mathrm{He}$ & $\mathrm{C}$ & 30 & $0.6727 \mathrm{e}+0$ & $0.6205 \mathrm{e}+0$ & $0.2234 \mathrm{e}+1$ & $-0.2715 \mathrm{e}+1$ & $(8)$ \\
${ }^{4} \mathrm{He}$ & $\mathrm{C}$ & 35 & $0.6894 \mathrm{e}+0$ & $0.6408 \mathrm{e}+0$ & $0.2248 \mathrm{e}+1$ & $-0.2792 \mathrm{e}+1$ & $(8)$ \\
${ }^{4} \mathrm{He}$ & $\mathrm{C}$ & 40 & $0.7213 \mathrm{e}+0$ & $0.6755 \mathrm{e}+0$ & $0.2261 \mathrm{e}+1$ & $-0.2875 \mathrm{e}+1$ & $(8)$ \\
${ }^{4} \mathrm{He}$ & $\mathrm{C}$ & 50 & $0.7727 \mathrm{e}+0$ & $0.7314 \mathrm{e}+0$ & $0.2270 \mathrm{e}+1$ & $-0.2995 \mathrm{e}+1$ & $(8)$ \\
${ }^{4} \mathrm{He}$ & $\mathrm{C}$ & 60 & $0.8031 \mathrm{e}+0$ & $0.7646 \mathrm{e}+0$ & $0.2288 \mathrm{e}+1$ & $-0.3091 \mathrm{e}+1$ & $(8)$ \\
${ }^{4} \mathrm{He}$ & $\mathrm{C}$ & 70 & $0.8818 \mathrm{e}+0$ & $0.8456 \mathrm{e}+0$ & $0.2273 \mathrm{e}+1$ & $-0.3185 \mathrm{e}+1$ & $(8)$ \\
${ }^{4} \mathrm{He}$ & $\mathrm{C}$ & 100 & $0.1133 \mathrm{e}+1$ & $0.1102 \mathrm{e}+1$ & $0.2223 \mathrm{e}+1$ & $-0.3384 \mathrm{e}+1$ & $(8)$ \\
${ }^{4} \mathrm{He}$ & $\mathrm{C}$ & 140 & $0.1379 \mathrm{e}+1$ & $0.1351 \mathrm{e}+1$ & $0.2246 \mathrm{e}+1$ & $-0.3600 \mathrm{e}+1$ & $(8)$ \\
${ }^{4} \mathrm{He}$ & $\mathrm{C}$ & 200 & $0.1337 \mathrm{e}+1$ & $0.1312 \mathrm{e}+1$ & $0.2312 \mathrm{e}+1$ & $-0.3741 \mathrm{e}+1$ & $(8)$ \\
${ }^{4} \mathrm{He}$ & $\mathrm{C}$ & 300 & $0.1305 \mathrm{e}+1$ & $0.1284 \mathrm{e}+1$ & $0.2393 \mathrm{e}+1$ & $-0.3891 \mathrm{e}+1$ & $(8)$ \\
${ }^{4} \mathrm{He}$ & $\mathrm{C}$ & 400 & $0.1192 \mathrm{e}+1$ & $0.1173 \mathrm{e}+1$ & $0.2362 \mathrm{e}+1$ & $-0.3859 \mathrm{e}+1$ & $(8)$ \\
${ }^{4} \mathrm{He}$ & $\mathrm{C}$ & 500 & $0.1315 \mathrm{e}+1$ & $0.1298 \mathrm{e}+1$ & $0.2318 \mathrm{e}+1$ & $-0.3906 \mathrm{e}+1$ & $(8)$ \\
${ }^{4} \mathrm{He}$ & $\mathrm{C}$ & 700 & $0.1297 \mathrm{e}+1$ & $0.1282 \mathrm{e}+1$ & $0.2293 \mathrm{e}+1$ & $-0.3928 \mathrm{e}+1$ & $(8)$ \\
${ }^{4} \mathrm{He}$ & $\mathrm{C}$ & 1000 & $0.1203 \mathrm{e}+1$ & $0.1191 \mathrm{e}+1$ & $0.2259 \mathrm{e}+1$ & $-0.3901 \mathrm{e}+1$ & $(8)$ \\
${ }^{4} \mathrm{He}$ & $\mathrm{C}$ & 2000 & $0.1086 \mathrm{e}+1$ & $0.1079 \mathrm{e}+1$ & $0.2265 \mathrm{e}+1$ & $-0.3967 \mathrm{e}+1$ & $(8)$ \\
${ }^{4} \mathrm{He}$ & $\mathrm{C}$ & 3000 & $0.1047 \mathrm{e}+1$ & $0.1042 \mathrm{e}+1$ & $0.2347 \mathrm{e}+1$ & $-0.4116 \mathrm{e}+1$ & $(8)$ \\
${ }^{4} \mathrm{He}$ & $\mathrm{C}$ & 5000 & $0.1014 \mathrm{e}+1$ & $0.1011 \mathrm{e}+1$ & $0.2652 \mathrm{e}+1$ & $-0.4552 \mathrm{e}+1$ & $(8)$ \\
${ }^{4} \mathrm{He}$ & $\mathrm{C}$ & 10000 & $0.1014 \mathrm{e}+1$ & $0.1013 \mathrm{e}+1$ & $0.2918 \mathrm{e}+1$ & $-0.5096 \mathrm{e}+1$ & $(8)$ \\
${ }^{4} \mathrm{He}$ & $\mathrm{C}$ & 20000 & $0.1026 \mathrm{e}+1$ & $0.1025 \mathrm{e}+1$ & $0.3527 \mathrm{e}+1$ & $-0.6128 \mathrm{e}+1$ & $(8)$ \\
\hline
\end{tabular}


Table 34: Constants for the angular dependence of the energy reflection coefficient at several energies.

\begin{tabular}{|c|c|c|c|c|c|c|c|}
\hline ion & solid & energy & $\mathrm{c} 1$ & c2 & c3 & $\mathrm{c} 4$ & fit \\
\hline $\mathrm{H}$ & $\mathrm{Al}$ & 13333 & $0.1010 \mathrm{e}+1$ & $0.1006 \mathrm{e}+1$ & $0.2688 \mathrm{e}+1$ & $-0.4822 \mathrm{e}+1$ & \\
\hline $\mathrm{H}$ & $\mathrm{Al}$ & 26667 & $0.9637 \mathrm{e}+0$ & $0.9624 \mathrm{e}+0$ & $0.3625 \mathrm{e}+1$ & $-0.6490 e+1$ & \\
\hline $\mathrm{H}$ & $\mathrm{Al}$ & 40000 & $0.9589 \mathrm{e}+0$ & $0.9581 \mathrm{e}+0$ & $0.4068 \mathrm{e}+1$ & $-0.7287 e+1$ & \\
\hline${ }^{4} \mathrm{He}$ & $\mathrm{Si}$ & 200 & $0.2273 \mathrm{e}+1$ & $0.2171 \mathrm{e}+1$ & $0.1839 \mathrm{e}+1$ & $-0.3442 \mathrm{e}+1$ & (8) \\
\hline${ }^{4} \mathrm{He}$ & $\mathrm{Si}$ & 2000 & $0.1578 \mathrm{e}+1$ & $0.1528 \mathrm{e}+1$ & $0.1931 \mathrm{e}+1$ & $-0.3568 \mathrm{e}+1$ & (8) \\
\hline${ }^{4} \mathrm{He}$ & $\mathrm{Si}$ & 3000 & $0.1412 \mathrm{e}+1$ & $0.1373 \mathrm{e}+1$ & $0.1909 \mathrm{e}+1$ & $-0.3515 e+1$ & (8) \\
\hline $\mathrm{Si}(\mathrm{KrC})$ & $\mathrm{Si}$ & 200 & $0.9802 \mathrm{e}+0$ & $0.9801 \mathrm{e}+0$ & $0.2861 \mathrm{e}+1$ & $-0.4471 \mathrm{e}+1$ & (8) \\
\hline $\mathrm{Si}(\mathrm{Mol})$ & $\mathrm{Si}$ & 200 & $0.9687 \mathrm{e}+0$ & $0.9687 \mathrm{e}+0$ & $0.2794 \mathrm{e}+1$ & $-0.4315 e+1$ & (8) \\
\hline $\mathrm{Si}(\mathrm{Si})$ & $\mathrm{Si}$ & 200 & $0.9719 \mathrm{e}+0$ & $0.9719 \mathrm{e}+0$ & $0.2677 \mathrm{e}+1$ & $-0.4638 e+1$ & (8) \\
\hline $\mathrm{Si}(\mathrm{ZBL})$ & $\mathrm{Si}$ & 200 & $0.1014 \mathrm{e}+1$ & $0.1013 e+1$ & $0.2867 \mathrm{e}+1$ & $-0.4564 e+1$ & (8) \\
\hline $\mathrm{Si}$ & $\mathrm{Si}$ & 500 & $0.1003 \mathrm{e}+1$ & $0.1002 \mathrm{e}+1$ & $0.2993 \mathrm{e}+1$ & $-0.4706 e+1$ & (8) \\
\hline${ }^{4} \mathrm{He}$ & $\mathrm{Ti}$ & 5000 & $0.3807 \mathrm{e}+0$ & $0.3213 \mathrm{e}+0$ & $0.1976 \mathrm{e}+1$ & $-0.2669 \mathrm{e}+1$ & (8) \\
\hline${ }^{4} \mathrm{He}$ & $\mathrm{Ti}$ & 100000 & $0.9891 \mathrm{e}+0$ & $0.9871 \mathrm{e}+0$ & $0.3355 \mathrm{e}+1$ & $-0.5839 e+1$ & (8) \\
\hline $\mathrm{Ne}$ & $\mathrm{Ti}$ & 38 & $0.7573 \mathrm{e}+0$ & $0.7147 \mathrm{e}+0$ & $0.1821 \mathrm{e}+1$ & $-0.2366 \mathrm{e}+1$ & (8) \\
\hline $\mathrm{Ne}$ & $\mathrm{Ti}$ & 380 & $0.1259 \mathrm{e}+1$ & $0.1236 \mathrm{e}+1$ & $0.2197 \mathrm{e}+1$ & $-0.3520 e+1$ & (8) \\
\hline $\mathrm{Ne}$ & $\mathrm{Ti}$ & 3800 & $0.1121 \mathrm{e}+1$ & $0.1109 \mathrm{e}+1$ & $0.2143 \mathrm{e}+1$ & $-0.3740 e+1$ & (8) \\
\hline $\mathrm{Ar}$ & $\mathrm{Ti}$ & 1050 & $0.1038 \mathrm{e}+1$ & $0.1036 \mathrm{e}+1$ & $0.2880 \mathrm{e}+1$ & $-0.4522 \mathrm{e}+1$ & $(8)$ \\
\hline $\mathrm{Ga}$ & $\mathrm{Ga}$ & 100 & $0.9671 \mathrm{e}+0$ & $0.9673 \mathrm{e}+0$ & $0.2582 \mathrm{e}+1$ & $-0.3916 e+1$ & (8) \\
\hline $\mathrm{Ga}$ & $\mathrm{Ga}$ & 200 & $0.9552 \mathrm{e}+0$ & $0.9549 \mathrm{e}+0$ & $0.2697 \mathrm{e}+1$ & $-0.4059 e+1$ & (8) \\
\hline $\mathrm{Ga}$ & $\mathrm{Ga}$ & 1000 & $0.1045 \mathrm{e}+1$ & $0.1044 \mathrm{e}+1$ & $0.2821 \mathrm{e}+1$ & $-0.4520 e+1$ & $(8)$ \\
\hline $\mathrm{Ar}$ & $\mathrm{Zr}$ & 1050 & $0.1616 \mathrm{e}+1$ & $0.1469 e+1$ & $0.1648 \mathrm{e}+1$ & $-0.2762 \mathrm{e}+1$ & (8) \\
\hline $\mathrm{Ar}$ & $\mathrm{Zr}$ & 150000 & $0.1037 \mathrm{e}+1$ & $0.1015 \mathrm{e}+1$ & $0.1787 \mathrm{e}+1$ & $-0.3062 e+1$ & (8) \\
\hline Ar & $\mathrm{Zr}$ & 900000 & $0.9908 \mathrm{e}+0$ & $0.9862 \mathrm{e}+0$ & $0.2410 \mathrm{e}+1$ & $-0.4040 e+1$ & (8) \\
\hline $\mathrm{H}$ & $\mathrm{Nb}$ & 1000 & $0.2555 \mathrm{e}+1$ & $0.2158 \mathrm{e}+1$ & $0.1679 \mathrm{e}+1$ & $-0.3303 e+1$ & (8) \\
\hline $\mathrm{H}$ & $\mathrm{Nb}$ & 2000 & $0.2077 \mathrm{e}+1$ & $0.1760 \mathrm{e}+1$ & $0.1407 \mathrm{e}+1$ & $-0.2737 \mathrm{e}+1$ & (8) \\
\hline $\mathrm{H}$ & $\mathrm{Nb}$ & 5000 & $0.1643 \mathrm{e}+1$ & $0.1442 \mathrm{e}+1$ & $0.1314 \mathrm{e}+1$ & $-0.2517 \mathrm{e}+1$ & (8) \\
\hline $\mathrm{H}$ & $\mathrm{Nb}$ & 10000 & $0.2077 \mathrm{e}+1$ & $0.1760 \mathrm{e}+1$ & $0.1407 \mathrm{e}+1$ & $-0.2737 e+1$ & (8) \\
\hline D & $\mathrm{Nb}$ & 12200 & $0.1443 \mathrm{e}+1$ & $0.1310 \mathrm{e}+1$ & $0.1424 \mathrm{e}+1$ & $-0.2536 \mathrm{e}+1$ & (8) \\
\hline${ }^{4} \mathrm{He}$ & $\mathrm{Nb}$ & 36500 & $0.1051 \mathrm{e}+1$ & $0.9741 \mathrm{e}+0$ & $0.1571 \mathrm{e}+1$ & $-0.2624 \mathrm{e}+1$ & (8) \\
\hline $\mathrm{Nb}$ & $\mathrm{Nb}$ & 60000 & $0.9917 \mathrm{e}+0$ & $0.9853 \mathrm{e}+0$ & $0.2046 \mathrm{e}+1$ & $-0.3398 e+1$ & (8) \\
\hline $\mathrm{Ar}$ & $\mathrm{Pd}$ & 1050 & $0.1820 \mathrm{e}+1$ & $0.1641 \mathrm{e}+1$ & $0.1558 \mathrm{e}+1$ & $-0.2629 e+1$ & $(8)$ \\
\hline $\mathrm{D}$ & $\mathrm{Ag}$ & 100 & $0.1152 \mathrm{e}+1$ & $0.6071 \mathrm{e}+0$ & $0.1261 \mathrm{e}+1$ & $-0.2078 e+1$ & $(8)$ \\
\hline $\mathrm{Ne}$ & $\mathrm{Ag}$ & 45000 & $0.1441 \mathrm{e}+1$ & $0.1339 \mathrm{e}+1$ & $0.1431 \mathrm{e}+1$ & $-0.2642 \mathrm{e}+1$ & (8) \\
\hline $\mathrm{Ar}$ & $\mathrm{Ag}$ & 1050 & $0.1845 \mathrm{e}+1$ & $0.1664 \mathrm{e}+1$ & $0.1521 \mathrm{e}+1$ & $-0.2657 e+1$ & (8) \\
\hline $\mathrm{Ar}$ & $\mathrm{Ag}$ & 150000 & $0.1032 \mathrm{e}+1$ & $0.9967 \mathrm{e}+0$ & $0.1718 \mathrm{e}+1$ & $-0.2912 \mathrm{e}+1$ & (8) \\
\hline Ar & $\mathrm{Ag}$ & 900000 & $0.9747 \mathrm{e}+0$ & $0.9678 \mathrm{e}+0$ & $0.2231 \mathrm{e}+1$ & $-0.3732 e+1$ & (8) \\
\hline $\mathrm{Kr}$ & $\mathrm{Ag}$ & 45000 & $0.1062 \mathrm{e}+1$ & $0.1042 \mathrm{e}+1$ & $0.1832 \mathrm{e}+1$ & $-0.3087 e+0$ & (8) \\
\hline $\mathrm{H}$ & In & 2000 & $0.4088 \mathrm{e}+1$ & $0.3918 \mathrm{e}+1$ & $0.1477 \mathrm{e}+1$ & $-0.3501 e+1$ & (8) \\
\hline In & In & 100 & $0.9682 \mathrm{e}+0$ & $0.9682 \mathrm{e}+0$ & $0.2587 \mathrm{e}+1$ & $-0.4006 \mathrm{e}+1$ & (8) \\
\hline In & In & 200 & $0.9391 \mathrm{e}+0$ & $0.9387 \mathrm{e}+0$ & $0.2658 \mathrm{e}+1$ & $-0.4090 e+1$ & (8) \\
\hline In & In & 1000 & $0.1052 \mathrm{e}+1$ & $0.1051 \mathrm{e}+1$ & $0.2757 \mathrm{e}+1$ & $-0.4484 \mathrm{e}+1$ & (8) \\
\hline
\end{tabular}


Table 35: Constants for the angular dependence of the energy reflection coefficient at several energies.

\begin{tabular}{|c|c|c|c|c|c|c|c|}
\hline ion & solid & energy & $\mathrm{c} 1$ & $\mathrm{c} 2$ & $\mathrm{c3}$ & $\mathrm{c} 4$ & fit \\
\hline $\mathrm{H}$ & $\mathrm{V}$ & 100 & $0.3185 \mathrm{e}+1$ & $0.2907 \mathrm{e}+1$ & $0.1431 \mathrm{e}+1$ & $-0.3076 e+1$ & (8) \\
\hline $\mathrm{H}$ & $\mathrm{V}$ & 120 & $0.3640 \mathrm{e}+1$ & $0.3372 \mathrm{e}+1$ & $0.1437 \mathrm{e}+1$ & $-0.3180 e+1$ & (8) \\
\hline $\mathrm{H}$ & V & 140 & $0.2954 \mathrm{e}+1$ & $0.2696 \mathrm{e}+1$ & $0.1439 e+1$ & $-0.3072 e+1$ & (8) \\
\hline $\mathrm{H}$ & V & 200 & $0.3595 \mathrm{e}+1$ & $0.3355 \mathrm{e}+1$ & $0.1490 \mathrm{e}+1$ & $-0.3280 e+1$ & (8) \\
\hline $\mathrm{H}$ & V & 400 & $0.3543 \mathrm{e}+1$ & $0.3342 \mathrm{e}+1$ & $0.1616 \mathrm{e}+1$ & $-0.3485 e+1$ & (8) \\
\hline $\mathrm{H}$ & V & 1000 & $0.3133 \mathrm{e}+1$ & $0.2989 \mathrm{e}+1$ & $0.1713 \mathrm{e}+1$ & $-0.3595 e+1$ & (8) \\
\hline $\mathrm{H}$ & V & 3000 & $0.2080 \mathrm{e}+1$ & $0.2006 \mathrm{e}+1$ & $0.1913 e+1$ & $-0.3710 e+1$ & (8) \\
\hline $\mathrm{H}$ & V & 10000 & $0.1120 \mathrm{e}+1$ & $0.1099 \mathrm{e}+1$ & $0.2190 \mathrm{e}+1$ & $-0.3901 e+1$ & (8) \\
\hline $\mathrm{D}$ & V & 55 & $0.1152 \mathrm{e}+1$ & $0.8617 \mathrm{e}+0$ & $0.1579 e+1$ & $-0.2482 \mathrm{e}+1$ & (8) \\
\hline $\mathrm{D}$ & V & 60 & $0.1260 \mathrm{e}+1$ & $0.9754 \mathrm{e}+0$ & $0.1551 \mathrm{e}+1$ & $-0.2537 \mathrm{e}+1$ & (8) \\
\hline $\mathrm{D}$ & V & 70 & $0.2085 \mathrm{e}+1$ & $0.1810 \mathrm{e}+1$ & $0.1437 \mathrm{e}+1$ & $-0.2778 \mathrm{e}+1$ & (8) \\
\hline $\mathrm{D}$ & V & 100 & $0.1333 \mathrm{e}+1$ & $0.1071 \mathrm{e}+1$ & $0.1591 \mathrm{e}+1$ & $-0.2692 \mathrm{e}+1$ & (8) \\
\hline $\mathrm{D}$ & $\mathrm{V}$ & 200 & $0.4865 \mathrm{e}+1$ & $0.4636 \mathrm{e}+1$ & $0.1473 e+1$ & $-0.3417 e+1$ & (8) \\
\hline $\mathrm{D}$ & V & 500 & $0.3914 \mathrm{e}+1$ & $0.3723 \mathrm{e}+1$ & $0.1624 \mathrm{e}+1$ & $-0.3561 e+1$ & (8) \\
\hline $\mathrm{D}$ & V & 1000 & $0.3056 \mathrm{e}+1$ & $0.2905 \mathrm{e}+1$ & $0.1697 \mathrm{e}+1$ & $-0.3551 e+1$ & (8) \\
\hline $\mathrm{D}$ & $\mathrm{V}$ & 3000 & $0.2201 \mathrm{e}+1$ & $0.2119 \mathrm{e}+1$ & $0.1697 \mathrm{e}+1$ & $-0.3443 e+1$ & (8) \\
\hline $\mathrm{D}$ & $\mathrm{V}$ & 10000 & $0.1338 \mathrm{e}+1$ & $0.1310 \mathrm{e}+1$ & $0.2053 \mathrm{e}+1$ & $-0.3763 e+1$ & (8) \\
\hline $\mathrm{T}$ & $\mathrm{V}$ & 40 & $0.9209 \mathrm{e}+0$ & $0.6500 \mathrm{e}+0$ & $0.1692 \mathrm{e}+1$ & $-0.2370 \mathrm{e}+1$ & (8) \\
\hline $\mathrm{T}$ & $\mathrm{V}$ & 50 & $0.1019 \mathrm{e}+1$ & $0.7608 \mathrm{e}+0$ & $0.1655 \mathrm{e}+1$ & $-0.2450 \mathrm{e}+1$ & (8) \\
\hline $\mathrm{T}$ & V & 70 & $0.1129 \mathrm{e}+1$ & $0.8848 \mathrm{e}+0$ & $0.1648 \mathrm{e}+1$ & $-0.2586 \mathrm{e}+1$ & (8) \\
\hline $\mathrm{T}$ & $\mathrm{V}$ & 100 & $0.1269 \mathrm{e}+1$ & $0.1038 \mathrm{e}+1$ & $0.1650 \mathrm{e}+1$ & $-0.2729 e+1$ & (8) \\
\hline $\mathrm{T}$ & $\mathrm{V}$ & 300 & $0.4806 \mathrm{e}+1$ & $0.4616 \mathrm{e}+1$ & $0.1558 \mathrm{e}+1$ & $-0.3548 e+1$ & (8) \\
\hline $\mathrm{T}$ & V & 1000 & $0.2927 \mathrm{e}+1$ & $0.2785 \mathrm{e}+1$ & $0.1704 \mathrm{e}+1$ & $-0.3537 \mathrm{e}+1$ & (8) \\
\hline $\mathrm{T}$ & $\mathrm{V}$ & 3000 & $0.2049 \mathrm{e}+1$ & $0.1966 \mathrm{e}+1$ & $0.1693 e+1$ & $-0.3388 e+1$ & (8) \\
\hline $\mathrm{T}$ & $\mathrm{V}$ & 10000 & $0.1430 \mathrm{e}+1$ & $0.1401 \mathrm{e}+1$ & $0.1948 \mathrm{e}+1$ & $-0.3640 \mathrm{e}+1$ & (8) \\
\hline${ }^{4} \mathrm{He}$ & V & 35 & $0.7651 \mathrm{e}+0$ & $0.4899 \mathrm{e}+0$ & $0.1912 \mathrm{e}+1$ & $-0.2225 e+1$ & (8) \\
\hline${ }^{4} \mathrm{He}$ & V & 40 & $0.8166 \mathrm{e}+0$ & $0.5603 \mathrm{e}+0$ & $0.1716 \mathrm{e}+1$ & $-0.2119 e+1$ & (8) \\
\hline${ }^{4} \mathrm{He}$ & $\mathrm{V}$ & 50 & $0.8266 \mathrm{e}+0$ & $0.5764 \mathrm{e}+0$ & $0.1871 \mathrm{e}+1$ & $-0.2356 e+1$ & (8) \\
\hline${ }^{4} \mathrm{He}$ & $\mathrm{V}$ & 70 & $0.9769 \mathrm{e}+0$ & $0.7483 \mathrm{e}+0$ & $0.1760 \mathrm{e}+1$ & $-0.2477 \mathrm{e}+1$ & (8) \\
\hline${ }^{4} \mathrm{He}$ & V & 100 & $0.8504 \mathrm{e}+0$ & $0.6329 \mathrm{e}+0$ & $0.1973 \mathrm{e}+1$ & $-0.2658 \mathrm{e}+1$ & (8) \\
\hline${ }^{4} \mathrm{He}$ & V & 300 & $0.3276 \mathrm{e}+1$ & $0.3107 \mathrm{e}+1$ & $0.1627 \mathrm{e}+1$ & $-0.3352 e+1$ & (8) \\
\hline${ }^{4} \mathrm{He}$ & V & 1000 & $0.3090 \mathrm{e}+1$ & $0.2960 \mathrm{e}+1$ & $0.1735 \mathrm{e}+1$ & $-0.3567 e+1$ & (8) \\
\hline${ }^{4} \mathrm{He}$ & $\mathrm{V}$ & 3000 & $0.2681 \mathrm{e}+1$ & $0.2594 \mathrm{e}+1$ & $0.1764 \mathrm{e}+1$ & $-0.3611 e+1$ & (8) \\
\hline${ }^{4} \mathrm{He}$ & V & 10000 & $0.1586 \mathrm{e}+1$ & $0.1547 \mathrm{e}+1$ & $0.1907 \mathrm{e}+1$ & $-0.3602 \mathrm{e}+1$ & (8) \\
\hline $\mathrm{Ar}$ & Mo & 160 & $0.8588 \mathrm{e}+0$ & $0.8199 \mathrm{e}+0$ & $0.1952 \mathrm{e}+1$ & $-0.2738 e+1$ & (8) \\
\hline $\mathrm{Ar}$ & Mo & 1601 & $0.1285 \mathrm{e}+1$ & $0.1263 \mathrm{e}+1$ & $0.2206 \mathrm{e}+1$ & $-0.3670 \mathrm{e}+1$ & (8) \\
\hline $\mathrm{Ar}$ & Mo & 16010 & $0.1082 \mathrm{e}+1$ & $0.1070 \mathrm{e}+1$ & $0.2034 \mathrm{e}+1$ & $-0.3640 \mathrm{e}+1$ & (8) \\
\hline Ar & Mo & 27500 & $0.1186 \mathrm{e}+1$ & $0.1174 \mathrm{e}+1$ & $0.2294 \mathrm{e}+1$ & $-0.4000 e+1$ & (8) \\
\hline Mo & Mo & 300 & $0.9406 \mathrm{e}+0$ & $0.9404 \mathrm{e}+0$ & $0.2803 e+1$ & $-0.4170 \mathrm{e}+1$ & (8) \\
\hline Mo & Mo & 350 & $0.9468 \mathrm{e}+0$ & $0.9466 \mathrm{e}+0$ & $0.2816 \mathrm{e}+1$ & $-0.4196 \mathrm{e}+1$ & (8) \\
\hline Mo & Mo & 1000 & $0.1040 \mathrm{e}+1$ & $0.1039 \mathrm{e}+1$ & $0.2779 \mathrm{e}+1$ & $-0.4371 \mathrm{e}+1$ & (8) \\
\hline Mo & Mo & 2000 & $0.1048 \mathrm{e}+1$ & $0.1047 \mathrm{e}+1$ & $0.2900 \mathrm{e}+1$ & $-0.4629 e+1$ & (8) \\
\hline
\end{tabular}


Table 36: Constants for the angular dependence of the energy reflection coefficient at several energies.

\begin{tabular}{|c|c|c|c|c|c|c|c|}
\hline ion & solid & energy & $\mathrm{c} 1$ & $\mathrm{c} 2$ & $\mathrm{c} 3$ & $\mathrm{c} 4$ & fit \\
\hline $\mathrm{H}$ & $\mathrm{Fe}$ & 4000 & $0.2069 \mathrm{e}+1$ & $0.2003 e+1$ & $0.1915 \mathrm{e}+1$ & $-0.3718 e+1$ & $(8)$ \\
\hline $\mathrm{H}$ & $\mathrm{Fe}$ & 8000 & $0.1331 \mathrm{e}+1$ & $0.1297 \mathrm{e}+1$ & $0.2073 e+1$ & $-0.3771 e+1$ & $(8)$ \\
\hline $\mathrm{H}$ & $\mathrm{Ni}$ & 450 & $0.3437 \mathrm{e}+1$ & $0.3221 e+1$ & $0.1591 \mathrm{e}+1$ & $-0.3416 e+1$ & $(8)$ \\
\hline $\mathrm{H}$ & $\mathrm{Ni}$ & 4000 & $0.1995 \mathrm{e}+1$ & $0.1924 \mathrm{e}+1$ & $0.1787 \mathrm{e}+1$ & $-0.3529 e+1$ & (8) \\
\hline $\mathrm{H}$ & $\mathrm{Ni}$ & 8000 & $0.1499 \mathrm{e}+1$ & $0.1462 \mathrm{e}+1$ & $0.2021 \mathrm{e}+1$ & $-0.3755 e+1$ & (8) \\
\hline $\mathrm{D}$ & $\mathrm{Ni}$ & 1000 & $0.2978 \mathrm{e}+1$ & $0.2801 \mathrm{e}+1$ & $0.1723 \mathrm{e}+1$ & $-0.3555 e+1$ & (8) \\
\hline D & $\mathrm{Ni}$ & 4000 & $0.2199 \mathrm{e}+1$ & $0.2114 \mathrm{e}+1$ & $0.1816 \mathrm{e}+1$ & $-0.3579 e+1$ & (8) \\
\hline D & $\mathrm{Ni}$ & 8000 & $0.1549 \mathrm{e}+1$ & $0.1502 \mathrm{e}+1$ & $0.1922 \mathrm{e}+1$ & $-0.3594 e+1$ & (8) \\
\hline${ }^{3} \mathrm{He}$ & $\mathrm{Ni}$ & 1000 & $0.3867 \mathrm{e}+1$ & $0.3703 \mathrm{e}+1$ & $0.1673 \mathrm{e}+1$ & $-0.3593 e+1$ & (8) \\
\hline${ }^{3} \mathrm{He}$ & $\mathrm{Ni}$ & 3000 & $0.2735 \mathrm{e}+1$ & $0.2622 \mathrm{e}+1$ & $0.1839 e+1$ & $-0.3697 e+1$ & (8) \\
\hline${ }^{3} \mathrm{He}$ & $\mathrm{Ni}$ & 8000 & $0.1869 \mathrm{e}+1$ & $0.1806 \mathrm{e}+1$ & $0.1945 \mathrm{e}+1$ & $-0.3687 e+1$ & (8) \\
\hline${ }^{3} \mathrm{He}$ & $\mathrm{Ni}$ & 25000 & $0.1272 \mathrm{e}+1$ & $0.1251 \mathrm{e}+1$ & $0.2178 \mathrm{e}+1$ & $-0.3947 e+1$ & (8) \\
\hline${ }^{4} \mathrm{He}$ & $\mathrm{Ni}$ & 100 & $0.7834 \mathrm{e}+0$ & $0.5353 e+0$ & $0.1976 \mathrm{e}+1$ & $-0.2468 \mathrm{e}+1$ & (8) \\
\hline${ }^{4} \mathrm{He}$ & $\mathrm{Ni}$ & 500 & $0.3260 \mathrm{e}+1$ & $0.3085 \mathrm{e}+1$ & $0.1642 \mathrm{e}+1$ & $-0.3384 \mathrm{e}+1$ & (8) \\
\hline${ }^{4} \mathrm{He}$ & $\mathrm{Ni}$ & 1000 & $0.2572 \mathrm{e}+1$ & $0.2420 \mathrm{e}+1$ & $0.1751 \mathrm{e}+1$ & $-0.3463 e+1$ & (8) \\
\hline${ }^{4} \mathrm{He}$ & $\mathrm{Ni}$ & 4000 & $0.2429 \mathrm{e}+1$ & $0.2334 \mathrm{e}+1$ & $0.1836 \mathrm{e}+1$ & $-0.3643 e+1$ & (8) \\
\hline${ }^{4} \mathrm{He}$ & $\mathrm{Ni}$ & 8000 & $0.1842 \mathrm{e}+1$ & $0.1782 \mathrm{e}+1$ & $0.1786 \mathrm{e}+1$ & $-0.3477 \mathrm{e}+1$ & (8) \\
\hline $\mathrm{Ne}$ & $\mathrm{Ni}$ & 1000 & $0.1352 \mathrm{e}+1$ & $0.1319 \mathrm{e}+1$ & $0.2216 \mathrm{e}+1$ & $-0.3601 e+1$ & (8) \\
\hline Ar & $\mathrm{Ni}$ & 40 & $0.6916 \mathrm{e}+0$ & $0.6848 \mathrm{e}+0$ & $0.2058 \mathrm{e}+1$ & $-0.2671 \mathrm{e}+1$ & (8) \\
\hline $\mathrm{Ar}$ & $\mathrm{Ni}$ & 50 & $0.7124 \mathrm{e}+0$ & $0.7052 \mathrm{e}+0$ & $0.2063 \mathrm{e}+1$ & $-0.2699 \mathrm{e}+1$ & (8) \\
\hline Ar & $\mathrm{Ni}$ & 70 & $0.7545 \mathrm{e}+0$ & $0.7472 \mathrm{e}+0$ & $0.2077 \mathrm{e}+1$ & $-0.2772 \mathrm{e}+1$ & (8) \\
\hline Ar & $\mathrm{Ni}$ & 100 & $0.6914 \mathrm{e}+0$ & $0.6827 \mathrm{e}+0$ & $0.2231 \mathrm{e}+1$ & $-0.2881 \mathrm{e}+1$ & (8) \\
\hline Ar & $\mathrm{Ni}$ & 300 & $0.8950 \mathrm{e}+0$ & $0.8873 \mathrm{e}+0$ & $0.2378 \mathrm{e}+1$ & $-0.3371 e+1$ & (8) \\
\hline Ar & $\mathrm{Ni}$ & 1000 & $0.1118 \mathrm{e}+0$ & $0.1113 e+0$ & $0.2583 \mathrm{e}+1$ & $-0.3987 e+1$ & (8) \\
\hline Ar & $\mathrm{Ni}$ & 3000 & $0.1093 e+1$ & $0.1089 e+1$ & $0.2710 \mathrm{e}+1$ & $-0.4322 e+1$ & (8) \\
\hline $\mathrm{Ar}$ & $\mathrm{Ni}$ & 30000 & $0.1037 \mathrm{e}+1$ & $0.1036 \mathrm{e}+1$ & $0.2720 \mathrm{e}+1$ & $-0.4605 e+1$ & (8) \\
\hline $\mathrm{Ni}$ & $\mathrm{Ni}$ & 500 & $0.9519 \mathrm{e}+0$ & $0.9516 \mathrm{e}+0$ & $0.2855 \mathrm{e}+1$ & $-0.4196 \mathrm{e}+1$ & (8) \\
\hline $\mathrm{Ni}$ & $\mathrm{Ni}$ & 2500 & $0.9998 \mathrm{e}+0$ & $0.9992 \mathrm{e}+0$ & $0.2978 \mathrm{e}+1$ & $-0.4691 e+1$ & (8) \\
\hline $\mathrm{Ni}$ & $\mathrm{Ni}$ & 5000 & $0.1018 \mathrm{e}+1$ & $0.1018 \mathrm{e}+1$ & $0.2934 \mathrm{e}+1$ & $-0.4805 e+1$ & (8) \\
\hline $\mathrm{Ni}$ & $\mathrm{Ni}$ & 10000 & $0.1009 \mathrm{e}+1$ & $0.1008 \mathrm{e}+1$ & $0.2996 \mathrm{e}+1$ & $-0.4915 e+1$ & $(8)$ \\
\hline $\mathrm{H}$ & $\mathrm{Cu}$ & 100 & $0.9580 \mathrm{e}+0$ & $0.6401 e+0$ & $0.1776 \mathrm{e}+1$ & $-0.2548 \mathrm{e}+1$ & $(8)$ \\
\hline $\mathrm{H}$ & $\mathrm{Cu}$ & 1000 & $0.3457 \mathrm{e}+1$ & $0.3289 \mathrm{e}+1$ & $0.1614 \mathrm{e}+1$ & $-0.3510 e+1$ & (8) \\
\hline $\mathrm{H}$ & $\mathrm{Cu}$ & 5000 & $0.1689 \mathrm{e}+1$ & $0.1628 \mathrm{e}+1$ & $0.1803 e+1$ & $-0.3479 e+1$ & (8) \\
\hline $\mathrm{H}$ & $\mathrm{Cu}$ & 13333 & $0.1295 \mathrm{e}+1$ & $0.1275 \mathrm{e}+1$ & $0.2028 \mathrm{e}+1$ & $-0.3794 e+1$ & (8) \\
\hline $\mathrm{H}$ & $\mathrm{Cu}$ & 26667 & $0.1046 \mathrm{e}+1$ & $0.1040 \mathrm{e}+1$ & $0.2045 \mathrm{e}+1$ & $-0.4052 e+1$ & (8) \\
\hline $\mathrm{H}$ & $\mathrm{Cu}$ & 40000 & $0.9035 \mathrm{e}+0$ & $0.8997 \mathrm{e}+0$ & $0.2328 \mathrm{e}+1$ & $-0.4504 \mathrm{e}+1$ & (8) \\
\hline $\mathrm{H}$ & $\mathrm{Cu}$ & 80000 & $0.9710 \mathrm{e}+0$ & $0.9694 e+0$ & $0.3318 \mathrm{e}+1$ & $-0.6015 e+1$ & (8) \\
\hline
\end{tabular}


Table 37: Constants for the angular dependence of the energy reflection coefficient at several energies.

\begin{tabular}{|c|c|c|c|c|c|c|c|}
\hline ion & solid & energy & c1 & c2 & c3 & c4 & fit \\
\hline $\mathrm{D}$ & $\mathrm{Cu}$ & 300 & $0.4009 \mathrm{e}+1$ & $0.3767 \mathrm{e}+1$ & $0.1510 \mathrm{e}+1$ & $-0.3358 e+1$ & (8) \\
\hline $\mathrm{D}$ & $\mathrm{Cu}$ & 1000 & $0.2971 \mathrm{e}+1$ & $0.2793 \mathrm{e}+1$ & $0.1586 e+1$ & $-0.3378 e+1$ & (8) \\
\hline $\mathrm{D}$ & $\mathrm{Cu}$ & 3000 & $0.2222 \mathrm{e}+1$ & $0.2120 \mathrm{e}+1$ & $0.1500 \mathrm{e}+1$ & $-0.3175 e+1$ & (8) \\
\hline $\mathrm{D}$ & $\mathrm{Cu}$ & 53333 & $0.9822 \mathrm{e}+0$ & $0.9791 \mathrm{e}+0$ & $0.2386 \mathrm{e}+1$ & $-0.4622 e+1$ & (8) \\
\hline $\mathrm{D}$ & $\mathrm{Cu}$ & 80000 & $0.9913 e+0$ & $0.9894 \mathrm{e}+0$ & $0.2903 e+1$ & $-0.5434 e+1$ & (8) \\
\hline $\mathrm{D}$ & $\mathrm{Cu}$ & 160000 & $0.9382 \mathrm{e}+0$ & $0.9374 \mathrm{e}+0$ & $0.2892 \mathrm{e}+1$ & $-0.5935 e+1$ & (8) \\
\hline${ }^{4} \mathrm{He}$ & $\mathrm{Cu}$ & 100 & $0.7784 \mathrm{e}+0$ & $0.5194 \mathrm{e}+0$ & $0.2017 \mathrm{e}+1$ & $-0.2518 e+1$ & (8) \\
\hline${ }^{4} \mathrm{He}$ & $\mathrm{Cu}$ & 1000 & $0.3213 e+1$ & $0.3053 \mathrm{e}+1$ & $0.1683 e+1$ & $-0.3505 e+1$ & (8) \\
\hline${ }^{4} \mathrm{He}$ & $\mathrm{Cu}$ & 4000 & $0.2889 \mathrm{e}+1$ & $0.2792 \mathrm{e}+1$ & $0.1693 e+1$ & $-0.3551 e+1$ & (8) \\
\hline${ }^{4} \mathrm{He}$ & $\mathrm{Cu}$ & 5000 & $0.2062 \mathrm{e}+1$ & $0.1975 \mathrm{e}+1$ & $0.1751 \mathrm{e}+1$ & $-0.3452 e+1$ & (8) \\
\hline $\mathrm{Ne}$ & $\mathrm{Cu}$ & 1000 & $0.1691 \mathrm{e}+1$ & $0.1652 \mathrm{e}+1$ & $0.2089 e+1$ & $-0.3578 e+1$ & (8) \\
\hline $\mathrm{Ne}$ & $\mathrm{Cu}$ & 45000 & $0.1142 \mathrm{e}+1$ & $0.1132 \mathrm{e}+1$ & $0.2317 \mathrm{e}+1$ & $-0.4063 e+1$ & (8) \\
\hline $\mathrm{Ar}$ & $\mathrm{Cu}$ & 14 & $0.7004 \mathrm{e}+0$ & $0.6937 \mathrm{e}+0$ & $0.1937 \mathrm{e}+1$ & $-0.2576 \mathrm{e}+1$ & (8) \\
\hline $\mathrm{Ar}$ & $\mathrm{Cu}$ & 16 & $0.7199 \mathrm{e}+0$ & $0.7130 \mathrm{e}+0$ & $0.1912 \mathrm{e}+1$ & $-0.2558 \mathrm{e}+1$ & (8) \\
\hline $\mathrm{Ar}$ & $\mathrm{Cu}$ & 18 & $0.7944 \mathrm{e}+0$ & $0.7875 e+0$ & $0.1871 \mathrm{e}+1$ & $-0.2576 \mathrm{e}+1$ & (8) \\
\hline $\mathrm{Ar}$ & $\mathrm{Cu}$ & 20 & $0.7684 \mathrm{e}+0$ & $0.7608 \mathrm{e}+0$ & $0.1867 \mathrm{e}+1$ & $-0.2545 e+1$ & (8) \\
\hline Ar & $\mathrm{Cu}$ & 25 & $0.7423 \mathrm{e}+0$ & $0.7334 \mathrm{e}+0$ & $0.1900 \mathrm{e}+1$ & $-0.2545 e+1$ & (8) \\
\hline $\mathrm{Ar}$ & $\mathrm{Cu}$ & 30 & $0.7457 \mathrm{e}+0$ & $0.7357 \mathrm{e}+0$ & $0.1915 \mathrm{e}+1$ & $-0.2557 e+1$ & (8) \\
\hline Ar & $\mathrm{Cu}$ & 40 & $0.7272 \mathrm{e}+0$ & $0.7157 \mathrm{e}+0$ & $0.1965 \mathrm{e}+1$ & $-0.2588 \mathrm{e}+1$ & (8) \\
\hline Ar & $\mathrm{Cu}$ & 50 & $0.7134 \mathrm{e}+0$ & $0.7012 \mathrm{e}+0$ & $0.2009 \mathrm{e}+1$ & $-0.2620 e+1$ & (8) \\
\hline $\mathrm{Ar}$ & $\mathrm{Cu}$ & 100 & $0.6997 \mathrm{e}+0$ & $0.6865 \mathrm{e}+0$ & $0.2178 \mathrm{e}+1$ & $-0.2827 e+1$ & (8) \\
\hline $\mathrm{Ar}$ & $\mathrm{Cu}$ & 300 & $0.8930 \mathrm{e}+0$ & $0.8824 \mathrm{e}+0$ & $0.2306 \mathrm{e}+1$ & $-0.3296 e+1$ & (8) \\
\hline $\mathrm{Ar}$ & $\mathrm{Cu}$ & 1050 & $0.1156 \mathrm{e}+1$ & $0.1148 \mathrm{e}+1$ & $0.2495 e+1$ & $-0.3910 e+1$ & (8) \\
\hline $\mathrm{Ar}$ & $\mathrm{Cu}$ & 20000 & $0.1068 \mathrm{e}+1$ & $0.1065 \mathrm{e}+1$ & $0.2508 \mathrm{e}+1$ & $-0.4319 e+1$ & (8) \\
\hline Ar & $\mathrm{Cu}$ & 27000 & $0.1064 e+1$ & $0.1062 \mathrm{e}+1$ & $0.2555 \mathrm{e}+1$ & $-0.4410 e+1$ & (8) \\
\hline $\mathrm{Ar}$ & $\mathrm{Cu}$ & 30000 & $0.1032 \mathrm{e}+1$ & $0.1029 \mathrm{e}+1$ & $0.2519 \mathrm{e}+1$ & $-0.4350 e+1$ & (8) \\
\hline $\mathrm{Ar}$ & $\mathrm{Cu}$ & 37000 & $0.1042 \mathrm{e}+1$ & $0.1041 \mathrm{e}+1$ & $0.2499 \mathrm{e}+1$ & $-0.4357 e+1$ & (8) \\
\hline $\mathrm{Ar}$ & $\mathrm{Cu}$ & 100000 & $0.1040 \mathrm{e}+1$ & $0.1039 \mathrm{e}+1$ & $0.2685 \mathrm{e}+1$ & $-0.4669 e+1$ & (8) \\
\hline Ar & $\mathrm{Cu}$ & 300000 & $0.1005 e+1$ & $0.1004 \mathrm{e}+1$ & $0.3041 \mathrm{e}+1$ & $-0.5261 e+1$ & (8) \\
\hline $\mathrm{Cu}$ & $\mathrm{Cu}$ & 100 & $0.9677 \mathrm{e}+0$ & $0.9679 \mathrm{e}+0$ & $0.2580 \mathrm{e}+1$ & $-0.3883 e+1$ & (8) \\
\hline $\mathrm{Cu}$ & $\mathrm{Cu}$ & 200 & $0.9708 \mathrm{e}+0$ & $0.9707 \mathrm{e}+0$ & $0.2688 \mathrm{e}+1$ & $-0.3916 e+1$ & (8) \\
\hline $\mathrm{Cu}$ & $\mathrm{Cu}$ & 300 & $0.9616 \mathrm{e}+0$ & $0.9612 \mathrm{e}+0$ & $0.2723 e+1$ & $-0.4003 e+1$ & (8) \\
\hline $\mathrm{Cu}$ & $\mathrm{Cu}$ & 1000 & $0.1018 \mathrm{e}+1$ & $0.1018 \mathrm{e}+1$ & $0.2940 \mathrm{e}+1$ & $-0.4458 e+1$ & (8) \\
\hline $\mathrm{Cu}$ & $\mathrm{Cu}$ & 3000 & $0.1014 \mathrm{e}+1$ & $0.1013 e+1$ & $0.3128 \mathrm{e}+1$ & $-0.4913 e+1$ & (8) \\
\hline $\mathrm{Cu}$ & $\mathrm{Cu}$ & 10000 & $0.9870 \mathrm{e}+0$ & $0.9867 \mathrm{e}+0$ & $0.2938 \mathrm{e}+1$ & $-0.4881 e+1$ & (8) \\
\hline
\end{tabular}


Table 38: Constants for the angular dependence of the energy reflection coefficient at several energies.

\begin{tabular}{|c|c|c|c|c|c|c|c|}
\hline ion & solid & energy & c1 & c2 & c3 & c4 & fit \\
\hline $\mathrm{H}$ & Mo & 210 & $0.3179 \mathrm{e}+1$ & $0.2880 \mathrm{e}+1$ & $0.1408 \mathrm{e}+1$ & $-0.3134 \mathrm{e}+1$ & (8) \\
\hline $\mathrm{H}$ & Mo & 220 & $0.5388 \mathrm{e}+1$ & $0.5089 \mathrm{e}+1$ & $0.1437 \mathrm{e}+1$ & $-0.3474 \mathrm{e}+1$ & (8) \\
\hline $\mathrm{H}$ & Mo & 230 & $0.4553 \mathrm{e}+1$ & $0.4256 \mathrm{e}+1$ & $0.1437 \mathrm{e}+1$ & $-0.3380 e+1$ & (8) \\
\hline $\mathrm{H}$ & Mo & 250 & $0.3747 \mathrm{e}+1$ & $0.3454 \mathrm{e}+1$ & $0.1440 \mathrm{e}+1$ & $-0.3280 e+1$ & (8) \\
\hline $\mathrm{H}$ & Mo & 300 & $0.4309 \mathrm{e}+1$ & $0.4027 \mathrm{e}+1$ & $0.1467 \mathrm{e}+1$ & $-0.3404 e+1$ & (8) \\
\hline $\mathrm{H}$ & Mo & 400 & $0.4611 \mathrm{e}+1$ & $0.4344 \mathrm{e}+1$ & $0.1522 \mathrm{e}+1$ & $-0.3530 e+1$ & (8) \\
\hline $\mathrm{H}$ & Mo & 700 & $0.3507 \mathrm{e}+1$ & $0.3271 \mathrm{e}+1$ & $0.1640 \mathrm{e}+1$ & $-0.3562 \mathrm{e}+1$ & (8) \\
\hline $\mathrm{H}$ & Mo & 1400 & $0.3298 \mathrm{e}+1$ & $0.3111 \mathrm{e}+1$ & $0.1710 \mathrm{e}+1$ & $-0.3639 e+1$ & (8) \\
\hline $\mathrm{H}$ & Mo & 2000 & $0.2950 \mathrm{e}+1$ & $0.2790 \mathrm{e}+1$ & $0.1646 \mathrm{e}+1$ & $-0.3525 e+1$ & (8) \\
\hline $\mathrm{H}$ & Mo & 3000 & $0.2609 \mathrm{e}+1$ & $0.2481 \mathrm{e}+1$ & $0.1702 \mathrm{e}+1$ & $-0.3520 \mathrm{e}+1$ & (8) \\
\hline $\mathrm{H}$ & Mo & 4000 & $0.2293 \mathrm{e}+1$ & $0.2186 \mathrm{e}+1$ & $0.1731 \mathrm{e}+1$ & $-0.3501 e+1$ & (8) \\
\hline $\mathrm{H}$ & Mo & 7000 & $0.1932 \mathrm{e}+1$ & $0.1862 \mathrm{e}+1$ & $0.1772 \mathrm{e}+1$ & $-0.3509 e+1$ & (8) \\
\hline $\mathrm{H}$ & Mo & 8000 & $0.1905 \mathrm{e}+1$ & $0.1842 \mathrm{e}+1$ & $0.1785 \mathrm{e}+1$ & $-0.3531 e+1$ & (8) \\
\hline $\mathrm{H}$ & Mo & 13333 & $0.1460 \mathrm{e}+1$ & $0.1423 \mathrm{e}+1$ & $0.1826 \mathrm{e}+1$ & $-0.3524 e+1$ & (8) \\
\hline $\mathrm{H}$ & Mo & 26667 & $0.1023 \mathrm{e}+1$ & $0.1015 \mathrm{e}+1$ & $0.2545 \mathrm{e}+1$ & $-0.4604 \mathrm{e}+1$ & (8) \\
\hline $\mathrm{H}$ & Mo & 40000 & $0.9689 \mathrm{e}+0$ & $0.9642 \mathrm{e}+0$ & $0.2807 \mathrm{e}+1$ & $-0.5054 \mathrm{e}+1$ & (8) \\
\hline $\mathrm{H}$ & Mo & 80000 & $0.9630 \mathrm{e}+0$ & $0.9610 \mathrm{e}+0$ & $0.3380 \mathrm{e}+1$ & $-0.6070 e+1$ & (8) \\
\hline $\mathrm{D}$ & Mo & 100 & $0.5113 \mathrm{e}+1$ & $0.4773 \mathrm{e}+1$ & $0.1316 \mathrm{e}+1$ & $-0.3233 e+1$ & (8) \\
\hline $\mathrm{D}$ & Mo & 110 & $0.1363 \mathrm{e}+1$ & $0.1023 e+1$ & $0.1495 \mathrm{e}+1$ & $-0.2614 e+1$ & (8) \\
\hline $\mathrm{D}$ & Mo & 120 & $0.1898 \mathrm{e}+1$ & $0.1565 \mathrm{e}+1$ & $0.1412 \mathrm{e}+1$ & $-0.2765 e+1$ & (8) \\
\hline $\mathrm{D}$ & Mo & 200 & $0.5156 \mathrm{e}+1$ & $0.4846 \mathrm{e}+1$ & $0.1388 \mathrm{e}+1$ & $-0.3374 \mathrm{e}+1$ & (8) \\
\hline D & Mo & 300 & $0.4099 \mathrm{e}+1$ & $0.3803 \mathrm{e}+1$ & $0.1475 \mathrm{e}+1$ & $-0.3391 e+1$ & (8) \\
\hline $\mathrm{D}$ & Mo & 450 & $0.3526 \mathrm{e}+1$ & $0.3253 \mathrm{e}+1$ & $0.1478 \mathrm{e}+1$ & $-0.3325 e+1$ & (8) \\
\hline $\mathrm{D}$ & Mo & 2000 & $0.2884 \mathrm{e}+1$ & $0.2705 \mathrm{e}+1$ & $0.1532 \mathrm{e}+1$ & $-0.3332 \mathrm{e}+1$ & (8) \\
\hline $\mathrm{D}$ & Mo & 8000 & $0.2888 \mathrm{e}+1$ & $0.2810 \mathrm{e}+1$ & $0.1613 e+1$ & $-0.3498 e+1$ & (8) \\
\hline $\mathrm{T}$ & Mo & 75 & $0.1012 \mathrm{e}+1$ & $0.6746 \mathrm{e}+0$ & $0.1615 \mathrm{e}+1$ & $-0.2441 \mathrm{e}+1$ & (8) \\
\hline $\mathrm{T}$ & Mo & 80 & $0.1013 \mathrm{e}+1$ & $0.6745 \mathrm{e}+0$ & $0.1667 \mathrm{e}+1$ & $-0.2518 e+1$ & (8) \\
\hline $\mathrm{T}$ & Mo & 90 & $0.1502 \mathrm{e}+1$ & $0.1179 \mathrm{e}+1$ & $0.1384 \mathrm{e}+1$ & $-0.2521 \mathrm{e}+1$ & (8) \\
\hline $\mathrm{T}$ & Mo & 100 & $0.1247 \mathrm{e}+1$ & $0.9208 \mathrm{e}+0$ & $0.1560 \mathrm{e}+1$ & $-0.2608 \mathrm{e}+1$ & (8) \\
\hline $\mathrm{T}$ & Mo & 170 & $0.3847 \mathrm{e}+1$ & $0.3546 \mathrm{e}+1$ & $0.1388 \mathrm{e}+1$ & $-0.3190 e+1$ & (8) \\
\hline $\mathrm{T}$ & Mo & 300 & $0.4156 \mathrm{e}+1$ & $0.3874 \mathrm{e}+1$ & $0.1481 \mathrm{e}+1$ & $-0.3399 e+1$ & (8) \\
\hline $\mathrm{T}$ & Mo & 1000 & $0.4645 \mathrm{e}+1$ & $0.4420 \mathrm{e}+1$ & $0.1553 \mathrm{e}+1$ & $-0.3607 e+1$ & (8) \\
\hline $\mathrm{T}$ & Mo & 3000 & $0.2952 \mathrm{e}+1$ & $0.2796 \mathrm{e}+1$ & $0.1616 \mathrm{e}+1$ & $-0.3464 e+1$ & (8) \\
\hline $\mathrm{T}$ & Mo & 10000 & $0.2003 \mathrm{e}+1$ & $0.1931 \mathrm{e}+1$ & $0.1648 \mathrm{e}+1$ & $-0.3343 e+1$ & (8) \\
\hline${ }^{3} \mathrm{He}$ & Mo & 90 & $0.1111 \mathrm{e}+1$ & $0.7806 \mathrm{e}+0$ & $0.1633 \mathrm{e}+1$ & $-0.2478 \mathrm{e}+1$ & (8) \\
\hline${ }^{3} \mathrm{He}$ & Mo & 100 & $0.1207 \mathrm{e}+1$ & $0.8839 \mathrm{e}+0$ & $0.1601 \mathrm{e}+1$ & $-0.2529 \mathrm{e}+1$ & (8) \\
\hline${ }^{3} \mathrm{He}$ & Mo & 140 & $0.1041 \mathrm{e}+1$ & $0.7313 \mathrm{e}+0$ & $0.1700 \mathrm{e}+1$ & $-0.2565 e+1$ & (8) \\
\hline${ }^{3} \mathrm{He}$ & Mo & 300 & $0.3957 \mathrm{e}+1$ & $0.3689 \mathrm{e}+1$ & $0.1455 \mathrm{e}+1$ & $-0.3271 e+1$ & (8) \\
\hline${ }^{3} \mathrm{He}$ & Mo & 1000 & $0.3636 \mathrm{e}+1$ & $0.3417 \mathrm{e}+1$ & $0.1628 \mathrm{e}+1$ & $-0.3534 e+1$ & (8) \\
\hline${ }^{3} \mathrm{He}$ & Mo & 3000 & $0.3151 \mathrm{e}+1$ & $0.2992 \mathrm{e}+1$ & $0.1626 \mathrm{e}+1$ & $-0.3503 e+1$ & (8) \\
\hline${ }^{3} \mathrm{He}$ & Mo & 10000 & $0.1829 \mathrm{e}+1$ & $0.1744 \mathrm{e}+1$ & $0.1633 \mathrm{e}+1$ & $-0.3279 e+1$ & (8) \\
\hline${ }^{4} \mathrm{He}$ & Mo & 70 & $0.9370 \mathrm{e}+0$ & $0.6093 e+0$ & $0.1737 e+1$ & $-0.2385 e+1$ & (8) \\
\hline${ }^{4} \mathrm{He}$ & Mo & 80 & $0.1159 \mathrm{e}+1$ & $0.8452 \mathrm{e}+0$ & $0.1571 \mathrm{e}+1$ & $-0.2428 \mathrm{e}+1$ & (8) \\
\hline${ }^{4} \mathrm{He}$ & Mo & 100 & $0.1029 \mathrm{e}+1$ & $0.7214 \mathrm{e}+0$ & $0.1700 \mathrm{e}+1$ & $-0.2502 \mathrm{e}+1$ & (8) \\
\hline${ }^{4} \mathrm{He}$ & Mo & 140 & $0.1039 \mathrm{e}+1$ & $0.7459 \mathrm{e}+0$ & $0.1722 \mathrm{e}+1$ & $-0.2589 e+1$ & (8) \\
\hline${ }^{4} \mathrm{He}$ & Mo & 1500 & $0.3859 \mathrm{e}+1$ & $0.3663 \mathrm{e}+1$ & $0.1683 \mathrm{e}+1$ & $-0.3657 \mathrm{e}+1$ & (8) \\
\hline${ }^{4} \mathrm{He}$ & Mo & 4000 & $0.2495 \mathrm{e}+1$ & $0.2353 \mathrm{e}+1$ & $0.1640 \mathrm{e}+1$ & $-0.3403 e+1$ & (8) \\
\hline${ }^{4} \mathrm{He}$ & Mo & 8000 & $0.2050 \mathrm{e}+1$ & $0.1951 \mathrm{e}+1$ & $0.1599 \mathrm{e}+1$ & $-0.3269 e+1$ & (8) \\
\hline
\end{tabular}


Table 39: Constants for the angular dependence of the energy reflection coefficient at several energies.

\begin{tabular}{|c|c|c|c|c|c|c|c|}
\hline ion & solid & energy & $\mathrm{c} 1$ & $\mathrm{c} 2$ & c3 & $\mathrm{c} 4$ & fit \\
\hline $\mathrm{H}$ & $\mathrm{W}$ & 10 & $0.7651 \mathrm{e}+0$ & $0.2142 \mathrm{e}+0$ & $0.1899 \mathrm{e}+1$ & $-0.1887 e+1$ & (8) \\
\hline $\mathrm{H}$ & W & 20 & $0.7932 \mathrm{e}+0$ & $0.2922 \mathrm{e}+0$ & $0.1803 e+1$ & $-0.2037 \mathrm{e}+1$ & (8) \\
\hline $\mathrm{H}$ & W & 50 & $0.8974 \mathrm{e}+0$ & $0.4566 \mathrm{e}+0$ & $0.1612 \mathrm{e}+1$ & $-0.2228 \mathrm{e}+1$ & (8) \\
\hline $\mathrm{H}$ & W & 100 & $0.1337 \mathrm{e}+1$ & $0.9425 \mathrm{e}+0$ & $0.1398 \mathrm{e}+1$ & $-0.2481 e+1$ & (8) \\
\hline $\mathrm{H}$ & W & 200 & $0.4873 \mathrm{e}+1$ & $0.4515 \mathrm{e}+1$ & $0.1349 \mathrm{e}+1$ & $-0.3326 e+1$ & (8) \\
\hline $\mathrm{H}$ & W & 300 & $0.4510 \mathrm{e}+1$ & $0.4172 \mathrm{e}+1$ & $0.1389 \mathrm{e}+1$ & $-0.3349 \mathrm{e}+1$ & (8) \\
\hline $\mathrm{H}$ & W & 500 & $0.4396 \mathrm{e}+1$ & $0.4085 \mathrm{e}+1$ & $0.1439 \mathrm{e}+1$ & $-0.3425 e+1$ & (8) \\
\hline $\mathrm{H}$ & W & 550 & $0.3931 \mathrm{e}+1$ & $0.3626 \mathrm{e}+1$ & $0.1454 \mathrm{e}+1$ & $-0.3384 \mathrm{e}+1$ & (8) \\
\hline $\mathrm{H}$ & W & 600 & $0.4576 \mathrm{e}+1$ & $0.4277 \mathrm{e}+1$ & $0.1438 \mathrm{e}+1$ & $-0.3454 \mathrm{e}+1$ & (8) \\
\hline $\mathrm{H}$ & W & 700 & $0.4234 \mathrm{e}+1$ & $0.3944 \mathrm{e}+1$ & $0.1415 \mathrm{e}+1$ & $-0.3382 e+1$ & (8) \\
\hline $\mathrm{H}$ & W & 800 & $0.4836 \mathrm{e}+1$ & $0.4558 \mathrm{e}+1$ & $0.1386 \mathrm{e}+1$ & $-0.3423 e+1$ & (8) \\
\hline $\mathrm{H}$ & W & 900 & $0.3422 \mathrm{e}+1$ & $0.3151 \mathrm{e}+1$ & $0.1418 \mathrm{e}+1$ & $-0.3274 \mathrm{e}+1$ & (8) \\
\hline $\mathrm{H}$ & W & 1000 & $0.3682 \mathrm{e}+1$ & $0.3418 \mathrm{e}+1$ & $0.1397 \mathrm{e}+1$ & $-0.3295 e+1$ & (8) \\
\hline $\mathrm{H}$ & W & 2000 & $0.3278 \mathrm{e}+1$ & $0.3053 \mathrm{e}+1$ & $0.1624 \mathrm{e}+1$ & $-0.3557 \mathrm{e}+1$ & (8) \\
\hline $\mathrm{H}$ & W & 4000 & $0.2785 \mathrm{e}+1$ & $0.2617 \mathrm{e}+1$ & $0.1608 \mathrm{e}+1$ & $-0.3440 \mathrm{e}+1$ & (8) \\
\hline $\mathrm{H}$ & W & 13333 & $0.1897 \mathrm{e}+1$ & $0.1823 e+1$ & $0.1597 \mathrm{e}+1$ & $-0.3289 e+1$ & (8) \\
\hline $\mathrm{H}$ & W & 26667 & $0.1179 \mathrm{e}+1$ & $0.1150 \mathrm{e}+1$ & $0.1908 \mathrm{e}+1$ & $-0.3554 \mathrm{e}+1$ & (8) \\
\hline $\mathrm{H}$ & W & 40000 & $0.1218 \mathrm{e}+1$ & $0.1199 \mathrm{e}+1$ & $0.2073 \mathrm{e}+1$ & $-0.3872 e+1$ & (8) \\
\hline $\mathrm{H}$ & W & 80000 & $0.1126 \mathrm{e}+1$ & $0.1118 \mathrm{e}+1$ & $0.2450 \mathrm{e}+1$ & $-0.4491 e+1$ & (8) \\
\hline $\mathrm{D}$ & W & 10 & $0.7857 \mathrm{e}+0$ & $0.2358 \mathrm{e}+0$ & $0.1625 \mathrm{e}+1$ & $-0.1697 e+1$ & (8) \\
\hline $\mathrm{D}$ & W & 20 & $0.7921 \mathrm{e}+0$ & $0.2793 \mathrm{e}+0$ & $0.1808 \mathrm{e}+1$ & $-0.2015 e+1$ & (8) \\
\hline $\mathrm{D}$ & W & 50 & $0.8864 \mathrm{e}+0$ & $0.4282 \mathrm{e}+0$ & $0.1665 \mathrm{e}+1$ & $-0.2251 \mathrm{e}+1$ & (8) \\
\hline $\mathrm{D}$ & W & 100 & $0.1296 \mathrm{e}+1$ & $0.8755 \mathrm{e}+0$ & $0.1444 \mathrm{e}+1$ & $-0.2520 \mathrm{e}+1$ & (8) \\
\hline $\mathrm{D}$ & W & 200 & $0.4358 \mathrm{e}+1$ & $0.3974 \mathrm{e}+1$ & $0.1347 \mathrm{e}+1$ & $-0.3268 \mathrm{e}+1$ & (8) \\
\hline $\mathrm{D}$ & W & 250 & $0.5077 \mathrm{e}+1$ & $0.4701 \mathrm{e}+1$ & $0.1374 \mathrm{e}+1$ & $-0.3401 e+1$ & (8) \\
\hline $\mathrm{D}$ & W & 270 & $0.5271 \mathrm{e}+1$ & $0.4898 \mathrm{e}+1$ & $0.1386 \mathrm{e}+1$ & $-0.3447 \mathrm{e}+1$ & (8) \\
\hline $\mathrm{D}$ & W & 300 & $0.4565 \mathrm{e}+1$ & $0.4201 \mathrm{e}+1$ & $0.1372 \mathrm{e}+1$ & $-0.3344 \mathrm{e}+1$ & (8) \\
\hline $\mathrm{D}$ & W & 350 & $0.4870 \mathrm{e}+1$ & $0.4507 \mathrm{e}+1$ & $0.1433 \mathrm{e}+1$ & $-0.3477 \mathrm{e}+1$ & (8) \\
\hline $\mathrm{D}$ & W & 400 & $0.4900 \mathrm{e}+1$ & $0.4547 \mathrm{e}+1$ & $0.1420 \mathrm{e}+1$ & $-0.3467 e+1$ & (8) \\
\hline $\mathrm{D}$ & W & 500 & $0.4164 \mathrm{e}+1$ & $0.3824 \mathrm{e}+1$ & $0.1456 \mathrm{e}+1$ & $-0.3425 e+1$ & (8) \\
\hline $\mathrm{D}$ & W & 600 & $0.4134 \mathrm{e}+1$ & $0.3801 \mathrm{e}+1$ & $0.1430 \mathrm{e}+1$ & $-0.3396 \mathrm{e}+1$ & (8) \\
\hline $\mathrm{D}$ & W & 700 & $0.4882 \mathrm{e}+1$ & $0.4562 \mathrm{e}+1$ & $0.1395 \mathrm{e}+1$ & $-0.3446 \mathrm{e}+1$ & (8) \\
\hline $\mathrm{D}$ & W & 1000 & $0.2881 e+1$ & $0.2581 \mathrm{e}+1$ & $0.1407 \mathrm{e}+1$ & $-0.3173 e+1$ & (8) \\
\hline $\mathrm{T}$ & W & 10 & $0.7731 e+0$ & $0.2290 \mathrm{e}+0$ & $0.1748 \mathrm{e}+1$ & $-0.1800 \mathrm{e}+1$ & (8) \\
\hline $\mathrm{T}$ & W & 20 & $0.7893 e+0$ & $0.2887 \mathrm{e}+0$ & $0.1765 \mathrm{e}+1$ & $-0.1981 e+1$ & (8) \\
\hline $\mathrm{T}$ & W & 50 & $0.8960 \mathrm{e}+0$ & $0.4457 \mathrm{e}+0$ & $0.1636 \mathrm{e}+1$ & $-0.2243 e+1$ & (8) \\
\hline $\mathrm{T}$ & W & 100 & $0.1231 \mathrm{e}+1$ & $0.8177 \mathrm{e}+0$ & $0.1475 \mathrm{e}+1$ & $-0.2506 \mathrm{e}+1$ & (8) \\
\hline $\mathrm{T}$ & W & 140 & $0.3030 \mathrm{e}+1$ & $0.2636 \mathrm{e}+1$ & $0.1303 e+1$ & $-0.2975 e+1$ & (8) \\
\hline $\mathrm{T}$ & W & 160 & $0.4308 \mathrm{e}+1$ & $0.3919 \mathrm{e}+1$ & $0.1298 \mathrm{e}+1$ & $-0.3183 e+1$ & (8) \\
\hline $\mathrm{T}$ & W & 170 & $0.4028 \mathrm{e}+1$ & $0.3641 \mathrm{e}+1$ & $0.1309 \mathrm{e}+1$ & $-0.3163 e+1$ & (8) \\
\hline $\mathrm{T}$ & W & 180 & $0.3965 \mathrm{e}+1$ & $0.3580 \mathrm{e}+1$ & $0.1322 \mathrm{e}+1$ & $-0.3174 \mathrm{e}+1$ & (8) \\
\hline $\mathrm{T}$ & W & 200 & $0.4788 \mathrm{e}+1$ & $0.4408 \mathrm{e}+1$ & $0.1304 \mathrm{e}+1$ & $-0.3262 \mathrm{e}+1$ & (8) \\
\hline $\mathrm{T}$ & W & 250 & $0.5119 \mathrm{e}+1$ & $0.4747 \mathrm{e}+1$ & $0.1355 \mathrm{e}+1$ & $-0.3376 e+1$ & (8) \\
\hline $\mathrm{T}$ & W & 300 & $0.3889 e+1$ & $0.3523 e+1$ & $0.1401 \mathrm{e}+1$ & $-0.3291 \mathrm{e}+1$ & (8) \\
\hline $\mathrm{T}$ & W & 400 & $0.5784 \mathrm{e}+1$ & $0.5429 \mathrm{e}+1$ & $0.1391 \mathrm{e}+1$ & $-0.3518 \mathrm{e}+1$ & (8) \\
\hline $\mathrm{T}$ & $\mathrm{W}$ & 500 & $0.4092 \mathrm{e}+1$ & $0.3750 \mathrm{e}+1$ & $0.1416 \mathrm{e}+1$ & $-0.3361 e+1$ & (8) \\
\hline $\mathrm{T}$ & W & 700 & $0.5005 \mathrm{e}+1$ & $0.4678 \mathrm{e}+1$ & $0.1414 \mathrm{e}+1$ & $-0.3491 e+1$ & (8) \\
\hline $\mathrm{T}$ & W & 1000 & $0.3379 e+1$ & $0.3074 \mathrm{e}+1$ & $0.1413 e+1$ & $-0.3272 \mathrm{e}+1$ & (8) \\
\hline
\end{tabular}


Table 40: Constants for the angular dependence of the energy reflection coefficient at several energies.

\begin{tabular}{|c|c|c|c|c|c|c|c|}
\hline ion & solid & energy & c1 & c2 & c3 & c4 & fit \\
\hline${ }^{4} \mathrm{He}$ & $\mathrm{W}$ & 10 & $0.8283 \mathrm{e}+0$ & $0.2399 \mathrm{e}+0$ & $0.1869 \mathrm{e}+1$ & $-0.1865 \mathrm{e}+1$ & $(8)$ \\
\hline${ }^{4} \mathrm{He}$ & W & 20 & $0.8137 \mathrm{e}+0$ & $0.2959 \mathrm{e}+0$ & $0.1836 \mathrm{e}+1$ & $-0.1962 e+1$ & (8) \\
\hline${ }^{4} \mathrm{He}$ & W & 50 & $0.8079 \mathrm{e}+0$ & $0.3622 \mathrm{e}+0$ & $0.1972 \mathrm{e}+1$ & $-0.2308 \mathrm{e}+1$ & (8) \\
\hline${ }^{4} \mathrm{He}$ & W & 100 & $0.8929 \mathrm{e}+0$ & $0.4916 \mathrm{e}+0$ & $0.1785 \mathrm{e}+1$ & $-0.2411 \mathrm{e}+1$ & (8) \\
\hline${ }^{4} \mathrm{He}$ & W & 125 & $0.9850 \mathrm{e}+0$ & $0.5994 \mathrm{e}+0$ & $0.1669 \mathrm{e}+1$ & $-0.2436 \mathrm{e}+1$ & (8) \\
\hline${ }^{4} \mathrm{He}$ & W & 130 & $0.9418 \mathrm{e}+0$ & $0.5570 \mathrm{e}+0$ & $0.1736 \mathrm{e}+1$ & $-0.2464 \mathrm{e}+1$ & (8) \\
\hline${ }^{4} \mathrm{He}$ & W & 140 & $0.9828 \mathrm{e}+0$ & $0.6020 \mathrm{e}+0$ & $0.1692 \mathrm{e}+1$ & $-0.2478 \mathrm{e}+1$ & (8) \\
\hline${ }^{4} \mathrm{He}$ & W & 150 & $0.9771 \mathrm{e}+0$ & $0.5973 \mathrm{e}+0$ & $0.1753 \mathrm{e}+1$ & $-0.2554 \mathrm{e}+1$ & (8) \\
\hline${ }^{4} \mathrm{He}$ & W & 170 & $0.1228 \mathrm{e}+1$ & $0.8602 \mathrm{e}+0$ & $0.1543 \mathrm{e}+1$ & $-0.2551 \mathrm{e}+1$ & (8) \\
\hline${ }^{4} \mathrm{He}$ & $\mathrm{W}$ & 200 & $0.1389 \mathrm{e}+1$ & $0.1031 \mathrm{e}+1$ & $0.1506 \mathrm{e}+1$ & $-0.2629 e+1$ & (8) \\
\hline${ }^{4} \mathrm{He}$ & W & 250 & $0.2594 \mathrm{e}+1$ & $0.2247 \mathrm{e}+1$ & $0.1392 \mathrm{e}+1$ & $-0.2952 e+1$ & (8) \\
\hline${ }^{4} \mathrm{He}$ & W & 300 & $0.3098 \mathrm{e}+1$ & $0.2759 \mathrm{e}+1$ & $0.1401 \mathrm{e}+1$ & $-0.3083 e+1$ & (8) \\
\hline${ }^{4} \mathrm{He}$ & $\mathrm{W}$ & 350 & $0.4605 e+1$ & $0.4272 \mathrm{e}+1$ & $0.1408 \mathrm{e}+1$ & $-0.3336 \mathrm{e}+1$ & (8) \\
\hline${ }^{4} \mathrm{He}$ & W & 400 & $0.4680 \mathrm{e}+1$ & $0.4354 \mathrm{e}+1$ & $0.1414 \mathrm{e}+1$ & $-0.3355 e+1$ & (8) \\
\hline${ }^{4} \mathrm{He}$ & W & 500 & $0.4093 \mathrm{e}+1$ & $0.3772 \mathrm{e}+1$ & $0.1493 \mathrm{e}+1$ & $-0.3404 e+1$ & (8) \\
\hline${ }^{4} \mathrm{He}$ & W & 600 & $0.3548 \mathrm{e}+1$ & $0.3236 \mathrm{e}+1$ & $0.1458 \mathrm{e}+1$ & $-0.3283 e+1$ & (8) \\
\hline${ }^{4} \mathrm{He}$ & W & 700 & $0.3837 \mathrm{e}+1$ & $0.3530 \mathrm{e}+1$ & $0.1472 \mathrm{e}+1$ & $-0.3361 e+1$ & (8) \\
\hline${ }^{4} \mathrm{He}$ & W & 1000 & $0.4905 \mathrm{e}+1$ & $0.4614 \mathrm{e}+1$ & $0.1489 \mathrm{e}+1$ & $-0.3539 e+1$ & (8) \\
\hline${ }^{4} \mathrm{He}$ & $\mathrm{W}$ & 1400 & $0.6573 e+1$ & $0.6300 \mathrm{e}+1$ & $0.1445 \mathrm{e}+1$ & $-0.3661 e+1$ & (8) \\
\hline${ }^{4} \mathrm{He}$ & W & 2000 & $0.4138 \mathrm{e}+1$ & $0.3886 \mathrm{e}+1$ & $0.1437 \mathrm{e}+1$ & $-0.3405 e+1$ & (8) \\
\hline${ }^{4} \mathrm{He}$ & W & 5000 & $0.3134 \mathrm{e}+1$ & $0.2943 e+1$ & $0.1320 \mathrm{e}+1$ & $-0.3120 \mathrm{e}+1$ & (8) \\
\hline${ }^{4} \mathrm{He}$ & W & 10000 & $0.2531 \mathrm{e}+1$ & $0.2388 \mathrm{e}+1$ & $0.1323 e+1$ & $-0.3024 e+1$ & (8) \\
\hline${ }^{4} \mathrm{He}$ & $\mathrm{W}$ & 20000 & $0.2286 \mathrm{e}+1$ & $0.2180 \mathrm{e}+1$ & $0.1686 \mathrm{e}+1$ & $-0.3446 \mathrm{e}+1$ & (8) \\
\hline${ }^{4} \mathrm{He}$ & W & 50000 & $0.1831 \mathrm{e}+1$ & $0.1780 \mathrm{e}+1$ & $0.1825 \mathrm{e}+1$ & $-0.3584 e+1$ & (8) \\
\hline $\mathrm{N}$ & W & 10 & $0.7019 \mathrm{e}+0$ & $0.2724 \mathrm{e}+0$ & $0.1731 \mathrm{e}+1$ & $-0.1855 e+1$ & (8) \\
\hline $\mathrm{N}$ & W & 20 & $0.7400 \mathrm{e}+0$ & $0.3403 e+0$ & $0.1653 \mathrm{e}+1$ & $-0.1834 e+1$ & (8) \\
\hline $\mathrm{N}$ & W & 40 & $0.7495 \mathrm{e}+0$ & $0.3967 \mathrm{e}+0$ & $0.1757 \mathrm{e}+1$ & $-0.20033+1$ & (8) \\
\hline $\mathrm{N}$ & W & 48 & $0.7593 \mathrm{e}+0$ & $0.4226 \mathrm{e}+0$ & $0.1723 e+1$ & $-0.1998 \mathrm{e}+1$ & (8) \\
\hline $\mathrm{N}$ & W & 50 & $0.7456 \mathrm{e}+0$ & $0.4084 \mathrm{e}+0$ & $0.1800 \mathrm{e}+1$ & $-0.2063 e+1$ & (8) \\
\hline $\mathrm{N}$ & W & 52 & $0.7487 \mathrm{e}+0$ & $0.4163 \mathrm{e}+0$ & $0.1780 \mathrm{e}+1$ & $-0.2053 e+1$ & (8) \\
\hline $\mathrm{N}$ & W & 55 & $0.7567 \mathrm{e}+0$ & $0.4311 \mathrm{e}+0$ & $0.1720 \mathrm{e}+1$ & $-0.2015 e+1$ & (8) \\
\hline $\mathrm{N}$ & W & 60 & $0.7418 \mathrm{e}+0$ & $0.4156 \mathrm{e}+0$ & $0.1827 \mathrm{e}+1$ & $-0.2105 e+1$ & (8) \\
\hline $\mathrm{N}$ & W & 70 & $0.7669 \mathrm{e}+0$ & $0.4575 \mathrm{e}+0$ & $0.1730 \mathrm{e}+1$ & $-0.2071 \mathrm{e}+1$ & (8) \\
\hline $\mathrm{N}$ & W & 80 & $0.7806 \mathrm{e}+0$ & $0.4777 \mathrm{e}+0$ & $0.1742 \mathrm{e}+1$ & $-0.2135 e+1$ & (8) \\
\hline $\mathrm{N}$ & W & 90 & $0.7665 \mathrm{e}+0$ & $0.4657 \mathrm{e}+0$ & $0.1845 \mathrm{e}+1$ & $-0.2233 e+1$ & (8) \\
\hline $\mathrm{N}$ & W & 100 & $0.7871 \mathrm{e}+0$ & $0.4947 \mathrm{e}+0$ & $0.1775 \mathrm{e}+1$ & $-0.2211 \mathrm{e}+1$ & (8) \\
\hline $\mathrm{N}$ & W & 120 & $0.8031 \mathrm{e}+0$ & $0.5236 \mathrm{e}+0$ & $0.1770 \mathrm{e}+1$ & $-0.2259 e+1$ & (8) \\
\hline $\mathrm{N}$ & W & 140 & $0.8233 \mathrm{e}+0$ & $0.5495 \mathrm{e}+0$ & $0.1795 \mathrm{e}+1$ & $-0.2347 \mathrm{e}+1$ & (8) \\
\hline $\mathrm{N}$ & W & 200 & $0.8580 \mathrm{e}+0$ & $0.5988 \mathrm{e}+0$ & $0.1817 \mathrm{e}+1$ & $-0.2472 \mathrm{e}+1$ & (8) \\
\hline $\mathrm{N}$ & W & 300 & $0.1061 \mathrm{e}+1$ & $0.8211 \mathrm{e}+0$ & $0.1688 \mathrm{e}+1$ & $-0.2587 e+1$ & (8) \\
\hline $\mathrm{N}$ & W & 500 & $0.1823 e+1$ & $0.1603 \mathrm{e}+1$ & $0.1574 \mathrm{e}+1$ & $-0.2909 e+1$ & (8) \\
\hline $\mathrm{N}$ & W & 1000 & $0.3171 \mathrm{e}+1$ & $0.2969 \mathrm{e}+1$ & $0.1600 \mathrm{e}+1$ & $-0.3345 e+1$ & (8) \\
\hline $\mathrm{Xe}$ & W & 9500 & $0.1064 \mathrm{e}+1$ & $0.1060 \mathrm{e}+1$ & $0.2616 \mathrm{e}+1$ & $-0.4318 \mathrm{e}+1$ & (8) \\
\hline $\mathrm{Xe}$ & W & 30000 & $0.1073 e+1$ & $0.1070 \mathrm{e}+1$ & $0.2501 \mathrm{e}+1$ & $-0.4307 \mathrm{e}+1$ & $(8)$ \\
\hline
\end{tabular}


Table 41: Constants for the angular dependence of the energy reflection coefficient at several energies.

\begin{tabular}{|c|c|c|c|c|c|c|c|}
\hline ion & solid & energy & c1 & c2 & c3 & c4 & fit \\
\hline $\mathrm{Ne}$ & $\mathrm{W}$ & 10 & $0.7772 \mathrm{e}+0$ & $0.3789 e+0$ & $0.1765 \mathrm{e}+1$ & $-0.2002 e+1$ & (8) \\
\hline $\mathrm{Ne}$ & W & 20 & $0.7932 \mathrm{e}+0$ & $0.4349 \mathrm{e}+0$ & $0.1646 e+1$ & $-0.1932 e+1$ & (8) \\
\hline $\mathrm{Ne}$ & W & 30 & $0.7899 \mathrm{e}+0$ & $0.4634 \mathrm{e}+0$ & $0.1614 \mathrm{e}+1$ & $-0.1915 e+1$ & (8) \\
\hline $\mathrm{Ne}$ & W & 40 & $0.7882 \mathrm{e}+0$ & $0.4791 \mathrm{e}+0$ & $0.1667 \mathrm{e}+1$ & $-0.1992 \mathrm{e}+1$ & (8) \\
\hline $\mathrm{Ne}$ & W & 45 & $0.7746 \mathrm{e}+0$ & $0.4732 \mathrm{e}+0$ & $0.1726 \mathrm{e}+1$ & $-0.2035 e+1$ & (8) \\
\hline $\mathrm{Ne}$ & W & 50 & $0.7772 \mathrm{e}+0$ & $0.4837 \mathrm{e}+0$ & $0.1712 \mathrm{e}+1$ & $-0.2041 e+1$ & (8) \\
\hline $\mathrm{Ne}$ & W & 60 & $0.7794 \mathrm{e}+0$ & $0.4967 \mathrm{e}+0$ & $0.1728 \mathrm{e}+1$ & $-0.2085 e+1$ & (8) \\
\hline $\mathrm{Ne}$ & W & 70 & $0.7768 \mathrm{e}+0$ & $0.5063 \mathrm{e}+0$ & $0.1740 \mathrm{e}+1$ & $-0.2111 \mathrm{e}+1$ & (8) \\
\hline $\mathrm{Ne}$ & W & 80 & $0.7712 \mathrm{e}+0$ & $0.5059 \mathrm{e}+0$ & $0.1803 e+1$ & $-0.2192 \mathrm{e}+1$ & (8) \\
\hline $\mathrm{Ne}$ & W & 100 & $0.7721 \mathrm{e}+0$ & $0.5210 \mathrm{e}+0$ & $0.1828 \mathrm{e}+1$ & $-0.2246 \mathrm{e}+1$ & (8) \\
\hline $\mathrm{Ne}$ & W & 140 & $0.8065 \mathrm{e}+0$ & $0.5739 \mathrm{e}+0$ & $0.1810 \mathrm{e}+1$ & $-0.2334 \mathrm{e}+1$ & (8) \\
\hline $\mathrm{Ne}$ & W & 200 & $0.8854 \mathrm{e}+0$ & $0.6689 \mathrm{e}+0$ & $0.1783 e+1$ & $-0.2463 e+1$ & (8) \\
\hline $\mathrm{Ne}$ & W & 300 & $0.1010 \mathrm{e}+1$ & $0.8115 \mathrm{e}+0$ & $0.1732 \mathrm{e}+1$ & $-0.2580 \mathrm{e}+1$ & (8) \\
\hline $\mathrm{Ne}$ & W & 400 & $0.1178 \mathrm{e}+1$ & $0.9893 \mathrm{e}+0$ & $0.1703 e+1$ & $-0.2709 e+1$ & (8) \\
\hline $\mathrm{Ne}$ & W & 500 & $0.1435 \mathrm{e}+1$ & $0.1254 \mathrm{e}+1$ & $0.1673 e+1$ & $-0.2843 e+1$ & (8) \\
\hline $\mathrm{Ne}$ & W & 700 & $0.2812 \mathrm{e}+1$ & $0.2641 \mathrm{e}+1$ & $0.1586 \mathrm{e}+1$ & $-0.3198 \mathrm{e}+1$ & (8) \\
\hline $\mathrm{Ne}$ & W & 1000 & $0.3147 \mathrm{e}+1$ & $0.2984 \mathrm{e}+1$ & $0.1645 e+1$ & $-0.3371 e+1$ & (8) \\
\hline $\mathrm{Ar}$ & W & 10 & $0.7652 \mathrm{e}+0$ & $0.5480 \mathrm{e}+0$ & $0.1665 \mathrm{e}+1$ & $-0.2085 e+1$ & (8) \\
\hline $\mathrm{Ar}$ & W & 20 & $0.7870 \mathrm{e}+0$ & $0.5780 \mathrm{e}+0$ & $0.1636 \mathrm{e}+1$ & $-0.2079 e+1$ & (8) \\
\hline $\mathrm{Ar}$ & W & 30 & $0.8039 \mathrm{e}+0$ & $0.6071 \mathrm{e}+0$ & $0.1616 \mathrm{e}+1$ & $-0.2088 \mathrm{e}+1$ & (8) \\
\hline $\mathrm{Ar}$ & W & 35 & $0.8157 \mathrm{e}+0$ & $0.6271 \mathrm{e}+0$ & $0.1595 \mathrm{e}+1$ & $-0.2083 e+1$ & (8) \\
\hline $\mathrm{Ar}$ & W & 40 & $0.8090 \mathrm{e}+0$ & $0.6239 e+0$ & $0.1619 \mathrm{e}+1$ & $-0.2104 e+1$ & (8) \\
\hline $\mathrm{Ar}$ & W & 45 & $0.7862 \mathrm{e}+0$ & $0.6045 \mathrm{e}+0$ & $0.1672 \mathrm{e}+1$ & $-0.2132 \mathrm{e}+1$ & (8) \\
\hline $\mathrm{Ar}$ & W & 50 & $0.7855 \mathrm{e}+0$ & $0.6100 \mathrm{e}+0$ & $0.1661 \mathrm{e}+1$ & $-0.2128 \mathrm{e}+1$ & (8) \\
\hline $\mathrm{Ar}$ & W & 55 & $0.7829 \mathrm{e}+0$ & $0.6097 \mathrm{e}+0$ & $0.1683 e+1$ & $-0.2157 e+1$ & (8) \\
\hline Ar & W & 60 & $0.7886 \mathrm{e}+0$ & $0.6179 \mathrm{e}+0$ & $0.1702 \mathrm{e}+1$ & $-0.2190 \mathrm{e}+1$ & (8) \\
\hline Ar & W & 70 & $0.8072 \mathrm{e}+0$ & $0.6456 \mathrm{e}+0$ & $0.1687 \mathrm{e}+1$ & $-0.2206 \mathrm{e}+1$ & (8) \\
\hline $\mathrm{Ar}$ & W & 80 & $0.7995 \mathrm{e}+0$ & $0.6409 e+0$ & $0.1715 e+1$ & $-0.2242 \mathrm{e}+1$ & (8) \\
\hline Ar & W & 100 & $0.8234 \mathrm{e}+0$ & $0.6748 \mathrm{e}+0$ & $0.1719 \mathrm{e}+1$ & $-0.2294 e+1$ & (8) \\
\hline $\mathrm{Ar}$ & W & 140 & $0.8297 \mathrm{e}+0$ & $0.6913 \mathrm{e}+0$ & $0.1790 \mathrm{e}+1$ & $-0.2419 \mathrm{e}+1$ & (8) \\
\hline $\mathrm{Ar}$ & W & 200 & $0.8744 \mathrm{e}+0$ & $0.7469 \mathrm{e}+0$ & $0.1826 \mathrm{e}+1$ & $-0.2550 \mathrm{e}+1$ & (8) \\
\hline $\mathrm{Ar}$ & W & 300 & $0.1005 \mathrm{e}+1$ & $0.8891 \mathrm{e}+0$ & $0.1816 \mathrm{e}+1$ & $-0.2703 e+1$ & (8) \\
\hline Ar & W & 500 & $0.1333 \mathrm{e}+1$ & $0.1232 \mathrm{e}+1$ & $0.1778 \mathrm{e}+1$ & $-0.2921 e+1$ & (8) \\
\hline $\mathrm{Ar}$ & W & 700 & $0.2044 \mathrm{e}+1$ & $0.1950 \mathrm{e}+1$ & $0.1736 \mathrm{e}+1$ & $-0.3179 e+1$ & (8) \\
\hline $\mathrm{Ar}$ & W & 1000 & $0.2246 \mathrm{e}+1$ & $0.2157 \mathrm{e}+1$ & $0.1773 e+1$ & $-0.3319 e+1$ & (8) \\
\hline Ar & W & 1005 & $0.2183 \mathrm{e}+1$ & $0.2095 \mathrm{e}+1$ & $0.1788 \mathrm{e}+1$ & $-0.3320 e+1$ & (8) \\
\hline $\mathrm{Ar}$ & W & 1050 & $0.2172 \mathrm{e}+1$ & $0.2084 \mathrm{e}+1$ & $0.1806 \mathrm{e}+1$ & $-0.3347 e+1$ & (8) \\
\hline $\mathrm{Ar}$ & W & 30000 & $0.1590 \mathrm{e}+1$ & $0.1540 \mathrm{e}+1$ & $0.2036 \mathrm{e}+1$ & $-0.3737 e+1$ & (8) \\
\hline W & W & 350 & $0.9262 \mathrm{e}+0$ & $0.9261 \mathrm{e}+0$ & $0.2696 \mathrm{e}+1$ & $-0.4122 \mathrm{e}+1$ & (8) \\
\hline W & W & 400 & $0.9196 \mathrm{e}+0$ & $0.9195 e+0$ & $0.2695 \mathrm{e}+1$ & $-0.4101 e+1$ & (8) \\
\hline W & W & 500 & $0.9199 \mathrm{e}+0$ & $0.9196 \mathrm{e}+0$ & $0.2717 \mathrm{e}+1$ & $-0.4143 e+1$ & (8) \\
\hline W & W & 800 & $0.9112 \mathrm{e}+0$ & $0.9107 \mathrm{e}+0$ & $0.2728 \mathrm{e}+1$ & $-0.4173 e+1$ & (8) \\
\hline W & W & 1000 & $0.9362 \mathrm{e}+0$ & $0.9356 \mathrm{e}+0$ & $0.2768 \mathrm{e}+1$ & $-0.4264 e+1$ & (8) \\
\hline W & W & 2500 & $0.1058 \mathrm{e}+1$ & $0.1058 \mathrm{e}+1$ & $0.2801 \mathrm{e}+1$ & $-0.4489 e+1$ & $(8)$ \\
\hline
\end{tabular}


Table 42: Constants for the angular dependence of the energy reflection coefficient at several energies.

\begin{tabular}{|c|c|c|c|c|c|c|c|}
\hline ion & solid & energy & $\mathrm{c} 1$ & $\mathrm{c} 2$ & c3 & $\mathrm{c} 4$ & fit \\
\hline${ }^{4} \mathrm{He}$ & $\mathrm{Ta}$ & 150000 & $0.1333 \mathrm{e}+1$ & $0.1310 \mathrm{e}+1$ & $0.2088 \mathrm{e}+1$ & $-0.3856 \mathrm{e}+1$ & $(8)$ \\
\hline $\mathrm{Ne}$ & $\mathrm{Ta}$ & 45000 & $0.2159 \mathrm{e}+1$ & $0.2091 \mathrm{e}+1$ & $0.1605 \mathrm{e}+1$ & $-0.3357 e+1$ & (8) \\
\hline Ar & $\mathrm{Ta}$ & 1050 & $0.2562 \mathrm{e}+1$ & $0.2477 \mathrm{e}+1$ & $0.1819 \mathrm{e}+1$ & $-0.3489 e+1$ & (8) \\
\hline $\mathrm{Kr}$ & $\mathrm{Ta}$ & 45000 & $0.1102 \mathrm{e}+1$ & $0.1091 \mathrm{e}+1$ & $0.2190 \mathrm{e}+1$ & $-0.3867 \mathrm{e}+1$ & (8) \\
\hline $\mathrm{H}$ & $\mathrm{Au}$ & 1000 & $0.4312 \mathrm{e}+1$ & $0.4036 \mathrm{e}+1$ & $0.1559 \mathrm{e}+1$ & $-0.3599 e+1$ & $(8)$ \\
\hline $\mathrm{H}$ & $\mathrm{Au}$ & 1333 & $0.4657 \mathrm{e}+1$ & $0.4399 \mathrm{e}+1$ & $0.1552 \mathrm{e}+1$ & $-0.3640 \mathrm{e}+1$ & (8) \\
\hline $\mathrm{H}$ & $\mathrm{Au}$ & 4000 & $0.3834 \mathrm{e}+1$ & $0.3659 \mathrm{e}+1$ & $0.1608 \mathrm{e}+1$ & $-0.3621 \mathrm{e}+1$ & (8) \\
\hline $\mathrm{H}$ & $\mathrm{Au}$ & 5000 & $0.3171 \mathrm{e}+1$ & $0.3021 \mathrm{e}+1$ & $0.1451 \mathrm{e}+1$ & $-0.3322 e+1$ & (8) \\
\hline $\mathrm{H}$ & $\mathrm{Au}$ & 10000 & $0.1788 \mathrm{e}+1$ & $0.1687 \mathrm{e}+1$ & $0.1567 \mathrm{e}+1$ & $-0.3185 e+1$ & (8) \\
\hline $\mathrm{D}$ & $\mathrm{Au}$ & 150 & $0.4788 \mathrm{e}+1$ & $0.4388 \mathrm{e}+1$ & $0.1299 \mathrm{e}+1$ & $-0.3257 e+1$ & (8) \\
\hline $\mathrm{D}$ & $\mathrm{Au}$ & 200 & $0.5367 \mathrm{e}+1$ & $0.4975 \mathrm{e}+1$ & $0.1361 \mathrm{e}+1$ & $-0.3414 e+1$ & (8) \\
\hline $\mathrm{D}$ & $\mathrm{Au}$ & 250 & $0.4989 \mathrm{e}+1$ & $0.4609 \mathrm{e}+1$ & $0.1406 \mathrm{e}+1$ & $-0.3432 \mathrm{e}+1$ & (8) \\
\hline $\mathrm{D}$ & $\mathrm{Au}$ & 300 & $0.4688 \mathrm{e}+1$ & $0.4315 \mathrm{e}+1$ & $0.1387 \mathrm{e}+1$ & $-0.3393 e+1$ & (8) \\
\hline $\mathrm{D}$ & $\mathrm{Au}$ & 500 & $0.4824 \mathrm{e}+1$ & $0.4477 \mathrm{e}+1$ & $0.1391 \mathrm{e}+1$ & $-0.3443 e+1$ & (8) \\
\hline $\mathrm{D}$ & $\mathrm{Au}$ & 750 & $0.4965 \mathrm{e}+1$ & $0.4628 \mathrm{e}+1$ & $0.1729 \mathrm{e}+1$ & $-0.3948 e+1$ & (8) \\
\hline $\mathrm{D}$ & $\mathrm{Au}$ & 1000 & $0.5561 \mathrm{e}+1$ & $0.5258 \mathrm{e}+1$ & $0.1338 \mathrm{e}+1$ & $-0.3457 e+1$ & (8) \\
\hline $\mathrm{D}$ & $\mathrm{Au}$ & 2500 & $0.4367 \mathrm{e}+1$ & $0.4123 \mathrm{e}+1$ & $0.1461 \mathrm{e}+1$ & $-0.3491 e+1$ & (8) \\
\hline $\mathrm{D}$ & $\mathrm{Au}$ & 3000 & $0.3636 \mathrm{e}+1$ & $0.3407 \mathrm{e}+1$ & $0.1399 \mathrm{e}+1$ & $-0.3317 \mathrm{e}+1$ & (8) \\
\hline $\mathrm{D}$ & $\mathrm{Au}$ & 5000 & $0.4603 e+1$ & $0.4419 \mathrm{e}+1$ & $0.1365 \mathrm{e}+1$ & $-0.3402 \mathrm{e}+1$ & (8) \\
\hline $\mathrm{D}$ & $\mathrm{Au}$ & 8300 & $0.3228 \mathrm{e}+1$ & $0.3086 \mathrm{e}+1$ & $0.1387 \mathrm{e}+1$ & $-0.3246 \mathrm{e}+1$ & (8) \\
\hline D & $\mathrm{Au}$ & 10000 & $0.2796 \mathrm{e}+1$ & $0.2670 \mathrm{e}+1$ & $0.1396 \mathrm{e}+1$ & $-0.3187 e+1$ & (8) \\
\hline${ }^{4} \mathrm{He}$ & $\mathrm{Au}$ & 5000 & $0.3603 e+1$ & $0.3391 \mathrm{e}+1$ & $0.1606 \mathrm{e}+1$ & $-0.3573 e+1$ & (8) \\
\hline $\mathrm{Ne}$ & $\mathrm{Au}$ & 6000 & $0.2527 \mathrm{e}+1$ & $0.2396 \mathrm{e}+1$ & $0.1670 \mathrm{e}+1$ & $-0.3428 e+1$ & (8) \\
\hline $\mathrm{Ne}$ & $\mathrm{Au}$ & 14000 & $0.2279 \mathrm{e}+1$ & $0.2165 \mathrm{e}+1$ & $0.1689 \mathrm{e}+1$ & $-0.3435 e+1$ & (8) \\
\hline Ar & $\mathrm{Au}$ & 1050 & $0.2312 \mathrm{e}+1$ & $0.2216 \mathrm{e}+1$ & $0.1773 e+1$ & $-0.3348 e+1$ & (8) \\
\hline Ar & $\mathrm{Au}$ & 3000 & $0.1961 \mathrm{e}+1$ & $0.1879 \mathrm{e}+1$ & $0.1903 e+1$ & $-0.3529 \mathrm{e}+1$ & (8) \\
\hline Ar & $\mathrm{Au}$ & 6000 & $0.2208 \mathrm{e}+1$ & $0.2132 \mathrm{e}+1$ & $0.1989 \mathrm{e}+1$ & $-0.3761 \mathrm{e}+1$ & (8) \\
\hline $\mathrm{Ar}$ & $\mathrm{Au}$ & 10000 & $0.2049 \mathrm{e}+1$ & $0.1978 \mathrm{e}+1$ & $0.1929 \mathrm{e}+1$ & $-0.3679 \mathrm{e}+1$ & (8) \\
\hline Ar & $\mathrm{Au}$ & 30000 & $0.1806 \mathrm{e}+1$ & $0.1752 \mathrm{e}+1$ & $0.1818 \mathrm{e}+1$ & $-0.3533 e+1$ & (8) \\
\hline $\mathrm{Xe}$ & $\mathrm{Au}$ & 10000 & $0.1070 \mathrm{e}+1$ & $0.1065 \mathrm{e}+1$ & $0.2413 \mathrm{e}+1$ & $-0.4088 \mathrm{e}+1$ & (8) \\
\hline $\mathrm{Kr}$ & $\mathrm{Hg}$ & 762 & $0.1412 \mathrm{e}+1$ & $0.1382 \mathrm{e}+1$ & $0.2050 \mathrm{e}+1$ & $-0.3484 \mathrm{e}+1$ & $(8)$ \\
\hline $\mathrm{H}$ & $\mathrm{U}$ & 2000 & $0.4335 \mathrm{e}+1$ & $0.4092 \mathrm{e}+1$ & $0.1440 \mathrm{e}+1$ & $-0.3478 \mathrm{e}+1$ & $(8)$ \\
\hline $\mathrm{Kr}$ & $\mathrm{U}$ & 17900 & $0.1263 e+1$ & $0.1233 e+1$ & $0.2294 \mathrm{e}+1$ & $-0.3949 e+1$ & (8) \\
\hline
\end{tabular}


Table 43: Ion-target combinations for which energy dependencies of the reflection coefficients have been calculated.

\begin{tabular}{|r|c|c|c|c|c|c|c|c|c|c|c|c|}
\hline ion & $\mathrm{H}$ & $\mathrm{D}$ & $\mathrm{T}$ & ${ }^{3} \mathrm{He}$ & ${ }^{4} \mathrm{He}$ & $\mathrm{N}$ & $\mathrm{O}$ & $\mathrm{Ne}$ & $\mathrm{Ar}$ & $\mathrm{Kr}$ & $\mathrm{Xe}$ & $\mathrm{selfb}$ \\
\hline target & & & & & & & & & & & & \\
\hline $\mathrm{Li}$ & $\mathrm{x}$ & $\mathrm{x}$ & $\mathrm{x}$ & & $\mathrm{x}$ & & & & & & & $\mathrm{x}$ \\
$\mathrm{Be}$ & $\mathrm{x}$ & $\mathrm{x}$ & $\mathrm{x}$ & $\mathrm{x}$ & $\mathrm{x}$ & $\mathrm{x}$ & & $\mathrm{x}$ & & & & $\mathrm{x}$ \\
$\mathrm{B}$ & $\mathrm{x}$ & $\mathrm{x}$ & $\mathrm{x}$ & & & & & & & & & $\mathrm{x}$ \\
$\mathrm{C}$ & $\mathrm{x}$ & $\mathrm{x}$ & $\mathrm{x}$ & $\mathrm{x}$ & $\mathrm{x}$ & $\mathrm{x}$ & & $\mathrm{x}$ & $\mathrm{x}$ & & & $\mathrm{x}$ \\
$\mathrm{Mg}$ & & & & & $\mathrm{x}$ & & & $\mathrm{x}$ & $\mathrm{x}$ & & & $\mathrm{x}$ \\
$\mathrm{Al}$ & $\mathrm{x}$ & $\mathrm{x}$ & $\mathrm{x}$ & $\mathrm{x}$ & $\mathrm{x}$ & $\mathrm{x}$ & & $\mathrm{x}$ & $\mathrm{x}$ & & & $\mathrm{x}$ \\
$\mathrm{Si}$ & $\mathrm{x}$ & $\mathrm{x}$ & $\mathrm{x}$ & $\mathrm{x}$ & $\mathrm{x}$ & $\mathrm{x}$ & & $\mathrm{x}$ & $\mathrm{x}$ & & & $\mathrm{x}$ \\
$\mathrm{Ti}$ & $\mathrm{x}$ & $\mathrm{x}$ & $\mathrm{x}$ & $\mathrm{x}$ & $\mathrm{x}$ & $\mathrm{x}$ & & $\mathrm{x}$ & $\mathrm{x}$ & & & $\mathrm{x}$ \\
$\mathrm{V}$ & $\mathrm{x}$ & $\mathrm{x}$ & $\mathrm{x}$ & $\mathrm{x}$ & $\mathrm{x}$ & $\mathrm{x}$ & & $\mathrm{x}$ & $\mathrm{x}$ & $\mathrm{x}$ & & $\mathrm{x}$ \\
$\mathrm{Cr}$ & & & & & $\mathrm{x}$ & & & $\mathrm{x}$ & $\mathrm{x}$ & $\mathrm{x}$ & & $\mathrm{x}$ \\
$\mathrm{Mn}$ & & & & & $\mathrm{x}$ & & & $\mathrm{x}$ & & & & \\
$\mathrm{Fe}$ & $\mathrm{x}$ & $\mathrm{x}$ & $\mathrm{x}$ & & $\mathrm{x}$ & & & $\mathrm{x}$ & $\mathrm{x}$ & $\mathrm{x}$ & & $\mathrm{x}$ \\
$\mathrm{Ni}$ & $\mathrm{x}$ & $\mathrm{x}$ & $\mathrm{x}$ & & $\mathrm{x}$ & $\mathrm{x}$ & $\mathrm{x}$ & $\mathrm{x}$ & $\mathrm{x}$ & $\mathrm{x}$ & & $\mathrm{x}$ \\
$\mathrm{Cu}$ & $\mathrm{x}$ & & $\mathrm{x}$ & & $\mathrm{x}$ & $\mathrm{x}$ & & $\mathrm{x}$ & $\mathrm{x}$ & $\mathrm{x}$ & $\mathrm{x}$ & $\mathrm{x}$ \\
$\mathrm{Zn}$ & & & & & $\mathrm{x}$ & & & $\mathrm{x}$ & $\mathrm{x}$ & $\mathrm{x}$ & & $\mathrm{x}$ \\
$\mathrm{Ga}$ & & $\mathrm{x}$ & $\mathrm{x}$ & & $\mathrm{x}$ & & & & & & & $\mathrm{x}$ \\
$\mathrm{Ge}$ & $\mathrm{x}$ & $\mathrm{x}$ & & & $\mathrm{x}$ & & & $\mathrm{x}$ & $\mathrm{x}$ & $\mathrm{x}$ & & $\mathrm{x}$ \\
$\mathrm{Se}$ & & & & & $\mathrm{x}$ & & & $\mathrm{x}$ & $\mathrm{x}$ & & & \\
$\mathrm{Zr}$ & $\mathrm{x}$ & $\mathrm{x}$ & $\mathrm{x}$ & $\mathrm{x}$ & $\mathrm{x}$ & & & $\mathrm{x}$ & $\mathrm{x}$ & $\mathrm{x}$ & $\mathrm{x}$ & $\mathrm{x}$ \\
$\mathrm{Nb}$ & $\mathrm{x}$ & $\mathrm{x}$ & $\mathrm{x}$ & $\mathrm{x}$ & $\mathrm{x}$ & & & $\mathrm{x}$ & $\mathrm{x}$ & $\mathrm{x}$ & $\mathrm{x}$ & $\mathrm{x}$ \\
$\mathrm{Mo}$ & $\mathrm{x}$ & $\mathrm{x}$ & $\mathrm{x}$ & $\mathrm{x}$ & $\mathrm{x}$ & $\mathrm{x}$ & $\mathrm{x}$ & $\mathrm{x}$ & $\mathrm{x}$ & $\mathrm{x}$ & $\mathrm{x}$ & $\mathrm{x}$ \\
$\mathrm{Ru}$ & $\mathrm{x}$ & $\mathrm{x}$ & & & $\mathrm{x}$ & & $\mathrm{x}$ & $\mathrm{x}$ & $\mathrm{x}$ & $\mathrm{x}$ & \\
$\mathrm{Rh}$ & $\mathrm{x}$ & $\mathrm{x}$ & & $\mathrm{x}$ & $\mathrm{x}$ & & & $\mathrm{x}$ & $\mathrm{x}$ & $\mathrm{x}$ & $\mathrm{x}$ & \\
$\mathrm{Pd}$ & $\mathrm{x}$ & & & $\mathrm{x}$ & $\mathrm{x}$ & & & $\mathrm{x}$ & $\mathrm{x}$ & $\mathrm{x}$ & $\mathrm{x}$ & $\mathrm{x}$ \\
$\mathrm{Ag}$ & $\mathrm{x}$ & $\mathrm{x}$ & $\mathrm{x}$ & $\mathrm{x}$ & $\mathrm{x}$ & $\mathrm{x}$ & $\mathrm{x}$ & $\mathrm{x}$ & $\mathrm{x}$ & $\mathrm{x}$ & $\mathrm{x}$ & $\mathrm{x}$ \\
$\mathrm{Cd}$ & & & & & $\mathrm{x}$ & & & $\mathrm{x}$ & $\mathrm{x}$ & $\mathrm{x}$ & & $\mathrm{x}$ \\
\hline
\end{tabular}


Table 44: Ion-target combinations for which energy dependencies of the reflection coefficients have been calculated.

\begin{tabular}{|r|c|c|c|c|c|c|c|c|c|c|c|c|}
\hline ion & $\mathrm{H}$ & $\mathrm{D}$ & $\mathrm{T}$ & ${ }^{3} \mathrm{He}$ & ${ }^{4} \mathrm{He}$ & $\mathrm{N}$ & $\mathrm{O}$ & $\mathrm{Ne}$ & $\mathrm{Ar}$ & $\mathrm{Kr}$ & $\mathrm{Xe}$ & $\mathrm{selfb}$ \\
\hline target & & & & & & & & & & & & \\
\hline $\mathrm{In}$ & $\mathrm{x}$ & & & & $\mathrm{x}$ & & & $\mathrm{x}$ & & $\mathrm{x}$ & & $\mathrm{x}$ \\
$\mathrm{Sn}$ & $\mathrm{x}$ & $\mathrm{x}$ & & $\mathrm{x}$ & $\mathrm{x}$ & & & $\mathrm{x}$ & $\mathrm{x}$ & $\mathrm{x}$ & $\mathrm{x}$ & $\mathrm{x}$ \\
$\mathrm{Sb}$ & & & & & $\mathrm{x}$ & & & $\mathrm{x}$ & & $\mathrm{x}$ & & \\
$\mathrm{Te}$ & & & & & $\mathrm{x}$ & & & $\mathrm{x}$ & $\mathrm{x}$ & & & \\
$\mathrm{Tm}$ & & & & & & & & & & $\mathrm{x}$ & & \\
$\mathrm{Tm}$ & $\mathrm{x}$ & & & & $\mathrm{x}$ & & & & $\mathrm{x}$ & & & \\
$\mathrm{Hf}$ & $\mathrm{x}$ & & & & $\mathrm{x}$ & & & & $\mathrm{x}$ & & & \\
$\mathrm{Ta}$ & $\mathrm{x}$ & $\mathrm{x}$ & & $\mathrm{x}$ & $\mathrm{x}$ & & & $\mathrm{x}$ & $\mathrm{x}$ & $\mathrm{x}$ & $\mathrm{x}$ & $\mathrm{x}$ \\
$\mathrm{W}$ & $\mathrm{x}$ & $\mathrm{x}$ & $\mathrm{x}$ & $\mathrm{x}$ & $\mathrm{x}$ & $\mathrm{x}$ & & $\mathrm{x}$ & $\mathrm{x}$ & $\mathrm{x}$ & $\mathrm{x}$ & $\mathrm{x}$ \\
$\mathrm{Re}$ & $\mathrm{x}$ & $\mathrm{x}$ & & & $\mathrm{x}$ & & & $\mathrm{x}$ & $\mathrm{x}$ & $\mathrm{x}$ & $\mathrm{x}$ & \\
$\mathrm{Os}$ & $\mathrm{x}$ & & & $\mathrm{x}$ & $\mathrm{x}$ & & & $\mathrm{x}$ & $\mathrm{x}$ & $\mathrm{x}$ & $\mathrm{x}$ & \\
$\mathrm{Ir}$ & $\mathrm{x}$ & & & $\mathrm{x}$ & $\mathrm{x}$ & & & $\mathrm{x}$ & $\mathrm{x}$ & $\mathrm{x}$ & $\mathrm{x}$ & \\
$\mathrm{Pt}$ & $\mathrm{x}$ & & & $\mathrm{x}$ & $\mathrm{x}$ & & $\mathrm{x}$ & $\mathrm{x}$ & $\mathrm{x}$ & $\mathrm{x}$ & $\mathrm{x}$ & $\mathrm{x}$ \\
$\mathrm{Au}$ & $\mathrm{x}$ & $\mathrm{x}$ & $\mathrm{x}$ & $\mathrm{x}$ & $\mathrm{x}$ & $\mathrm{x}$ & $\mathrm{x}$ & $\mathrm{x}$ & $\mathrm{x}$ & $\mathrm{x}$ & $\mathrm{x}$ & $\mathrm{x}$ \\
$\mathrm{Hg}$ & & & & & & & & & & $\mathrm{x}$ & & \\
$\mathrm{Tl}$ & & & & & & & & & & $\mathrm{x}$ & & \\
$\mathrm{Pb}$ & $\mathrm{x}$ & & & & $\mathrm{x}$ & & & $\mathrm{x}$ & $\mathrm{x}$ & $\mathrm{x}$ & $\mathrm{x}$ & $\mathrm{x}$ \\
$\mathrm{Bi}$ & & & & & & & & $\mathrm{x}$ & $\mathrm{x}$ & & \\
$\mathrm{Th}$ & & & & & $\mathrm{x}$ & & & $\mathrm{x}$ & $\mathrm{x}$ & $\mathrm{x}$ & $\mathrm{x}$ & \\
$\mathrm{U}$ & $\mathrm{x}$ & $\mathrm{x}$ & & & & & & & & & \\
\hline
\end{tabular}


Table 45: Ion-target combinations for which angular dependencies of the reflection coefficients have been calculated.

\begin{tabular}{|r|c|c|c|c|c|c|c|c|c|c|c|}
\hline ion & $\mathrm{H}$ & $\mathrm{D}$ & $\mathrm{T}$ & ${ }^{3} \mathrm{He}$ & ${ }^{4} \mathrm{He}$ & $\mathrm{N}$ & $\mathrm{Ne}$ & $\mathrm{Ar}$ & $\mathrm{Kr}$ & $\mathrm{Xe}$ & $\mathrm{selfb}$ \\
\hline target & & & & & & & & & & & \\
\hline $\mathrm{Be}$ & $\mathrm{x}$ & $\mathrm{x}$ & $\mathrm{x}$ & & $\mathrm{x}$ & $\mathrm{x}$ & $\mathrm{x}$ & $\mathrm{x}$ & & & $\mathrm{x}$ \\
$\mathrm{B}$ & & $\mathrm{x}$ & & & & & & & & & $\mathrm{x}$ \\
$\mathrm{C}$ & $\mathrm{x}$ & $\mathrm{x}$ & $\mathrm{x}$ & & $\mathrm{x}$ & $\mathrm{x}$ & $\mathrm{x}$ & $\mathrm{x}$ & & & $\mathrm{x}$ \\
$\mathrm{Al}$ & $\mathrm{x}$ & & & & & & & $\mathrm{x}$ & & & \\
$\mathrm{Si}$ & & & & & $\mathrm{x}$ & & & $\mathrm{x}$ & & & $\mathrm{x}$ \\
$\mathrm{Ti}$ & & & & & $\mathrm{x}$ & & $\mathrm{x}$ & $\mathrm{x}$ & & & \\
$\mathrm{V}$ & $\mathrm{x}$ & $\mathrm{x}$ & $\mathrm{x}$ & & $\mathrm{x}$ & & & & & & \\
$\mathrm{Fe}$ & $\mathrm{x}$ & & & & & & & & & & \\
$\mathrm{Ni}$ & $\mathrm{x}$ & $\mathrm{x}$ & & $\mathrm{x}$ & $\mathrm{x}$ & & $\mathrm{x}$ & $\mathrm{x}$ & & & $\mathrm{x}$ \\
$\mathrm{Cu}$ & $\mathrm{x}$ & & & & $\mathrm{x}$ & & $\mathrm{x}$ & $\mathrm{x}$ & $\mathrm{x}$ & $\mathrm{x}$ & $\mathrm{x}$ \\
$\mathrm{Ga}$ & & & & & & & & & & & $\mathrm{x}$ \\
$\mathrm{Zr}$ & & & & & & & $\mathrm{x}$ & & & \\
$\mathrm{Nb}$ & $\mathrm{x}$ & $\mathrm{x}$ & & & $\mathrm{x}$ & & & & & & $\mathrm{x}$ \\
$\mathrm{Mo}$ & $\mathrm{x}$ & $\mathrm{x}$ & $\mathrm{x}$ & $\mathrm{x}$ & $\mathrm{x}$ & & & $\mathrm{x}$ & & $\mathrm{x}$ & $\mathrm{x}$ \\
$\mathrm{Pd}$ & & & & & & & & $\mathrm{x}$ & & & \\
$\mathrm{Ag}$ & & $\mathrm{x}$ & & & & & $\mathrm{x}$ & $\mathrm{x}$ & $\mathrm{x}$ & & \\
$\mathrm{In}$ & $\mathrm{x}$ & & & & & & & & & & $\mathrm{x}$ \\
$\mathrm{Ta}$ & & & & & $\mathrm{x}$ & & $\mathrm{x}$ & $\mathrm{x}$ & $\mathrm{x}$ & & \\
$\mathrm{W}$ & $\mathrm{x}$ & $\mathrm{x}$ & $\mathrm{x}$ & & $\mathrm{x}$ & $\mathrm{x}$ & $\mathrm{x}$ & $\mathrm{x}$ & & $\mathrm{x}$ & $\mathrm{x}$ \\
$\mathrm{Au}$ & $\mathrm{x}$ & $\mathrm{x}$ & & & $\mathrm{x}$ & & $\mathrm{x}$ & $\mathrm{x}$ & & $\mathrm{x}$ & \\
$\mathrm{Hg}$ & & & & & & & & $\mathrm{x}$ & & \\
$\mathrm{U}$ & $\mathrm{x}$ & & & & & & & $\mathrm{x}$ & & \\
\hline
\end{tabular}

